\title{
Evoluation and technological advances in ablation of complex atrial and ventricular arrhythmias
}

Citation for published version (APA):

Wolf, M. (2020). Evoluation and technological advances in ablation of complex atrial and ventricular arrhythmias. [Doctoral Thesis, Maastricht University]. Maastricht University. https://doi.org/10.26481/dis.20201002mw

Document status and date:

Published: 01/01/2020

DOI:

10.26481/dis.20201002mw

Document Version:

Publisher's PDF, also known as Version of record

\section{Please check the document version of this publication:}

- A submitted manuscript is the version of the article upon submission and before peer-review. There can be important differences between the submitted version and the official published version of record. People interested in the research are advised to contact the author for the final version of the publication, or visit the DOI to the publisher's website.

- The final author version and the galley proof are versions of the publication after peer review.

- The final published version features the final layout of the paper including the volume, issue and page numbers.

Link to publication

\footnotetext{
General rights rights.

- You may freely distribute the URL identifying the publication in the public portal. please follow below link for the End User Agreement:

www.umlib.nl/taverne-license

Take down policy

If you believe that this document breaches copyright please contact us at:

repository@maastrichtuniversity.nl

providing details and we will investigate your claim.
}

Copyright and moral rights for the publications made accessible in the public portal are retained by the authors and/or other copyright owners and it is a condition of accessing publications that users recognise and abide by the legal requirements associated with these

- Users may download and print one copy of any publication from the public portal for the purpose of private study or research.

- You may not further distribute the material or use it for any profit-making activity or commercial gain

If the publication is distributed under the terms of Article $25 \mathrm{fa}$ of the Dutch Copyright Act, indicated by the "Taverne" license above, 


\section{EVOLUTION AND TECHNOLOGICAL ADVANGES IN ABLATION OF COMPLEX ATRIAL AND VENTRICULAR ARRHYTHMIAS}

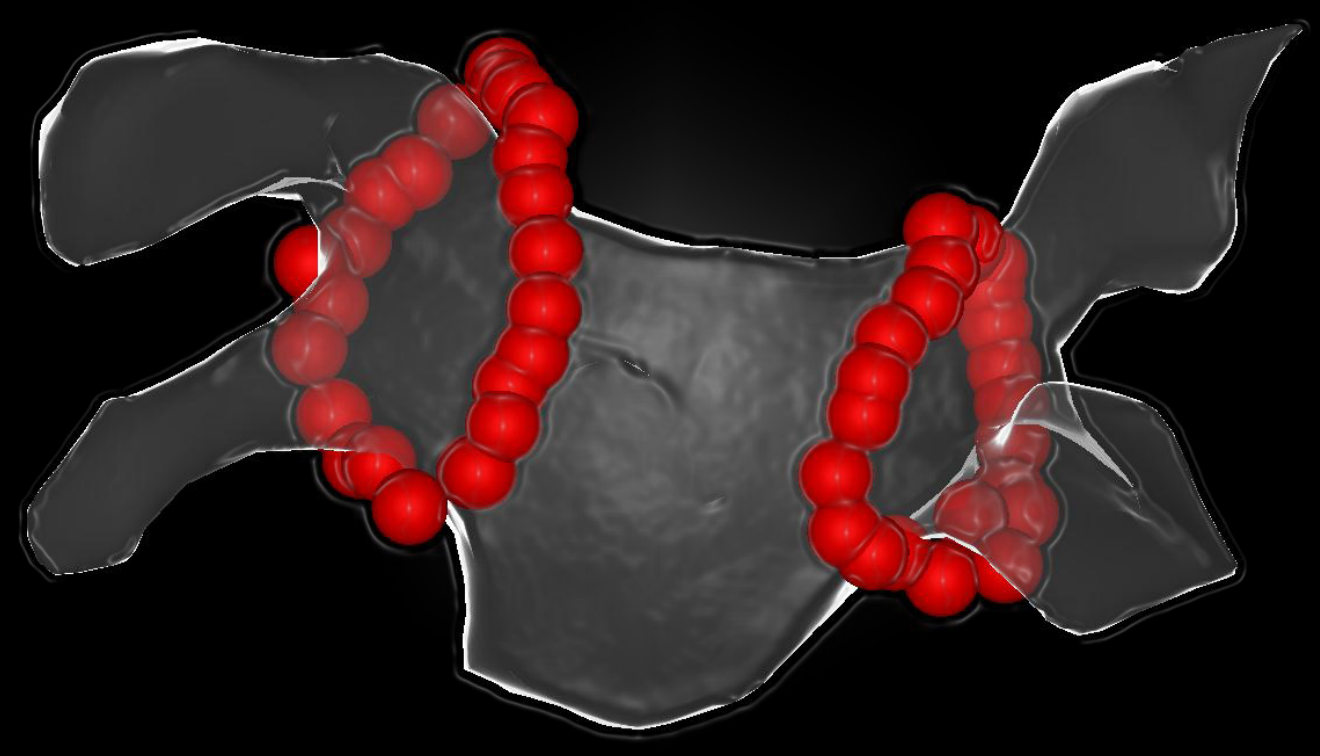

MICHAEL WOLF 

Evolution and technological advances in ablation of complex atrial and ventricular arrhythmias.

Michael Wolf

2020 



\section{Evolution and technological advances in ablation of complex atrial and ventricular arrhythmias.}

\section{Proefschrift}

ter verkrijging van de graad van doctor aan de Universiteit Maastricht, op gezag van de Rector Magnificus,

Prof. dr. Rianne M. Letschert,

volgens het besluit van het College van Decanen, in het openbaar te verdedigen

op vrijdag 2 oktober 2020 om 10:00 uur

door

\section{Michael Wolf}

geboren op 13 januari 1983 te Lier 


\section{Promotores}

Prof. dr. H.J.G.M. Crijns

Prof. dr. M. Duytschaever (AZ Sint-Jan, Brugge, België)

\section{Beoordelingscommissie}

Prof. dr. U. Schotten (Voorzitter)

Prof. dr. H.P. Brunner-La Rocca

Prof. dr. L.R. Dekker (TU Eindhoven/Catharina Ziekenhuis Eindhoven)

Prof. dr. L. Pison (Ziekenhuis Oost-Limburg, Genk, België)

Prof. dr. P.G.A.Volders 
To my incredible family

To all the extraordinary friends and colleagues I was lucky to meet all over the world 



\section{TABLE OF CONTENTS}

Chapter 1 General introduction

PART I

ABLATION OF ATRIAL FIBRILLATION AND ATRIAL TACHYCARDIA 23

PART IA

Conventional contact force-guided pulmonary vein isolation

Chapter 2 Prospective comparison between contact force vs. non-contact force guided 25 pulmonary vein isolation after the learning period with contact force catheters.

PART IB A protocol for pulmonary vein isolation with contiguous and optimized 45 radiofrequency applications: "CLOSE-PVI"

Chapter 3 Evaluation of optimal ablation parameters for radiofrequency PVI: inter-tag distance and ablation index.

Chapter 4 Comparison of CLOSE-PVI to conventional contact force-guided PVI.

Chapter 5 Evaluation of clinical outcome of CLOSE-PVI in a larger patient cohort.

Chapter 6 Safety evaluation of CLOSE-PVI concerning oesophageal and mediastinal lesions. 109

Chapter 7 Evaluation of durability of PV isolation in CLOSE-PVI.

Chapter 8 Long-term impact of catheter ablation on arrhythmia burden in low-risk patients 145 with paroxysmal atrial fibrillation: the CLOSE to CURE study

\section{PART IC Optimizing left atrial linear ablation}

Chapter 9 Contiguous and optimized radiofrequency applications for linear lesions.

PART ID Mapping of drivers in persistent atrial fibrillation 187

Chapter 10 Direct contact activation mapping in persistent atrial fibrillation

PART II ABLATION OF VENTRICULAR TACHYCARDIA.

Chapter 11 Evaluation of long-term outcomes of substrate modification for post-myocardial 209 infarction ventricular tachycardia

PART III GENERAL DISCUSSION AND SUMMARY.

Chapter 12 General discussion. 
Summary - Samenvatting

Valorization

Aknowledgement - Dankwoord

Curriculum vitae - List of publications 



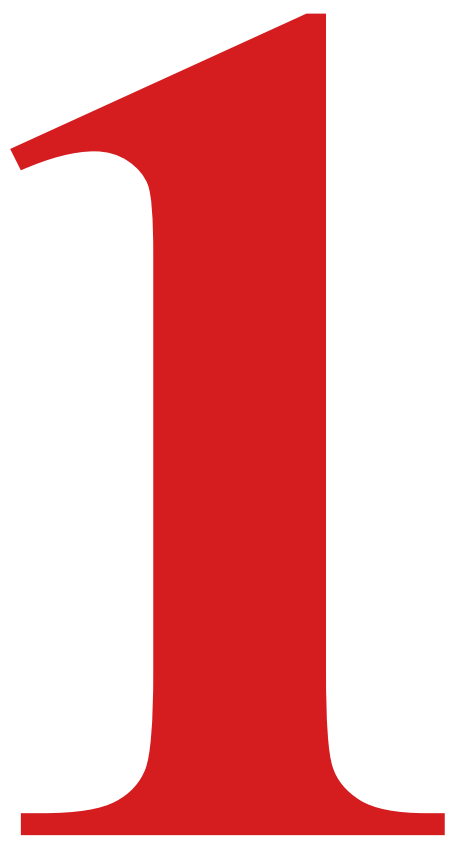




\section{Chapter 1}

General introduction 


\section{Background}

The interventional treatment of complex arrhythmias continues to grow in importance within our health care system.

\section{ATRIAL FIBRILLATION AND ATRIAL TACHYCARDIA}

Par excellence, atrial fibrillation (AF) is a serious public health problem because of its increasing incidence and prevalence in the aging population ${ }^{1}$ and its association with elevated risks of cardiovascular events and death. ${ }^{2-7}$ Since the initial description of triggers in the pulmonary veins that initiate paroxysmal $\mathrm{AF}$, catheter ablation of $\mathrm{AF}$ has become a common treatment to prevent recurrent AF. This is primarily achieved through isolation of the pulmonary veins, probably requiring complete isolation for full effectiveness. ${ }^{8}$ Over the past 20 years, the development of AF ablation technologies has been a constant effort to improve the pulmonary vein isolation (PVI) technique. The goal has been to improve patient outcomes, reducing the need for repeat procedures, to reduce procedure times and to increase the safety of the procedure. However, despite these advances and two decades of experience, long-term procedural success rates at curing AF are still suboptimal and may require repeat procedures or hybrid therapy with antiarrhythmic drugs to improve outcome. The reason for these suboptimal results is twofold; on one side the pathophysiological mechanism of AF is still not completely elucidated, and on the other side the ablation tools and techniques still fail to deliver durable and transmural ablation lesions. Even though fatal complications after AF ablation are rare, 2 $-3 \%$ will experience life threatening but usually manageable complications. ${ }^{9-11}$

\section{Pulmonary vein isolation}

The cornerstone of ablation of AF is to achieve durable electrical isolation of the pulmonary veins. ${ }^{12}$ Radiofrequency (RF) ablation, the most established ablation technique, aims to achieve PVI by applying circumferential contiguous and transmural point-by-point ablation lesions. ${ }^{13}$ Despite achieving nearly always acute procedural PVI, early and late PV reconnections are frequent and result in AF recurrences and the need for repeat ablation procedures. Indeed, with standard irrigated tip catheters only $65 \%$ of PV pairs remain isolated after adenosine-challenge during the index procedure $^{14}$; only $72 \%$ of PV circles remain isolated at three months ${ }^{15}$ and the reported 1-year freedom of AF after a single procedure amounts $66 \%{ }^{16}$

\section{Contact force-sensing catheters}

Tip-tissue contact has shown to be an important factor in lesion creation and firm and stable contact force (CF) a prerequisite for durable, transmural RF lesions. ${ }^{17}$ As estimation of $\mathrm{CF}$ through tactile feedback or indirect measurements has been shown to be a poor predictor of actual tip-tissue contact, the utilization of catheters with embedded CF measuring sensors has led to improved outcomes and 
fewer repeat procedures. ${ }^{18-20}$ Both acute adenosine-proof PV isolation and one-year freedom of AF increased to $85 \% .^{14,21-22}$

PART IA of this thesis investigated if the routine use of CF sensing catheters led to a better estimation of good catheter tip-tissue contact and improved PVI results when the experienced operator is blinded to the $\mathrm{CF}$.

A protocol for pulmonary vein isolation with contiguous and optimized radiofrequency applications (PART IB)

In addition to $\mathrm{CF}$, lesions depth and quality are determined by the ablation power and application time. The combination of these 3 factors in a logarithmic function (the Force-Power-Time Index or Ablation Index (AI)) was found to accurately describe the lesion depth. ${ }^{23-24}$ Therefore minimal AI targets would be more reliable is predicting lesion transmurality and lesion durablility. Other data showed that lesion contiguity was another important determinant in obtaining durable PVI. ${ }^{25-26}$

PART IB of this thesis consists of the development of a protocol aiming to improve the point-by-point RF PVI technique by combining strict criteria for both RF application contiguity and quality. In Chapter 3 we analysed optimal cut-off criteria for maximum distance between neighbouring RF applications and minimum AI targets associated with durable PV isolation.

\section{Acute $P V$ isolation and freedom of atrial tachyarrhythmia outcome results in paroxysmal AF}

The analysed criteria for inter-tag distance and AI were incorporated in a new PVI protocol, aiming at delivering stable, relatively high-power, short-duration RF applications relatively close to the left atrial-PV junction. The acute and mid-term clinical results of this 'CLOSE' (Contiguous Lesion Optimized pre-Specified Encircling) protocol were prospectively analysed in Chapter 4 and 5.

\section{Safety evaluation of the 'CLOSE' protocol}

Although rare, atrio-oesophageal fistula (AEF) is one of the most feared complications of PVI because of its high fatality rate. Whereas the incidence of AEF is low $(0.02$ to $0.11 \%)$, ${ }^{12}$ there is a high (2 to $30 \%$ ) incidence of subclinical oesophageal or peri-oesophageal injury on endoscopy performed early after PVI procedures. ${ }^{27-38}$ It was established that the only risk factor associated with the occurrence of oesophageal injury or ulcer is intra-oesophageal temperature rise (ITR ${ }^{27}$ and that an oesophageal ulcer is a necessary precursor for $\mathrm{AEF} .^{27}$

In Chapter 6, we assessed the incidence of oesophageal injury (and potential risk of AEF) by performing echo-endoscopy in those patients revealing ITR during PVI according 'CLOSE' protocol.

\section{Long-term efficacy outcomes of CLOSE-PVI for paroxysmal AF}

Even though acute isolation of all PVs is generally always achieved by RF point-by-point PVI, the likelihood of finding four isolated veins at repeat procedures for AF recurrence has been very low 
(varying from $41 \%$ to as low as $0 \%$ ). ${ }^{39-53}$ In Chapter 7 we evaluated the durability of PV isolation in patients undergoing repeat ablation for AF recurrence after undergoing an index CLOSE-PVI procedure. AF burden is a new and clinically relevant endpoint shown to be related to symptoms, heart failure and stroke. ${ }^{54-58}$ In Chapter 8 we studied the impact of CLOSE-PVI on AF burden before and during the first two years after CLOSE-PVI using insertable cardiac monitors.

\section{Mapping and ablation of the left atrial substrate}

In more complex atrial arrhythmias, such as (long-standing) persistent atrial fibrillation and atrial tachycardia, the pathophysiological mechanism involves the left atrial substrate.

Linear ablation lesions are often applied in ablation of macro-re-entrant atrial tachycardia to interrupt the re-entrant circuit, but also in ablation of (long-standing) persistent AF in order to replicate various aspects of the surgical MAZE procedure because of the high recurrence rate observed in these patients when only PVI is performed. ${ }^{12}$ However, performing linear ablation is challenging. Indeed, bidirectional block across LA lines should be the ablation endpoint (HRS/EHRA/ECAS recommendation ${ }^{12}$ ) but this is achieved in a limited amount of patients ${ }^{59-63}$ and incomplete electrical block can have pro-arrhythmic consequences and cause atrial tachycardia recurrence..$^{3-4}$ On the other hand, increased success in completing linear block is often associated with an increased complication rate from higher ablation power and a higher risk of steam pop, perforation, and tamponade. ${ }^{64}$ In Chapter 9 we focused on the optimization of left atrial linear ablation by applying the same elements of the CLOSE protocol, i.e. stable applications respecting strict criteria for maximal inter-application distance and minimal AI values.

During persistent AF, multiple atrial wavelets and localized (focal or re-entrant) sources have been reported to contribute to the maintenance of $A F{ }^{65-67}$ In the last few years, there has been progress in mapping AF sources and mechanisms. Recently, AF propagation maps based on direct contact mapping using a multi-electrode basket catheter and dedicated software (CARTOFINDER ${ }^{\mathrm{TM}}$, Biosense Webster Inc) were able to reliably and reproducibly identify repetitive atrial activation patterns (RAAPS) in the majority of persistent AF patients. ${ }^{68-72}$ Nevertheless, because the basket catheter has a long inter-electrode distance and therefore can't reach stable contact with whole atrial surface, some regions and therefore some repetitive activation patterns were probably missed. In Chapter 10 we sought to evaluate a new mapping method to identify and characterize these RAAPS during on-going AF based upon the automated annotation of unipolar electrograms (Cartofinder software) directly obtained by sequential recordings with a regional high-density contact mapping catheter (PentaRay ${ }^{\mathrm{TM}}$, Biosense Webster Inc) covering the entire bi-atrial endocardial surface. 


\section{VENTRICULAR TACHYCARDIA}

The second part of this thesis focuses on the evolution and technical advances in ablation of ventricular tachycardia, more specifically in ischemic cardiomyopathy patients.

Catheter ablation for sustained monomorphic ventricular tachycardia (VT) after myocardial infarction (MI) has emerged as an effective tool for the control of VT recurrence. ${ }^{72}$ Recently few studies have reported long-term follow-up data of VT ablation in ischemic cardiomyopathy (ICMP) patients, guided predominantly by activation and entrainment mapping of inducible and hemodynamically tolerated VTs. ${ }^{73-74}$ However, alternative strategies predominantly based on substrate modification have gained prominence. These strategies not only have the advantage of identifying targets for ablation in patients in whom VT is not inducible or not tolerated, but they may also provide a more comprehensive treatment of the substrate for present but also future VT, as they are not limited to inducible or "clinical" types of VT. Long-term results of substrate modification strategies have not been reported. ${ }^{75-77}$

In Chapter 11 we reported long-term outcomes after single and multiple ablation procedures with elimination of local abnormal ventricular activities (LAVA) for VT ablation in post-MI patients. We also investigated the impact of strategy variants to perform and achieve LAVA elimination, more specifically the added value of multi-electrode mapping catheters and of real-time integration of preprocedural imaging with delayed enhancement-magnetic resonance imaging or multi-detector computed tomography in post-MI VT ablation. 


\section{References}

1. Go AS, et al. Prevalence of diagnosed atrial fibrillation in adults: national implications for rhythm management and stroke prevention: the AnTicoagulation and Risk Factors in Atrial Fibrillation (ATRIA) Study. JAMA. 2001; 285:2370-2375.

2. Wolf PA, et al. Atrial fibrillation as an independent risk factor for stroke: the Framingham Study.Stroke. 1991; 22:983-988.

3. Wang TJ, et al. Temporal relations of atrial fibrillation and congestive heart failure and their joint influence on mortality: the Framingham Heart Study.Circulation. 2003; 107:2920-2925.

4. Benjamin EJ, et al. Impact of atrial fibrillation on the risk of death: the Framingham Heart Study.Circulation. 1998; 98:946-952.

5. Chen LY, et al. Atrial fibrillation and the risk of sudden cardiac death: the Atherosclerosis Risk in Communities Study and Cardiovascular Health Study.JAMA Intern Med. 2013; 173:29-35.

6. Soliman EZ, et al. Atrial fibrillation and the risk of myocardial infarction. JAMA Intern Med. 2014; 174:107-114.

7. Soliman EZ, et al. Atrial fibrillation and risk of ST-segment-elevation versus non-ST-segmentelevation myocardial infarction: the Atherosclerosis Risk in Communities (ARIC) study. Circulation. 2015; 131:1843-1850.

8. Kuck KH, et al - AFNET 1 Investigators. Impact of Complete Versus Incomplete Circumferential Lines Around the Pulmonary Veins During Catheter Ablation of Paroxysmal Atrial Fibrillation: Results From the Gap-Atrial Fibrillation-German Atrial Fibrillation Competence Network 1 Trial. Circ Arrhythm Electrophysiol. 2016;9:e003337.

9. Cappato R, et al. Updated worldwide survey on the methods, efficacy, and safety of catheter ablation for human atrial fibrilla- tion. Circ Arrhythm Electrophysiol. 2010;3:32-38.

10. Dagres N, et al. Complications of atrial fibrillation ablation in a high-volume center in 1,000 procedures: still cause for concern? J Cardiovasc Electrophysiol. 2009; 20:1014-1019.

11. Gupta A, et al. Complications of catheter ablation of atrial fibrillation: a systematic review. Circ Arrhythm Electrophysiol. 2013; 6:1082-1088.

12. Calkins H, et al. $2017 \mathrm{HRS} / \mathrm{EHRA} / \mathrm{ECAS} / \mathrm{APHRS} / \mathrm{SOLAECE}$ expert consensus statement on catheter and surgical ablation of atrial fibrillation: Executive summary. Europace. 2018; 20:157208.

13. Ouyang F, et al. Complete isolation of left atrium surrounding the pulmonary veins: new insights from the double-Lasso technique in paroxysmal atrial fibrillation. Circulation. 2004;110:20902096.

14. Andrade JG, et al. Pulmonary vein isolation using "contact force" ablation: the effect on dormant conduction and long-term freedom from recurrent atrial fibrillation - a prospective study. Heart Rhythm. 2014; 11:1919-1924.

15. Neuzil $\mathrm{P}$, et al. Electrical reconnection after pulmonary vein isolation is contingent on contact force during initial treatment: results from the EFFICAS I study. Circ Arrhythm Electrophysiol. 2013; 6:327-333.

16. Wilber DJ, et al. Thermo- Cool AF Trial Investigators. Comparison of antiarrhythmic drug therapy and radiofrequency catheter ablation in patients with paroxysmal atrial fibrillation: a randomized controlled trial. JAMA. 2010; 303:333-340.

17. Haines DE. Determinants of lesion size during radiofrequency catheter ablation: the role of electrode-tissue contact pressure and duration of energy delivery. J Cardiovasc Electrophysiol. 1991; 2:509-515.

18. Kuck KH, et al. "A novel radiofrequency ablation catheter using contact force sensing: Toccata study." Heart Rhythm. 2012; 9:18-23. 
19. Reddy $\mathrm{V}$, et al. The relationship between contact force and clinical outcome during radiofrequency catheter ablation of atrial fibrillation in the TOCCATA study. Heart Rhythm. 2012; 9:1789-1795.

20. Kautzner J, et al. EFFICAS II: Optimization of catheter contact force improves outcome of pulmonary vein isolation for paroxysmal atrial fibrillation. Europace. 17, 1229-1235.

21. Marijon E, et al. Real-time contact force sensing for pulmonary vein isolation in the setting of parox- ysmal atrial fibrillation: procedural and 1-year results. J Cardiovasc Electrophysiol. 2014; 25:130-137.

22. Natale A, et al. Paroxysmal AF catheter ablation with a contact force sensing catheter: results of the prospective, multicenter SMART-AF trial. J Am Coll Cardiol. 2014; 64:647-656.

23. Nakagawa H, et al. The Role Of Contact Force In Atrial Fibrillation Ablation. J Atr Fibrillation 2014; 7:1027.

24. Nakagawa $\mathrm{H}$, et al. Prospective study to test the ability to create RF lesions at predicted depths of 3, 5, 7 and $9 \mathrm{~mm}$ using formula incorporating contact force, radiofrequency power and application time (force-power-time index) in the beating canine heart. Heart Rhythm. 2013; 10:S481.

25. Park CI, et al. Mechanisms of pulmonary vein reconnection after radiofrequency ablation of atrial fibrillation: the deterministic role of contact force and interlesion distance. J Cardiovasc Electrophysiol. 2014; 1:1-8.

26. Miller MA, et al. Acute electrical isolation is a necessary but insufficient endpoint for achieving durable PV isolation: the importance of closing the visual gap. Europace. 2012; 14:653-660.

27. Halbfass P, et al. Progression From Esophageal Thermal Asymptomatic Lesion to Perforation Complicating Atrial Fibrillation Ablation: A Single-Center Registry. Circ Arrhythm Electrophysiol. 2017; 10(8).pii:e005233.

28. Yarlagadda B, et al. Temporal Relationships between Esophageal Injury Type and Progression in Patients Undergoing Atrial Fibrillation Catheter Ablation. Heart Rhythm. 2018; pii:S15475271(18)31010-5.

29. Martinek $\mathrm{M}$, et al. Identification of a high-risk population for esophageal injury during radiofrequency catheterablation of atrial fibrillation: procedural and anatomical considerations. Heart Rhythm. 2010; 7:1224-1230.

30. Blockhaus C, et al. Low Incidence of Esophageal Lesions After Pulmonary Vein Isolation Using Contact-Force Sensing Catheter Without Esophageal Temperature Probe. Int Heart J 2017; 58:880-884.

31. Halbfass $\mathrm{P}$, et al. Impact of surround flow catheter tip irrigation in contact force ablation on the incidence of asymptomatic oesophageal lesions after atrial fibrillation ablation: a prospective comparative study. Europace. 2017; 19:1116-1122.

32. Di Biase L, et al. Esophageal capsule endoscopy after radiofrequency catheter ablation for atrial fibrillation: documented higher risk of luminal esophageal damage with general anesthesia as compared with conscious sedation. Circ Arrhythm Electrophysiol. 2009; 2:108-112.

33. Müller P, et al. Higher incidence of esophageal lesions after ablation of atrial fibrillation related to the use of esophageal temperature probes. Heart Rhythm. 2015; 12:1464-1469.

34. Halbfass $\mathrm{P}$, et al. Incidence of asymptomatic oesophageal lesions after atrial fibrillation ablation using an oesophageal temperature probe with insulated thermocouples: a comparative controlled study. Europace. 2016; 19:385-391.

35. Contreras-Valdes FM, et al. Severity of esophageal injury predicts time to healing after radiofrequency catheter ablation for atrial fibrillation. Heart Rhythm 2011; 8:1862-1868.

36. Knopp $\mathrm{H}$, et al. Incidental and ablation-induced findings during upper gastrointestinal endoscopy in patients after ablation of atrial fibrillation: a retrospective study of 425 patients. Heart Rhythm. 2014; 11:574-578. 
37. Fürnkranz A, et al. Luminal esophageal temperature predicts esophageal lesions after secondgeneration cryoballoon pulmonary vein isolation. Heart Rhythm 2013; 10:789-793.

38. Fürnkranz A, et al. Reduced incidence of esophageal lesions by luminal esophageal temperatureguided second-generation cryoballoon ablation. Heart Rhythm 2015; 12:268-274.

39. Nanthakumar K, et al. Resumption of electrical conduction in previously isolated pulmonary veins: rationale for a different strategy? Circulation. 2004; 109:1226-1229.

40. Ouyang F, et al. Recovered pulmonary vein conduction as a dominant factor for recurrent atrial tachyarrhythmias after complete circular isolation of the pulmonary veins: lessons from double Lasso technique. Circulation. 2005; 111:127-135.

41. Bordignon S, et al. High rate of durable pulmonary vein isolation after second-generation cryoballoon ablation: analysis of repeat procedures. Europace 2015; 17:725-731.

42. Callans DJ, et al. Efficacy of repeat pulmonary vein isolation procedures in patients with recurrent atrial fibrillation. J Cardiovasc Electrophysiol. 2004; 15:1050-1055.

43. Cappato R, et al. Prospective assessment of late conduction recurrence across radiofrequency lesions producing electrical disconnection at the pulmonary vein ostium in patients with atrial fibrillation. Circulation. 2003; 108:1599-1604.

44. Heeger $\mathrm{CH}$, et al. Once isolated, always isolated? Incidence and characteristics of pulmonary vein reconduction after second-generation cryoballoon-based pulmonary vein isolation. Circ Arrhythm Electrophysiol. 2015; 8:1088-1094.

45. Jiang RH, et al. Incidence of pulmonary vein conduction recovery in patients without clinical recurrence after ablation of paroxysmal atrial fibrillation: mechanistic implications. Heart Rhythm. 2014; 11:969-976.

46. Kuck KH, et al., for the Gap-AF-AFNET 1 Investigators. Impact of complete versus incomplete circumferential lines around the pulmonary veins during catheter ablation of paroxysmal atrial fibrillation: results from the Gap-Atrial Fibrillation-German Atrial Fibrillation Competence Network 1 Trial. Circ Arrhythm Electrophysiol. 2016; 9:e003337.

47. Martins RP, et al. The second generation cryoballoon has improved durable isolation of left but not right pulmonary veins: new insights from a multicentre study. Europace. 2018; 20:1115-1121.

48. Ouyang F, et al. Long-term results of catheter ablation in paroxysmal atrial fibrillation: lessons from a 5-year follow-up. Circulation 2010; 122:2368-2377.

49. Verma A, et al. Response of atrial fibrillation to pulmonary vein antrum isolation is directly related to resumption and delay of pulmonary vein conduction. Circulation. 2005; 112:627-635.

50. Willems S, et al. Persistence of pulmonary vein isolation after robotic remote-navigated ablation for atrial fibrillation and its relation to clinical outcome. J Cardiovasc Electrophysiol. 2010; 21:1079-84.

51. Buist TJ, et al. Arrhythmia-free survival and pulmonary vein reconnection patterns after secondgeneration cryoballoon and contact-force radiofrequency pulmonary vein isolation. Clin Res Cardiol. 2018; 107:498-506.

52. Shah S, et al. Recurrent atrial fibrillation after initial long-term ablation success: electrophysiological findings and outcomes of repeat ablation procedures. Circ Arrhythm Electrophysiol. 2018; 11:e05785.

53. Baldinger $\mathrm{SH}$, et al. Recurrence of atrial arrhythmias despite persistent pulmonary vein isolation after catheter ablation for atrial fibrillation: a case series. J Am Coll Cardiol EP. 2016; 2:723-731.

54. Mantovan R, et al. Relationship of quality of life with procedural success of atrial fibrillation (AF) ablation and postablation AF burden: substudy of the STAR AF randomized trial. Can J Cardiol 2013; 29:1211-1217. 
55. Bjorkenheim A, et al. Patient-Reported Outcomes in Relation to Continuously Monitored Rhythm Before and During 2 Years After Atrial Fibrillation Ablation Using a Disease-Specific and a Generic Instrument. Journal of the American Heart Association 2018;7: pii: e008362.

56. Glotzer TV, et al The relationship between daily atrial tachyarrhythmia burden from implantable device diagnostics and stroke risk: the TRENDS study. Circ Arrhythm Electrophysiol. 2009; 2:474-480.

57. Go A, et al. Association of Burden of Atrial Fibrillation With Risk of Ischemic Stroke in Adults With Paroxysmal Atrial Fibrillation. JAMA Cardiology. 2018; 3:601.

58. Marrouche N, et al. Catheter Ablation for Atrial Fibrillation with Heart Failure. N Engl J Med. $2018 ; 378: 417-427$.

59. Jais $\mathrm{P}$, et al. Technique and results of linear ablation at the mitral isthmus. Circulation 2004; 110:2996-3002.

60. Knecht $\mathrm{S}$, et al. Left atrial linear lesions are required for successful treatment of persistent atrial fibrillation. Eur Heart J 2008; 29:2359-2366.

61. Matsuo S, et al. Completion of mitral isthmus ablation using a steerable sheath: Prospective randomized comparison with a nonsteerable sheath. J Cardiovasc Electrophysiol. 2011; 22:13311338.

62. Miyazaki S, et al. Preprocedural clinical parameters determining perimitral conduction time during mitral isthmus line ablation. Circ Arrhythm Electrophysiol 2011; 4:287-294.

63. Arbelo $\mathrm{E}$, et al. Benefit of left atrial roof linear ablation in paroxysmal atrial fibrillation: a prospective, randomized study. J Am Heart Assoc. 2014; 3:e00877.

64. Wong KC, et al. A review of mitral isthmus ablation. Indian Pacing Electrophysiol J. 2012; $12: 152-170$.

65. Konings KT, et al. High-density mapping of electrically induced atrial fibrillation in humans. Circulation. 1994; 89:1665-1680.

66. Jalife J, et al. Mother rotors and fibrillatory conduction: a mechanism of atrial fibrillation. Cardiovasc Res. 2002; 54:204-216.

67. Haissaguerre M, et al. Localized sources maintaining atrial fibrillation organized by prior ablation. Circulation. 2006; 113:616-625.

68. Daoud EG, et al. Identification of Repetitive Activation Patterns Using Novel Computational Analysis of Multielectrode Recordings During Atrial Fibrillation and Flutter in Humans. JACC Clin Electrophysiol. 2017; 3:207-216.

69. Honarbakhsh S, et al. A Novel Mapping System for Panoramic Mapping of the Left Atrium: Application to Detect and Characterize Localized Sources Maintaining Atrial Fibrillation. JACC Clin Electrophysiol. 2018; 4:124-134.

70. Honarbakhsh S, et al. Characterization of drivers maintaining atrial fibrillation: Correlation with markers of rapidity and organization on spectral analysis. Heart Rhythm. 2018; 15:1296-1303.

71. Verma A, et al. Characterization and significance of localized sources identified by a novel automated algorithm during mapping of human persistent atrial fibrillation. J Cardiovasc Electrophysiol. 2018; 29:1480-1488.

72. Sapp JL, et al Ventricular Tachycardia Ablation versus Escalation of Antiarrhythmic Drugs. N Engl J Med. 2016; 375:111-121.

73. Kumar S, et al. Long-term outcomes after catheter ablation of ventricular tachycardia in patients with and without structural heart disease. Heart Rhythm. 2016; 13:1957-1963.

74. Marchlinski FE, et al. Long-Term Success of Irrigated Radiofrequency Catheter Ablation of Sustained Ventricular Tachycardia: Post-Approval THERMOCOOL VT Trial. J Am Coll Cardiol. 2016; 67:674-683. 
75. Kumar S, et al. Substrate-Based Ablation Versus Ablation Guided by Activation and Entrainment Mapping for Ventricular Tachycardia: A Systematic Review and Meta-Analysis. J Cardiovasc Electrophysiol. 2016; 27:1437-1447.

76. Proietti R, et al. Substrate-guided ablation of haemodynamically tolerated and untolerated ventricular tachycardia in patients with structural heart disease: effect of cardiomyopathy type and acute success on long-term outcome. Europace. 2015; 17:461-467.

77. Silberbauer J, et al. Noninducibility and late potential abolition: a novel combined prognostic procedural end point for catheter ablation of postinfarction ventricular tachycardia. Circ Arrhythm Electrophysiol. 2014; 7:424-435. 


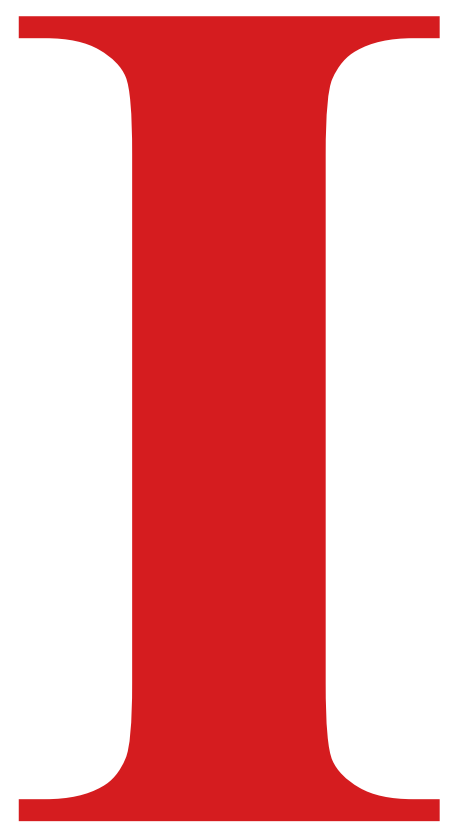




\section{Part I}

\section{ABLATION OF ATRIAL FIBRILLATION AND ATRIAL TACHYCARDIA}

\section{Part IA}

Conventional contact force-guided pulmonary vein isolation 


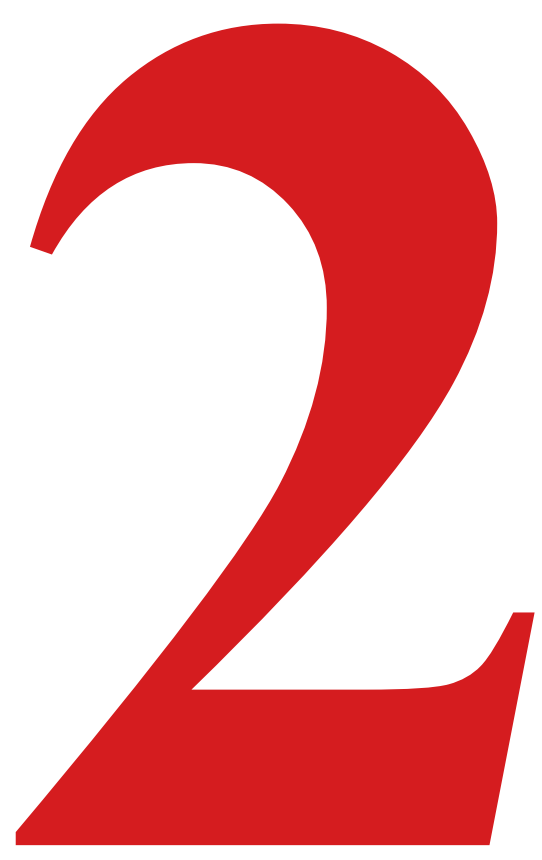




\section{Chapter 2}

\section{Prospective comparison between contact force vs. non-contact force guided pulmonary vein isolation after the learning period with contact force catheters}

Michael Wolf, Johan Saenen, Wim Bories, Hielko Miljoen, Sara Nullens, Christiaan Vrints, Andrea Sarkozy 


\begin{abstract}
Purpose: Use of online contact force (CF) measurement during circumferential pulmonary vein (PV) isolation (CPVI) for atrial fibrillation (AF) has demonstrated improvements in procedural parameters and mid-term clinical outcome. However it is unknown if experience gained with CF measuring catheters improves the efficacy of subsequent CPVI procedures performed without $\mathrm{CF}$ measurement.
\end{abstract}

Methods \& results: This prospective trial compared procedural results of CPVI performed without a $\mathrm{CF}$ measuring catheter to a control group performed with a CF measuring catheter, by an operator with prior experience with CF technology. Thirty-six eligible paroxysmal $(n=27)$ or persistent $(n=9)$ AF patients were consecutively enrolled. Twelve patients underwent CPVI with the non-CF catheter (CFgroup) in a recall period and 24 with the $\mathrm{CF}$ catheter ( $\mathrm{CF}+$ group). After the first circumferential lesion set the number of $\mathrm{PV}$ pairs requiring additional touch-up lesions to achieve electrical isolation was significantly less in the CF+ group ( 2 of $48(4.2 \%)$ vs. 7 of 24 (29.2\%) in the CF+ and CF- group respectively, $\mathrm{p}=0.005)$. The procedure time was significantly lower in the $\mathrm{CF}+$ group $(117.9 \pm 23.3$ $\min$ vs. $134.1 \pm 25.3 \mathrm{~min}, \mathrm{p}=0.033)$. RF and fluoroscopy time did not differ between groups $(31.5 \pm$ $7.1 \mathrm{~min}$ vs. $31.8 \pm 7.0 \mathrm{~min}$ and $11.8 \pm 5.6 \mathrm{~min}$ vs. $11.0 \pm 5.8 \mathrm{~min}$ in the $\mathrm{CF}+$ and the CF- group, respectively).

Conclusion: With the use of online CF measurement PV isolation is more frequently complete following the first circumferential lesion set. A previous learning period with direct $\mathrm{CF}$ feedback is not a substitute for real-time direct CF measurement to maintain this advantage. 


\section{Introduction}

The clinical efficacy of pulmonary vein isolation (PVI) is limited by arrhythmia recurrences thought to occur predominantly because of reconnections of the isolated pulmonary veins (PVs) [1]. The reconnections in $\mathrm{PV}$ conduction indicate a lack of durable transmural lesion formation during the ablation procedure. A firm contact between the tissue and the catheter tip during radiofrequency (RF) ablation has shown to be an important determinant of lesion size and quality [2-8]. Estimation of contact through tactile feedback or indirect measurements have been shown to be poor predictors of actual tip-tissue contact [9-10]. As a result, preliminary studies showed a high variability of applied contact force $(\mathrm{CF})$ both between operators and within individual operator when blinded to $\mathrm{CF}$ measurements during the ablation [11-12]. Incorporation of a CF measuring sensor at the distal tip of the ablation catheter provides precise and real-time CF feedback. Six non-randomized and one randomized small clinical studies compared circumferential PVI (CPVI) performed with CF sensing catheters to standard open-irrigated-tip catheters. The use of CF sensing catheters was associated with improvement of procedural parameters and outcome. A significant reduction in procedure time [9, 1317] and RF ablation time [13-15], as well as of fluoroscopy time [9, 14-15] and acute PV reconnection rate $[14,18]$ were observed. Furthermore a mid-term clinical benefit with a significant reduction of the AF recurrence rate at 12 months after PVI was recently demonstrated in 2 studies [15-16].

Following promising preliminary clinical results in early 2012, the Thermocool $^{\circledR}$ SmartTouch $^{\mathrm{TM}}$ catheter (Biosense Webster, Diamond Bar, CA, USA) became available for clinical use in European countries and quickly found its way into routine clinical practice. Up to the enrollment in the current prospective registry, the single operator involved in this study gained 18 months experience performing more than 100 CPVI procedures using online CF measurement with the Thermocool ${ }^{\mathbb{B}}$ SmartTouch $^{\mathrm{TM}}$ catheter. In September 2013 the Thermocool ${ }^{\circledR}$ SmartTouch $^{\mathrm{TM}}$ catheter was temporarily withdrawn worldwide from clinical use due to technical defaults leading to safety concerns in a small number of catheters. In this transient recall period, the operator involved in the current study returned to the use of the irrigated-tip ablation catheter without CF measurement technology (NaviStar ${ }^{\circledR}$ Thermocool ${ }^{\circledR}$ ablation catheter).

The prospective comparison of procedural parameters of consecutive CPVI procedures performed without $\mathrm{CF}$ measurements during the recall period to a control group undergoing CPVI with $\mathrm{CF}$ measurement technology might offer further unique insights for several reasons. First, the recall setting allows the comparison of CPVI with or without CF measurements beyond the learning curve period of the new technique. Second, it is unknown if operators' experience with routine use of CF sensing catheters leads to a better estimation of good catheter tip-tissue contact and improved CPVI results with ablation catheters without CF measurement. Additionally, a registry allows the assessment of benefit of CF measurement technology in a real world clinical setting. 


\section{Methods}

\section{Patient population and study design}

Patients were enrolled in a prospective, non-randomized, single-center registry from September 2013 to June 2014. The registry protocol was approved by the ethical committee of the University Hospital of Antwerp. All consecutive patients with symptomatic paroxysmal or persistent AF refractory to at least one antiarrhythmic drug, who underwent a first ablation procedure during the recall period from September to the beginning of December 2013 were included. CPVI in these patients was performed without CF measuring technology (NaviStar ${ }^{\circledR}$ Thermocool $^{\circledR}$ ablation catheter) (CF- group). Following the reintroduction of the SmartTouch ${ }^{\mathrm{TM}}$ catheter in early December 2013, all patients were consecutively included in the registry as the $\mathrm{CF}+$ control group in a 1:2 ratio (patients were controlled for AF type and operator). Patients with long-standing persistent AF were excluded. Definitions of paroxysmal and persistent AF, success and failure and complications of the CPVI procedure are based on the HRS/EHRA/ECAS consensus statement on catheter and surgical ablation of AF [1].

\section{Procedure}

All ablation procedures were performed by a single, experienced operator ( $>300$ CPVI procedures and $>100$ CPVI procedures with the Thermocool ${ }^{\circledR}$ SmartTouch $^{\mathrm{TM}}$ catheter) (AS). All procedures were performed under general anesthesia and under uninterrupted warfarin or novel anticoagulants (last dose 24 hours before procedure). A decapolar coronary sinus catheter was introduced via the right femoral vein. Double transseptal puncture was performed with conventional long sheaths (St.-Jude Medical, SL0). Intravenous heparin was administered to achieve an activated clotting time of $>300$ seconds. A circular mapping catheter (LASSO ${ }^{\circledR}$, Biosense Webster) was used to create an anatomical shell of the left atrium with fast anatomical mapping (FAM) guided by CT images acquired the day before the procedure. For ablation a $3.5 \mathrm{~mm}$ irrigated tip ablation catheter (Thermocool ${ }^{\mathbb{B}} \mathrm{NaviStar}^{\mathbb{B}}$ ) with and without CF sensing technology was used with power settings between 30-40 Watts with irrigation rates from 17 to $30 \mathrm{ml} / \mathrm{min}$. After placing the $\mathrm{LASSO}^{\circledR}$ catheter in the ostium of the PVs contiguous circumferential antral ablations were performed around both the left and right sided veins in pairs $\geq 1 \mathrm{~cm}$ from the ostium of the pulmonary veins. In the anterior left PV - atrial appendage region RF was applied on the ridge. Along the posterior and inferior left atrial (LA) wall a power setting of 30 Watts and on the anterior wall a power setting of 35 Watts were used. At each application RF energy was delivered for 20 to 60 seconds. On the posterior wall $30 \mathrm{sec}$ and on the anterior wall 60 seconds application duration was targeted with an impedance drop of $10 \mathrm{Ohms}$. The applications were stopped prematurely after 20 seconds on the posterior and after 40 seconds on the anterior wall in case of a $>80 \%$ local diminution of bipolar voltage or $>25$ Ohms impedance drop or $>10$ Ohms impedance rise was observed. Attempts were made to make contiguous point-by-point applications during stable catheter position without jumps between the lesions. Without CF measurements, applications were only performed with stable near-field intracardiac signals, tactile feedback and 3D position suggesting 
stable catheter position with observations of the baseline impedance value, in order to avoid edema due to initial low contact applications. Special attention for the confirmation of good contact was paid in the anterior LA appendage-PV ridge and posterior and inferior right $\mathrm{PV}$ antrum region. In these regions the threshold to prolong RF duration and sometimes to increase RF power was lower, in case in spite of catheter adjustments the contact was felt to be suboptimal. For these applications on the anterior wall a power increase up to 40 Watts or RF duration to maximum 65 seconds and on the posterior wall a power of 35 Watts or a maximum RF duration of 35 seconds was allowed. When utilizing the NaviStar ${ }^{\circledR}$ Thermocool $^{\circledR}$ SmartTouch $^{\mathrm{TM}}$ catheter, applications were administered with a $\mathrm{CF}$ aimed between 10-30g and a stable CF curve throughout the application. No applications were made with a contact force $<5 \mathrm{~g}$, whereas power and RF time duration adjustment was allowed in the range between 5-10g following several attempts to increase CF above $10 \mathrm{~g}$. For safety outcome CF was aimed not to exceed 50g during application. Following circumferential ablation of a unilateral PV pair, entry and exit block of both PVs was assessed. If the PVs were not isolated, local bipolar voltage and pacing thresholds were mapped along the ablation line focusing at the region of the earliest $\mathrm{PV}$ potentials on the $\mathrm{LASSO}^{\circledR}$ catheter. Antral touch-up lesions were performed at sites of local bipolar voltage of $>0.5 \mathrm{mV}$ and/or if capture with $10 \mathrm{~mA} @ 2 \mathrm{~ms}$ bipolar pacing. If all attempts to antrally close the remaining gaps and isolate the PVs failed, more ostial applications were made. Following entry and exit block of one unilateral PV pair, the same protocol was repeated on the contralateral side. Following entry and exit block of all $4 \mathrm{PVs}$, after sequential reinsertion of the Lasso ${ }^{\circledR}$ catheter into each PV, adenosine was administered (with boli to induce atrioventricular block, with a minimum dose of $12 \mathrm{mg}$ and maximum dose of $24 \mathrm{mg}$ ) in an attempt to unmask dormant conduction. In case of adenosine-induced PV reconnection, antral remapping of the previous lesion was performed as previously described. Adenosine injection was repeated in case of entry and exit block of the PV, absence of local bipolar voltage $>0.5 \mathrm{mV}$ and local non-capture with $10 \mathrm{~mA} @ 2 \mathrm{~ms}$ pacing at the suspected gap sites. Complete adenosine-proof electrical isolation of all PVs was considered the procedural endpoint.

\section{Follow-up}

The day following the procedure, all patients underwent a transthoracic echocardiography to assess the amount of pericardial effusion.

\section{Primary and secondary endpoints}

The primary endpoint was the incidence of touch-up lesions following the initial circumferential ablation lesion set for antral circumferential PV pair isolation with entry and exit block. Secondary endpoints were the incidence of adenosine-induced PV reconnection, the total procedure time (defined as the time from venous access to adenosine-proof PVI), the LA procedure time (defined as the time from completion of transseptal puncture to adenosine-proof PVI), the RF time and the fluoroscopy 
time. The following procedural characteristics were also collected: mean and maximum power, mean and maximum temperature and mean contact force. The duration of hospitalization, the amount of pericardial effusion one day post-PVI, and procedural complications up tot 6 weeks after the PVI procedure were also assessed.

\section{Statistical analysis}

Continuous variables were expressed as means \pm standard deviation, and categorical values as frequencies (percentage). The two-sided unpaired Student's t-test was used to compare continuous variables when values were normally distributed or its non-parametric equivalent (Mann-Whitney U) when variables were not normally distributed. The Chi-square or Fisher's exact test was used to compare categorical variables. A p-value $\leq 0.05$ was considered to be statistically significant. IBM SPSS Statistics version 20 (SPSS Inc, Chicago, IL, USA) was used for all statistical analysis.

\section{Results}

\section{Study population}

A total of 36 patients were enrolled in the registry from September 2013 to June 2014. During the recall period 12 patients were treated without the CF sensing technology (CF- group) and 24 patients were treated with the SmartTouch ${ }^{\mathrm{TM}}$ catheter $(\mathrm{CF}+$ group). The baseline patient characteristics are detailed in Table 1. Overall no significant differences were observed between the CF- and the CF+ control group, except for a statistically higher percentage of patients treated with vitamin $\mathrm{K}$ antagonists in the CF- group. 
Table 1. Patient characteristics.

\begin{tabular}{|c|c|c|c|}
\hline & $\begin{array}{l}\text { CF- group } \\
(n=12)\end{array}$ & $\begin{array}{l}\text { CF+ group } \\
(n=24)\end{array}$ & P-value \\
\hline \multicolumn{4}{|l|}{ Demographics } \\
\hline Age (years) & $62.2 \pm 8.5$ & $58.6 \pm 11.3$ & 0.341 \\
\hline Range & $48-76$ & $29-76$ & \\
\hline Men, n (\%) & $11(91.7)$ & $19(79.2)$ & 0.640 \\
\hline \multicolumn{4}{|l|}{ Medical history, $\mathrm{n}(\%)$} \\
\hline Arterial hypertension & $6(50.0)$ & $8(33.3)$ & 0.471 \\
\hline Coronary artery disease & $1(8.3)$ & $5(20.8)$ & 0.640 \\
\hline History of myocardial infarction & $0(0)$ & $3(12.5)$ & 0.536 \\
\hline Valvular heart disease & $0(0)$ & $3(12.5)$ & 0.536 \\
\hline Dyslipidemia & $2(16.7)$ & $3(12.5)$ & 1.000 \\
\hline Diabetes mellitus & $0(0)$ & $2(8.3)$ & 0.543 \\
\hline \multicolumn{4}{|l|}{ Ejection fraction } \\
\hline$\%$ & $58.1 \pm 8.0$ & $56.0 \pm 7.9$ & 0.471 \\
\hline Range & $45-68$ & $37-68$ & \\
\hline $\begin{array}{l}\text { Mild to moderate LV dysfunction } \\
(\text { LVEF } \leq 45 \%), \mathrm{n}(\%)\end{array}$ & $1(8.3)$ & $1(4.2)$ & 1.000 \\
\hline \multicolumn{4}{|l|}{ Atrial fibrillation, $\mathrm{n}(\%)$} \\
\hline Paroxysmal AF & $9(75.0)$ & $18(75.0)$ & 1.000 \\
\hline Persistent AF & $3(25.0)$ & $6(75.0)$ & \\
\hline CHADSVASc score & $1.5 \pm 0.9$ & $1.6 \pm 1.6$ & 0.824 \\
\hline \multicolumn{4}{|l|}{ Left atrium configuration } \\
\hline Diameter $(\mathrm{mm})^{\star}$ & $43.0 \pm 4.3$ & $42.0 \pm 3.6$ & 0.448 \\
\hline Range & $34-48$ & $36-50$ & \\
\hline Volume FAM map $(\mathrm{ml})^{\star *}$ & $164.3 \pm 24.8$ & $157.9 \pm 28,3$ & 0.506 \\
\hline Range & $127-204$ & $117-221$ & \\
\hline Classic 4 PV configuration, $\mathrm{n}(\%)$ & $11(91.7)$ & $24(100)$ & 0.333 \\
\hline Accessory right middle PV & $1(8.3)$ & $0(0)$ & \\
\hline \multicolumn{4}{|l|}{ Antiarrhythmic drugs, n (\%) } \\
\hline Number of drugs tried & $1.5 \pm 0.9$ & $1.4 \pm 0.9$ & 0.728 \\
\hline Class I AAD & $8(66.7)$ & $12(50.0)$ & 0.481 \\
\hline Sotalol & $7(58.3)$ & $12(50.0)$ & 0.732 \\
\hline Amiodarone & $3(25.0)$ & $7(29.2)$ & 1.000 \\
\hline \multicolumn{4}{|l|}{ Anticoagulative drugs, $n(\%)$} \\
\hline Vitamin $\mathrm{K}$ antagonist & $11(91.7)$ & $13(54.2)$ & 0.031 \\
\hline Novel oral anticoagulant & $1(8.3)$ & $5(20.8)$ & 0.640 \\
\hline Aspirin derivative & $0(0)$ & $2(8.3)$ & 0.543 \\
\hline
\end{tabular}

Data are presented as mean \pm standard deviation or absolute number $(n)+$ percentage $(\%)$ AAD: antiarrhythmic drugs; AF: atrial fibrillation; CF-: group in which CPVI is performed with the NaviStar ${ }^{\circledR}$ Thermocool ${ }^{\circledR}$ ablation catheter; CF+: control group in which CPVI is performed with the Thermocool ${ }^{\circledR}$ SmartTouch ${ }^{\mathrm{TM}}$ catheter; FAM: fast anatomical map; LV: left ventricle; PV: pulmonary vein.

* Left atrial diameter on transthoracic echocardiography posterior long axis view. 
** Left atrial volume of fast anatomical map with Carto® system.

${ }^{* \star *}$ Antiarrhythmic and anticoagulant drug treatment prior to the ablation procedure.

\section{Procedural results}

The procedural endpoint (complete bidirectional electrical adenosine-proof isolation of all PVs with entry and exit block) was reached in $100 \%$ of performed procedures. The mean total lesion set length, as well as the mean lesion set length of left and right-sided PV circles were similar between both groups (Table 2).

The number of PV pairs requiring touch-up to achieve electrical isolation after the first circumferential lesion set was significantly less in the CF+ group (2 of $48 \mathrm{PV}$ pairs (4.2\%) compared to 7 of $24 \mathrm{PV}$ pairs $(29.2 \%)$ in the CF- group $(\mathrm{p}=0.005)$ (Table 2). Adenosine-induced acute PV reconnection occurred only in 1 patient (CF- group), who had also required touch-up applications after the first circumferential lesion set. If touch-ups were required, bidirectional electrical PV isolation was achieved with additional antral RF applications; no ostial touch-up applications were necessary. 
Table 2. Procedural parameters.

\begin{tabular}{|c|c|c|c|}
\hline & $\begin{array}{l}\text { CF- group } \\
(n=12)\end{array}$ & $\begin{array}{l}\text { CF+ group } \\
(n=24)\end{array}$ & P-value \\
\hline $\begin{array}{l}\text { Number of } P V \text { pairs requiring touch-up } \\
(n, \%)\end{array}$ & $7 / 24(29.2)$ & 2/48 (4.2) & 0.005 \\
\hline $\begin{array}{l}\text { Number of patients requiring touch-up } \\
(\mathrm{n}, \%)\end{array}$ & $7 / 12(58.3)$ & $2 / 24(8.3)$ & 0.002 \\
\hline $\begin{array}{l}\text { Number of adenosine-induced } \\
\text { reconnected PV pairs }(n, \%)\end{array}$ & $1 / 24(4.2)$ & $0 / 48(0)$ & 0.333 \\
\hline Total procedure time (min) & $167.8 \pm 26.0$ & $151.8 \pm 24.8$ & 0.075 \\
\hline Total left atrial time (min) & $134.1 \pm 25.3$ & $117.9 \pm 23.3$ & 0.033 \\
\hline RF time (min) & $31.8 \pm 7.0$ & $31.5 \pm 7.1$ & 0.919 \\
\hline Fluoroscopy time (min) & $11.0 \pm 5.8$ & $11.8 \pm 5.6$ & 0.708 \\
\hline Mean power delivered (Watts) & $30.4 \pm 1.2$ & $29.2 \pm 1.3$ & 0.024 \\
\hline Maximum power delivered (Watts) & $35.6 \pm 1.6$ & $35.3 \pm 1.1$ & 0.787 \\
\hline Mean temperature delivered $\left({ }^{\circ} \mathrm{C}\right)$ & $35.9 \pm 0.7$ & $38.4 \pm 1.9$ & $<0.001$ \\
\hline Maximum temperature delivered $\left({ }^{\circ} \mathrm{C}\right)$ & $46.2 \pm 2.8$ & $44.5 \pm 11.0$ & 0.672 \\
\hline Mean contact force applied (grams) & & $17.8 \pm 2.2$ & \\
\hline Total lesion set length (mm) & $267.8 \pm 14.1$ & $265.7 \pm 31.1$ & 0.831 \\
\hline Lesion set length left PV pair (mm) & $122.5 \pm 13,4$ & $123.1 \pm 22.0$ & 0.937 \\
\hline Lesion set length right $\mathrm{PV}$ pair (mm) & $145.3 \pm 10.9$ & $142.6 \pm 14.0$ & 0.583 \\
\hline
\end{tabular}

Data are presented as mean \pm standard deviation or absolute number $(n)+$ percentage $(\%)$ CF-: group in which CPVI is performed with the NaviStar $^{(B)}$ Thermocool $^{(8)}$ ablation catheter;

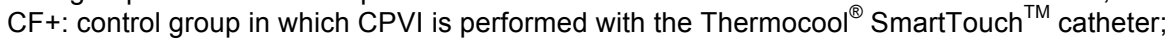
$\mathrm{PV}$ : pulmonary vein; RF: radiofrequency

The touch-ups characteristics and distribution are detailed in Table 3a and 3b. Touch-up applications of the left PV were necessary in 4 patients (11.1\%) and of the right PV in 5 patients (13.9\%). The most frequent locations of 22 gaps following the first lesion set were on the left PV pair the anterior segment (4 patients, 11.1\%) and on the right PV pair the posterior segment (4 patients, $11.1 \%$ ). The frequency of gaps in the right anterior $(\mathrm{CF}+: 0 / 24$ vs. $\mathrm{CF}-: 3 / 12, \mathrm{p}=0.031)$ and the right inferior segment (CF+: $0 / 24$ vs. CF-: $3 / 12, p=0.031)$ was significantly higher in the CF- group. The frequency of gaps in the other PV segments did not significantly differ between the two groups. 
Table 3a. Touch-up characteristics

\begin{tabular}{|c|c|c|c|c|c|c|c|c|}
\hline \# & $\begin{array}{c}\text { CF } \\
\text { group }\end{array}$ & $\begin{array}{l}\text { LPV/RPV } \\
\text { pair }\end{array}$ & $\begin{array}{l}\text { Antral touch-up } \\
\text { region }\end{array}$ & $\begin{array}{l}\text { RF time first } \\
\text { lesion set } \\
\text { (single PV } \\
\text { pair) }\end{array}$ & $\begin{array}{c}\text { RF time } \\
\text { touch-up } \\
\text { (single PV } \\
\text { pair) }\end{array}$ & $\begin{array}{l}\text { Adenosine- } \\
\text { induced PV } \\
\text { reconnection }\end{array}$ & $\begin{array}{l}\text { RF time } \\
\text { touch-up } \\
\text { after } \\
\text { adenosine }\end{array}$ & $\begin{array}{l}\text { Mean } \\
\text { CF }(g)\end{array}$ \\
\hline 1 & $\mathrm{CF}+$ & LPV & $\begin{array}{l}\text { Left superior } \\
\text { Left anterior } \\
\text { Left posterior }\end{array}$ & $13^{\prime} 06 "$ & 6'05" & & & 14.8 \\
\hline 2 & $\mathrm{CF}+$ & RPV & Right posterior & $14^{\prime} 25 "$ & 3' 51" & & & 15.6 \\
\hline 3 & CF- & RPV & $\begin{array}{l}\text { Right anterior } \\
\text { Right inferior }\end{array}$ & $12^{\prime} 52^{\prime \prime}$ & 4' 10" & & & 1 \\
\hline 4 & CF- & RPV & $\begin{array}{l}\text { Right superior } \\
\text { Right anterior } \\
\text { Right posterior }\end{array}$ & $10^{\prime} 27^{\prime \prime}$ & $11^{\prime} 06 "$ & & & 1 \\
\hline 5 & CF- & LPV & Left anterior & $14^{\prime} 05^{\prime \prime}$ & $1 ' 14 "$ & & & 1 \\
\hline 6 & CF- & RPV & $\begin{array}{l}\text { Right superior } \\
\text { Right inferior } \\
\text { Right posterior }\end{array}$ & $13^{\prime} 57^{\prime \prime}$ & $13^{\prime} 25^{\prime \prime}$ & & & I \\
\hline 7 & CF- & RPV & $\begin{array}{l}\text { Right posterior } \\
\text { Right anterior } \\
\text { Right inferior }\end{array}$ & $14^{\prime} 09^{\prime \prime}$ & 4' 16" & & & I \\
\hline 8 & CF- & LPV & $\begin{array}{l}\text { Left anterior } \\
\text { Left superior } \\
\text { Left posterior }\end{array}$ & 9' 53" & $10^{\prime} 21 "$ & $\begin{array}{l}\text { LPV: } \\
\text { Left anterior \& } \\
\text { Left posterior }\end{array}$ & 7' 30" & I \\
\hline 9 & CF- & LPV & $\begin{array}{l}\text { Left anterior } \\
\text { Left superior } \\
\text { Left posterior }\end{array}$ & $12^{\prime} 42^{\prime \prime}$ & $16^{\prime} 02^{\prime \prime}$ & & & 1 \\
\hline
\end{tabular}

AF: atrial fibrillation; CF: contact force; LPV: left pulmonary veins;; vein; RPV: right pulmonary veins;

RF: radiofrequency

Table 3b. Distribution of conduction gaps requiring touch-ups.

\section{CF-group $(n=12) \quad C F+$ group $(n=24) \quad P$-value}

\begin{tabular}{|c|c|c|c|}
\hline \multicolumn{4}{|c|}{ Right pulmonary veins } \\
\hline Anterior & $3(25.0)$ & 0 & 0.031 \\
\hline Posterior & $3(25.0)$ & $1(4.2)$ & 0.253 \\
\hline Superior & $2(16.7)$ & 0 & 0.105 \\
\hline Inferior & $3(25.0)$ & 0 & 0.031 \\
\hline \multicolumn{4}{|c|}{ Left pulmonary veins } \\
\hline Anterior & $3(25.0)$ & $1(4.2)$ & 0.098 \\
\hline Posterior & $2(16.7)$ & $1(4.2)$ & 0.253 \\
\hline Superior & $2(16.7)$ & $1(4.2)$ & 0.253 \\
\hline Inferior & 0 & 0 & $\mathrm{n} / \mathrm{a}$ \\
\hline
\end{tabular}


The left atrial procedure time was significantly lower in the $\mathrm{CF}+$ group $(\mathrm{CF}+: 117.9 \pm 23.3 \mathrm{~min}$ vs. CF-: $134.1 \pm 25.3 \mathrm{~min}, \mathrm{p}=0.033)$. RF time $(\mathrm{CF}+: 31.5 \pm 7.1 \mathrm{~min}$ vs. $\mathrm{CF}-: 31.8 \pm 7.0 \mathrm{~min})$ and fluoroscopy time $(\mathrm{CF}+: 11.8 \pm 5.6 \mathrm{~min}$ vs. $\mathrm{CF}-\mathrm{:} 11.0 \pm 5.8 \mathrm{~min})$ did not differ between groups.

The mean applied CF was $17.8 \pm 2.2$ grams. The mean delivered temperature was higher in the $\mathrm{CF}+$ group $(\mathrm{CF}+: 38.4 \pm 1.9$ vs. $\mathrm{CF}-: 35.9 \pm 0.7, \mathrm{p}=<0.001)$, with a lower mean power $(\mathrm{CF}+: 29.2 \pm 1.3$ vs. $\mathrm{CF}-\mathrm{:} 30.4 \pm 1.2 \mathrm{~W}, \mathrm{p}=0.024)$.

One patient (CF+ group) developed anterior ST elevation near the end of the PVI due to an underlying LAD stenosis and percutaneous coronary intervention (PCI) was performed immediately following the PVI. In one patient ( $\mathrm{CF}+$ group) a small arterio-venous fistula was discovered following the inguinal puncture, which was treated conservatively. One patient ( $\mathrm{CF}+$ group) had complaints of pericarditis the day following the procedure. No patients experienced cerebral ischemic events or pericardial perforation or tamponade.

Pericardial effusion was assessed on transthoracic echocardiography the day after the procedure. None of the patients had a hemodynamically significant pericardial effusion. There was no difference in the periprocedural hospitalization duration between the two groups $(\mathrm{CF}+: 4.0 \pm 1.6$ vs. $\mathrm{CF}$-: $4.0 \pm 2.3$ days, $\mathrm{p}=0.679$ ).

\section{Discussion}

\section{Superior efficacy of online CF measurement in CPVI}

The TOCCATA [19] and EFFICAS-I studies [20] demonstrated a positive correlation between applied $\mathrm{CF}$ and clinical efficacy endpoints as the occurrence of pulmonary vein reconnection and recurrence of AF after PVI. Based on these results current strategies to achieve durable PVI target higher CF values by adjusting catheter position to improve contact [19-20]. If in spite of repositioning a low CF remains, effective lesion formation might be achieved by increasing the power and/or duration of the RF application. Recently, 6 small non-randomized and 1 randomized clinical studies compared online CF measurement with CF sensing catheters to standard open-irrigated-tip catheters for PVI. In 5 of these studies the SmartTouch ${ }^{\mathrm{TM}}$ catheter $[13-15,17-18]$ and in 2 studies the TactiCath $^{\circledR}$ catheter (Endosense SA, Geneva, Switzerland) $[9,16]$ was used. In these studies the use of CF sensing catheters was associated with better procedural parameters and outcome as a significant reduction in procedure time [9, 13-17] and RF ablation time [13-15], as well as of fluoroscopy time [9, 14-15] and acute PV reconnection rate $[14,18]$ were observed. A mid-term clinical benefit with a significant reduction of the AF recurrence rate at 12 months after PVI was recently demonstrated in 2 studies [15-16]. 


\section{Requirement of touch-up lesions}

The EFFICAS-I trial recently demonstrated that in spite of acute complete PV isolation conduction recurs at sites with low $\mathrm{CF}$ application. It was suggested that edema formation during the first low force applications might prevent durable transmural lesion formation of subsequent repeat applications at the same site. At these sites conduction gaps recurred [20-21]. Based on these results current strategies include first ablation attempts with optimal CF to achieve lesion transmurality with the first application [20]. PVI with entry and exit block following the first circumferential lesion set as the primary endpoint in the current study reflects this strategy. The main finding of our study is that the use of CF technology significantly reduced the need for touch-up lesions to achieve electrical isolation after the first circumferential lesion set from $29 \%$ to $4 \%$ per PV pair. The most frequent gap locations were in the regions previously reported prone to low contact (the left anterior and right posterior PV segments). Touch-up applications in the right anterior and right inferior PV segments were more frequent in non-CF guided CPVI procedures.

In a recently published prospective, non-randomized, single-center study, Marijon et al. compared CPVI with the SmartTouch ${ }^{\mathrm{TM}}$ catheter to a regular irrigated-tip ablation catheter in 60 patients with paroxysmal AF. In their study bidirectional PV isolation using solely an anatomical approach was achieved in $80.0 \%$ (24/30) of patients with CF technology compared to only $36.7 \%$ (11/30) of patients when CF measurement was not used. The use of online CF measurement was also associated with a reduction of AF recurrence at 12 months of follow up [15]. In a similar study Haldar et al. included 40 patients undergoing CPVI. 20 patients underwent CF guided and 20 patients CF blinded procedure. After 60 min waiting and adenosine administration $70 \%$ of patients ( $21 \%$ of the veins) had acute reconnection in the CF blinded vs. $15 \%$ of patients ( $4 \%$ of veins) in the CF guided group.

In the current study bidirectional PV isolation was achieved with the first circumferential lesion set in $95.8 \%$ of the PV pairs and in $91.6 \%$ of the patients with CF technology compared to $70.8 \%$ of the PV pairs and in $41.6 \%$ of the patients without CF. The PV isolation rates with first circumferential lesion set in both the CF+ and CF- group in our study are very similar to the previous two studies $(92 \% \mathrm{vs}$. $80 \%$ and $85 \%$, and $42 \%$ vs. $37 \%$ and $30 \%$ of patients in the CF+ and CF- group respectively). The slight differences between the studies might be explained by the slightly different ablation strategies. In our study the electrograms from the Lasso catheter within the PV are displayed during PV isolation, allowing longer applications at the site of PV delay or isolation similar to the study by Haldar et al. as opposed to the study by Marijon et al. In our approach the power settings were higher between 30 and 40 Watts as opposed to 25 to 30 Watts in the study by Marijon et al. and 30 Watts in the study by Haldar. Additionally, in our study we adjusted the power settings according to obtained CF.

In addition to improving CF before RF delivery, CF guided ablation allows recognition of application sites where the obtained CF remains low after attempts to improve contact. Despite the lower CF (between 5 and $10 \mathrm{~g}$ ) a durable transmural lesion could still be achieved by increasing the RF duration along with RF power for these specific applications. This strategy explains the higher RF time of the 
first circumferential lesion set in the $\mathrm{CF}+$ group as compared to the $\mathrm{CF}$ - group $(\mathrm{CF}+: 31.4 \pm 7.1 \mathrm{~min}$ vs. CF-: $25.6 \pm 2.5 \mathrm{~min}, \mathrm{p}=0.015$ ). The study of Haldar et al. reported similar findings; total mean RF time was (non-significantly) higher in the $\mathrm{CF}$ guided procedures $(\mathrm{CF}+$ : $60.7 \pm 20.6 \mathrm{~min}$ vs. $52.9 \pm 23.1$ min, $\mathrm{p}=0.29$ ) despite the lower incidence of acute PV reconnection requiring touch-up ablations.

In addition to the online $\mathrm{CF}$ feedback, the higher RF duration of first lesion set might have resulted in the lower incidence of touch-up applications in CF guided procedures.

\section{Sustainable superior efficacy of online CF measurement after the learning period}

CF sensing catheters allow online tactile feedback at different CF values, which might teach the operators to better estimate contact quality in the absence of online CF measurement. Experience with the CF catheters might also teach the operators which catheter and sheath maneuvers result in improved tip-tissue contact. The use of CF measuring catheters identified which PV segments are prone to low CF applications if no online CF feedback is available [19, 20]. Individual experience with these catheters also has the potential to identify operator specific CF differences. For these reasons in the current study we tested the hypothesis whether an extensive training period with realtime direct $\mathrm{CF}$ feedback from the SmartTouch ${ }^{\mathrm{TM}}$ catheter could replace the use of CF sensing catheters.

The large majority of the previously mentioned studies were performed early after the introduction of the CF measuring catheters, thus operators had no or little experience with the CF technology. In only one study by Haldar et al. the operators had medium-term experience with the SmartTouch ${ }^{\mathrm{TM}}$ catheter before enrollment [18]. Furthermore, in most of the non-randomized trials the control group procedures without CF measurement were performed before the procedures with $\mathrm{CF}$ technology [9, 13-14, 16]. Hence, in contrast to the current study, no learning effect from procedures with online CF measurement feedback on procedures without availability of CF technology was present in these studies. The recall period of the SmartTouch ${ }^{\mathrm{TM}}$ catheter allowed us to compare CPVI with and without $\mathrm{CF}$ measurements beyond the learning curve period of the CF technology. The second main finding of our study is that even after gaining experience in CPVI with CF measuring catheters, procedural parameters remain superior with this technology. An extensive training period with real-time direct $\mathrm{CF}$ feedback from the SmartTouch ${ }^{\mathrm{TM}}$ catheter did not result in equal quality CPVI when returning to standard open-irrigated-tip RF ablation catheters with adjusting catheter position, RF power or time in regions typically known for difficulty to achieve good CF. This indicates that even after a training period with direct CF feedback, mere tactile feedback remains unreliable to estimate firm tip-to-tissue contact as it may be influenced by the catheter shaft, deflection, torque, and by the different sheaths used. 


\section{Adenosine-induced acute $P V$ reconnection}

The incidence of adenosine-unmasked dormant conduction was very low in our study. Adenosine provoked PV reconnection occurred only in 1 patient in the CF- group ( $8 \%$ of patients in non-CF guided procedures). In a published, prospective non-randomized two-center study Andrade et al. compared the prevalence of dormant conduction after CPVI with a non-CF sensing catheter $\left(\right.$ Thermocool $\left.^{\circledR}\right)$ to CPVI with a CF sensing catheter $\left(\right.$ SmartTouch $\left.^{\mathrm{TM}}\right)$ in 75 paroxysmal AF patients [22]. The use of CF catheters led to a significant reduction of adenosine-induced acute PV reconnection. Adenosine-unmasked dormant conduction was present in 4 out of $50 \mathrm{PV}$ pairs (8\%) in 4 out of 25 patients (16\%) in the CF\% group, and in 35 of $100 \mathrm{PV}$ pairs (35\%) and 26 of 50 patients $(52 \%)$ in the CF- group ( $\mathrm{p}=0.0004$ per PV pair, $\mathrm{p}=0.0029$ per patient). The lower incidence of adenosine-induced acute PV reconnection in our study could be partly explained by a difference in RF parameters. In our study applications were made with a power of 35 Watts along the anterior wall and 30 Watts along the posterior wall as opposed to 30-35 watts on the anterior and 25 Watts on the posterior wall in the study by Andrade et al. Moreover in CF guided procedures if it was not possible to attain $C F \geq 10 \mathrm{~g}$, in our study an extension of the duration of the RF application was applied, along with a power increase up to 40 Watts on the anterior wall and 35 Watts on the posterior wall. Also we aimed the CF between 10 and $30 \mathrm{~g}$ as opposed to a targeted CF between 5 and $50 \mathrm{~g}$ in the study of Andrade et al. An additional reason for the low adenosine reconnection in our study is likely the shorter waiting time. In the study of Andrade et al. adenosine was administered at least 20 minutes after obtaining PV pair isolation, while in our study adenosine-induced reconnection was checked 30 minutes after isolation of left PVs and only 10 minutes after isolation of the right PVs. Lastly for the non-CF guided procedures, it is possible that the experience gained with using $\mathrm{CF}$ catheters improved the results of procedures without CF measurements, resulting in a lower incidence of adenosineunmasked dormant conduction in our study.

\section{Procedural parameters}

In our study the procedure time (the LA procedure time) of CPVI was significantly reduced with the CF technology (CF+ group: $117.9 \pm 23.3 \mathrm{~min}$ vs. $\mathrm{CF}$ - group $134.1 \pm 25.3 \mathrm{~min}, \mathrm{p}=0.033)$, largely because looking for residual gaps (for touch-up lesion target) after completion of first circumferential lesion set is time consuming. Although less additional touch-up RF applications were required in the $\mathrm{CF}+$ group, the total $\mathrm{RF}$ time was equal in both groups $(\mathrm{CF}+: 31.5 \pm 7.1 \mathrm{~min}$ vs. $\mathrm{CF}-: 31.8 \pm 7.0 \mathrm{~min}$, $\mathrm{p}=0.919$ ). This is because in the $\mathrm{CF}+$ group ablation points with lower $\mathrm{CF}$, due to difficulty in the catheter position, were compensated with longer RF duration of the RF application.

The use of CF did not result in a reduction in fluoroscopy time in our study $(\mathrm{CF}+$ : $11.8 \pm 5.6 \mathrm{~min}$ vs. CF-: $11.0 \pm 5.8 \mathrm{~min})$. However, the fluoroscopy time in both groups was low and was mostly used for the catheter insertion and the transseptal puncture, which are not affected by CF technology.

The mean delivered temperature was higher in the $\mathrm{CF}+$ group $\left(\mathrm{CF}+: 38.4 \pm 1.9^{\circ} \mathrm{C}\right.$ vs. $\mathrm{CF}-\mathrm{s} 35.9 \pm$ 
$\left.0.7^{\circ} \mathrm{C}, \mathrm{p}=<0.001\right)$, with a lower mean power (CF+: $29.2 \pm 1.3$ Watts vs. CF-: $30.4 \pm 1.2$ Watts, $\mathrm{p}=$ 0.024). This finding suggests that higher temperatures are achieved with less power when optimal $\mathrm{CF}$ is pursued. In general catheter tip temperature in irrigated-tip catheters is not considered an indicator of neither tissue temperature nor contact.

\section{Pulmonary vein isolation with operators blinded to contact force measurements}

Because in the CF- group CPVI was performed with a conventional non-CF sensing catheter in a clinical scenario when the SmartTouch ${ }^{\mathrm{TM}}$ catheter was unavailable, assessment of the obtained CF in the CF- group was not possible. Two previous clinical studies compared CPVI performed with CF sensing catheters with operators guided by vs. blinded to the $\mathrm{CF}$ measurements. In the previously mentioned study, Haldar et al. compared CF guided to CF blinded CPVI in 40 non-randomized patients [18]. CF guided CPVI reduced the rate of PV reconnection after a waiting period of 1 hour and adenosine challenge after PV isolation was achieved. The mean CF was significantly lower in the CF blinded group as compared to the CF guided group (11.6g (10.5-12.9g) vs. $14.4 \mathrm{~g}(13.3-15,7 \mathrm{~g})$ ), and mean contact force was significantly lower at the segments that experienced reconnection (almost all in the blinded group) (14.5g (13.3-15.7g) vs. 19.6g (14.6-26.3g). The majority of reconnection sites were on the left side the anterior and superior segments and on the right side the carina. In our study we report similar gap locations.

In another recently published single-center study, Kimura et al. compared CF to non-CF guided CPVI in 38 randomized patients with paroxysmal (28) and non-paroxysmal (10) AF [17]. All procedures were performed with the SmartTouch ${ }^{\mathrm{TM}}$ catheter, but in the non-CF group the operators were blinded to the online $\mathrm{CF}$ measurement. Blinding to $\mathrm{CF}$ measurement lead to a significant reduction in the mean applied CF (5.9 \pm 4.5 vs. $11.1 \pm 4.3 \mathrm{~g}$ in blinded and unblinded procedures respectively). In this study, online CF feedback reduced the mean number of residual conduction gaps requiring touch-up applications after the first circumferential lesion set to achieve PV isolation from $6.3 \pm 3.0$ to $2.8 \pm 1.9$ per patient $(\mathrm{p}<0.001)$. In addition procedure time (defined from initial the RF application to completion of CPVI) was significantly shorter in CF guided procedures (59 \pm 16 vs. $96 \pm 39$ min in blinded and unblinded procedures, $\mathrm{p}<0.001$ ). The results in the CF blinded groups in these 2 studies suggest that the inferior efficacy in our CF- group is likely due to uncorrected lower contact during CPVI. In contrast to our study, all patients in the study of Kimura et al. had conduction gaps after the first circumferential lesion set, although significantly less in the CF guided CPVI group. These important differences in results could be explained by different ablation strategies. In our approach point-by-point applications ranging from 20 to 60 seconds were applied as opposed to dragging lesion applications of 20-25 seconds in the study of Kimura et al. The power settings in our study were higher between 30 and 40 Watts as opposed to 25 to 30 Watts. We used a long sheath in the left atrium. We aimed a contact force between 10 and $30 \mathrm{~g}$ as opposed to a targeted CF between 10 and 20g. The mean obtained CF in the CF guided group was in our study significantly higher as compared 
to the study of Kimura et al. $(17.8 \pm 2.2$. vs. $11.1 \pm 4.3 \mathrm{~g})$.

In addition in the study of Kimura et al. the operators blinded to CF had a learning curve of 5 cases. In a subsequent phase of their study an additional 20 patients underwent CF guided CPVI. In these patients the mean CF and number of gaps was not significantly different as compared to the first 20 patients suggesting that there was no learning curve effect between the first and second $20 \mathrm{CF}$ guided cases. However, Kimura et al. also examined the CF at each segment of the left PV under CF blinded $\mathrm{CPVI}$ in 5 cases, performed by operators having experienced $>30 \mathrm{CF}$ guided procedures. The CFs were in all segments similar to those in the CF guided CPVI suggesting a learning effect. Although only the left sided CF in a small number of cases was investigated, the authors hypothesized that accumulating experience with CF guided CPVI may favorably affect outcomes in non-CF guided CPVI. In our study we report that even if this improvement is present CF guided CPVI remains superior to CF blinded CPVI even after an extensive learning curve.

Although the blinded studies demonstrate that applied CF was lower if operators were blinded to the $\mathrm{CF}$ measurements, it must be noted that due to the incorporation of the $\mathrm{CF}$ sensor, the distal $3 \mathrm{~cm}$ of the SmartTouch ${ }^{\mathrm{TM}}$ catheter is stiffer compared to the NaviStar ${ }^{\circledR}$ Thermocool $^{\circledR}$ catheter. Without the online CF feedback, tactile sensation of CF might be further impaired by the increased catheter stiffness in the blinded study group procedures with the SmartTouch ${ }^{\mathrm{TM}}$ catheter compared to the NaviStar $^{\circledR}$ Thermocool $^{\circledR}$ catheter. Additionally the CF sensing catheter has a significantly higher cost price. Given these differences, it is a clinically relevant question if after the learning curve the $\mathrm{CF}$ sensing catheter could be replaced by a non-CF sensing catheter without risking the efficacy of the procedure. Our study compared two different commercially available catheters, with and without CF sensing, as opposed to the two blinded studies where the same CF catheter is used.

\section{Limitations}

Our study has several limitations. The number of patients included in both groups was relatively small. The study was non-randomized and we did not include clinical follow-up. Further as a single-center, single-operator trial it is not known to what extend these results can be generalized. However, it is reasonable to speculate that the benefits of CF sensing catheters, both regarding efficacy and safety, are only greater with less experienced operators. Additionally, the study design did not allow assessment of the obtained CF in the CF- group. Finally, we did not compare procedural parameters without online CF measurements between patients undergoing PVI prior to patients undergoing PVI after the introduction of the CF sensing catheters. It cannot be excluded that the experience gained with using $\mathrm{CF}$ catheters improved the results of procedures without $\mathrm{CF}$ measurements. 


\section{Conclusion}

Our results suggest that with the use of online CF measurement PVI is more frequently complete following the first circumferential lesion set. After gaining experience with $\mathrm{CF}$-measuring catheters, procedural CPVI parameters remain superior with this technology when compared to non-CF guided CPVI procedures. An extensive training period with real-time direct CF-feedback was not a substitute for real-time direct CF measurement to maintain this advantage. 


\section{References}

1. Calkins H., Kuck K.H., Cappato R., Brugada J., Camm A.J., Chen S.A., et al. (2012). 2012 HRS/EHRA/ECAS Expert Consensus Statement on Catheter and Surgical Ablation of Atrial Fibrillation: recommendations for patient selection, procedural techniques, patient management and follow-up, definitions, endpoints, and research trial design. Hearth Rhythm, 9, 632-696.

2. Haines D.E. (1991). Determinants of lesion size during radiofrequency catheter ablation: the role of electrode-tissue contact pressure and duration of energy delivery. J Cardiovasc Electrophysiol, 2, 509-515.

3. Avitall B., Mughal K., Hare J., Helms R., Krum D. (1997). The effects of electrode-tissue contact on radiofrequency lesion generation. Pacing Clin Electrophysiol, 20, 2899-2910.

4. Strickberger S.A., Vorperian V.R., Man K.C., Williamson B.D., Kalbfleisch S.J., Hasse C., et al. (1994). Relation between impedance and endocardial contact during radiofrequency catheter ablation. Am Heart J, 128, 226-229.

5. Zheng X., Walcott G.P., Hall J.A., Rollins D.L., Smith W.M., Kay G.N., et al. (2000). Electrode impedance: an indicator of electrode-tissue contact and lesion dimensions during linear ablation. $\mathrm{J}$ Interv Card Electrophysiol, 4, 645-654.

6. Nath S., DiMarco J.P., Gallop R.G., McRury I.D., Haines D.E. (1996). Effects of dispersive electrode position and surface area on electrical parameters and temperature during radiofrequency catheter ablation. Am J Cardiol, 77, 765-767.

7. Eick O.J., Wittkampf F.H., Bronneberg T., Schumacher B. (1998). The LETR-Principle: a novel method to assess electrode-tissue contact in radiofrequency ablation. J Cardiovasc Electrophysiol, 9, 1180-1185.

8. Wittkampf F.H., Nakagawa H. (2006). RF catheter ablation: Lessons on lesions. Pacing Clin Electrophysiol, 29, 1285-1297.

9. Wakili R., Clauss S., Schmidt V., Ulbrich M., Hahnefeld A., Schüssler F., Siebermair J., Kääb S., Estner H.L. (2014). Impact of real-time contact force and impedance measurement in pulmonary vein isolation procedures for the treatment of atrial fibrillation. Clin Res Cardiol, 103, 97-106.

10. Di Biase L., Paoletti Perini A., Mohanty P., Goldenberg A.S., Grifoni G., Santangeli P., et al. (2014). Visual, tactile, and contact force feedback: which one is more important for catheter ablation? Results from an in vitro experimental study. Heart Rhythm, 11, 506-513.

11. Kuck K.H., Reddy V.Y., Schmidt B., Natale A., Neuzil P., Saoudi N., et al. (2012). A novel radiofrequency ablation catheter using contact force sensing: Toccata study. Heart Rhythm, 9, 1823.

12. Park C.I., Lehrmann H., Keyl C., Weber R., Schiebeling J., Allgeier J., et al. (2014). Mechanisms of pulmonary vein reconnection after radiofrequency ablation of atrial fibrillation: the deterministic role of contact force and interlesion distance. J Cardiovasc Electrophysiol, 25, 701708.

13. Martinek M., Lemes C., Sigmund E., Derndorfer M., Aichinger J., Winter S., et al. (2012). Clinical impact of an open-irrigated radiofrequency catheter with direct force measurement on atrial fibrillation ablation. Pacing Clin Electrophysiol, 35, 1312-1318.

14. Sciarra L., Golia P., Natalizia A., De Ruvo E., Dottori S., Scarà A., et al. (2014). Which is the best catheter to perform atrial fibrillation ablation? A comparison between standard ThermoCool, SmartTouch, and Surround Flow catheters. J Interv Card Electrophysiol, 39, 193-200.

15. Marijon E., Fazaa S., Narayanan K., Guy-Moyat B., Bouzeman A., Providencia R., et al. (2013). Real-Time Contact Force Sensing for Pulmonary Vein Isolation in the Setting of Paroxysmal Atrial Fibrillation: Procedural and 1-Year Results. J Cardiovasc Electrophysiol 2013 [Epub ahead 
of print]. doi: $10.1111 /$ jce. 12303

16. Wutzler A., Huemer M., Parwani A.S., Blaschke F., Haverkamp W., Boldt L.H. (2014) Contact force mapping during catheter ablation for atrial fibrillation: procedural data and one-year followup. Arch Med Sci, 10, 266-272.

17. Kimura M., Sasaki S., Owada S., Horiuchi D., Sasaki K., Itoh T., et al. (2014). Comparison of lesion formation between contact force-guided and non-guided circumferential pulmonary vein isolation: a prospective, randomized study. Heart Rhythm, 11, 984-991.

18. Haldar S., Jarman J.W., Panikker S., Jones D.G., Salukhe T., Gupta D., et al. (2013). Contact force sensing technology identifies sites of inadequate contact and reduces acute pulmonary vein reconnection; a prospective case control study. Int J Cardiol, 168, 1160-1166.

19. Reddy V.Y., Shah D., Kautzner J., Schmidt B., Saoudi N., Herrera C., et al. (2012). The relationship between contact force and clinical outcome during radiofrequency catheter ablation of atrial fibrillation in the TOCCATA study. Heart Rhythm, 9, 1789-1795.

20. Neuzil P., Reddy V.Y., Kautzner J., Petru J., Wichterle D., Shah D., et al. (2013). Electrical reconnection after pulmonary vein isolation is contingent on contact force during initial treatment: results from the EFFICAS I study. Circ Arrhythm Electrophysiol, 6, 327-333.

21. Ren J.F., Callans D.J., Schwartzman D., Michele J.J., Marchlinski F.E. (2001). Changes in local wall thickness correlate with pathologic lesion size following radiofrequency catheter ablation: an intracardiac echocardiographic imaging study. Echocardiography, 18, 503-507.

22. Andrade J.G., Monir G., Pollak S.J., Khairy P., Dubuc M., Roy D., Talajic M., Deyell M., Rivard L., Thibault B., Guerra P.G., Nattel S., Macle L. (2014). Pulmonary vein isolation using "contact force" ablation: the effect on dormant conduction and long-term freedom from recurrent atrial fibrillation--a prospective study. Heart Rhythm, 11, 1919-1924. 


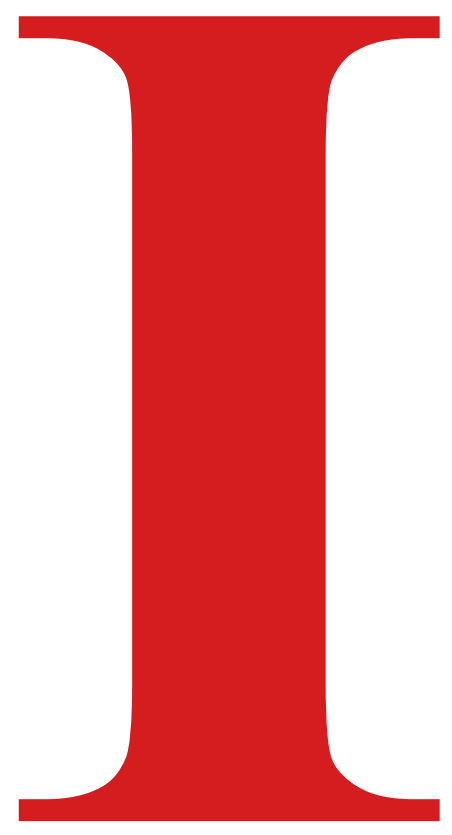




\section{Part I}

\section{ABLATION OF ATRIAL FIBRILLATION AND ATRIAL TACHYCARDIA}

\section{Part IB}

A protocol for pulmonary vein isolation with contiguous and optimized radiofrequency applications: "CLOSE-PVI" 


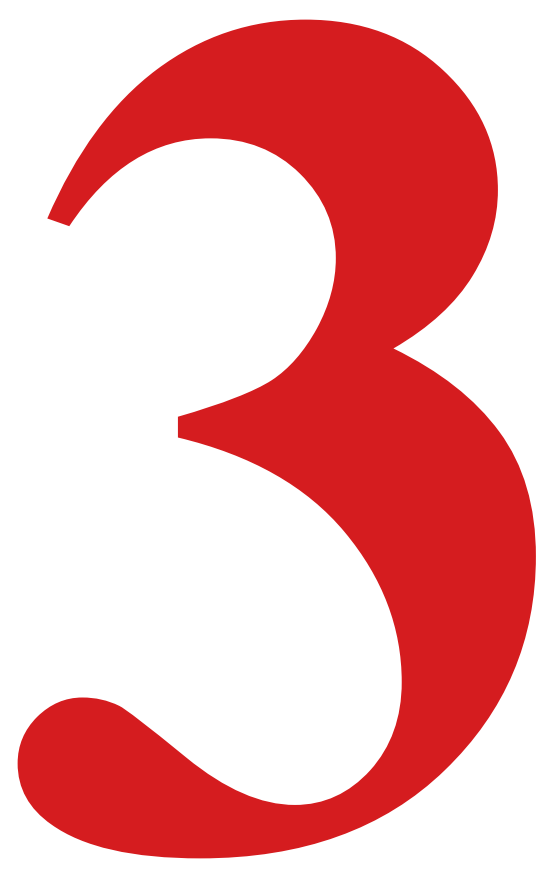




\section{Chapter 3}

\section{Evaluation of optimal ablation parameters for radiofrequency PVI: inter-tag distance and ablation index}

Milad El Haddad, Philippe Taghji, Thomas Phlips, Michael Wolf, Anthony Demolder, Rajin Choudhury, Sébastien Knecht, Yves Vandekerckhove, René Tavernier, Hiroshi Nakagawa, Mattias Duytschaever

Circulation: Arrhythmia \& Electrophysiology 2017; 10:pii: e004867. doi:10.1161/CIRCEP.116.004867 


\section{ABSTRACT}

Background: Pulmonary vein reconnection (PVR) still determines recurrences of atrial fibrillation (AF) after contact force (CF)-guided PV isolation (PVI). We studied whether acute PVR (adenosine and/or waiting time) and late PVR (at repeat) are explained by incomplete transmurality and/or contiguity within the deployed RF circle.

Methods: We analysed 42 CF-guided ipsilateral PVI procedures. For each RF tag within the circle, we collected data on transmurality (time of application, power, impedance drop ( $\Delta$-Imp), CF, forcetime integral (FTI), ablation index (AI)) and contiguity (automated interlesion distance, AILD). Ablation line contiguity index (ALCI) was developed as a novel automated algorithm combining transmurality and contiguity into one single criterion. Each circle was subdivided into 10 segments. For each segment we determined its "weakest link" by annotating: Time $e_{\min }$, Power $_{\min }, \Delta-\mathrm{Imp}_{\min }, \mathrm{CF}_{\min }$, FTI $_{\min }, \mathrm{AI}_{\min }, \mathrm{AILD}_{\max }, \mathrm{ALCI}_{\min }$.

Results: Compared to segments without PVR ( $\mathrm{n}=758)$, segments with PVR ( $\mathrm{n}=44)$ were characterized by lower $\Delta$-Imp min $_{\text {(n) }}\left(4.8\right.$ vs. $7.4 \Omega$ ), $\mathrm{CF}_{\min }\left(8.5\right.$ vs. $11.8 \mathrm{~g}$ ), $\mathrm{FTI}_{\min }$ (351 vs. $473 \mathrm{gs}$ ), $\mathrm{AI}_{\min }$ (367 vs. 408 arbitrary unit) and higher $\mathrm{AILD}_{\max }(6.8 \mathrm{vs} .5 .5 \mathrm{~mm})$, all $\mathrm{p}<0.001$. $\mathrm{ALCI}_{\min }$ was significantly lower in segments with PVR (74 vs. 104\%, $\mathrm{p}<0.001$ ) and was associated with the highest accuracy to predict durable segments (AUC $=0.73)$.

Conclusion: In CF-guided PVI, PVR is explained by lack of both transmurality and contiguity within the deployed RF circle. ALCI, a novel parameter combing contiguity and transmurality, is the most accurate predictor for durable segments. By avoiding weak links in the ablation chain, ALCI-guided ablation is expected to improve success rate of point-by-point RF ablation. 


\section{INTRODUCTION}

Catheter based pulmonary vein isolation (PVI) is associated with a single procedure freedom of atrial fibrillation (AF) in $\approx 70 \%$ of patients with paroxysmal AF.[1-2] Lack of durability of PVI, a major determinant of $\mathrm{AF}$ recurrence, is evidenced by acute $\mathrm{PV}$ reconnection at the time of the procedure and late PVR during follow-up.[3-5] The use of contact force (CF) sensing catheters, allowing to better control stability and lesion depth during point-by-point PV encircling, has improved freedom of AF now amounting to $\approx 80 \%$.[6-8] Nevertheless acute and late PVR still occur and data on outcome are not unequivocal.[9]

We aimed to investigate whether acute and late PVR in CF-guided ablation are due to incomplete transmural or discontiguous RF lesions within the deployed PV circle and whether criteria can be set forward as targets to avoid weak links in the ablation chain. Criteria for transmurality implied time of application, median delivered power, impedance drop, average $\mathrm{CF}$, force-time integral and ablation index.[10-11] Contiguity was assessed by calculating automated interlesion distance (AILD). Ablation line contiguity index (ALCI) was developed as a novel automated algorithm to assess both transmurality and contiguity and was defined as the ratio of the estimated width of a transmural lesion over its distance to the closest transmural lesion.

\section{METHODS}

\section{Study population}

From august 2013 all patients undergoing CF-guided ablation for AF are followed in a prospective database approved by the local institutional review committee. This database implies collecting patients' informed consent, a detailed case report form of the procedure and follow-up at 1, 3, 6 and 12 months. In the present manuscript we present retrospective analysis of 42 patients that underwent CFguided catheter ablation for drug-resistant or drug-intolerant symptomatic paroxysmal AF between December 2013 and July 2014 in the St Jan Hospital Bruges.

\section{Contact force-guided pulmonary vein isolation}

PVI was performed under conscious sedation or general anaesthesia. In patients undergoing anaesthesia, transesophageal echocardiogram and thermal probing (SensiTherm ${ }^{\mathrm{TM}}$, St Jude Medical Inc, Minnesota, US) were performed. After femoral access, intravenous heparin (100 IU/kg) was administered and continuously infused (ACT $>300 \mathrm{sec}$ ). After double transseptal puncture, a decapolar Lasso catheter and an $8 \mathrm{~F}$ open-tip irrigated RF catheter with tip-integrated $\mathrm{CF}$ sensor (Thermocool SmartTouch ${ }^{\circledR}$, Biosense-Webster Inc., Diamond Bar, CA, USA) were positioned in the left atrium. Calibration of CF catheter and respiratory gating were performed. After reconstructing 3D- 
geometry (Carto System ${ }^{\circledR}$, BSW), point-by-point ipsilateral PVI was performed using real-time automated display of RF applications (Visitag®, Biosense-Webster Inc.), with predefined settings of catheter stability ( $3 \mathrm{~mm}$ for $8 \mathrm{sec}$ ) and minimum CF (30\% of time $>4 \mathrm{gr}$ ). RF was delivered in a power-controlled mode with maximal $25 \mathrm{~W}$ for $30 \mathrm{~s}$ on the posterior wall and roof, and maximal $35 \mathrm{~W}$ for $60 \mathrm{~s}$ on the anterior wall (irrigation flow of 17 to $30 \mathrm{cc} / \mathrm{min}$ ). According to data from the EFFICAS studies, CF target was $>10$ g.[12-13] In case of chest pain or significant intra-esophageal $\mathrm{T}^{\circ}$ rise (> $38.5^{\circ} \mathrm{C}$ ) during posterior wall ablation, RF delivery was stopped prematurely. No strict criteria for contiguity were available at that time. Ablation index and ALCI were not available to the operators.

After electrical isolation within the ipsilateral circle (i.e. entrance block verified by Lasso catheter) we waited for 20 minutes after which adenosine (dose resulting in atrio-ventricular block) was given. In case of reconnection during waiting or after adenosine (acute PVR), ablation was continued until PVI was resistant to subsequent waiting and adenosine. The anatomical site of reconnection within the circle (i.e. site of earliest activity and where touch-up RF eliminated PVR or markedly changed activation pattern) were carefully annotated.

\section{Follow-up}

After ablation, anticoagulation and previously failing anti-arrhythmic drugs were continued. At 3 months, anticoagulation was continued according to the stroke risk. Clinical evaluation and ECG were performed at 1, 3, 6 and 12months. Holter was performed at 3 month $(24 \mathrm{~h}), 6$ month $(24 \mathrm{~h})$ and 12 month ( 7 day) or in case of symptoms. In case of recurrence, defined as any documented atrial tachyarrhythmia lasting more than 30 seconds, repeat ablation procedure was advised. In case reconnection was observed at the beginning of the repeat procedure (late PVR), ablation was performed until PVI was obtained. The sites of reconnection within the circle (i.e. site of earliest activity and where RF eliminated PVR) were annotated.

\section{Criteria for transmurality and contiguity}

Each procedure was exported and analyzed offline. For each RF tag within the circle we determined offline criteria for transmurality: time of application (s), median delivered power (W), impedance drop $(\Delta$-Imp, $\Omega$ ), average CF (CF, g), force-time integral (FTI, gs) and ablation index (AI, arbitrary unit, $\mathrm{au})$. AI is an indirect parameter for prediction of lesion quality based on a logarithmic formula integrating power and $\mathrm{CF}$ over time, which was available as an offline research tool.[12,13]. In addition, we determined contiguity by measuring interlesion distance in between neighboring ablation tags (center-to-center distance, $\mathrm{mm}$ ). To combine both transmurality and contiguity in one single criterion we developed in Matlab (Mathworks, Natick, MA, USA) a new algorithm to assess ablation line contiguity (ALC). ALC index (ALCI) is defined as the ratio of the estimated width of a 
transmural lesion (determined by the $\mathrm{AI}$ ) over its distance to the closest transmural lesion and calculated as

$A L C I_{i j} \%=\frac{O C x \operatorname{Con} v x \frac{A I_{i}+A I_{j}}{2}}{100 x A I L D_{i j}} \times 100$; where 'OC' is the overlapping coefficient of the 2 neighboring tags and 'Conv' is the conversion factor specific to anatomical location (anterior or posterior wall).

\section{Determination of the weakest link within the deployed RF circle}

Each PV circle was divided into 10 anatomical segments (Fig.1, top panel). For each segment, the "weakest link" was defined as the minimal or maximal value for each criterion (Time ${ }_{\min }$, Power $_{\min }, \Delta-$ $\mathrm{Imp}_{\min }, \mathrm{CF}_{\min }, \mathrm{FTI}_{\min }, \mathrm{AI}_{\min }, \mathrm{AILD}_{\max }, \mathrm{ALCI}_{\min }$ ). A representative example of the "weakest link" analysis is shown in (Fig.1, lower panel). In this patient (without acute or late PVR), the weakest link in segment 9 (anterior segment of the right circle encompassing $4 \mathrm{RF}$ tags) was characterized by Time $_{\min }$ of $60 \mathrm{~s}$, Power $\min$ of $30 \mathrm{~W}, \Delta-\mathrm{Imp}_{\min }$ of $11 \Omega, \mathrm{CF}_{\min }$ of $8.6 \mathrm{~g}, \mathrm{FTI}_{\min }$ of $515 \mathrm{gs}, \mathrm{AI}_{\min }$ of $529 \mathrm{au}$, AILD $_{\max }$ of $6.4 \mathrm{~mm}$ and $\mathrm{ALCI}_{\min }$ of $95 \%$.

Ablation tags belonging to additional RF applications delivered to treat acute PV were ignored for analysis in case of acute PVR, but were included in the analysis of late PVR.

The weakest link values of segments revealing acute or late PVR were compared to those values within segments without PVR. Out of 84 circles, 4 (40 PV segments) were excluded from analysis due to incomplete RF/VisiTag ${ }^{\circledR}$ data. The remaining 80 circles ( 800 PV segments) were analyzed. Results are presented for all segments, per segment and for posterior wall (segments 1-2-3-4-5 and roof segment 10) and anterior wall (segments 6-7-8-9) separately. 


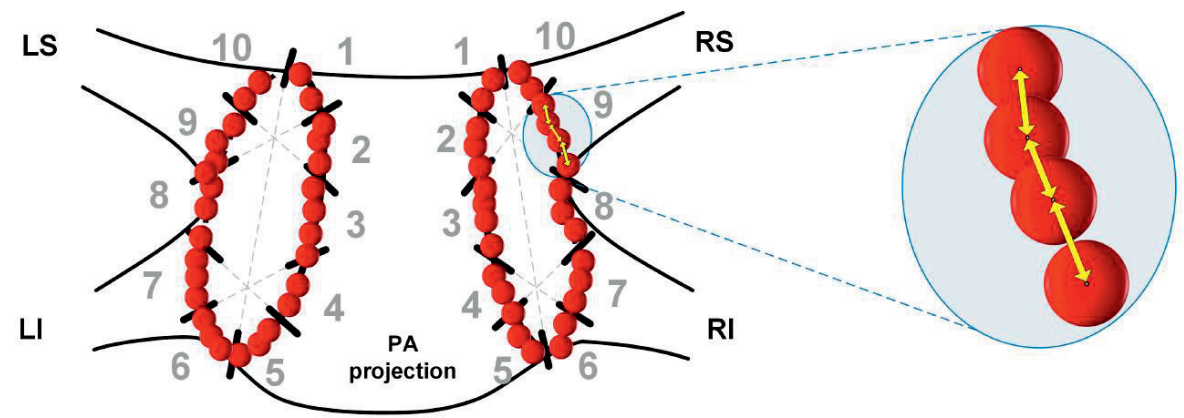

\begin{tabular}{|c|c|c|c|c|c|c|c|}
\hline$\underset{(s)}{\operatorname{Time}}$ & $\begin{array}{l}\text { Power } \\
\text { (W) }\end{array}$ & $\underset{(\Omega)}{\Delta-\operatorname{Imp}^{\prime}}$ & $\begin{array}{l}\text { CF } \\
(\mathrm{g})\end{array}$ & $\begin{array}{l}\text { FTI } \\
\text { (gs) }\end{array}$ & $\begin{array}{l}\text { Al } \\
(a u)\end{array}$ & $\underset{(\mathrm{mm})}{\text { ILD }}$ & $\underset{(\%)}{A L C I}$ \\
\hline 60 & 35 & 17 & 9.9 & 597 & 607 & \multirow{4}{*}{$\begin{array}{l}4.5 \\
4.2 \\
6.4\end{array}$} & \multirow{4}{*}{$\begin{array}{l}147 \\
151 \\
95\end{array}$} \\
\hline 60 & 35 & 16 & 8.6 & 515 & 571 & & \\
\hline 60 & 30 & 11 & 12.3 & 740 & 555 & & \\
\hline 60 & 30 & 21 & 9.1 & 554 & 529 & & \\
\hline Time $_{\min }$ & Power $_{\min }$ & $\Delta-\operatorname{Imp} p_{\min }$ & $\mathrm{CF}_{\min }$ & $\mathrm{FTI}_{\min }$ & $\mathbf{A} \mathbf{l}_{\min }$ & ILD $_{\max }$ & $\mathrm{ALCl}_{\min }$ \\
\hline 60 & 30 & 11 & 8.6 & 515 & 529 & 6.4 & 95 \\
\hline
\end{tabular}

Figure 1: A representative example of "weakest link" analysis in a given segment. Each circle is divided into 10 segments (upper panel). Per segment we defined the minimal value for time (Time $\min _{\text {), }}$ power (Power min $_{\text {) }}$, impedance drop $\left(\Delta\right.$-Imp $\left.\mathrm{Imin}_{\mathrm{m}}\right)$, contact force $\left(\mathrm{CF}_{\min }\right)$, force-time integral $\left(\mathrm{FTI}_{\min }\right)$, ablation index $\left(\mathrm{AI}_{\min }\right)$ and ablation line contiguity index $\left(\mathrm{ALCI}_{\min }\right)$ and maximal value for automated interlesion distance $\left(\mathrm{AILD}_{\max }\right)$. RS, right superior pulmonary vein; RI, right inferior pulmonary vein; LS, left superior pulmonary vein; LI, left inferior pulmonary vein.

\section{Statistics}

Continuous variables are expressed as mean $\pm \mathrm{SD}$ (or medians with IQR for non-normal distributions) and dichotomous variables as percentage. Normality of data distribution was tested with Shapiro-Wilk test. Mann-Whitney U test or Kruskal-Wallis test were used to evaluate significant difference between segments with and without PVR. For parameters showing significant difference, receiver operator characteristics (ROC) curves were plotted and analyzed to determine the diagnostic accuracy to predict durable isolation at different clinical cutoffs.[11,12,14] Multivariable logistic regression was used to assess the association between the different parameters and PVR. P values $<0.05$ were considered statistically significant. All statistical analyses were performed in SPSS Statistics 22 (IBM Corporation, Armonk, New York, USA). 


\section{RESULTS}

\section{Patients, procedure and follow-up}

Clinical characteristics are given in Table 1. All patients had paroxysmal AF. General anesthesia was used in 15 procedures (36\%). Procedure time was $189 \pm 41 \mathrm{~min}$, RF time per procedure was $55.0 \pm$ $11.8 \mathrm{~min}$ and RF time per circle was $27.6 \pm 7.7 \mathrm{~min}$. All ipsilateral pairs of veins ( 84 circles) were electrically isolated at the end of the procedure.

In 7 patients recurrence of $\mathrm{AF}$ was documented (freedom of $\mathrm{AF} 81 \%$ ). In 6 patients, a repeat ablation was performed. Mean time from the first PVI procedure to repeat procedure was $287 \pm 17$ days.

Table 1: Clinical characteristics of study subjects

\begin{tabular}{lc}
\hline Characteristic & CF -guided PVI (n=42) \\
\hline Mean age, y & $63 \pm 10$ \\
Male, n (\%) & $24(57 \%)$ \\
Mean BMI, kg/m² & $27 \pm 5$ \\
HTN, n (\%) & $16(38 \%)$ \\
CHA2DS2-VASc score & \\
$\quad \leq 1, \mathrm{n}(\%)$ & $20(48 \%)$ \\
$2-3, \mathrm{n}(\%)$ & $20(48 \%)$ \\
$\quad \geq 4, \mathrm{n}(\%)$ & $2(4 \%)$ \\
Time of first AF episode to PVI, months & $24[$ range $3-28]$ \\
Mean LVEF, \% & $63 \pm 3$ \\
Mean LAD, mm & $40 \pm 5$ \\
\hline
\end{tabular}

\section{Comparison of segments with PVR vs. durable segments without PVR}

In total, acute PVR occurred in 25 segments (14 circles in 11 patients) whereas late PVR occurred in 19 segments ( 6 circles in 6 patients). All patients at repeat revealed at least one reconnected circle. The spatial distribution of acute and late PVR is given for each segment in Fig.2. Out of 44 PVR, we observed 17 PVR at anterior wall vs. 27 PVR at the posterior wall $(\mathrm{p}=0.143)$, and 25 PVR at the right circle vs. 19 at the left circle $(\mathrm{p}=0.176)$. No PVR was seen at the superior and inferior segments of the posterior left circle (segment 1, 4 and 5). Out of 19 segments with late PVR, only 2 were also characterized by acute PVR. 
Acute PVR distribution per segment

$(n=25)$

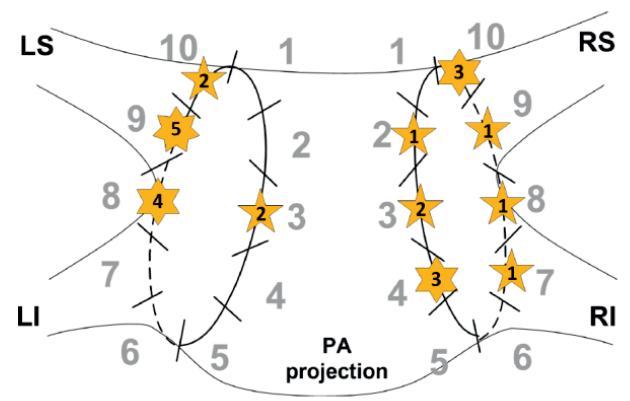

\section{Late PVR distribution per segment}

$(n=19)$

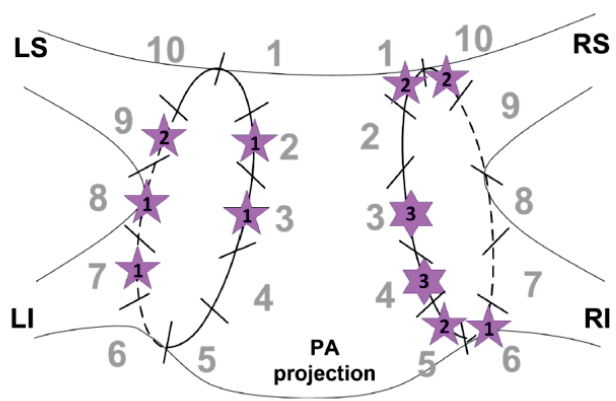

Figure 2: Number of acute (orange stars) and late (purple stars) pulmonary vein reconnections (PVR) observed for each segment. See text for further explanation. PA, postero-anterior projection.

In those 44 segments with acute or late PVR (vs. 758 segments without PVR) we observed lower $\Delta$ $\mathrm{Imp}_{\min }, \mathrm{CF}_{\min }, \mathrm{FTI}_{\min }, \mathrm{AI}_{\min }$, higher $\mathrm{AILD}_{\max }$ and lower $\mathrm{ALCI}_{\min }$. A representative example is given in Fig.3. In this patient, adenosine revealed acute PVR at the left circle with the site of reconnection in segment 8 (anterior). The weakest link in segment 8 was characterized by the lowest $\operatorname{ALCI}_{\min }(51 \%)$ due to the combination of low $\mathrm{AI}_{\min }$ and high $\mathrm{AILD}_{\max }(6.8 \mathrm{~mm})$. 


\begin{tabular}{|c|c|c|c|c|c|c|c|c|}
\hline Segment & $\begin{array}{c}\text { Time }_{\text {min }} \\
\text { (s) }\end{array}$ & $\begin{array}{l}\text { Power }_{\text {min }} \\
\text { (W) }\end{array}$ & $\begin{array}{c}\Delta-\operatorname{Im}_{\min } \\
(\Omega)\end{array}$ & $\begin{array}{c}\mathbf{C F}_{\min } \\
(\mathrm{g})\end{array}$ & $\begin{array}{c}\mathbf{F T I}_{\min } \\
\text { (gs) }\end{array}$ & $\begin{array}{c}\mathrm{Al}_{\text {min }} \\
\text { (au) }\end{array}$ & $\begin{array}{l}\text { ILD }_{\max } \\
(\mathrm{mm})\end{array}$ & $\begin{array}{c}\mathrm{ALCI}_{\min } \\
(\%)\end{array}$ \\
\hline 1 & 31 & 20 & 6 & 12 & 370 & 356 & 4.9 & 111 \\
\hline 2 & 33 & 20 & 5 & 10 & 350 & 363 & 3.7 & 149 \\
\hline 3 & 26 & 20 & 7 & 15 & 403 & 369 & 2.9 & 192 \\
\hline 4 & 33 & 25 & 2 & 5 & 330 & 413 & 5.1 & 123 \\
\hline 5 & 39 & 25 & 2 & 5 & 236 & 383 & 5.3 & 79 \\
\hline 6 & 64 & 30 & 3 & 5 & 329 & 460 & 5.2 & 132 \\
\hline 7 & 59 & 35 & 9 & 7 & 433 & 558 & 6.1 & 102 \\
\hline 8 & 23 & 27 & 10 & 6 & 149 & 338 & 6.8 & 51 \\
\hline 9 & 36 & 27 & 4 & 7 & 411 & 502 & 4.3 & 122 \\
\hline 10 & 54 & 18 & 11 & 11 & 585 & 393 & 4.4 & 128 \\
\hline
\end{tabular}

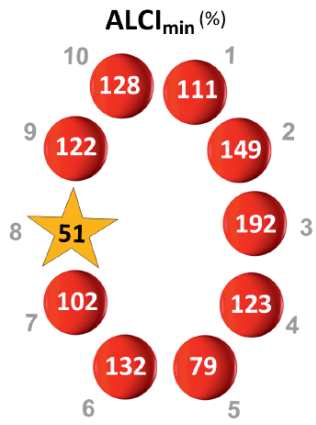

Acute PVR of the LSPV during adenosine challenge

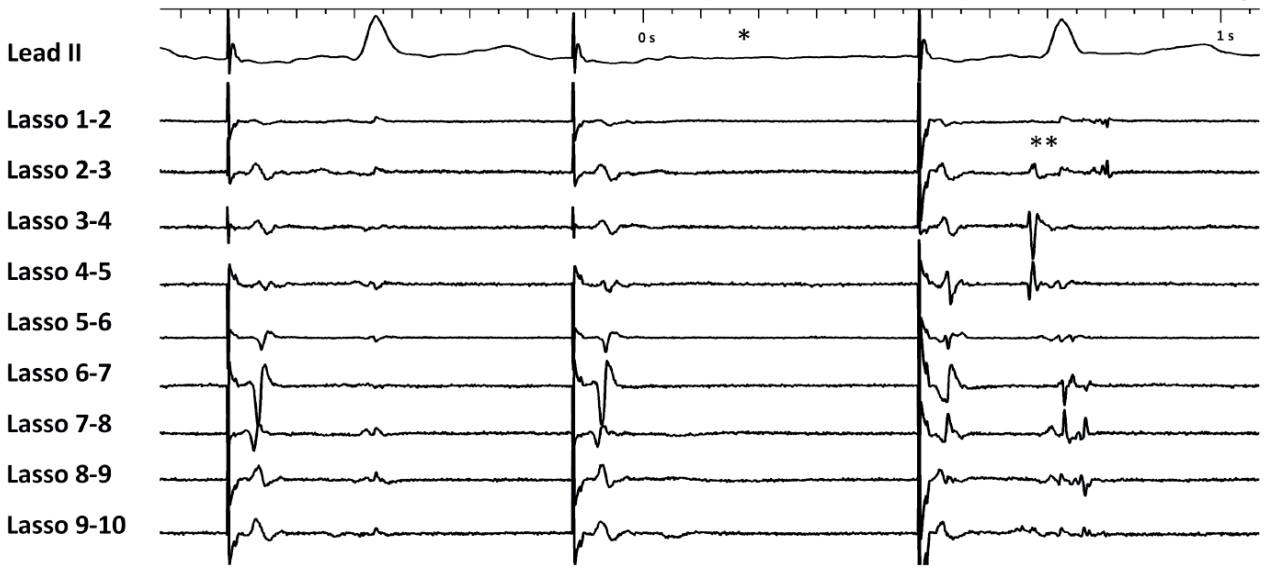

Figure 3: Acute pulmonary vein reconnections (PVR) in the left circle, eliminated by touch-up ablation in segment 8 . This segment is characterized by a low minimal ALCI value. In the lower panel, bipolar electrograms from the circular mapping catheter at the LSPV together with surface ECG Lead II are presented. * AV block induced by Adenosine. ** PVR. Abbreviations similar to Figure 1.

Overall results are summarized in Figure 4. Compared to segments without PVR $(n=758)$, segments with PVR $(\mathrm{n}=44)$ had comparable Time $\min _{\text {in }}(33 \mathrm{~s}$ vs. $35 \mathrm{~s}, \mathrm{p}=0.054)$ and Power $_{\min }(20$ vs. $22 \mathrm{~W}, \mathrm{p}=$ 0.513). In contrast, segments with PVR were characterized by significantly lower $\Delta$-Imp $\operatorname{Imin}_{\text {(n) }}(4.8 \mathrm{vs} .7 .4$ $\Omega, \mathrm{p}=0.002), \mathrm{CF}_{\min }(8.5$ vs. $11.8 \mathrm{~g}, \mathrm{p}=0.001), \mathrm{FTI}_{\min }(351 \mathrm{vs.} 473 \mathrm{gs}, \mathrm{p}<0.001)$ and $\mathrm{AI}_{\min }(367 \mathrm{vs}$. $408 \mathrm{au}, \mathrm{p}=0.001)$. Furthermore, segments with PVR were characterized by higher AILD $_{\max }$ (6.8 vs. $5.5 \mathrm{~mm}, \mathrm{p}<0.001)$. Minimal ALCI per segment was significantly lower in segments with PVR (74 vs. $104 \%, \mathrm{p}<0.001)$.

Differences (except of $\Delta$-Imp) remained significant when analyzed for acute PVR and late PVR separately (Table 2). When analyzed per segment and circle separately (Tables $3 \mathrm{a}$ and $3 \mathrm{~b}$ ), a similar trend was observed although statistically not invariably significant (due to low number of PVR within a segment ranging from 7 to as low as 0 ). 

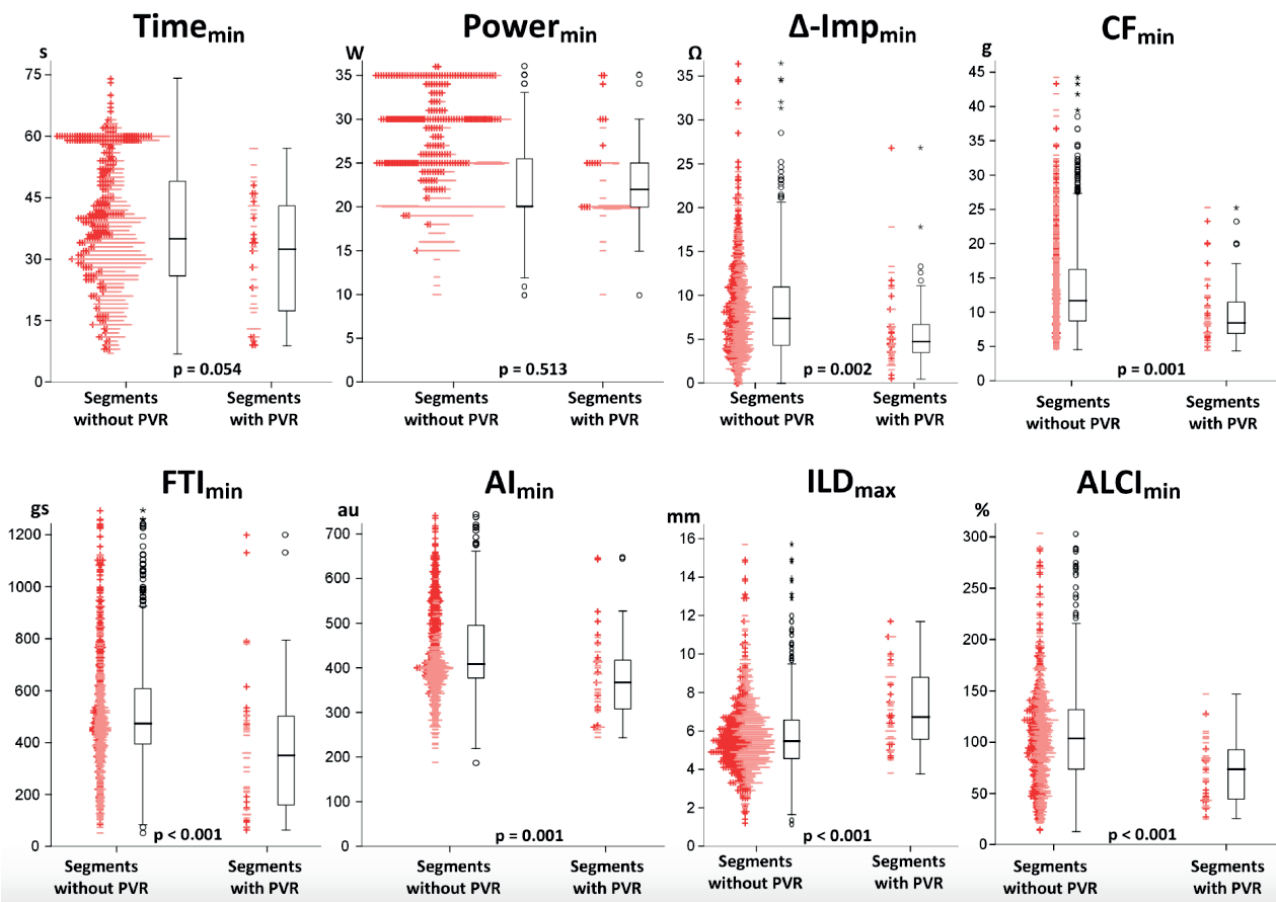

Figure 4: Difference in Time $_{\min }$, Power ${ }_{\min }, \Delta-\mathrm{Imp}_{\min }, \mathrm{CF}_{\min }, \mathrm{FTI}_{\min }, \mathrm{AI}_{\min }, \mathrm{AILD}_{\max }$, and $\mathrm{ALCI}_{\min }$ between all segments without PVR vs. all segments with PVR. Red scatter markers represent anterior segments. Pink scatter markers represent posterior segments. Abbreviations similar to Figure 1. See text for further explanation. 


\section{Comparison of segments with or without PVR in anterior and posterior segments}

Weakest link analysis was performed on anterior wall and posterior wall separately (box and whisker plots, Fig. 5). Compared to durable anterior segments $(n=303)$, anterior segments with PVR $(n=27)$ were characterized by significantly lower Time $\min _{\min }(\mathrm{p}<0.001)$, lower $\Delta-\operatorname{Imp}_{\min }(\mathrm{p}=0.005), \operatorname{FTI}_{\min }(\mathrm{p}=$ $0.005), \mathrm{AI}_{\min }(\mathrm{p}<0.001)$, higher $\operatorname{AILD}_{\max }(\mathrm{p}=0.001)$ and lower $\mathrm{ALCI}_{\min }(\mathrm{p}<0.001)$. Compared to durable posterior segments $(n=455)$, posterior segments with PVR $(n=17)$ were characterized by significantly lower Power $r_{\text {min }}(p=0.02), \mathrm{CF}_{\min }(\mathrm{p}=0.003), \operatorname{FTI}_{\min }(\mathrm{p}=0.011)$, higher $\operatorname{AILD}_{\max }(\mathrm{p}<$ $0.001)$ and lower $\mathrm{ALCI}_{\min }(\mathrm{p}=0.001)$.
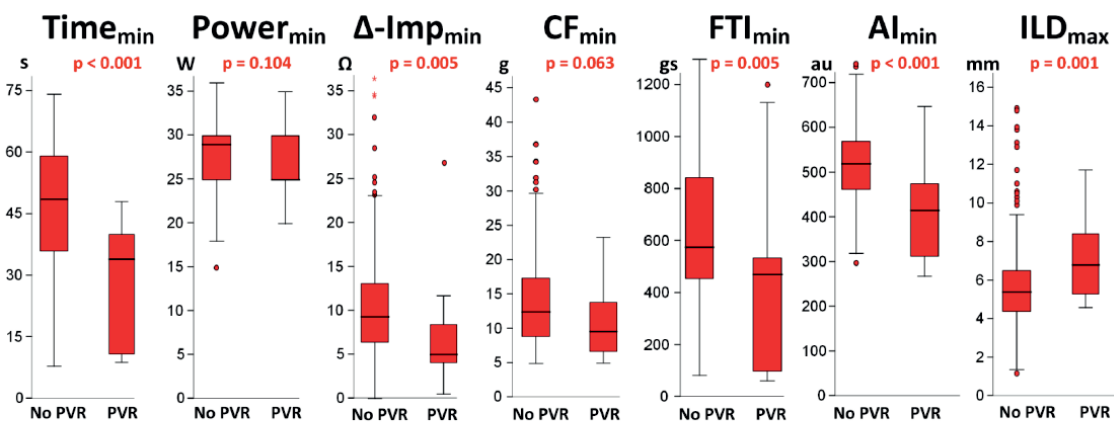

$\mathrm{ALCl}_{\min }$
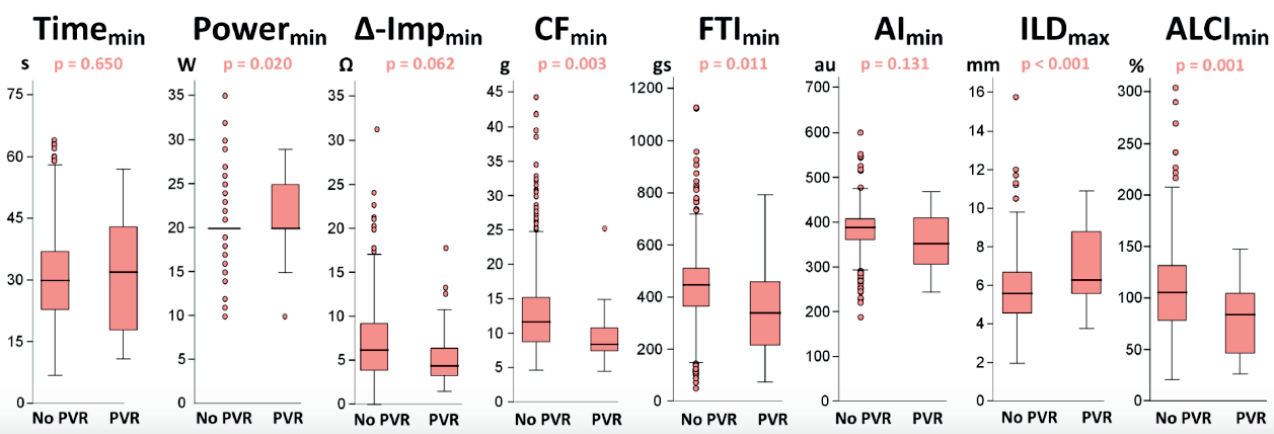

Figure 5: Upper panels: difference in Time ${ }_{\min }$, Power ${ }_{\min }, \Delta$-Imp $_{\min }, \mathrm{CF}_{\min }, \mathrm{FTI}_{\min }, \mathrm{AI}_{\min }$, $\mathrm{AILD}_{\max }$, and ALCI $\mathrm{min}_{\min }$ between anterior segments without PVR vs. anterior segments with PVR. Lower panels: idem for posterior segments. Abbreviations similar to Figure 1. 


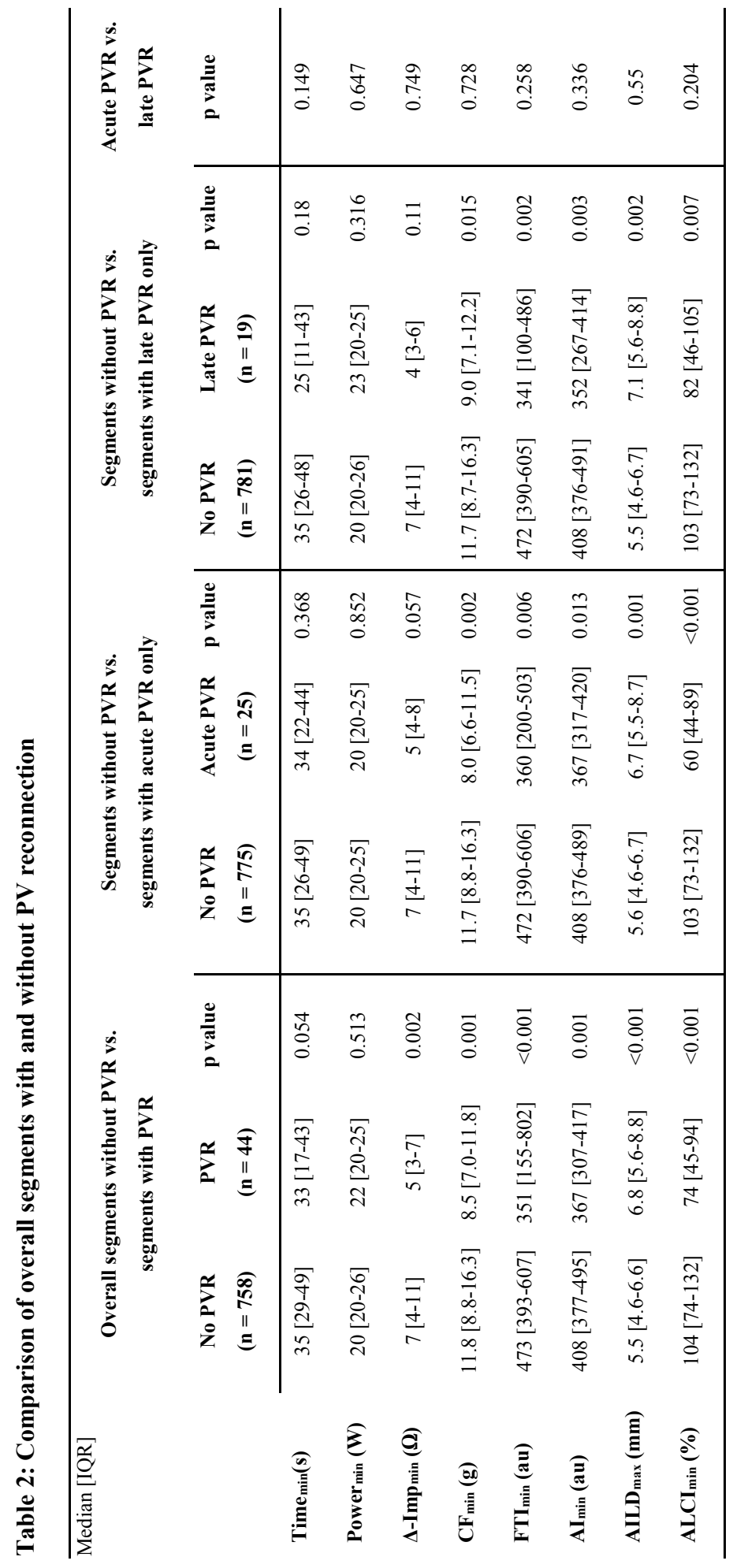




\begin{tabular}{|c|c|c|c|c|c|c|c|c|c|c|c|}
\hline 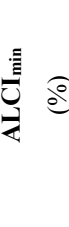 & 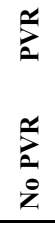 & 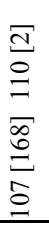 & 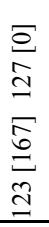 & 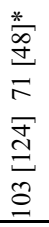 & 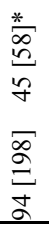 & 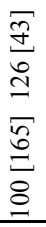 & 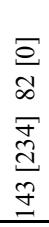 & 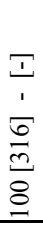 & 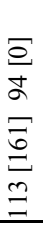 & 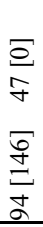 & 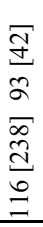 \\
\hline 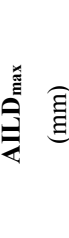 & 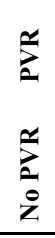 & 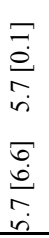 & 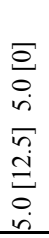 & 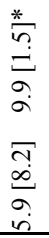 & 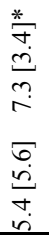 & 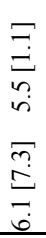 & 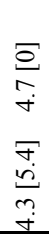 & $\begin{array}{l}\overline{0} \\
0 \\
i \\
i \\
0 \\
\dot{0} \\
\infty \\
\dot{n} \\
\dot{n}\end{array}$ & 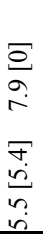 & 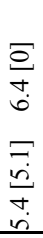 & 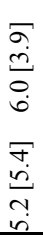 \\
\hline$\frac{E}{4}$ & 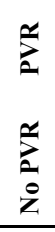 & 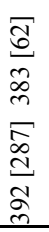 & 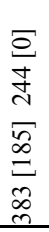 & 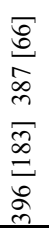 & 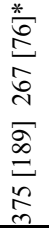 & 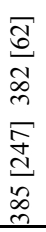 & $\begin{array}{l} \\
0 \\
0 \\
0 \\
n \\
0 \\
0 \\
0 \\
n \\
n \\
n \\
n\end{array}$ & 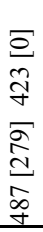 & 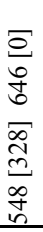 & 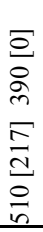 & 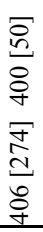 \\
\hline 庖 & 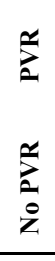 & 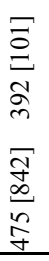 & 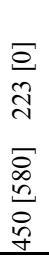 & 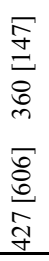 & 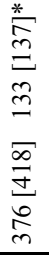 & 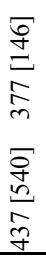 & $\begin{array}{l}\bar{\sigma} \\
\infty \\
\infty \\
\therefore \\
\widetilde{J} \\
\Xi \\
\Xi \\
0 \\
\infty \\
\infty\end{array}$ & 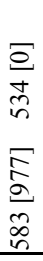 & 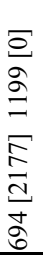 & 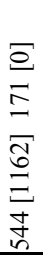 & 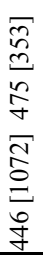 \\
\hline 竞 & 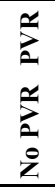 & $\begin{array}{l}\sqrt{n} \\
\infty \\
\stackrel{\sigma}{\simeq} \\
\simeq\end{array}$ & 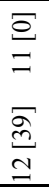 & $\begin{array}{l}\underset{\infty}{\Xi} \\
\underset{d}{d} \\
\sigma\end{array}$ & $\begin{array}{l}\sqrt{n} \\
\sim \\
\vdots \\
\vdots \\
a\end{array}$ & 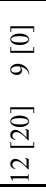 & 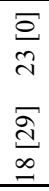 & $\begin{array}{l}\underset{\Xi}{J} \\
\Xi\end{array}$ & $\begin{array}{l}\text { o } \\
\text { 인 }\end{array}$ & $\begin{array}{l}\curvearrowleft \\
\Xi \\
\Xi\end{array}$ & $\underset{\infty}{\Xi}$ \\
\hline 言 & $\begin{array}{l}\frac{1}{2} \\
2 \\
\frac{1}{2} \\
2 \\
z\end{array}$ & $\begin{array}{l}\underset{\sigma}{\sigma} \\
\Xi \\
\Xi \\
\Xi\end{array}$ & 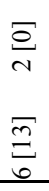 & 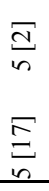 & $\begin{array}{l}\Xi \\
\Xi \\
\sigma \\
\sigma \\
\sigma\end{array}$ & $\underset{\sigma}{\Xi}$ & 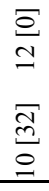 & $\underset{a}{\infty}$ & $\begin{array}{l}\Xi \\
\Xi \\
\Xi\end{array}$ & $\begin{array}{l}\vec{\Xi} \\
m \\
\vec{\sigma} \\
\stackrel{\sigma}{\sigma}\end{array}$ & 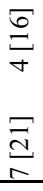 \\
\hline ڤ & 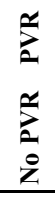 & 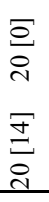 & $\begin{array}{l}\stackrel{*}{\circ} \\
\stackrel{\circ}{\circ} \\
\text { ప্ } \\
\stackrel{\sim}{\circ}\end{array}$ & 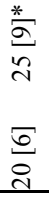 & 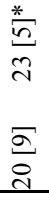 & $\begin{array}{l}\text { 을 } \\
\text { ¿ }\end{array}$ & $\begin{array}{l}\sqrt{2} \\
\approx \\
\sim\end{array}$ & $\begin{array}{l}\sqrt{\Xi} \\
\stackrel{a}{2}\end{array}$ & $\begin{array}{l}\Xi \\
\Xi \\
\end{array}$ & $\begin{array}{l}\sqrt{0} \\
ٍ \\
a\end{array}$ & $\begin{array}{l}\stackrel{\overrightarrow{\mathrm{d}}}{\circ} \\
\stackrel{\mathrm{d}}{ }\end{array}$ \\
\hline 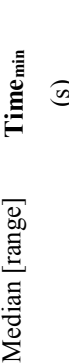 & 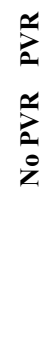 & 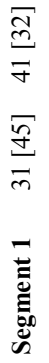 & 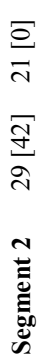 & 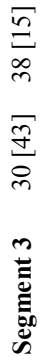 & 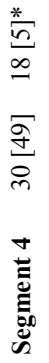 & 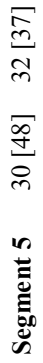 & 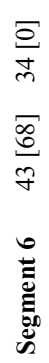 & 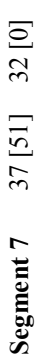 & 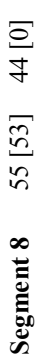 & 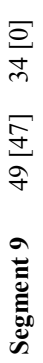 & 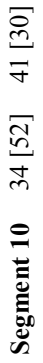 \\
\hline
\end{tabular}




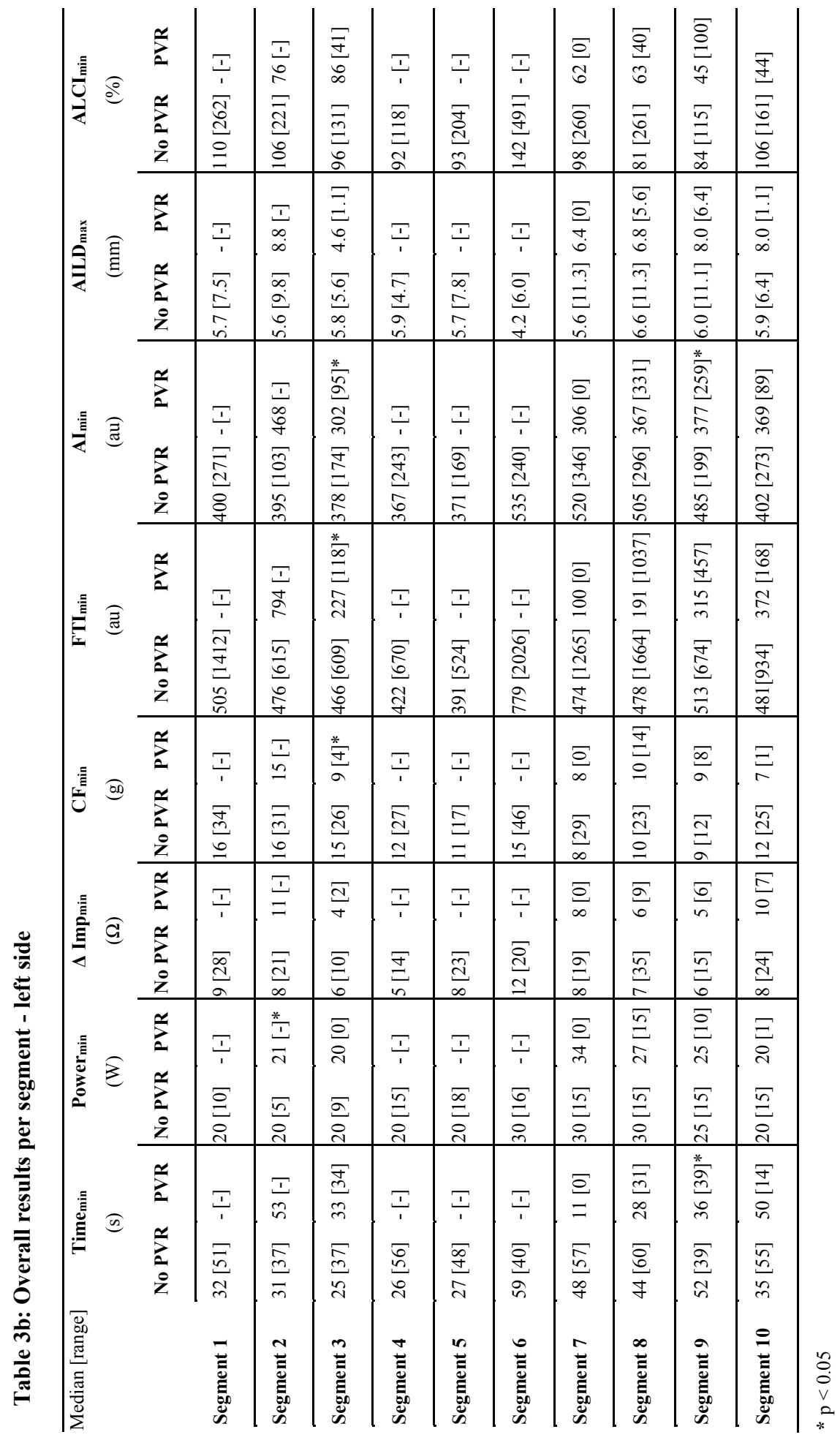




\section{Diagnostic accuracy of transmurality/contiguity criteria to predict a durable segment}

In multivariable analysis, all parameters significantly predicted durability except for time and power.

Overall, ROC curve analysis for all segments together showed the highest diagnostic accuracy for ALCI (area under the curve, AUC = 0.73), compared to $\operatorname{AILD}(0.70, p=0.601), \operatorname{FTI}(0.69, p=0.460)$, $\mathrm{CF}(0.68, \mathrm{p}=0.339), \mathrm{AI}(0.67, \mathrm{p}=0.331)$, and $\Delta-\operatorname{Imp}(0.64, \mathrm{p}=0.126)$.

ROC curve analysis for anterior and posterior wall separately is given in Figure 6. In the lower panels, we plotted the specificity, sensitivity, positive predictive value (PPV), and negative predictive value (NPV) to predict durable segments at known clinical cutoffs.[11,12,14] Both at the anterior and posterior wall, ALCI was invariably associated with the highest specificity and PPV to predict a durable segment.

The relatively low sensitivity of each parameter is evident from the significant overlap in values below or above the cutoffs of segments with and without PVR (Fig. 5).

Anterior wall

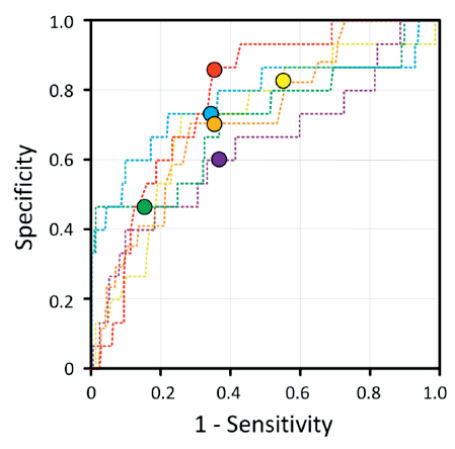

\begin{tabular}{|c|c|c|c|c|c|}
\hline Parameter & $\begin{array}{l}\text { Clinical } \\
\text { cutoff }\end{array}$ & $\begin{array}{c}\text { Specificity } \\
(\%)\end{array}$ & $\begin{array}{c}\text { Sensitivity } \\
(\%)\end{array}$ & $\begin{array}{l}\text { PPV } \\
(\%)\end{array}$ & $\begin{array}{l}\text { NPV } \\
(\%)\end{array}$ \\
\hline$\Delta-I m p_{\min }$ & $>10 \Omega^{14}$ & 82 & 45 & 72 & 60 \\
\hline $\mathrm{CF}_{\text {min }}$ & $>10 \mathrm{~g}^{12}$ & 59 & 65 & 61 & 63 \\
\hline $\mathrm{FTI}_{\text {min }}$ & $>400 \mathrm{gs}^{12}$ & 47 & 84 & 61 & 75 \\
\hline$A I_{\min }$ & $>480 a^{11}$ & 76 & 67 & 74 & 70 \\
\hline ILD ${ }_{\max }$ & $<6 \mathrm{~mm}$ & 71 & 66 & 69 & 67 \\
\hline $\mathrm{ALCl}_{\text {min }}$ & $>100 \%$ & 85 & 66 & 81 & 71 \\
\hline
\end{tabular}

Posterior wall

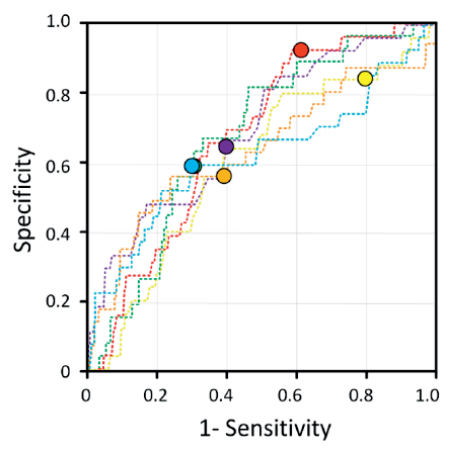

Figure 6: Diagnostic accuracy of transmurality and contiguity parameters to predict durable isolation within a given segment for the anterior wall (left upper panel) and posterior wall (right upper panel). In the lower panels we plotted the specificity, sensitivity, positive predictive value (PPV), and negative predictive value (NPV) to predict durable segments at known clinical cutoffs. [11,12,14]

\begin{tabular}{|c|c|c|c|c|c|}
\hline Parameter & $\begin{array}{l}\text { Clinical } \\
\text { cutoff }\end{array}$ & $\begin{array}{c}\text { Specificity } \\
(\%)\end{array}$ & $\begin{array}{c}\text { Sensitivity } \\
(\%)\end{array}$ & $\begin{array}{l}\text { PPV } \\
\text { (\%) }\end{array}$ & $\begin{array}{l}\text { NPV } \\
(\%)\end{array}$ \\
\hline$\Delta-I m p_{\min }$ & $>10 \Omega^{14}$ & 84 & 21 & 57 & 52 \\
\hline $\mathrm{CF}_{\min }$ & $>10 \mathrm{~g}^{12}$ & 67 & 63 & 66 & 65 \\
\hline $\mathrm{FTI}_{\min }$ & $>400 \mathrm{gs}^{12}$ & 59 & 66 & 62 & 64 \\
\hline$A I_{\min }$ & $>370 \mathrm{au}^{11}$ & 59 & 68 & 62 & 65 \\
\hline ILD $_{\max }$ & $<6 \mathrm{~mm}$ & 56 & 61 & 58 & 59 \\
\hline$A L C I_{\text {min }}$ & $>100 \%$ & 92 & 38 & 83 & 60 \\
\hline
\end{tabular}




\section{DISCUSSION}

\section{Main findings}

This study is the first to objectively show that criteria for both transmurality and contiguity are major determinants of durable PVI in CF guided ablation. The combination of interlesion distance and parameters of lesion depth (power, time and contact force) into a unique variable, the ablation line contiguity index (ALCI), provides the best criterion to predict durable PVI. Therefore, an ALCIguided ablation procedure has the potential to further reduce significantly the need for repeat procedures after ablation for paroxysmal AF.

\section{Time of RF application, power and contact force as targets for RF ablation}

The depth of RF lesions is dependent on the time and delivered power of the RF application.[15-16] The present observation that segments with acute or late PVR are not characterized by shorter time nor minimal power of RF application, can be explained by the nature of the ablation protocol allowing only limited variation in time and power at the anterior and posterior wall. Also contact force (or force-time integral) has been shown as a major determinant of RF lesion volume in experimental studies.[17-19] In the EFFICAS I study, the site of late PVR correlated strongly with minimal CF and FTI. Minimal CF of $20 \mathrm{~g}$ and minimal FTI $>400$ gs at each PV segment correlated with a $95 \%$ probability of durable PV isolation (at 3 months after index procedure).[12] Likewise Park et al showed that a cutoff value of $10 \mathrm{~g}$ CF (and a minimum FTI of $400 \mathrm{gs}$ ) has high sensitivity and specificity for acute durability of PVI.[20] Our data confirm that segments with acute or late PVR are characterized by RF applications with lower minimal CF and FTI. However our data suggest that FTI $>400$ gs is associated with a poor accuracy to predict durable segments (specificity of $47 \%$ at anterior wall and $59 \%$ at the posterior wall). Other limitations speak against the use of CF and FTI to guide point-by-point RF ablation for PVI. Practically it is impossible to reach 10 to $20 \mathrm{~g}$ at each RF site (as evidenced by the current distribution of CF values) and, by targeting $20 \mathrm{~g}$, one might introduce catheter dislocation, unnecessary tissue compression, and longer procedure time. Moreover calculation of contact force (and FTI) might be inaccurate due to inadequate calibration, contact with neighboring electrodes or the angle of incidence (underestimation when parallel contact).[21] Finally, FTI as a marker of lesion quality suffers from two major limitations: first, FTI (calculated as the product of force by application time) suggests a linear lesion formation during ablation whereas it is known that lesion volume growth describes a mono-exponential curve.[15] Second, FTI ignores the important and evident role of power delivery in lesion creation. Guerra et al. showed that lesions with an FTI value of $300 \mathrm{gs}$ at $35 \mathrm{~W}$ had a volume more than three times that of the lesion created with $20 \mathrm{~W}$.[22] As such it was surprising that FTI 400gs was found to be predictive for gaps in a multi-center study in which power was reported to vary from 10 to $40 \mathrm{~W}$.[12] 


\section{Impedance drop}

In experimental studies, initial impedance decrease is a good correlate for RF lesion volume [15-19] and in patients, initial impedance decrease is an indicator of good catheter contact.[23] Chinitz et al showed that areas of late PVR were associated with sites with small impedance decrease $(<10 \Omega)$ during point-by-point PVI.[14] The present data - obtained in exclusively paroxysmal AF undergoing only CF-guided PVI - confirm these findings and imply that obtaining/targeting a minimal impedance decrease of $>10 \Omega$ is likely to result in a durable segment. The fact that impedance decrease is often an observation (rather than a modifiable parameter) speaks against the use of impedance decrease as an optimal ablation target.

\section{Ablation index and its putative role as ablation target values}

Ablation index is based upon the experimental work of Nakagawa et al.[10] This index, incorporating contact force, power and time in a weighted formula, predicted lesion depth in the canine ventricle with high accuracy. By taking into account the greater contribution of power (over contact force) and of the initial time of ablation, it differs from force-power-time integral calculated at the multiplication of force, power and time.[20] Gupta el al recently showed that ablation index correlated with impedance drop and that minimal ablation index within a segment was predictive of reconnection at that segment at repeat EP study.[11] Our data confirm that there is a significant difference in minimal AI values required to obtain durable segments in the anterior part of the circle ( $>644 \mathrm{au}$ ) compared with the posterior segments $(>437 \mathrm{au})$, suggestive of thicker atrial tissue in anterior regions. In the present study the accuracy of AI was at the higher end of the spectrum but in absolute values not so much higher compared to CF and FTI. AI-guided ablation might have the following advantages; (1) the target can be reached readily at each site within the circle (e.g. by increasing power at sites with low but stable contact). (2) In contrast to FTI, lesion quality is expected to be similar at a certain AI value (despite differences in power, force and time of application required to reach that value). Several observations speak against the ablation index as the sole target: (1) it does not take into account characteristics of underlying atrial tissue (composition, thickness), (2) it ignores the importance of lesion contiguity.

\section{Contiguity of RF applications}

One of the key findings of this study is that interlesion distance is predictive of reconnection of that segment. Automated interlesion distance is a new and objective criterion defined as the center-tocenter distance between two, automatically acquired, neighboring RF tags. Our data suggest that 
targeting AILD's of $<5 \mathrm{~mm}$ might be the 'missing link' towards durable PVI. Prior studies already hinted to the importance of contiguous lesions to obtain acute durable isolation. Reddy et al showed that closing the "visual" gap was essential for acute durable PVI, [24] whereas Park et al showed that acute PVR despite CF $\geq 10 \mathrm{~g}$ was explained by ablation sites with a long interlesion distance $(\geq 5$ $\mathrm{mm}$ ).[20] Those studies however were limited by the fact that RF tags were taken manually throughout the procedure, whereas in the present study tags were acquired automatically based upon preset criteria of force and stability (Visitag $\left.{ }^{\circledR}\right)$. The observation that segments can be durable despite higher AILD values (limited sensitivity), might be explained by inaccuracy in 3D electro-anatomical mapping, or the presence of underlying tissue not relevant to atrio-venous conduction.

\section{Ablation line contiguity index}

In the present study we introduced ablation line contiguity index (ALCI), as a novel marker to detect and/or avoid the weakest link in the ablation chain. Based upon AI and AILD, this index combines contiguity and transmurality to assess integrity of the PV circle. In a multivariable model, ALCI remained independently predictive of segment reconnection. Moreover ROC curve analysis suggested that ALCI (target $\approx 100 \%$ ) has the highest accuracy to predict durable segments. ALCI as ablation target has the following advantages; 1) ALCI cutoff value is similar for the anterior and posterior wall. 2) In keeping with the concept that the chain of ablation lesions is only as strong as its weakest link, we hypothesize that integrity of the complete RF circle can be quantified by one single, fullyautomated value (i.e. lowest ALCI value within the circle). As such it is expected to enhance outcome (and its reproducibility) after CF guided ablation. Obvious limitations are that it is dependent on CF, AI, AILD (and their inherent disadvantages) and that it still oversimplifies the holistic nature of lesion formation in atrial tissue.

\section{Clinical implications}

We hypothesize that AILD and ALCI-guided ablation will increase freedom of AF after CF-guided PVI. Whether this will be associated with higher reproducibility in outcome and safety, is currently being evaluated in a multi-center study. The present findings are an impetus for further research: the moderate sensitivity of ablation targets points to oversimplification of the holistic nature of lesion formation an points to ignoring intra- and inter-patient variation.

\section{Limitations}

Sample size of this study was small, which limits the strength of the analysis per segment. The chosen analysis methodology did not take clustering of effects by patients into account. Finally it is possible 
that segments were misclassified as durable, knowing that some patients free of AF (not undergoing repeat ablation) have reconnected PVs.

The present clinical cutoffs are all associated with moderate sensitivity. This implies that RF applications with values below the target (or above in case of AILD) might still be associated with durability. As such, cutoff-guided ablation strategies might result in durable isolation at the cost of overshoot and potential safety issue. This needs to be evaluated in prospective clinical studies.

\section{Conclusions}

Minimal ablation line contiguity index, a novel ablation quality marker integrating interlesion distance into a weighed formula, is independently predictive of PV reconnection. Similar ALCI values are required to prevent reconnection of anterior and posterior segments. ALCI-guided ablation warrants prospective evaluation for improving freedom of AF. 


\section{REFERENCES}

1. Calkins H, Kuck KH, Cappato R, et al.; Heart Rhythm Society Task Force on Catheter and Surgical Ablation of Atrial Fibrillation. 2012 HRS/EHRA/ECAS Expert Consensus Statement on Catheter and Surgical Ablation of Atrial Fibrillation. Heart Rhythm 2012;9:632-696.

2. Kuck KH, Brugada J, Fürnkranz A, et al.; FIRE AND ICE Investigators. Cryoballoon or Radiofrequency Ablation for Paroxysmal Atrial Fibrillation. N Engl J Med 2016;374:2235-2245.

3. Cappato R, Negroni S, Pecora D, et al. Prospective assessment of late conduction recurrence across radiofrequency lesions producing electrical disconnection at the pulmonary vein ostium in patients with atrial fibrillation. Circulation 2003;108:1599-1604.

4. Nanthakumar K, Plumb VJ, Epstein AE, et al. Resumption of electrical conduction in previously isolated pulmonary veins: rationale for a different strategy? Circ 2004;109:1226-1229.

5. Ouyang F, Tilz R, Chun J, et al. Long-term results of catheter ablation in paroxysmal atrial fibrillation: lessons from a 5-year follow-up. Circulation 2010;122:2368-2377.

6. Andrade JG, Monir G, Pollak SJ, et al. Pulmonary vein isolation using contact force ablation: The effect on dormant conduction and long-term freedom from recurrent atrial fibrillation. A prospective study. Heart Rhythm 2014;11:1919-1924.

7. Marijon E, Fazaa S, Narayanan K, et al. Real-time contact force sensing for pulmonary vein isolation in the setting of paroxysmal atrial fibrillation: procedural and 1-year results. $\mathrm{J}$ Cardiovasc Electrophysiol 2014;25:130-137.

8. Afzal MR, Chatta J, Samanta A, et al. Use of contact force sensing technology during radiofrequency ablation reduces recurrence of atrial fibrillation: A systematic review and metaanalysis. Heart Rhythm 2015;12:1990-1996.

9. Ullah W, McLean A, Tayebjee MH, et al.; UK Multicentre Trials Group. Randomized trial comparing pulmonary vein isolation using the SmartTouch catheter with or without real-time contact force data. Heart Rhythm 2016;13:1761-1767.

10. Nakagawa $\mathrm{H}$, Ikeda A, Constantine G, et al. Controlling lesion size and incidence of steam pop by controlling contact force, radiofrequency power and application time (Force-Power-Time Index) in canine beating heart. Heart Rhythm 2012; 9:S5.

11. Das M, Loveday JJ, Wynn GJ, et al. Ablation index, a novel marker of ablation lesion quality: prediction of pulmonary vein reconnection at repeat electrophysiology study and regional differences in target values. Europace. 2016 May 31. pii: euw105. [Epub ahead of print]

12. Neuzil P, Reddy VY, Kautzner J, et al. Electrical reconnection after pulmonary vein reconnection is contingent on contact force during initial treatment: results from the Efficas I study. Circ Arrhythm Electrophysiol 2013;6:327-333.

13. Kautzner J, Neuzil P, Lambert H, et al. Optimization of catheter contact force improves outcome of pulmonary vein isolation for paroxysmal atrial fibrillation (EFFICAS II). Europace 2015;17:1229-1235.

14. Chinitz JS, Kapur S, Barbhaiya C, et al. Sites With Small Impedance Decrease During Catheter Ablation for Atrial Fibrillation Are Associated With Recovery of Pulmonary Vein Conduction. J Cardiovasc Electrophysiol. 2016 Sep 1. doi: 10.1111/jce.13095. [Epub ahead of print] 
15. Nath and Haines. Biophysics and Pathology of Catheter Energy Delivery Systems. Progress in Cardiovascular Diseases, Vol XXXVII, No 4 (January/February), 1995: pp 185-204.

16. Wittkampf FH, Nakagawa H. Radiofrequency catheter ablation: lessons on lesions. Pacing Clin Electrophysiol 2006;29:1285-1297.

17. Yokoyama K, Nakagawa H, Shah DC, et al. Novel contact force sensor incorporated in irrigated radiofrequency ablation catheter predicts lesion size and incidence of steam pop and thrombus. Circ Arrhythm Electrophysiol 2008;1:354-362.

18. Ikeda A, Nakagawa $\mathrm{H}$, Lambert $\mathrm{H}$, et al. Relationship between catheter contact force and radiofrequency lesion size and incidence of steam pop in the beating canine heart: electrogram amplitude, impedance, and electrode temperature are poor predictors of electrode-tissue contact force and lesion size. Circ Arrhythm Electrophysiol 2014;7:1174-1180.

19. Shah DC, Lambert H, Nakagawa H, et al. Area under the real-time contact force curve (forcetime integral) predicts radiofrequency lesion size in an in vitro contractile model. J Cardiovasc Electrophysiol 2010;21:1038-1043.

20. Park CI, Lehrmann H, Keyl C, et al. Mechanisms of pulmonary vein reconnection after radiofrequency ablation of atrial fibrillation: the deterministic role of contact force and interlesion distance. J Cardiovasc Electrophysiol 2014;1:1-8.

21. Bourier F, Hessling G, Ammar-Busch S, et al. Electromagnetic Contact-Force Sensing Electrophysiological Catheters: How Accurate is the Technology? J Cardiovasc Electrophysiol 2016;27:347-350.

22. Guerra JM, Jorge E, Raga S, et al. Effects of open-irrigated radiofrequency ablation catheter design on lesion formation and complications: in vitro comparison of 6 different devices. $\mathrm{J}$ Cardiovasc Electrophysiol 2013;24:1157-1162.

23. Knecht S, Reichlin T, Pavlovic N, et al. Contact force and impedance decrease during ablation depends on catheter location and orientation: insights from pulmonary vein isolation using a contact force-sensing catheter. J Interv Card Electrophysiol 2015;43:297-306.

24. Miller MA, d'Avila A, Dukkipati SR, et al. Acute electrical isolation is a necessary but insufficient endpoint for achieving durable PV isolation: the importance of closing the visual gap. Europace 2012;14:653-660. 


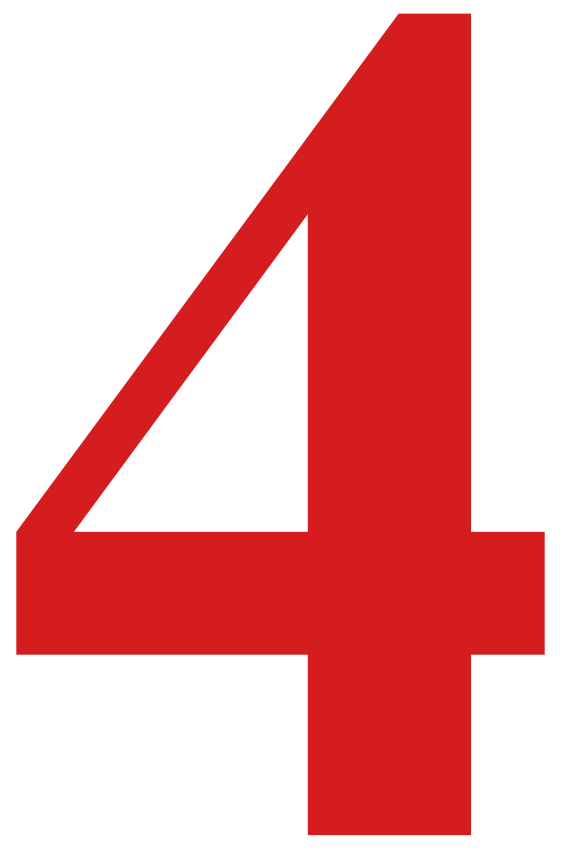




\section{Chapter 4}

\section{Comparison of CLOSE-PVI to conventional contact force-guided PVI}

Thomas Phlips, Philippe Taghji, Milad El Haddad, Michael Wolf, Sébastien Knecht, Yves Vandekerckhove, René Tavernier, Mattias Duytschaever 


\begin{abstract}
Background: We have recently shown that a contact force (CF) guided ablation protocol respecting region-specific criteria of lesion contiguity and lesion depth ('CLOSE' protocol) is associated with high incidence of acute durable pulmonary vein (PV) isolation and a high single-procedure arrhythmia-free survival at 1 year. In the present study, we compared efficiency, safety and efficacy of 'CLOSE'-guided PVI to conventional CF guided PVI.
\end{abstract}

Methods: 50 consecutive paroxysmal atrial fibrillation (AF) patients underwent PV encircling using a CF-sensing catheter targeting an interlesion distance (ILD) $\leq 6 \mathrm{~mm}$ and ablation index $\geq 400$ and $\geq 550$ at posterior and anterior wall ('CLOSE' group). Results were compared to the last 50 patients undergoing conventional CF-guided PVI ('CONV-CF' group). All patients underwent adenosine testing after PVI. Arrhythmia recurrence was defined as any atrial tachyarrhythmia (ATA) $>30$ s on Holter at 3, 6 and 12 months.

Results: Clinical characteristics did not differ. Contact force variability was comparable in between both groups (proportion of applications with intermittent contact $2 \%$ in 'CLOSE' vs $1 \%$ in CONV-CF, $\mathrm{p}=0.67$ ). In the 'CLOSE' group, procedure time and RF time per circle were shorter (respectively $149 \pm 33 \mathrm{~min}$ vs $192 \pm 42 \mathrm{~min}, \mathrm{p}<0.0001$ and $18 \pm 4 \mathrm{~min}$ vs $28 \pm 7.5 \mathrm{~min}, \mathrm{p}<0.0001$ ) and incidence of adenosine-proof isolation was higher $(97 \%$ vs $82 \%, \mathrm{p}<0.001)$. No complications were observed in the 'CLOSE' group, 1 tamponade in the 'CONV-CF' group. At 12 months, single-procedure freedom from ATA was $94 \%$ in 'CLOSE' vs $80 \%$ in 'CONV CF' group $(\mathrm{p}<0.05)$. In both groups, the majority of reconnections at repeat were associated with either ILD $>6 \mathrm{~mm}$ and/or $\mathrm{AI}<400 / 550(100 \%$ vs $83 \%$, $\mathrm{p}=0.99$ ).

Conclusions: 'CLOSE'-guided PVI improves procedural and 1-year outcome in CF-guided PVI while shortening procedure time. Improvement cannot be explained by differences in $\mathrm{CF}$ variability and is most likely due to the strict application of criteria for contiguity and ablation index. A randomized controlled trial is needed to exclude the possible contribution of a learning curve. 


\section{Introduction}

Pulmonary vein isolation (PVI) is the cornerstone for successful treatment of paroxysmal atrial fibrillation (AF) ${ }^{1,2}$ We have recently shown that a contact force (CF)-guided ablation protocol aiming to enclose the veins with contiguous (interlesion distance $\leq 6 \mathrm{~mm}$, ILD) and optimized RF lesions (ablation index $\geq 400 / 550, \mathrm{AI}$ ) is associated with a high incidence of acute durable PV isolation and a high single-procedure arrhythmia-free survival at 1 year (the 'CLOSE' protocol).,

Randomized data on the superiority of 'CLOSE'-guided PVI are lacking. In the present study, we provided comparative data on the efficiency, safety and efficacy of 'CLOSE'-guided PVI to a historical control group of conventional CF guided PVI.

\section{Methods}

\section{Study subjects}

From August 2013 all patients undergoing CF-guided ablation for AF at the St-Jan Hospital Bruges are followed in a prospective database approved by the local institutional review committee. This database implies collecting patients' written informed consent, a detailed case report form of the procedure and follow-up with Holter-ECGs at 3, 6 and 12 months. In this manuscript we present the analysis of the first 50 cases performed according to the 'CLOSE'-criteria. Results were compared to the last 50 cases performed with conventional CF-guided PVI (in which the operator was blinded to $\mathrm{AI}$ and no formal ILD criterion was put forward).

\section{'CLOSE'-guided PVI}

PVI was performed by 4 operators. In anaesthesized patients esophageal temperature monitoring (SensiTherm ${ }^{\mathrm{TM}}$, St Jude Medical Inc, 76 Minnesota, US) was performed. To avoid discomfort, we did not use temperature monitoring under conscious sedation. Intravenous heparin (100IU/kg) was administered and continuously infused (ACT $>300 \mathrm{sec})$. After transseptal puncture, a decapolar Lasso catheter and a CF catheter (Thermocool SmartTouch ${ }^{\circledR}$, Biosense-Webster Inc., Diamond Bar, CA, USA) were positioned in the left atrium and calibration of CF and respiratory gating were performed. Then we predefined the RF circle around the PVs (nephroid shape) on the 3D-geometry (Carto System ${ }^{\circledR}$, Biosense Webster Inc.). Point-by-point RF delivery was performed during sinus rhythm aiming for a contiguous circle enclosing the veins. Real-time automated display of RF applications (Visitag ${ }^{\circledR}$, Biosense Webster Inc.) was used with predefined settings of catheter stability ( $3 \mathrm{~mm}$ for $8 \mathrm{sec}$ ) and minimum CF (30\% of time $>4 \mathrm{gr}$ ). RF was delivered (EP Shuttle ST-3077, Stockert GmbH, Freiburg, Germany) in a power-controlled mode (without ramping) aiming for $35 \mathrm{~W}$ (irrigation flow up to $30 \mathrm{cc} / \mathrm{min}$ ) irrespective of posterior or anterior ablation. Reasons for occasional lowering of RF power were high $\mathrm{CF}$ at baseline $(\geq 30 \mathrm{~g})$ and increase in the tip $\mathrm{T}^{\circ}$ during $\mathrm{RF}\left(>40^{\circ}\right)$. $\mathrm{RF}$ was delivered until an $\mathrm{AI}$ of $\geq 400$ at the posterior wall/roof and $\geq 550$ at the anterior wall (Fig.1, right panel). Once the target AI was reached, the RF application was stopped and the catheter was moved to an adjacent 
spot. Only after verifying stability, contact force and ILD, a new application was started (no catheter dragging). In case of instability leading to an RF tag not reaching the AI target, a new RF application reaching the AI target was applied. Maximal ILD between two neighboring lesions was $\leq 6 \mathrm{~mm}$ (Fig.1, right panel). In case of chest pain or intra-esophageal $\mathrm{T}^{\circ}$ rise $>38.5^{\circ} \mathrm{C}$ during posterior wall ablation, RF delivery was stopped at an AI of 300 (but not before). In the absence of first-pass isolation (i.e. no isolation after completing the circle), touch-up ablation was delivered until PVI. After PVI we waited for 20 minutes after which adenosine (dose resulting in atrio-ventricular block) was given 4 times (with the Lasso in its corresponding position). In case of reconnection during waiting time or during adenosine (acute PVR), the site of reconnection was allocated and treated with touch-up ablation until PVI was resistant to subsequent adenosine challenge. In case of pre-procedural or procedural documentation of typical flutter, cavotricuspid isthmus (CTI) ablation was performed until bidirectional block.

\section{Conventional CF-guided PVI procedure}

Ablation was performed by the same 4 operators, using the same anesthesia protocol, transseptal access and catheters. Also identical were calibration of CF catheter, respiratory gating, reconstructing of 3D-geometry, the predefined nephroid RF circle, Visitag ${ }^{\circledR}$ settings, RF generator, and RF mode. In contrast to 'CLOSE', RF was delivered for $30 \mathrm{sec}$ (at $25 \mathrm{~W}$ ) at the posterior wall and roof, and for 60 $\sec ($ at $35 \mathrm{~W}$ ) at the anterior wall. According to data from the EFFICAS studies, CF target was $>10 \mathrm{~g}$ (Fig.1, left panel). No specific force-time-integral (FTI) threshold nor strict criteria for contiguity were targeted. AI was not available to the operators at the time of the procedure. In case of chest pain or intra-esophageal $\mathrm{T}^{\circ}$ rise $>38.5^{\circ} \mathrm{C}$ during posterior wall ablation, RF delivery was stopped prematurely. Assessment and management of first-pass isolation and acute PVR was identical as for the 'CLOSE' group. CTI ablation was performed if appropriate.

\section{Conventional CF guided PVI}

\section{CLOSE guided PVI}
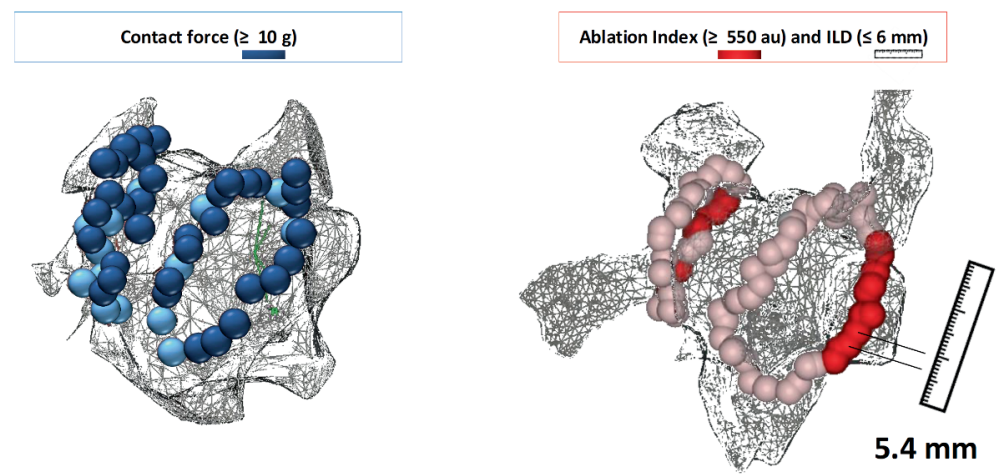

Figure 1: Left panel: Conventional CF-guided PVI group, Right panel: 'CLOSE'-guided PVI. See text for explanation. In the 'CLOSE' protocol, $42 \%$ of circles are characterized by one or more applications with an AI target of 300 at the posterior wall. PVI $=\mathrm{PV}$ isolation, ILD=interlesion distance. 


\section{Off-line analysis of criteria to assess integrity of the deployed RF circle}

Each procedure was exported and analyzed offline. For each RF tag within the circle we determined time of application (s), median delivered power (W), impedance drop (D-Imp, $\Omega$ ), average CF (CF, g), force-time integral (FTI, gs) and AI (arbitrary unit, au). Custom-made software was used to automatically determine the path of encircling, the perimeter of the RF circle, ILD (center-to-center distance, $\mathrm{mm}$ ) and ablation line contiguity index (ALCI). ALCI was developed as a criterion to algorithmically score the combination of contiguity and lesion depth and is defined as the ratio of the estimated width of a transmural lesion (determined by the AI) over its distance (ILD) to the closest transmural lesion.

To determine the weakest link in a given RF circle, each circle was divided into 10 segments, each encompassing 3 to 5 RF tags. [13] For each segment we determined for each measure the 130 minimal $\left(\right.$ Time $_{\text {min }}$, Power $\left._{\text {min }}, \mathrm{D}^{-\mathrm{Imp}_{\min }}, \mathrm{CF}_{\min }, \mathrm{FTI}_{\min }, \mathrm{AI}_{\min }, \mathrm{ALCI}_{\min }\right)$ or maximal value $\left(\mathrm{ILD}_{\max }\right)$. In the CF group 10 procedures were not available for off-line analysis.

We analyzed CF variability using custom-made algorithm (Fig.2). For each application thereconstructed instantaneous $\mathrm{CF}$ curve and histogram were analyzed. ${ }^{5,6}$ Constant contact was defined as $\mathrm{CF} 60 \%$ variability of absolute $\mathrm{CF} \leq 10 \mathrm{~g}$. Variable contact was defined as the $\mathrm{CF} 60 \%$ variability of absolute $\mathrm{CF}>10 \mathrm{~g}$. Intermittent contact was classified when the percentage of absolute $\mathrm{CF}$ dropping below $3 \mathrm{~g}$ is more than $25 \%$ of the application.
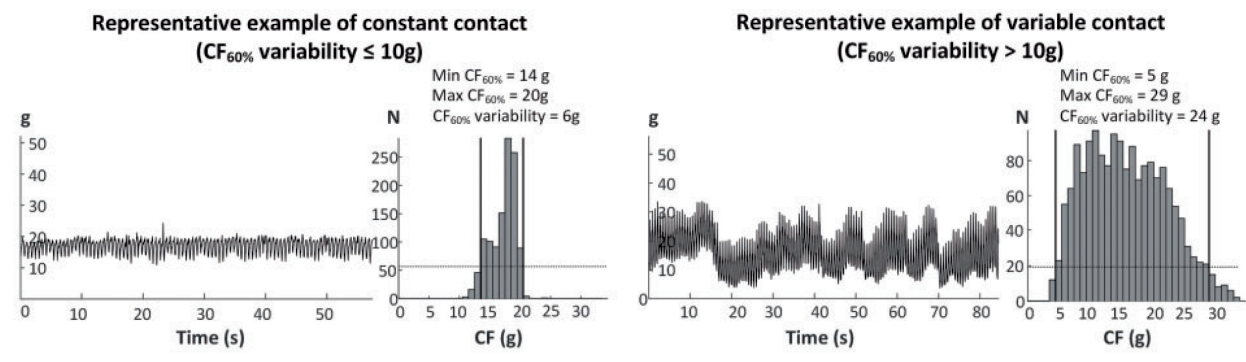

Representative example of intermittent contact (variable contact and $\mathbf{2} \mathbf{2 5} \%$ CF less than $\mathbf{3 g}$ )

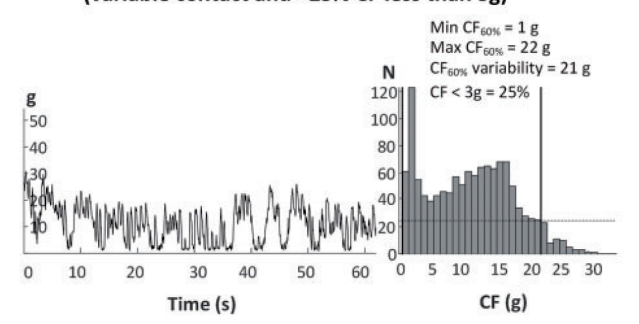

Proportion of stable, variable and intermittent contact

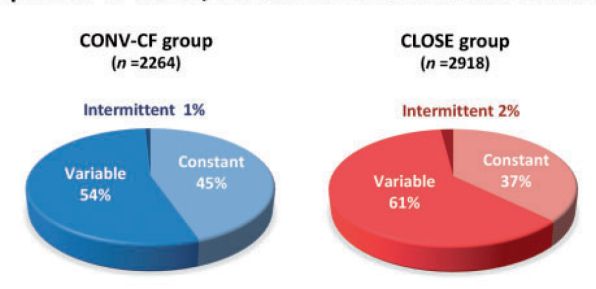

Figure 2: Representative examples of applications with constant contact, variable contact and intermittent contact. Right lower panel: the proportion of applications with intermittent contact is equally low in both groups. $\mathrm{CF}$, contact force; CONV-CF, conventional contact force. 


\section{Follow-up}

Complications were reported on the CRF and collected during follow-up. After ablation, anticoagulation and previously failing anti-arrhythmic drug therapy (ADT) were continued. At 3 months, anticoagulation was continued according to stroke risk, whereas ADT was continued at the discretion of the treating physician. Clinical evaluation and ECG were performed at 1, 3, 6 and 12 months. Holter-ECG was performed at 3 months (24h), 6 months (24h) and 12 months (7 day) or in case of symptoms. Freedom from recurrence was defined as 1 -year absence from AF/AT/AFL >30 seconds beyond a 3-month blanking period.

\section{Statistical analysis}

Normality of data distribution was tested with Shapiro-Wilk test. Continuous variables are expressed as mean $\pm \mathrm{SD}$ if normally distributed, medians with IQR if non-normally distributed, and dichotomous variables as percentage. Respectively, variables were compared using Student's t test, Mann-Whitney U test, and Fisher's exact test. Linear mixed model tests were used to evaluate difference of ablation parameters between the CF-group versus the CLOSE group. Kaplan-Meier estimate was used to compare freedom from AF between both groups during 12 months follow-up period. Multivariable logistic regression was used to assess the association between the different parameters and PVR. P values $<0.05$ were considered statistically significant. All statistical analyses were performed in SPSS Statistics (IBM Corporation, Armonk, New York, USA).

\section{Results}

\section{Patient characteristics}

All patients had paroxysmal AF. There were no differences in baseline clinical characteristics between two groups (Table 1). 
Table 1. Patient characteristics

\begin{tabular}{|c|c|c|c|}
\hline & $\begin{array}{l}\text { Conventional CF group } \\
\qquad(n=50)\end{array}$ & $\begin{array}{l}\text { CLOSE group } \\
(n=50)\end{array}$ & $p$ value \\
\hline Paroxysmal AF & $50(100 \%)$ & $50(100 \%)$ & $>0.99$ \\
\hline Age, $y$ & $62 \pm 10$ & $61 \pm 11$ & 0.466 \\
\hline Male, n & $31(62 \%)$ & $36(72 \%)$ & 0.395 \\
\hline BMI, $\mathrm{kg} / \mathrm{m}^{2}$ & $26.6 \pm 4.3$ & $27.0 \pm 3.7$ & 0.678 \\
\hline Arterial hypertension, $n$ & $20(40 \%)$ & $15(30 \%)$ & 0.402 \\
\hline Structural heart disease, $n$ & $7(14 \%)$ & $3(6 \%)$ & 0.318 \\
\hline Diabetes, n & $0(0 \%)$ & $2(4 \%)$ & 0.495 \\
\hline $\mathrm{CHA}_{2} \mathrm{DS}_{2} \mathrm{VASc}$ score $\geq 2, \mathrm{n}$ & $25(50 \%)$ & $16(32 \%)$ & 0.068 \\
\hline Left atrial diameter, mm & $40.4 \pm 4.4$ & $39.9 \pm 3.9$ & 0.597 \\
\hline Electrical cardioversion, $n$ & $7(14 \%)$ & $3(22 \%)$ & 0.436 \\
\hline $1^{\text {st }}$ AF episode to PVI, months & $24[12-72]$ & $24[12-84]$ & 0.623 \\
\hline Resistance to ADT, n & $47(94 \%)$ & $45(90 \%)$ & 0.715 \\
\hline
\end{tabular}

\section{$R F$ characteristics and integrity of the $R F$ circle}

Two representative cases illustrating the difference in RF parameters and integrity of the deployed RF circles are given in Fig.3. Compared to the conventional CF-guided procedure (left panel), the 'CLOSE' procedure (right panel) is characterized by applications with shorter duration (ranging from 11 to $61 \mathrm{~s})$, a higher rate of applications with high power $(\geq 30 \mathrm{~W})$, a higher rate of RF tags with a marked impedance drop ( $\geq 7 \mathrm{~W}$ ), applications with lower FTI values (ranging from 184 to $875 \mathrm{gs}$ ) and a higher rate of applications with an $\mathrm{AI}$ of $\geq 550$. The range of obtained $\mathrm{CF}$ values ( 5 to $37 \mathrm{~g}$ ) is not different from the conventional CF case. Finally, whereas the perimeter of the circles is comparable, the 'CLOSE'-guided circles are characterized by enclosing lesions all with 172 and ILD $\leq 6 \mathrm{~mm}$ and an ALCI of $>100 \%$. 


\section{Conventional CF guided PVI}
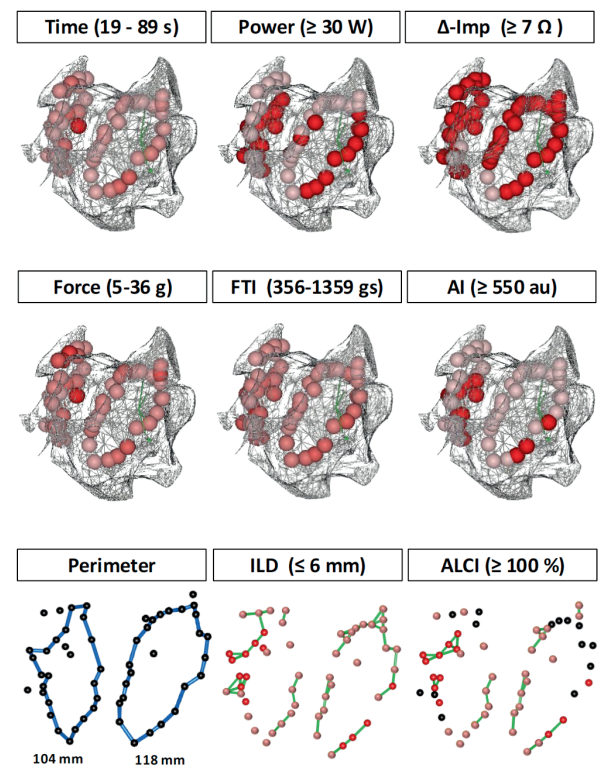

\section{CLOSE guided PVI}
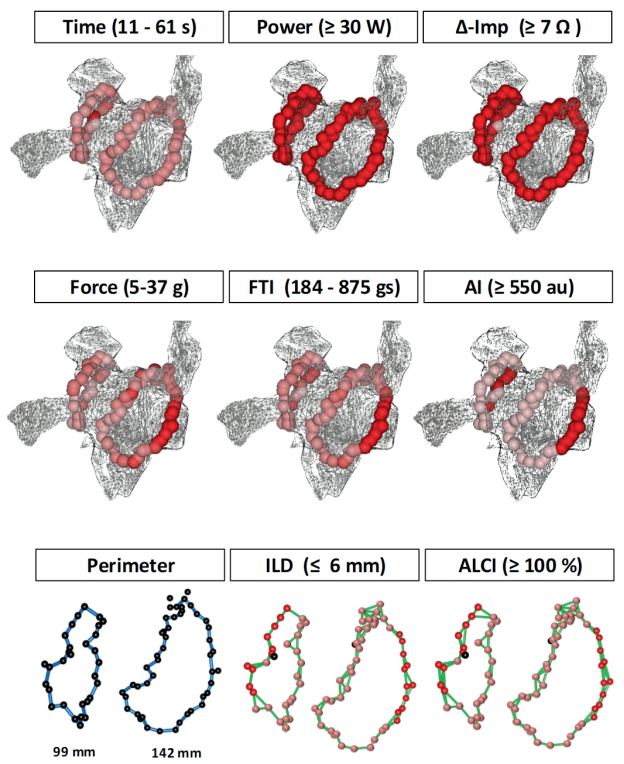

Figure 3: RF characteristics obtained during CF-guided PVI procedure (left panels) vs 'CLOSE'-guided PVI procedure (right panels). The deployed circles are represented color-coded (dichotomous or continuous) for each of the following criteria: application time, power, impedance drop (Delta-Imp), contact force (CF), force-time integral (FTI), ablation index (AI), perimeter, interlesion distance (ILD) and ablation index contiguity index (ALCI). See text for further explanation.

Weakest link analysis for all segments is summarized in Fig.4. Compared to the CF group, segments in the 'CLOSE' group are characterized by lower application time $\min _{\min }(\mathrm{p}<0.001)$, higher delivered power $_{\min }(\mathrm{p}<0.001)$, higher obtained $\Delta$-Imp $_{\min }(\mathrm{p}<0.001)$, similar $\mathrm{CF}_{\min }(\mathrm{p}=0.401)$, lower $\mathrm{FTI}_{\min }$ $(p<0.001)$, higher $\mathrm{AI}_{\min }(\mathrm{p}<0.001)$, lower $\operatorname{ILD}_{\max }(\mathrm{p}=0.032)$, and higher $\mathrm{ALCI}_{\min }(\mathrm{p}<0.001)$. RF characteristics for the posterior and anterior segments separately are illustrated in the scatter plots.

Compared to the CF group, applications in the 'CLOSE' group were characterized by the same proportion of applications with intermittent contact ( $2 \% \mathrm{vs} 1 \%, \mathrm{p}=0.67)$ (Figure 2, right panels).

In the 'CLOSE' group, applications at the posterior wall lasted $23 \pm 6$ s overall. In 42 out of 100 circles (42\%), there was at least one application with an AI target of 300. In those 'low AI183 target' lesions, applications lasted for $13 \pm 4$ s only. 

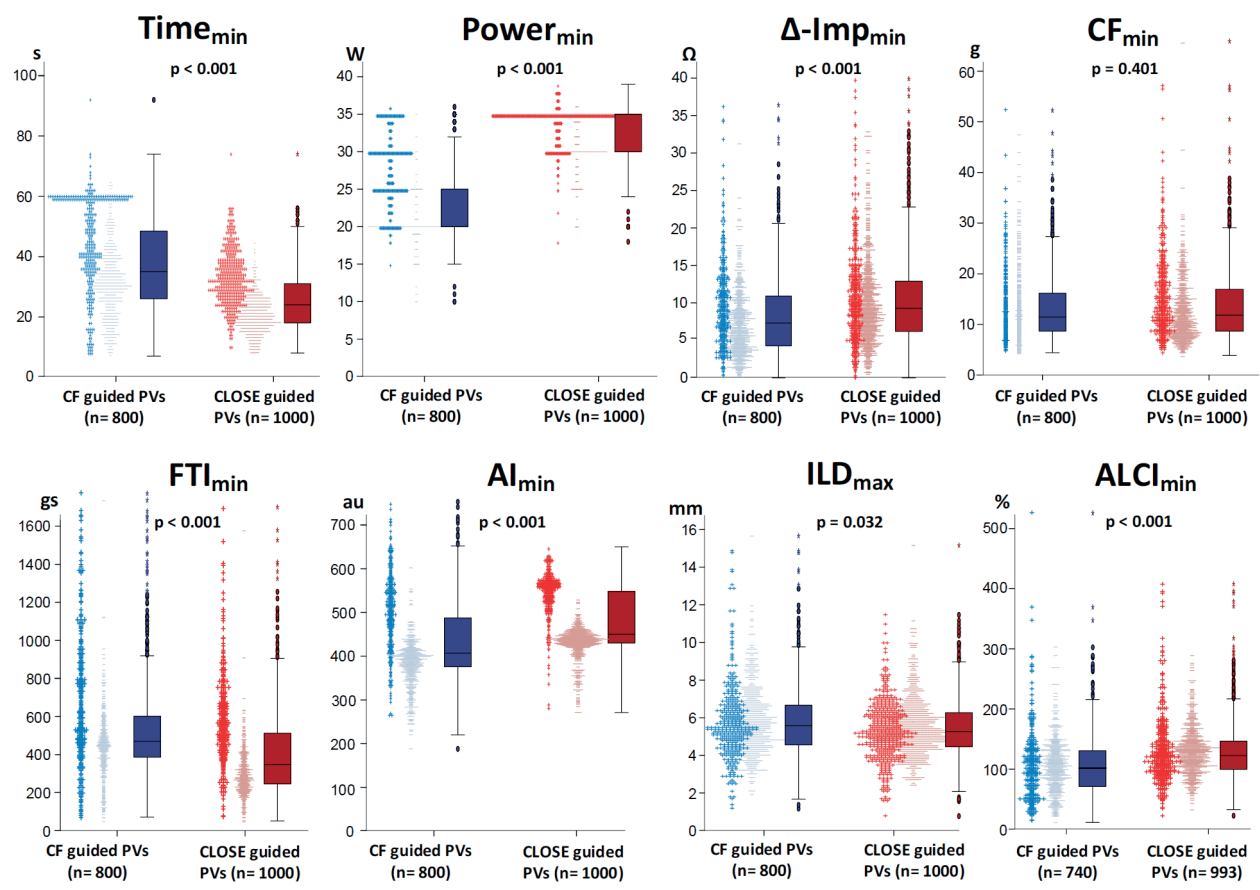

Figure 4: Weakest link analysis. Difference in Time $\mathrm{min}_{\min }, \mathrm{Power}_{\min }, \mathrm{D}-\mathrm{Imp}_{\min }, \mathrm{CF}_{\min }, \mathrm{FTI}_{\min }, \mathrm{AI}_{\min }, \mathrm{ILD}_{\max }$, and $\mathrm{ALCI}_{\text {min }}$ between segments from the CF group (blue box) vs segments from the 'CLOSE' group (red box). Scatter markers represent anterior (blue and red) and posterior (light blue and pink) segments separately. Abbreviations similar to Figure 2. See text for further explanation.

\section{Procedural characteristics}

Characteristics are given in Table 2. PVI was obtained in all circles. CTI ablation was performed in 11 patients in the CF group and 4 in the 'CLOSE' group ( $p=0.091)$. The use of general anesthesia was comparable between groups. Compared to CONV-CF group, 'CLOSE' group was associated with a shorter procedure time $(149 \pm 33 \mathrm{~min}$ vs $192 \pm 42 \mathrm{~min}, \mathrm{p}<0.0001)$ (Fig.5). Perimeter of the right and left circles and number of applications per circle were not different. Compared to CONV-CF group, 'CLOSE' group was associated with shorter RF time per circle ( $17 \pm 3 \mathrm{~min}$ vs $29 \pm 8 \mathrm{~min}, \mathrm{p}<0.0001$ for the right circles, $19 \pm 4 \mathrm{~min}$ vs $26 \pm 6 \mathrm{~min}, \mathrm{p}<0.001$ for the left circles). 
Table 2. Procedure characteristics

\begin{tabular}{lccc}
\hline & $\begin{array}{c}\text { Conventional CF group } \\
(\mathbf{n}=50)\end{array}$ & $\begin{array}{c}\text { CLOSE group } \\
(\mathbf{n}=50)\end{array}$ & p value \\
\hline PV isolation, $\mathbf{n}$ & $50(100 \%)$ & $50(100 \%)$ & $>0.99$ \\
CTI ablation, $\mathbf{n}$ & $11(22 \%)$ & $4(8 \%)$ & 0.091 \\
General anesthesia, $\mathbf{n}$ & $18(36 \%)$ & $25(50 \%)$ & 0.225 \\
Procedure time, min & $192 \pm 42$ & $149 \pm 33$ & $<0.001$ \\
Fluoroscopy time, min & $21.3 \pm 9.0$ & $26.4 \pm 7.8$ & 0.003 \\
Right circles & & & \\
Perimeter, mm & $138 \pm 24$ & $129 \pm 19$ & 0.068 \\
RF tags, $\mathbf{n}$ & $41 \pm 10$ & $38 \pm 8$ & 0.077 \\
RF time, min & $29 \pm 8$ & $17 \pm 3$ & $<0.001$ \\
First-pass isolation, $\mathbf{n}$ & $25(50 \%)$ & $50(100 \%)$ & $<0.001$ \\
Waiting/adeno. proof isolation, $\mathbf{n}$ & $41(82 \%)$ & $49(98 \%)$ & 0.008 \\
Left circles & & & \\
Perimeter, mm & $123 \pm 26$ & $122 \pm 27$ & 0.985 \\
RF tags, $\mathbf{n}$ & $37 \pm 10$ & $40 \pm 10$ & 0.068 \\
RF time, min & $26 \pm 6$ & $19 \pm 4$ & $<0.001$ \\
First-pass isolation, $\mathbf{n}$ & $29(58 \%)$ & $48(96 \%)$ & $<0.001$ \\
Waiting/adeno. proof isolation, $\mathbf{n}$ & $41(82 \%)$ & $48(96 \%)$ & 0.025
\end{tabular}
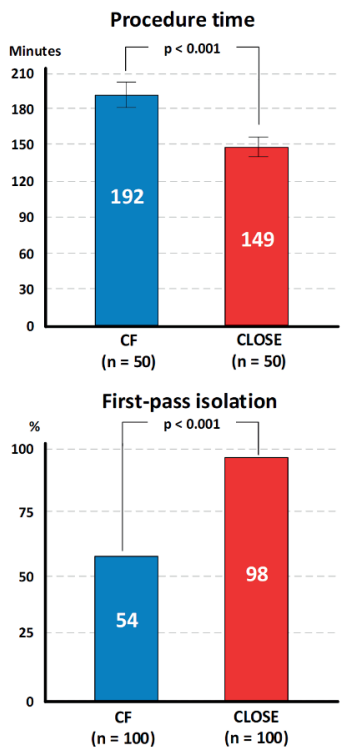

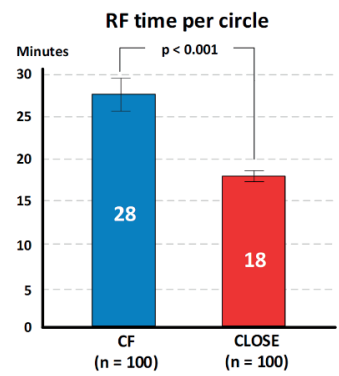

Waiting/adenosine-proof isolation

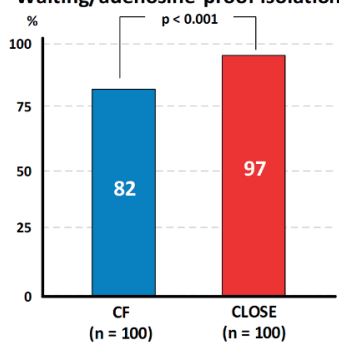

Figure 5: Upper panels. Procedure time and radiofrequency (RF) time per circle. Lower panels. Incidence of first-pass isolation and waiting time/adenosine- proof isolation in both groups. See text for further explanation. 


\section{First-pass and waiting time/adenosine-proof isolation}

Results are summarized in Fig.5. A limited number patients did not receive adenosine because of a history of asthma or COPD (5 in the CONV-CF group vs 3 in the 'CLOSE'-group). Compared to CONV-CF group, 'CLOSE' group was associated with higher incidence of first pass isolation (98\% vs $54 \%, \mathrm{p}<0.0001)$ and waiting time/adenosine-proof isolation ( $97 \%$ vs $82 \%, \mathrm{p}<0.001)$.

\section{1-year freedom from $A F / A T / A F L$}

Kaplan Meier analysis was plotted in Fig.6. At the end of the blanking period 23 patients continued ADT in the CONV-CF group (46\%) vs 18 patients in the 'CLOSE' group (36\%). Overall, singleprocedure 12 month freedom from AF/AT/AFL was $94 \%$ in CLOSE compared to $80 \%$ in CONV-CF group $(\mathrm{p}<0.05)$. Both in patients with and without ADT, 'CLOSE' was associated with a higher freedom from AF/AT/AFL (Fig.6, right panels).

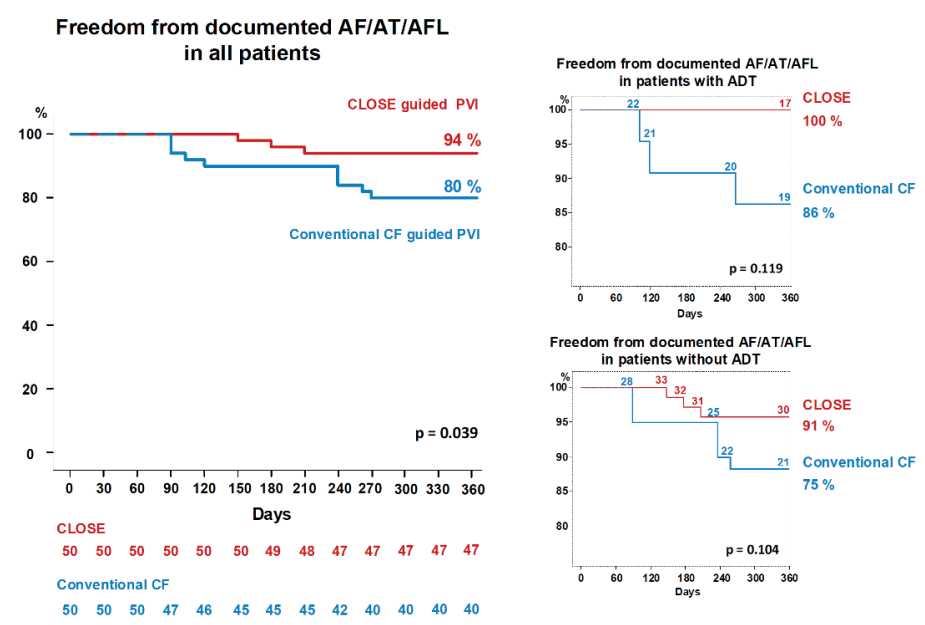

Figure 6: Left panel: Kaplan-Meier curves depicting time to first recurrence of atrial fibrillation (AF), atrial flutter (AFL), or atrial tachycardia (AT) (including a 3-month blanking period) in patients undergoing 'CLOSE'guided ablation and conventional CF-guided ablation. Right panels: Arrhythmia-free survival for patients that continued anti-arrhythmic drug therapy (ADT) at the end of the blanking period (upper panel) and patients without ADT (lower panel).

\section{Safety of 'CLOSE'-guided vs conventional CF-guided PVI}

No symptomatic complications were observed in the 'CLOSE' group. In the conventional CF group we observed 1 tamponade during transseptal puncture requiring pericardiocentesis and prolonged hospitalization. Finally there was no significant difference in hospitalization length. 


\section{Predictors of AF recurrence and mechanism of reconnections at repeat}

Compared to patients with successful 1-year outcome $(n=87)$, patients with recurrence $(n=13)$ had similar baseline clinical characteristics. Use of 'CLOSE' protocol was an independent predictor for maintenance of sinus rhythm ( $54 \%$ vs $23 \%$; OR=3.917; 95\% CI: $1.008-15.220)$.

In the CONV-CF group (7 patients undergoing repeat) there were 21623 sites of reconnection in 18 veins, in the CLOSE group ( 3 patients undergoing repeat), we observed 7 sites of reconnection in 7 veins. We observed no difference in spatial distribution of reconnection sites in between both groups ( $43 \%$ posterior in the CLOSE group vs $48 \%$ posterior in the CF group) (Figure 7). In both groups majority of reconnections were associated with either ILD $>6 \mathrm{~mm}$ and/or AI $<400 / 550$ (7 out or 7 , $100 \%$ in 'CLOSE' vs 19 out of $23,83 \%$ in 'CONV-CF', $p=0.99$ ).
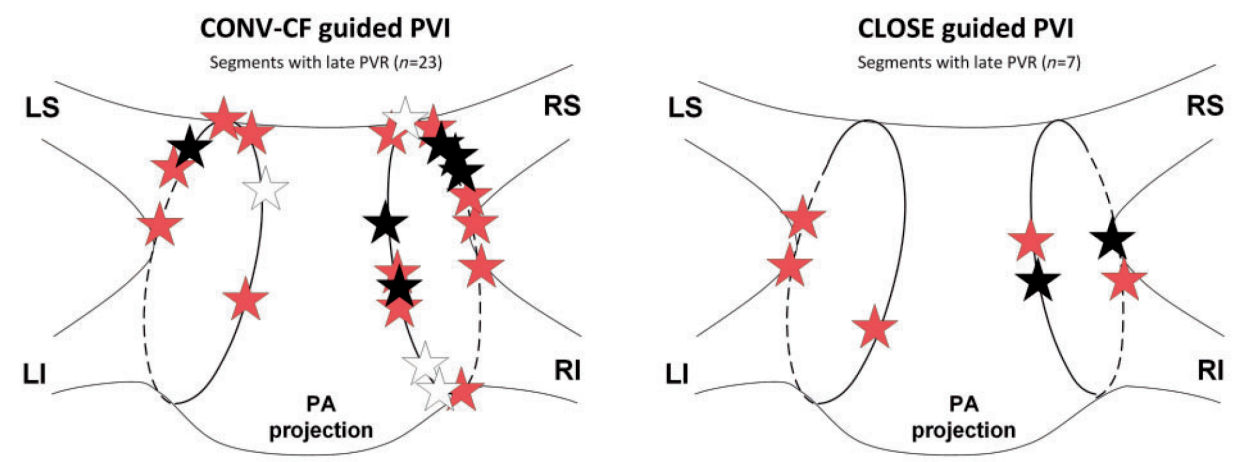

Interlesion distance (ILD) $>6 \mathrm{~mm}$

Ablation index (AI) $<\mathbf{4 0 0}$ (posterior), or

Ablation index $(\mathrm{AI})<\mathbf{5 5 0}$ (anterior)

Neither ILD nor A

Figure 7: Spatial distribution of the sites of reconnection in both treatment groups and their putative mechanism for reconnection. See text for explanation. CONV-CF, conventional contact force; LI, left inferior; LS, left superior; PA, postero-anterior; PVI, pulmonary vein isolation; PVR, pulmonary vein reconnection; RI, right inferior; RS, right superior.

\section{Discussion}

\section{Main findings}

This study shows that, compared to conventional CF-guided ablation, an ablation strategy aiming to enclose the PVs with contiguous and optimized RF lesions results in a higher rate of adenosine-proof isolation and higher single-procedure arrhythmia free survival. A single procedure freedom of $94 \%$ at 1 -year is obtained without compromising safety and with shorter procedure and RF times. Whereas in the absence of randomization this study should be interpreted as hypothesis generating only, our data might provide the necessary base to setup a randomized control trial. 


\section{Single-procedure freedom of AF after point-by-point RF PVI}

The recent FIRE and ICE study confirmed a 64\% single-procedure freedom from AF after point-bypoint RF ablation. ${ }^{7,8}$ Since the introduction of CF-sensing technology in ablation catheters, several studies suggested enhanced 1-year freedom from $\mathrm{AF} .{ }^{9-12}$ In a 2-centre prospective parallel cohort study, CF-guidance was associated with better 1-year arrhythmia free survival $(88 \% \mathrm{v} 66 \%){ }^{9}$ In a single center controlled study including 60 paroxysmal AF patients, CF- guidance was associated with higher 1-year freedom from $\mathrm{AF}(90 \%$ vs $67 \%){ }^{10}$

Although randomized controlled studies failed to demonstrate a beneficial effect of CF239 guidance on AF free survival at 1 year, ${ }^{13-14}$ our data confirm the $\approx 80 \%$ single-procedure freedom of AF after CF-guided PVI (historical control group).

More importantly, the present data suggest that further optimization of CF-guided ablation by adding objective criteria (the 'CLOSE' criteria of ILD and AI) further improves acute durable isolation rates and 1-year single-procedure outcome. Several observations speak for the reliability of the currently reported $>90 \%$ single-procedure freedom of AF after 'CLOSE'-guided PVI. First, this high success rate is 245 in line with the hypothesis that avoiding weak links within the deployed RF circle is the key to durable PVI and clinical success. ${ }^{3}$ Second, also other research groups, respecting a contiguous encircling, reported a $>90 \%$ single-procedure arrhythmia-free survival in CF-guided PVI. A recent sub-analysis of SMART-AF in 40 paroxysmal AF patients showed $93 \%$ freedom from arrhythmia in those patients with short ILD. ${ }^{15}$ Likewise, Itoh et al reported single-procedure freedom of $96 \%$ at 1 year after a procedure in which CF-guided PVI was performed using catheter tip dragging in steps of $\approx 2 \mathrm{~mm} .{ }^{16}$ Finally, the currently reported high single-procedure freedom after PVI for paroxysmal AF is in line with what one can expect after durable PVI. On the one hand it is known that non-PV triggers account for $<10 \%$ of paroxysmal $\mathrm{AF},{ }^{17}$ on the other hand a strategy of aiming for durable PVI with repeat ablations is known to cumulate in a $>90 \%$ freedom of $\mathrm{AF}{ }^{18}$

Conducting a randomized controlled trial confirming the superiority of the 'CLOSE'-protocol will be challenging. In a prospective control group claiming 'conventional CF' only, it seems impossible to avoid the bias of previous experience with AI and ILD. Also other study designs using other control groups will face challenges. Although no randomized trial showed incremental benefit of CF-sensing catheters, it seems unethical to design a control group using catheters without CF sensing. Finally, a control group using catheters with other energy sources will be prone to the obvious criticism of bias introduced by operator experience or preference. Therefore, we believe that the current database of 100 consecutive cases offers a unique cross-sectional view allowing the comparison of both strategies. 


\section{What could explain the higher success rate of the 'CLOSE' protocol?}

The improved procedural and 1-year outcome in 'CLOSE' are in line with the weakest link hypothesis. ${ }^{3}$ Whereas in the conventional CF group there were no objective ranges (not even 268 for contact force), the RF circle in the 'CLOSE' protocol was characterized by lesions all with an ILD $\leq$ $6 \mathrm{~mm}$ and an $\mathrm{AI} \geq 400(300)$ at posterior 269 and 550 at anterior wall. Those target values, resulting in an ablation line contiguity index of $100 \%$, where found to be predictive for durable isolation in CFguided PVI.3 The observation that the majority of reconnections in both groups were due to either ILD $>6 \mathrm{~mm}$ and/or $\mathrm{AI}<400 / 550$ also supports this hypothesis.

Nevertheless, improved outcome might be (partly) due other factors beyond ILD and AI as well. First, one might speculate that the improvement in the 'CLOSE' protocol is mediated by the use of higher power $(35 \mathrm{~W}$ at the posterior wall instead of $25 \mathrm{~W})$ and shorter application times to obtain AI targets. Although we did not observe any major shift in the spatial distribution of gaps at repeat in between both groups and although there exists preclinical evidence that lesion size is similar at a certain AI value (despite differences in power, force and time of application to reach that value), ${ }^{19}$ no clinical study compared quality of lesions being delivered with different settings. Second, one might hypothesize that improved outcome is due to reduced contact force variability during RF delivery in the 'CLOSE' protocol. Stability of lesion delivery, as shown by Shah et al. is a critical determinant of lesion size ${ }^{5-6}$ and one might expect less CF variability during shorter applications. Of interest however, we did not observe any difference in CF variability in between both groups. In fact, the proportion of applications with intermittent contact was equally low in both groups, due to the use of combined stability and force criteria in VisiTag module. Finally, improved outcome could be (partly) due to a learning curve promoted by the experience of technology and algorithm application. In the absence of a randomized trial this hypothesis can never be refuted.

\section{Safety precautions during 'CLOSE'-guided ablation at the posterior wall}

The present patient cohort is too small to draw any definitive conclusions regarding safety in 'CLOSE'-guided PVI. In a recent study, CF catheters were associated with a high incidence of atrialesophageal fistula. ${ }^{20}$ Therefore one could argue that a CF-guided strategy with higher power ablation on the posterior wall aiming at fixed AI target values might be imprudent. So far however we did not detect any clinically relevant esophageal injury using the 'CLOSE' protocol. ${ }^{4}$ This might be due to the following reasons: (1) in the 'CLOSE' protocol, we strive for high stability rather than high contact force during RF delivery. Avoiding high force might avoid pushing the posterior wall towards the esophagus; (2) In our approach, RF is delivered at the left atrial-PV junction rather than at the thin free wall of the posterior left atrium; (3) AI target was reduced to 300 at the posterior wall in the presence of pain perception or temperature rise (general anesthesia). This lower target was also maintained for the rest of the posterior wall lesions of that circle. As a result, $42 \%$ of 'CLOSE' circles were characterized by one or more lesions with an $\mathrm{AI}<400$; (4) Finally, due to the use of relatively higher 
power $(35 \mathrm{~W})$, the 'CLOSE' protocol is associated with short applications at the posterior wall (23 \pm 6 s), even as short as $13 \pm 4 \mathrm{sec}$ in case of possible signs of esophageal injury. Short applications might be associated with a lower risk of deep penetration of conductive heating to adjacent structures such as the esophagus.

Of course, safety (and its putative determinants) need to be investigated in properly powered clinical trials or in studies investigating esophageal thermal asymptomatic lesions using esophageal endoscopy.

\section{Towards a short and standardized PVI procedure}

In the present study, we observed that 'CLOSE'-guided PVI is associated with a significant $30 \%$ reduction in procedure time and nearly $50 \%$ reduction in RF time. A shorter procedure time in the 'CLOSE' protocol is explained by the absence of PV reconnections (no time spent chasing gaps at the junction) and a reduction in RF time. Shorter RF time is explained by the markedly shorter applications (use of AI allowing delivery of higher power) and a trend towards less applications. Although the $192 \pm 42$ min procedure time and $56 \pm 11$ min total RF time, as observed in the 'CONVCF' group, are in line with procedure time and RF time previously reported in CF-guided procedures (223 $\pm 86 \mathrm{~min}$ and $61 \pm 36 \mathrm{~min} 318$ in the SMART-AF study, ${ }^{11}$ and $194 \pm 23 \mathrm{~min}$ and $41 \pm 7 \mathrm{~min}$ in the multicenter UK study ${ }^{13}$ ) we can never exclude the contribution of a learning curve.

Finally, we believe that the 'CLOSE' protocol offers the base for a straightforward and standardized PVI procedure. Rather than isolation of the veins, the endpoint for the procedure is a perfect circle leading invariably to PVI. Introduction of objective and verifiable criteria (AI and ILD) should lead to reproducible outcome across centers and operators.

\section{Limitations}

Although 100 patients were enrolled in this pragmatic parallel cohort study, several limitations need to be underlined: our study is monocentric, non-randomized and small-sized. Despite a $24 \mathrm{~h}-$ Holter ECG at 3, 6 months and a 7-day Holter-ECG at 12 months and the symptomatic nature of PAF, the discontinuous nature of monitoring can underestimate AF recurrence. Finally anti-arrhythmic drug therapy was not systematically withdrawn after the blanking period, and may have improved clinical success in both groups. The limitations of discontinuous monitoring and single-center nature of the study are being tackled by two ongoing clinical studies (a study monitoring arrhythmia-free survival using subcutaneous loop recorders and a multicenter clinical trial in $>300$ patients). Finally, the present comparison is too small to draw any definitive conclusions regarding safety. 


\section{Conclusions}

In the present study we evaluated the feasibility of an ablation protocol aiming to enclose the pulmonary veins with contiguous and optimised RF lesions. Compared to conventional CF guided PVI, we observed a shorter procedure time, significant improvement in acute PVI durability and a higher single-procedure arrhythmia free survival at 1 year. 


\section{REFERENCES}

1. Calkins H, Kuck KH, Cappato R, Brugada J, Camm J, Chen SA, et al. Heart Rhythm Society Task Force on Catheter and Surgical Ablation of Atrial Fibrillation. 2012 HRS/EHRA/ECAS Expert Consensus Statement on Catheter and Surgical Ablation of Atrial Fibrillation. Heart Rhythm. 2012; 9:632-96

2. Kirchhof P, Benussi S, Kotecha D, Ahlsson A, Atar D, Casadei B, et al. 2016 ESC Guidelines for the management of atrial fibrillation developed in collaboration with EACTS. Eur Heart J. 2016; 37:2893-962.

3. El Haddad M, Taghji P, Phlips T, Wolf M, Demolder A, Choudhury R, et al. Determinants of Acute and Late Pulmonary Vein Reconnection in Contact Force Guided Pulmonary Vein Isolation. Circ Arrhythmia Electrophysiol 2017; 10:e004867.

4. Taghji P, El Haddad M, Phlips T, Wolf M, Knecht S, Vandekerckhove Y, et al. Evaluation of a Strategy Aiming to Enclose the Pulmonary Veins with Contiguous and Optimized Radiofrequency Lesions in Paroxysmal Atrial Fibrillation: A Pilot Study. JACC Clinical Electrohysiology; 4:99108.

5. Shah DC, Lambert H, Nakagawa H, Langenkamp A, Aeby N, Leo G. Area under the real-time contact force curve (force-time integral) predicts radiofrequency lesion size in an in vitro contractile model. J Cardiovasc Electrophysiol. 2010; 21:1038.

6. Sarkozy A, Shah D, Saenen J, Sieira J, Phlips T, Bories W, et al. Contact Force in Atrial Fibrillation: Role of Atrial Rhythm and Ventricular Contractions: Co-Force Atrial Fibrillation Study. Circ Arrhythm Electrophysiol. 2015; 8:1342-50.

7. Kuck KH, Brugada J, Fürnkranz A, Metzner A, Ouyang F, Chun KR, et al.; FIRE AND ICE Investigators. Cryoballoon or Radiofrequency Ablation for Paroxysmal Atrial Fibrillation. N Engl J Med. 2016; 374:2235-45.

8. Wilber DJ, Pappone C, Neuzil P, De Paola A, Marchlinski F, Natale A, et al. Comparison of antiarrhythmic drug therapy 368 and radiofrequency catheter ablation in patients with paroxysmal atrial fibrillation: a randomized controlled trial. JAMA 2010; 303:333-40.

9. Andrade JG, Monir G, Pollak SJ, Khairy P, Dubuc M, Roy D, et al. Pulmonary vein isolation using contact force ablation: The effect on dormant conduction and long-term freedom from recurrent atrial fibrillation. A prospective study. Heart Rhythm 2014; 11:1919-24.

10. Marijon E, Fazaa S, Narayanan K, Guy-Moyat B, Bouzeman A, Providencia R, et al. Real-time contact force sensing for pulmonary vein isolation in the setting of paroxysmal atrial fibrillation: procedural and 1-year results. J Cardiovasc Electrophysiol 2014; 25:130-7.

11. Natale A, Reddy V, Monir G, Wilber D, Lindsay B, McElderry H, et al. Paroxysmal AF catheter ablation with a contact force sensing catheter: results of the prospective, multicenter SMART-AF trial. J Am Coll Cardiol 2014; 64:647-56.

12. Afzal MR, Chatta J, Samanta A, Waheed S, Mahmoudi M, Vukas R, et al. Use of contact force sensing technology during radiofrequency ablation reduces recurrence of atrial fibrillation: A systematic review and meta-analysis. Heart Rhythm. 2015; 12:1990-6.

13. Ullah W, McLean A, Tayebjee MH, Gupta D, Ginks MR, Haywood GA, et al.; UK Multicentre Trials Group. Randomized trial comparing pulmonary vein isolation using the SmartTouch catheter with or without real-time contact force data. Heart Rhythm 2016; 13:1761-7.

14. Reddy V, Dukkipati S, Neuzil P, Natale A, Albenque J, Kautzner J, et al. Randomized controlled trial of the safety and effectiveness of a contact-force sensing irrigated catheter for ablation of paroxysmal atrial fibrillation (Toccastar study). Circulation 2015; 132:907-15.

15. Reddy V, Pollak S, Lindsay B, McElderry H, Natale A, Kantipudi C, et al. Relationship between catheter stability and 12-month success after pulmonary vein isolation: a subanalysis of the SMART-AF trial. J Am Coll Cardiol EP. 2016: 6:691-9. 
16. Itoh T, Kimura M, Tomita H, Sasaki S, Owada S, Horiuchi D, et al. Reduced residual conduction gaps and favourable outcome in contact force-guided circumferential pulmonary vein isolation. Europace. 2016; 18:531-7.

17. Tsai CF, Tai CT, Hsieh MH, Lin WS, Yu WC, Ueng KC, et al. Initiation of atrial fibrillation by ectopic beats originating from the superior vena cava: electrophysiological characteristics and results of radiofrequency ablation. Circulation 2000; 102:67-74.

18. De Greef Y, Tavernier R, Vandekerckhove Y, Duytschaever M. Triggering pulmonary veins: a paradoxical predictor for atrial fibrillation recurrence after PV isolation. J Cardiovasc Electrophysiol 2010; 21:381-8.

19. Nakagawa H, Ikeda A, Govari A, et al. Prospective study to test the ability to create RF lesions at predicted depths of 3,5, 7 and $9 \mathrm{~mm}$ using a new formula incorporating contact force, radiofrequency power and application time (Force-Power-Time Index) in the beating canine heart. Heart Rhythm 2013; 10:S481.

20. Black-Maier E, Pokorney SD, Barnett AS, Zeitler EP, Sun AY, Jackson KP et al. Risk of atrioesophageal fistula formation with contact force-sensing catheters. Heart Rhythm. 2017; 14:1328-3.

21. Halbfass P, Nentwich K, Krug J, Roos M, Sonne K, Ene E et al. Impact of surround flow catheter tip irrigation in contact force ablation on the incidence of asymptomatic oesophageal lesions after atrial fibrillation ablation: a prospective comparative study. Europace 2017; 19:1116-22. 


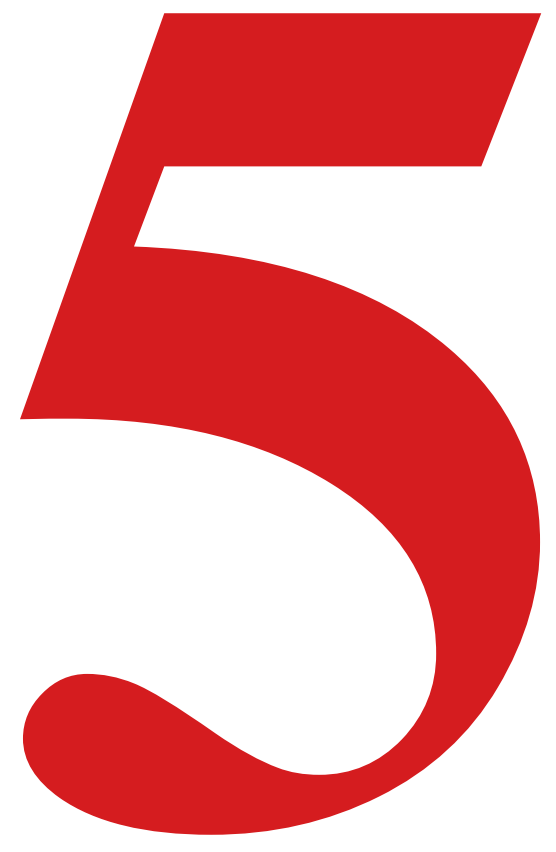




\section{Chapter 5}

\section{Evaluation of clinical outcome of CLOSE-PVI in a larger patient cohort}

Philippe Taghji, Milad El Haddad, Thomas Phlips, Michael Wolf, Sébastien Knecht, Yves Vandekerckhove, René Tavernier, Hiroshi Nakagawa, Mattias Duytschaever. 


\begin{abstract}
Background: Reconnections after PV isolation (PVI) are explained by insufficient lesion depth and/or discontiguity of radiofrequency (RF) lesions.

Objectives: To evaluate safety, acute and 1-year outcome of an ablation protocol aiming to enclose the PVs with a contiguous and optimized RF circle by targeting region-specific criteria for (1) lesion depth assessed by ablation index and (2) interlesion distance (ILD).
\end{abstract}

Methods: 130 consecutive patients with paroxysmal atrial fibrillation (AF) underwent PV encircling using a contact force $(\mathrm{CF})$-sensing catheter. RF was delivered targeting ILD $\leq 6 \mathrm{~mm}$ and ablation index $\geq 400$ at posterior and $\geq 550$ at anterior wall. Recurrence was defined as any AF, atrial tachycardia or flutter $(\mathrm{AF} / \mathrm{AT} / \mathrm{AFL}>30 \mathrm{~s})$ on Holter at 3, 6 and 12 months.

Results: Procedure and RF time per circle were $155 \pm 28 \mathrm{~min}$ and $17 \pm 5 \mathrm{~min}$ respectively. Incidence of first-pass and adenosine-proof isolation were $98 \%$ and $98 \%$ respectively. One short-lived TIA was observed. At 12 months, single-procedure freedom from AF/AT/AFL was 91.3\% in those 104 patients off antiarrhythmic drug therapy (ADT) and $96.2 \%$ in those 26 patients on ADT. Single-procedure freedom from both AF/AT/AFL and ADT was 73.1\%.

Conclusions: This study suggests that an ablation protocol respecting strict criteria for lesion depth and contiguity results in acute durable PVI followed by a high single-procedure arrhythmia-free survival at 1 year. A prospective, multi-center trial is ongoing. 


\section{Introduction}

Single procedure freedom from atrial fibrillation (AF) after radiofrequency (RF) pulmonary vein isolation (PVI) is $\approx 70 \%$ in patients with paroxysmal AF.(1-3) PV reconnection (PVR) is the major determinant of AF recurrence during follow-up.(4-6) The use of contact force (CF) sensing catheters, allowing to better control energy delivery during point-by-point PV encircling, seems to improve freedom from AF to $\approx 80 \%$.(7-10) Nevertheless, acute and late PVR still occur, and data on 1 year outcome are not consistent across studies.(11-12)

We recently showed that acute and late PV reconnections after CF-guided PVI are explained by lack of contiguity and insufficient lesion depth, as assessed by ablation index, within the deployed RF circle.(13-15) Ablation index is based upon the experimental work of Nakagawa et al.(14) This index, incorporating contact force, power and time in a weighted formula, predicted lesion depth in the canine ventricle with high accuracy.

The 'CLOSE' protocol is a new approach aiming to enclose the PVs with contiguous and optimized RF lesions by targeting an ILD $\leq 6 \mathrm{~mm}$ and ablation index (AI) $\geq 400$ at the posterior wall and $\geq 550$ on the anterior wall. We evaluated safety, acute and 1-year single-procedure outcome after 'CLOSE'guided PVI in 130 patients with paroxysmal AF.

\section{Methods}

\section{Study subjects}

From August 2013, all patients undergoing CF-guided ablation for AF at the St Jan Hospital Bruges are followed in a prospective database approved by the local institutional review committee. This database implies collecting patients' written informed consent, a detailed case report form of the procedure and follow-up with Holter-ECGs at 3, 6 and 12 months. In this manuscript, we present the analysis of 130 consecutive cases of PVI for paroxysmal AF using the 'CLOSE' protocol.

\section{'CLOSE'-guided PVI}

PVI was performed by 4 operators under conscious sedation or general anesthesia. In anesthesized patients esophageal temperature monitoring (SensiTherm ${ }^{\mathrm{TM}}$, St Jude Medical Inc, Minnesota, US) was performed. After transseptal puncture (SL0, St Jude Medical), a Lasso catheter and CF catheter (Thermocool SmartTouch ${ }^{\circledR}$, Biosense-Webster Inc., Diamond Bar, CA, USA) were positioned in the left atrium and calibration of $\mathrm{CF}$ and respiratory gating were performed. Then we predefined the RF circle around the PVs (nephroid shape) on the 3D-geometry (Carto System ${ }^{\circledR}$, Biosense Webster Inc.). Point-by-point RF delivery was performed during sinus rhythm aiming for a contiguous circle 
enclosing the veins. Real-time automated display of RF applications (Visitag ${ }^{\circledR}$, Biosense Webster Inc.) was used with predefined settings of catheter stability $(3 \mathrm{~mm}$ for $8 \mathrm{sec})$ and minimum CF (30\% of time $>$ 4gr). RF was delivered (EP Shuttle ST-3077, Stockert GmbH, Freiburg, Germany) in powercontrolled mode (without ramping) with 25 to $35 \mathrm{~W}$ (irrigation flow up to $30 \mathrm{cc} / \mathrm{min}$ ). RF was delivered until an $\mathrm{AI}$ of $\geq 400$ at the posterior wall/roof and $\geq 550$ at the anterior wall (Figure 1). In case of dislocation, a new RF application reaching the AI target was applied. Maximal ILD between two neighboring lesions was $\leq 6 \mathrm{~mm}$. In case of chest pain or intra-esophageal $\mathrm{T}^{\circ}$ rise $>38.5^{\circ} \mathrm{C}$ during posterior wall ablation, RF delivery was stopped at an $\mathrm{AI}$ of 300. In the absence of first-pass isolation (i.e. no isolation after completing the circle), touch-up ablation was delivered until PVI. After PVI we waited for 20 minutes after which adenosine (dose resulting in atrio-ventricular block) was given 4 times (with the Lasso in its corresponding position). In case of reconnection during waiting time or during adenosine, the site of reconnection was located and treated with touch-up ablation until PVI was resistant to subsequent adenosine challenge. In case of (pre-)procedural documentation of typical flutter, cavotricuspid isthmus (CTI) ablation was performed.

\section{CLOSE guided PVI}

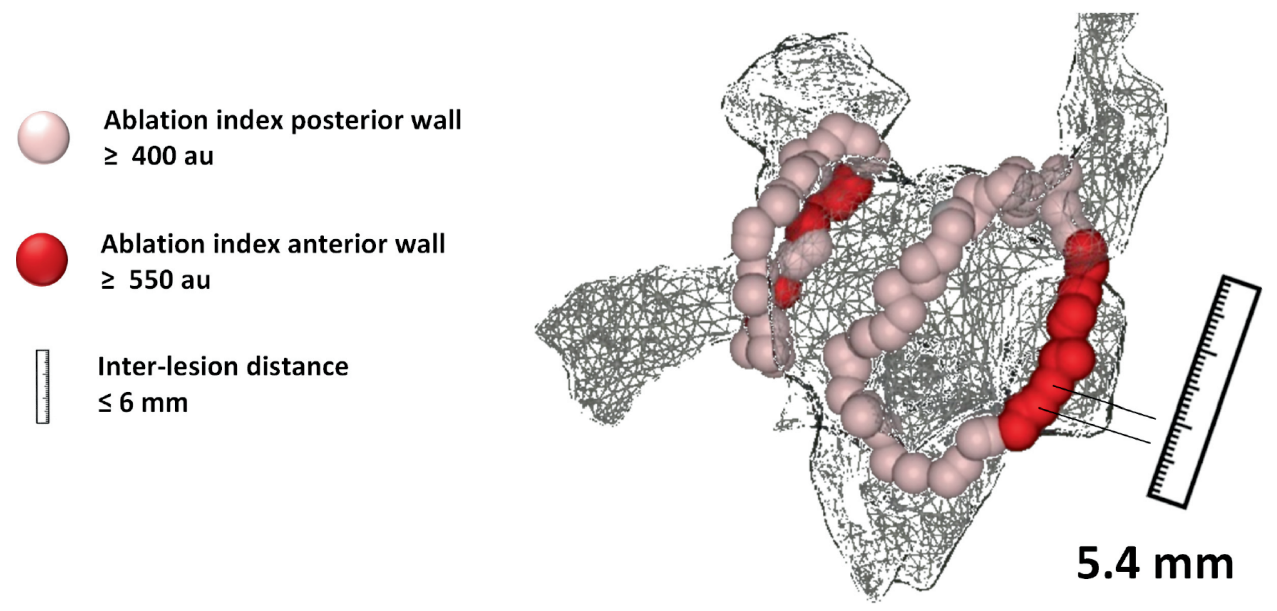

Figure 1: Illustration of 'CLOSE'-guided PVI. PVI=pulmonary vein isolation, ILD=interlesion distance. 


\section{Offline analysis of criteria to assess integrity of the deployed RF circle}

Each procedure was analyzed offline. For each RF tag within the circle we determined time of application (s), median delivered power (W), impedance drop (D-Imp, $\Omega$ ), average CF (CF, g), forcetime integral (FTI, gs) and ablation index (AI, arbitrary unit, au). Custom-made software was used to automatically determine the path of encircling, perimeter of the RF circle, ILD (center-to-center distance, $\mathrm{mm}$ ) and ablation line contiguity index (ALCI). ALCI was developed as a criterion to algorithmically score the combination of contiguity and lesion depth.(13)

To describe the overall quality for a given circle, we determined for each circle the median value of all analyzed ablation parameters. Four circles from 2 patients were not available for off-line analysis.

To describe the weakest link in a given circle we determined for each circle the minimal value for ablation index and the maximal value for ILD.

\section{Follow-up}

Complications were reported on the CRF and collected during follow-up. After ablation, anticoagulation and anti-arrhythmic drug therapy (ADT) were continued. At 3 months, anticoagulation was continued according to stroke risk, whereas ADT was continued at the discretion of the treating physician. Clinical evaluation and ECG were performed at 1, 3,6 and 12months. Holter-ECG was performed at 3 and 6 months (24h) and 12 months (7 day) or in case of symptoms. Freedom from recurrence was defined as 1-year absence from AF/AT/AFL $>30$ seconds beyond a 3-month blanking period.

\section{Statistical analysis}

Normality of data distribution was tested with Shapiro-Wilk test. Continuous variables are expressed as mean $\pm \mathrm{SD}$ if normally distributed, medians with IQR if non-normally distributed, and dichotomous variables as percentage. Kaplan-Meier estimate with $95 \%$ confidence intervals ( \pm 1.96 standard error) was used to assess freedom from AF during 12-month follow-up period. All statistical analyses were performed in SPSS Statistics 24 (IBM Corporation, Armonk, New York, USA). 


\section{Results}

\section{Patient characteristics}

All 130 patients had paroxysmal AF. Baseline clinical characteristics are given in Table 1.

Table 1. Patient characteristics $(n=130)$

\begin{tabular}{lc}
\hline Paroxysmal AF, n (\%) & $130(100 \%)$ \\
Age, $\mathrm{y}$ & $59 \pm 13$ \\
Male, $\mathrm{n}(\%)$ & $84(65 \%)$ \\
BMI, kg/m & $26.5 \pm 4.1$ \\
Arterial hypertension, $\mathrm{n}(\%)$ & $42(32 \%)$ \\
Structural heart disease, $\mathrm{n}(\%)$ & $11(9 \%)$ \\
Diabetes, $\mathrm{n}(\%)$ & $5(4 \%)$ \\
CHA DS 2 VASc score $\geq 2, \mathrm{n}(\%)$ & $47(36 \%)$ \\
Left atrial diameter, mm & $39.7 \pm 3.7$ \\
Electrical cardioversion, $\mathrm{n}(\%)$ & $26(20 \%)$ \\
$1^{\text {st }}$ AF episode to PVI, months & $24[9-84]$ \\
Resistance to ADT, $\mathrm{n}(\%)$ & $103(79 \%)$ \\
\hline
\end{tabular}

\section{Procedural characteristics}

Characteristics are given in Table 2. PVI was obtained in all cases. CTI ablation was performed in 5 patients $(3.8 \%)$. General anesthesia was used in 72 out of 130 patients $(55 \%)$. Procedure time was $155 \pm 28 \mathrm{~min}$ (Figure 2, panel A). RF time per circle was $17 \pm 5 \mathrm{~min}$ (panel B). Perimeter of the deployed circle was $115.7 \pm 22.3 \mathrm{~mm}$ (panel C). The number of RF tags to enclose the PVs was $28 \pm 5$ (panel D). The number of dislocations per circle was $3 \pm 3$. Procedural characteristics for the right and left circles separately are given in Table 2 . 
Table 2. Procedure characteristics $(n=130)$

\begin{tabular}{lc} 
PV isolation, $\mathrm{n}$ & $130(100 \%)$ \\
CTI ablation, $\mathrm{n}$ & $5(4 \%)$ \\
General anesthesia, $\mathrm{n}$ & $72(55 \%)$ \\
Procedure time, min & $155 \pm 28$ \\
Fluoroscopy time, min & $16.7 \pm 7.1$ \\
Dose area product (DAP), mGy/cm ${ }^{2}$ & $11314 \pm 8687$ \\
Total hospitalization time, hrs & $39 \pm 12$ \\
Right circles & $123 \pm 19$ \\
Perimeter, mm & $29 \pm 5$ \\
RF tags, $\mathrm{n}$ & $128(99 \%)$ \\
RF time, min & $130(100 \%)$ \\
First-pass isolation, $\mathrm{n}$ & $128(99 \%)$ \\
Waiting/adeno. proof isolation, $\mathrm{n}$ & \\
Left circles & $108 \pm 23 \pm 5$ \\
Perimeter, mm & \\
RF tags, $\mathrm{n}$ & \\
\hline
\end{tabular}

Compared to conscious sedation, procedures with general anesthesia were characterized by shorter procedure time $(147 \pm 22 \mathrm{~min}$ vs. $164 \pm 31 \mathrm{~min}, \mathrm{p}=0.001)$, shorter fluoroscopy time $(16 \pm 7 \mathrm{~min}$ vs. $17 \pm 8 \mathrm{~min}, \mathrm{p}=0.725)$, lower dose area product (DAP) value $(9312 \pm 7538 \mathrm{mGy} / \mathrm{cm} 2$ vs. $15850 \pm 9644 \mathrm{mGy} / \mathrm{cm} 2, \mathrm{p}=0.014)$ and shorter $\mathrm{RF}$ time for the right circle $(15 \pm 3 \mathrm{~min}$ vs $18 \pm 4, \mathrm{p}<0.001)$ and left circle $(16 \pm 4$ vs $21 \pm 7, \mathrm{p}<0.001)$. 


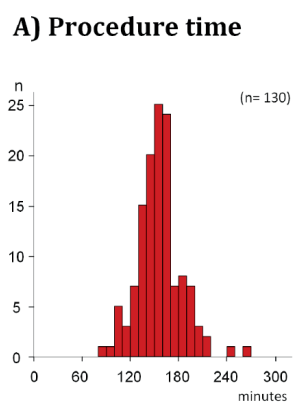

\section{B) RF time per circle}

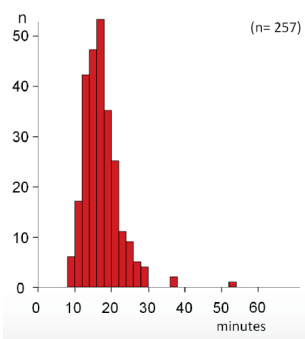

C) Perimeter of deployed

$$
\text { RF circle }
$$

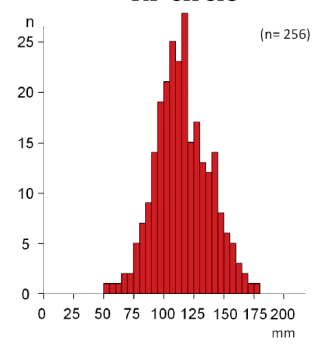

D) Number of RF tags in a circle

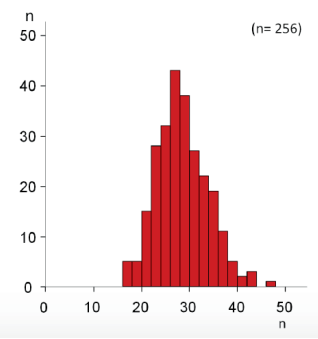

E) Minimal ablation index $\left(\mathbf{A} \mathbf{I}_{\text {min }}\right)$

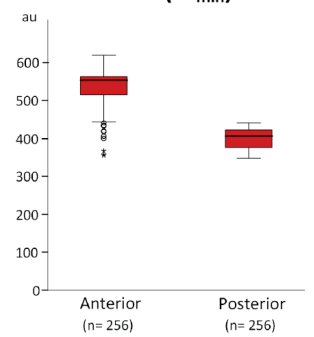

F) Maximal inter-lesion distance (ILD $\left.{ }_{\text {max }}\right)$

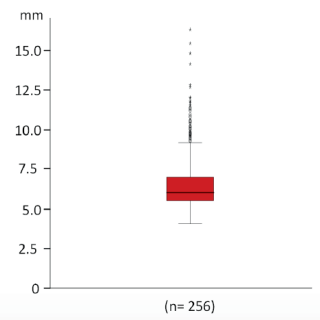

G) First-pass isolation

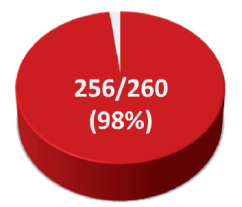

H) Waiting/adenosine proof

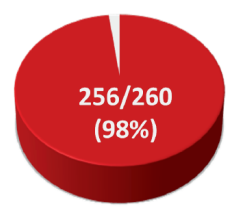

Figure 2: RF characteristics and incidence of acute durable $P V$ isolation

Procedure time (A). RF time per circle (B). Perimeter of the deployed circle (C). Number of RF tags within the circle (D). Obtained minimal ablation index per circle (E). Obtained maximal ILD (F). Incidence of first-pass isolation (G). Incidence of waiting time/adenosine-proof isolation (H). See text for further explanation.

\section{RF characteristics and integrity of the RF circle}

A representative case illustrating the integrity of the RF circle given in Figure 3. The 'CLOSE' procedure is characterized by applications with relatively short duration (ranging from 11 to 61s), a high rate of applications with high power $(\geq 30 \mathrm{~W})$ and a high rate of RF tags with a marked impedance drop $(\geq 7 \Omega$ ). There is a wide range of obtained CF values (5 to $37 \mathrm{~g}$ ) and FTI values (ranging from 184 to $875 \mathrm{gs}$ ). Due to the nature of the protocol, applications at the anterior wall are all with an AI of $\geq 550$. Finally, the 'CLOSE'-guided circle is characterized by enclosing lesions all with ILD $\leq 6 \mathrm{~mm}$ and ALCI of $>100 \%$. 

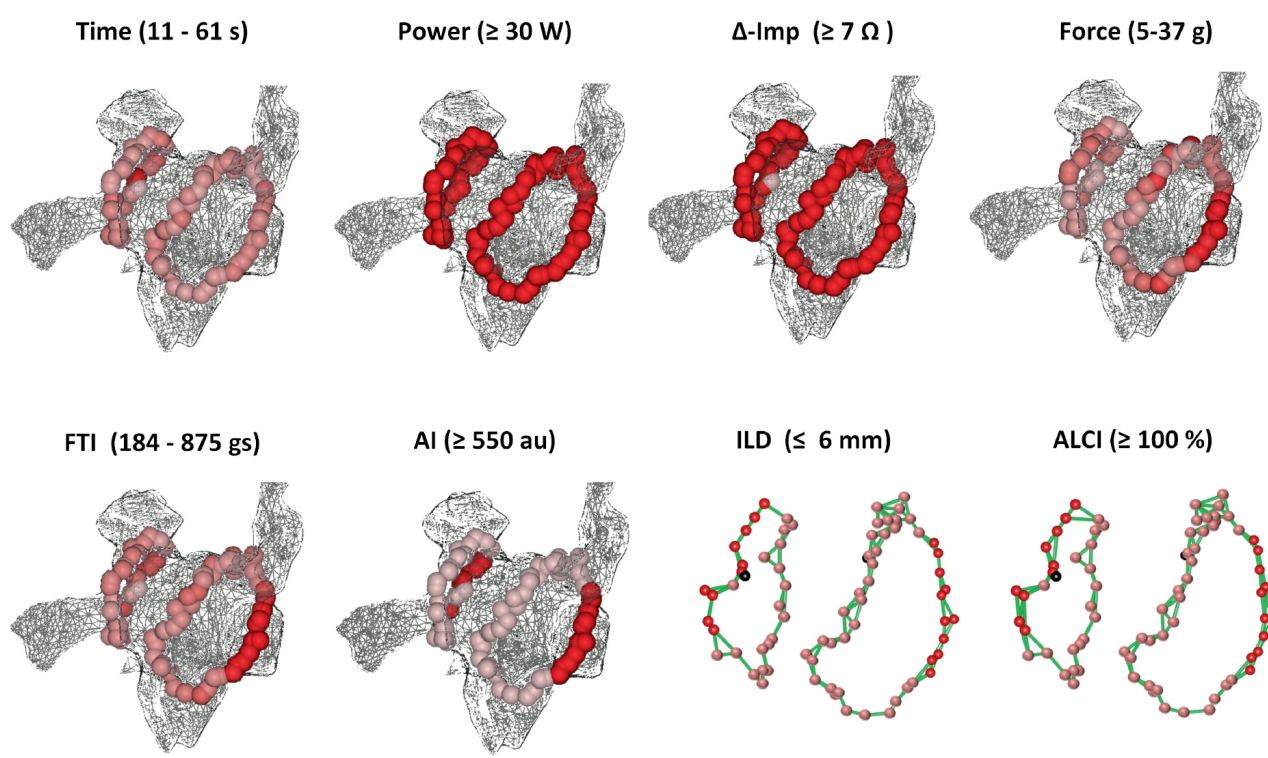

$\mathrm{ALCl}(\geq 100 \%)$

Figure 3: Representative examples of RF characteristics in 'CLOSE'-guided PVI

The deployed circles are represented color-coded (dichotomous or continuous) for each of the following criteria: application time, power, impedance drop (D-Imp), contact force (CF), force-time integral (FTI), ablation index (AI), perimeter, interlesion distance (ILD) and ablation index contiguity index (ALCI). See text for further explanation.

Overall, RF characteristics of lesion quality were as follows; median application time $=25 \mathrm{~s}$, [23-35], median power $=35 \mathrm{~W},[35-35]$, median obtained $\Delta-\mathrm{Imp}=12.7 \Omega$, [10.6-14.5], median $\mathrm{CF}=15.0 \mathrm{~g}$, [13.417.4], median FTI 375gs, [326-446], median AI 456 au, [449-469], median ILD 4.1mm, [3.8-4.4] and median ALCI 163, [150-179].

Overall, weakest link analysis (Figure 2, panels E and F) showed a minimal ablation index of 408au, [378-425] and 556au, [517-565] at the posterior and anterior wall separately, whereas maximal ILD was $6.5 \mathrm{~mm},[5.9-8.0]$.

Of interest, in 105 out of 260 circles (40\%), we did not target/reach the 400 AI value at the posterior wall because of chest pain and/or intra-esophageal $\mathrm{T}^{\circ}$ rise.

\section{First-pass and waiting time/adenosine-proof isolation}

Results are summarized in Figure 2 (panel G and H). Only 5 patients did not receive adenosine because of a history of asthma/COPD. The 'CLOSE'-protocol was associated with high incidence of 
first-pass isolation (98\%) and waiting time/adenosine-proof isolation (98\%).

\section{1-year freedom from AF/AT/AFL after 'CLOSE'-guided PVI}

Kaplan-Meier analysis was plotted in Figure 4 and 5. Overall, single-procedure 12-month freedom from AF/AT/AFL was $92.3 \%$ (Figure 4). Throughout the course of the study, 26 patients were on ADT. Those patients had continued taking ADT at the end of the blanking period. Conversely, no patient was taking ADT to treat a recurrence documented while being off ADT. In those 26 patients on ADT, freedom from AF/AT/AFL was 96.2\% (Figure 5, left lower panel). In those 104 patients off ADT, freedom from AF/AT/AFL was 91.3\% (Figure 5, left upper panel). Single-procedure freedom from both recurrence and ADT was $73.1 \%$.

Freedom from documented AF/AT/AFL in all

patients $(n=130)$

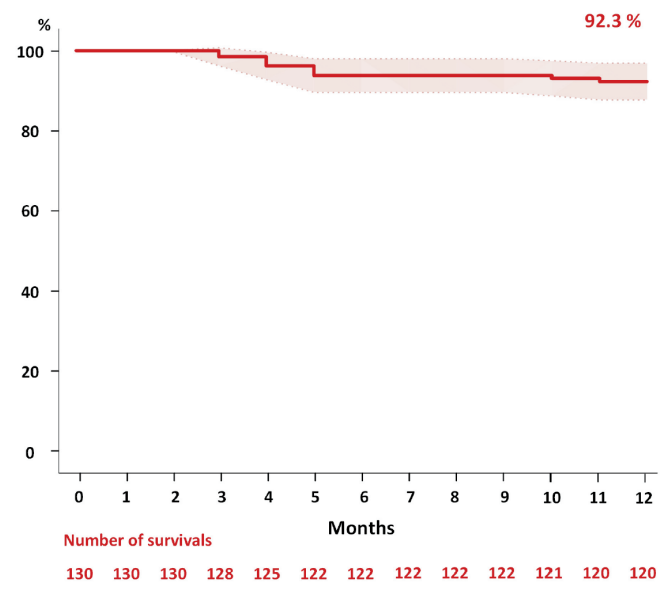

Figure 4: Survival plot in 'CLOSE'-guided PVI

Kaplan-Meier curve depicting time to first recurrence of atrial fibrillation (AF), atrial flutter (AFL), or atrial tachycardia (AT) (including a 3-month blanking period) in all 130 patients undergoing 'CLOSE'-guided ablation. 


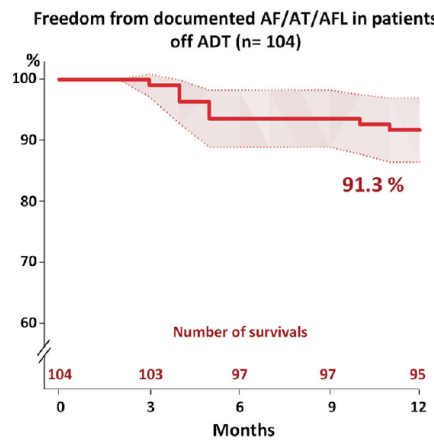

Freedom from documented AF/AT/AFL in patients without arrhythmia during the 3 months blanking $(n=114)$

Freedom from documented AF/AT/AFL in patients on ADT $(n=26)$
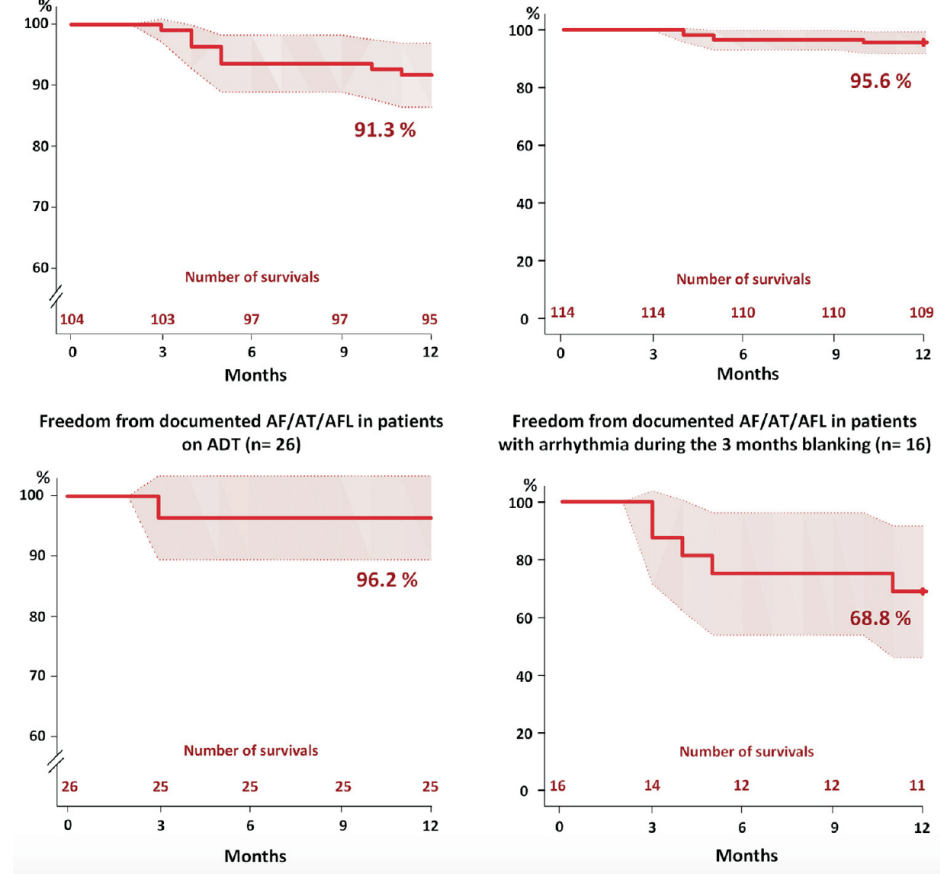

Freedom from documented AF/AT/AFL in patients with arrhythmia during the 3 months blanking $(n=16)$

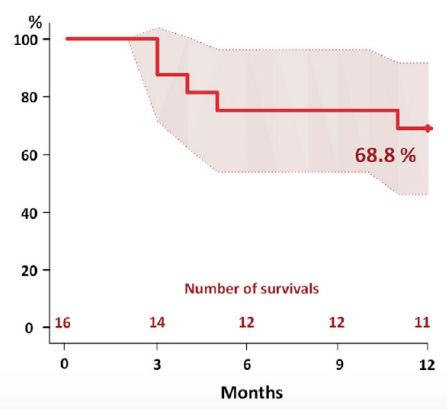

Figure 5: Survival plots for patient subgroups

Kaplan-Meier curves depicting time to first recurrence of atrial fibrillation (AF), atrial flutter (AFL), or atrial tachycardia (AT) in patients undergoing 'CLOSE'-guided ablation for subgroup of patients not taking ADT (left upper) or taking ADT (left lower), and without arrhythmia during the 3 months blanking (right upper) or with arrhythmia during blanking (right lower).

Single-procedure freedom from AF/AT/AFL was comparable amongst operators $(92.3 \pm 1.3 \%)$ and type of anesthesia $($ general $=90.3 \%$, conscious sedation $=94.8 \%, \mathrm{p}=0.511)$.

Of interest, 16 patients (12\%) showed early recurrence during the 3 months blanking period. KaplanMeier analysis for patients with $(\mathrm{n}=16)$ and without $(\mathrm{n}=114)$ arrhythmia during the blanking period is given in Figure 5 (right panels).

\section{Safety of 'CLOSE'- guided PVI}

One short-lived event, diagnosed as TIA, occurred at day 8 after the ablation. Mean hospitalization length was $39 \pm 12$ hrs. 


\section{Findings at repeat ablation}

All 10 patients with documented recurrence underwent repeat ablation. Out of 10 patients, 9 revealed a normal bipolar voltage map during high-density mapping ( $>200$ points). In 6 out of 10 patients $(60 \%)$ we observed a status of permanent isolation of all veins. In those 4 patients with reconnection of the veins we observed 5 gaps in 4 out of 8 circles. Relative to the RF characteristics of the first procedure, 1 gap could not be explained (adequate AI and ILD according to the 'CLOSE' criteria), whereas 4 gaps were explained by a weak link in the initial ablation circle (all IL distance $>6 \mathrm{~mm}$ ).

\section{Discussion}

\section{Main findings}

This study, evaluating the 'CLOSE' protocol in 130 patients, shows that an ablation strategy aiming to enclose the PVs with a contiguous ablation line with optimized RF lesions results in a high rate of adenosine-proof isolation. This is translated into a single-procedure arrhythmia-free survival of $91 \%$ at 1 -year without ADT. These results are obtained without compromising safety and with relatively short procedure and RF times. The above findings are in line with the hypothesis that avoiding weak links within the deployed RF circle is the key to durable PVI and clinical success.(13)

\section{Single-procedure AF freedom after point-by-point RF ablation}

PVI is simultaneously the cornerstone and Achilles heel of ablation for paroxysmal AF. Despite acute isolation, late $\mathrm{PV}$ reconnection results in $\mathrm{AF}$ recurrences with the ensuing need for repeat ablations. The recent FIRE and ICE study confirmed a 64\% single-procedure freedom from AF after point-bypoint RF ablation. $(2,3)$

Since the introduction of CF-sensing technology, several studies suggested enhanced acute durability of PVI and 1-year freedom from paroxysmal AF.(7-10) In a parallel cohort study, CF-guidance was associated with lower incidence of dormant conduction ( $8 \%$ vs 35\%) and better 1-year arrhythmia-free survival ( $88 \%$ v 66\%).(7) In a single-center controlled study, CF-guidance was associated with higher acute procedural success ( $80 \%$ vs 36\%) and 1-year AF-freedom (90\% vs 67\%).(8)

The above studies are in line with work from Michaud et al, showing $84 \%$ AF-freedom if PVI is guided by initial impedance decrease, as an indicator of good catheter contact.(9) Improved outcome is 
also in line with experimental data showing that for a given stable catheter position and for a given power, lesion depth, width and volume increase with higher CF.(18-22)

However, data on 1-year outcome are not consistent across all studies.(11-12) A recent multicenter randomized controlled study in 117 paroxysmal AF patients, failed to demonstrate a beneficial effect of CF-guidance on AF-free survival. (11) Also Reddy et al failed to demonstrate a benefit of CFguided ablation in a multicenter randomized trial.(12) Only in the subgroup of patients with at least $90 \%$ of RF applications $\geq 10 \mathrm{~g}$, AF-freedom was superior compared to non-optimal CF ( $76 \%$ vs $58 \%$ at 1-year).

On top of these conflicting results, even in the most promising scenario, still $\approx 20 \%$ of the patients require a repeat procedure after CF-guided PVI.

\section{Determinants of gap in CF-guided PVI}

The conflicting results indicate that $\mathrm{CF}$ alone cannot preclude the presence of weak links in the ablation chain. Recently, our group demonstrated that in CF-guided PVI, acute and late PVR are explained by discontiguity (interlesion distance) and/or insufficient lesion depth (ablation index) of the lesions within the deployed RF circle.(13)

Prior studies already hinted to the importance of contiguous lesions to obtain acute durable isolation. Miller et al showed that closing the "visual" gap was essential for acute durable PVI, (23) whereas Park et al showed that acute PVR despite CF $\geq 10$ g was explained by ablation sites with an ILD of $\geq 5$ mm.(24) A recent sub-analysis of SMART-AF in 40 paroxysmal AF patients also showed that ILD correlates with success rate (1-year outcome ranging from $33 \%$ to $93 \%$ ).(25)

The above findings support the concept of superiority of contiguous overlapping lesions with sufficient energy delivery. This concept is also in line with human MRI studies showing that acute isolation can be achieved by a combination of ablation-induced reversible (edema) and irreversible atrial injury (necrosis).(26) Likewise, experimental data showed that tissue heating can lead to acute changes in electrophysiological properties allowing for larger gaps to have conduction block, but as the tissue recovers, to become conductive again.(27) This reversible atrial injury is more likely to occur at the periphery of the lesion (where the tissue temperature is lower) but can also occur at the center of the ablation point when insufficient energy is delivered.

The key observation that lower ablation index values are associated with reconnection - even in segments characterized by closely spaced lesions - suggests that ablation index can be used to titrate delivery of sufficient energy.(13) By taking into account the greater contribution of power (over 
contact force) and of the initial time of ablation,(28) ablation index differs from force-power-time integral calculated as the multiplication of force, power and time.(29) Of interest, El Haddad et al observed that there is a significant difference in minimal AI values required to obtain durable segments in the anterior part of the circle compared with the posterior segments, suggestive of thicker atrial tissue in anterior regions.(13)

Based upon the findings by El Haddad et al, we hypothesized that, in order to obtain durable PVI, maximum ILD should not exceed $6 \mathrm{~mm}$ and that minimal lesion depth defined by the AI should reach the value of at least 400 at the posterior wall and 550 at the anterior wall (the 'CLOSE' criteria).

\section{Strengths and weaknesses of the 'CLOSE' protocol}

In the present relatively large patient cohort, we observed a high rate of acute durable isolation and a high 91\% single-procedure, 1-year freedom from AF/AT/AFL without ADT. If continuing ADT (without evidence of recurrence) is considered as failure,(1) single-procedure freedom from both arrhythmia recurrence and ADT was $73.1 \%$. Also the $60 \%$ prevalence of permanent and complete PV isolation at repeat is at the higher end compared to prior studies reporting on the status of complete PV isolation in patients undergoing a repeat procedure for arrhythmia recurrence.(4-6, 30) Taken together, these data suggest that the 'CLOSE' protocol results in acute and late durable PVI.

The 'CLOSE' protocol describes a method to obtain PVI with objective and quantifiable criteria (AI, ILD and ALCI). Although integrity of the deployed RF circle is expected to similar when all parameters are met, reproducibility across centers requires further study.

We surmise that the 'CLOSE' protocol is different from currently used 'dragging' or point-by-point RF strategies. Although anecdotally some operators already pursue a contiguous ablation approach with overlapping lesions, this claim is not justified by objective measures. In the SMART-AF subanalysis - most likely reflecting expert use of CF-guided PVI - more than half of the patients were treated with RF circles characterized by any ILD of $>10.6 \mathrm{~mm}$.(25) Lack of verification of ILD is reflected in the ongoing need for touch-up ablation after first encirclement or adenosine challenge.

Also the use of ablation index to optimize RF delivery is different from conventional CF- or FTIguided strategies. In the 'CLOSE' protocol, low CF values per se do not preclude delivery of RF at that site. In contrast to prior studies, obtained FTI values at the posterior wall are as low as 200gs. $(16,17)$ The use of higher power implies that lesions can be deployed with a relatively short application time (resulting in few dislocations), even at sites with low but stable contact. Finally, in contrast to FTI or CF targets, lesion quality is expected to be similar at a certain AI value (despite 
differences in power, force and time of application required to reach that value).(29)

Our data suggest that durable PV isolation can be obtained with relatively short procedure and RF times. This is explained by the fact that AI allows use of higher power during RF delivery (shorter applications) and by the fact that no time is lost for chasing and managing acute PV reconnection. Although our data suggest that procedural efficiency is higher using general anesthesia, outcome was found to be similar.

The present population is too small to draw any definitive conclusions regarding safety. There is no pre-clinical data to support the safety of any strategy using higher power RF delivery. Nevertheless, in those 130 patients undergoing the 'CLOSE' protocol we observed no complications, especially no audible steam pop, no signs of clinical PV stenosis, no cardiac perforation, permanent stroke, atrioesophageal fistula or death. Absence of esophageal injury despite relatively high power can be explained by short application times at the posterior wall $(\max 20 \mathrm{~s})$ and esophageal $\mathrm{T}^{\circ}$ monitoring.

Finally, we retain considerable challenges for future RF-guided PVI. (1) Our strategy requires validation and optimization using other ablation catheters and/or ablation parameters settings (lower irrigation, higher power...). (2) Although we observed a limited number of dislocations during encircling, it remains to be seen whether this is reproducible across centers. (3) The 'CLOSE' protocol seems appropriate to obtain durable PVI using this strategy of deploying $\approx 30 \mathrm{RF}$ lesions following a 10 to $12 \mathrm{~cm}$ path around the PVs. Whether the 'CLOSE' criteria (AI, ILD and ALCI) might offer an ideal platform to guide wider encirclement of the PVs requires further study. (4) Cutoff-guided ablation strategies, as advocated in the present study, might result in durable isolation at the cost of overshoot and potential safety issue. The current observation of acute durability despite the delivery of AI values lower than 400 at the posterior wall, might be a first step towards avoiding this overshoot. Moreover AI is an estimate and not a direct measure of lesion depth. To minimize RF delivery, each application should be individualized according to on-line in vivo characterization of the tissue before, during and after RF delivery. Only then the unique advantage of point-by-point ablation will be exploited to its full potential.

\section{Limitations}

Although 130 patients were enrolled, several limitations need to be underlined.

First, as many pilot studies, this study was monocentric. We believe however that this feasibility study in a relatively large cohort of 130 patients undergoing 'CLOSE'-PVI was a first and necessary step towards adequately powered multi-center studies. 
Despite a 24h-Holter ECG at 3, 6 months and a 7-day Holter-ECG at 12 months and the symptomatic nature of PAF, the discontinuous nature of monitoring can underestimate AF recurrence.

In the present study, we did not perform voltage mapping at baseline. No pre-ablation imaging with CT or MRI was performed. Therefore we cannot exclude the possibility of an aberrant PV.

Finally, ADT was not systematically withdrawn after the blanking period. Due to the large sample size however, we could report on outcome in 104 'CLOSE' patients not taking ADT.

The above limitations are tackled by ongoing clinical studies evaluating the safety and efficacy of the 'CLOSE' protocol (a multicenter trial, and a study using subcutaneous loop recorders).

\section{Conclusions}

In the present study, we evaluated the feasibility of an ablation protocol aiming to enclose the pulmonary veins with contiguous and optimized RF lesions. We observed a relatively short procedure time, high rate of acute PVI durability and a high single-procedure arrhythmia free survival at 1 year. 


\section{REFERENCES}

1. Calkins H, Kuck KH, Cappato R, et al. Heart Rhythm Society Task Force on Catheter and Surgical Ablation of Atrial Fibrillation. 2012 HRS/EHRA/ECAS Expert Consensus Statement on Catheter and Surgical Ablation of Atrial Fibrillation. Heart Rhythm. 2012;9:632-96.

2. Wilber DJ, Pappone C, Neuzil P, et al. Comparison of antiarrhythmic drug therapy and radiofrequency catheter ablation in patients with paroxysmal atrial fibrillation: a randomized controlled trial. JAMA 2010;303:333-40.

3. Kuck KH, Brugada J, Fürnkranz A, et al. FIRE AND ICE Investigators. Cryoballoon or Radiofrequency Ablation for Paroxysmal Atrial Fibrillation. N Engl J Med. 2016;374:2235-45.

4. Cappato R, Negroni S, Pecora D, et al. Prospective assessment of late conduction recurrence across radiofrequency lesions producing electrical disconnection at the pulmonary vein ostium in patients with atrial fibrillation. Circulation 2003;108:1599-604.

5. Nanthakumar K, Plumb VJ, Epstein AE, Veenhuyzen GD, Link D, Kay GN. Resumption of electrical conduction in previously isolated pulmonary veins: rationale for a different strategy? Circ 2004;109:1226-9.

6. Ouyang F, Tilz R, Chun J, et al. Long-term results of catheter ablation in paroxysmal atrial fibrillation: lessons from a 5-year follow-up. Circulation. 2010;122:2368-77.

7. Andrade JG, Monir G, Pollak SJ, et al. Pulmonary vein isolation using contact force ablation: The effect on dormant conduction and long-term freedom from recurrent atrial fibrillation. A prospective study. Heart Rhythm 2014;11:1919-24.

8. Marijon E, Fazaa S, Narayanan K, et aL. Real-time contact force sensing for pulmonary vein isolation in the setting of paroxysmal atrial fibrillation: procedural and 1-year results. J Cardiovasc Electrophysiol 2014;25:130-7.

9. Reichlin T, Lane C, Nagashima K, et al. Feasibility, efficacy, and safety of radiofrequency ablation of atrial fibrillation guided by monitoring of the initial impedance decrease as a surrogate of catheter contact. J Cardiovasc Electrophysiol. 2015;26:390-6.

10. Afzal MR, Chatta J, Samanta A, et al. Use of contact force sensing technology during radiofrequency ablation reduces recurrence of atrial fibrillation: A systematic review and metaanalysis. Heart Rhythm. 2015;12:1990-6.

11. Ullah W, McLean A, Tayebjee MH, et al.; UK Multicentre Trials Group. Randomized trial comparing pulmonary vein isolation using the SmartTouch catheter with or without real-time contact force data. Heart Rhythm 2016;13:1761-7.

12. Reddy VY, Dukkipati SR, Neuzil P, et al. Randomized controlled trial of the safety and effectiveness of a contact-force sensing irrigated catheter for ablation of paroxysmal atrial fibrillation (Toccastar study). Circulation 2015;132:907-15.

13. El Haddad M, Taghji P, Phlips T, et al. Determinants of acute and late pulmonary vein reconnection in contact force-guided pulmonary vein isolation: identifying the weakest link in the ablation chain. Circ Arrhythm Electrophysiol. 2017;10. pii: e00486.

14. Nakagawa H, Ikeda A, Constantine G, et al. Controlling lesion size and incidence of steam pop by controlling contact force, radiofrequency power and application time (Force-Power-Time Index) in canine beating heart. Heart Rhythm 2012;9:S5.

15. Das M, Loveday JJ, Wynn GJ, et al. Ablation index, a novel marker of ablation lesion quality: 
prediction of pulmonary vein reconnection at repeat electrophysiology study and regional differences in target values. Europace. 2017;19:775-83.

16. Neuzil P, Reddy VY, Kautzner J, et al. Electrical reconnection after pulmonary vein reconnection is contingent on contact force during initial treatment: results from the Efficas I study. Circ Arrhythm Electrophysiol. 2013;6:327-33.

17. Kautzner J, Neuzil P, Lambert H, et al. Optimization of catheter contact force improves outcome of pulmonary vein isolation for paroxysmal atrial fibrillation (EFFICAS II). Europace 2015;17:1229-35.

18. Nath and Haines. Biophysics and Pathology of Catheter Energy Delivery Systems. Progress in Cardiovascular Diseases, Vol XXXVII, No 4 (January/February), 1995: pp 185-204.

19. Wittkampf FH, Nakagawa H. Radiofrequency catheter ablation: lessons on lesions. Pacing Clin Electrophysiol 2006;29:1285-97.

20. Yokoyama K, Nakagawa H, Shah DC, et al. Novel contact force sensor incorporated in irrigated radiofrequency ablation catheter predicts lesion size and incidence of steam pop and thrombus. Circ Arrhythm Electrophysiol. 2008;1:354-362.

21. Ikeda A, Nakagawa H, Lambert H, et al. Relationship between catheter contact force and radiofrequency lesion size and incidence of steam pop in the beating canine heart: electrogram amplitude, impedance, and electrode temperature are poor predictors of electrode-tissue contact force and lesion size. Circ Arrhythm Electrophysiol. 2014; 7:1174-80.

22. Shah DC, Lambert H, Nakagawa H, et al. Area under the real-time contact force curve (forcetime integral) predicts radiofrequency lesion size in an in vitro contractile model. J Cardiovasc Electrophysiol. 2010;21:1038-43.

23. Miller MA, d'Avila A, Dukkipati SR, et al. Acute electrical isolation is a necessary but insufficient endpoint for achieving durable PV isolation: the importance of closing the visual gap. Europace. 2012;14:653-60.

24. Park CI, Lehrmann H, Keyl C, et al. Mechanisms of pulmonary vein reconnection after radiofrequency ablation of atrial fibrillation: the deterministic role of contact force and interlesion distance. J Cardiovasc Electrophysiol 2014;1:1-8.

25. Reddy VY, Pollak S, Lindsay BD, et al. Relationship between catheter stability and 12-month success after pulmonary vein isolation: a subanalysis of the SMART-AF trial. J Am Coll Cardiol EP. 2016:6:691-699.

26. Arujuna A, Karim R, Caulfield D, et al. Acute pulmonary vein isolation is achieved by a combination of reversible and irreversible atrial injury after catheter ablation: evidence from magnetic resonance imaging. Circ Arrhythm Electrophysiol. 2012;5:691-700.

27. Ranjan R, Kato R, Zviman MM, et al. Gaps in the ablation line as a potential cause of recovery from electrical isolation and their visualization using MRI. Circ Arrhythm Electrophysiol. $2011 ; 4: 279-86$.

28. Haines D. Determinants of Lesion Size During Radiofrequency Catheter Ablation: The Role of Electrode-Tissue Contact Pressure and Duration of Energy Delivery. Journal of Cardiovascular Electrophysiology. 1991,2:509-515.

29. Nakagawa H, Ikeda A, Govari A, et al. Prospective study to test the ability to create RF lesions at predicted depths of 3,5, 7 and $9 \mathrm{~mm}$ using a new formula incorporating contact force, 
radiofrequency power and application time (Force-Power-Time Index) in the beating canine heart. Heart Rhythm 2013;10:S481.

30. Heeger $\mathrm{CH}$, Wissner $\mathrm{E}$, Mathew $\mathrm{S}$, et al. Once Isolated, always isolated? Incidence and characteristics of pulmonary vein reconduction after second-generation cryoballoon-based pulmonary vein isolation. Circ Arrhythm Electrophysiol. 2015;8:1088-94. 


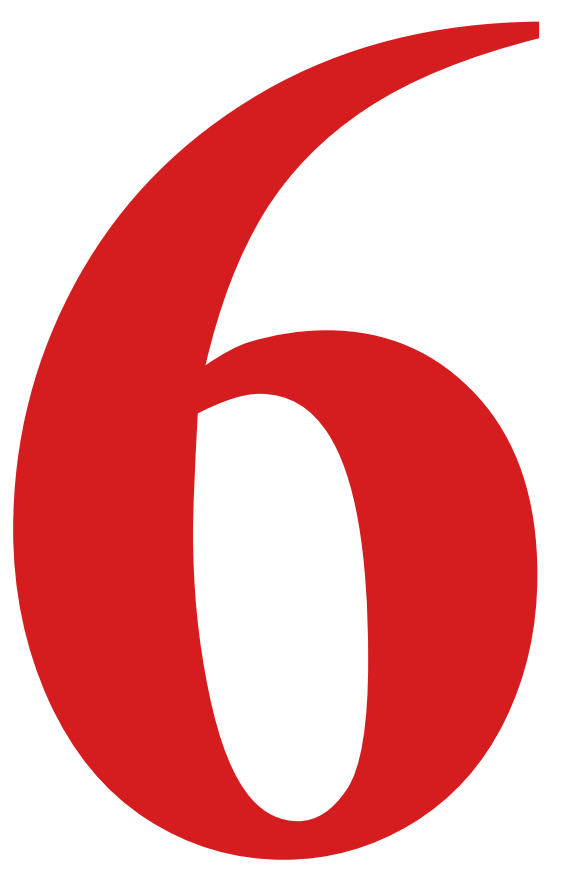




\section{Chapter 6}

\section{Safety evaluation of CLOSE-PVI concerning oesophageal and mediastinal lesions}

Michael Wolf, Milad El Haddad, Vincent De Wilde, Thomas Phlips, Jan De Pooter, Alexandre Almorad, Teresa Strisciuglio, Yves Vandekerckhove, René Tavernier, Harry Crijns, Sébastien Knecht, Mattias Duytschaever 


\begin{abstract}
Background: The incidence of endoscopically detected esophageal lesions after pulmonary vein isolation (PVI) is as high as $18 \%$. Intra-esophageal temperature rise (ITR) during ablation is a predictor for esophageal injury.
\end{abstract}

Objective: We recently described an ablation strategy aiming to enclose the PVs with contiguous, stable and optimized RF applications (referred to as CLOSE-PVI). We evaluated esophageal and periesophageal injury with endoscopy in patients revealing ITR during CLOSE-PVI.

Methods: 85 patients with ITR during CLOSE-PVI underwent endoscopy of the esophagus (with ultrasound in 38 patients). PVI consisted of contact force (CF)-guided encircling of the veins using $35 \mathrm{~W}$ applications respecting strict criteria of intertag distance $(\leq 6 \mathrm{~mm})$ and ablation index (AI 550au anterior wall, 400au posterior wall, 300au if $\operatorname{ITR}>38.5^{\circ} \mathrm{C}$ ).

Results: Endoscopy was performed $9 \pm 4$ days after PVI. At the posterior wall, median power was $35 \mathrm{~W}$

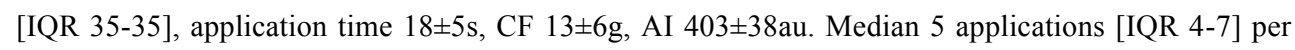
patient over a length of $21.8 \pm 6.8 \mathrm{~mm}$ resulted in ITR $>38.5^{\circ} \mathrm{C}$ (median $39.9^{\circ} \mathrm{C}$, IQR $39.2-41.2^{\circ} \mathrm{C}$, range 38.6-50. $0^{\circ} \mathrm{C}$ ). For these applications median power was $35 \mathrm{~W}$ [IQR 30-35], application time $14 \pm 3 \mathrm{~s}, \mathrm{CF}$ $12 \pm 5 \mathrm{~g}$, AI $351 \pm 38 \mathrm{au}$. The incidence of esophageal erythema/erosion on endoscopy was $1 / 85(1.2 \%)$ and of ulceration $0 / 85(0 \%)$. The incidence of mediastinal or esophageal injury on ultrasound was $0 / 38$ $(0 \%)$.

Conclusions: The occurrence of esophageal or peri-esophageal injury after CLOSE-PVI is markedly low $(1.2 \%)$. Absence of esophageal ulceration in patients with ITR suggests that this strategy of delivering contiguous, relatively high-power, and short-duration applications at the posterior wall is safe. 


\section{Introduction}

Atrio-esophageal fistula (AEF) is a life-threatening complication of pulmonary vein isolation (PVI) for treatment of atrial fibrillation (AF). ${ }^{1}$ Whereas the incidence of AEF is low $(0.02$ to $0.11 \%),{ }^{2}$ there is a high ( 2 to $30 \%$ ) incidence of subclinical esophageal or peri-esophageal injury on endoscopy performed within 7 days after radiofrequency or cryoenergy ablation. ${ }^{3-14}$ Halbfass et al showed that the only risk factor associated with the occurrence of esophageal injury or ulcer is intra-esophageal temperature rise (ITR). ${ }^{3}$ Furthermore they found that an esophageal ulcer is a necessary precursor for $\mathrm{AEF}^{3}$

We recently introduced a new strategy to enclose the veins with contiguous and optimized RF applications. ${ }^{15-17}$ This strategy referred to as CLOSE-PVI is characterized by relatively high-power (35Watts), short-duration applications to target a prespecified ablation index (AI) and is associated with high rate of adenosine-proof, first-pass isolation, favorable 1-year freedom of AF and high rate of durable isolation at repeat procedure. ${ }^{16-18}$ To assess the incidence of esophageal injury (and potential risk of AEF) using this ablation strategy, we performed echo-endoscopy in those patients revealing ITR during CLOSE-PVI.

\section{Methods}

\section{Patient population}

All patients undergoing AF ablation in the St-Jan Hospital Bruges are followed in a prospective database approved by the local institutional review committee. This database implies collecting patients' written informed consent and detailed case report form. From September 2017 to May 2018, 185 patients with paroxysmal or persistent AF underwent a first CLOSE-guided PVI monitored with a luminal esophageal temperature probe. According to institutional protocol (implemented after the study by Halbfass et al), ${ }^{3}$ all patients revealing ITR $>38.5^{\circ} \mathrm{C}$ were advised to undergo endoscopic evaluation of the esophagus within the first 2 weeks after PVI.

\section{PVI according the CLOSE protocol}

The CLOSE-PVI protocol was previously described. ${ }^{16-17}$ Under general anesthesia double transseptal puncture (SL0, St. Jude Medical, St. Paul, Minnesota) was guided by trans-esophageal echocardiography (TEE). After puncture, TEE was removed and a temperature probe with 5 electrodes (SensiTherm, St. Jude Medical) was advanced into the esophagus until positioned at the LA level. Each of the 3 intermediate electrodes has a thermocouple for independent detection of intraluminal temperature. After 3-dimensional-reconstruction of LA geometry (Carto System, Biosense Webster, Diamond Bar, California), a Lasso and contact force (CF) catheter (Thermocool SmartTouch, Biosense Webster,) were used to predefine the RF circle around the PVs (nephroid shape). Identification of the LA-PV junction was based upon anatomy, CF, CF vector, catheter jump during 
pull-back maneuvers, position relative to the Lasso, impedance and local electrogram characteristics (fusion of atrial and PV potentials).

Point-by-point RF delivery was performed aiming for a contiguous circle enclosing the PVs (maximal inter-tag distance between 2 neighboring applications $\leq 6 \mathrm{~mm}$ ). Real-time automated display of RF applications (Visitag, Biosense Webster) was used with predefined settings of catheter stability ( $3 \mathrm{~mm}$ for $8 \mathrm{~s}$ ) and minimum CF ( $30 \%$ of time $>4 \mathrm{~g}$ ). RF was delivered in power-controlled mode (without ramping) at $35 \mathrm{~W}$ (irrigation flow $30 \mathrm{ml} / \mathrm{min}$ ) targeting $\mathrm{AI}$ of $\geq 550 \mathrm{au}$ at the anterior wall and $\mathrm{AI}$ of $\geq 400$ au at the posterior wall/roof. In case of dislocation, a new RF application reaching the prespecified AI target was applied. During encirclement of PVs, the position of the temperature probe was manually adjusted in order to place the 3 sensors as closely as possible to the respective ablation site (Figure 1). In case of early ITR $>38.5^{\circ} \mathrm{C}$, RF was continued until target $\mathrm{AI} \geq 300 \mathrm{au}$ (Figure 2). In case of ITR $>38.5^{\circ} \mathrm{C}$ observed between AI 300 and $400 \mathrm{au}$, RF was stopped, in case of ITR $>38.5^{\circ} \mathrm{C}$ after reaching 400au, the site was noted. Before applying RF energy to the next adjacent position, we waited for temperature dropping $<38.5^{\circ} \mathrm{C}$. During this waiting time, the operator could stay on site and wait for temperature drop (preferred strategy if minor ITR and quick decline of temperature after RF cessation) or choose to ablate at a remote locus before going to the adjacent position (preferred strategy if high ITR and/or slow decline). RF power, as previously described, was only reduced in case of catheter tip temperature rise $>43^{\circ} \mathrm{C}$ or in case of high $\mathrm{CF}(\geq 20 \mathrm{~g})$ that might lead to overshoot of the AI target. In the absence of first-pass isolation, touch-up ablation was delivered guided by the earliest PV potential on the Lasso-catheter until PVI was achieved.

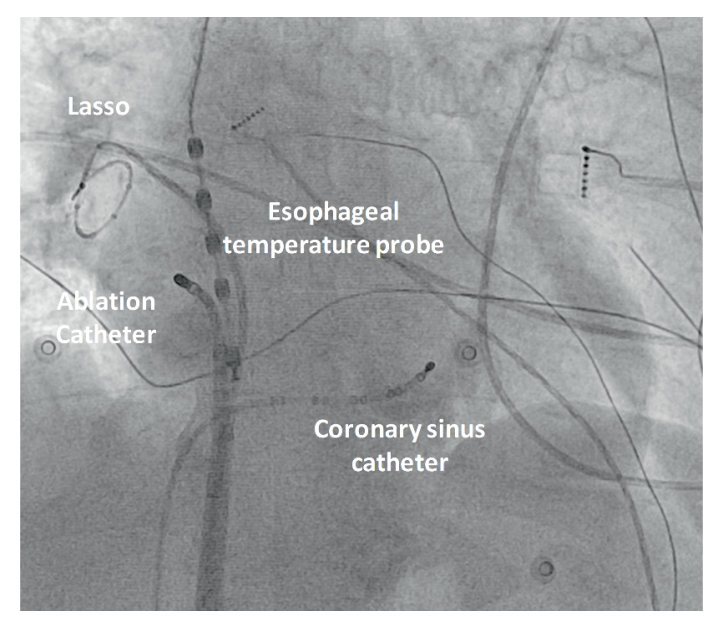

Figure 1: Luminal esophageal temperature probe.

During encirclement of the right veins, a circular mapping catheter is positioned in the right superior pulmonary vein. The position of a luminal esophageal temperature probe (SensiTherm, St. Jude Medical, St. Paul, Minnesota) is adjusted so that the 3 thermosensors are as close as possible to the respective ablation site. 


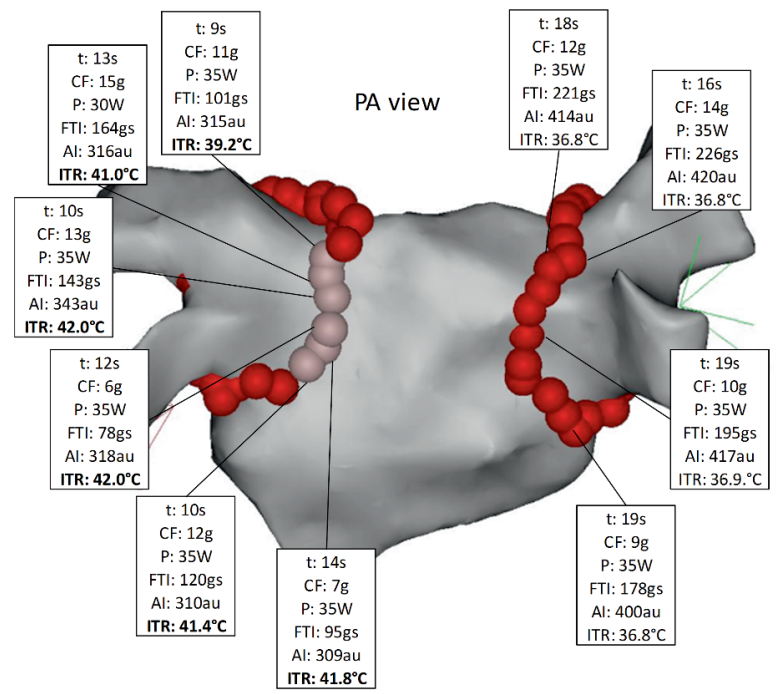

Figure 2: CLOSE-guided PVI.

Representative example of CLOSE-PVI and off-line analysis. In this patient, applications on the posterior wall of the right circle did not result in temperature rise, whereas six applications on the posterior wall of the left circle resulted in intra-esophageal temperature rise $>38.5^{\circ} \mathrm{C}$. The ablation index target was reduced to $\geq 300 \mathrm{au}$ (pink Visitags). (AI: ablation index; CF: contact force; FTI: force-time integral; ITR: intra-esophageal temperature rise; P: power; $t$ : radiofrequency time)

\section{Post-procedural care and follow-up}

Complications were reported and collected during follow-up. According to institutional protocol all PVI patients were prescribed omeprazole 40mg daily for 1 month. Follow-up consisted of clinical visit at 1, 3, 6 and 12 months.

\section{Endoscopic evaluation of the esophagus}

Endoscopic examinations were scheduled $<14$ days after PVI and performed by 4 experienced operators. All patients were kept fasting overnight prior to examination. As standard of care all patients underwent clinical evaluation of symptoms (chest pain, dysphagia, fever or neurological symptoms) before endoscopy. They were examined in left lateral position under conscious sedation with midazolam $2.5 \mathrm{mg}$ and/or alfentanyl $0.5 \mathrm{mg}$ IV. Oxygen saturation, pulse rate and blood pressure were monitored. Evaluation of the esophagus, stomach and duodenum was performed using a standard video endoscope (Pentax EG 29-i10 and Olympus GIF-H180). Endoscopic lesions were attributed to $\mathrm{AF}$ ablation if they were located on the anterior wall of the mid-esophagus. Esophageal lesions were classified into erythema/erosion, ulcer or perforation.,

Endoscopic ultrasound investigation (EUS) was performed using a radial scanning ultrasound endoscope (Pentax EG 3670URK) and the Hi Vision AVIUS ultrasound system from Hitachi. Careful 
examination of mediastinum and esophageal wall was performed to assess mucosal and periesophageal/mediastinal lesions.

\section{Off-line analysis of the deployed RF circle}

Procedures from all patients with ITR were analyzed off-line. For each RF tag on the posterior wall we determined application time (s), power (W), CF (g), force-time integral (FTI) (gs), AI (arbitrary unit, au) and the maximum intra-esophageal temperature $\left({ }^{\circ} \mathrm{C}\right)$ (Figure 2). To anatomically allocate sites with ITR, the posterior hemisphere of each circle was divided into 5 segments. To assess the lesion length associated with ITR, we calculated the distance between most cranial and caudal RF tags causing ITR.

\section{Results}

\section{Patient population and procedural characteristics}

From October 2017 to May 2018, 185 patients underwent first-time CLOSE-guided PVI with luminal esophageal temperature probe. ITR $>38.5^{\circ} \mathrm{C}$ was observed in 133 procedures $(72 \%) .85$ patients consented for endoscopic evaluation. In the first 38 patients, additional EUS was performed. Patient and procedural characteristics are detailed in Supplementary Table S1. Mean number of applications per circle was $57 \pm 15$, mean number of dislocations was $2 \pm 1$, and mean number of dislocations on the posterior wall was $0 \pm 1$. First-pass isolation was obtained in $162 / 170$ circles $(95 \%)$. During a median follow-up of 8.9 months [IQR 7.1-11.1] no complications of esophageal fistula, stroke or death were noted.

\section{Characteristics of the RF applications}

A representative example is given in Figure 2. Results are summarized in Table 1. At the posterior wall, median power was $35 \mathrm{~W}$ [IQR 35-35], application time $18 \pm 5 \mathrm{~s}$, CF $13 \pm 6 \mathrm{~g}$, AI $403 \pm 38$ au. Due to high baseline CF, power was reduced to $<35 \mathrm{~W}$ (25W [IQR 25-30] in median 1 [IQR 0-3] RF application per patient. For the left PV circle median 10 [IQR 9-12] and for the right PV circle median 12 [IQR 10-14] RF applications were located on the posterior wall. Median 5 [IQR 4-7] RF applications per patient resulted in ITR $>38.5^{\circ} \mathrm{C}$, covering a mean length of $21.8 \pm 6.8 \mathrm{~mm}$ along the posterior wall. 
Table 1: RF characteristics of all applications on the posterior wall and of applications with intra-esophageal temperature rise

\begin{tabular}{|l|c|c|}
\hline & $\begin{array}{c}\text { Applications } \\
\text { posterior wall }\end{array}$ & $\begin{array}{c}\text { Applications } \\
\text { with ITR }\end{array}$ \\
\hline Number of tags, $\mathrm{n}$ & $22[19-26]$ & $5[4-7]$ \\
\hline RF time, $\mathrm{s}$ & $18 \pm 5$ & $14 \pm 3$ \\
\hline CF, $\mathrm{g}$ & $13 \pm 6$ & $12 \pm 5$ \\
\hline Power, $\mathrm{W}$ & $35[35-35]$ & $35[30-35]$ \\
\hline FTI, gs & $219 \pm 88$ & $165 \pm 77$ \\
\hline AI, au & $403 \pm 38$ & $351 \pm 38$ \\
\hline
\end{tabular}

AI: ablation index; CF: contact force; fore-time integral; ITR: intra-esophageal temperature rise $>38.5^{\circ} \mathrm{C}$

The spatial distribution of sites with ITR is shown in Figure 3. In 53 patients ITR was observed deploying the left PV circle and in 36 patients deploying the right (in 4 patients in both circles). Baseline esophageal temperature at those sites was $36.2 \pm 0.3^{\circ} \mathrm{C}$. Maximal temperature observed was $39.9^{\circ} \mathrm{C}\left(\left[\mathrm{IQR} 39.2-41.2^{\circ} \mathrm{C}\right]\right.$ and range $38.6-50.0^{\circ} \mathrm{C}$, Figure 4$)$. Of interest, out of $405 \mathrm{RF}$ applications resulting in ITR, only $36(8.9 \%)$ were characterized by delayed ITR (i.e. occurring only after stopping $\mathrm{RF}$ at the AI target of 400au).

For applications resulting in ITR, median power was $35 \mathrm{~W}$ [IQR 30-35], application time $14 \pm 3 \mathrm{~s}$, CF $12 \pm 5 \mathrm{~g}$, AI $351 \pm 38 \mathrm{au}$. In case of ITR, the median time in between neighboring applications was $22 \mathrm{~s}$ [IQR 14-34]. In 14 patients with ITR, the operator chose to ablate at a remote locus (3 [IQR 2-4] applications) before resuming RF at the neighboring spot.

Spatial distribution of intra-esophageal temperature rise $>38.5^{\circ} \mathrm{C}$ in 85 patients

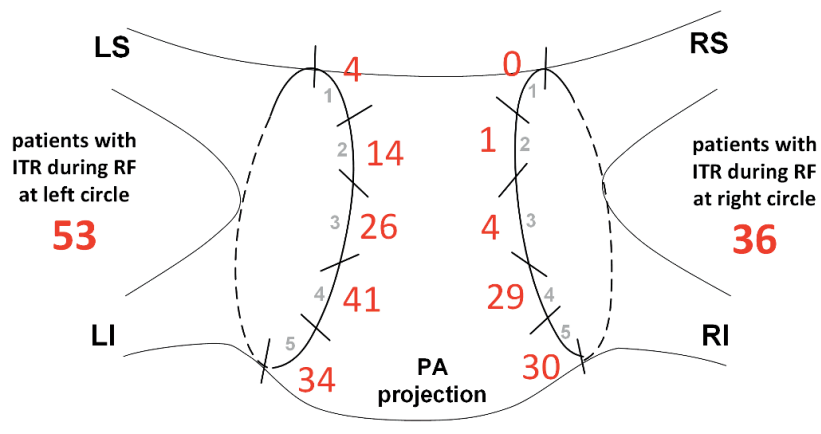

Figure 3: Spatial distribution of intra-esophageal temperature rise in 85 patients.

Number of patients showing ITR $>38.5^{\circ} \mathrm{C}$ : frequency per circle and per segment. 


\section{RF applications with ITR $>38.5^{\circ} \mathrm{C}$}

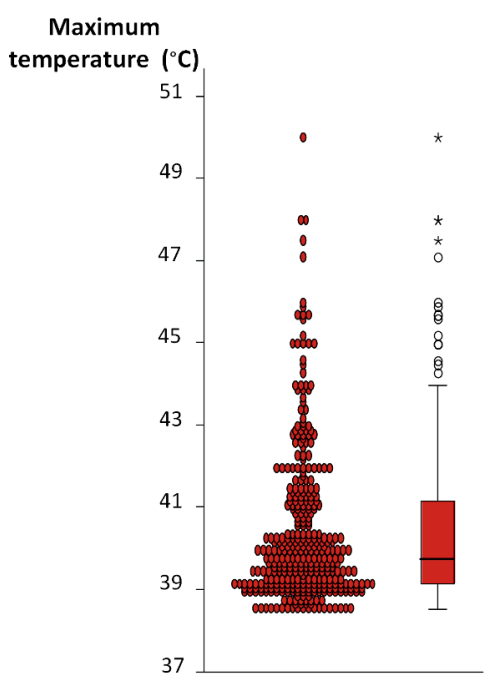

Figure 4: Maximum intra-esophageal temperature rise.

All applications resulting in ITR $>38.5^{\circ} \mathrm{C}$ and their respective maximum intra-esophageal temperature.

\section{Endoscopic evaluation}

Endoscopy was performed after mean $9 \pm 4$ days after PVI. In none of the 85 patients an esophageal ulcer was detected. In one patient we observed two erythematous lesions at day 4 after ablation (Figure 5). In this patient RF was applied on the posterior wall for 26 applications (RF duration 8.4 min). ITR was observed during three applications along the left inferior PV (max. ITR 39.5, 40.1 and $41.2^{\circ} \mathrm{C}$ respectively).

Furthermore endoscopy revealed following findings in 28 of 85 patients: peptic esophagitis in 16 patients (grade A, B, C and D in respectively $n=13, n=1, n=1, n=1$ ), short Barrett esophagus in 3 patients, esophageal moniliasis in 3 patients, gastric antritis/gastritis in 8 patients, gastric erosion/ulcer in 4 patients, duodenal erosion/ulcer in 2 patients, an ampulloma in 1 patient. Findings were classified as incidental, and not related to the ablation procedure, based upon the anatomical location (distant from the left atrial wall). 


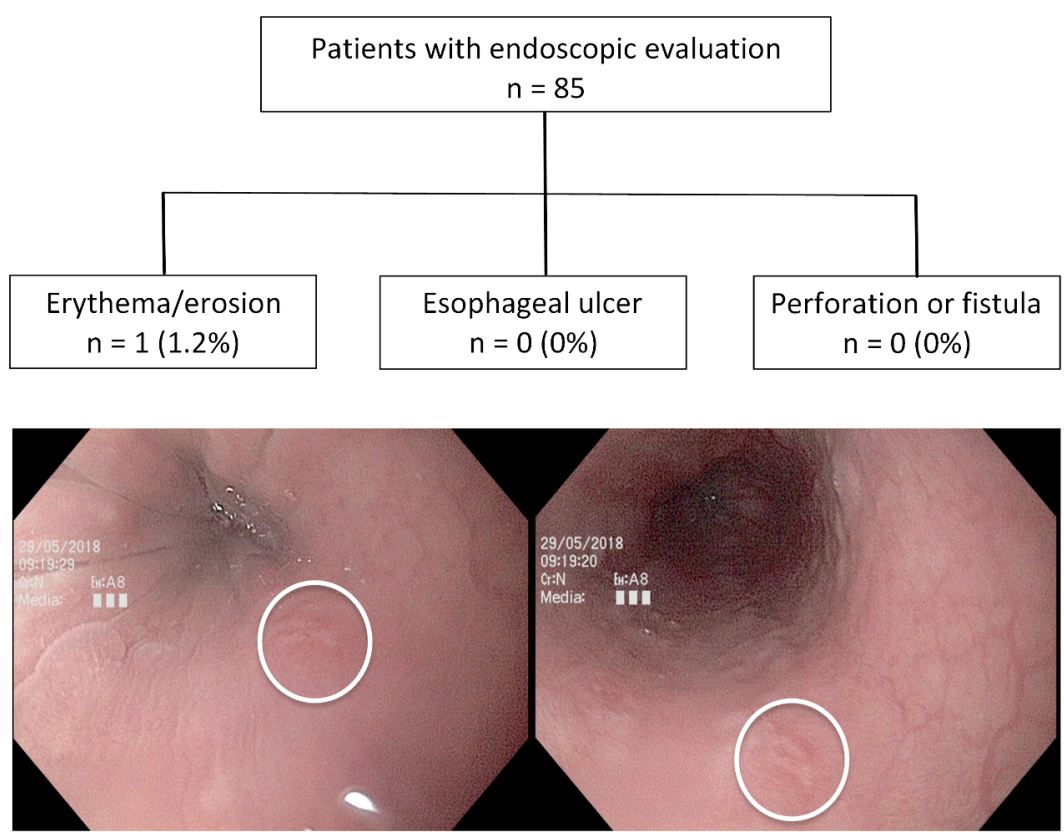

Figure 5: Results of endoscopic evaluation.

In only $1 / 85$ patients endoscopy revealed two erythematous lesions at day 4 after ablation.

\section{Ultrasound evaluation}

In none of the 38 patients undergoing additional sonographic evaluation, we observed direct signs of injury to the esophageal wall. Peri-esophageal lymph nodes at the atrial level were observed in 13 patients, minimal pleural effusion in 17 patients, physiological pericardial effusion in 7 patients and focal pleural thickening in 2 patients.

\section{8 patients not undergoing endoscopy}

In the present study, 48 ITR patients did not undergo endoscopy because of decline by the patient (large distance home-hospital, undesired absence from work or aversion for endoscopy). Of interest, clinical, RF and ITR characteristics were not different from the 85 patients undergoing endoscopy. More specifically, at the posterior wall, median power was $35 \mathrm{~W}$ [IQR 35-35], application time 18 $\pm 6 \mathrm{~s}$, CF $12 \pm 6 \mathrm{~g}, \mathrm{AI} 404 \pm 44 \mathrm{au}$, with a median of 4 [IQR 4-6] RF applications per patient resulting in ITR. Finally in none of the patients an adverse event was noted at follow-up. 


\section{Discussion}

\section{Main findings}

The incidence of esophageal injury on endoscopy after CLOSE-PVI is low (1.2\%) despite significant ITR during the procedure. Absence of esophageal ulceration and lack of changes on ultrasound suggest that this specific strategy of delivering relatively high-power, short-duration optimized applications at the posterior wall might be safe. These findings require confirmation in a larger patient cohort.

\section{Prior studies reporting on the incidence of esophageal injury on endoscopy}

The incidence of endoscopically detected esophageal injury after catheter ablation is high, both after PVI guided by cryoballoon (from 3 to $19 \%)^{13-14}$ and by RF energy (from 2 to $30 \%$ ). ${ }^{3-12}$ The majority of reports on esophageal injury are based upon endoscopy performed within two to three days after PVI and describe both erythema and ulceration. In the present discussion we focus on the incidence of ulceration (1) because ulceration and not erythema is a known precursor of fistula and (2) because erythema (in contrast to ulceration) is potentially underestimated in the present paper due to relatively late timing of endoscopy (see below). Several papers reported on injury after RF-guided PVI performed with $\leq 25 \mathrm{~W}$ on the posterior wall without esophageal temperature probe. Whereas Martinek et $\mathrm{al}^{5}$ (267 patients, non CF) and Blockhaus et $\mathrm{al}^{6}$ (70 patients, CF range 10-40g) reported injury in only $2.2 \%$ (1.5 ulceration) and $2.9 \%$ (1.4\% ulceration) respectively, Halbfass et al (100 patients, CF $10-40 \mathrm{~g}$ ) reported an incidence of $13 \%$ (with $8 \%$ ulceration). ${ }^{7}$ The incidence is equally high after PVI in which RF delivery is guided by an esophageal temperature probe and ITR. Di Biase et al (50 patients, $35 \mathrm{~W}$ over the posterior wall with discontinuation of $\mathrm{RF}$ in case of ITR $>39^{\circ} \mathrm{C}$ ) reported esophageal injury in $26 \%$ of patients. ${ }^{8}$ Muller et al ( 40 patients, $\leq 25 \mathrm{~W}$ posterior wall, CF target 10 $40 \mathrm{~g}$, discontinuation of RF delivery if ITR $\geq 39.5^{\circ} \mathrm{C}$ ) reported an incidence of esophageal injury in $30 \%$ (7.5\% ulceration). ${ }^{9}$ Halbfass et al (40 patients, $\leq 25 \mathrm{~W}, \mathrm{CF} 10-20 \mathrm{~g}$ and RF time $\leq 20 \mathrm{~s}$ on posterior wall, discontinuation of RF delivery at ITR $\left.>39^{\circ} \mathrm{C}\right)$ reported an incidence of $7.5 \%$ (2.5\% ulceration). ${ }^{10}$ The high incidence of endoscopically detected esophageal injury after PVI is reflected in a recent meta-analysis including 3921 patients (mainly RF-ablation). ${ }^{4}$ The overall incidence of endoscopic injury after ablation was $15 \%$ with $5.2 \%$ incidence of erythema, 5.7\% superficial ulceration and $3.6 \%$ deep ulceration. The high incidence is also in line with the recently published largest single-center registry (832 patients), in which Halbfass et al demonstrated that endoscopically detected esophageal injury early after RF ablation (performed within 7 days) is as high as $18 \%$ (12\% erythema, $6 \%$ ulceration). ${ }^{3}$

There is only limited data on predictors for esophageal injury. Smaller studies have identified lower body mass index ${ }^{19}$, general anesthesia ${ }^{8}$, use of a nasogatric tube for visualisation of the esophagus ${ }^{20}$, use of TEE probe ${ }^{21}$ and maximal energy at the posterior wall ${ }^{20}$ to be predictors of esophageal injury. In the study from Halbfass et al, the only risk factor associated with the occurrence of esophageal injury 
was ITR $>40.5^{\circ} \mathrm{C}^{3}$ This study underscores the relevance of performing and reporting on endoscopy in those specific patients with ITR during ablation.

\section{Performing endoscopy in patients with intra-esophageal temperature rise during ablation}

We observed a low incidence of esophageal injury after CLOSE-PVI with ITR (1.2\%), despite reaching a median temperature of $39.9^{\circ} \mathrm{C}$ and despite using relatively high power. This incidence is at the lower end compared to prior studies on esophageal injury, ${ }^{3-12}$ and more specifically compared to prior reports on endoscopy performed in ITR patients.

Contreras-Valdes et al ( 82 patients with ITR $\geq 39^{\circ} \mathrm{C}, 25 \mathrm{~W}$ on the posterior wall, discontinuation of RF delivery at ITR $\left.>38^{\circ} \mathrm{C}\right)$ reported esophageal injury in $27 \%\left(8.5 \%\right.$ deep ulceration) of ITR patients. ${ }^{11}$ Knopp et al. ( 425 patients with ITR $>41^{\circ} \mathrm{C}, 30 \mathrm{~W}$ on the posterior wall in proximity to the esophageal temperature probe) found thermal esophageal lesions in $11 \%$ of ITR patients. ${ }^{12}$

One might speculate that a protocol of performing endoscopy only in patients with ITR underestimates the true incidence of injury after all CLOSE-PVI procedures. It seems however unlikely that the incidence of injury would be higher in patients without ITR. Indeed, Halbfass et al determined ITR as the only risk factor for injury. Moreover, due to careful positioning of the probe, patients with ITR represent the large majority of CLOSE-PVI patients ( $72 \%$ of cases).

Finally, it seems unlikely that we missed esophageal injury (and more specifically ulcer) due to the relatively late timing of endoscopy ( $9 \pm 4$ days). In those patients with endoscopy within 7 days we also did not observe any ulceration. Moreover, also in the study by Halbfass et al, reporting $18 \%$ of injury, endoscopy was performed up to 7 days after ablation. ${ }^{3}$ Finally in that latter study, 47 patients with documented esophageal injury underwent a control endoscopy at $12.3 \pm 8.9$ days. Whereas in those patients with initial erythema/erosion, the site of injury was still detectable in $21 \%$ of patients, in those patients with initial ulcer the site of injury was still apparent in $83 \%$ of patients. As such it is unlikely that ulceration was missed in the present study. This hypothesis is further supported by the absence of esophageal or peri-esophageal wall injury on ultrasound. A prior study has shown that ultrasound has the potential to detect thermal injury-induced changes to the esophagus (submucosal and mucosal) and to the peri-esophageal tissue in patients with normal endoscopy. ${ }^{22}$

\section{What explains the present findings?}

Most likely the low incidence after CLOSE-PVI is explained by the ablation protocol itself. (1) Due to the automated ablation tag, stability and contiguity criteria (resulting in limited dislocations and high incidence of first-pass isolation), there is a minimal amount of applications required to isolate the PVs. On average 10 applications per circle cross the posterior wall and only 5 of those are associated with ITR. (2) In contrast to prior RF strategies, CLOSE-PVI is characterized by short-duration, relatively higher power $(35 \mathrm{~W})$ applications. Due to the pre-specified AI targets, RF applications at the posterior wall can be as short as 10s (mean 18s) for AI 400au and as short as $8 \mathrm{~s}$ (mean 14s) for AI 300au. It has 
been hypothesized that short duration-high power (SD-HP) applications favor the balance in resistive to convective heating thus preventing the risk of collateral damage. ${ }^{23}$ At least in experimental models, SD-HP applications result in more shallow lesions. ${ }^{24,25}$ (3) Also inherent to CLOSE-PVI, in contrast to prior RF studies, is the concept of striving for high stability rather than high CF. Excessive CF by the ablation catheter (applied in non-CF guided procedures or in earlier CF studies) might result in inadvertent displacement of the posterior wall towards the esophagus. ${ }^{26}$ (4) Finally CLOSE-PVI is characterized by ablation at the LA-PV junction. Avoiding a wider encirclement reduces potential overlap with the esophagus and limits the number of procedures in which ITR is observed during both the left and right circle. Indeed only in 4 patients we observed ITR during both left and right PV encirclement.

\section{Clinical implications}

Esophageal injury and AEF are obstacles in the search for durable PVI. In the past, powerful energy to isolate the veins was associated with fistulisation. ${ }^{27}$ In order to avoid thermal esophageal lesions, the 2017 expert consensus statement advises 1) reduction of RF power along the posterior wall (class I indication) and 2) use of an intraluminal temperature probe to monitor ITR to help guide energy delivery (class IIa indication). ${ }^{2}$ Reduction of RF power however might lead to non-durable PVI and more repeat ablations. Moreover, during the procedure itself lower power might lead to nonadenosine-proof or non-first-pass isolation with the ensuing need for touch-up applications (with possible accumulation of RF delivery and conductive heating at particular locations, even paradoxically increasing the risk for AEF formation).

The findings of the present study suggest that CLOSE-guided ablation could offer a strategy in which efficacy and safety go hand in hand despite relatively higher power at the posterior wall. Indeed, in a prospective cohort study CLOSE-PVI was associated with a very high rate of first-pass and adenosineproof PV isolation and a single-procedure arrhythmia-free survival of $92.3 \%$ at 1-year for paroxysmal AF. ${ }^{17}$ The present data suggest that CLOSE-PVI is safe from the esophageal perspective, because the risk for AEF development is unlikely in absence of ulceration. The low incidence of erythema/erosion and the absence of ultrasound lesions suggest that our observations are not a matter of sample size.

These initial results however need confirmation. The safety and efficacy of the CLOSE-PVI protocol are currently being investigated in a multi-center randomized controlled trial (the VISTAX-study, ClinicalTrials.gov Identifier: NCT03062046). Furthermore it remains to be seen whether our data can be extrapolated to other patient populations (dilated atria, more thin-walled atria, fibrosis) and other strategies using higher power applications (but without following specific criteria inherent to the CLOSE-PVI protocol like contiguity, temperature monitoring, optimized AI target). 


\section{Limitations}

1) The number of patients evaluated with endoscopy was limited $(n=85)$. Furthermore, to prevent long waiting time between PVI and endoscopy, evaluation by ultrasound was not continued after the first series of 38 patients. The absence of ulcer and lack of morphological changes in the periesophageal connective tissue evaluated with EUS however are reassuring compared to other studies with the same sample size.

2) Only patients with ITR were evaluated as per institutional protocol. Those patients however represent the majority of CLOSE-PVI cases (ITR was observed in $72 \%$ of the cases). This is explained by dynamic, optimal positioning of the esophageal temperature probe. Moreover ITR has been shown to be a predictor of injury.

3) Only one type of luminal temperature probe (a non-insulated multi-electrode with fast response time) was used in our study. Non-insulated probes have been shown to increase esophageal injury. $3,9,10,28,29,30$

4) As the temperature probe was only used in anesthetized patients, the conclusion of safety of the CLOSE-PVI protocol with regards to esophageal injury cannot be generalized to unmonitored and awake patients.

5) In none of the patients we did perform pre-procedural imaging to define LA-PV junction or esophagus. Ablation closer to the PVs might explain the minimal number of applications required to isolate the veins and lack of esophageal injury.

6) Finally, 48 patients with ITR did not undergo endoscopy. It is unlikely however that those patients would have a different risk for esophageal injury because the reason not to perform EGD was not procedure or operator related, but simply due to decline of the patient. This is in line with the observation that those 48 patients had similar clinical, procedural and ITR characteristics.

\section{Conclusion:}

The absence of esophageal ulceration after CLOSE- and ITR-guided PVI suggests that this strategy of delivery of contiguous relatively high-power and short-duration applications is safe. 


\section{References}

1. Barbhaiya CR, Kumar S, John RM, et al. Global survey of esophageal and gastric injury in atrial fibrillation ablation: incidence, time to presentation, and outcomes. J Am Coll Cardiol 2015;65:1377-1378.

2. Calkins H, Hindricks G, Cappato R, et al. 2017 HRS/EHRA/ECAS/APHRS/SOLAECE expert consensus statement on catheter and surgicalablation of atrial fibrillation. Heart Rhythm 2017;14:e275-e444.

3. Halbfass P, Pavlov B, Müller P, et al. Progression From Esophageal Thermal Asymptomatic Lesion to Perforation Complicating Atrial Fibrillation Ablation: A Single-Center Registry. Circ Arrhythm Electrophysiol 2017;10(8).pii:e005233.

4. Yarlagadda B, Deneke T, Turagam M, et al. Temporal Relationships between Esophageal Injury Type and Progression in Patients Undergoing Atrial Fibrillation Catheter Ablation. Heart Rhythm 2018; pii:S1547-5271(18)31010-5.

5. Martinek M, Meyer C, Hassanein S, et al. Identification of a high-risk population for esophageal injury during radiofrequency catheterablation of atrial fibrillation: procedural and anatomical considerations. Heart Rhythm 2010;7:1224-1230.

6. Blockhaus C, Müller P, Vom Dahl S, et al. Low Incidence of Esophageal Lesions After Pulmonary Vein Isolation Using Contact-Force Sensing Catheter Without Esophageal Temperature Probe. Int Heart J 2017;58:880-884.

7. Halbfass P, Nentwich K, Krug J, et al. Impact of surround flow catheter tip irrigation in contact force ablation on the incidence of asymptomatic oesophageal lesions after atrial fibrillation ablation: a prospective comparative study. Europace 2017;19:1116-1122.

8. Di Biase L, Saenz LC, Burkhardt DJ, et al. Esophageal capsule endoscopy after radiofrequency catheter ablation for atrial fibrillation: documented higher risk of luminal esophageal damage with general anesthesia as compared with conscious sedation. Circ Arrhythm Electrophysiol 2009;2:108-112.

9. Müller P, Dietrich JW, Halbfass P, et al. Higher incidence of esophageal lesions after ablation of atrial fibrillation related to the use of esophageal temperature probes. Heart Rhythm 2015;12:1464-1469.

10. Halbfass P, Müller P, Nentwich K, et al. Incidence of asymptomatic oesophageal lesions after atrial fibrillation ablation using an oesophageal temperature probe with insulated thermocouples: a comparative controlled study. Europace 2016;19:385-391.

11. Contreras-Valdes FM, Heist EK, et al. Mansour M. Severity of esophageal injury predicts time to healing after radiofrequency catheter ablation for atrial fibrillation. Heart Rhythm 2011;8:18621868 .

12. Knopp H, Halm U, Lamberts R, et al. Incidental and ablation-induced findings during upper gastrointestinal endoscopy in patients after ablation of atrial fibrillation: a retrospective study of 425 patients. Heart Rhythm 2014;11:574-578.

13. Fürnkranz A, Bordignon S, Schmidt B, et al. Luminal esophageal temperature predicts esophageal lesions after second-generation cryoballoon pulmonary vein isolation. Heart Rhythm 2013;10:789793.

14. Fürnkranz A, Bordignon S, Böhmig M, et al. Reduced incidence of esophageal lesions by luminal esophageal temperature-guided second-generation cryoballoon ablation. Heart Rhythm 2015;12:268-274.

15. El Haddad M, Taghji P, Phlips T, et al. Determinants of Acute and Late Pulmonary Vein Reconnection in Contact Force-Guided Pulmonary Vein Isolation: Identifying the Weakest Link in the Ablation Chain. Circ Arrhythm Electrophysiol 2017;10(4).pii:e004867. 
16. Phlips T, Taghji P, El Haddad M, et al. Improving procedural and one-year outcome after contact force-guided pulmonary vein isolation: the role of interlesion distance, ablation index, and contact force variability in the 'CLOSE'-protocol. Europace 2018 doi:10.1093/europace/eux376. [Epub ahead of print]

17. Taghji P, El Haddad M, Phlips T, et al. Evaluation of a strategy aiming to enclose the pulmonary veins with contiguous 2 and optimized RF lesions in paroxysmal atrial fibrillation: a pilot study. $\mathrm{J}$ Am Coll Cardiol EP 2018;4:99-108.

18. De Pooter J, Strisciuglio T, El Haddad M, et al. Pulmonary vein reconnection no longer occurs in the majority of patients after a single pulmonary vein isolation procedure. J Am Coll Cardiol EP 2018 (in press).

19. Rillig A, Meyerfeldt U, Birkemeyer R, et al. Oesophageal temperature monitoring and incidence of oesophageal lesions after pulmonary vein isolation using a remote robotic navigation system. Europace 2010;12:655-661.

20. Martinek M, Bencsik G, Aichinger J, et al. Esophageal damage during radiofrequency ablation of atrial fibrillation: impact of energy settings, lesion sets, and esophageal visualization. J Cardiovasc Electrophysiol 2009;20:726-733.

21. Kumar S, Brown G, Sutherland F, et al. The transesophageal echo probe may contribute to esophageal injury after catheter ablation for paroxysmal atrial fibrillation under general anesthesia: a preliminary observation. J Cardiovasc Electrophysiol $2015 ; 26: 119-126$.

22. Zellerhoff S, Ullerich H, Lenze F, et al. Damage to the esophagus after atrial fibrillation ablation: Just the tip of the iceberg? High prevalence of mediastinal changes diagnosed by endosonography. Circ Arrhythm Electrophysiol 2010;3:155-159.

23. Barkagan M, Contreras-Valdes FM, Leshem E, Buxton AE, Nakagawa H, Anter E. High-power and short-duration ablation for pulmonary vein isolation: Safety, efficacy, and long-term durability. J Cardiovasc Electrophysiol 2018;29:1287-1296.

24. Leshem E, Zilberman I, Tschabrunn CM, et al. High-Power and Short Duration Ablation for Pulmonary Vein Isolation: Biophysical Characterization. JACC Clin Electrophysiol 2018;4:467479.

25. Bourier F, Duchateau J, Vlachos K, et al. High-power short-duration versus standard radiofrequency ablation: Insights on lesion metrics. J Cardiovasc Electrophysiol 2018;29:15701575 .

26. Chelu MG, Morris AK, Kholmovski EG, et al. Durable lesion formation while avoiding esophageal injury during ablation of atrial fibrillation: Lessons learned from late gadolinium MR imaging. J Cardiovasc Electrophysiol 2018;29:385-392.

27. Neven K, Schmidt B, Metzner A, et al. Fatal end of a safety algorithm for pulmonary vein isolation with use of high-intensity focused ultrasound.Circ Arrhythm Electrophysiol 2010;3:260265.

28. Carroll BJ, Contreras-Valdes FM, Heist EK, et al. Multi-sensor esophageal temperature probe used during radiofrequency ablation for atrial fibrillation is associated with increased intraluminal temperature detection and increased risk of esophageal injury compared to single-sensor probe. $\mathbf{J}$ Cardiovasc Electrophysiol 2013;24:958-964.

29. Pérez JJ, D'Avila A, Aryana A, Berjano E. Electrical and thermal effects of esophageal temperature probes on radiofrequency catheterablation of atrial fibrillation: results from a computational modeling study. J Cardiovasc Electrophysiol 2015;26:556-564.

30. Berger RD. Lightning rods that don't protect. Heart Rhythm 2015;12:2170-2171. 


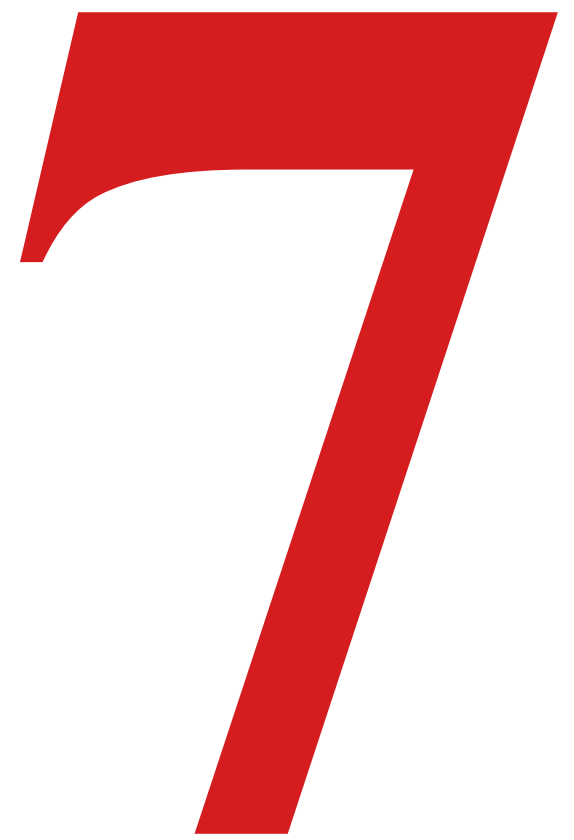




\section{Chapter 7}

\section{Evaluation of the durability of pulmonary vein isolation in CLOSE-PVI}

Jan De Pooter, Teresa Strisciuglio, Milad El Haddad, Michael Wolf, Thomas Phlips, Yves Vandekerckhove, René Tavernier, Sebastien Knecht, Mattias Duytschaever

JACC Clin Electrophysiol. 2019; 5: 295-305.

doi: 10.1016/j.jacep.2018.11.020. 


\begin{abstract}
Objectives: This study sought to determine the prevalence of patients with 4 isolated veins at repeat ablation after 'CLOSE'-guided pulmonary vein isolation (PVI), a strategy based upon delivery of contiguous and optimized radiofrequency lesions.
\end{abstract}

Background: The likelihood of finding 4 isolated veins at a repeat ablation for atrial fibrillation (AF) recurrence after a first PVI is low.

Methods: Patients undergoing repeat ablation for AF recurrence after first 'CLOSE'-guided PVI were included. At repeat, 1) the status of the PVs was evaluated and 2) high density voltage mapping was performed. In case of pulmonary vein reconnection (PVR), veins were re-isolated. In patients with 4 isolated veins, empirical trigger or substrate ablation was performed.

Results: Out of 326 patients undergoing CLOSE-guided PVI for paroxysmal AF, 45 patients

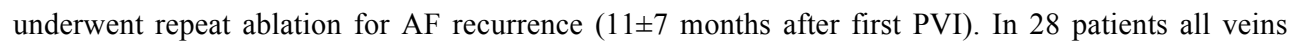
were still isolated (62\%). They showed similar clinical characteristics and similar time from first PVI to $\mathrm{AF}$ recurrence $(8 \pm 7$ versus $6 \pm 6$ months respectively, $\mathrm{p}=0.453)$ compared to patients with PVR. In contrast, they were characterized by a higher incidence of low voltage $(57 \%$ versus $17 \%, \mathrm{p}=0.033)$. Patients with 4 isolated veins showed a lower 12-month freedom from AF after repeat ablation compared to patients treated for PVR ( $61 \%$ vs $88 \%, \mathrm{p}=0.045)$.

Conclusion: After 'CLOSE' guided ablation, PVR is no longer the rule in patients with AF recurrence. Patients with AF recurrence and 4 isolated veins present with a similar clinical profile and time to recurrence that compared to patients with PVR. 


\section{Introduction}

Pulmonary vein isolation (PVI) is considered the cornerstone for successful ablation in atrial fibrillation (AF). (1) AF recurrence after PVI is assumed to be mediated by PV reconnection (PVR). (2-4) This assumption is based upon the observation that (1) the likelihood of finding 4 isolated veins at repeat ablation is extremely low (varying from $41 \%$ to as low as $0 \%$ ) and (2) upon the good outcome after re-isolation of reconnected veins. (3-17)

The 'CLOSE'-protocol is a contact force guided ablation protocol aiming to enclose the veins with stable, contiguous, and optimized (ablation index-guided) radiofrequency (RF) applications. (18) This new approach of PVI is associated with a high rate of first-pass and adenosine-proof isolation and a high single-procedure arrhythmia-free survival at 1 year. (18-20)

In the present study we determined the prevalence of patients presenting with 4 isolated veins at repeat ablation for AF recurrence after 'CLOSE'-guided PVI. This endpoint is a relevant and objective parameter to evaluate effectiveness of any PVI strategy. We further investigated whether those patients (with non-PV mediated AF recurrence) are characterized by specific clinical, first procedural or post-procedural characteristics and whether outcome of repeat ablation is different from patients with reconnected veins.

\section{Methods}

\section{Patient recruitment}

All patients undergoing ablation for AF at the St-Jan Hospital Bruges are followed in a prospective database approved by the local ethical committee. This study enrolled all consecutive patients undergoing repeat ablation for AF recurrence after a first CLOSE-guided PVI for paroxysmal AF. All patients were recruited at the St-Jan Hospital Bruges between November 2014 and December 2017. AF recurrence was detected at ambulatory ECG, Holter or implantable loop recorder.

\section{'CLOSE'-guided pulmonary vein isolation}

First PVI was performed under general anesthesia or conscious sedation. The CLOSE strategy has been described in detail previously. (18) Esophageal temperature monitoring (SensiTherm ${ }^{\mathrm{TM}}$, St Jude Medical Inc., St. Paul, MN, USA) was used in anaesthetized patients. Intravenous heparin bolus $(100 \mathrm{IU} / \mathrm{kg})$ and continuous infusion (ACT $>300 \mathrm{~s})$ was administered after groin puncture. After transseptal puncture, a decapolar lasso catheter and a contact force guided ablation catheter (Thermocool Smart Touch ${ }^{\circledR}$, Biosense-Webster Inc., Diamond Bar, CA, USA) were positioned in the left atrium (LA). Calibration of contact force and respiratory gating were subsequently performed. A 3D-geometry of the LA was constructed using the CartoSystem ${ }^{\circledR}$ (Biosense-Webster Inc.) and the RF circle around the pulmonary veins was predefined on this $3 \mathrm{D}$-geometry. Point by point RF delivery 
was performed aiming for a contiguous circle enclosing the ipsilateral veins, respecting a maximal interlesion distance (ILD) of $\leq 6 \mathrm{~mm}$. Real-time automated display of RF applications (Visitag ${ }^{\circledR}$, Biosense Webster Inc.) was used with predefined settings of catheter stability ( $3 \mathrm{~mm}$ for $8 \mathrm{sec}$ ) and minimum CF (30\% of time $>4 \mathrm{gr}$ ). RF was delivered (EP Shuttle ST-3077, Stockert GmbH, Freiburg, Germany) in a power-controlled mode aiming for $35 \mathrm{~W}$ (irrigation flow up to $30 \mathrm{cc} / \mathrm{min}$ ). RF was delivered until an ablation index (AI) target of $\geq 550$ at the anterior wall and $\geq 400$ at the posterior wall. In case of chest discomfort or intra-esophageal temperature rise $\left(>38.5^{\circ}\right)$, AI target was limited to 300 at the posterior wall. After encircling the ipsilateral veins, PVI was ensured using the decapolar lasso catheter at the ostium of all four veins. In case of remaining PV potentials, touch-up ablation was delivered until PVI. Intravenous adenosine was administered to assess dormant PV conduction in the majority of patients. Voltage mapping was not systematically performed at the time of the first procedure.

\section{Repeat procedure: high-density voltage mapping and ablation strategy}

Repeat ablation was performed using the same modalities as the first procedure (general anesthesia or conscious sedation, double transseptal puncture, 3D-geometry of the LA, use of a Thermocool Smart Touch $^{\circledR}$ catheter and decapolar lasso catheter, ablation index and ILD targets).

At repeat procedure, high-density, point-by-point, bipolar voltage mapping of the LA was performed by acquiring $>300$ points during sinus rhythm homogenously distributed around the surface of the LA (pre-specified color fill of 5 and after exclusion of the pulmonary veins). To ensure reliable mapping during automated acquisition of the points (Confidense, Biosense-Webster Inc) pre-specified filter settings were used: a mapping window set to the sinus rhythm cycle length minus or plus $100 \mathrm{~ms}$, minimum contact force of 4 grams and LAT stability of maximal $3 \mathrm{~ms}$. Off-line we determined, the area $\left(\mathrm{cm}^{2}\right)$ with low bipolar voltage $(<0.5 \mathrm{mV})$ using the area measurement tool embedded in the Carto system $^{\circledR}$. A low voltage area was defined as an area of $>1 \mathrm{~cm}^{2}$ with bipolar voltage $<0.5 \mathrm{mV}$.

$\mathrm{PV}$ reconnection was defined as residual PV potentials at the LA-PV junction detected by the decapolar lasso catheter or by the Thermocool Smart Touch catheter. Patients with PVR underwent reisolation of the reconnected vein(s). Patients revealing four isolated veins were treated by ablation of the empirical trigger or substrate. Empirical trigger ablation consisted of superior vena cava isolation and/or widening of the circle around the veins. Adenosine or isoproterenol to elicit triggers was not systematically used. Substrate ablation was preferred in patients with ongoing AF at the time of procedure or in case of AF episodes lasting longer than 24hours. Empirical substrate ablation consisted of deployment of linear lesions (i.e. CLOSE-guided linear ablation at the roof, mitral isthmus and anterior wall) during sinus rhythm or AF. Ablation was continued until proven block over the roof and mitral isthmus line. 


\section{Radiofrequency characteristic of the ablation circles during the first PVI procedure}

For each repeat patient, we exported the first procedure and analyzed offline the RF characteristics of the initial RF circles using custom made software. (19) For each RF tag within the circle we calculated the time of RF delivery (Time, s), ablation index (AI, arbitrary unit, au), force time integral (FTI, gs), interlesion distance (ILD, mm), mean contact force (CF, g), and drop in impedance ( $\Delta$ Impedance, $\Omega$ ). To assess quality of RF applications, we determined for each circle the median value

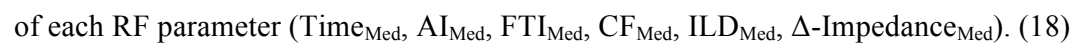

\section{Follow up after repeat procedure}

All patients had standard follow up with clinical evaluation and 12-lead electrocardiograms at 1,6 and 12 months and Holter at 6 months after repeat procedure. In case of symptoms, additional Holter analyses were performed. Recurrence of AF was registered after a 3 month blanking window. Of interest, 12 (27\%) patients had continuous rhythm evaluation by implantable loop recorders (ILR).

\section{Statistical Analysis}

Continuous variables are expressed as mean \pm standard deviation or median [interquartile range (IQR)] if data were non-Gaussian distributed. Categorical variables are expressed as absolute number with percentage (\%). Comparison among groups for continuous variables is done by the Student $\mathrm{T}$ test or Mann-Whitney U test (in case of non-Gaussian distribution). Comparison of categorical variables among groups is performed by Chi square test. Kaplan-Meier estimate was used to compare freedom of AF after repeat ablation. Statistical significance is set at a 2-tailed probability level of $<0.05$. All statistical analysis is performed using SPSS software (Version 24.0, IBM, Armonk, NY, US).

\section{Results}

Status of the pulmonary veins at repeat ablation for AF recurrence after first 'CLOSE'-guided PVI

Of 326 patients undergoing first CLOSE-guided PVI for paroxysmal AF, 45 patients underwent repeat ablation for AF recurrence (Figure 1). Out of those 45 patients, 28 (62\%) patients revealed 4 isolated veins. In patients with PVR $(n=17)$ the mean number of reconnected veins was $2 \pm 1$ with no predilection sites of PV reconnection (Figure 2, left). 


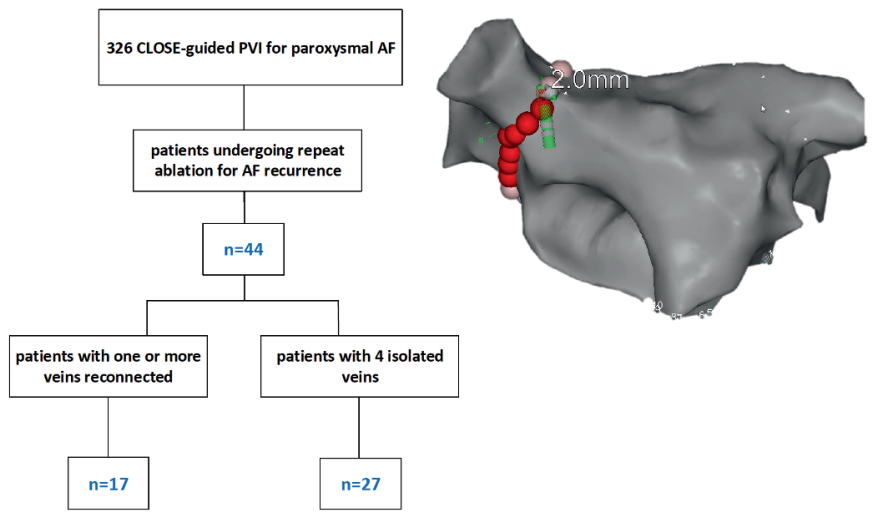

Figure 1: High incidence $(28 / 45,62 \%)$ of four isolated veins in patients with atrial fibrillation recurrence after first CLOSE-guided pulmonary vein isolation.

\section{Localisation of gaps in patients with PV reconnection} ( $n=30$ gaps, 17 patients)

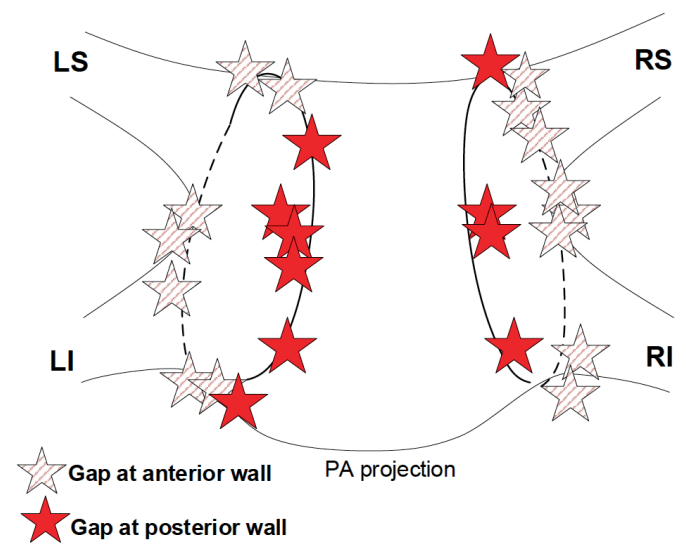

\section{Quality analysis of the radiofrequency circle} (circles with versus without reconnection)

\begin{tabular}{|c|c|c|c|}
\hline $\begin{array}{l}\text { Radiofrequency } \\
\text { characteristics }\end{array}$ & $\begin{array}{l}\text { Circles with PV } \\
\text { reconnection }\end{array}$ & $\begin{array}{c}\text { Circles with } \\
\text { isolated veins }\end{array}$ & $\mathrm{p}$ value \\
\hline Time $_{\text {Med }}(\mathrm{s})$ & $\begin{array}{c}29 \\
{[27: 31]}\end{array}$ & $\begin{array}{c}29 \\
{[26: 32]}\end{array}$ & 0.262 \\
\hline Ablation index $\operatorname{Med}_{\mathrm{Med}}(\mathrm{au})$ & $\begin{array}{c}483 \\
{[477: 487]}\end{array}$ & $\begin{array}{c}488 \\
{[478: 496]}\end{array}$ & 0.540 \\
\hline Force Time integral $\left.\right|_{\text {Med }}(\mathrm{gs})$ & $\begin{array}{c}412 \\
{[370: 448]}\end{array}$ & $\begin{array}{c}428 \\
\text { [380:499] }\end{array}$ & 0.244 \\
\hline Inter lesion distance ${ }_{\text {Med }}(\mathrm{mm})$ & $\begin{array}{c}4.1 \\
{[3.7: 4.6]}\end{array}$ & $\begin{array}{c}3.9 \\
{[3.7: 4.3]}\end{array}$ & 0.112 \\
\hline Contact force $_{\text {Med }}(\mathrm{g})$ & $\begin{array}{c}15 \\
{[13: 18]}\end{array}$ & $\begin{array}{c}15 \\
{[13: 18]}\end{array}$ & 0.172 \\
\hline$\Delta$ Impedance $_{\text {Med }}(\mathrm{ohm})$ & $\begin{array}{c}11 \\
{[10: 16]}\end{array}$ & $\begin{array}{c}12 \\
{[11: 13]}\end{array}$ & 0.740 \\
\hline
\end{tabular}

Figure 2: Left panel: Localization of gaps in the ablation circles among patients (17 patients, 27 gaps) with AF recurrence and pulmonary vein reconnection (PVR). No predilection sites of PVR could be identified. Right panel: Circles with gaps showed similar lesion quality compared to circles with durable pulmonary vein isolation.

Patients with four isolated veins: clinical characteristics, first procedural characteristics and time to recurrence after first PVI

Baseline clinical characteristics of all patients undergoing repeat ablation are listed in Table 1. Patients revealing 4 isolated veins at repeat had similar clinical characteristics compared to patients with PVR. Also the arrhythmic profile of the initial paroxysmal AF was not different, with similar AF burden, both in terms of frequency and duration of AF episodes. 
Compared to patients with PVR, patients revealing four isolated veins had also similar first procedural and RF characteristics (Table 2). We did not observe any difference in the radiofrequency

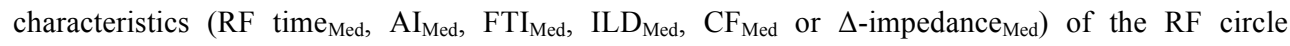
deployed during the first procedure. Comparing circles with PVR to circles without reconnection could also not identify any differences in $\mathrm{RF}$ time $\mathrm{Med}_{\mathrm{M}}, \mathrm{AI}_{\mathrm{Med}}, \mathrm{FTI}_{\mathrm{Med}}, \mathrm{ILD}_{\text {Med }}, \mathrm{CF}_{\text {Med }}$ or $\Delta$-impedance -imed (Figure 2, right).

Finally, also the mean time to recurrence after prior PVI did not differ among both groups ( $8 \pm 7$ vs $6 \pm 6$ months, $p=0.453$, Figure 3). Of interest, patients revealing four isolated veins at repeat (i.e. non-PV mediated AF recurrence) presented with a wide variation in time to AF recurrence after initial PVI.

Table 1: Clinical characteristics of patients undergoing repeat procedure

\begin{tabular}{|c|c|c|c|c|}
\hline & $\begin{array}{c}\text { All repeat } \\
\text { patients } \\
(n=45)\end{array}$ & $\begin{array}{c}\text { Patients with } \\
4 \text { isolated } \\
\text { veins }(n=28)\end{array}$ & $\begin{array}{c}\text { Patients with } \\
\text { reconnected veins } \\
(n=17)\end{array}$ & $\underset{\text { value }}{p}$ \\
\hline \multicolumn{5}{|l|}{ Patient characteristics } \\
\hline Age, yrs & $61 \pm 10$ & $63 \pm 10$ & $59 \pm 10$ & 0.153 \\
\hline Male gender, n (\%) & $23(51)$ & $12(43)$ & $11(65)$ & 0.221 \\
\hline BMI, kg/m2 & $27 \pm 5$ & $26 \pm 5$ & $28 \pm 4$ & 0.271 \\
\hline Obesity $(\mathrm{BMI}>25), \mathrm{n}(\%)$ & $26(58)$ & $15(54)$ & $11(65)$ & 0.543 \\
\hline $\mathrm{CHA}_{2} \mathrm{DS}_{2}$-VASc-score & $1 \pm 1$ & $1 \pm 1$ & $1 \pm 1$ & 0.617 \\
\hline Arterial Hypertension, n(\%) & $13(29)$ & $9(32)$ & $4(24)$ & 0.737 \\
\hline Diabetes, n(\%) & $2(4)$ & $1(4)$ & $1(6)$ & 1.00 \\
\hline Obstructive sleep apnea, n(\%) & $2(4)$ & $0(0)$ & $2(12)$ & 0.137 \\
\hline Left atrial diameter, $\mathrm{mm}$ & $41 \pm 4$ & $41 \pm 5$ & $41 \pm 4$ & 0.811 \\
\hline \multicolumn{5}{|l|}{ Characteristics of paroxysmal AF before PVI } \\
\hline Paroxysmal AF, n (\%) & $45(100)$ & $27(100)$ & $17(100)$ & 1.00 \\
\hline AF duration, months & $67 \pm 86$ & $75 \pm 100$ & $55 \pm 61$ & 0.916 \\
\hline ADT resistant, n (\%) & $39(87)$ & 25(89) & $14(82)$ & 1.00 \\
\hline \multicolumn{5}{|l|}{ Frequency of AF episodes, n (\%) } \\
\hline$<1$ episode/ month & $20(45)$ & $14(50)$ & $6(35)$ & 0.336 \\
\hline$>1$ episode/month & $16(36)$ & $8(29)$ & $8(47)$ & 0.209 \\
\hline$>1$ episode/week & $3(7)$ & $2(7)$ & $1(6)$ & 0.869 \\
\hline$>1$ episode/day & $6(13)$ & $4(14)$ & $2(12)$ & 0.809 \\
\hline \multicolumn{5}{|l|}{ Duration of AF episodes, $\mathrm{n}(\%)$} \\
\hline$>24$ hours & $8(18)$ & $4(14)$ & $4(24)$ & 0.431 \\
\hline 12-24 hours & $7(15)$ & $2(7)$ & $4(24)$ & 0.116 \\
\hline $1-12$ hours & $16(36)$ & $13(47)$ & $4(24)$ & 0.124 \\
\hline$<1$ hour & $14(31)$ & $9(32)$ & $5(29)$ & 0.848 \\
\hline \multicolumn{5}{|l|}{ Characteristics of recurrence of AF after first PVI } \\
\hline Time to recurrence, months & $7 \pm 7$ & $8 \pm 7$ & $6 \pm 6$ & 0.453 \\
\hline Initial responder ( $>1$ month free of AF), $\mathrm{n}(\%)$ & $28(62)$ & $18(64)$ & $10(59)$ & 0.759 \\
\hline
\end{tabular}


Table 2: Characteristics of the first PVI procedure in patients revealing 4 isolated veins at repeat

\begin{tabular}{|c|c|c|c|c|}
\hline & $\begin{array}{c}\text { All repeat } \\
\text { patients }(n=45)\end{array}$ & $\begin{array}{c}\text { Patients with } 4 \\
\text { isolated veins }(n=28)\end{array}$ & $\begin{array}{c}\text { Patients with } \\
\text { reconnected veins }(n=17)\end{array}$ & $\begin{array}{c}\mathbf{p} \\
\text { value }\end{array}$ \\
\hline \multicolumn{5}{|l|}{ Procedure characteristics } \\
\hline General Anaesthesia, n (\%) & $30(67)$ & $20(74)$ & $9(53)$ & 0.193 \\
\hline Common pulmonary vein, $\mathrm{n}(\%)$ & $4(9)$ & $2(7)$ & $2(12)$ & 0.626 \\
\hline Procedure duration (min) & $162 \pm 37$ & $158 \pm 41$ & $168 \pm 29$ & 0.378 \\
\hline $\mathrm{N}$ of RF applications (points) & $71 \pm 13$ & $68 \pm 13$ & $75 \pm 13$ & 0.114 \\
\hline Total time of RF energy (min) & $33 \pm 7$ & $32 \pm 6$ & $35 \pm 7$ & 0.076 \\
\hline Fluoroscopy time (min) & $15 \pm 8$ & $13 \pm 6$ & $16 \pm 9$ & 0.211 \\
\hline DAP $\left(\mathrm{mGy} / \mathrm{cm}^{2}\right)$ & $7569 \pm 7249$ & $7157 \pm 7366$ & $8252 \pm 7244$ & 0.398 \\
\hline $\mathrm{AK}(\mathrm{mGy})$ & $36 \pm 35$ & $35 \pm 37$ & $38 \pm 31$ & 0.353 \\
\hline \multicolumn{5}{|l|}{ RF circle characteristics } \\
\hline $\operatorname{Time}_{\mathrm{Med}}(\mathrm{min})$ & $29[26: 32]$ & $29[26: 33]$ & $28[27: 31]$ & 0.365 \\
\hline Ablation index $\mathrm{xed}_{\mathrm{Me}}(\mathrm{au})$ & $486[478: 494]$ & $484[478: 496]$ & $487[479: 494]$ & 0.689 \\
\hline Force time integral $_{\mathrm{Med}}(\mathrm{gs})$ & $426[379: 484]$ & $426[370: 501]$ & $426[386: 451]$ & 0.567 \\
\hline Inter lesion distance ${ }_{\mathrm{Med}}(\mathrm{mm})$ & $3.9[3.7: 4.4]$ & $4.1[3.7: 4.4]$ & $3.9[3.7: 4.3]$ & 0.907 \\
\hline Contact force $_{\text {Med }}(g)$ & $15[13: 18]$ & $15[13: 18]$ & $16[14: 18]$ & 0.716 \\
\hline$\Delta$ Impedance $_{\text {Med }}(\mathrm{ohm})$ & $12[11: 13]$ & $12[11: 14]$ & $12[11: 13]$ & 0.772 \\
\hline
\end{tabular}




\section{7 patients revealing one or more reconneted vein at repeat}

\section{Timing of the first AF recurrence after initial PVI}

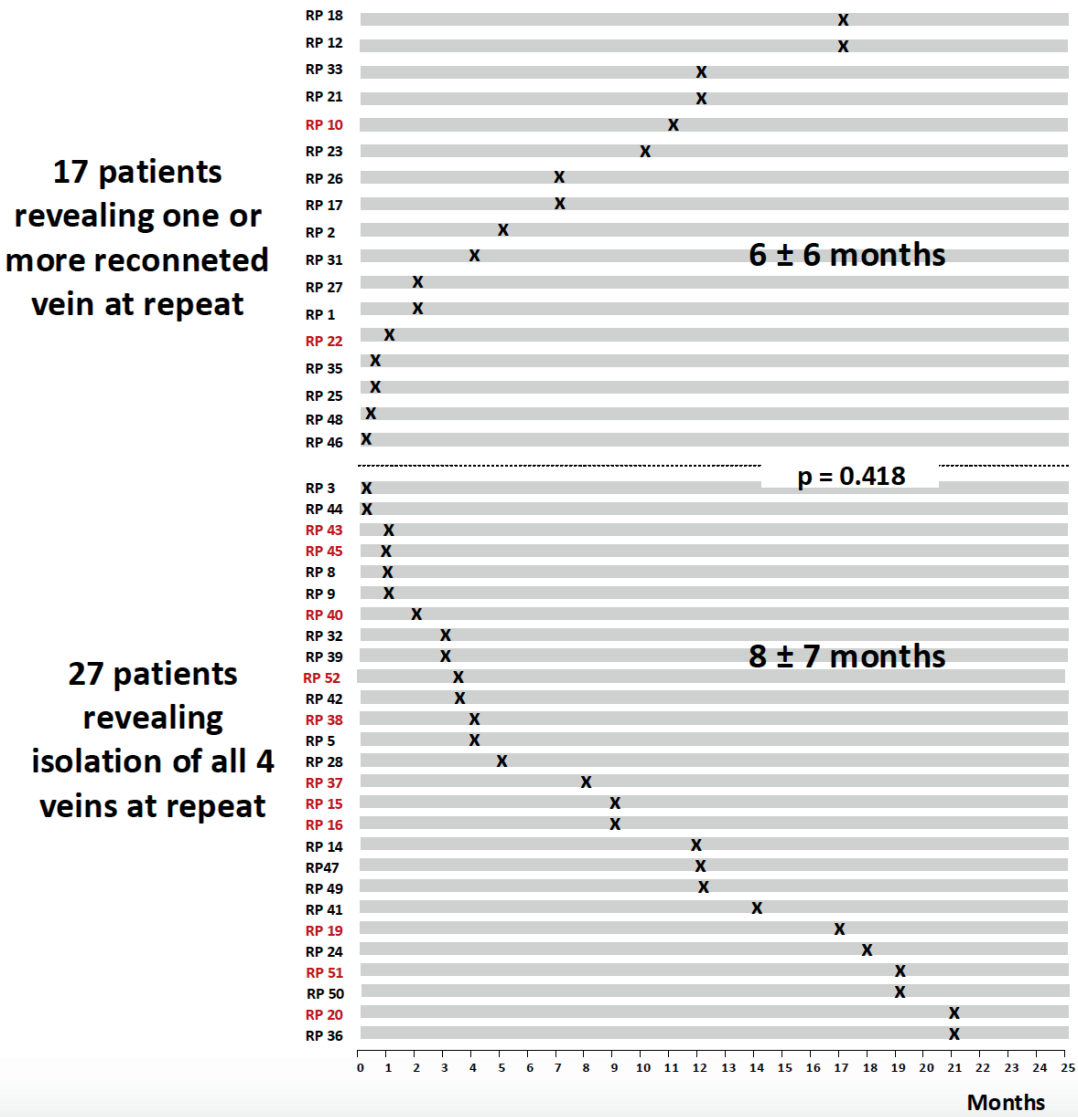

Figure 3: Time course to AF recurrence after first CLOSE-guided pulmonary vein isolation. Patients with a status of four veins being isolated, showed similar time course to first AF recurrence compared to patients with pulmonary vein reconnection. Of interest, patients with low voltage (color-coded in red) present with both early and late recurrence after first PVI.

\section{High-density bipolar voltage mapping of the left atrium}

Compared to patients with PVR, patients with 4 isolated veins were characterized by a higher incidence of low atrial voltage in the residual left atrial surface. Representative examples are given in Figure 4 (left). Whereas the patient with AF recurrence and PVR showed no low voltage area in the posterior LA, the patient with AF recurrence and complete PV isolation presented with a distinct area of low voltage at the posterior LA $\left(8.3 \mathrm{~cm}^{2}\right)$.

Overall results are summarized in the right panels of Figure 4. In patients with AF recurrence and four isolated veins, the incidence of low voltage was significantly higher $(57 \%$ vs $17 \%$ in PVR patients, $\mathrm{p}=0.033)$ and the area of low voltage was significantly larger $\left(4.6[2.3-7.9] \mathrm{cm}^{2}\right.$ versus $0.0[0.0-0.0] \mathrm{cm}^{2}$ 
in PVR patients, $\mathrm{p}=0.049$ ). As can be seen in Figure 3 (in which we color-coded in red those patients with low voltage), patients with low voltage present with both early and late recurrence after first PVI. In 10 out of 45 patients, we could compare the presence or absence of low voltage at repeat to a baseline high-density voltage map created during the first PVI procedure. None of these patients revealed any change in low voltage characteristics between baseline and repeat at $7 \pm 6$ months. Representative maps are plotted in Figure 5 (left). Overall, in 3 patients presenting with low voltage at baseline, the surface of low voltage remained identical over time. Likewise, none of the 7 patients presenting with normal voltage at baseline developed low voltage (Figure 5, right).

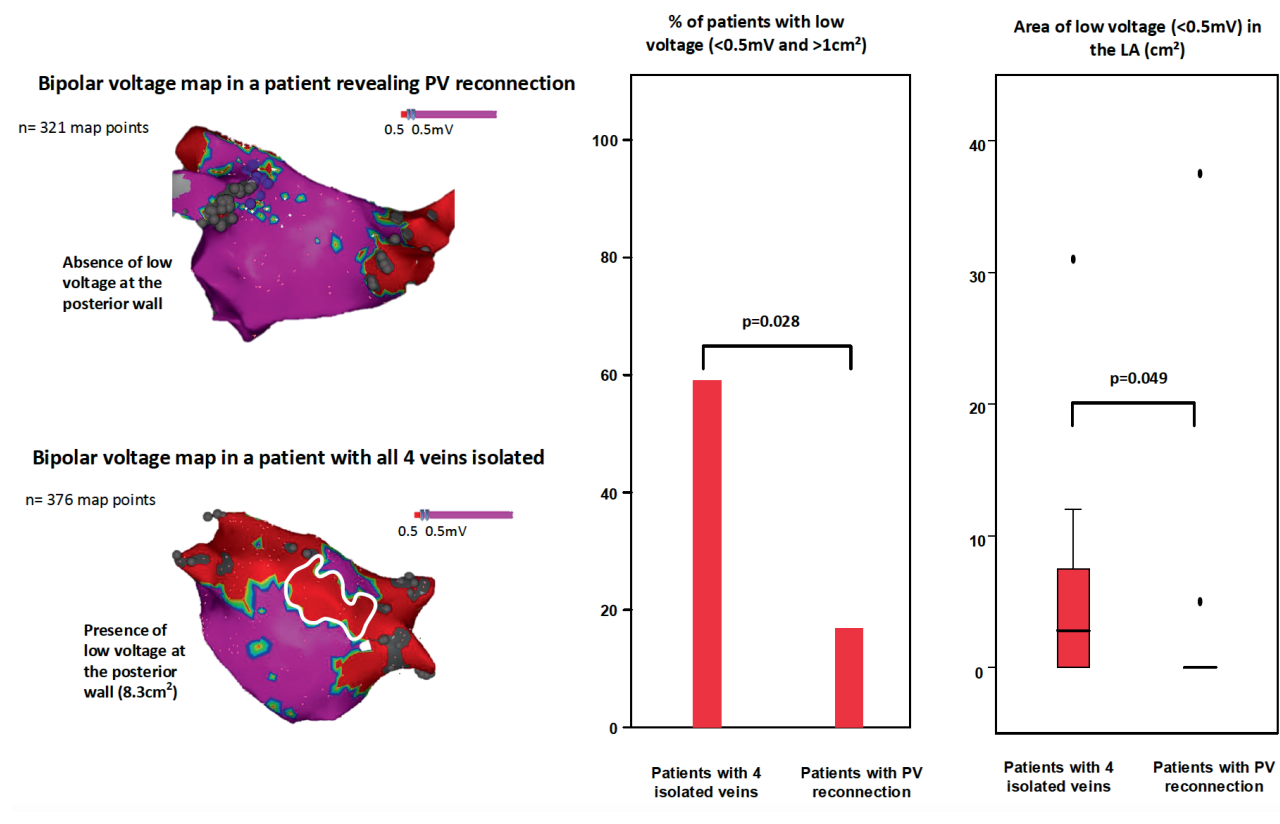

Figure 4: Left panel: Representative examples of left atrial (LA) voltage mapping (bipolar voltage cut off $0.5 \mathrm{mV}$ ) in two patients with atrial fibrillation (AF) recurrence after first CLOSE-guided pulmonary vein isolation. The upper left panel represents a patient with reconnection of the left inferior pulmonary vein. The LA shows a normal bipolar voltage with absence of low voltage zones. The lower left panel shows the voltage map of a patient with AF recurrence despite all four veins isolated. A large area of low voltage at the posterior wall is documented. Right panel: Overall, patients with AF recurrence despite isolated veins have more often areas $\left(>1 \mathrm{~cm}^{2}\right)$ of bipolar voltage $<0.5 \mathrm{mV}$ and have larger areas of low voltage compared to patients with pulmonary vein reconnection. 


\section{Patient with presence of low voltage at baseline}
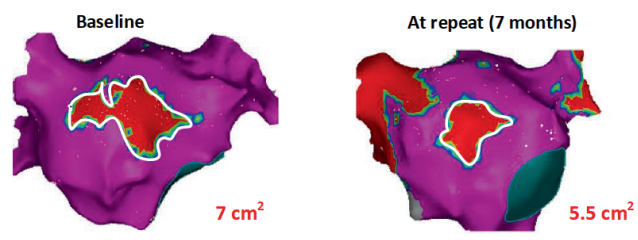

Patient with absence of low voltage at baseline
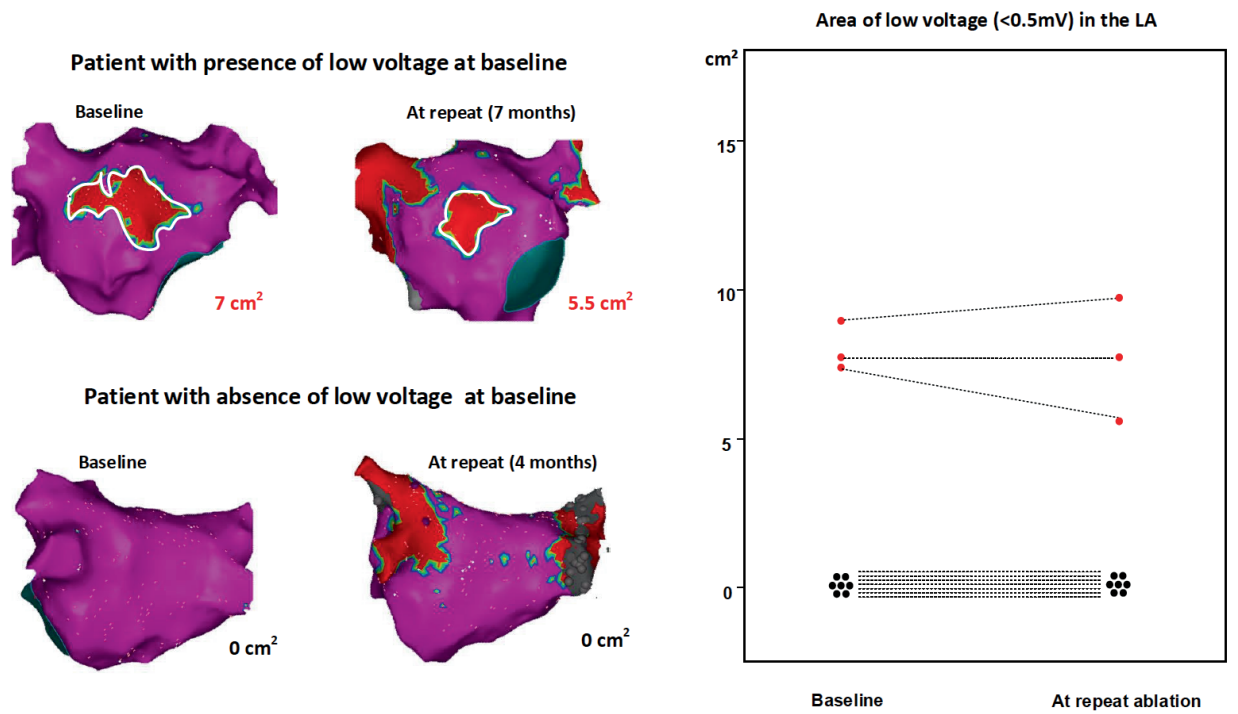

Figure 5: Comparison of the presence or absence of low voltage at repeat to a baseline high-density voltage map created during the first PVI procedure. The patient in the upper left panel presented with area of $7 \mathrm{~cm}^{2}$ on the posterior wall, but showed no progression of this low voltage area 7 months later at repeat procedure. The patient (lower left panel) without left atrial low voltage at baseline did not develop low voltage over a period of 4 months. Right panel: Overall, in 3 patients presenting with low voltage at baseline, the surface of low voltage remained identical over time. Likewise, none of the 7 patients presenting with normal voltage at baseline developed low voltage.

\section{Outcome after repeat ablation}

In patients with PVR, all reconnected veins were re-isolated. Out of 28 patients with 4 isolated veins, $15(54 \%)$ patients underwent empirical ablation of the trigger and $13(46 \%)$ patients underwent ablation of the empirical substrate.

Median follow-up after repeat ablation was $13 \pm 11$ months. Patients with AF recurrence and complete vein isolation, tended to have a lower freedom of AF compared to patients with PVR (61\% vs $88 \%$, $\mathrm{p}=0.045$ ) (Figure 6). Time to AF recurrence after repeat procedure was $6.5 \pm 4.8$ months in the PVR group versus $5.7 \pm 2.1$ months in patients with a status of four isolated veins $(\mathrm{p}=1.00)$. 
Kaplan-Meier for recurrence of atrial fibrillation after repeat ablation strategies

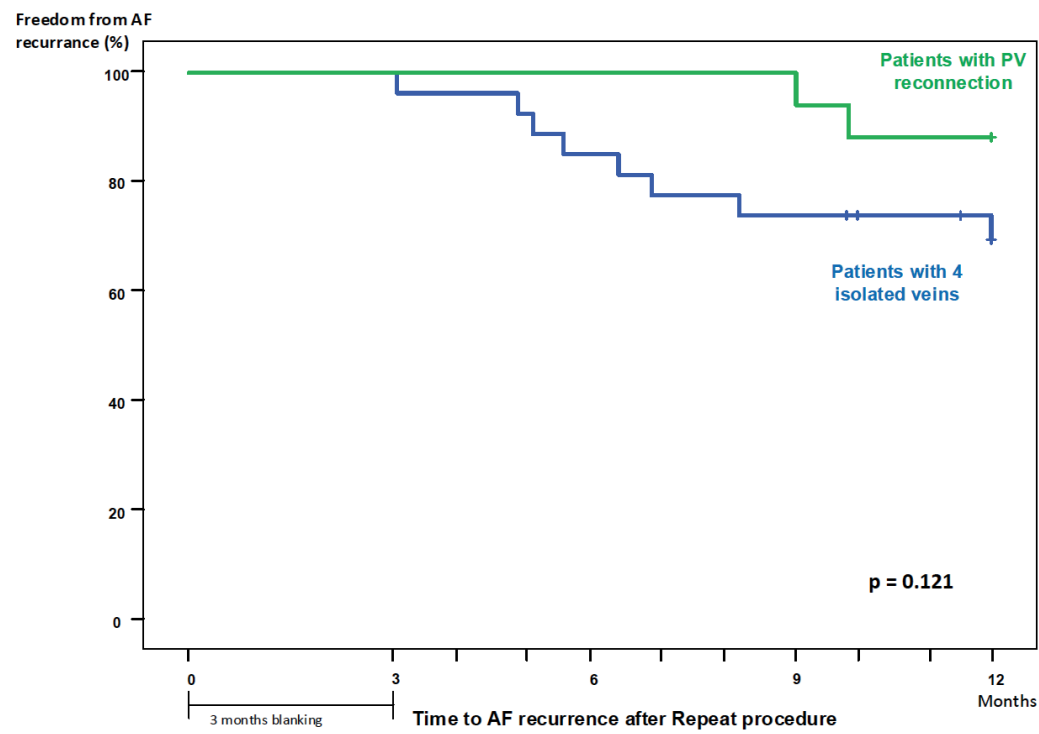

Figure 6: Kaplan-Meier curves depicting time to AF recurrence after repeat ablation for patients with and without pulmonary vein reconnection at repeat ablation. Patients with all four veins being isolated (treated with either ablation of the empirical trigger or substrate) showed worse outcome.

\section{Repeat ablation in patients with implantable loop recorders}

Of interest, from the twelve patients who were implanted with an ILR, AF recurrence was paroxysmal in $10(83 \%)$ patients and persistent in $2(17 \%)$. Of these ILR patients, $5(42 \%)$ patients presented with PVR, whereas 7 (58\%) of those patients presented with a status of all veins being isolated. In patients with ILR undergoing re-isolation of all reconnected PVs, $80 \%$ remained free of $\mathrm{AF}$, whereas in patients with ILR who underwent substrate or trigger ablation $57 \%$ remained free of $\mathrm{AF}(\mathrm{p}=0.636)$.

\section{Discussion}

\section{Main findings}

First, the likelihood of finding 4 isolated veins at the time of repeat ablation for AF recurrence after 'CLOSE'-guided PVI is $62 \%$ - higher than previously reported- and implying that AF recurrence after PVI now is more likely to point to non-PV mediated AF. Second, patients presenting with AF recurrence and 4 isolated veins (i.e. non-PV mediated AF recurrence) have no distinct clinical or first procedural characteristics and also time to AF recurrence after the first PVI is not different from patients presenting with PVR. Third, the association with low voltage and the moderate outcome after empirical ablation suggest that a patient-specific repeat ablation might be required to optimize outcome in patients presenting with complete vein isolation. 


\section{AF recurrence does not longer imply $\mathrm{PV}$ reconnection at repeat}

It is generally accepted that AF recurrence after PVI is due to reconnection of 1 or more PVs. This is based upon the good outcome after isolation of reconnected veins with repeat ablation for AF and numerous observations that recurrence after PVI is almost invariably associated with reconnection of the vein(s).

Prior studies, predominantly in patients with paroxysmal AF, reported indeed a low incidence of a status of complete vein isolation at repeat ablation for AF recurrence (Figure 7). (3-9,11-17) In the earlier years (using non-irrigated or irrigated non-contact force RF or first generation cryoballoon to isolate the veins) the likelihood of complete isolation was as low as $0 \%$ and certainly not exceeding $20 \%$. The likelihood of finding 4 isolated veins at repeat ablation for AF recurrence after predominantly second-generation cryoballoon PVI ranged from $26 \%$ to $41 \%$. $(5,8,11,17)$ More recent data on RF-guided PVI still show high reconnection rates. (15-16) Shah et al. showed that the incidence of isolated veins in patients with late AF recurrence ( $>36$ months after first PVI) was only $19 \%$. (16) Of interest, although contact force-guided ablation is associated with higher percentage of complete isolation in study patients undergoing invasive re-evaluation at 3 months regardless of symptoms, $(21,22)$ Buist et al. reported a low incidence of finding 4 veins isolated at repeat for contact force- guided RF (only 3\%). (15) Finally, a recent meta-analysis by Nery et al. reported an overall $14 \%$ likelihood of finding 4 isolated veins in case of a clinically-indicated repeat ablation after PVI.

Incidence of isolation of all veins in patients with AF recurrence undergoing first PVI

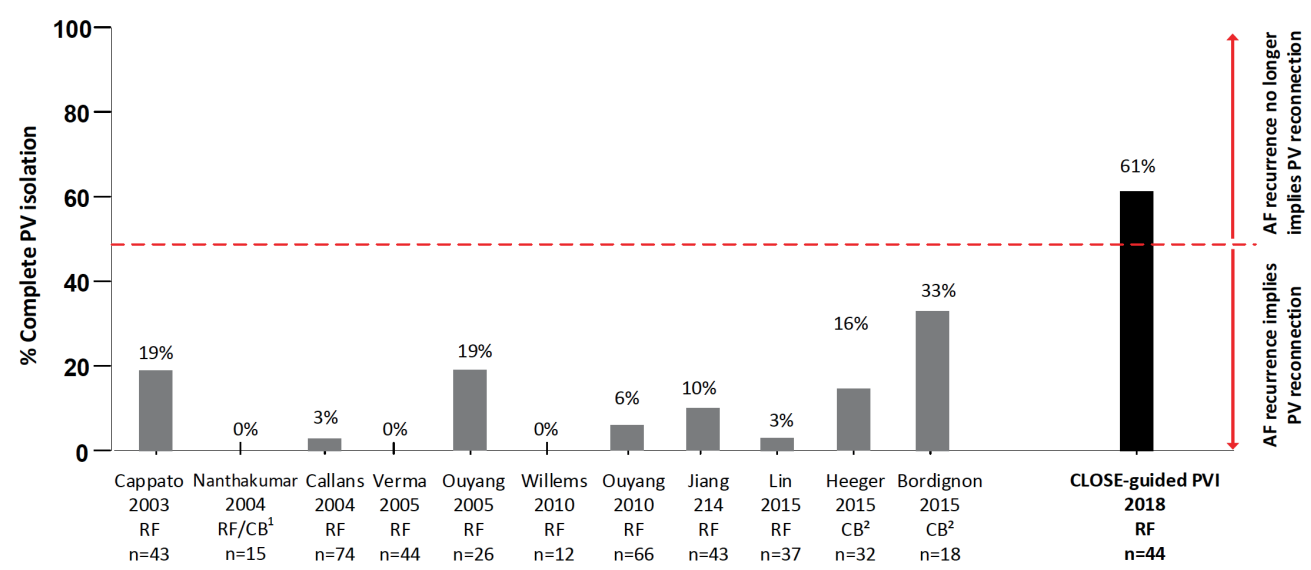

Figure 7: Overview of studies assessing the likelihood of finding four isolated veins after single pulmonary vein isolation (PVI). After CLOSE-guided PVI, atrial fibrillation (AF) recurrence no longer implies pulmonary vein reconnection. 
The present observation that $62 \%$ of repeat patients reveal a status of complete isolation confirms that 'CLOSE'-guided PVI, by avoiding weak links in the ablation chain, is associated with durable isolation. This finding is in line with prior data from our group reporting favorable acute and 1-year outcome after a PVI strategy based upon stable, contiguous, and optimized RF applications. $(18,20)$ Of interest, the $62 \%$ complete isolation is in line with the $64 \%$ incidence of complete isolation in 14 patients undergoing repeat ablation for recurrence after a prior video-assisted thoracoscopic PVI (using bipolar RF clamp to ensure transmural and durable isolation). (23)

All of the preceding percentages (Fig. 7) apply to the likelihood of finding 4 isolated veins in patients undergoing repeat study for AF recurrence (clinically-indicated repeat study). Although it is wellknown from protocol-mediated repeat studies that $\mathrm{PV}$ reconnection can occur in patients free of $\mathrm{AF}$, $(7,9,10,13,14,21)$ the likelihood of finding 4 isolated veins is expected to be higher when evaluating all patients, also those without AF recurrence. Indeed in a recent meta-analysis (combining data of eleven studies) by Nery et al., the likelihood of finding complete isolation was $41.4 \%$ in patients without AF recurrence, markedly higher than the reported $14.5 \%$ in patients with AF recurrence. (2)

\section{Can we identify upfront those patients that will present with isolated veins at repeat procedure?}

Predicting upfront those patients that will represent with four isolated veins at repeat might optimize communication with the patients (expectations regarding repeat ablation outcome, length of the procedure), and repeat procedural planning (cath lab time, mapping strategies, ablation tools). It might even avoid a repeat procedure and favor non-ablative treatment (anti-arrhythmic drugs). (24)

We showed however that patients with four isolated veins (i.e. non-PV mediated AF recurrence) have no distinct clinical characteristics. Also the phenotype of paroxysmal AF before PVI did not show any specific characteristics. These findings are in line with prior studies on non-PV mediated AF, demonstrating that clinical characteristics (i.e. age, gender, LA size) do not differ between patients with PV and non-PV mediated AF. $(25,26)$

Furthermore we observed no difference in the time to AF recurrence after PVI among both groups, with both early and late recurrence in patients with non-PV mediated AF. One might have anticipated that patients with non-PV mediated AF recurrence would be more likely to present with early recurrence after PVI. We retain two hypotheses to explain why patients presenting with 4 isolated veins at repeat would reveal a late recurrence after initially responding well to PVI; (1) development of non-PV mediated AF at a later stage, with an initial good response because of the initial PVmediated AF or (2) non-PV mediated AF from the very start, with an initial good response to PVI due to a secondary but transient effect of ablation (like autonomic modulation). This latter hypothesis could explain the striking similarity in the variation in time to recurrence in both patient groups i.e. in 
the reconnected group, PV reconnection occurs early, but the time to recurrence is mediated by absence or recovery from autonomic modulation.

We could not detect any difference in procedural characteristics or lesion characteristics between patients/circles with reconnection vs patients/circles with permanent isolation. Lack of difference might be explained by the fact that ablation is meticulously performed according to well-controlled strict criteria (precluding variation in those parameters). The high freedom of AF after first PVI and the currently reported high incidence of complete isolation suggest that those 'CLOSE'-criteria were well-chosen. In case of reconnection after 'CLOSE', PVR must be due to other, unmeasured variables like tissue thickness or tissue composition. Therefore markers of direct tissue contact as intra cardiac echocardiography or sound mapping might further improve CLOSE-guided PVI.

\section{Low voltage assessment at the time of repeat procedure.}

We observed that patients with non-PV mediated AF recurrence after first PVI were characterized by a higher prevalence of low voltage areas in the left atrium. Those low voltage areas are suggestive for atrial fibrosis either as a manifestation of structural heart disease or as a result of an intrinsic fibrotic atrial cardiomyopathy. $(27,28)$ Our findings are in line with prior studies reporting lower single procedure outcome after first PVI in patient presenting with fibrosis or low voltage areas in the LA. $(29,30)$ Marrouche et al. showed that both the presence and extent of atrial fibrosis (as detected by delayed enhancement on magnetic resonance imaging), was independently associated with a higher likelihood of AF recurrence. (29) In the study of Verma et al., the presence of left atrial fibrosis (as assessed by electro-anatomical mapping) was the strongest predictor for AF recurrence. (30) It is unknown to what extent low voltage is a risk factor or marker for patients with non-PV mediated substrate and/or trigger. Of interest, Chao et al. reported that patients with higher CHADS2 scores presented with more low left atrial voltage (31) and subsequently reported a higher likelihood of nonPV triggers in patients with a higher CHADS2 score. (32)

\section{Outcome after repeat ablation in patients with 4 isolated veins}

Most studies that provided data about outcome after repeat ablation reported outcome data in those patients undergoing re-isolation of reconnected veins with freedom of AF varying between 81 and $97 \%$. $(3,6,7,13,14)$ Two studies reported on outcome in all repeat patients (both patients with reisolation of reconnected veins and patients undergoing non-PV ablation in case of isolated veins). In these studies long-term freedom of $\mathrm{AF}$ was observed in $53 \%$ and $66 \%$ of the patients undergoing repeat ablation. $(12,33)$ Only 2 studies reported specifically on outcome data after repeat ablation for AF recurrence in patients with a status of all veins being isolated. $(4,17)$ In the first study by Ouyang et al., 5 out of 26 patients revealed a status of isolated veins and underwent repeat ablation (non-PV triggers, focal AT and linear lesions). (4) After a blanking period of one month, all 5 patients remained free of AF recurrence. In a second study by Baldinger et al., freedom of arrhythmia after repeat 
ablation depended on whether patients with isolated veins presented with AF recurrence $(20 \%$ freedom after a repeat strategy consisting of lines and/or antral extension and/or defractionation) or with AT recurrence (67\% freedom after a repeat strategy targeting activation time). (17)

The present study showed that outcome after repeat ablation in patients with complete isolation $(n=27)$ was inferior to patients presenting with PVR. Worse outcome was seen independent of whether we performed empirical ablation of either the non-PV trigger or substrate. Poor outcome and its association with low voltage suggests that ablation outcome could be improved by adding patientspecific ablation strategies at repeat ablation.

\section{Study limitations}

The major limitations of the study are the small sample size and that we did not report systematically on status of PVs in all patients. Although the number of patients seems limited it is important to state that the sample size is comparable to prior published studies and that it is (and will be more) challenging to provide data on 45 patients given the low probability of recurrence/repeat after durable PVI for paroxysmal AF. Our data on outcome after repeat ablation should be considered as a hypothesis generating only as there was no predefined randomization amongst strategies. Similarly, the data on low voltage require further study by evaluating a larger patient cohort and by assessing its relation with clinical characteristics.

\section{Conclusions}

After pulmonary vein isolation with stable, contiguous and optimized (ablation index-guided) radiofrequency lesions (CLOSE-protocol), PV reconnection is no longer the rule in patients experiencing AF recurrence. Patients with AF recurrence and 4 isolated veins present with a clinical profile and time to recurrence that is not different from patients with reconnected veins. The presence of low voltage regions and moderate outcome after repeat suggest that a patient-tailored strategy is required to treat patients with non-PV mediated AF. 


\section{References}

1. Calkins H, Hindricks G, Cappato R et al. 2017 HRS/EHRA/ECAS/APHRS/SOLAECE expert consensus statement on catheter and surgical ablation of atrial fibrillation. Heart Rhythm 2017; 14:e275-e444.

2. Nery PB, Belliveau D, Nair GM et al. Relationship Between Pulmonary Vein Reconnection and Atrial Fibrillation Recurrence. A Systematic Review and Meta-Analysis. JACC: Clinical Electrophysiology 2016;2:474-83.

3. Nanthakumar K, Plumb VJ, Epstein AE, Veenhuyzen GD, Link D, Kay GN. Resumption of electrical conduction in previously isolated pulmonary veins: rationale for a different strategy? Circulation 2004;109:1226-9.

4. Ouyang F, Antz M, Ernst S et al. Recovered pulmonary vein conduction as a dominant factor for recurrent atrial tachyarrhythmias after complete circular isolation of the pulmonary veins: lessons from double Lasso technique. Circulation 2005;111:127-35.

5. Bordignon S, Furnkranz A, Perrotta L et al. High rate of durable pulmonary vein isolation after second-generation cryoballoon ablation: analysis of repeat procedures. Europace 2015;17:725-31.

6. Callans DJ, Gerstenfeld EP, Dixit S et al. Efficacy of repeat pulmonary vein isolation procedures in patients with recurrent atrial fibrillation. J Cardiovasc Electrophysiol 2004; 15:1050-5.

7. Cappato R, Negroni S, Pecora D et al. Prospective assessment of late conduction recurrence across radiofrequency lesions producing electrical disconnection at the pulmonary vein ostium in patients with atrial fibrillation. Circulation 2003;108:1599-604.

8. Heeger $\mathrm{CH}$, Wissner E, Mathew $\mathrm{S}$ et al. Once Isolated, Always Isolated? Incidence and Characteristics of Pulmonary Vein Reconduction After Second-Generation CryoballoonBased Pulmonary Vein Isolation. Circulation Arrhythmia and electrophysiology 2015;8:108894.

9. Jiang RH, Po SS, Tung R et al. Incidence of pulmonary vein conduction recovery in patients without clinical recurrence after ablation of paroxysmal atrial fibrillation: mechanistic implications. Heart Rhythm 2014;11:969-76.

10. Kuck KH, Hoffmann BA, Ernst $\mathrm{S}$ et al. Impact of Complete Versus Incomplete Circumferential Lines Around the Pulmonary Veins During Catheter Ablation of Paroxysmal Atrial Fibrillation: Results From the Gap-Atrial Fibrillation-German Atrial Fibrillation Competence Network 1 Trial. Circulation Arrhythmia and electrophysiology 2016;9:e003337.

11. Martins RP, Galand V, Cesari O et al. The second generation cryoballoon has improved durable isolation of left but not right pulmonary veins: new insights from a multicentre study. Europace 2018;20:1115-21.

12. Ouyang F, Tilz R, Chun J et al. Long-term results of catheter ablation in paroxysmal atrial fibrillation: lessons from a 5-year follow-up. Circulation 2010;122:2368-77.

13. Verma A, Kilicaslan F, Pisano E et al. Response of atrial fibrillation to pulmonary vein antrum isolation is directly related to resumption and delay of pulmonary vein conduction. Circulation 2005;112:627-35.

14. Willems S, Steven D, Servatius $\mathrm{H}$ et al. Persistence of pulmonary vein isolation after robotic remote-navigated ablation for atrial fibrillation and its relation to clinical outcome. J Cardiovasc Electrophysiol 2010;21:1079-84.

15. Buist TJ, Adiyaman A, Smit JJJ, Ramdat Misier AR, Elvan A. Arrhythmia-free survival and pulmonary vein reconnection patterns after second-generation cryoballoon and contact-force radiofrequency pulmonary vein isolation. Clinical research in cardiology : official journal of the German Cardiac Society 2018;107:498-506. 
16. Shah S, Barakat AF, Saliba WI et al. Recurrent Atrial Fibrillation After Initial Long-Term Ablation Success: Electrophysiological Findings and Outcomes of Repeat Ablation Procedures. Circulation Arrhythmia and electrophysiology 2018;11:e005785.

17. Baldinger SH, Chinitz JS, Kapur S et al. Recurrence of Atrial Arrhythmias Despite Persistent Pulmonary Vein Isolation After Catheter Ablation for Atrial Fibrillation: A Case Series. JACC Clin Electrophysiol 2016;2:723-31.

18. Taghji P, El Haddad M, Phlips T et al. Evaluation of a Strategy Aiming to Enclose the Pulmonary Veins With Contiguous and Optimized Radiofrequency Lesions in Paroxysmal Atrial Fibrillation: A Pilot Study. J Am Coll Cardiol EP 2018;4:99-108

19. El Haddad M, Taghji P, Phlips T et al. Determinants of Acute and Late Pulmonary Vein Reconnection in Contact Force-Guided Pulmonary Vein Isolation: Identifying the Weakest Link in the Ablation Chain. Circulation Arrhythmia and electrophysiology 2017;10e004867.

20. Phlips T, Taghji P, El Haddad M et al. Improving procedural and one-year outcome after contact force-guided pulmonary vein isolation: the role of interlesion distance, ablation index, and contact force variability in the 'CLOSE'-protocol. Europace 2018;20:f419-27.

21. Das M, Wynn GJ, Saeed Y, et al. Pulmonary Vein Re-Isolation as a Routine Strategy Regardless of Symptoms: The PRESSURE Randomized Controlled Trial. JACC Clin Electrophysiol 2017;3:602-11.

22. Neuzil P, Reddy VY, Kautzner J et al. Electrical reconnection after pulmonary vein isolation is contingent on contact force during initial treatment: results from the EFFICAS I study. Circulation Arrhythmia and electrophysiology 2013;6:327-33.

23. Velagic V, C DEA, Mugnai G et al. Repeat Procedures After Hybrid Thoracoscopic Ablation in the Setting of Longstanding Persistent Atrial Fibrillation: Electrophysiological Findings and 2-Year Clinical Outcome. J Cardiovasc Electrophysiol 2016;27:41-50.

24. Duytschaever M, Demolder A, Phlips T et al. PulmOnary vein isolation With vs. without continued antiarrhythmic Drug trEatment in subjects with Recurrent Atrial Fibrillation (POWDER AF): results from a multicentre randomized trial. Eur Heart J 2018;39:1429-37.

25. Higuchi K, Yamauchi Y, Hirao K et al. Superior vena cava as initiator of atrial fibrillation: factors related to its arrhythmogenicity. Heart Rhythm 2010;7:1186-91.

26. Tsai CF, Tai CT, Hsieh $\mathrm{MH}$ et al. Initiation of atrial fibrillation by ectopic beats originating from the superior vena cava: electrophysiological characteristics and results of radiofrequency ablation. Circulation 2000;102:67-74.

27. Kottkamp H, Berg J, Bender R, Rieger A, Schreiber D. Box Isolation of Fibrotic Areas (BIFA): A Patient-Tailored Substrate Modification Approach for Ablation of Atrial Fibrillation. J Cardiovasc Electrophysiol 2016;27:22-30.

28. Sanders P, Morton JB, Davidson NC et al. Electrical remodeling of the atria in congestive heart failure: electrophysiological and electroanatomic mapping in humans. Circulation 2003;108:1461-8.

29. Marrouche NF, Wilber D, Hindricks G et al. Association of atrial tissue fibrosis identified by delayed enhancement MRI and atrial fibrillation catheter ablation: the DECAAF study. JAMA 2014;311:498-506.

30. Verma A, Wazni OM, Marrouche NF et al. Pre-existent left atrial scarring in patients undergoing pulmonary vein antrum isolation: an independent predictor of procedural failure. $\mathrm{J}$ Am Coll Cardiol 2005;45:285-92.

31. Chao TF, Cheng CC, Lin WS et al. Associations among the CHADS(2) score, atrial substrate properties, and outcome of catheter ablation in patients with paroxysmal atrial fibrillation. Heart Rhythm 2011;8:1155-9. 
32. Chao TF, Ambrose K, Tsao HM et al. Relationship between the CHADS(2) score and risk of very late recurrences after catheter ablation of paroxysmal atrial fibrillation. Heart Rhythm 2012;9:1185-91.

33. Heeger CH, Wissner E, Knoll M et al. Three-Year Clinical Outcome After 2nd-Generation Cryoballoon-Based Pulmonary Vein Isolation for the Treatment of Paroxysmal and Persistent Atrial Fibrillation- A 2-Center Experience. Circ J 2017;81:974-980. 


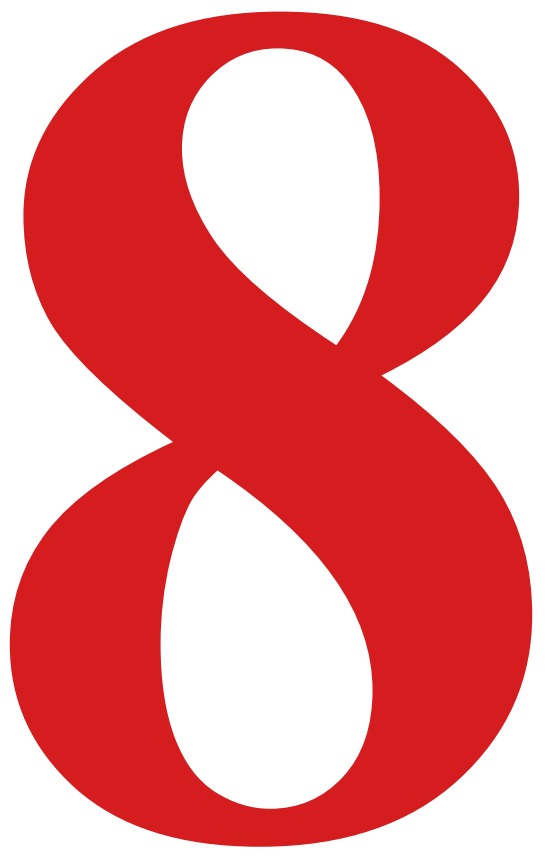




\section{Chapter 8}

Long-term impact of catheter ablation on arrhythmia burden in low-risk patients with paroxysmal atrial fibrillation: the CLOSE to CURE study

Mattias Duytschaever, Jan De Pooter, Anthony Demolder, Milad El Haddad, Thomas Phlips, Teresa Strisciuglio, Philippe Debonnaire, Michael Wolf, Yves Vandekerckhove, Sebastien Knecht, René Tavernier 


\section{ABSTRACT}

Background: Few studies have evaluated the impact of catheter ablation (CA) on atrial tachyarrhythmia (ATA) burden in paroxysmal atrial fibrillation (AF).

Objective: In the prospective, patient-controlled CLOSE to CURE study we studied longer-term impact of optimized CA on ATA burden using insertable cardiac monitors (ICM).

Methods: 105 symptomatic PAF patients were implanted with an ICM 65 [61-78] days before CA. CA consisted of contact force guided pulmonary vein isolation (PVI) targeting an intertag distance $\leq 6 \mathrm{~mm}$ and a region-specific ablation index. Primary endpoint was reduction in ICM-detected ATA burden (relative time spent in ATA), secondary endpoints were single-procedure freedom from ATA, quality of life (QOL), and adverse events.

Results: Mean age was 62 \pm 8 y, CHA2DS2-VASc score 1[1-2], left atrial (LA) diameter 43 [39-43] $\mathrm{mm}$. After PVI (1.13 \pm 0.39 procedure per patient), ATA burden decreased from 2.68 [0.09-15.02] \% at baseline to $0[0-0] \%$ during the first year and $0[0-0] \%$ during the second 2-year (reduction in ATA burden $100[100-100] \%, \mathrm{p}<0.001)$. Single-procedure freedom from any ATA was $87 \%$ at 1 year and $78 \%$ at 2-year. QOL improved significantly across all scores. Adverse events occurred in $5(4.8 \%)$ patients.

Conclusions: CA has become an effective procedure in paroxysmal AF with a major impact on ICMdetected ATA burden. Whereas conventional survival analysis suggests progressive decline in efficacy, we observed that burden reduction is maintained at longer follow-up. These data imply that ATA burden is a more optimal endpoint for assessing ablation efficacy. 


\section{Introduction}

Catheter ablation (CA) is recommended in patients with drug-resistant symptomatic paroxysmal atrial fibrillation (PAF).(1,2) In PAF, pulmonary vein isolation (PVI) results in a 65\% single-procedure oneyear freedom from atrial tachyarrhythmia (ATA),(3-6) with progressive decline of efficacy at longer follow-up and recurrence of ATA mainly due to non-durable PV isolation.(7)

Few studies evaluated the impact of PVI on ATA burden in PAF.(8,9) ATA burden, defined as \% of time spent in ATA, $(1,2,10)$ is associated with AF-related symptoms, heart failure and stroke.(11-15) In the prospective, patient-controlled CLOSE to CURE (C2C) study we determine the longer-term impact of PVI on ATA burden in PAF. To reliably quantify ATA burden all patients were implanted with an insertable cardiac monitor (ICM) at least 2 months before PVI. Ablation consisted of a pointby-point contact force (CF)-guided RF approach aiming to enclose the PVs with contiguous, stable and optimized RF lesions. (16-19)

\section{Methods}

\section{Study design}

The C2C study (NCT02925624) is a single-center, patient-controlled, prospective cohort study. Enrollment started July 2016 until July 2017 at the St Jan Hospital Bruges. Written informed consent was obtained before patient inclusion. The study was approved by the ethics review board.

\section{Patient population}

Patients were eligible if there was a history of symptomatic ECG-proven PAF (either resistant, intolerant or unwilling to take ADT) with at least $\geq 3 \mathrm{AF}$ episodes (anamnestic or documented) in the last 3 months.

Exclusion criteria were persistent AF, prior AF ablation, left atrial (LA) diameter $>50 \mathrm{~mm}$, ejection fraction $<35 \%$, AF secondary to reversible causes, unstable angina or uncontrolled heart failure, myocardial infarction or $\mathrm{CABG}$ within the last 3 months, awaiting cardiac surgery, diagnosed atrial myxoma or thrombus, acute illness, blood clotting or bleeding abnormalities, life expectancy $<12$ months, pregnant or breastfeeding women, and enrollment in any other study.

\section{Implantation and programming of the ICM}

All ICMs (Reveal LINQTM, Medtronic Inc.) were inserted parasternal, over the 4th intercostal space, aiming for R-wave $>0.3 \mathrm{mV}$. Programming of ATA detection was standardized across all patients (AF/AT detection on; sensitivity: balanced; ectopy rejection: nominal; AF/AT recording threshold: all episodes). This algorithm, based upon R-R interval stability and P-wave detection, has an overall accuracy for AF and non-AF detection of $99.4 \% .(20,21)$ Because the algorithm makes a rhythm classification within a 2-min window, the minimal length of an ICM-detected ATA is 2-min. To avoid memory overflow and loss of intracardiac electrograms, patients were asked to weekly transmit ICM 
data to the Medtronic Care Link® Network.

\section{Mechanical and electrical properties of the LA}

All patients underwent at baseline an advanced transthoracic echocardiographic study (Vivid E9, GEVingmed, Milwaukee,WI) to determine LA diameter (parasternal long-axis view), LA volume (Simpson's biplane method at end-systole) and LA reservoir strain function assessed by 2-dimensional speckle tracking echocardiography (EchoPAC, GE, Norway, version 110.2). Prior to PVI, we determined atrial refractoriness, AF inducibility, electrogram voltage, and intra-atrial conduction velocity. (22) An area of low voltage was defined as an area of $>1 \mathrm{~cm} 2$ with $<0.5 \mathrm{mV}$.

\section{Catheter ablation}

PVI was performed by 6 operators. The CLOSE procedure was previously described in extenso.(17) By preference the procedure was perfomed under general anesthesia with an esophageal temperature monitor (SensiTherm ${ }^{\mathrm{TM}}$, St Jude Medical Inc, Minnesota, US) Further set-up consisted of a contactforce (CF) catheter (Thermocool SmartTouch ${ }^{\circledR}$, Biosense-Webster Inc., Diamond Bar, CA, USA) and a circular mapping catheter (CMC) (Figure 1, left panel). Identification of LA-PV junction was based upon anatomy, $\mathrm{CF}, \mathrm{CF}$ vector, catheter jump during pull-back maneuvers, position relative to the $\mathrm{CMC}$, impedance and local electrogram characteristics. Maximal intertag distance between two neighboring lesions was $\leq 6 \mathrm{~mm}$. In case of (pre-)procedural documentation of typical flutter, cavotricuspid isthmus (CTI) ablation was performed.
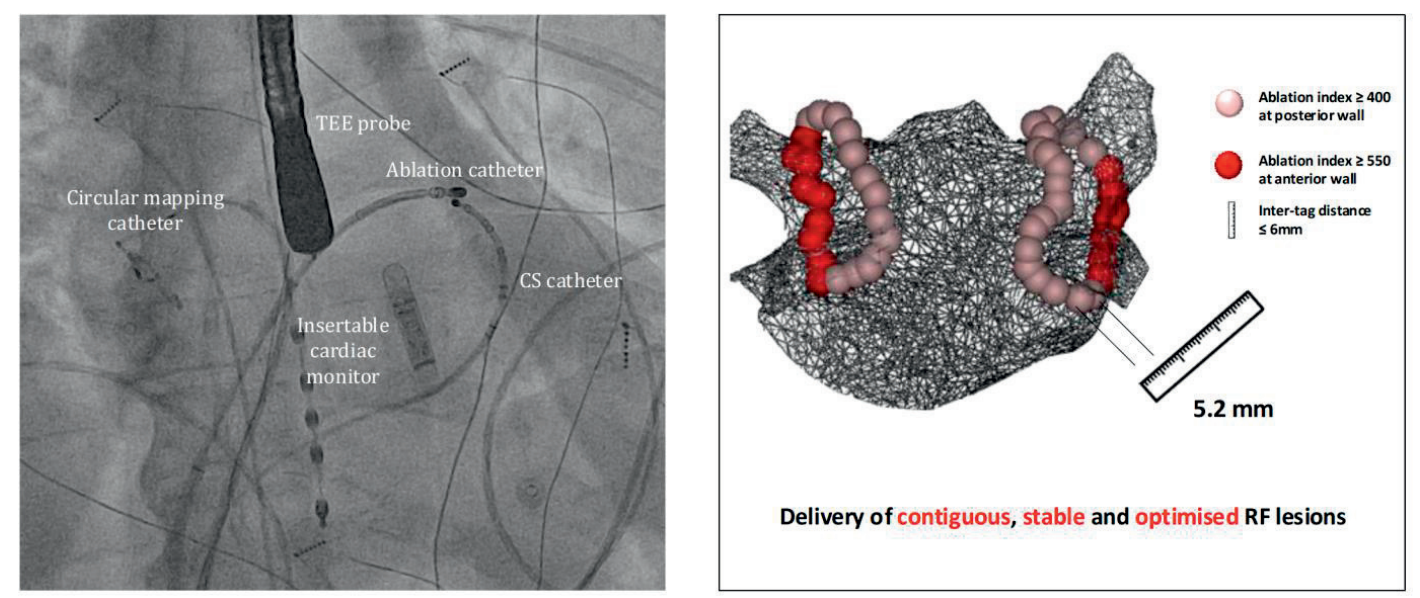

Figure 1: Catheter ablation.

Left panel: Fluoroscopic image showing position of the circular mapping catheter and ablation catheter during CA. Right panel: Tags are represented color-coded according to ablation index target. An intertag distance of $\leq 6 \mathrm{~mm}$ is respected. 


\section{Study visits and follow-up after CA}

Clinical visits, 12-lead ECG and ICM data review were performed at enrollment, at 1, 3, 6, 12, 18 and 24 months after CA or in case of symptoms. Three months after CA, ADT was stopped whereas anticoagulation was continued according to stroke risk. Quality of life (QOL) was assessed before and every 6 months after CA via the Short Form 36 Health Survey, (23) AF Symptom Checklist,(24) and EHRA symptom score. Repeat ablation was advised in case of symptomatic ATA recurrence and consisted of re-isolation or an empirical trigger or substrate ablation.(18)

\section{Primary endpoint}

The primary endpoint, ICM-detected ATA burden, was defined as the \% of time spent in ATA (hours of ATA/hours of monitoring) (Figure 2). ATA burden before PVI was compared to ATA burden during the first (excluding a 3-month blanking window) and second year after PVI. All ICM-detected ATA episodes and their corresponding electrograms were revised on a weekly basis by two independent investigators (JDP, MD). This allowed to adjudicate false detection of ATA due to artefact, premature atrial or ventricular beats or sinus arrhythmia (Figure 2, upper panels). For data analysis, all true episodes and their duration were exported to a custom-made C2C-ATA database (Figure 2, lower panels). This allowed to calculate for each patient the daily burden of ATA (black bars) and the relative time spent in ATA (\%).

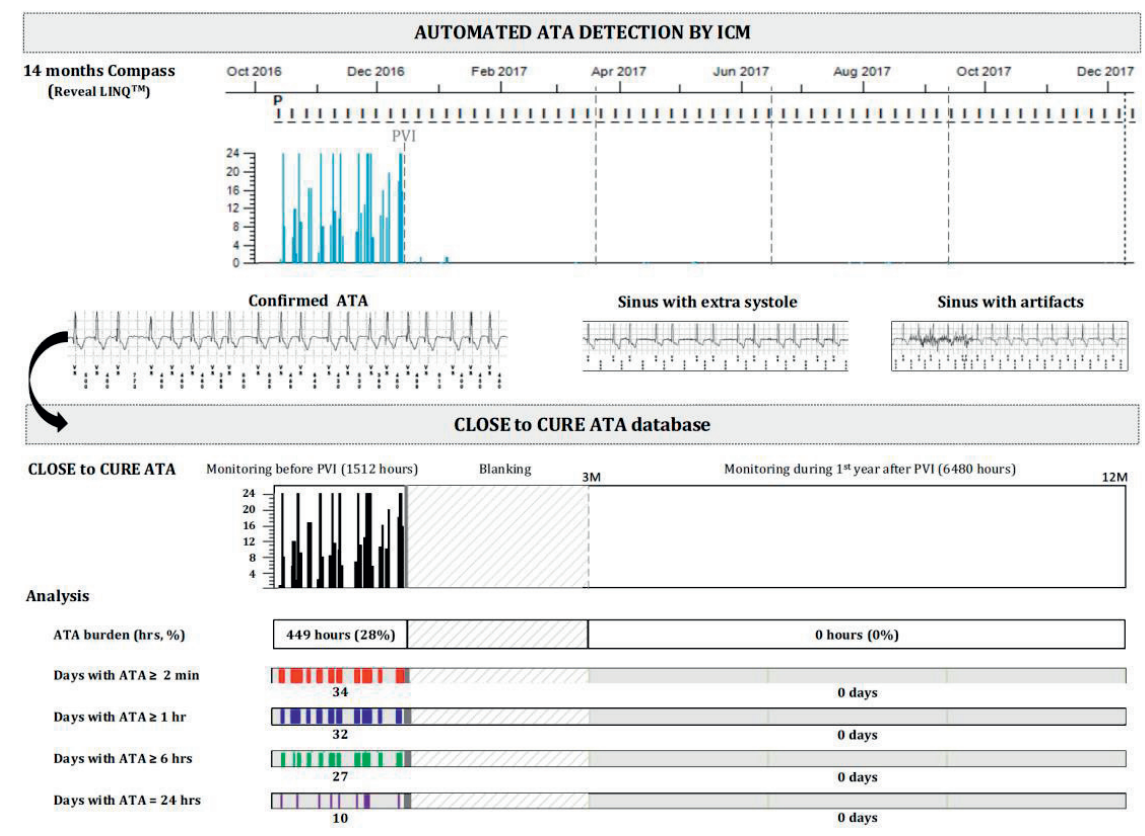

Figure 2: Analysis of atrial tachyarrhythmia burden.

Determining atrial tachyarrhythmia (ATA) burden from the insertable cardiac monitor. 


\section{Secondary endpoints}

Secondary endpoints included alternative measures of ATA burden: ATA burden before adjudication (according to raw ICM data), proportion of patients with reduction in ATA burden (by $>50 \%,>75 \%$, $>90 \%$ and $>95 \%$ ) and with residual ATA burden $>0.5 \%$ after CA. In addition, we calculated for each patient the number of days characterized by ATA using different cut-offs i.e. daily burden of $\geq 2$-min, $\geq 1$-hour, $\geq 6$-hours or 24-hours. Other secondary endpoints were single-procedure freedom from any ICM-detected ATA ( $\geq 2-\mathrm{min})$ at 1 and 2 years, QOL among the different scores and ablation-related adverse events.

\section{Sample size and statistical analysis}

Normality of data distribution was tested with Shapiro-Wilk test. Continuous variables were expressed as mean \pm standard deviation or medians with interquartile range. Dichotomous variables were expressed as frequency (\%). Group comparisons for continuous variables were performed using the paired T test or paired Wilcoxon Signed Rank test. Group comparisons for categorical variables were performed using the Chi square test. Kaplan-Meier survival curves were used to assess time to first documented ATA recurrence. For analysis of QOL, SF 36 data were normalized.(23-25) Statistical significance was set at 0.05 for two tailed tests.

For analysis of ATA burden and its reduction, data were given for all patients $(n=105)$ and for those patients with actual ICM-detected ATA during the baseline monitoring period $(n=84)$. Simple linear regression models using baseline variables were calculated to predict ATA burden after PVI. A multivariable analysis was performed adjusting for potentially confounding variables by selecting variables with at least $\mathrm{p}<0.2$ from the univariable analysis. All analysis was performed using SPSS software (Version 23.0, IBM, Armonk, NY, US).

\section{Results}

\section{Clinical characteristics}

The study included 105 patients (Table 1). The medium number of monitoring days was 65 [61-78]. Overall, 21 patients did not reveal any ATA episode during monitoring. In these patients the time from diagnosis to CA was 9.0 [6.5-20] months and the last ECG documented-AF episode was 17 [9-28] days before enrollment. 
Table 1: Baseline and procedural characteristics $(n=105)$

\begin{tabular}{|c|c|}
\hline \multicolumn{2}{|l|}{ Baseline characteristics } \\
\hline Age, yrs & $62 \pm 8$ \\
\hline Male gender, $\mathrm{n}(\%)$ & $65(62)$ \\
\hline $\mathrm{BMI}, \mathrm{kg} / \mathrm{m} 2$ & $27 \pm 4$ \\
\hline $\mathrm{CHA}_{2} \mathrm{DS}_{2}$-VASc-score & $1[1,2]$ \\
\hline Arterial hypertension, $\mathrm{n}(\%)$ & $35(33)$ \\
\hline Diabetes, n (\%) & $8(8)$ \\
\hline Structural heart disease, $\mathrm{n}(\%)$ & $13(12)$ \\
\hline Coronary artery disease, $\mathrm{n}(\%)$ & $11(10)$ \\
\hline Valvular heart disease, $\mathrm{n}(\%)$ & $2(2)$ \\
\hline Paroxysmal AF, n (\%) & $105(100)$ \\
\hline Time from $1^{\text {st }}$ AF episode to PVI (months) & $15[9,28]$ \\
\hline Left atrial diameter, $\mathrm{mm}$ & $43[39,43]$ \\
\hline Left atrial volume, $\mathrm{ml}$ & $84 \pm 22$ \\
\hline ADT resistant, $\mathrm{n}(\%)$ & $63(60)$ \\
\hline ADT intolerance or unwillingness, $n(\%)$ & $42(40) \mid$ \\
\hline \multicolumn{2}{|l|}{ Procedural characteristics } \\
\hline General anaesthesia, $n(\%)$ & $102(97)$ \\
\hline Procedure duration, $\min$ & $143 \pm 31$ \\
\hline Isolation of all PVs, $n$ (\%) & $105(100)$ \\
\hline Additional ablation of CTI, $n(\%)$ & $6(6)$ \\
\hline First pass isolation right circle, $n(\%)$ & $98(93)$ \\
\hline First pass isolation left circle, $n$ (\%) & $101(96)$ \\
\hline Adenosine challenge performed, $n(\%)$ & $97(92)$ \\
\hline Adenosine proof isolation, $n(\%)$ & $93(96)$ \\
\hline RF energy, number of applications & $59 \pm 11$ \\
\hline RF energy, total time of delivery, min & $28 \pm 6$ \\
\hline $\operatorname{DAP}\left(\mathrm{mGy} / \mathrm{cm}^{2}\right)$ & $4769 \pm 3378$ \\
\hline
\end{tabular}

\section{Mechanical and electrical properties of the LA}

Results are summarized in Figure 3. In 10 patients (9.5\%) we observed a low-voltage area. During premature stimulation, self-terminating ATA was induced in 8 patients $(7.6 \%)$. 


\section{Mechanical properties of the LA}
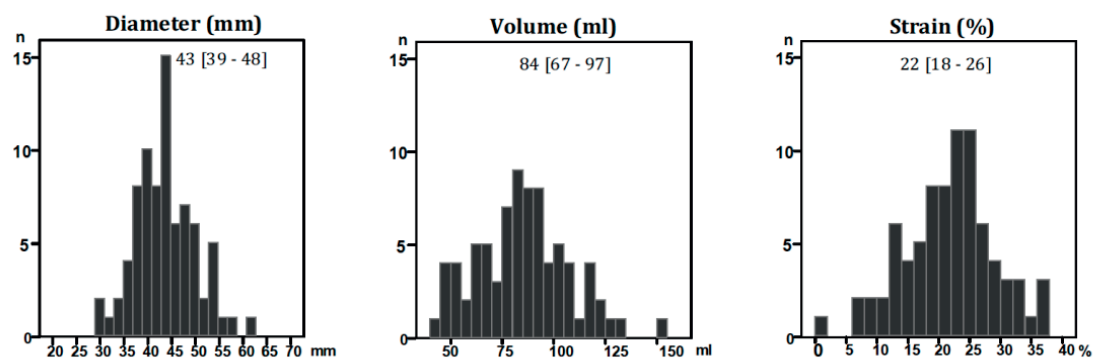

Electrical properties of the LA
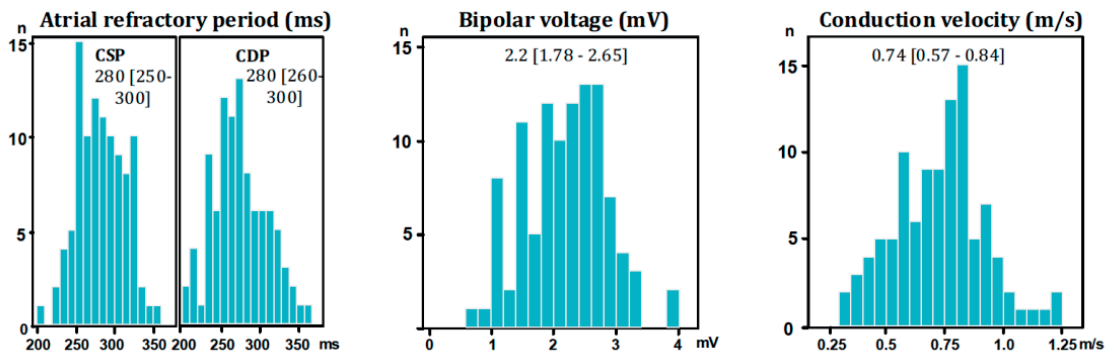

Figure 3: Mechanical and electrical left atrial properties

Histogram plots depicting mechanical (upper panels) and electrical properties (lower panels) of the left atrium (LA).

\section{Procedural characteristics of CA and follow-up}

Results are given in Table 1. All patients except one were discharged the day after CA. Within the two-year follow-up period, 0 patients received clzss I or III antiarrhythmic drugs or cardioversion, 14 patients received a repeat procedure at 150 [100-513] days after PVI.

\section{Primary endpoint: ATA burden before and after ablation}

Results for ATA burden for the entire study population are given in the upper panels of Figure 4. After PVI (1.13 \pm 0.39 procedure per patient throughout the 2 year follow-up), ATA burden decreased from 2.68 [0.09-15.02] \% at baseline to 0 [0-0] \% during the first year (reduction in ATA burden 100 [100$100] \%, \mathrm{p}<0.001$, left panel) and $0[0-0] \%$ during the second 2-year (reduction in ATA burden 100 $[100-100] \%, \mathrm{p}<0.001$, right panel). Burden reduction was seen both in patients without (black bars) and with any 2-min ATA recurrence (red and green bars). None of the patients progressed to persistent $\mathrm{AF}$ after $\mathrm{CA}$. Results for the subset of 84 patients with documented ATA during the monitoring period were similar (Figure 4, lower panels). 


\section{Secondary ATA burden-related endpoints}

Results for the 84 patients are given in Table 2. ATA burden without adjudication decreased from 6.61 [1.80-19.00] \% to 0 [0-0.03] \% during the first 12 months after PVI and to 0 [0-0.03] \% during the second year ( $<<0.001$ for both). The proportion of patients with $>95 \%$ reduction in ATA burden was $94 \%$ and $96 \%$ at 1 and 2-y FU. Finally, throughout the first and second year after ablation, only 5 $(6 \%)$ and $1(1 \%)$ had a residual ATA burden $>0.5 \%$.

In Figure 5 we plotted the AF calendar plots for the entire study population. Using a cumulative daily ATA burden of 2-min (red), 1-hour (blue), 6-hours (green) and 24-hours (purple), the number of days per patient characterized by ATA decreased significantly (all paired tests $\mathrm{p}<0.001$ ). Of interest, of the 14 patients with ATA during the first year, only 4 patients showed ATA during the second year. Vice versa, of the 13 patients with ATA during the second year, only 4 patients showed ATA during the first year.

A significant but weak regression was found between ATA burden after PVI and ATA burden before PVI (Table 3). In a multivariable model during the 1st year adjusting for both ATA burden before PVI and male gender, only ATA burden before PVI remained statistically significant. 

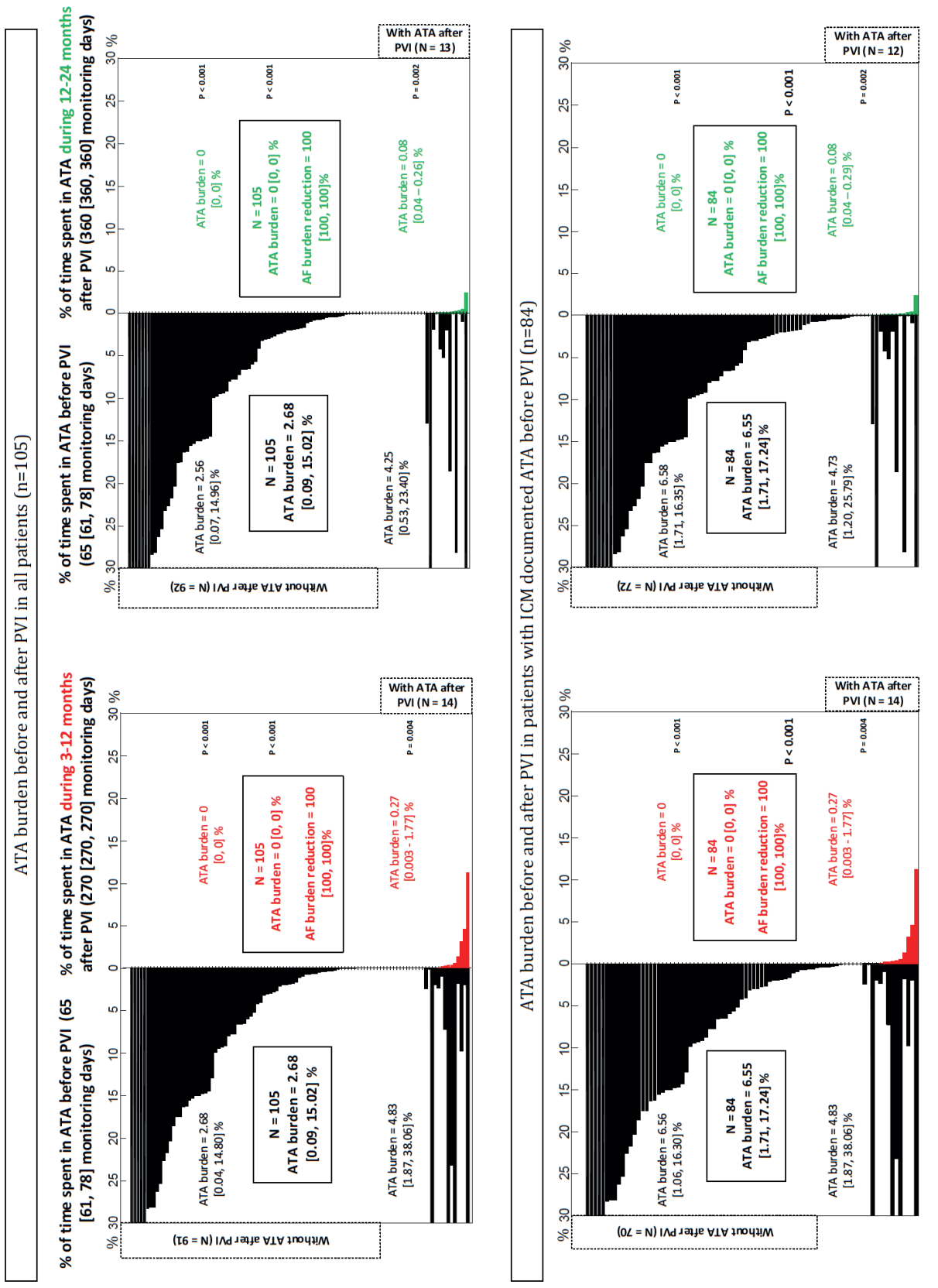

京.

ऐั

으몽

홍

氜

을

范

कृ

ฮิ

흘 응

产

.

苛

¿

氖

로

$\exists$

g

司

$\circ$

总完

हैं

苛

원

通

卷范

흔

这芒

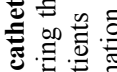

๖

范

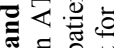

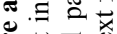

흥

8 क 5

흠응

छ

ㅇํㄹ

웡

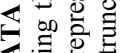

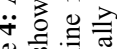

के

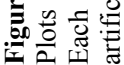


Table 2: ATA burden before and after PVI $(n=84)$

\begin{tabular}{|c|c|c|c|c|}
\hline & $\begin{array}{c}\text { ATA burden before } \\
\text { PVI }\end{array}$ & $\begin{array}{c}\text { ATA burden } \\
\text { throughout 3-12 } \\
\text { months after PVI }\end{array}$ & $\begin{array}{c}\text { ATA burden } \\
\text { throughout 12-24 } \\
\text { months after PVI }\end{array}$ & $P$ value \\
\hline $\begin{array}{l}\text { ATA burden (relative time spent in } \\
\text { ATA), unadjudicated \% }\end{array}$ & $6.61[1.80,19.00]$ & $0[0,0.03]$ & $0\left[\begin{array}{ll}0 & 0.03\end{array}\right]$ & $<0.001^{*}$ \\
\hline $\begin{array}{l}\text { ATA burden (relative time spent in } \\
\text { ATA), } \%\end{array}$ & $6.56[1.71,17.24]$ & $0[0,0]$ & $0[0,0]$ & $<0.001^{*}$ \\
\hline Reduction in ATA burden, \% & - & $100[100,100]$ & $100[100,100]$ & \\
\hline \multicolumn{5}{|l|}{$\begin{array}{l}\text { Proportion of patients with } \\
\text { reduction in ATA burden, } n(\%)\end{array}$} \\
\hline by $>\mathbf{5 0} \%$ & - & $82 / 84(98)$ & $82 / 84(98)$ & - \\
\hline by $>75 \%$ & - & $81 / 84(96)$ & $81 / 84(96)$ & - \\
\hline by > $90 \%$ & - & $79 / 84(94)$ & $81 / 84(96)$ & - \\
\hline by > $95 \%$ & - & $79 / 84(94)$ & $81 / 84(96)$ & - \\
\hline \multicolumn{5}{|l|}{$\begin{array}{l}\text { Proportion of patients with ATA } \\
\text { burden after PVI, } n(\%)\end{array}$} \\
\hline$>0.5$ and $\leq 1 \%$ & - & $1(1)$ & $O(0)$ & - \\
\hline$>1$ and $\leq 5 \%$ & - & $3(3)$ & $1(1)$ & - \\
\hline$>5$ and $\leq 10 \%$ & - & $0(0)$ & $0(0)$ & - \\
\hline$>10 \%$ & - & $1(1)$ & $O(0)$ & - \\
\hline
\end{tabular}

* Applies to ATA before PVI vs 3-12M and to ATA before PVI vs 12-24M

Table 3: Linear regression of ATA burden after PVI versus baseline characteristics

3-12 months after PVI

\begin{tabular}{|c|c|c|c|c|c|}
\hline \multicolumn{2}{|c|}{ Simple linear regression } & \multicolumn{2}{|c|}{ Multiple linear regression } & \multicolumn{2}{|c|}{ Simple linear regression } \\
\hline Coefficient $(95 \% \mathrm{CI})$ & P-value & Coefficient $(95 \% \mathrm{Cl})$ & P-value & Coefficient $(95 \% \mathrm{Cl})$ & P-value \\
\hline $\begin{array}{c}0.005 \\
(-0.033,0.043)\end{array}$ & 0.797 & & & $\begin{array}{c}0.001 \\
(-0.006,0.008)\end{array}$ & 0.769 \\
\hline $\begin{array}{c}-0.404 \\
(-1.005,0.198)\end{array}$ & 0.186 & $\begin{array}{c}-0.585 \\
(-1.178,0.009)\end{array}$ & 0.053 & $\begin{array}{c}-0.059 \\
(-0.176,0.058)\end{array}$ & 0.318 \\
\hline $\begin{array}{c}0.021 \\
(-0.058,0.101)\end{array}$ & 0.594 & & & $\begin{array}{c}0.009 \\
(-0.006,0.025)\end{array}$ & 0.234 \\
\hline $\begin{array}{c}-0.042 \\
(-0.3,0.217)\end{array}$ & 0.75 & & & $\begin{array}{c}-0.008 \\
(-0.058,0.042)\end{array}$ & 0.737 \\
\hline $\begin{array}{c}-0.136 \\
(-0.771,0.499)\end{array}$ & 0.671 & & & $\begin{array}{c}-0.043 \\
(-0.166,0.080)\end{array}$ & 0.487 \\
\hline $\begin{array}{c}-0.215 \\
(-1.368,0.938)\end{array}$ & 0.712 & & & $\begin{array}{c}-0.033 \\
(-0.257,0.190)\end{array}$ & 0.766 \\
\hline $\begin{array}{c}-0.297 \\
(-1.212,0.619)\end{array}$ & 0.521 & & & $\begin{array}{c}-0.041 \\
(-0.219,0.136)\end{array}$ & 0.644 \\
\hline $\begin{array}{c}0 \\
(-0.059,0.06)\end{array}$ & 0.988 & & & $\begin{array}{c}0.002 \\
(-0.009,0.014)\end{array}$ & 0.699 \\
\hline $\begin{array}{c}-0.003 \\
(-0.019,0.013)\end{array}$ & 0.716 & & & $\begin{array}{c}0.001 \\
(-0.003,0.004)\end{array}$ & 0.746 \\
\hline $0.011(-0.598,0.619)$ & 0.972 & & & $\begin{array}{c}0.038 \\
(-0.079,0.156)\end{array}$ & 0.519 \\
\hline $\begin{array}{c}-0.163 \\
(-1.419,1.092)\end{array}$ & 0.796 & & & $\begin{array}{c}-0.032 \\
(-0.275,0.211)\end{array}$ & 0.792 \\
\hline $\begin{array}{c}-0.004 \\
(-0.012,0.005)\end{array}$ & 0.411 & & & $\begin{array}{c}-0.000 \\
(-0.001,0.001)\end{array}$ & 0.818 \\
\hline $\begin{array}{c}0 \\
(-0.014,0.014)\end{array}$ & 0.608 & & & $\begin{array}{c}0.000 \\
(-0.003,0.003)\end{array}$ & 0.904 \\
\hline $\begin{array}{c}0.015 \\
(0.002,0.027)\end{array}$ & 0.021 & $\begin{array}{c}0.018 \\
(0.005,0.030)\end{array}$ & 0.007 & $\begin{array}{c}0.003 \\
(0.001,0.006)\end{array}$ & 0.010 \\
\hline
\end{tabular}



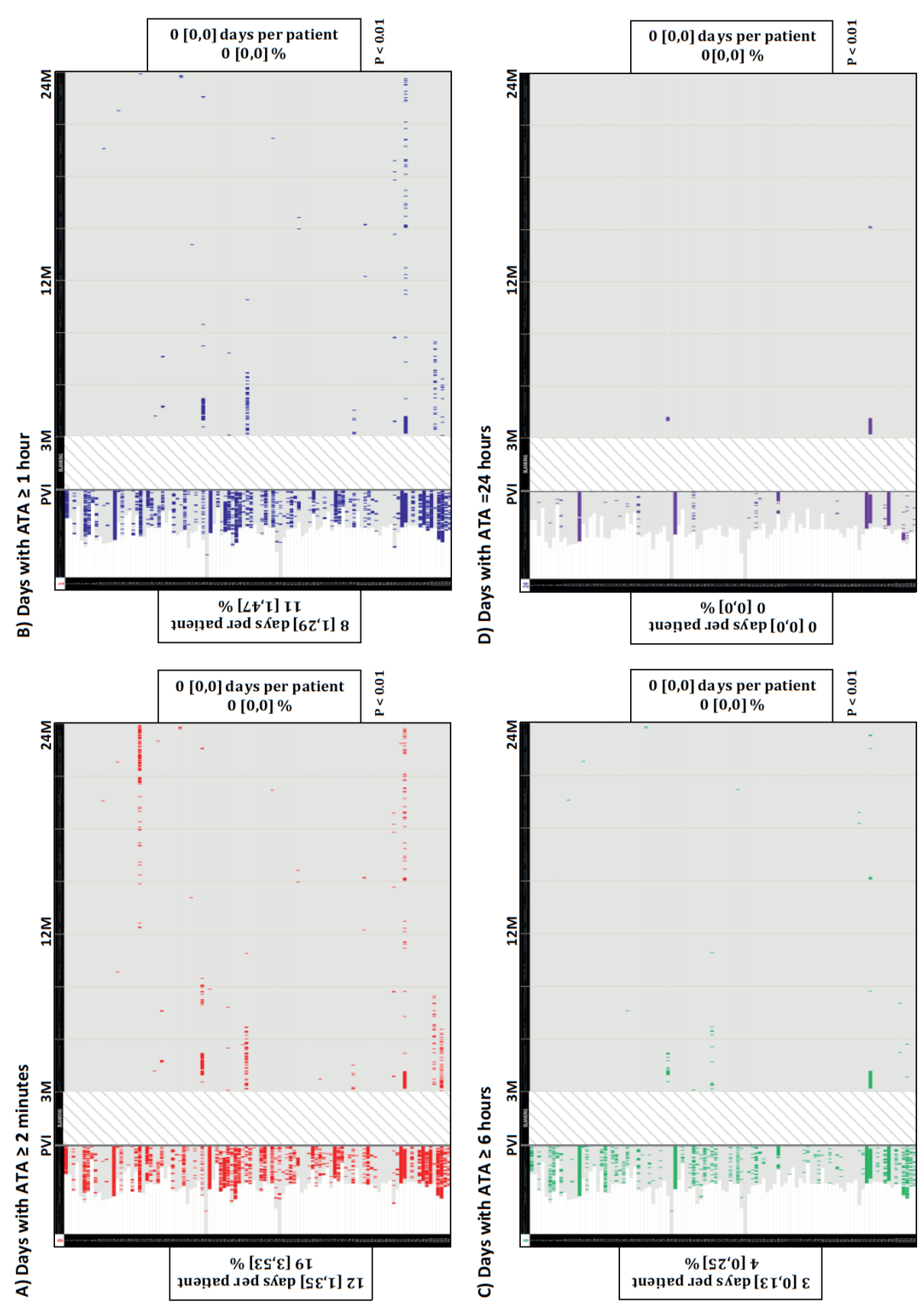


\section{Single-procedure, off ADT freedom from ATA}

In Figure 6 we plotted the time to the first day with any 2-min ATA for the entire population (blue curve) and subpopulation (red curve). Single-procedure, off-ADT freedom from any ATA declined from $87 \%$ after 1 year to $78 \%$ after 2 years $(\mathrm{p}=0.343)$.

Freedom from any ICM documented ATA at 2 years follow-up

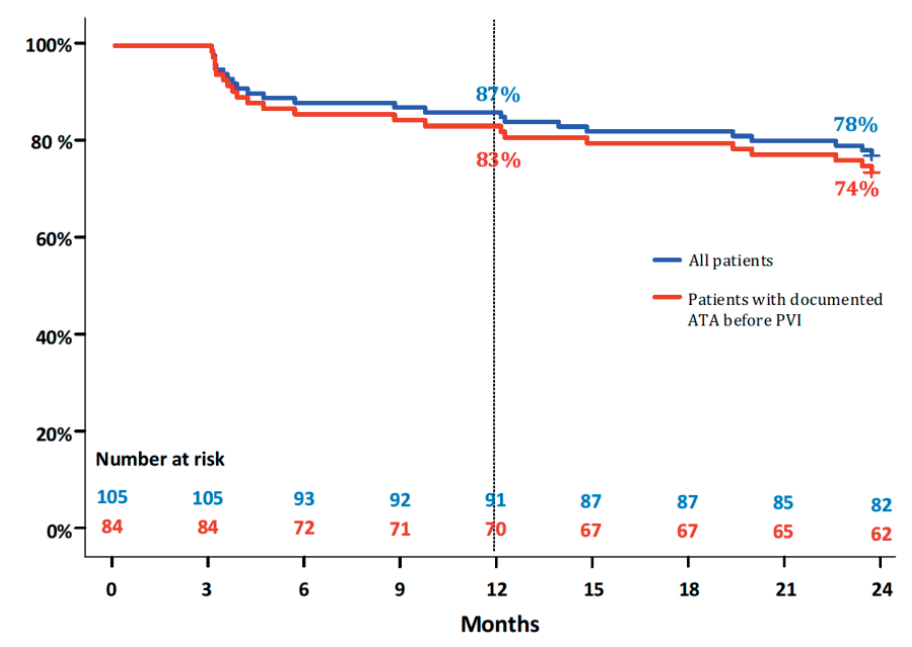

Figure 6: ATA free survival plot

Kaplan-Meier curves depicting time to first recurrence of any ATA during the first 2 years after PVI (blue curve: entire study population; red curve: 84 patients).

\section{Quality of Life}

Results are summarized in Table 4. Physical and mental Health SF36 score, symptom frequency and severity scores, and EHRA score all improved significantly at 1 and 2 year $(\mathrm{p}<0.001)$. Improvement in QOL was comparable among patients with and without ATA recurrence. 


\begin{tabular}{|c|c|c|c|c|c|}
\hline & $\begin{array}{l}\text { At baseline } \\
\text { before PVI }\end{array}$ & At 12 months after PVI & $P$ value & At 24 months after PVI & $P$ value \\
\hline \multicolumn{6}{|l|}{ SF 36 normalised scores } \\
\hline Physical health score & $47.6 \pm 7.4$ & $51.0 \pm 6.8$ & $<0.0001$ & $49.45 \pm 7.96$ & 0.192 \\
\hline $\mathrm{N}$ of fully completed forms & 97 & 95 & & 96 & \\
\hline Mental health score & $48.7 \pm 9.1$ & $52.1 \pm 7.9$ & 0.002 & $52.16 \pm 8.31$ & $<0.001$ \\
\hline $\mathrm{N}$ of fully completed forms & 97 & 95 & & 96 & \\
\hline \multicolumn{6}{|l|}{ Symptoms scores } \\
\hline Symptoms frequency score & $18.3 \pm 8.7$ & $9.8 \pm 7.4$ & $<0.0001$ & $10.76 \pm 8.50$ & $<0.0001$ \\
\hline $\mathrm{N}$ of fully completed forms & 102 & 99 & & 102 & \\
\hline Symptoms severity score & $13.9 \pm 7.4$ & $7.12 \pm 7.0$ & $<0.0001$ & $7.81 \pm 7.30$ & $<0.0001$ \\
\hline $\mathrm{N}$ of fully completed forms & 95 & 93 & & 96 & \\
\hline \multicolumn{6}{|l|}{ EHRA score } \\
\hline Median score [IQR] & $3[2,3]$ & $1[1,1]$ & $<0.001$ & $1[1,1]$ & $<0.001$ \\
\hline
\end{tabular}

\section{Safety}

Ablation-related adverse events were observed in 5 patients $(4.7 \%)$ : three patients with groin-site hematoma (all treated conservatively by mechanical pressure), one patient with femoral pseudoaneurysm (requiring surgery) and one patient with symptomatic left PV stenosis (treated with percutaneous stenting at 104 days after PVI with resolution of symptoms throughout 2-year FU). In none of the patients undergoing repeat ablation narrowing of PVs was observed.

\section{Discussion}

\section{Main Findings}

The $\mathrm{C} 2 \mathrm{C}$ study shows that an optimized CA results in a major overall reduction in ICM-detected ATA burden in patients with PAF. Whereas survival analysis based upon freedom from any ATA recurrence suggests progressive decline of efficacy over time, the impact of CA on ATA burden is maintained at longer-term FU. These data imply that ATA burden is a more optimal endpoint for assessing ablation efficacy.

\section{PAF patients in the $\mathrm{C} 2 \mathrm{C}$ study}

In the present study, patients were relatively young, had limited structural heart disease, low stroke risk, near-normal left atrial diameter and a relatively short time from diagnose to CA. This low-risk profile however does not differ from the PAF patient enrolled in CA trials,(3-6) or from patients referred for CA in real-life.(26,27). Likewise the ATA burden and its variation (median 2.68\%) is not different from prior studies reporting on ATA burden in PAF. $(8,9,14,28)$ Finally, C2C patients were characterized by left atrial electrical and mechanical properties in line with prior studies in 
PAF. $(29,30)$ Also the finding of low-voltage areas in $\approx 10 \%$ of apparently healthy patients is in line with prior studies in PAF.(2-32) All together, above data suggest that the $\mathrm{C} 2 \mathrm{C}$ population reflects the relatively young, symptomatic and otherwise relatively healthy patient referred for ablation of PAF both in studies and real-life.

\section{ATA burden before and after CA: are we close to curing PAF?}

Efficacy of CA is commonly expressed as single-procedure freedom from any ATA (>30s).(1) This definition (in which one single, even short episode implies permanent failure) most likely underestimates clinically relevant success after CA.(1) In this regard, ATA burden, defined as \% of time spent in ATA and preferably measured by continuous monitoring, seems a more reliable estimate of clinically relevant success after CA.(1,2,10) Indeed, prior studies suggested a dose-effect relation between ATA burden and symptoms, heart failure and stroke.(11-15) Glotzer et al showed that ATA burden $\geq 5.5$ hours per day $(\geq 20 \%$ ) was associated with a double stroke risk(14), whereas Go et al showed that only the highest tertile of $\operatorname{AF}$ burden $(\geq 11.4 \%)$ was associated with a $>3$-fold higher adjusted rate of thromboembolism in PAF patients.(13)

A limited number of prospective studies reported on patient-controlled ATA burden after CA. In the DISCERN AF study, CA reduced mean ICM-detected ATA burden from $8.3 \%$ to $1.25 \%$ after 18 months.(9) In MANTRA-PAF, CA reduced estimated ATA burden from a 90th ATA burden of 30\% to $13 \%$ throughout the first 24 months.(27) In the CAPTAF study, CA reduced ICM-detected ATA burden from $24.9 \pm 37 \%$ to $5.5 \pm 18.1 \%$ at 12 months ( $\mathrm{p}<0.001$ ), not different from medical therapy.(8) In the present $\mathrm{C} 2 \mathrm{C}$ study, optimized CA had a marked and maintained impact on ATA burden (with a low number or repeat procedures and without ADT). In a PAF population in which one quarter of the patients presented with ATA burden $>15.02 \%$, median ATA burden in the 1st two years after CA was 0 [IQR 0-0] \% (Figure 4) and also in patients with some ATA recurrence, ATA burden was significantly reduced (because episodes were short-lasting and isolated in nature). The good results of the $\mathrm{C} 2 \mathrm{C}$ study, most likely, are explained by durable PV isolation,(19) rather than patient selection (in view of the patient characteristics comparable to prior trials). Also an early intervention strategy during the first year from AF diagnosis (although performed in only $26 \%$ of patients) might contribute to improved outcome. Whether reduction in ATA burden by CA might impact AF-related morbidity and mortality requires further study, especially in a sicker population. Marrouche et al recently showed that reduction of burden improves outcome in heart failure patients.(15) Likewise, reduction of burden might be expected to improve symptoms, $(11,12)$ and reduce stroke. $(13,14)$

\section{Single-procedure freedom from ATA, QOL, and safety after CA for PAF}

In the $\mathrm{C} 2 \mathrm{C}$ study freedom from any ATA was $\approx 85 \%$ at 1 -year. This relatively high success rate (in the setting of continuous monitoring) (33) is in line with 1) data from the CLOSE-PILOT study,(17) 2) 
prior studies reporting a $\approx 90 \% 1$-year success rate after multiple PVI procedures, $(3,4)$ and 3 ) the knowledge that non-PV triggers account for $\approx 10 \%$ of PAF.(34) The $\mathrm{C} 2 \mathrm{C}$ data at 2-year (suggesting decline in efficacy as previosuly reported) (7) however underscore that this definition of success is not optimal to describe longer-term efficacy of CA.

Prior studies showed that CA improves QOL in AF patients, especially but not exclusively in patients free from ATA. $(3,5,6)$ Recent studies showed that residual ATA burden (i.e. $>4 \%$ assessed by Holter; or $>0.5 \%$ assessed by ICM) determines poor QOL after CA.(11,12) Also in the CAPTAF study, general health was related to actual ATA burden.(8) Due to the low residual burden after CA in the C2C study, QOL significantly improved both in patients with and without ATA recurrence.

The $\mathrm{C} 2 \mathrm{C}$ study confirms the overall safety profile of CA.(3-6) Whereas we did not observe death, stroke or tamponade, there was a limited number of groin-site hematomas, one pseudoaneurysm and one PV stenosis requiring stenting. None of the patients undergoing repeat ablation revealed PV narrowing. The safety profile is in line with prior safety data after CLOSE-PVI.(17,35) Moreover, improvement in safety is underappreciated because safety of CA also depends on the number of repeat procedures required for durable isolation.

\section{Implications of the present study}

(1) The C2C study shows that PVI, if meticulously performed, markedly impacts ATA burden and QOL on the longer-term. It suggests that any novel CA strategy resulting in durable isolation will have similar impact; (2) Despite a class I indication, despite superiority over ADT,(3,5,6) and excellent outcome after CA combined with ADT,(24) there is underutilization and late referral of ablative therapy in PAF. The current longer-term data may lower the threshold for CA; (3) Finally, C2C data suggest that ATA burden is more useful measure of the outcome of the ablation than to censor an ablation as a failure after a single 30 -second recurrence as is currently recommended by the guidelines. (1) Because of the short duration of false-positive ATA episodes, ATA burden is not sensitive to adjudication. Because of the shorter and isolated nature of late recurrences, ATA burden (in contrast to survival analysis) is more appropriate to assess longer-term efficacy of CA. In this regard, C2C analysis can serve as a methodological guide for future studies on ATA burden, either assessed by implanted or wearable devices.

\section{Limitations}

(1) Despite the importance of patient-controlled data, present results require confirmation by multicentric large studies; (2) ATA burden is dependent on the accuracy of ICM detection. Although one can correct for false positive ATA and although adjudication did not affect results on ATA burden, one cannot correct for false negative findings (like asymptomatic AT with slow regular ventricular rate); (3) We did not advise patients to record symptoms in a standardized diary. Therefore the $\mathrm{C} 2 \mathrm{C}$ study is less suited to address the issue of symptomatic vs asymptomatic ATA.(9,10); (4) Finally, 21 
patients did not have ICM-documented ATA during the monitoring phase. The fact that those patients had a median time from diagnosis to CA of 9.0 [6.5-20] months together with at least 3 episodes before enrollment favors the hypothesis that absence of ATA is the result of the probabilistic nature of PAF rather than spontaneous resolution of the arrhythmia.

\section{Conclusions}

We observed that CA aiming for durable pulmonary vein isolation markedly reduces ATA burden assessed by insertable cardiac monitors; in a PAF population in which one quarter of the patients presented with ATA burden $>15.02 \%$, median ATA burden in the first two years after CA was 0 [IQR $0-0] \%$. Finally, our data make a strong case that the "survival" approach to assessing efficacy with time to first recurrence underestimates the benefit of ablation in reducing burden. 


\section{REFERENCES}

1. Calkins H., Hindricks G., Cappato R., et al. 2017 HRS/EHRA/ECAS/APHRS/SOLAECE expert consensus statement on catheter and surgical ablation of atrial fibrillation. Heart Rhythm 2017; 14:e275-e444.

2. 2. Kirchhof P, Benussi S, Kotecha D, et al. 2016 ESC Guidelines for the management of atrial fibrillation developed in collaboration with EACTS. European Journal of Cardio-Thoracic Surgery. 2016;50:e1-e88.

3. Jaïs P, Cauchemez B, Macle L, et a. Catheter ablation versus antiarrhythmic drugs for atrial fibrillation: the A4 study. Circulation 2008;118:2498-505.

4. Kuck K, Brugada J, Fürnkranz A, et al. Cryoballoon or Radiofrequency Ablation for Paroxysmal Atrial Fibrillation. New England Journal of Medicine. 2016;374:2235-45.

5. Packer D, Kowal R, Wheelan K, et al. Cryoballoon Ablation of Pulmonary Veins for Paroxysmal Atrial Fibrillation. Journal of the American College of Cardiology. 2013;61:1713-23.

6. Wilber D, Pappone C, Neuzil P, et al. ThermoCool AF Trial Investigators. Comparison of Antiarrhythmic Drug Therapy and Radiofrequency Catheter Ablation in Patients With Paroxysmal Atrial Fibrillation. JAMA. 2010;303:333.

7. Ouyang F, Tilz R, Chun J, et al. Long-Term Results of Catheter Ablation in Paroxysmal Atrial Fibrillation. Circulation. 2010;122:2368-77.

8. Blomström-Lundqvist C, Gizurarson S, Schwieler J, et al. Effect of Catheter Ablation vs Antiarrhythmic Medication on Quality of Life in Patients With Atrial Fibrillation. JAMA. 2019;321:1059.

9. Verma A, Champagne J, Sapp J, et al. Discerning the Incidence of Symptomatic and Asymptomatic Episodes of Atrial Fibrillation Before and After Catheter Ablation (DISCERN AF). JAMA Internal Medicine. 2013;173:149.

10. Chen L, Chung M, Allen L, et al. Atrial Fibrillation Burden: Moving Beyond Atrial Fibrillation as a Binary Entity: A Scientific Statement From the American Heart Association. Circulation. 2018;137(20).

11. Mantovan R, Macle L, De Martino, et al. Relationship of quality of life with procedural success of atrial fibrillation (AF) ablation and postablation AF burden: substudy of the STAR AF randomized trial. The Canadian journal of cardiology 2013;29:1211-7.

12. Bjorkenheim A, Brandes A, Magnuson A, et al. Patient-Reported Outcomes in Relation to Continuously Monitored Rhythm Before and During 2 Years After Atrial Fibrillation Ablation Using a Disease-Specific and a Generic Instrument. Journal of the American Heart Association $2018 ; 7$.

13. Glotzer TV, Daoud EG, Wyse DG, et al. The relationship between daily atrial tachyarrhythmia burden from implantable device diagnostics and stroke risk: the TRENDS study. Circulation Arrhythmia and electrophysiology 2009;2:474-80.

14. Go A, Reynolds K, Yang J, et al. Association of Burden of Atrial Fibrillation With Risk of Ischemic Stroke in Adults With Paroxysmal Atrial Fibrillation. JAMA Cardiology. 2018;3:601.

15. Marrouche N, Brachmann J, Andresen D, et al. Catheter Ablation for Atrial Fibrillation with Heart Failure. New England Journal of Medicine. 2018;378:417-27.

16. El Haddad M, Taghji P, Phlips T, et al. Determinants of Acute and Late Pulmonary Vein Reconnection in Contact Force-Guided Pulmonary Vein Isolation. Circulation: Arrhythmia and Electrophysiology. 2017;10.

17. Taghji P, El Haddad M, Phlips T, et al. Evaluation of a Strategy Aiming to Enclose the Pulmonary Veins With Contiguous and Optimized Radiofrequency Lesions in Paroxysmal Atrial Fibrillation. JACC: Clinical Electrophysiology. 2018;4:99-108.

18. Phlips T, Taghji P, El Haddad M, et al. Improving procedural and one-year outcome after contact 
force-guided pulmonary vein isolation: the role of interlesion distance, ablation index, and contact force variability in the 'CLOSE'-protocol. EP Europace. 2018;20:f419-27.

19. De Pooter J, Strisciuglio T, El Haddad M, et al.Pulmonary vein reconnection no longer occurs in the majority of patients after a single pulmonary vein isolation procedure. J Am Coll Cardiol EP 2019;5:295-305.

20. Lee R, Mittal S. Utility and limitations of long-term monitoring of atrial fibrillation using an implantable loop recorder. Heart Rhythm 2018;15:287-95.

21. Sanders P, Pürerfellner H, Pokushalov E, et al. Performance of a new atrial fibrillation detection algorithm in a miniaturized insertable cardiac monitor: Results from the Reveal LINQ Usability Study. Heart Rhythm. 2016;13:1425-30.

22. Anter E, Duytschaever M, Shen C, et al. Activation Mapping With Integration of Vector and Velocity Information Improves the Ability to Identify the Mechanism and Location of Complex Scar-Related Atrial Tachycardias. Circulation: Arrhythmia and Electrophysiology. 2018;11.

23. Ware JE, Jr., Sherbourne CD. The MOS 36-item short-form health survey (SF-36). I. Conceptual framework and item selection. Med Care 1992;30:473-83.

24. Bubien RS, Knotts-Dolson SM, Plumb VJ, et al. Effect of radiofrequency catheter ablation on health-related quality of life and activities of daily living in patients with recurrent arrhythmias. Circulation 1996;94:1585-91.

25. Duytschaever M, Demolder A, Phlips T, et al. PulmOnary vein isolation With vs. without continued antiarrhythmic Drug trEatment in subjects with Recurrent Atrial Fibrillation (POWDER AF): results from a multicentre randomized trial. European Heart Journal. 2017;39:1429-37.

26. Arbelo E, Brugada J, Hindricks G, et al. The Atrial Fibrillation Ablation Pilot Study: an European Survey on Methodology and results of catheter ablation for atrial fibrillation conducted by the European Heart Rhythm Association. European Heart Journal. 2014;35:1466-78.

27. Schmidt M, Dorwarth U, Andresen D, et al. German ablation registry: Cryoballoon vs radiofrequency ablation in paroxysmal atrial fibrillation - One-year outcome data. Heart Rhythm. 2016;13:836-44.

28. Cosedis Nielsen J, Johannessen A, Raatikainen P, et al. Radiofrequency ablation as initial therapy in paroxysmal atrial fibrillation. The New England journal of medicine 2012;367:1587-95.

29. Stiles MK, John B, Wong CX, et al. Paroxysmal lone atrial fibrillation is associated with an abnormal atrial substrate: characterizing the "second factor". J Am Coll Cardiol 2009;53:1182-91.

30. Walters T, Nisbet A, Morris G, et al. Progression of atrial remodeling in patients with high-burden atrial fibrillation: Implications for early ablative intervention. Heart Rhythm. 2016;13:331-9.

31. Rolf S, Kircher S, Arya A, Eitel C, Sommer P, Richter S, Gaspar T, Bollmann A, Altmann D, Piedra C, Hindricks G, Piorkowski C. Tailored Atrial Substrate Modification Based on LowVoltage Areas in Catheter Ablation of Atrial Fibrillation. Circulation: Arrhythmia and Electrophysiology. 2014;7:825-33.

32. Kottkamp H, Bender R, Berg J. Catheter ablation of atrial fibrillation: how to modify the substrate? J Am Coll Cardiol 2015;65:196-206.

33. Hindricks G, Piorkowski C, Tanner H, et al. Perception of atrial fibrillation before and after radiofrequency catheter ablation: relevance of asymptomatic arrhythmia recurrence. Circulation 2005;112:307-13.

34. Tsai CF, Tai CT, Hsieh MH, et al. Initiation of atrial fibrillation by ectopic beats originating from the superior vena cava: electrophysiological characteristics and results of radiofrequency ablation. Circulation 2000;102:67-74.

35. Wolf M, El Haddad M, De Wilde V, et al. Endoscopic evaluation of the esophagus after catheter ablation of atrial fibrillation using contiguous and optimized radiofrequency applications. Heart Rhythm. 2019;6:1013-20. 


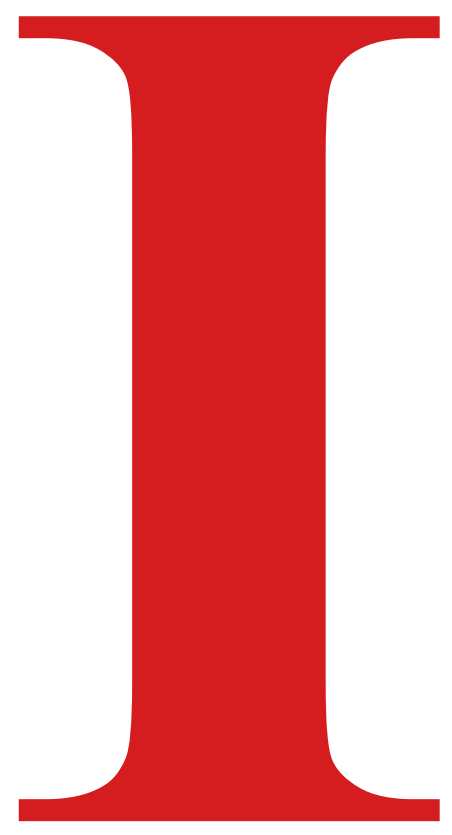




\section{Part I}

\section{ABLATION OF ATRIAL FIBRILLATION AND ATRIAL TACHYCARDIA}

Part IC

Optimizing left atrial linear ablation 


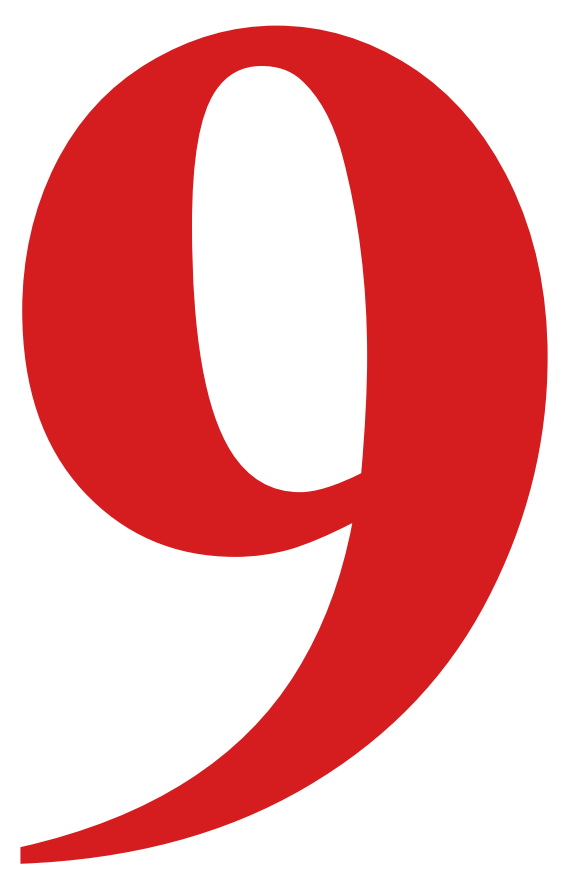




\section{Chapter 9}

\section{Contiguous and optimized radiofrequency applications for linear lesions.}

Michael Wolf, Milad El Haddad, Joël Fedidia, Philippe Taghji, Katarina Van Beeumen, Teresa Strisciuglio, Jan De Pooter, Caroline Lepièce, Yves Vandekerckhove, René Tavernier, Mattias Duytschaever, Sébastien Knecht 


\begin{abstract}
Aims: Achieving block across linear lesions is challenging. We prospectively evaluated radiofrequency (RF) linear ablation at the roof and mitral isthmus (MI) using point-by-point contiguous and optimized RF lesions.

Methods: 41 consecutive patients with symptomatic persistent AF underwent stepwise CF-guided catheter ablation during ongoing AF. A single linear set of RF lesions was delivered at the roof and posterior MI according to the 'ALINE'-criteria, i.e. point-by-point RF delivery (up to $35 \mathrm{~W}$ ) respecting strict criteria of contiguity (inter-lesion distance $\leq 6 \mathrm{~mm}$ ) and indirect lesion depth assessment (Ablation Index $\geq 550$ ). We assessed incidence of bidirectional block across both lines only after restoration of sinus rhythm.
\end{abstract}

Results: After a median RF time of 7min [IQR 5-9], first-pass block across roof lines was observed in $38 / 41$ patients (93\%). Final bidirectional roof block was achieved in 40/41 patients (98\%). First-pass block was observed in 8/35 MI lines (23\%), after a median RF time of 8min [IQR 7-12]. Additional endo- and epicardial (54\% of patients) RF applications resulted in final bidirectional MI block in 28/35 patients (80\%). During a 396-day [IQR 310-442] median follow-up, 12 patients underwent repeat procedures, with conduction recovery in $4 / 12$ and $5 / 10$ previously blocked roof lines and MI lines respectively. No complications occurred.

\title{
Conclusion:
}

Anatomical linear ablation using contiguous and optimized RF lesions results in a high rate of firstpass block at the roof, but not at the MI. Due to its complex 3D architecture, the MI frequently requires additional endo- and epicardial lesions in order to be blocked. 


\section{Introduction}

Left atrial (LA) linear lesions are one of the main techniques described for ablation of persistent atrial fibrillation (AF) and are also frequently performed for left atrial macroreentrant tachycardia. However, performing linear ablation remains challenging. Indeed, bidirectional block across LA lines should be the ablation endpoint (HRS/EHRA/ECAS recommendation ${ }^{1}$ ) but this is achieved in a limited amount of patients ${ }^{2-6}$ and incomplete electrical block can have pro-arrhythmic consequences and cause atrial tachycardia recurrence..$^{3-4}$ On the other hand, increased success in completing linear block is often associated with an increased complication rate from higher ablation power and a higher risk of steam pop, perforation, and tamponade. ${ }^{7}$

New studies have recently introduced a new ablation approach of anatomical point-by-point radiofrequency (RF) ablation based upon indirect evaluation of lesion depth (estimated by a specific formula i.e. "Force-Power-Time Index or Ablation Index") and delivery of contiguous RF lesions. ${ }^{8-9}$ During pulmonary vein isolation (PVI), it has been showed that acute and late pulmonary vein (PV) reconnection resulted from an insufficient Ablation Index ( $<550$ anteriorly and $<400$ posteriorly) and/or from a too long interlesion distance $(>6 \mathrm{~mm}){ }^{8}$

However, these criteria on minimal Ablation Index and maximal interlesion distance have never been evaluated and validated for linear lesions yet. Therefore, we sought to assess the efficacy of performing point-by-point left Atrial LINEar RF lesions (the ALINE study) during AF at the roof and at the mitral isthmus (MI) only using criteria for contiguity (interlesion distance $\leq 6 \mathrm{~mm}$ ) and Ablation Index ( $\geq 550)$, i.e. the ALINE criteria.

\section{Methods}

\section{Patient population}

From October 2015 to May 2016, all patients undergoing ablation for drug-resistant symptomatic persistent AF were prospectively included in the study if a roof line and/or a MI line was required. LA lines were always performed during ongoing AF to avoid bias from electrogram interpretation, and sinus rhythm or atrial tachycardia were exclusion criteria. Patients with previous linear or complex fractionated atrial electrogram ablation in the LA were also excluded. All patients were followed in a prospective database approved by the local institutional review committee. This database implies collecting patients' informed consent, a detailed case report form of the procedure, and follow-up at 1 , 3,6 and 12 months.

\section{Procedure}

All procedures were performed by 4 different operators, under general anesthesia or conscious sedation, and under novel anticoagulants (last dose taken 24 hours before procedure) or uninterrupted warfarin. Antiarrhythmic drugs, with the exception of amiodarone (6 patients), were withdrawn 24 hours before the procedure. No antiarrhythmic medication was administered during the procedure. In 
patients under general anesthesia, an esophageal temperature monitoring probe (SensiTherm ${ }^{\mathrm{TM}}$, St Jude Medical Inc, Minnesota US) was placed at the discretion of the operator. Intravenous heparin was administered after femoral vein access to achieve an activated clotting time $>300 \mathrm{~s}$. A decapolar coronary sinus (CS) catheter and quadripolar His catheter were introduced via the right femoral vein and double transseptal puncture was performed with conventional long sheaths (SL0, St. Jude Medical Inc). A decapolar circular mapping catheter (Lasso ${ }^{\circledR}$, Biosense-Webster Inc, Diamond Bar, CA, USA) and an $8 \mathrm{~F}$ open-tip irrigated RF catheter with tip-integrated CF sensor (Thermocool SmartTouch ${ }^{\circledR}$, Biosense-Webster Inc) were positioned in the LA. Then calibration of CF catheter, respiratory gating and 3D-geometry of the LA (Carto System ${ }^{\circledR}$, Biosense Webster Inc) were performed. Patients in persistent AF undergoing a first catheter ablation procedure underwent PVI first. In patients undergoing a repeat ablation procedure, electrical isolation of the PVs was checked with the Lasso catheter and electrical re-isolation of the PVs was performed if reconnection had occurred. If AF persisted after PVI, LA linear ablation at the roof and MI was subsequently performed.

\section{Protocol for roof and mitral lines using the ALINE criteria}

After confirmation of electrical PVI, a single linear lesion at the roof and subsequently the MI was performed during ongoing AF respecting strict criteria for contiguity (interlesion distance $\leq 6 \mathrm{~mm}$ ) and Ablation Index $(\geq 550)$. MI lines were only performed if AF continued after PVI and roof line, and if the persistent AF duration was $>1$ month (operator's preference). Roof lines were carried out at the shortest distance between the superior margins of right and left superior PVs as cranial as possible. The MI line was drawn at the shortest distance between the posterolateral mitral annulus (A/V ratio of 1:1) and the left inferior PV ostium. ALINE-guided ablation consisted of point-by-point RF delivery (EP Shuttle ST-3077, Stockert GmbH, Freiburg, Germany) in a power-controlled mode (without ramping) with a power setting of $35 \mathrm{~W}$ (adapted based on catheter tip temperature feedback) and with $30 \mathrm{~mL} / \mathrm{min}$ irrigation. Applications targeted if possible a contact force (CF) between 10-30g with stable CF curve during RF delivery with support of a long non-steerable sheath. At each point, energy was delivered in order to reach an Ablation Index (Hiroshi formula ${ }^{10-11}$, Biosense Webster Inc) of $\geq 550$. No applications were started with a $\mathrm{CF}$ value $<5 \mathrm{~g}$. RF applications were depicted on the anatomical map with automated tagging technology (Carto VisiTag ${ }^{\mathrm{TM}}$, Biosense Webster Inc). Attempts were made to create a single line of VisiTag applications with a maximum interlesion distance $\leq 6 \mathrm{~mm}$ (center-to-center). In case of instability leading to an inadequate RF tag (not reaching the Ablation Index target), the lesion was discarded and a new RF tag reaching the Ablation Index target was applied in order to obtain a contiguous linear ablation set of applications with Ablation Index $\geq 550$ (Figure 1). In case of chest pain or significant intra-esophageal temperature rise $\left(>38.5^{\circ} \mathrm{C}\right)$ RF delivery was ceased, and no other application with Ablation Index $\geq 550$ was attempted at this spot. In some patients, additional substrate ablation was performed, but delivery close to the roof or MI 
lines or in the CS was not allowed on or close to the lines which were previously drawn and if necessary, electrical cardioversion was carried out.

After conversion to sinus rhythm (by ablation or electrical cardioversion), first-pass bidirectional block across roof and MI line was assessed by conventional methods consisting of both differential pacing maneuvers and documenting widely separated double potentials across the entire length of the line. ${ }^{2-7}$ In patients without documentation of double potentials despite of bidirectional block established by differential pacing, high-density activation mapping was additionally performed. ${ }^{2-7}$ Complete block of mitral and roof lines were confirmed by observing the reversal of the expected activation sequence at one side of the line while pacing from the other side as follows: while pacing from LAA, activation of the LA posterior wall ascends with a complete roof line (instead of descending with an incomplete line $)^{6}$ and the CS sequence is activated proximal to distal with a complete mitral line (instead of distal to proximal if incomplete). ${ }^{2,3}$ Differential pacing was then also systematically used to exclude slow conduction on both lines. ${ }^{2}$

In sinus rhythm, if block was not achieved after a single linear lesion, additional applications were made by mapping the earliest activity during pacing from the contralateral part of the line and until bidirectional block was achieved. For the posterior MI, both additional RF ablation at the endocardium and in the CS (with a power setting of 15-25 Watts, 30ml/min irrigation, CF 10-30g, Ablation Index $\geq 400$ ) were performed. A maximum total duration of RF application of 30min was allowed, after which failure of block was accepted. Bidirectional conduction block was re-assessed $\geq 20 \mathrm{~min}$ after the last ablation lesion along the line.

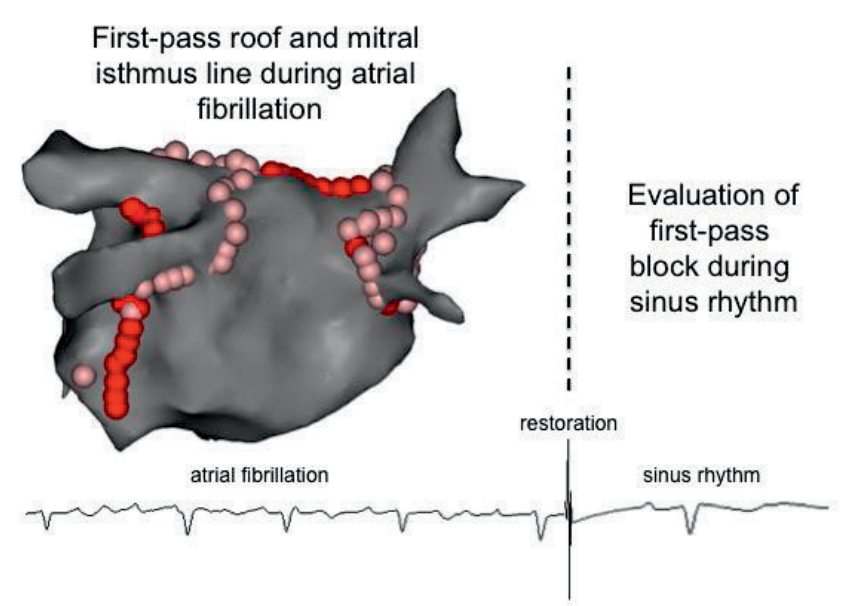

Figure 1: 'ALINE'-guided first-pass ablation of roof and posterior mitral isthmus line during ongoing AF. Attempts were made to create a single anatomical line of contiguous VisiTag applications with a maximum interlesion distance $\leq 6 \mathrm{~mm}$ (center-to-center) and Ablation Index (AI) $\geq 550$ (red Visitags). After restoration of sinus rhythm, first-pass block was assessed. 


\section{Off-line analysis of RF characteristics of roof and MI lines}

Each procedure was exported and analyzed offline. For each RF tag at the roof and MI, we determined time of application (s), median delivered power (W), impedance drop ( $\Delta$-Imp, $\Omega$ ), average contact force (CF, g), force-time integral (FTI, gs) and Ablation Index (AI, no unit). In addition, custom-made software was used to automatically determine the interlesion distance in between neighboring ablation tags (center-to-center distance, $\mathrm{mm}$ ) and the ablation line contiguity index (ALCI). The ALCI has been previously evaluated during PVI and has been shown to be the most accurate predictor for durable RF segments. ${ }^{8}$ The ALCI algorithmically combines interlesion distance and Ablation Index into one single criterion, and is defined as the ratio of the mean Ablation Index of 2 neighboring RF lesions (multiplied by a 1.5 conversion factor referring to the thickness of the tissue) over their interlesion distance. ${ }^{8}$ The length of the line (as measured on the $3 \mathrm{D}$ navigation system) was also measured.

\section{Post-procedural care and follow-up.}

Clinical evaluation and ECG were performed at 1, 3,6 and 12 months or in case of symptoms. Holter recordings were performed at 6 and 12 months. Recurrence of AF and atrial tachycardia ( $\geq 30 \mathrm{~s}$ ) was evaluated considering a blanking period of 8 weeks. Repeat procedures were performed based on caseby-case evaluation, but repeat ablation was preferred in patients with atrial tachycardia recurrence.

\section{Statistical analysis}

Continuous variables are expressed as mean $\pm \mathrm{SD}$ (or medians with interquartile range (IQR, 25th75th percentile) for non-normal distributions) and dichotomous variables as count and percentage. Normality of data distribution was tested with the Shapiro-Wilk test. Parametric (Student's t-test) or non-parametric tests (Mann-Whitney $U$ ) were used when appropriate to evaluate differences in continuous clinical and ablation parameters between groups with bidirectional block vs. remaining conduction after first-pass RF delivery at the roof and MI respectively. Categorical variables were compared with the $\chi^{2}$ test or Fisher's exact test when appropriate. Univariable and multivariable predictors were examined using logistic regression analysis. Variables with a univariable p-value of 0.1 were included in the multivariable model. All reported p-values were 2-sided, and a p-value of 0.05 was considered significant. All statistical analyses were performed in SPSS Statistics 24 (IBM Corporation, Armonk, New York, USA).

\section{Results}

\section{Patient population and procedural characteristics.}

The study population included 41 consecutive patients (41 patients with a roof line and 35 with a MI line) according to the pre-established inclusion criteria. The mean age of the study population was $65 \pm 9$ years, and $68 \%$ of patients were male. The mean left atrial volume was $130 \pm 37 \mathrm{~mL}$. Further 
patient characteristics are summarized in Table 1. Procedures were performed under general anesthesia in 30 patients (73\%). An esophageal temperature probe was used in 19 patients $(46 \%)$. There were no peri-procedural complications.

Table 1: Patient and procedural characteristics

\begin{tabular}{|c|c|}
\hline \multicolumn{2}{|l|}{ Patient characteristics } \\
\hline Total number & 41 \\
\hline Age, y & $64.8 \pm 8.5$ \\
\hline Male gender, n (\%) & $28(68)$ \\
\hline BMI, $\mathrm{kg} / \mathrm{m}^{2}$ & $27.7 \pm 5.0$ \\
\hline $\mathrm{CHA}_{2} \mathrm{DS}_{2} \mathrm{VASc}$-score & $1.6 \pm 1.4$ \\
\hline Left atrial volume, mL (Carto System $\left.{ }^{\circledR}\right)$ & $129.6 \pm 37.4$ \\
\hline Left atrial diameter, mm (ultrasound) & $44.1 \pm 6.1$ \\
\hline \multicolumn{2}{|l|}{ Atrial fibrillation } \\
\hline persistent $(<12 \mathrm{mo}), \mathrm{n}(\%)$ & $32(78)$ \\
\hline long standing persistent (>12 mo), n (\%) & $9(22)$ \\
\hline atrial fibrillation cycle length, $\mathrm{ms}$ & $172 \pm 24$ \\
\hline Prior pulmonary vein isolation, $\mathrm{n}(\%)$ & $8(20)$ \\
\hline \multicolumn{2}{|l|}{ Procedural characteristics } \\
\hline General anesthesia, $\mathrm{n}(\%)$ & $30(73)$ \\
\hline Esophageal temperature probe, n (\%) & $19(46)$ \\
\hline \multicolumn{2}{|l|}{$\begin{array}{l}\text { Pulmonary vein anatomy } \\
\text { Plat }\end{array}$} \\
\hline 4 pulmonary veins, $\mathrm{n}(\%)$ & $39(95)$ \\
\hline LCPV, n $(\%)$ & $2(5)$ \\
\hline
\end{tabular}

\section{Roof line using ALINE criteria}

A roof line was performed in 41 patients. The mean length of the lines was $39 \pm 12 \mathrm{~mm}$. The median number of RF applications to create a first-pass blocked roof line was 11 [IQR 8 - 13] with a median RF time of $7 \min$ [IQR 5 - 9]. The median number of dislocations before reaching the Ablation Index target was 0 [IQR $0-1]$ per patient. First-pass bidirectional block was observed in 38 of the 41 roof lines (93\%) (Figure 2). Additional RF (5 - 16 applications, with $2-7$ min duration) resulted in block across 2 of the 3 remaining unblocked roofs. Conduction gaps were located at the middle of the roof in one patient, and near the right PVs in the second patient. Final bidirectional block was achieved in 40 of 41 patients (98\%) (Figure 2) and was still present in all patients after a waiting period of $\geq 20 \mathrm{~min}$. In one patient, RF delivery was ceased before reaching the target Ablation Index due to an esophageal temperature rise $>38.5^{\circ} \mathrm{C}$.

Comparison of clinical and RF lesion characteristics between lines with bidirectional block vs. conduction after first-pass RF are summarized in Table 2 and Figure 3A. Increased length of the roof 
was a significant predictor of failure of first-pass roof line block in univariable (OR 0.81, 95\% CI 0.66 -0.99 , p-value 0.044$)$, but not in multivariable analysis. No clinical or procedural parameters differed between both groups (Table 2). The characteristics of the $\geq 550$ Ablation Index tags (RF time, delivered power, impedance drop, average CF, FTI, AI, interlesion distance and ALCI) did not differ between first-pass blocked and unblocked roof lines (Figure 3A).

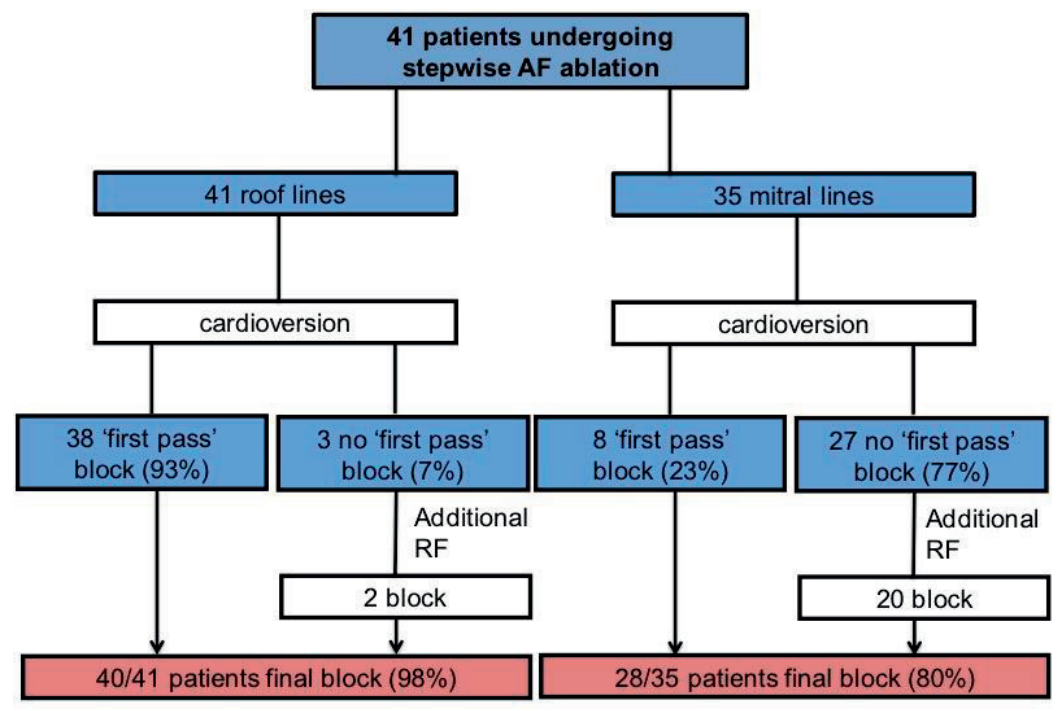

Figure 2: Results of first-pass and final roof and mitral isthmus block.

\section{Mitral isthmus lines using ALINE criteria}

Posterior MI lines were performed in 35 patients. The mean length of the MI line was $39 \pm 13 \mathrm{~mm}$. The median number of RF applications to create a first-pass MI line was 12 [IQR 9 - 14] with a median RF time of $8 \mathrm{~min}$ [IQR 7 - 12]. The median number of dislocations prior to reaching the Ablation Index target was 0 [IQR $0-2$ ] per patient. An example of a case is illustrated in Figure 4. First-pass bidirectional block was observed in only 8 of 35 MI lines (23\%) (Figure 2). Preferential conduction gaps after first-pass RF are shown in Figure 5. In 11 patients no clear preferential gap could be determined and more extensive additional endo- and epicardial ablation was needed in order to obtain block. Additional RF at the epicardial/CS side (median of 7 applications [IQR 5 - 14], during 4min [IQR 3 - 7]) in 19 patients, and at the endocardial side (median of 8 applications [IQR 6 - 15], during $6 \mathrm{~min}$ [IQR $4-9]$ ) in 14 patients resulted in block of 20 out of 27 remaining unblocked MI lines. Final bidirectional MI block was achieved in 28 of 35 patients (80\%), after a median total RF duration of $15 \mathrm{~min}[10-20]$ and was still present in all patients after a waiting period of $\geq 20 \mathrm{~min}$. In two patients, 
cannulation of the distal CS was not feasible. An esophageal temperature rise $>38.5^{\circ} \mathrm{C}$ (or pain) was never observed during applications along the posterior MI.

Comparison of clinical and RF lesion characteristics between lines with bidirectional block vs. conduction after first-pass RF are summarized in Table 2 and Figure 3B. LA volume was statistically significant in univariable (OR 1.04, 95\% CI 1.00-1.07, p-value 0.033), but not in multivariable analysis. No clinical or procedural parameters differed between both groups (Table 2). No difference was observed in the characteristics of the $\geq 550$ Ablation Index tags between first-pass blocked and unblocked MI lines (Figure 3B). 


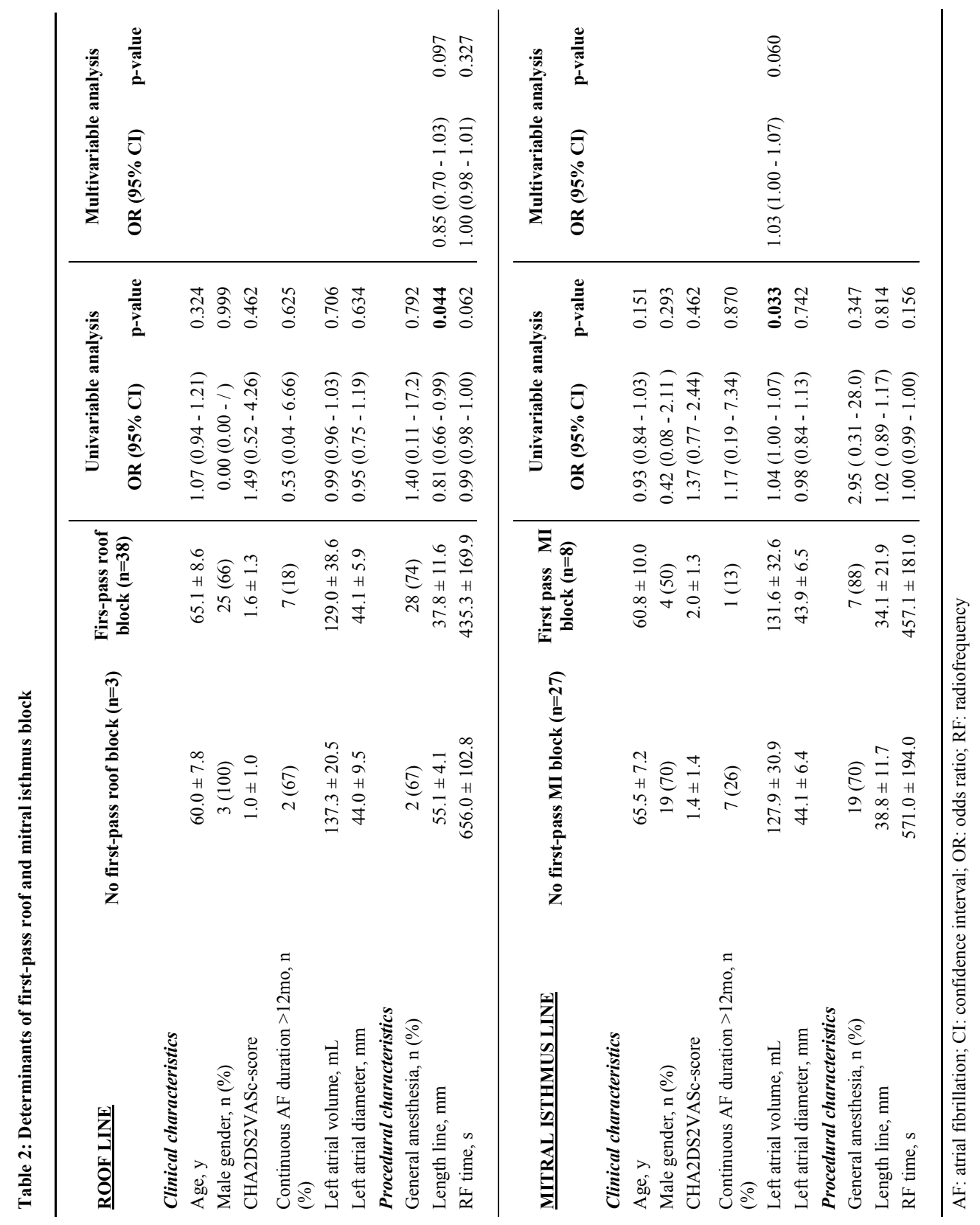


A
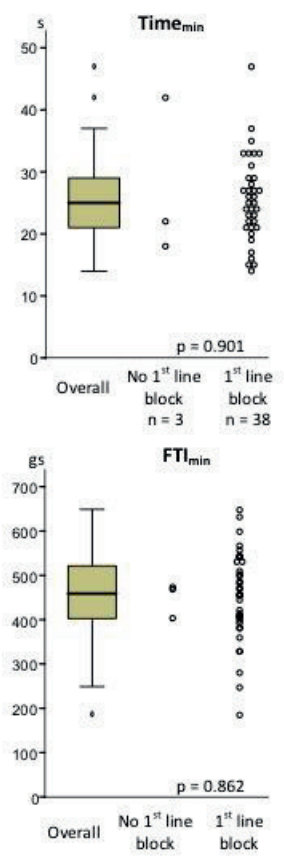

B
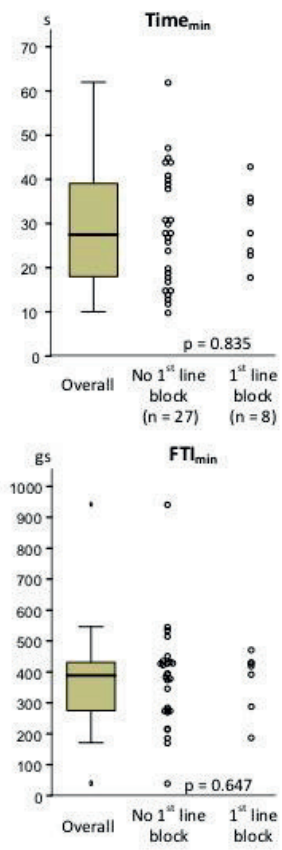
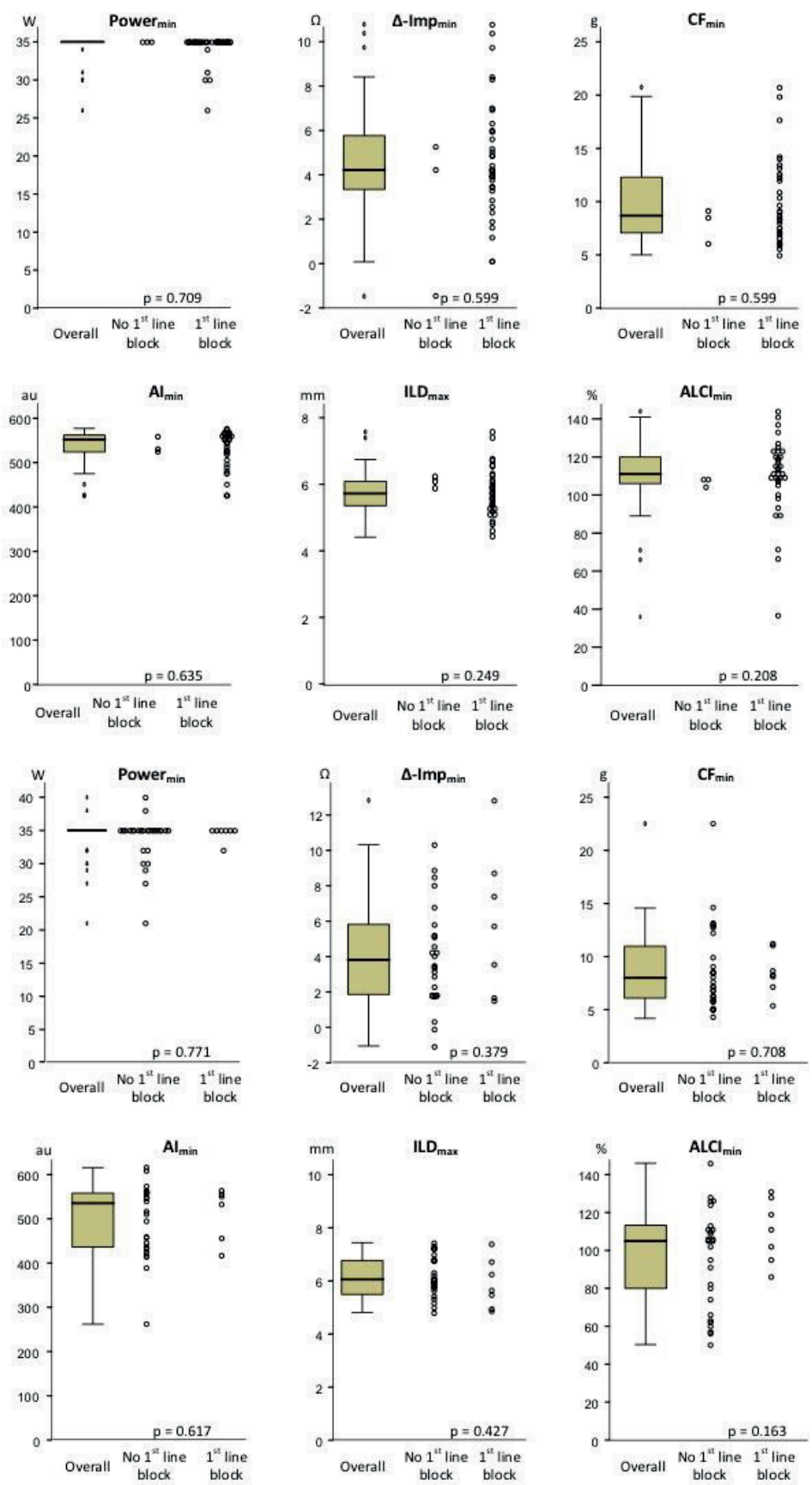

Figure 3: Comparison between lines with vs. without first-pass conduction block at the roof (A) and mitral isthmus (B) concerning the minimal value from all effective RF lesions for the following parameters: application time $\left(\right.$ Time $\left._{\min }\right)$, power $\left(\right.$ Power $\left._{\min }\right)$, impedance drop $\left(\Delta-\mathrm{Imp}_{\min }\right)$, contact force $\left(\mathrm{CF}_{\mathrm{min}}\right)$, force-time integral $\left(\mathrm{FTI}_{\mathrm{min}}\right)$, Ablation Index $\left(\mathrm{AI}_{\min }\right)$ and ablation index contiguity index $\left(\mathrm{ALCI}_{\mathrm{min}}\right)$, and the maximal value of interlesion distance $\left(\mathrm{ILD}_{\max }\right.$ ) . RF points with dislocation during application were excluded from the analysis. 

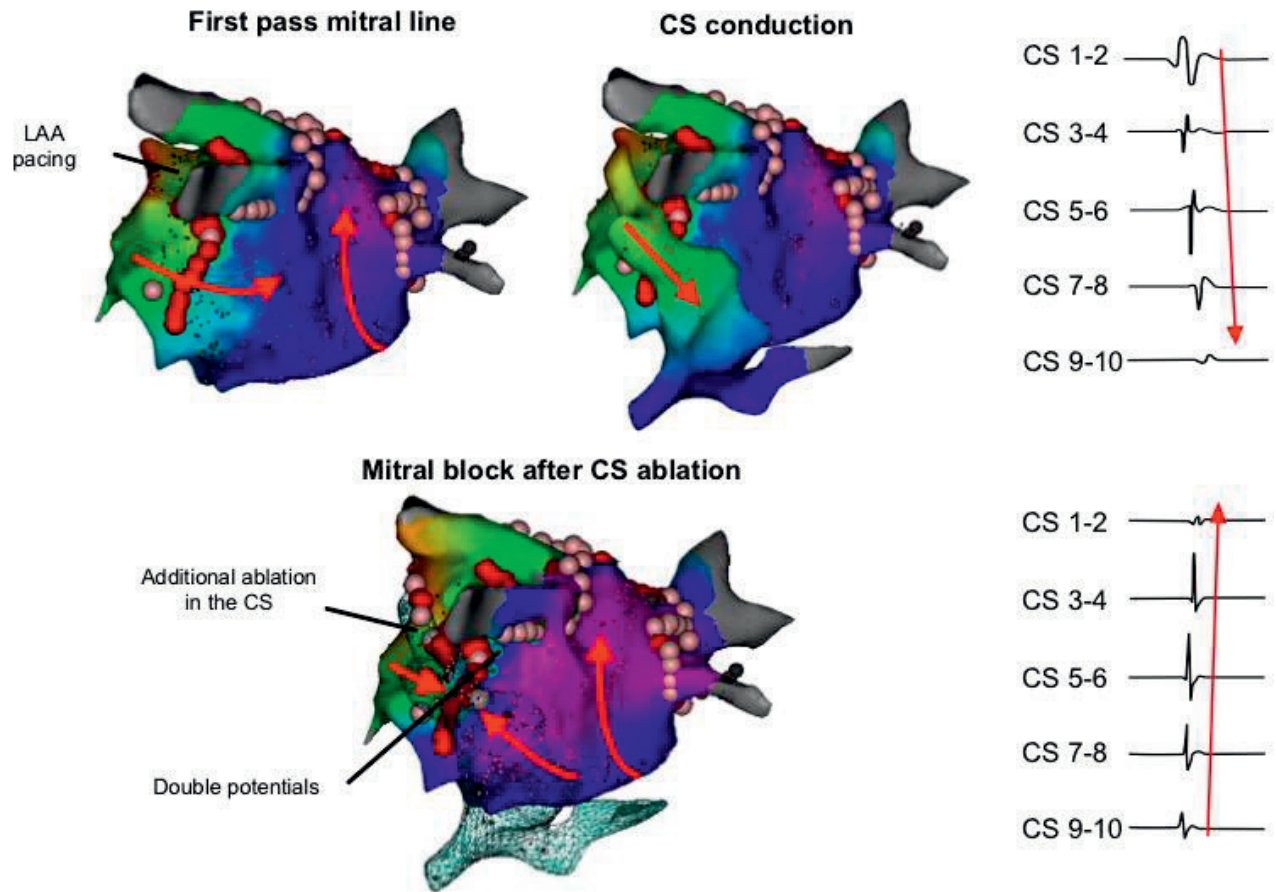

Figure 4: Upper panels: Evaluation of conduction block after first-pass roof and posterior mitral isthmus (MI) line. High density activation map during left atrial appendage (LAA) pacing shows propagation across the MI (arrows), with probable epicardial conduction through the coronary sinus (CS). Distal-to-proximal CS activation is concordant with MI conduction. The roof was blocked after first-pass RF delivery.

Lower panels: After additional RF applications in the CS, MI block was obtained, evidenced by pattern of activation (arrows), presence of widely-spaced double potentials across the MI line and proximal-to-distal activation along the CS catheter.

\section{Follow-up}

During a median follow up of 396 days [IQR 310 - 442], 17/41 patients (41\%) experienced atrial arrhythmia recurrence (14 patients with AT, 3 with AF) with 22 patients (56\%) continuing antiarrhythmic drugs (10 pts class Ic, 5 pts sotalol and 7 pts amiodarone). There was no difference in achievement of final block across roof and MI lines between patients with and without atrial arrhythmia recurrence $(19 / 24(79 \%)$ vs. $14 / 17$ (82\%) pts, $p=1.000)$. Repeat ablation procedures with assessment of persistent conduction block across roof and MI were performed at $7 \pm 4$ months after the index procedure in 12 of 14 patients with atrial tachycardia recurrence.

\section{Repeat evaluation of roof lines}

Of the 12 evaluated roof lines, all had been blocked after first-pass RF delivery during the index procedure. There was persistent block in $8 / 12$ and there was re-conduction in 4/12 (33\%). Conduction 
gaps were small, located at the middle of the roof or at the transition between the middle towards the right PVs (Figure 5) and were responsible for roof-dependent macroreentry in 3 of the 4 patients with re-conducting roof lines. Re-ablation using the ALINE criteria resulted in block in all patients after a short duration of application (1.6 $\pm 0.6 \mathrm{~min})$.

\section{Repeat evaluation of mitral isthmus lines}

Of the 12 re-evaluated MI lines, 2 were not blocked during the index procedure and were still conducting. Of the remaining 10 evaluated lines (with block during the index procedure of which 2 with first-pass block), 5 MI lines showed persistent block while 5 had re-conduction (Figure 6). Conduction gaps were short and located in the mid-isthmus region (4 patients) and in the CS (1 patient). Block was re-achieved after endocardial ablation only in 4 patients; in one patient, additional applications in the distal CS were required (Figure 5). Six of the 7 patients with conduction across the MI at re-evaluation presented with a procedural perimitral atrial tachycardia. All re-ablation using the ALINE criteria resulted in block after short application duration (3.1 $\pm 0.9 \mathrm{~min})$.

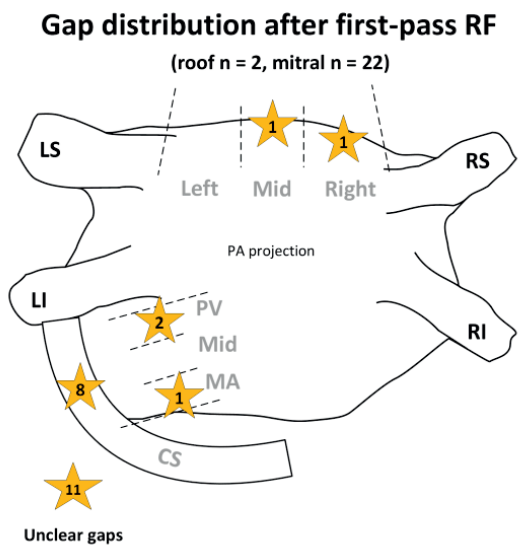

\section{Gap distribution during repeat procedures}

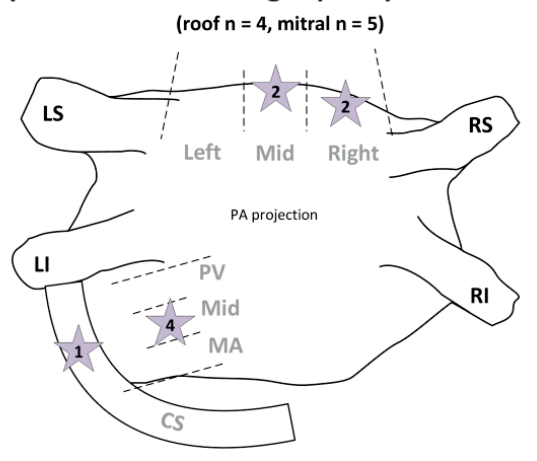

Unclear gaps

Figure 5: Distribution of conduction gaps on the roof and mitral isthmus (MI) after first-pass radiofrequency lesions respecting criteria of contiguity and lesion depth (shown for patients with final block) (left panel). Distribution of conduction gaps at the roof and MI in patients with recovered conduction during repeat ablation procedures (right panel).

\section{Discussion}

\section{Main findings}

Our study shows that performing LA linear lesions with contiguous and optimized RF lesions results in a very high rate of first-pass bidirectional block at the roof but not at the mitral isthmus line. During repeat procedures, limited RF delivery was necessary to achieve conduction block and was successful in all re-ablated patients for both lines. 


\section{Additional benefit of both contiguous and optimized RF lesions}

In studies on RF ablation in the canine heart, Nakagawa et al found that the RF lesion depth was accurately $( \pm 1 \mathrm{~mm})$ described by a logarithmic function of CF, RF power and application time (i.e. the Force-Power-Time Index or Ablation Index). ${ }^{10-11}$ In a recent retrospective analysis, our research group emphasized that in CF-guided PVI, both indirect lesion depth assessment (as measured via Ablation Index) and lesion contiguity are essential to obtain a durable ablation lesion. El Haddad et al demonstrated that for durable PVI, the maximum interlesion distance should not exceed $6 \mathrm{~mm}$ and the minimal Ablation Index should be $\geq 400$ at the posterior and $\geq 550$ at the anterior wall, reflecting the difference in wall thickness between the anterior and posterior PV junction. ${ }^{8}$ Additionally in a prospective cohort study, an ablation strategy respecting contiguous and optimized RF lesions resulted in a very high rate of first-pass and adenosine-proof PV isolation and a single-procedure arrhythmiafree survival of $92 \%$ at 1 -year, without any complications. ${ }^{9}$ Based upon these findings, we hypothesized that linear ablation respecting strict criteria of contiguity and indirect lesion depth assessment based on the Ablation Index would also improve achievement of both acute and durable block across LA linear lesions.

\section{Conduction block with left atrial linear ablation: technically challenging but essential}

Roof and MI lines are the most commonly performed LA linear lesions. Even though achieving bidirectional conduction block is challenging ${ }^{2-6}$ (reported block across roof lines of 81 to $95 \% \%^{3,6,12}$ and for the MI line between 57 to $92 \%^{2-4,13-14}$ ), it is well known that incomplete bidirectional conduction block has been associated with an increase in subsequent arrhythmia during follow-up. ${ }^{3-4}$

In our study, even respecting the criteria for contiguity and Ablation Index, we achieved complete block in the roof line in $93 \%$ of patients after a single linear lesion set, but only in $23 \%$ at the MI line (without CS ablation) confirming this difference for both lines. The very high success rate of first-pass block on the roof in our study can probably be explained by the favorable and uniform anatomy of the roof, which renders the roof quite suitable for a strategy aiming at transmurality and contiguity of RF lesions. This was emphasized by the low number of dislocations before reaching Ablation Index target value. In the case of absence of block after the first-pass roof line and during the redo procedures, all conduction gaps were located in the middle of the roof or near the right PVs (never at the left side of the roof). These locations are characterized by lower stability during RF delivery and also by thicker tissue with interatrial muscular bridges. ${ }^{15}$

Conversely, the success rate for achieving bidirectional conduction block after a first pass linear lesion at the MI was low. This can probably be explained by the anatomical characteristics of the MI region. Post mortem anatomical and in vivo studies have shown that the atrial myocardium at the left mitral line has a wall thickness of up to $10 \mathrm{~mm}$ at the ostium of the left inferior PV. ${ }^{16}$ In comparison, the LA roof is thinner with a range between 3.5 and $6 \mathrm{~mm} .{ }^{15}$ The distance between the endocardium and the overlying inner surface of the CS is even larger than the MI thickness, due to the adipose tissue overlaying the CS along the mitral valve, sometimes with interlaying circumflex artery branches. ${ }^{13,17}$ 
Numerous recesses and cavities along the left mitral isthmus have also been reported ${ }^{18}$ as well as epicardial muscular connections necessitating ablation within the CS in the majority of patients. ${ }^{2,17-18}$ Blood flow in the CS and circumflex artery may also act as a 'heat sink' and reduce the efficacy of radiofrequency ablation. ${ }^{17}$ Length of the MI line, increased MI thickness and interposition of the circumflex artery between the CS and the MI were independent predictors of incomplete MI block in previous studies ${ }^{13-14}$ and the need for epicardial CS ablation in order to obtain MI block has been reported in 60 to $84 \%$ of cases $^{2,13-14}$ (as compared to $54 \%$ in our study).

Recovery of conduction has been reported to occur in up to $90 \%$ of MI line and $79 \%$ of the roof lines during repeat evaluation for atrial arrhythmia recurrence ${ }^{19,20}$. In our study, $50 \%$ of the MI lines and $33 \%$ of the roof lines that were blocked at the end of the index procedure had recovered conduction. Interestingly, among the 8 patients with first-pass MI block in the index procedure, 2 of them had a redo procedure and reevaluation of the MI line showed persistent complete conduction block. The other 6 patients did not have atrial arrhythmia recurrence. Even if the number of patients is really low, these findings might suggest that if first-pass MI block is achieved, the probability of durable MI block is higher, possibly due to favorable patient's MI anatomy.

\section{Limitations}

(1) The criteria for Ablation Index $(\geq 550)$ and interlesion distance $(\leq 6 \mathrm{~mm})$ were arbitrarily applied for both lines, even if this may oversimplify the complex anatomy of the roof and MI region. These values were selected based on previous studies ${ }^{8-9}$ and local experience, to minimize the risk of complication and to minimize the risk of dislocation (during longer RF applications) which could have impacted the interpretation of the data. In the future, higher Ablation Index or shorter interlesion distance should be evaluated for the MI line given the low amount of first pass block. (2) Pre-evaluation with imaging technologies might also improve the results by allowing adaptation of the criteria depending on individual anatomical variations and helping avoid RF under- and overshooting. (3) The Ablation Index has been shown to be an accurate marker for lesion depth, but we did not measure the actual lesion depth with imaging or histology. (4) Although no safety-related issues were observed, larger studies are necessary to evaluate the safety and efficacy of this protocol for linear LA lesions. (5) Because evaluation of persistent roof and MI line block was only performed in a limited number of patients who presented with atrial tachycardia recurrence, the rate of conduction recurrence might be underestimated. (6) Previous ablation for PVI may impact the atrial tissue at the roof and MI differently if ostial or antral ablation is performed. However, in all study patients, PVI was performed close to the PV junction. ${ }^{9}$ (7) The use of a steerable sheath might have improved the results mainly by increasing optimizing contact, catheter stability and by decreasing catheter dislocation ${ }^{4}$; however this happened only sporadically in our study. 


\section{Conclusion}

Anatomical ablation using contiguous and optimized RF lesions is very effective for the roof line but not for the mitral isthmus line. The complex 3D architecture of the mitral isthmus region frequently necessitates epicardial and additional endocardial RF lesions in order to obtain block. 


\section{References}

1. Calkins H, Kuck KH, Cappato R, Brugada J, Camm AJ, Chen SA, et al. 2012 HRS/EHRA/ECAS Expert Consensus Statement on Catheter and Surgical Ablation of Atrial Fibrillation: Recommendations for patient selection, procedural techniques, patient management and follow-up, definitions, endpoints, and research trial design. Europace 2012;14:528-606.

2. Jais P, Hocini M, Hsu LF, Sanders P, Scavee C, Weerasooriya R, et al. Technique and results of linear ablation at the mitral isthmus. Circulation 2004;110:2996-3002.

3. Knecht S, Hocini M, Wright M, Lellouche N, O’Neill MD, Matsuo S, et al. Left atrial linear lesions are required for successful treatment of persistent atrial fibrillation. Eur Heart J 2008;29:2359-66.

4. Matsuo S, Yamane T, Date T, Hioki M, Narui R, Ito K, et al. Completion of mitral isthmus ablation using a steerable sheath: Prospective randomized comparison with a nonsteerable sheath. $J$ Cardiovasc Electrophysiol 2011;22:1331-8.

5. Miyazaki S, Shah AJ, Liu X, Jadidi AS, Nault I, Wright M, et al. Preprocedural clinical parameters determining perimitral conduction time during mitral isthmus line ablation. Circ Arrhythm Electrophysiol 2011;4:287-94.

6. Arbelo E, Guiu E, Ramos P, Bisbal F, Borras R, Andreu D, et al. Benefit of left atrial roof linear ablation in paroxysmal atrial fibrillation: a prospective, randomized study. $J$ Am Heart Assoc 2014;3:e00877.

7. Wong KC, Betts TR. A review of mitral isthmus ablation. Indian Pacing Electrophysiol $J$ 2012;12:152-70.

8. El Haddad M, Taghji P, Phlips T, Wolf M, Demolder A, Choudhury R, et al. Determinants of acute and late pulmonary vein reconnection in contact force-guided pulmonary vein isolation: Identifying the weakest link in the ablation chain. Circ Arrhythm Electrophysiol 2017;10(4). pii: e004867.

9. Taghji P, El Haddad M, Phlips T, Wolf M, Knecht S, Vandekerckhove Y, et al. Evaluation of a strategy aiming to enclose the pulmonary veins with contiguous 2 and optimized RF lesions in paroxysmal atrial fibrillation: a pilot study. J Am Coll Cardiol EP 2017; (in press).

10. Nakagawa H, Jackman WM. The Role Of Contact Force In Atrial Fibrillation Ablation. J Atr Fibrillation 2014;7:1027.

11. Nakagawa H, Ikeda A, Govari A, Thanassis $P$, Constantine G, Bar-Tal M, et al. Prospective study to test the ability to create RF lesions at predicted depths of 3, 5, 7 and $9 \mathrm{~mm}$ using formula incorporating contact force, radiofrequency power and application time (force-power-time index) in the beating canine heart. Heart Rhythm 2013; 10:S481.

12. Wong KC, Sadarmin PP, De Bono J, Qureshi N, Jones M, Rajappan K, et al. Local activation times at the high posterior wall of the left atrium during left atrial appendage pacing predict roof line block with high specificity and sensitivity. Europace 2011;13:1243-9.

13. Latcu DG, Squara F, Massaad Y, Bun SS, Saoudi N, Marchlinski FE. Electroanatomic characteristics of the mitral isthmus associated with successful mitral isthmus ablation. Europace 2016;18:274-80.

14. Scherr D, Derval N, Sohal M, Pascale P, Wright M, Jadidi A, et al. Length of the Mitral Isthmus But Not Anatomical Location of Ablation Line Predicts Bidirectional Mitral Isthmus Block in Patients Undergoing Catheter Ablation of Persistent Atrial Fibrillation: A Randomized Controlled Trial. $J$ Cardiovasc Electrophysiol 2015;26:629-34.

15. Ho SY, Sanchez-Quintana D, Cabrera JA, Anderson RH. Anatomy of the left atrium: implications for radiofrequency ablation or atrial fibrillation. J Cardiovasc Electrophysiol 1999;10:1525-33.

16. Becker AE. Left atrial isthmus: anatomic aspects relevant for linear catheter ablation procedures in humans. J Cardiovasc Electrophysiol 2004;15:809-12.

17. Wittkampf FH, van Oosterhout MF, Loh P, Derksen R, Vonken EJ, Slootweg PJ, et al. Where to draw the mitral isthmus line in catheter ablation of atrial fibrillation: histological analysis. Eur Heart $J$ 2005;26:689-95.

18. Fassini G, Riva S, Chiodelli R, Trevisi N, Berti M, Carbucicchio C, et al. Long-term results of a 
prospective randomized study. J Cardiovasc Electrophysiol 2005;16:1150-6.

19. Rostock T, O'Neill MD, Sanders P, Rotter M, Jaïs P, Hocini M, et al. Characterization of conduction recovery across left atrial linear lesions in patients with paroxysmal and persistent atrial fibrillation. $J$ Cardiovasc Electrophysiol. 2006;17:1106-11.

20. Kim TH, Park J, Uhm JS, Kim JY, Joung B, Lee MH, Pak HN. Challenging Achievement of Bidirectional Block After Linear Ablation Affects the Rhythm Outcome in Patients With Persistent Atrial Fibrillation. J Am Heart Assoc 2016;5. pii: e003894. 


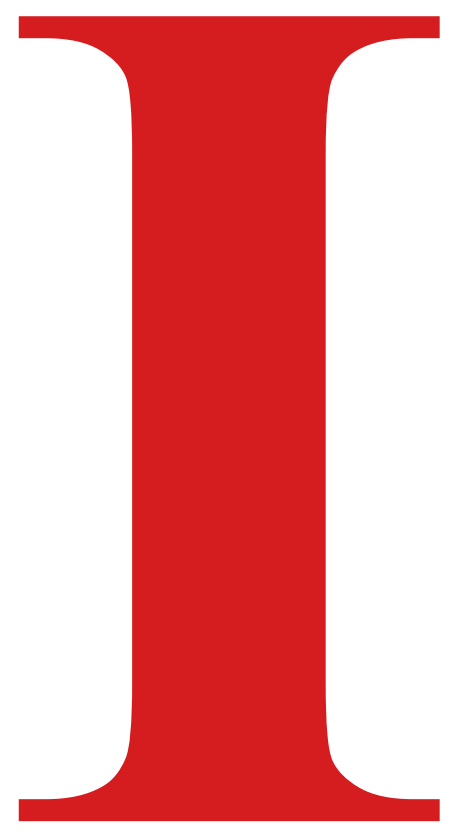




\section{Part I}

\section{ABLATION OF ATRIAL FIBRILLATION AND ATRIAL TACHYCARDIA}

Part ID

Mapping of drivers in persistent atrial fibrillation 

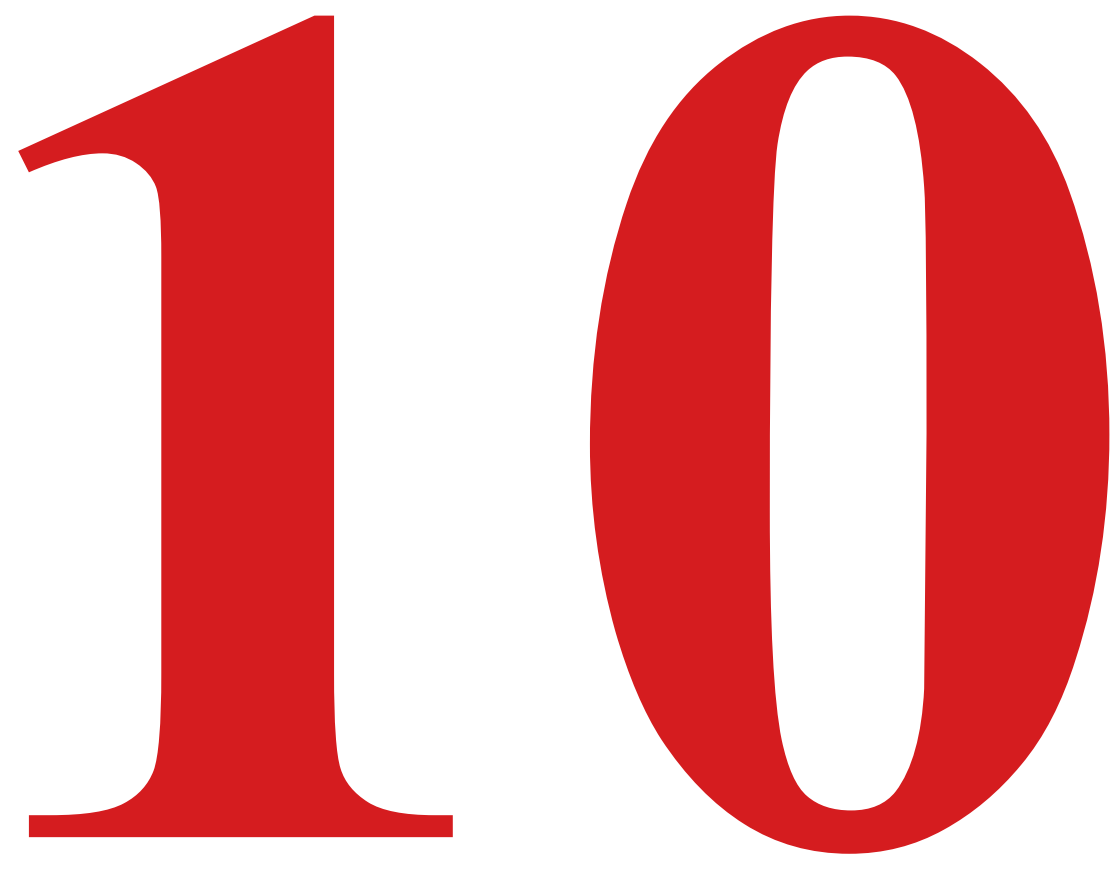


\section{Chapter 10}

\section{Direct contact activation mapping in persistent atrial fibrillation}

Michael Wolf, René Tavernier, Ziad Zeidan, Milad El Haddad, Yves Vandekerckhove, Jan De Pooter, Thomas Phlips, Teresa Strisciuglio, Alexandre Almorad, Maria Kyriakopoulou, Michelle Lycke, Mattias Duytschaever, Sébastien Knecht 


\begin{abstract}
Introduction:

Recent studies have characterized drivers in persistent atrial fibrillation using automated algorithm detection with panoramic endocardial mapping by means of basket catheters. We aimed to identify repetitive atrial activation patterns (RAAPs) during ongoing AF based upon automated annotation of unipolar electrograms recorded with a high-density regional endocardial contact mapping catheter.
\end{abstract}

\title{
Methods:
}

In 14 persistent $\mathrm{AF}$ patients, high resolution electrograms (EGMs) were recorded for 30 seconds at sequential PentaRay ${ }^{\mathrm{TM}}$ (Biosense Inc) positions covering the entire bi-atrial surface. All recordings were reviewed off-line with dedicated software allowing automated annotation of the local activation time of the unipolar fibrillatory EGMs (CARTOFINDER ${ }^{\mathrm{TM}}$, Biosense Inc).

RAAPs were defined as a consistent activation pattern (for $\geq 3$ consecutive beats) of either focal activity with centrifugal spread $\left(\mathrm{RAAP}_{\text {focal }}\right)$ or rotational activity across the PentaRay splines spanning the AF cycle length (RAAP rotational $_{1}$.

\section{Results:}

498 PentaRay recordings were analyzed (35.6 \pm 7.6 per patient). The number of PentaRay recordings displaying RAAP was 9.8 \pm 3.1 per patient (range 3-15), of which 2.4 \pm 2.4 RAAP $_{\text {rotational }}$ (range 0-7) and 7.4 $\pm 4.4 \mathrm{RAAP}_{\text {focal }}$ (range 1-13). 77\% of RAAPs portrayed focal firing. The median number of repetitions per 30 second recording was 11 (range 3-225) per recording. RAAPs were observed both in the RA (35\%) and LA (65\%), with the majority being near the left PVs/appendage (35\% of all RAAPs) and the SVC/right appendage (23\% of all RAAPs).

\section{Conclusions:}

High-resolution, sequential endocardial EGM-based mapping allows identification of RAAPs in persistent AF. In our series, focal firing was the most frequently observed pattern. 


\section{Introduction}

During persistent atrial fibrillation (AF), multiple atrial wavelets and localized (focal or reentrant) sources have been reported to contribute to the maintenance of $\mathrm{AF} .^{1-3}$ In the last few years, there has been progress in mapping AF sources and mechanisms, mostly using phase-based analysis of epicardial or endocardial potentials. ${ }^{4-10}$ However, phase mapping has limitations as it is known to generate false rotational activities, particularly in cases of low voltage, variable contact and when farfield signals are present. ${ }^{11-12}$

Recently, AF propagation maps based on direct contact mapping (without phase transformation) with a dedicated software (CARTOFINDER ${ }^{\mathrm{TM}}$, Biosense Webster Inc, Diamond Bar, CA) have also been tested. ${ }^{13-16}$ Using a multi-electrode basket catheter, the authors were able to reliably and reproducibly identify repetitive activation patterns in the majority of patients. Nevertheless, the basket catheter had long interelectrode distance and could not reach stable contact with the atrial wall in some regions and therefore, some repetitive activation patterns were probably missed.

This study sought to evaluate a new mapping method for the Cartofinder software, using a regional high-density contact mapping catheter (PentaRay ${ }^{\mathrm{TM}}$, Biosense Webster Inc). For the present work, we characterized repetitive atrial activation patterns (RAAPs) during ongoing AF based upon the automated annotation of unipolar electrograms (EGMs) directly obtained by these sequential recordings of the entire bi-atrial endocardial surface.

\section{Methods}

\section{Patient population}

During a two-fold period of availability of the Cartofinder software in our center (from May to October 2017 and from May to June 2019) consecutive patients who were referred to our institution for a first ablation of ongoing persistent $\mathrm{AF}$ and who consented to participate in the protocol, were enrolled in the study. Persistent AF was defined as continuous AF from 7 days to 12 months. Exclusion criteria were 1) sinus rhythm or atrial tachycardia at the onset of the procedure, 2) prior pulmonary vein isolation or atrial substrate ablation (linear lesions or ablation of fragmented signals), 3) presence of intra-cardiac leads, mechanical heart valves, an inter-atrial septum or a left atrial appendage occluder devices, 4) contra-indication for general anesthesia. The study protocol was approved by our institutional Ethics Committee.

\section{Procedure and contact mapping}

All procedures were performed under general anesthesia and under novel anticoagulants (last dose taken 24 hours before procedure) or uninterrupted warfarin by three operators (RT, SK and MD). Antiarrhythmic drugs, with the exception of amiodarone, were withdrawn at least five half-lives before the procedure. Intravenous heparin was administered after femoral vein access to achieve an activated clotting time $>300 \mathrm{~s}$. A decapolar coronary sinus catheter was introduced via the right 
femoral vein and double transseptal puncture was performed with conventional long sheaths (SL0, St. Jude Medical Inc). An irrigated mapping catheter with five soft radiating splines with 10 pairs of $1 \mathrm{~mm}$ electrodes separated by 2-6-2mm interelectrode spacing (PentaRay, Biosense Webster Inc) and an 8F irrigated RF catheter with contact force sensor (Thermocool SmartTouch ${ }^{\circledR}$, Biosense-Webster Inc) were positioned in the LA. Then calibration of contact force catheter and respiratory gating (Carto System ${ }^{\circledR}$, Biosense Webster Inc) were performed. After reconstruction of $3 \mathrm{D}$-geometry of the left atrium with the PentaRay catheter, high resolution EGMs during ongoing AF were recorded for 30 seconds at sequential PentaRay positions covering the entire left atrial surface, with each position overlapping the previous recording position with the radius of the PentaRay (Figure 1). Recordings were only obtained after optimal positioning of the PentaRay catheter, aiming maximal and even spread of the five splines with optimal electrode-tissue contact (also guided by the Tissue Proximity Indicator (Biosense Webster Inc), which determines the electrode proximity to cardiac tissue based on impedance measurements). After sequential mapping of the left atrium, 1 transseptal sheath was pulled back and sequential mapping of the entire right atrium was then performed. After EGM acquisition, RF ablation was performed with a lesion set consisting of pulmonary vein isolation with entrance block and linear lesions (roof, mitral and cavotricuspid isthmus). Ablation was not guided by the Cartofinder analysis. 


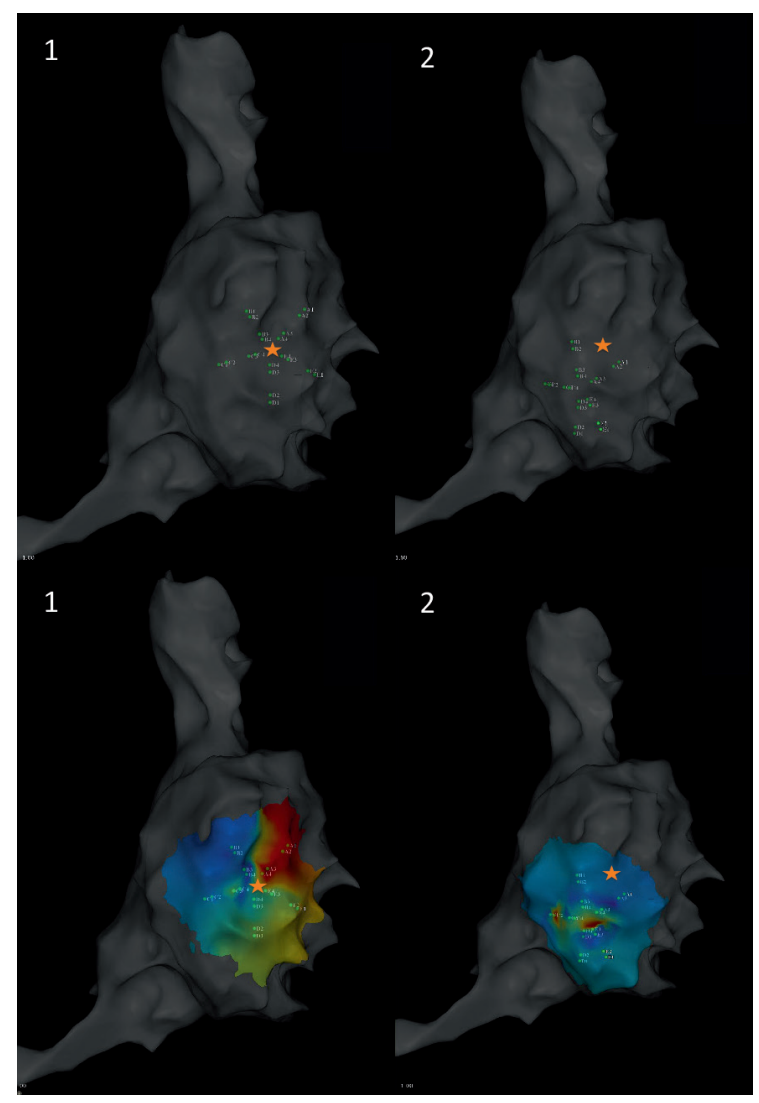

Figure 1: Sequential PentaRay recording positions covering the entire bi-atrial surface. Recordings were only obtained after optimal positioning of the PentaRay catheter, aiming maximal and even spread of the five splines with optimal electrode-tissue contact. Sequential positions overlap the previous recording position with the radius of the PentaRay: the center of position 1 (star) becomes the radius of position 2 (top panels). Overlapping recordings enhance detection of repetitive atrial patterns (rotational in position 1 and focal in position 2, bottom panels).

\section{EGM analysis and translation to activation mapping.}

All recordings were reviewed off-line with dedicated software allowing automated annotation of the local activation time of the fibrillatory EGMs (CARTOFINDER ${ }^{\mathrm{TM}}$, Biosense Webster Inc). Off-line analysis consisted of the following two parts: 1) identification and characterization of RAAPs, 2) Assessment of manual RAAP identification on Cartofinder activation maps and agreement between reviewers. 


\section{Part 1: identification of repetitive atrial activation patterns}

Cartofinder is based on the assessment of the local activation time of atrial signals during ongoing $\mathrm{AF}^{13-17}$. To accurately determine the activation time, the automated Cartofinder software entails the following: 1) first the algorithm filters out the far-field ventricular signals. 2) Then for each electrode, 2 bipolar atrial signals are created by using the nearest two electrodes, which are determined by the Carto matrix (impedance based). For each electrode, the bipolar EGM window is then established based on measurement of the earliest onset to latest offset of its 2 bipolar electrograms. This bipolar EGM window establishes the window to record the unipolar atrial signal from each electrode. 3) Next, a wavelet analysis of unipolar signals recorded from the bipolar electrogram window is performed. This analysis uses a proprietary algorithm to annotate only high-quality unipolar signals ${ }^{13,17}$. For each electrode the local activation time is established as the maximal negative slope of the unipolar electrogram within its predefined bipolar window, with the purpose of blanking out negative slopes due to far-field potentials. The accuracy of automated annotation of atrial local activation time and $\mathrm{V}$ far-field subtraction has been previously validated by experienced electrophysiologists (EPs) as part of the development process and used for the FDA submission, and were not routinely manually verified in our study.

After automated EGM analysis, RAAPs were identified on a 3D propagation map, which was automatically generated for each recording (Figure 2 and 3 and supplementary video recording $A$ and B). RAAPs were defined as a consistent activation pattern (for $\geq 3$ consecutive beats) of either focal activity with centrifugal spread along the PentaRay splines (RAAP focal $_{\text {) }}$ (Figure 2) or rotational activity across the PentaRay splines spanning the AF cycle length (RAAP ${ }_{\text {rotational }}$ (Figure 3). To classify the spatial distribution of RAAPs, the bi-atrial surface was divided in 7 regions (Figure 4). Four regions were defined in the left atrium, 2 in the right atrium, and 1 in the anterior interatrial septum. Because of their proximity, left PVs were grouped with the left appendage into 1 region en bloc. Similarly, the right atrial appendage was grouped with the upper right atrium. Type, number and location of RAAPs were cataloged per patient. 


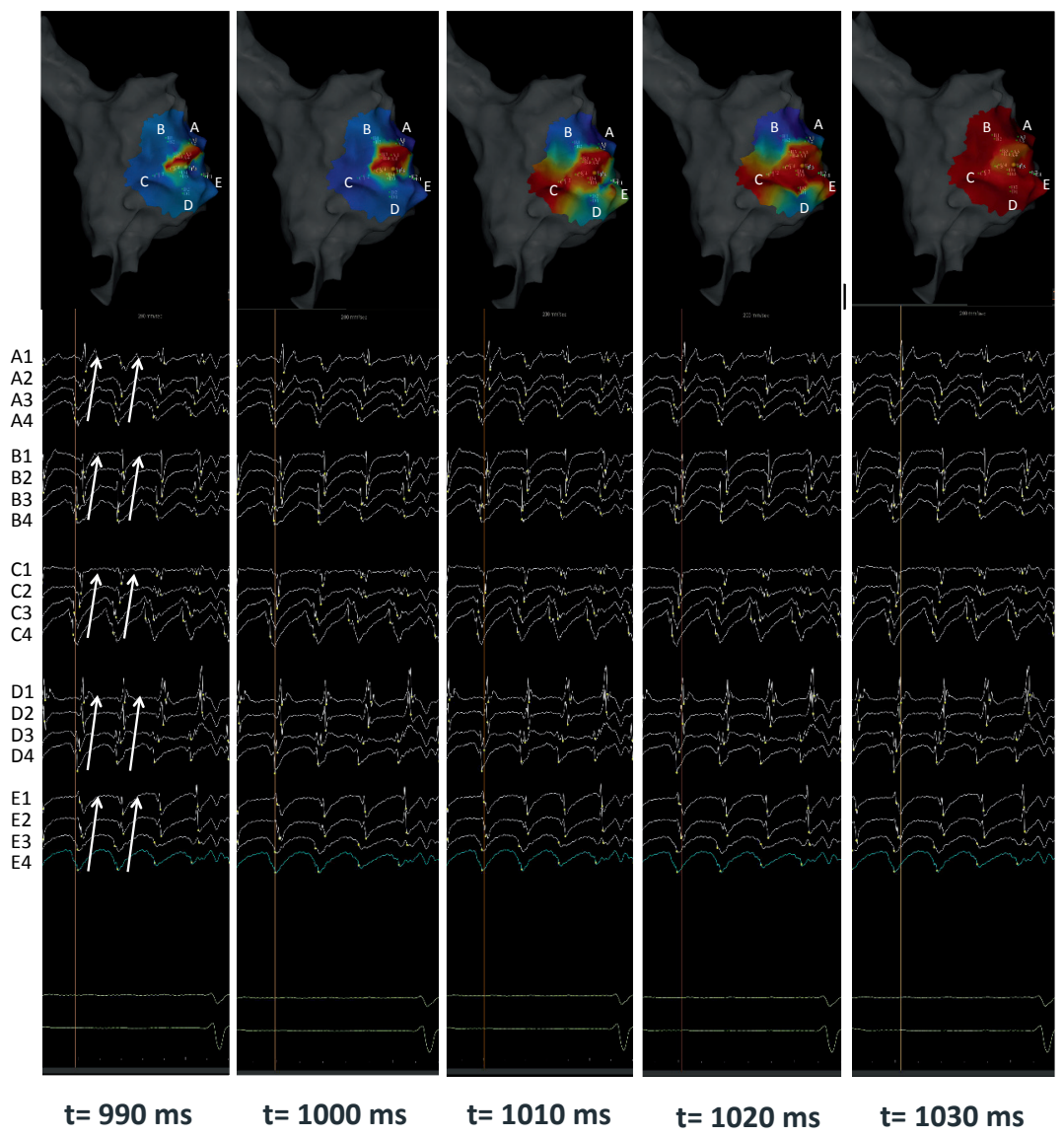

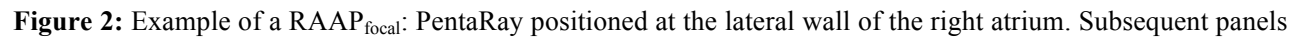
show the propagation during a time window of 990 to $1030 \mathrm{~ms}$ of a $30 \mathrm{~s}$ registration during ongoing atrial fibrillation. The color-coding, as well as the unipolar fibrillatory EGMs recorded at each electrode (here organized per PentaRay spline (A to E) with electrode 4 as the most proximal and electrode 1 as the most distal per spline) shows a centrifugal propagation consistent with focal firing ( $\left.\mathrm{RAAP}_{\text {focal }}\right)$. 


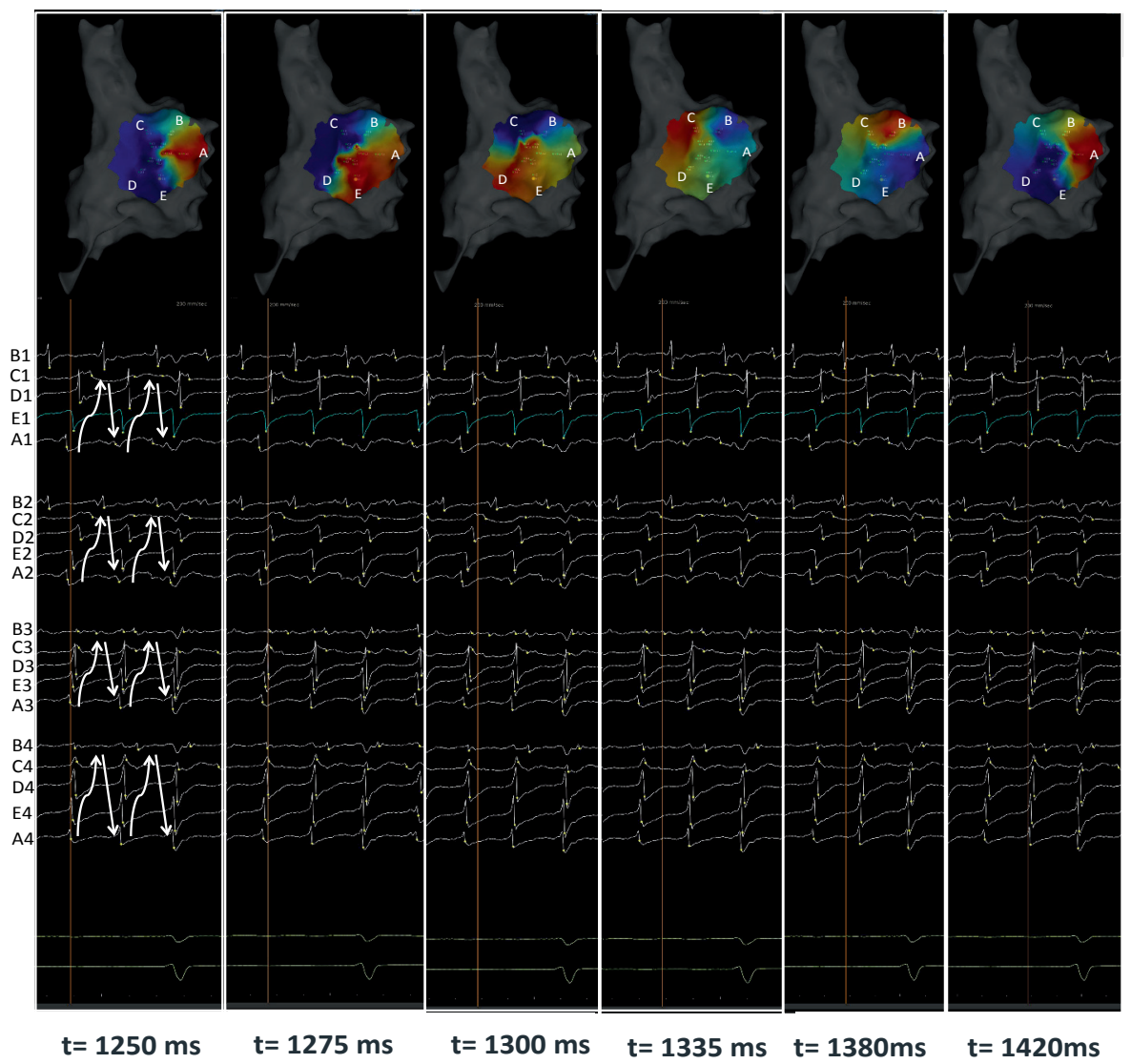

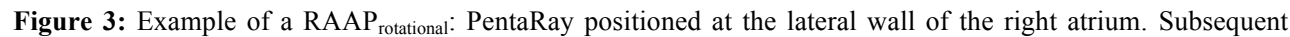
panels show the propagation during a time window of 1250 to $1420 \mathrm{~ms}$ of a $30 \mathrm{~s}$ registration during ongoing atrial fibrillation. The color-coding, as well as the unipolar fibrillatory EGMs recorded at each electrode (here organized per radius with electrode 4 as the most proximal and electrode 1 as the most distal per spline A to E), show a repetitive rotational propagation consistent with a $\mathrm{RAAP}_{\text {rotational. }}$ 


\section{Part 2: Validation of RAAP identification from the Cartofinder software}

This part of the study sought to validate the propagation map as generated by the Cartofinder system with the EGM analysis obtained from automated EGM annotation and reviewed by 4 experienced EPs who were blinded to the Cartofinder results. 46 out of 498 recordings (annotated EGMs and propagation maps) were randomly selected (21 with identified $\mathrm{RAAP}_{\text {focal }}, 18$ with identified RAAP $_{\text {rotational }}$ and 7 recordings without RAAP identification). Agreement among the individual classification by 4 reviewers was documented and compared to the propagation map generated by the Cartofinder software.

\section{$\underline{\text { Statistics }}$}

Continuous variables are expressed as mean $\pm \mathrm{SD}$ (or medians with range for non-normal distributions) and dichotomous variables as count and percentage. Normality of data distribution was tested with the Shapiro-Wilk test. Intraclass correlation coefficient was calculated to determine the agreement amongst the 4 operators. All statistical analyses were performed in SPSS Statistics 24 (IBM Corporation, Armonk, New York, USA).

\section{Results}

\section{Part 1: identification of repetitive atrial activation patterns}

The characteristics of the 14 patients are detailed in Table 1. In total 498 PentaRay recordings were achieved and analyzed (35.6 \pm 7.6 recordings per patient, $13.3 \pm 3.5$ in the RA and $22.1 \pm 3.2$ in the LA). The duration to perform all recordings was $44 \pm 6$ min. Radiation exposure during mapping was $6 \pm 3$ min. The mean AF cycle per recording was $172 \pm 25 \mathrm{~ms}$.

The mean number of PentaRay recordings displaying RAAP was 9.8 \pm 3.1 per patient (range 3-15) (6.4 \pm 2.5 in the LA and $3.4 \pm 2.1$ in the RA). Per RAAP, the median number of consecutive cycle repetitions during the $30 \mathrm{~s}$ recording was 11 (range 3-225). 


\section{Table 1: Patient characteristics}

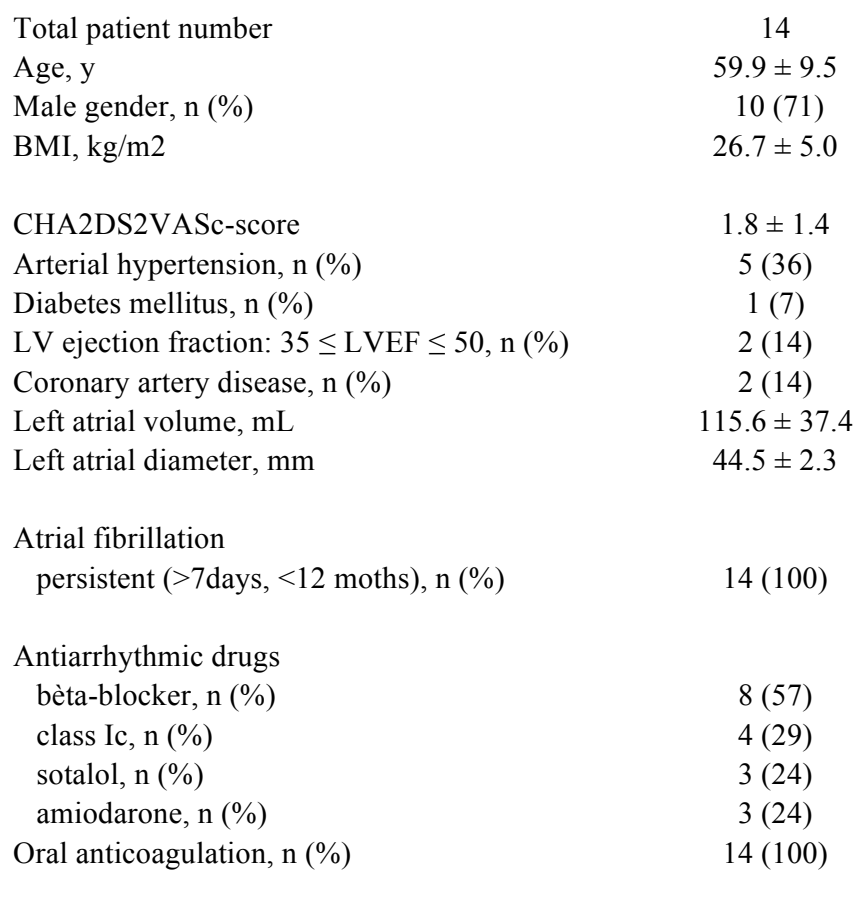

BMI: body mass index; LV: left ventricular

\section{Focal firing vs. rotational activity:}

Per patient, a mean of 7.4 $\pm 4.4 \mathrm{RAAP}_{\text {focal }}$ (range 1-13) and 2.4 $\pm 2.4 \mathrm{RAAP}_{\text {rotational }}$ (range 0-7) were observed. In total $77 \%$ of RAAPs showed focal spread and in 7 out of 14 patients only RAAP focal $_{\text {f }}$ could be observed.

\section{Distribution in left and right atrium:}

The distribution of RAAPs is displayed in Figure 4. RAAPs were identified in a mean of $4.5 \pm 1.1$ regions per patient, in both the right (35\%) and the left atrium (65\%). RAAPs were most frequently located near the left PV / left atrial appendage region ( $\mathrm{n}=47$ or $35 \%$ of all RAAPs) and in the superior $\mathrm{RA} /$ right atrial appendage region ( $\mathrm{n}=31$ or $23 \%$ of all RAAPs).

The distribution of $\mathrm{RAAP}_{\text {rotational }}$ and $\mathrm{RAAP}_{\text {focal }}$ were similar. In the RA, per patient a mean of $0.9 \pm 1.2$ $\mathrm{RAAP}_{\text {rotational }}$ (range 0-3) and 2.4 $\pm 2.3 \mathrm{RAAP}_{\text {focal }}$ (range 0-8) were observed. In the LA, per patient a mean of $1.5 \pm 1.8$ RAAP $_{\text {rotational }}$ (range $0-5$ ) and 4.9 $\pm 3.1 \mathrm{RAAP}_{\text {focal }}$ (range 1-11) were identified. 
A)

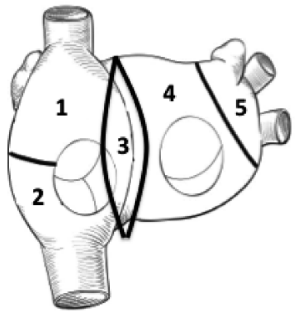

Segmentation of the bi-atrial surface

C)
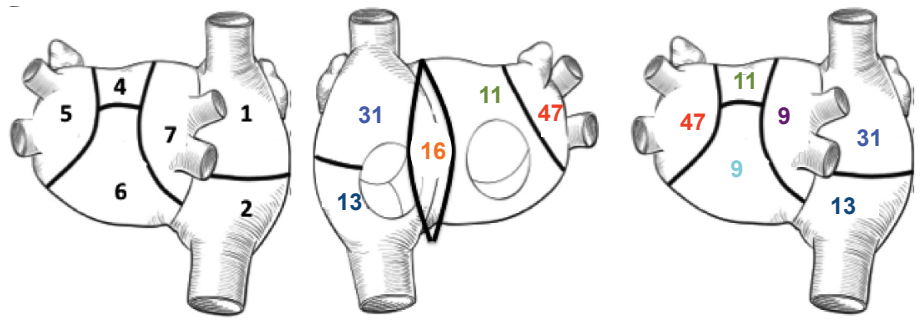

Distribution of $\operatorname{RAP}_{\text {rotational }}(\mathrm{n})$
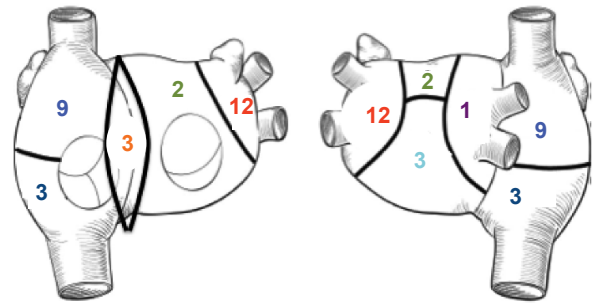

D)

Distribution of $\operatorname{RAP}_{\text {focal }}(\mathrm{n})$
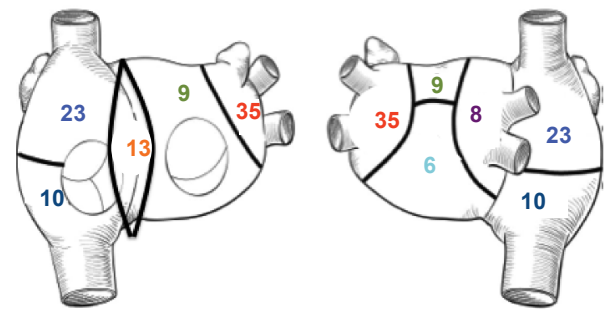

Figure 4: Panel A: To classify the spatial distribution of RAAPs, the bi-atrial surface was divided in 7 regions. Four regions were defined in the left atrium, 2 in the right atrium, and 1 in the anterior interatrial septum. Because of their proximity, left PVs were grouped with the left appendage into 1 region en bloc. Similarly, the right atrial appendage was grouped with the upper right atrium. Panel B: total number of identified RAAPs per

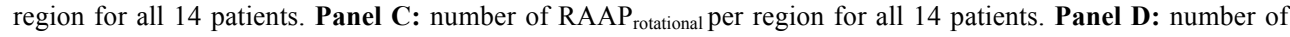
$\mathrm{RAAP}_{\text {focal }}$ per region for all 14 patients.

\section{Part 2: Validation of RAAP identification from the Cartofinder software}

There was good statistical agreement amongst the 4 operators in identifying RAAP (intraclass correlation $=0.725$ [95\% CI: 0.571, 0.835], $\mathrm{p}<0.001$ ).

In the majority of the selected recordings ( 37 of 46 or $80 \%$ ), an agreement between reviewers was obtained. This includes a full agreement amongst the 4 reviewers in 19 (41\%) recordings and an agreement amongst 3 reviewers in 18 (39\%) recordings. In 9 recordings (20\%), agreement was achieved amongst only 2 of the 4 reviewers. More precisely, for rotational activity, agreement amongst 4 and 3 reviewers was present in 44 and 50\% respectively. For focal activity, agreement amongst 4 and 3 reviewers was present in 53 and $40 \%$ respectively. In the 7 recordings without RAAP, agreement amongst 4 and 3 reviewers was present in $43 \%$ and $43 \%$ respectively. We added two examples of the agreement between reviewers to the supplementary appendix. 


\section{Discussion}

\section{Main findings}

This study shows that in persistent AF, using a high-density direct contact mapping catheter, the Cartofinder software consistently identifies numerous RAAPs (and predominantly focal firing sources), based on an automated acquisition of unipolar fibrillatory EGMs. Sequential high-density mapping may detect a greater number of RAAPs due to better coverage and mapping density in comparison with basket catheters ${ }^{13-17}$.

\section{Localized high-density vs. panoramic mapping using the Cartofinder software.}

Recent studies have investigated the feasibility of the Cartofinder software (Biosense Webster) to identify and characterize RAAPs during AF. However, all of those studies used an endocardial 64electrode basket-catheter (Constellation, Boston Scientific) to cover the entire LA ${ }^{13-16}$ except for one in which the basket catheter was evaluated in combination with the PentaRay catheter ${ }^{17}$. In this study, focusing on the $\mathrm{LA}^{17}$, focal RAAPs detected by the PentaRay catheter were less frequent as compared to our study. This could be explained by the fact that there were less recordings per patient (mean of 13 recordings as compared to 22 in our study) and that all included patients had long-standing persistent $\mathrm{AF}$, while patients with $\mathrm{AF}>12$ months were excluded in our trial.

Using the basket catheter, there has been some inconsistency concerning the amount of RAAP which could be identified by the software: from $\sim 1^{14,15,17}$ to $\sim 6^{16}$ RAAP, as compared to $\sim 10$ in our study (all of the patients had $\geq 3$ RAAPs). Moreover, while some investigators used a prolonged recording time (1-minute vs. 30 seconds in our study $)^{13}$, and although RAAP definition was sometimes more flexible

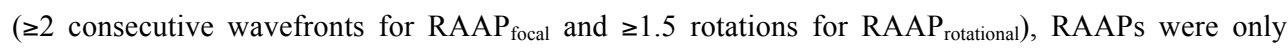
present in $55-65 \%$ of the patients in some of those studies using the basket catheter ${ }^{14,15}$. In addition, RAAPs were mostly rotational ${ }^{14}$, while focal RAAPs were more prevalent our study.

With the basket catheter, the interelectrode spacing is larger and there could be an issue with electrode-tissue contact and a predilection to position the basket towards the anterior wall. ${ }^{15,16}$ This can result in a suboptimal coverage of the atrial surface (54 to $72 \%$ ) ${ }^{15,16}$, with possible underestimation of RAAPs.

Sequential global mapping using a regional high-density mapping catheter can potentially overcome these issues. The PentaRay catheter used in our study has already demonstrated its usefulness in the analysis of activation patterns during ongoing AF. In a study on AF mapping, complex fractionated electrogram (CFAE) areas were demonstrated to be mostly passive as there was a simultaneous activation in all PentaRay splines in $84 \%$ of the CFAE areas ${ }^{18}$. In another study, this high-density mapping catheter was used to localize and ablate focal activity in the left atrium during AF, with 
encouraging results ${ }^{19}$. More recently, focal drivers that were detected in AF on sequential PentaRay recordings were more consistent, showed greater temporal stability and a higher recurrence rate compared with drivers that were not identified as "region of interest" using the basket catheter ${ }^{17}$. Furthermore, ablation at these RAAP sites identified with sequential PentaRay maps was more likely to terminate AF than drivers identified on global wavefront maps alone ${ }^{17}$.

While localized mapping allows for mapping both entire atria, it is crucial that the catheter remains stable, and with good contact of all 5 catheter splines. In addition, it cannot be excluded that meandering rotors were counted repeatedly with the overlapping mapping approach, resulting in a higher number of detected RAAPs.

\section{Detection of in vivo AF drivers derived from phase transformation analysis}

The mechanistic role of RAAPs as AF drivers was first supported by modeling and animal studies. ${ }^{20,21}$ Recently, new technologies have been developed that also allow direct visualization of the mechanisms driving AF in humans. Both the RhythmView (Topera, Menlo Park, CA, USA) and the ECVUE $^{\mathrm{TM}}$ system (CardioInsight Technologies Inc, Cleveland, OH) have been developed for automated intra-procedural mapping of RAAPs, and are both based on filtering and phase analysis of a wide-field of view (biatrial) of fibrillatory electograms. Both systems apply phase transformation to the unipolar fibrillatory electrograms to facilitate the description of complex spatiotemporal fibrillation patterns. This phase transformation potentially results in "falsely" increased detection of temporal and spatial organization during fibrillation, and could facilitate the visualization of nonexistent rotors. ${ }^{23}$ Furthermore, the basket catheter used with the Topera system has a lack of spatial resolution, which could also potentially result in the detection of false rotors when phase transformation is applied, ${ }^{6,23}$ because data interpolation from the surrounding electrodes is needed. These limitations can potentially explain the mitigated results of ablation strategies based on systems using phase transformation ${ }^{72}$. Importantly, no randomized clinical study using the Cartofinder software has yet evaluated the impact of RAAP ablation during AF and the system therefore has to be validated in the future.

\section{Location of RAAPS}

In our study, RAAPs were mostly located in the left PV/left appendage region and the right atrial appendage region, but were not often observed near the right PVs. Also, in the AFACART multicenter study on non-invasive mapping with phase conversion of persistent AF, the right PVs were a much less frequent origin of AF drivers, especially of focal sources, compared to the left PVs and the appendage region ${ }^{7}$. This is in contrast to the findings of Haïssaguerre et al. who identified drivers with ECGi and phase transformation near both left and right PV pairs in $>95 \%$ of persistent AF patients without prior PV isolation ${ }^{6}$. In addition, foci in the inferior LA and on the roof/anterior wall were less frequent in our study. 


\section{Clinical perspectives for ablation of atrial fibrillation}

While the pulmonary veins are the predominant source of triggers responsible in the pathogenesis of paroxysmal AF, electrical and structural remodeling is fundamental in the progression to persistent $\mathrm{AF}$, in which multiple atrial wavelets and localized (focal or reentrant) sources have been reported to contribute to the substrate ${ }^{1-3}$.

Therefore, durable electrical isolation of the pulmonary veins is an effective ablation strategy for paroxysmal AF, but yields less favorable results in persistent AF. In order to improve the outcome in persistent AF patients, several different ablation techniques have been developed to target the atrial substrate including ablation of complex fractionated electrogram (CFAEs) in both atria, left atrial linear lesions, and the so-called 'stepwise' approach. ${ }^{25}$ Outcomes from ablation of persistent AF using these strategies have given modest results, and each approach lacks specificity, sometimes resulting in extensive ablation with potentially deleterious effects (on atrial hemodynamic function or with proarrhythmic consequences). ${ }^{25}$ This would explain why empirical substrate ablation (with linear or CFAE ablation) on top of PVI did not seem to improve outcome ${ }^{26}$. These pitfalls encourage development of patient tailored ablation strategies targeting patient-specific AF drivers.

This study shows that direct endocardial high-density contact mapping EGM analysis during ongoing AF allows identification of RAAPs. The automated annotation software has the potential to provide on-line detection of potential bi-atrial AF drivers annotated on the 3D-geometric bi-atrial shell, making patient-tailored substrate ablation possible. Further studies are needed to investigate if ablation of RAAPs as detected by the system might improve clinical outcome.

\section{Limitations}

1) It was not always possible to equally spread the 5 spines of the mapping catheter (mostly on the anterior septum), which could have affected the results. 2) Procedures were performed with the PentaRay with 2-6-2 mm interelectrode spacing. We cannot certify that use of the 4-4-4 mm interelectrode spacing would have rendered identical results. 3) It is possible that the detection of rotational RAAPs is decreased by the narrow field-of-view of the PentaRay catheter. 4) Automated electrogram annotations were not manually verified and this could have led to some annotation errors. 5) Although this study suggests the universal existence of RAAPs in persistent AF patients, this study does not clarify whether RAAPs have a mechanistic role in AF maintenance or if they are just an epiphenomenon. The effect of ablation of RAAP was not investigated in our study. A randomized study about the potential benefit of driver ablation as depicted by the Cartofinder software should be performed. 


\section{Conclusions}

High-resolution and sequential endocardial EGM-based mapping shows the presence of repetitive activation patterns during persistent $\mathrm{AF}$ in all patients. In our series, focal firing was the most frequently observed pattern. The PentaRay high-density mapping catheter has the ability to reach all atrial regions and could potentially enhance RAAP detection. 


\section{References}

1. Konings KT, Kirchhof CJ, Smeets JR, Wellens HJ, Penn OC, Allessie MA. High-density mapping of electrically induced atrial fibrillation in humans. Circulation 1994;89:1665-1680.

2. Jalife J, Berenfeld O, Mansour M. Mother rotors and fibrillatory conduction: a mechanism of atrial fibrillation. Cardiovasc Res 2002;54:204-216.

3. Haissaguerre M, Hocini M, Sanders P, Takahashi Y, Rotter M, Sacher F, Rostock T, Hsu LF, Jonsson A, O’Neill MD, Bordachar P, Reuter S, Roudaut R, Clementy J, Jais P. Localized sources maintaining atrial fibrillation organized by prior ablation. Circulation 2006;113:616-625.

4. Narayan SM, Krummen DE, Rappel WJ. Clinical mapping approach to diagnose electrical rotors and focal impulse sources for human atrial fibrillation. J Cardiovasc Electrophysiol 2012;23:447454.

5. Narayan SM, Baykaner T, Clopton P, Schricker A, Lalani GG, Krummen DE, Shivkumar K, Miller JM. Ablation of rotor and focal sources reduces late recurrence of atrial fibrillation compared with trigger ablation alone: extended follow-up of the CONFIRM trial (Conventional Ablation for Atrial Fibrillation With or Without Focal Impulse and Rotor Modulation). J Am Coll Cardiol 2014;63:1761-1768.

6. Haissaguerre M, Hocini M, Denis A, Shah AJ, Komatsu Y, Yamashita S, Daly M, Amraoui S, Zellerhoff S, Picat MQ, Quotb A, Jesel L, Lim H, Ploux S, Bordachar P, Attuel G, Meillet V, Ritter P, Derval N, Sacher F, Bernus O, Cochet H, Jais P, Dubois R. Driver domains in persistent atrial fibrillation. Circulation 2014;130:530-538.

7. Knecht S, Sohal M, Deisenhofer I, Albenque JP, Arentz T, Neumann T, Cauchemez B, Duytschaever M, Ramoul K, Verbeet T, Thorsten S, Jadidi A, Combes S, Tavernier R, Vandekerckhove Y, Ernst S, Packer D, Rostock T. Multicentre evaluation of noninvasive biatrial mapping for persistent atrial fibrillation ablation: the AFACART study. Europace 2017;19:1302-1309.

8. Kuklik P, Zeemering S, van Hunnik A, Maesen B, Pison L, Lau DH, Maessen J, Podziemski P, Meyer C, Schaffer B, Crijns H, Willems S, Schotten U. Identification of Rotors during Human Atrial Fibrillation Using Contact Mapping and Phase Singularity Detection: Technical Considerations. IEEE Trans Biomed Eng. 2017;64:310-318.

9. Kik C, Mouws EMJP, Bogers AJJC, de Groot NMS. Intra-operative mapping of the atria: the first step towards individualization of atrial fibrillation therapy? Expert Rev Cardiovasc Ther. 2017; 15:537-545.

10. Pathik B, Kalman JM, Walters T, Kuklik P, Zhao J, Madry A, Prabhu S, Nalliah C, Kistler P, Lee G. Transient Rotor Activity During Prolonged 3-Dimensional Phase Mapping in Human Persistent Atrial Fibrillation. JACC Clin Electrophysiol. 2018;4:72-83.

11. Benharash P, Buch E, Frank P, Share M, Tung R, Shivkumar K, Miller JM. Quantitative analysis of localized sources identified by focal impulse and rotor modulation mapping in atrial fibrillation. Circ Arrhythm Electrophysiol 2015;8:554-561.

12. Nattel S, Xiong F, Aguilar M. Demystifying rotors and their place in clinical translation of atrial fibrillation mechanisms. Nat Rev Cardiol 2017;14:509-520.

13. Daoud EG, Zeidan Z, Hummel JD, Weiss R, Houmsse Mahmoud, Augostini R, Kalbfleish SJ. Identification of Repetitive Activation Patterns Using Novel Computational Analysis of Multielectrode Recordings During Atrial Fibrillation and Flutter in Humans. JACC Clin Electrophysiol 2017;3:207-216.

14. Honarbakhsh S, Schilling RJ, Dhillon G, Ullah W, Keating E, Providencia R, Chow A, Earley MJ, Hunter RJ. A Novel Mapping System for Panoramic Mapping of the Left Atrium: Application to Detect and Characterize Localized Sources Maintaining Atrial Fibrillation. JACC Clin Electrophysiol. 2018;4:124-134. 
15. Honarbakhsh S, Schilling RJ, Providencia R, Keating E, Chow A, Sporton S, Lowe M, Earley MJ, Lambiase PD, Hunter RJ. Characterization of drivers maintaining atrial fibrillation: Correlation with markers of rapidity and organization on spectral analysis. Heart Rhythm. 2018;15:1296-1303.

16. Verma A, Sarkozy A, Skanes A, Duytschaever M, Bulava A, Urman R, Amos YA, Potter T. Characterization and significance of localized sources identified by a novel automated algorithm during mapping of human persistent atrial fibrillation. J Cardiovasc Electrophysiol. 2018;29:14801488 .

17. Honarbakhsh S, Schilling RJ, Providencia R, Keating E, Sporton S, Lowe M, Lambiase PD, Chow A, Earley MJ, Hunter RJ. Automated detection of repetitive focal activations in persistent atrial fibrillation: Validation of a novel detection algorithm and application through panoramic and sequential mapping. J Cardiovasc Electrophysiol. 2019;30:58-66.

18. Rostock T, Rotter M, Sanders P, Takahashi Y, Jaïs P, Hocini M, Hsu LF, Sacher F, Clémenty J, Haïssaguerre M. High-density activation mapping of fractionated electrograms in the atria of patients with paroxysmal atrial fibrillation. Heart Rhythm 2006;3:27-34.

19. Takahashi Y, Hocini M, O'Neill MD, Sanders P, Rotter M, Rostock T, Jonsson A, Sacher F, Clémenty J, Jaïs P, Haïssaguerre M. Sites of focal atrial activity characterized by endocardial mapping during atrial fibrillation. J Am Coll Cardiol 2006;47:2005-2012.

20. Nattel S, Shiroshita-Takeshita A, Brundel BJ, Rivard L. Mechanisms of atrial fibrillation: lessons from animal models. Prog Cardiovasc Dis 2005;48:9-28.

21. Mandapati R, Skanes A, Chen J, Berenfeld O, Jalife J. Stable microreentrant sources as a mechanism of atrial fibrillation in the isolated sheep heart. Circulation 2000;101:194-199.

22. Gray RA, Pertsov AM, Jalife J. Spatial and temporal organization during cardiac fibrillation. Nature 1998;392:75-78.

23. Umapathy K, Nair K, Masse S, Krishnan S, Rogers J, Nash MP, Nanthakumar K. Phase mapping of cardiac fibrillation. Circ Arrhythm Electrophysiol 2010;3:105-114.

24. Buch E, Share M, Tung R, Benharash P, Sharma P, Koneru J, Mandapati R, Ellenbogen KA, Shivkumar K. Long-term clinical outcomes of focal impulse and rotor modulation for treatment of atrial fibrillation: a multicenter experience. Heart Rhythm 2016;13:636-641.

25. Brooks AG, Stiles MK, Laborderie J, Lau DH, Kuklik P, Shipp NJ, Hsu LF, Sanders P. Outcomes of long-standing persistent atrial fibrillation ablation: a systematic review. Heart Rhythm 2010;7:835-846.

26. Verma A, Jiang CY, Betts TR, Chen J, Deisenhofer I, Mantovan R, Macle L, Morillo CA, Haverkamp W, Weerasooriya R, Albenque JP, Nardi S, Menardi E, Novak P, Sanders P; STAR AF II Investigators. Approaches to catheter ablation for persistent atrial fibrillation. N Engl J Med 2015;372:1812-22. 


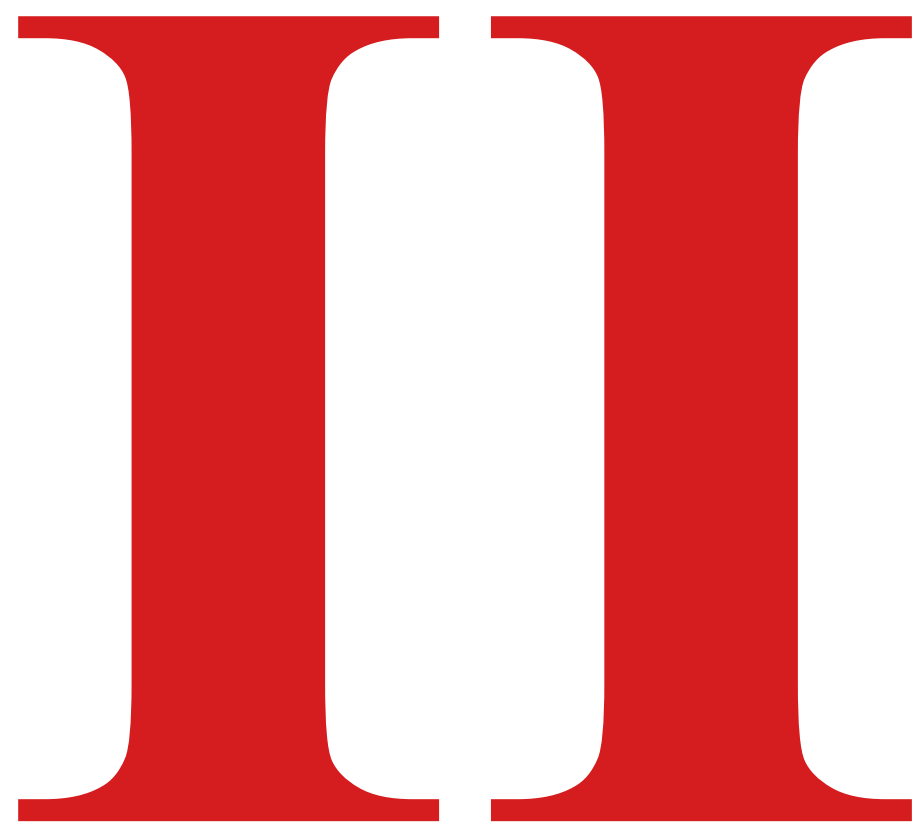




\section{Part II}

\section{ABLATION OF VENTRICULAR TACHYCARDIA}



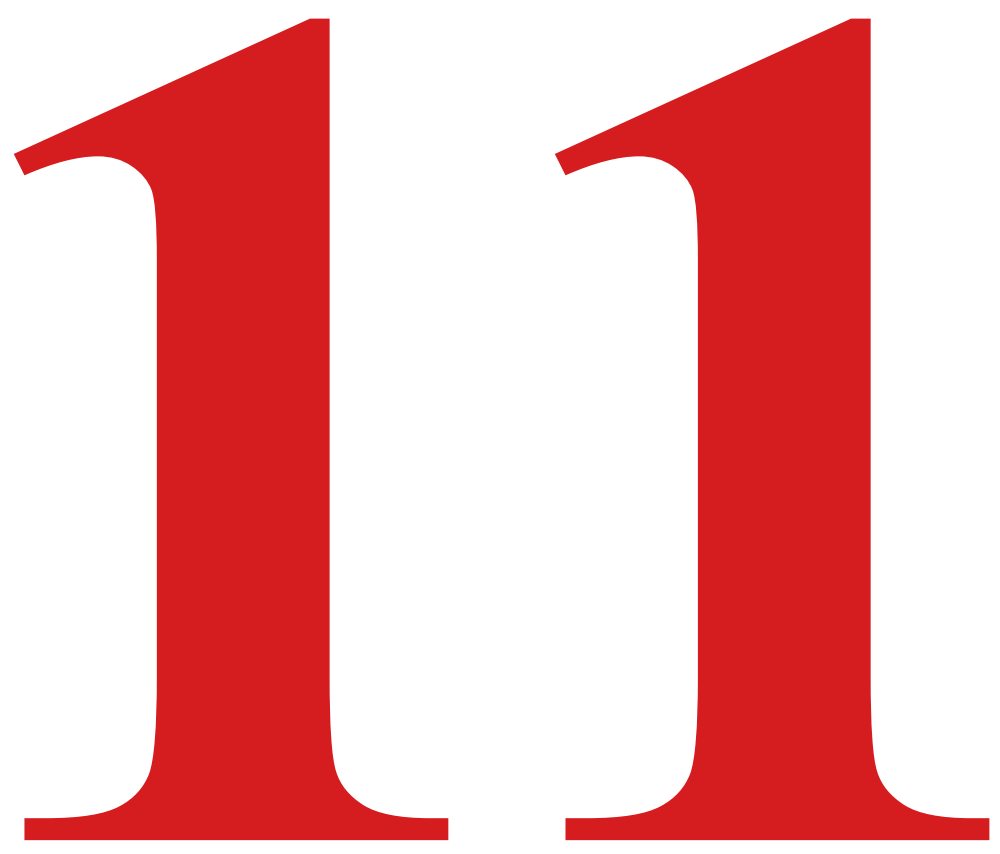


\section{Chapter 11}

\section{Evaluation of long-term outcomes of substrate modification for post- myocardial infarction ventricular tachycardia.}

Michael Wolf, Frédéric Sacher, Hubert Cochet, Takeshi Kitamura, Masateru Takigawa, Seigo Yamashita, Konstantinos Vlachos, Ghassen Cheniti, Antonio Frontera, Ruairidh Martin, Nathaniel Thompson, Grégoire Massoullié, Anna Lam, Claire Martin, Florent Collot, Josselin Duchateau, Thomas Pambrun, Arnaud Denis, Nicolas Derval, Mélèze Hocini, Michel Haïssaguerre, Pierre Jaïs

Circulation: Arrhythmia \& Electrophysiology. 2018;11:e005635.

doi: 10.1161/CIRCEP.117.005635. 


\begin{abstract}
Background: Long-term results of substrate modification for ablation of ventricular tachycardia (VT) have not been reported. We report long-term outcomes of substrate elimination targeting local abnormal ventricular activities (LAVA) for post-myocardial infarction (MI) VT.

Methods \& Results: 159 consecutive patients undergoing first ablation were included (65 $\pm 11 \mathrm{y}, 92 \%$ ICDs, 54\% storms and 73\% appropriate shocks). LAVA were identified in $92 \%$ and VT was inducible in $73 \%$. Complete LAVA elimination and non-inducibility after ablation were achieved in $64 \%$ and $85 \%$. During a median follow-up of 47 months (IQR 34-82), single procedure ventricular arrhythmia (VA)-free survival was 55\% (10\% storms and 19\% shocks). The VA-free survival was 73, 68, 61, 55 and $49 \%$ after 1, 2, 3, 4 and 5 years. Complete LAVA elimination was associated with improved outcomes: VA-free survival of $82 \%$ at 1 and $61 \%$ at 5 years. In the subgroup treated with multielectrode (ME) mapping and real-time image integration, VA-free survival was 86 and $65 \%$ at 1 and 4 years respectively. Including repeat procedures in $18 \%$ of pts $(1.3 \pm 0.6$ ablations $/ \mathrm{pt})$ outcomes improved to $69 \%$ VA-free survival (2\% storms and $9 \%$ shocks) during median 46 months follow-up. Overall survival was 91 at 1 and $77 \%$ at 5 years of follow-up. Conclusions: In this mono-centric study, substrate modification targeting LAVA for post-MI VT resulted in a substantial reduction of VT storm and ICD shocks and up to $49 \%$ of patients free from arrhythmia at 5 years after a single procedure. Complete LAVA elimination, ME mapping and realtime integration were associated with improved VA-free survival.
\end{abstract}




\section{Introduction}

Catheter ablation for sustained monomorphic ventricular tachycardia (VT) after myocardial infarction (MI) has emerged as an effective tool for the control of VT recurrence. ${ }^{1}$ Recently few studies have reported long-term follow-up data of VT ablation in ischemic cardiomyopathy (ICMP) patients, guided predominantly by activation and entrainment mapping of inducible and hemodynamically tolerated VTs. ${ }^{2-3}$ However, alternative strategies predominantly based on substrate modification have gained prominence. These strategies not only have the advantage of identifying targets for ablation in patients in whom VT is not inducible or not tolerated, but they may also provide a more comprehensive treatment of the substrate for present but also future VT, as they are not limited to inducible or "clinical" types of VT. Long-term results of substrate modification strategies have not been reported..$^{4-6}$ The aim of our study was to report the long-term outcomes after single and multiple ablation procedures with elimination of local abnormal ventricular activities (LAVA) for VT ablation in post-MI patients.

\section{Methods}

The data, analytic methods, and study materials have been made available to other researchers for purposes of reproducing the results or replicating the procedure.

\section{Definition of LAVA}

Our definition of LAVA has been described previously. ${ }^{7}$ LAVA incorporate all abnormal local electrical signals arising from pathological slowly conducting/poorly coupled ventricular tissue, hence potential VT isthmuses. LAVA are defined as sharp high-frequency ventricular potentials, possibly of low amplitude, distinct from the far-field ventricular electrogram occurring anytime during or after the far-field ventricular electrogram in sinus rhythm, or before the far-field ventricular electrogram during VT. They sometimes display fractionation or double or multiple components separated by very-lowamplitude signals or an isoelectric interval and are poorly coupled to the rest of the myocardium.

\section{Study population}

From May 2006 to September 2015, all consecutive patients referred to our center for a first catheter ablation procedure for post-MI VT were included. Inclusion criteria consisted of repetitive episodes of drug-resistant sustained monomorphic VT in patients with a history of MI, documented by surface electrocardiography or implantable cardioverter-defibrillator (ICD) tracings. Patients were excluded if ventricular arrhythmias were attributable to an acute or reversible cause or if another type of cardiomyopathy co-existed. Sustained VT was defined as VT lasting $\geq 30$ seconds or terminated by external or internal cardioversion or antitachypacing (ATP). VT storm was defined as $\geq 3$ VT episodes in 24 hours. Patients with repetitive premature ventricular contractions or non-sustained VT in the 
absence of sustained VT were excluded. The study was approved by the Institutional Review Board and all patients gave written informed consent.

\section{Left ventricular access and electrophysiological study}

The electrophysiological study and ablation were conducted with conscious sedation with midazolam $0.02 \mathrm{mg} / \mathrm{kg}$ before femoral puncture, morphine sulfate 0.1 to $0.2 \mathrm{mg} / \mathrm{kg}$, and supplementary analgesia (sufentanil $0.05-0.15 \mu \mathrm{g}$ ) under supervision of an anesthesiologist for pericardial approach when needed. Non-invasive or intra-arterial blood pressure monitoring and digital pulse oximetry were performed continuously. ICD therapies were inactivated prior to femoral puncture. Antiarrhythmic drug treatment was continued until the ablation procedure. Vascular sheaths were inserted into the right femoral vein (2-3), right femoral artery (1), and/or subxiphoid area (1) under local anesthesia (bupivacaine). A steerable quadripolar/decapolar catheter (Xtrem, Sorin France, or Dynamic, Boston Scientific Inc, Cambridge, LA) was placed in the right ventricular apex or coronary sinus. The left ventricle was accessed by a transseptal (BRK needle, Agilis sheath, St. Jude Medical, St. Paul, MN) and/or retrograde aortic approach with or without additional epicardial mapping (Tuohy Needle, Agilis sheath short curve, St. Jude Medical). The mode of access to the left ventricle (LV) was at the operator's discretion, however dual (transseptal and retrograde) or triple (transseptal, retrograde and epicardial) access was encouraged. In the absence of contraindications, epicardial mapping was specifically encouraged if an epicardial origin was suspected (preprocedural imaging, 12-lead ECG, absence of endocardial LAVA, and/or persistent LAVA after endocardial ablation). Contraindications to epicardial access included previous cardiac surgery, pericardial adhesions, and the use of double antiplatelet therapy or continued oral anticoagulation. Our method of percutaneous pericardial access through anterior subxiphoid puncture has been described previously. ${ }^{7}$ After LV access was obtained, a $50 \mathrm{U} / \mathrm{kg}$ heparin bolus was administered intravenously and repeated as necessary to achieve an activated clotting time $>250$ seconds.

\section{Substrate mapping}

Twelve-lead ECG and bipolar intracardiac electograms were recorded continuously (LabSystem Pro, Bard Electrophysiology, Lowell, MA, or Siemens Axiom Sensis XP Siemens, Munich, Germany). Signals were sampled at $1 \mathrm{kHz}$ and filtered at 0.1 to $50 \mathrm{~Hz}$ for surface ECGs and 30 to $250 \mathrm{~Hz}$ for intracardiac signals, displayed at an amplification of $0.1 \mathrm{mV} / \mathrm{cm}$. Use of 3D-electroanatomical mapping (EAM) systems, multi-electrode (ME) mapping catheters and real-time image integration (RTII) was integrated into the workflow once available for clinical use (if no contra-indication to contrast-enhanced multidetector computed tomography (MDCT) and magnetic resonance imaging (MRI)). Mapping was performed with either a $3.5 \mathrm{~mm}$ open-irrigated ablation catheter (Biosense Webster, Diamond Bar, CA), but preferably a multipolar high-density mapping catheter (Pentaray 2-62mm spacing, Biosense Webster, or Intellamap Orion, Boston Scientific). Peak-to-peak amplitudes of 
0.5 to $1.5 \mathrm{mV}$ and $<0.5 \mathrm{mV}$ were used to define low-voltage zones and dense scar zone (adjusted voltage range of 0.2 to $0.8 \mathrm{mV}$ was used in 6 patients mapped with the Intellamap Orion ${ }^{8}$ ). When a 3DEAM system was used (CARTO3, Biosense Webster; NavX, St. Jude Medical; Rhythmia, Boston Scientific) mapping was performed during sinus rhythm to create a voltage map and annotate LAVA. For RTII, MDCT or MRI images were processed using dedicated software (MUSIC, Liryc Université de Bordeaux / Inria - Sophia Antipolis, France) to render patient-specific meshes of the cardiac chambers, epicardium, coronary sinus, coronary arteries and left phrenic nerve. Structural substrate (scar) was segmented on imaging as areas of delayed gadolinium enhancement (DE) on MRI and/or areas of wall thinning $\leq 5 \mathrm{~mm}$ on MDCT (Figure 1).

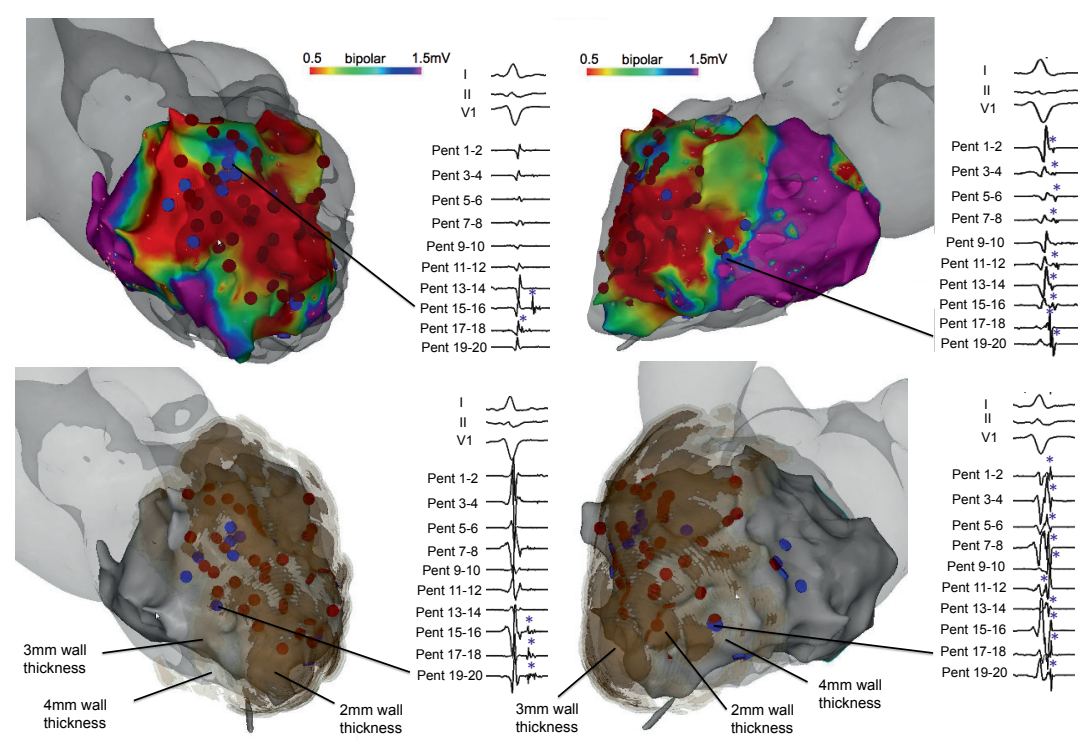

Figure 1: LAVA elimination using high-density multi-electrode mapping and real-time image integration in a case of post-anterior myocardial infarction ventricular tachycardia. Upper panels: A large anterior scar $(<0.5 \mathrm{mV})$ is demonstrated on endocardial bipolar voltage maps (modified RAO and LAO view). Lower panels: Integration of $2 \mathrm{~mm}$ (dark brown), $3 \mathrm{~mm}$ and $4 \mathrm{~mm}$ myocardial wall thinning layers derived from multi-detector computed tomography, showing good correlation with scar and border zone on voltage maps. LAVA (*) were recorded with multi-electrode mapping (Pentaray) mostly in scar and border zone (blue tags). Ablation (red tags) resulted in complete LAVA elimination.

\section{VT inducibility}

Prior to ablation, VT induction was attempted with programmed stimulation from at least two sites (right ventricular apex or outflow tract, and/or LV apex) at two drive trains (600 and 400ms), with up to 3 extrastimuli decremented to ventricular refractoriness or $200 \mathrm{~ms}$. Isoprenalin was not used for the purpose of inducibility. If hemodynamically tolerated VT was induced or originated during substrate 
mapping, conventional activation and entrainment mapping and ablation at the critical isthmus was performed first. Induced VTs were classified as clinical or non-clinical based on comparison of the twelve-lead ECG morphology and cycle length to documented tracings of the clinical VTs. When no 12-lead tracings were available, classification was based on the cycle length on ICD tracings. In case of non-inducible or hemodynamically untolerated VT, ablation targeting LAVA elimination was performed in sinus rhythm.

\section{Catheter ablation}

Ablation in sinus rhythm targeting complete LAVA elimination was the procedural endpoint in addition to non-inducibility of any VT. Ablation was delivered with open-irrigated catheter (25-50W endocardial and 25-35W epicardial) until targeted sites were rendered electrically unexcitable with pacing at $20 \mathrm{~mA}$ with $2 \mathrm{~ms}$ pulse width output. If a sequence of activation of LAVA was mapped, the earliest signals were targeted first. If both endo- and epicardial LAVA were present, ablation from the endocardial side was performed first with subsequent epicardial ablation if LAVA persisted. After ablation, the ventricles were remapped with the original mapping catheter and additional substrate ablation would be performed if residual LAVA were identified and/or if any VT remained inducible. Inducibility testing after ablation was avoided if patients were unstable or if they had required $\geq 2$ cardioversions for hemodynamically unstable VT induced during the procedure. In case of epicardial ablation, proximity of coronary arteries and phrenic nerve to the targeted ablation sites was derived from RTII when available. Coronary arteries were injected only if the distance between the ablation catheter and the coronary image was $10 \mathrm{~mm}$ or less. High-output pacing for the phrenic was also used following the same rule.

\section{Post-procedural care}

Venous and arterial sheaths were withdrawn immediately. Pericardial sheaths were retracted after a dry pericardial aspirate was confirmed. ICD therapies were reactivated at the end of the procedure. Patients were monitored in hospital for at least 2 to 5 days. Preprocedural antiarrhythmic drugs were continued unless contraindicated or not tolerated.

\section{Follow-up}

Patients were followed up at 1, 3, 6 and 12 months during the first year and every 6 months thereafter. ICD were interrogated at each visit and arrhythmia logs were retrieved. In addition, 64 of 146 (44\%) patients with ICD were followed through remote monitoring with weekly transmissions. A detailed history, ECG and Holter monitoring were performed in symptomatic patients without ICD. At each follow-up consultation, reduction or discontinuation of antiarrhythmic drugs was evaluated. The primary endpoint was the ventricular arrhythmia (VA)-free survival. Any documented monomorphic 
or polymorphic VT sustaining $\geq 30$ s or terminated by appropriate ICD therapy (ATP or shock) was considered a recurrence. In patients with ICD implanted for $\geq 1$ year prior to ablation, the VT burden was compared 1 year before and after ablation. Secondary endpoints were recurrence of VT storm, appropriate ICD shocks, all-cause mortality and discontinuation of antiarrhythmic drugs. Mortality during follow-up was categorized as either cardiovascular arrhythmogenic, cardiovascular nonarrhythmogenic or non-cardiovascular. ICDs of all deceased patients were analyzed to exclude an arrhythmogenic cause (monitor zones were programmed to detect to slow VT). Mortality was only adjudicated as non-cardiovascular if a clear non-cardiac cause, directly related to the death could be withheld. Indications for repeat procedures were evaluated on a case-by-case basis.

\section{Statistical analysis}

SPSS version 24 (IBM Corp, Armonk, NY) was used for statistical analysis. Continuous variables are summarized by mean \pm standard deviation or median \pm interquartile range (IQR, 25-75 ${ }^{\text {th }}$ percentile), depending on the normality of distribution. Categorical variables are presented by frequencies and percentages. Kaplan-Meier analysis with log-rank tests were used to calculate VA-free and overall survival over time and compare survival rates between subgroups. Univariable Cox proportional hazards regression was used to identify predictors of VA-free and overall survival (Supplemental Table). Variables significant at the 0.2 level in univariate analyses were considered in an automated stepwise multivariate Cox regression model. All statistical tests were 2-tailed. A p-value $<0.05$ was considered statistically significant.

\section{Results}

\section{Population (Figure 2, Table 1)}

During the study period, 842 ablations for any ventricular arrhythmia were performed in 707 patients. Five hundred and seven pts were excluded because of non-qualifying diagnoses ( $\mathrm{n}=206$ non-sustained VT or idiopathic ventricular fibrillation, $n=99$ idiopathic VT, $n=177$ non-ischemic, $n=6$ mixed cardiomyopathy, n=19 ICMP without MI). An additional 31 patients were excluded due to prior VT ablation and 10 patients did not consent. The study population consisted of 159 consecutive patients referred for first ablation of post-MI VT. Eighty-six (54\%) had VT storm and 84 of 115 patients with ICD prior to ablation (73\%) had appropriate shock(s). Most $(n=118,74 \%)$ were treated with amiodarone prior to ablation (usual daily dose $200 \mathrm{mg}$ ). 


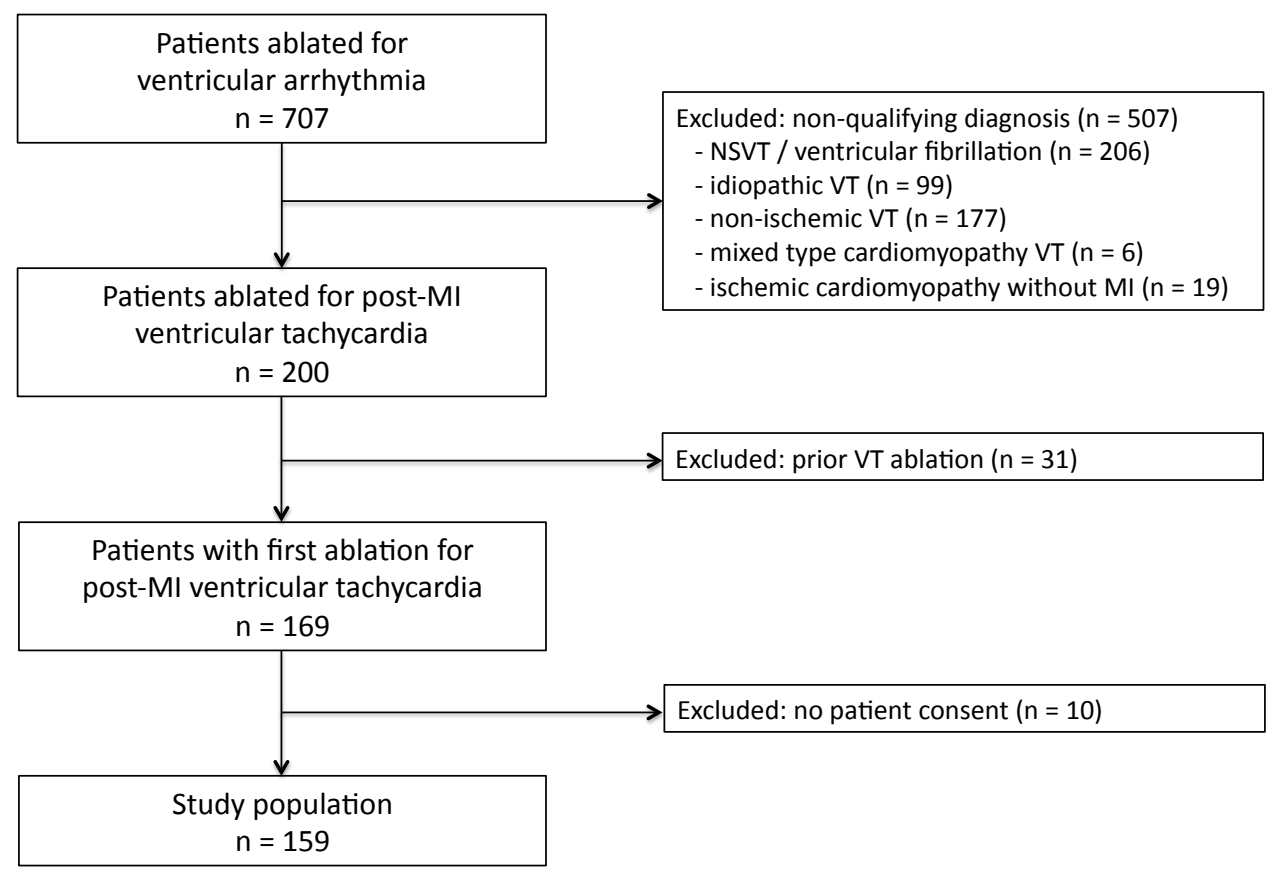

Figure 2: Flow diagram of the study population. 
Table 1: Characteristics of the study population $(n=159)$

Age, y

Male gender

$152(96 \%)$

Coronary risk factors

Arterial hypertension

$99(62 \%)$

Diabetes

$35(22 \%)$

Dyslipidemia

$123(77 \%)$

Smoking

$90(57 \%)$

LVEF, \%

$34 \pm 11$

LVEF $\leq 30 \%$

$74(47 \%)$

Percutaneous coronary intervention

$93(58 \%)$

Coronary artery bypass grafting

$39(25 \%)$

NYHA class

I/II

$127(80 \%)$

III/IV

$32(20 \%)$

Serum creatinin, $\mu \mathrm{mol} / 1$

$111 \pm 56$

Creatinin clearance CKD-EPI, $\mathrm{ml} / \mathrm{min} / 1.73 \mathrm{~m}^{2}$

$71 \pm 29$

Antiarrhythmic drugs

number

$1.8 \pm 0.6$

beta-blocker

$153(96 \%)$

sotalol/class I

$17(11 \%)$

amiodarone

$118(74 \%)$

Other cardiovascular drugs

ACE-inhibitor/angiotensin receptor blocker

$125(79 \%)$

mineralocorticoid receptorblocker

$61(38 \%)$

statin

$127(80 \%)$

Cardiac device

ICD

$146(92 \%)$

ICD only implanted after ablation

$31 / 146$

CRT

$41(26 \%)$

Indication for ablation

VT storm

$86(54 \%)$

Appropriate ICD shock

$84 / 115(73 \%)$

ACE: angiotensin-converting enzyme; CRT: cardiac resynchronization therapy;

ICD: implantable cardioverter defibrillator; LVEF: left ventricular ejection fraction; NYHA: New York Heart Association; VT: ventricular tachycardia 


\section{Procedural characteristics (Table 2)}

LV access was obtained by transseptal and retrograde approach in 141 (89\%) and 51 (32\%) patients. Dual transseptal and retrograde access was performed in 35 (22\%) and percutaneous pericardial access in $46(29 \%)$ patients. In two patients presenting with refractory VT storm requiring urgent ablation, only pericardial access was obtained due to LV thrombus.

ME mapping catheters were used in 89 (56\%), 3D-EAM in 119 (75\%), and RTII in 57 (36\%) patients. RTII consisted of MDCT in 53 and MRI data in 23 cases (combined in 19 patients).

LAVA were identified in 146 patients ( $92 \%$, endocardially $n=141 / 157$; epicardially $n=36 / 46)$. In 13 patients (8\%) no LAVA were mapped (4 of them with both endo- and epicardial access).

In 39 patients (25\%) VT was mechanically induced during substrate mapping. In 116 of the remaining 120 patients, inducibility by programmed stimulation was tested at baseline. In 77 of them, VT was inducible. Seventy-four patients had some degree of VT mapped and in total, a mean of 1.6 \pm 1.6 VTs were observed during the ablation procedure. Clinical VT was observed in 89 (56\%) and non-clinical in $75(47 \%)$ patients. A total of 39 pts had no inducible VT and in 4 patients, inducibility was not tested because of hemodynamic instability. 


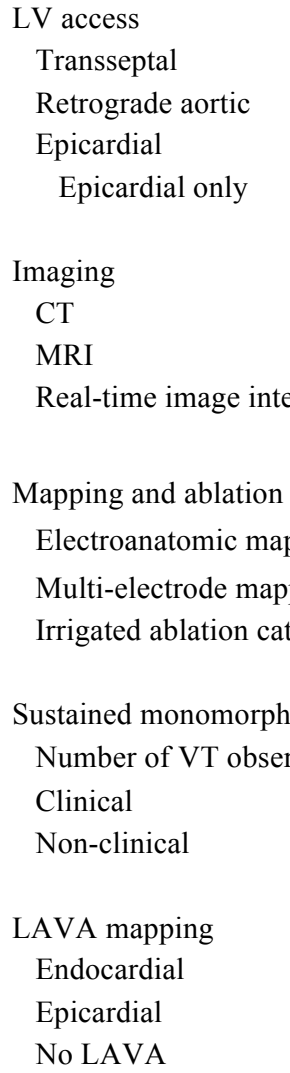

$141(89 \%)$

$51(32 \%)$

$46(29 \%)$

$2(1 \%)$

$100(63 \%)$

87 (55\%)

38 (24\%)

$57(36 \%)$

$119(75 \%)$

$89(56 \%)$

$159(100 \%)$

$116(73 \%)$

$1.6 \pm 1.6$

$89(56 \%)$

75 (47\%)

$141 / 157(90 \%)$

$36 / 46(78 \%)$

$13 / 159(8 \%)$

CT: computed tomography; LAVA: local abnormal ventricular activities; LV: left ventricular; MRI: magnetic resonance imaging

\section{Acute procedural outcome (Table 3)}

Complete LAVA elimination was achieved in 93 of 146 patients (64\%) using a mean RF time of $36 \pm 20 \mathrm{~min}$. In one patient with septal involvement, additional transcoronary ethanol ablation was performed during the index procedure in order to achieve complete VT/LAVA elimination. In 53 pts (36\%), LAVA elimination was incomplete. In 7 cases this was due to anatomical reasons (epicardial proximity of coronary arteries in 2 and septal localization in 5), in 3 cases the procedure was shortened to due hemodynamic instability and in the remaining cases, some LAVA persisted despite extensive ablation. In 13 patients without LAVA, ablation was based on activation/entrainment mapping of induced VT $(n=7)$, and on voltage maps combined with pace mapping if non-inducible $(n=6)$. 
Non-inducibility after ablation was achieved in 94 of 110 tested patients (85\%). In 16 patients (7 with complete LAVA elimination), a non-clinical monomorphic VT (cycle length $<270 \mathrm{~ms}$ ) remained inducible.

Epicardial ablation was performed in 27 of the 36 patients with epicardially mapped LAVA (in 2 patients no epicardial RF was performed due to proximity of coronary artery and in the remaining patients epicardial LAVA were eliminated after endocardial ablation).

Table 3: Procedural results $(\mathbf{n}=159)$

\section{Outcome defined by LAVA elimination}

Complete elimination

$93 / 146(64 \%)$

Incomplete elimination

$53 / 146(36 \%)$

No LAVA mapped

$13 / 159(8 \%)$

\section{Outcome defined by inducibility}

Inducibility tested after ablation

Non-inducibility of any VT

$94 / 110(85 \%)$

Clinical VT inducible

$0 / 110(0 \%)$

Only non-clinical VT inducible

$16 / 110(15 \%)$

\section{Procedural parameters}

Radiofrequency time, min $35.9 \pm 20.4$

Epicardial ablation performed, $\mathrm{n}$

Epicardial radiofrequency time, $\min$

$9.8 \pm 11.7$

Fluoroscopy time, min

$48.2 \pm 23.7$

Procedure time, $\mathrm{min}$

$247.9 \pm 78.2$

LAVA: local abnormal ventricular activities; VT: ventricular tachycardia

\section{Adverse events}

Complications were observed in 12 patients (7.5\%). Nine patients experienced epicardial bleeding, of which 4 required surgical intervention. In 2 patients ablation resulted in complete AV block and 1 patient required non-invasive ventilation due to pulmonary edema shortly after the procedure. Two patients with advanced heart failure who presented with VT storm died within 24 hours following the procedure, one as a result of low-flow state and one as a result of low flow and VT storm recurrence. 


\section{Clinical outcomes (Table 4)}

Arrhythmia recurrence after a single procedure:

During a median follow-up of 47 months (IQR 33 - 82), the single procedure ventricular arrhythmia (VA)-free survival was $55 \%$. The VA-free survival declined from $73 \%$ at 1 year to $68,61,55$ and $49 \%$ after 2, 3, 4 and 5 years respectively (Figure 3).
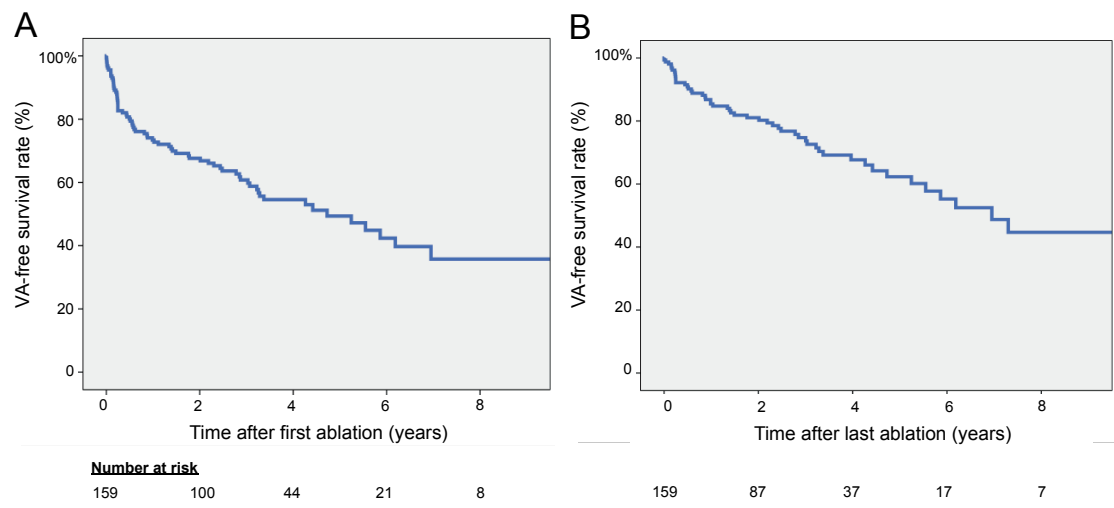

Figure 3: Ventricular arrhythmia (VA)-free survival after single (A) and including repeat (B) ablation procedures.

The majority (58\%) of first VA recurrence occurred during the first year (median 217 days, IQR 79899 ) post-ablation, but in $25 \%$ of patients first recurrence only occurred after 2.5 years, up to 6.9 years. The proportion of patients experiencing VT storm decreased from $54 \%$ before to $10 \%$ after a single procedure. The proportion of ICD patients experiencing appropriate shocks declined from $73 \%$ to $19 \%$ after single ablation. In 74 ICD patients implanted at least one year before ablation and who survived at least one year, the VT burden was significantly reduced from a median of 20 episodes (IQR 8-58) the year before to a median of 0 (IQR 0-0) episodes during the year after ablation. In $91 \%$ of these 74 pts, the reduction in VT burden was more than $87 \%$ (Figure 4). In none of the patient, recurrent ischemia occurred prior to the first VA-recurrence. In two patients intercurrent heart failure occurred prior, but remote (5 and 7 months) to the first VA-recurrence. During follow-up, antiarrhythmic drugs were discontinued in $52 \%$ of patients, with reduction of amiodarone use from 118 to 54 patients. 


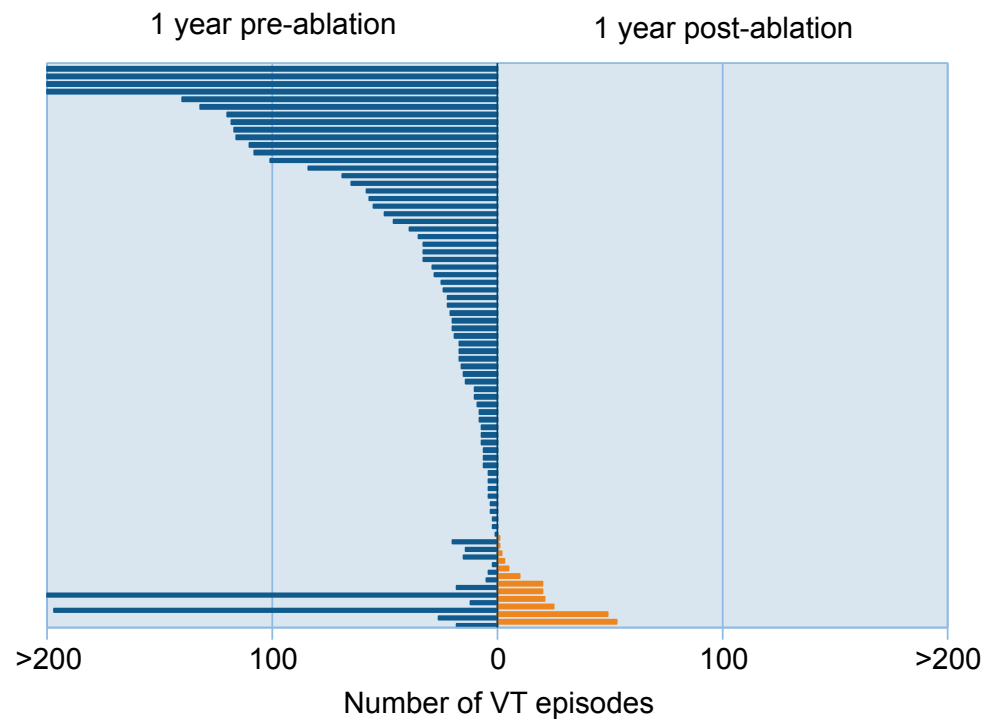

Figure 4: There were 74 patients with implantable cardioverter-defibrillator (ICD) at least 1 year prior to ablation who survived for 1 year. Each line represents an individual patient; blue lines indicate pre-ablation and orange lines post-ablation. Upper boundaries are truncated at 200 episodes. Patients are arranged by recurrences during follow-up, ranging from greatest (bottom) to least (top). In $91 \%$ of these 74 patients, the reduction in VT burden was more than $87 \%$.

\section{Arrhythmia recurrence including redo procedures:}

Repeat procedures were performed in 29 patients (18\%) (mean total procedures $1.3 \pm 0.6 /$ patient). All repeat procedures were performed with the same strategy and procedural endpoints, except in two patents in whom repeat ablation consisted of transcoronary ethanol injection. Including repeat procedures the VA-free survival increased to $69 \%$, and recurrence of storm decreased to $2 \%$ and appropriate shocks to $9 \%$ of ICD patients, during a median follow-up of 46 months (IQR $32-81$ ). Including repeat procedures, the VA-free survival was $85,81,75,68$ and $62 \%$ at $1,2,3,4$ and 5 years respectively (Figure 3).

\section{Overall survival}

The overall survival was $91,86,84,80$ and $77 \%$ at 1, 2, 3, 4 and 5 years (Figure 5). During follow-up 40 patients died: 3 patients died from arrhythmia despite implanted ICD, 20 from cardiovascular morbidity (terminal heart failure), and 17 from non-cardiovascular causes. Two patients received cardiac transplant during follow-up. 


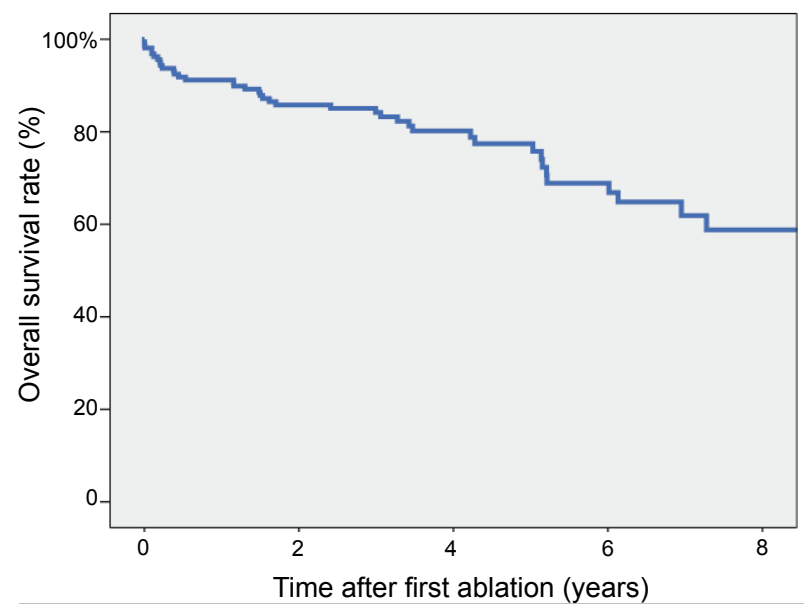

Number at risk

159

122

61

34

16

Figure 5: Overall survival timed from the first ablation procedure (including repeat procedures in $18 \%$ of patients). 
Table 4: Clinical outcome data $(n=159)$

\section{After first procedure:}

Ventricular arrhythmia-free survival

$88(55 \%)$

VT storm

$16(10 \%)$

Appropriate shock in ICD patients

$28 / 146(19 \%)$

\section{Including repeat procedures:}

Ventricular arrhythmia-free survival

$109(69 \%)$

VT storm

$3(2 \%)$

Appropriate shock in ICD patients

$13 / 146(9 \%)$

Number of procedures

1 procedure

$130(82 \%)$

2 procedures

$20(13 \%)$

3 procedures

$7(4 \%)$

4 procedures

$2(1 \%)$

Mean number of procedures

$1.3 \pm 0.6$

Interval between first and last procedure, days

197 (IQR 98-885)

\section{All-cause mortality}

Arrhythmogenic cardiac death

$40(25 \%)$

Cardiovascular death

$3(2 \%)$

Non-cardiovascular death

$20(13 \%)$

$17(11 \%)$

Heart transplant

$2(1 \%)$

\section{Antiarrhythmic drugs use at last follow-up}

amiodarone

$54(34 \%)$

sotalol/class I drug

$11(7 \%)$

beta-blocker

$145(91 \%)$

ICD: implantable cardioverter defibrillator; VT: ventricular tachycardia

\section{Subgroup analysis}

\section{Impact of technological evolution}

The use of ME mapping catheters and real-time image integration was implemented based on availability of the technology over the study period, independently from patient characteristics. They significantly improved the VA-free survival, and with trend to improve overall survival as well (Figure 6). Use of ME mapping combined with RTII resulted in a VA-free survival of $86 \%$ at 1 and $65 \%$ at 4 years follow-up, compared to 57 and $46 \%$ with conventional mapping ( $p=0.043$ ). Similarly 
overall survival improved to $94 \%$ at 1 and $91 \%$ at 4 years, compared to 83 and $74 \%$ with conventional mapping $(\mathrm{p}=0.062)$.
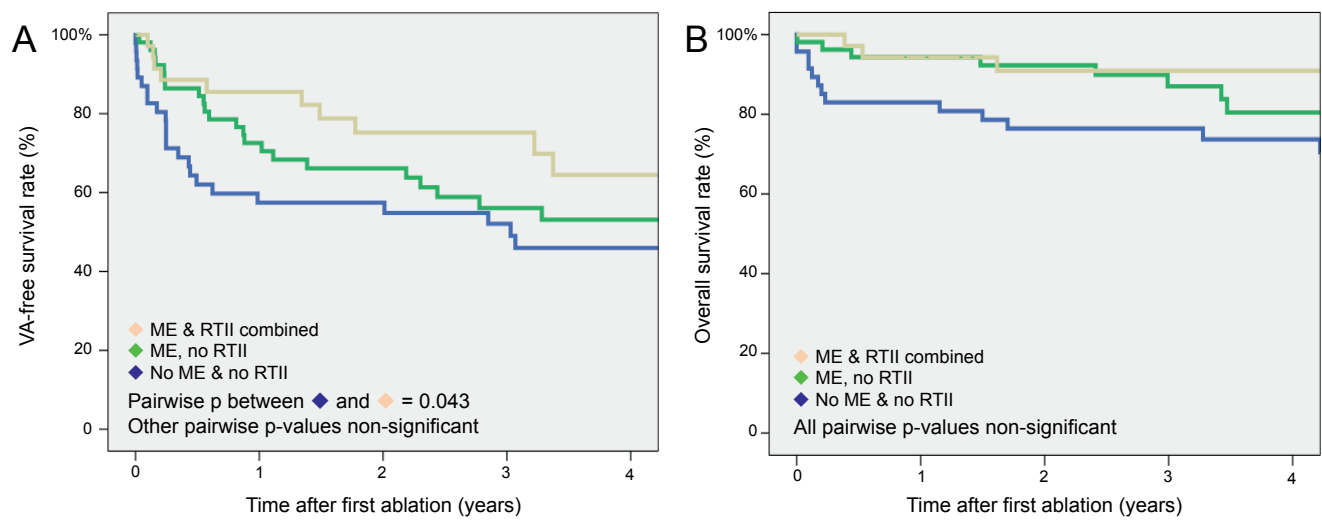

Figure 6: Impact of different mapping technologies. Ventricular arrhythmia (VA)-free (A) and overall (B) survival rate in procedures performed without multielectrode (ME) mapping and real-time image integration (RTII) (blue, $\mathrm{n}=35$ ), with ME mappig but without RTII (green, $\mathrm{n}=53$ ), and with combined ME mapping and RTII (yellow, n=47).

\section{LAVA elimination}

Achievement of complete LAVA elimination was also associated with improved outcome. At a median follow-up of 47 months after a single procedure, VA-free survival was $63 \%$ if complete LAVA elimination was achieved, compared to $44 \%$ if LAVA elimination was incomplete or if no LAVA were mapped $(\mathrm{p}=0.001)$ (Figure 7). Further, complete LAVA elimination was associated with less recurrence of VT storm ( 5 vs. 17\%, p=0.030). Recurrence of at least one ICD shock and overall mortality did not differ significantly. Including repeat procedures, the VA-free survival was $73 \%$ at median follow-up of 46 months if complete LAVA elimination was achieved after the final procedure, compared to $57 \%$ if LAVA persisted $(\mathrm{p}=0.009)$ (Figure 8 ).

The long-term arrhythmia-free survival of other subgroups is detailed in Table 5. 


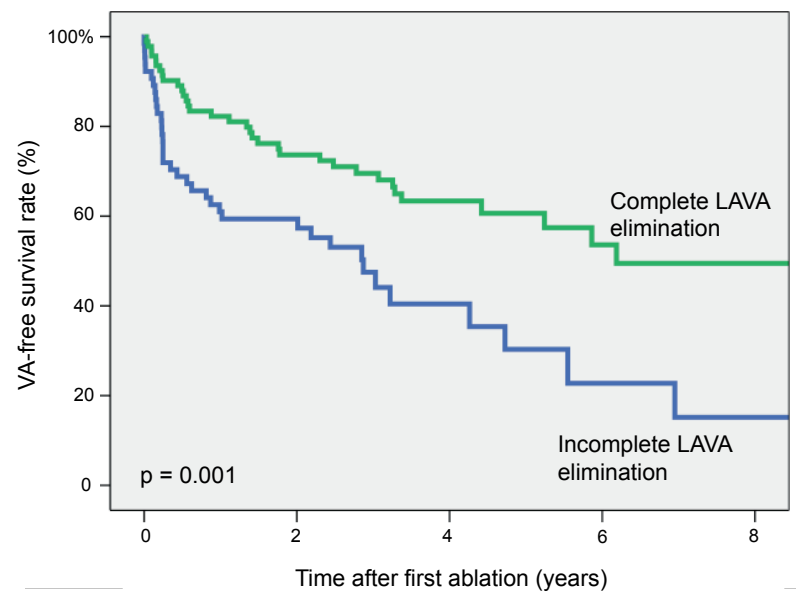

$\begin{array}{lrrrrr}\text { Number at risk } & & & & \\ \text { Complete LAVA elimination } & 93 & 58 & 28 & 14 & 5 \\ \text { Incomplete LAVA elimination } & 66 & 29 & 9 & 3 & 2\end{array}$

Figure 7: Ventricular arrhythmia (VA)-free survival rate in procedures with and without complete elimination of local abnormal ventricular activities (LAVA) after a single ablation procedure.

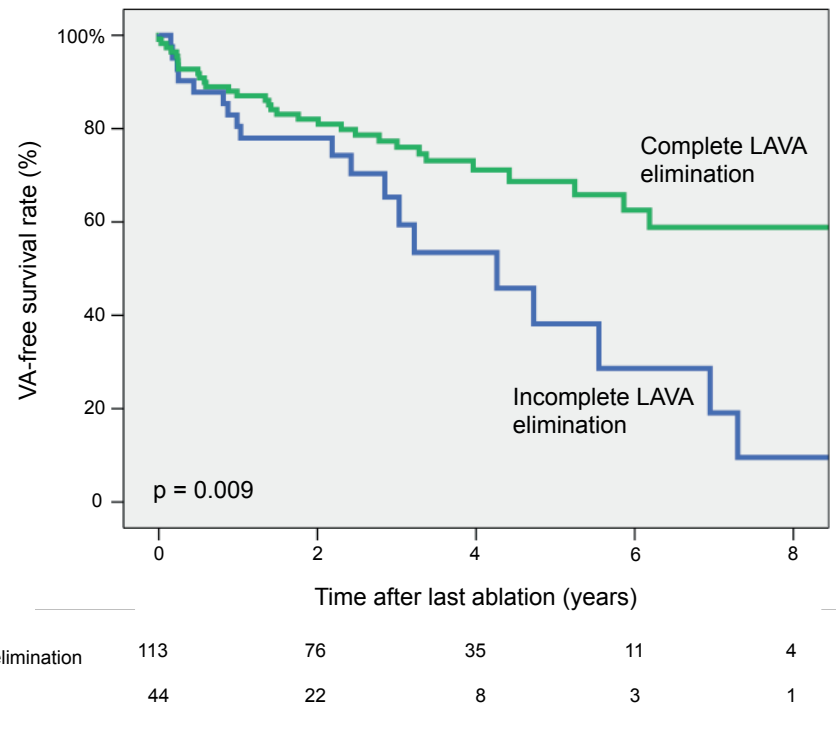

Figure 8: Ventricular arrhythmia (VA)-free survival rate in procedures with and without complete elimination of local abnormal ventricular activities (LAVA) after the last ablation procedure (including repeat ablation procedures in $18 \%$ of patients). 


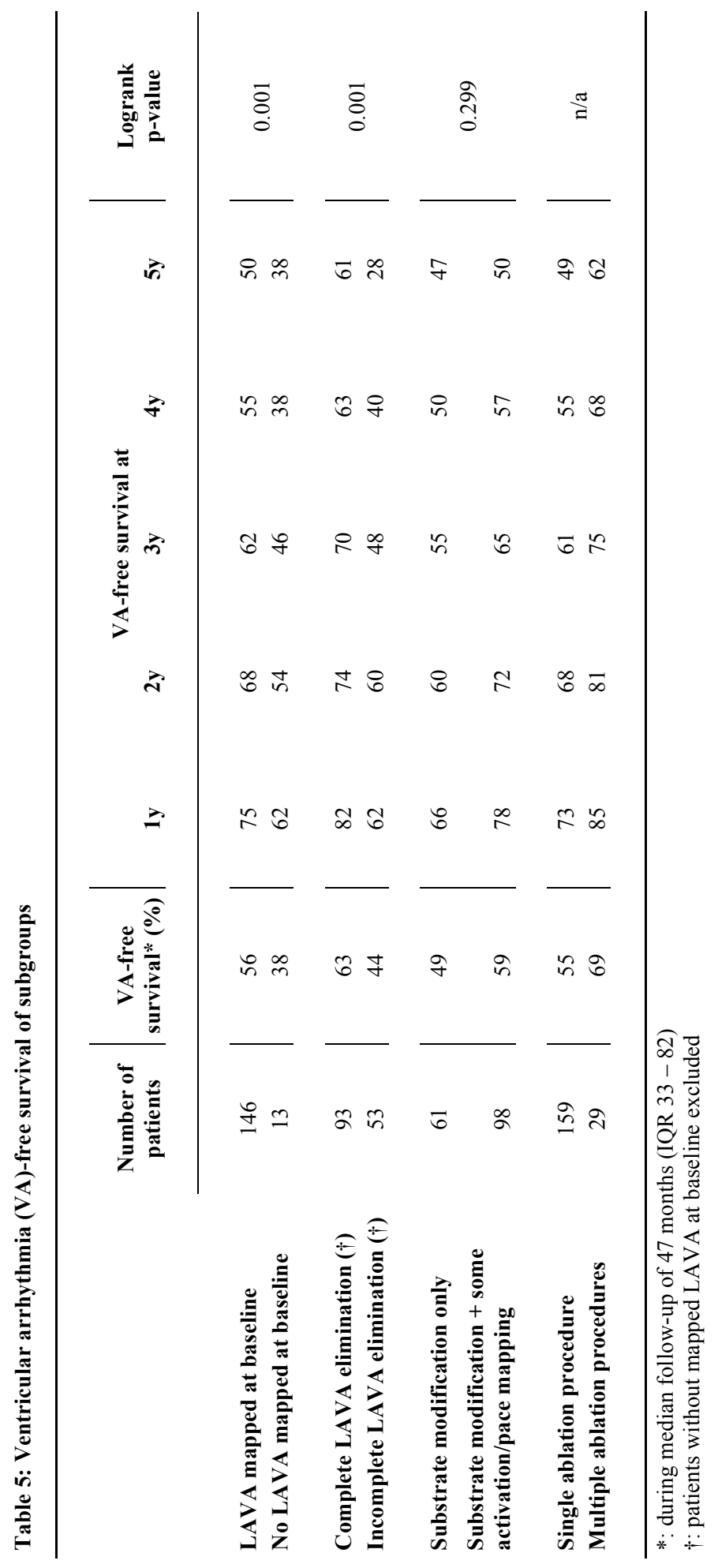




\section{Substrate comparison between index and repeat procedures}

Twenty-seven patients underwent a repeat RF ablation procedure. In none of the patients, recurrent ischemia occurred between the index and repeat procedures. Between the index and first repeat procedure, scar areas were unchanged, but induced VTs were mostly different (Figure 9A). Only in 2 patients, 1 of the induced VTs in the repeat procedure had the same axis with similar morphology (but different cycle length) as in the index procedure. LAVA patterns in repeat procedures were mostly due to recurrence of previously successfully ablated LAVA or residual LAVA from the index procedure (Figure 9B). In 1 patient septal LAVA were de novo mapped (with the same 3D mapping system and ME mapping catheter) and ablated in the repeat procedure.

A

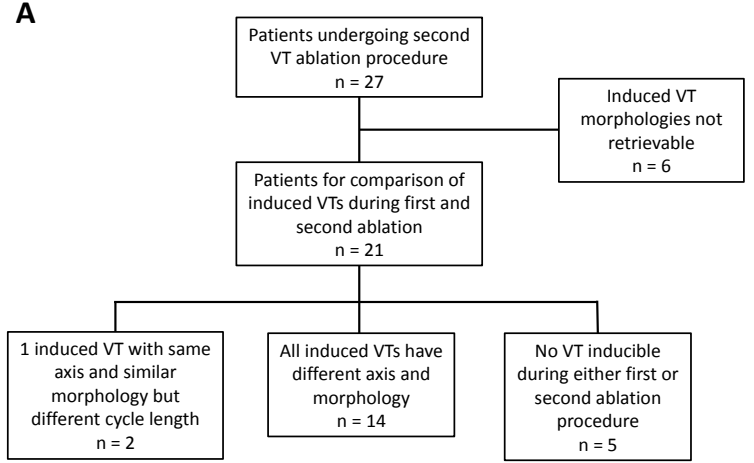

B

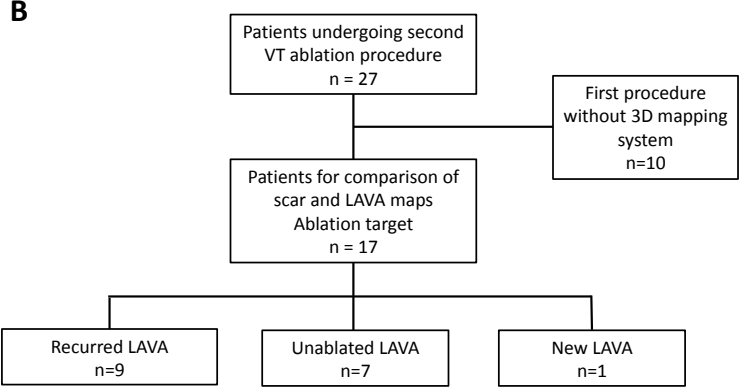

Figure 9: Comparison between index ablation and first repeat ablation procedure of (A) induced ventricular tachycardia morphologies and (B) characteristics of local abnormal ventricular activities.

\section{Discussion}

Our study is the first to report the long-term results of substrate-based catheter ablation of drugrefractory post-MI VT, targeting LAVA elimination. The main findings are:

- LAVA are successfully identified in the vast majority of patients (92\%) referred for post-MI VT ablation, when using a strategy of meticulous mapping. The endpoint of LAVA elimination in addition to non-inducibility is feasible and effective for arrhythmia control.

With a single procedure, $73 \%$ of the patients are free from arrhythmia at 1 year. This success rate decreases over time, but $49 \%$ of patients are still free from any ventricular arrhythmia at 5 years. At the modest cost of repeat procedures in $18 \%$ of pts, this result is improved to $85 \%$ arrhythmiafreedom at 1 year, remaining $62 \%$ after 5 years. In addition only $9 \%$ of pts had appropriate shocks over a mean of 4 years follow-up. The reduction in VT burden was more than $87 \%$ in $91 \%$ of patients after a single procedure. This strategy allowed for a substantial discontinuation of 
antiarrhythmic drugs and amiodarone use was reduced by $54 \%$, this benefit being maintained at 5 years.

- Complete LAVA elimination, which is possible in $64 \%$ of patients, is associated with better outcome. Improved mapping by implementing modern technologies such as ME mapping catheters and integration of high-definition imaging of scar and anatomy improved both short and long-term outcome, with freedom of VA recurrence in $86 \%$ of patients at 1 year and $65 \%$ at 4 years follow-up.

- First VT recurrence occurred at median 217 days, but extended up to 6.9 years after the first procedure. In fact $25 \%$ of first VT recurrence occurred only 2.5 years after the first ablation procedure, i.e. longer than the follow-up period of most previous studies on substrate-based VT ablation.

- Albeit the all-cause mortality of this patient population was high, amounting $23 \%$ at 5 years, the incidence of arrhythmogenic death was low. Fifty percent of mortality was caused by terminal heart failure.

- In repeat VT ablation procedures, the arrhythmogenic substrate and scar remained generally unchanged. Incomplete or insufficient index substrate ablation was found in $94 \%$ of redo procedures.

\section{Activation and entrainment mapping vs. substrate modification}

Initial strategies for ablation of post-MI VT predominantly used activation and/or entrainment mapping to identify the critical isthmus as the ideal target for RF delivery. ${ }^{9}$ Limited linear ablation transecting these isthmuses would interrupt reentry, terminate the mapped VT and render the targeted VT non-inducible. This strategy and the endpoint of non-inducibility are hampered by several limitations, such as baseline inducibility, sustainability and hemodynamic tolerance of the VT, reproducibility of inducibility, etc... Despite all these limitations, non-inducibility has been reported as an interesting endpoint, correlated with improved clinical outcomes, with non-inducibility of both clinical and non-clinical VT being superior to non-inducibility of clinical VT only. ${ }^{2}$ Inducible nonclinical VTs observed after such ablation suggests the persistence of a substrate able to sustain VT and likely to be clinically relevant in the future, as shown by superior outcomes when ablated. In the only randomized trial comparing extensive substrate-based ablation to a strategy only targeting clinical and stable VTs in patients with ICMP, the substrate-based approach resulted in a superior VT-free survival at 1 year follow-up $(84.5 \%$ vs. $51.7 \%) .{ }^{10}$ For these reasons a substrate-based ablation strategy, has gained prominence in the current era of VT ablation. Several different methods of substrate modification have been described, such as elimination of LAVA, ${ }^{7}$ late potential abolition, ${ }^{6}$ scar dechanneling, ${ }^{11}$ scar core isolation ${ }^{12}$ and scar homogenization. ${ }^{10,13}$ 


\section{Differences in substrate modification techniques}

Several groups have tried to modify potentially arrhythmogenic surviving myocardial tissue by targeting late potentials in scar regions. ${ }^{14-16}$ In routine clinical practice, electroanatomic mapping is typically performed in sinus rhythm or during right ventricular pacing. However it has recently been demonstrated that the activation direction of the wavefront impacts the voltage amplitude. ${ }^{17}$ Tung et al performed voltage maps with at least two different activation wavefronts and found that $18 \%$ of critical VT isthmuses were within low-voltage areas during 1 wavefront, but were within normal amplitude $(>1.5 \mathrm{mV})$ during another activation wavefront. ${ }^{17}$ The timing of near-field signals from poorly coupled surviving bundles is also dependent on the activation wavefront, and sometimes ventricular (and decremental) pacing is needed to unmask them. ${ }^{14}$ Hence substrate-based ablation targeting solely late potentials or confined to voltage-defined scar might miss part of the arrhythmogenic substrate. The LAVA approach aims to overcome these limitations, by not being limited to late potentials (occurring after the QRS) or confined to low-voltage regions. In fact earlier coupled LAVA are targeted first in our approach and can result in elimination of later LAVA using limited RF delivery, as they may indicate a channel entrance to the scar.

\section{Comparison with other outcome studies}

Data on outcomes of VT ablation beyond 24 months of follow-up are scarce. Our study compared favorably to 2 recently published studies reporting long-term outcomes of VT ablation in ICMP patients, predominantly guided by activation and entrainment mapping of inducible and hemodynamically tolerated VTs. ${ }^{2-3}$ In the largest study, Kumar et al presented a single center retrospective series of 358 patients with ICMP undergoing VT ablation. ${ }^{2}$ Including repeat ablation in $25 \%$ of pts (mean $1.3 \pm 0.6$ procedures/pt), the VA-free and overall survival were around 54 and $77 \%$ respectively at 5 years FU, compared to 62 and $77 \%$ in our study. The prospective multicenter PostApproval THERMOCOOL VT trial, reported follow-up up to 3 years after EAM-system-guided VT ablation in 249 patients with coronary artery disease $\left(85.4 \%\right.$ with previous MI). ${ }^{3}$ While acute procedural success, defined as non-inducibility of the targeted VT, was achieved in $75.9 \%$, the singleprocedure freedom from arrhythmia was considerably lower when compared to our study amounting only $41 \%$ after 3 years (with possible underreporting of asymptomatic VT episodes due to recall bias). The overall survival was $75 \% 3$ years post-ablation. The HELP-VT study reported medium-term outcomes 164 patients undergoing VT ablation predominantly guided by activation/entrainment mapping. ${ }^{18}$ VT-free survival amounted $57 \%$ at 12 months and $43 \%$ at a mean follow-up of 27 (16-37) months.

Outcomes from substrate-based VT ablation studies have only been reported with limited follow-up. ${ }^{4-}$ 6,10,12-13 Silberbauer et al reported 160 patients undergoing substrate modification (20\% epicardial mapping) targeting late potential abolition combined with non-inducibility for post-MI VT. ${ }^{6}$ Sixty- 
five percent had baseline LP, of which 77\% underwent successful abolition. After a mean follow-up time of 18.8 (12.2-29.0) months, VT-free survival was 68\%. Proietti et al reported outcomes of 87 patients with ICMP undergoing EAM-guide substrate-based VT ablation targeting LP in scar regions and regions in the scar-border zone with good pace-map correlation to clinical VTs. ${ }^{5}$ Non-inducibility of any VT was achieved in $69 \%$ of patients after a mean RF time of $96 \pm 51$ minutes, but in $21 \%$ of patients clinical VT remained inducible at the end of the procedure. After a mean follow-up of $21 \pm 10$ months, the VA-free survival was $74 \%$ with overall survival of $91 \%$.

Better short-term results were reported after more extensive substrate modification targeting not only delayed but all abnormal potentials within and around scar ("scar homogenization"). Di Biase et al reported outcomes of endo- and epicardial scar homogenization in 43 patients with ICMP and electrical storm. ${ }^{10}$ Epicardial access was obtained in all of them, but epicardial ablation was only performed in $33 \%$ if abnormal potentials remained after endocardial ablation. The acute procedural endpoint of non-inducibility of any VT was achieved in 100\%. At a mean follow-up of 21 months the VT-free survival was $81 \%$. Despite extensive ablation (mean RF time $74 \pm 21 \mathrm{~min}$ ) and systematic pericardial access, no major complications were reported. The results of this ablation approach were confirmed in the VISTA Randomized Multicenter Trial, in which 58 patients underwent extensive ablation of all abnormal electograms in endocardial scar, but epicardial access was limited to $10 \%$ of patients in whom VT remained inducible after endocardial ablation. ${ }^{13}$ After a mean RF time of $86 \pm 21 \mathrm{~min}$, all abnormal potentials within scar were successfully eliminated in $84 \%$ of cases. At 12 months follow-up, the VT-free survival was $84.5 \%$ and the overall-survival $88.1 \%$. Both the scar homogenization and LAVA elimination strategy aim to modify the substrate more comprehensively, although the LAVA strategy may allow for less extensive ablation ( $36 \pm 20$ vs. $74 \pm 21$ and $86 \pm 21 \mathrm{~min}$ ), especially if earlier coupled LAVAs are targeted first. Short-term freedom from arrhythmia was however $>80 \%$ with the scar homogenization strategy, compared to 73 and $68 \%$ at 1 and 2 years follow-up after a single procedure in our patient series.

\section{Impact of strategy variants}

In our approach, identification of the substrate is contingent on the detectability of LAVA by the mapping catheters used. Our group previously demonstrated that the use of ME mapping catheters is associated with increased LAVA detection due to reduction of the far-field signal and magnification of the near-field component. ${ }^{19}$ In our study, ME mapping catheters were used in only $56 \%$ of patients. Of note, ME catheters were not used in any of the above-mentioned studies of substrate based VT ablation.

Our research group also reported the added value of real-time integration of preprocedural imaging with DE-MRI or MDCT in post-MI VT ablation..$^{20}$ Both DE on MRI as well as wall thinning on MDCT correlate well with low voltage areas on EAM and the presence of LAVA. ${ }^{21-22}$ After substrate 
mapping, real-time integration of image-derived substrate has been shown to motivate the operator to perform additional mapping in the majority of cases with extra LAVA detection in all of them. In addition, in some patients, pre-procedural imaging influenced the decision to perform epicardial access and RTII modified epicardial ablation strategy due to proximity of coronary arteries or phrenic nerve. $^{20}$

In our most recent approach of LAVA ablation, RTII and ME mapping catheters are therefore consistently implemented and resulted in significantly improved outcomes with a VA-free survival of $86 \%$ at 1 year, remaining $65 \%$ at 4 years of follow-up and overall survival of 94 and $91 \%$ at 1 and 4 years respectively. With the contemporary LAVA-strategy, (short-term) results comparable to the scar homogenization strategy are achieved, but with more limited RF delivery. ${ }^{10,13}$ Given these results, a randomized, head-to-head comparison of the contemporary LAVA elimination approach and the scar homogenization approach could be of interest.

\section{Reasons for VA recurrence}

Potential reasons for VA recurrence are 1. inadequate mapping of the substrate, 2. inadequate ablation with lesion regression and reconnection of critical isthmuses, 3. inadequate ablation due to anatomic limitations (mid-myocardial substrate, substrate in vicinity of the coronary arteries or phrenic nerve), 4. progression of the substrate, and 5. progression of neurohormonal and autonomic remodeling. ${ }^{23}$ While inadequate mapping can be improved by triple (both retrograde and transseptal endocardial, and epicardial) LV access and use of ME mapping catheters and RTII, intramyocardial infusion-needle and bipolar irrigated RF catheter ablation may offer benefit when conventional RF ablation lesions are of insufficient depth to reach a mid-myocardial substrate. ${ }^{24,25}$ Comparable to previously published series in post-MI VT ablation ${ }^{26}$, recurrent VTs had new morphologies in our study. During repeat ablation, $\geq 1$ VT with different morphology from the index procedure was induced in all evaluable patients and only in a minority, a VT with a same exit as during the index procedure was induced. Despite different VT morphologies, the arrhythmogenic substrate generally remained unchanged between index and repeat procedures. In redo procedures, LAVA were generally located in the same area as during the index ablation, either due to recurrence of previously ablated LAVA or residual, unablated LAVA from the index procedure (in several cases possibly missed by mapping with the ablation catheter in the index, but detected by ME mapping in the redo procedure). The recurrence of previously ablated LAVA advocates the importance of effective and durable RF lesions. Without recurrent ischemia, substrate progression in post-MI VT appears to be the exception, as scar areas were unchanged between index and repeat procedures and 'new' LAVA in repeat procedures were very rare.

\section{Limitations}

Our study is a single-center, non-randomized study and represents the experience of skilled operators. Outcomes may therefore not be generalized to lower volume centers. Although the endpoint of the 
ablation procedure remained unchanged, additional mapping technologies were implemented based upon availability over the study period resulting in improved outcomes. Therefore, the outcomes of the entire study cohort were hampered by the procedures performed at the beginning of the study period, but this was clarified by providing subgroup analysis. The discontinuation of antiarrhythmic drugs was not part of a study protocol and therefore up to the discretion of the treating physician. As antiarrhythmic drugs were not systematically discontinued at the first follow-up consultation, outcomes of ablation have to be interpreted as adjunct to medical therapy, but reflect the real-world clinical setting.

\section{Conclusions}

In this mono-centric study, substrate modification by LAVA elimination for post-MI VT resulted in a substantial reduction of VT storm, VT burden and need for ICD shocks. Both short- and long-term outcomes were improved by repeat ablation in a limited number of patients. Complete LAVA elimination was associated with improved VA-free survival. When combined with ME mapping and RTII, LAVA ablation was very successful at one year, with an annual 5\% attrition rate. 


\section{References}

1. Sapp JL, Wells GA, Parkash R, Stevenson WG, Blier L, Sarrazin JF, Thibault B, Rivard L, Gula L, Leong-Sit P, Essebag V, Nery PB, Tung SK, Raymond JM, Sterns LD, Veenhuyzen GD, Healey JS, Redfearn D, Roux JF, Tang AS. Ventricular Tachycardia Ablation versus Escalation of Antiarrhythmic Drugs. N Engl J Med. 2016;375:111-121.

2. Kumar S, Romero J, Mehta NK, Fujii A, Kapur S, Baldinger SH, Barbhaiya CR, Koplan BA, John RM, Epstein LM, Michaud GF, Tedrow UB, Stevenson WG. Long-term outcomes after catheter ablation of ventricular tachycardia in patients with and without structural heart disease. Heart Rhythm. 2016;13:1957-1963.

3. Marchlinski FE, Haffajee CI, Beshai JF, Dickfeld TM, Gonzalez MD, Hsia HH, Schuger CD, Beckman KJ, Bogun FM, Pollak SJ, Bhandari AK. Long-Term Success of Irrigated Radiofrequency Catheter Ablation of Sustained Ventricular Tachycardia: Post-Approval THERMOCOOL VT Trial. J Am Coll Cardiol. 2016;67:674-683.

4. Kumar S, Baldinger SH, Romero J, Fujii A, Mahida SN, Tedrow UB, Stevenson WG. SubstrateBased Ablation Versus Ablation Guided by Activation and Entrainment Mapping for Ventricular Tachycardia: A Systematic Review and Meta-Analysis. J Cardiovasc Electrophysiol. 2016;27:1437-1447.

5. Proietti R, Essebag V, Beardsall J, Hache P, Pantano A, Wulffhart Z, Juta R, Tsang B, Joza J, Nascimento T, Pegoraro V, Khaykin Y, Verma A. Substrate-guided ablation of haemodynamically tolerated and untolerated ventricular tachycardia in patients with structural heart disease: effect of cardiomyopathy type and acute success on long-term outcome. Europace. 2015; 17:461-467.

6. Silberbauer J, Oloriz T, Maccabelli G, Tsiachris D, Baratto F, Vergara P, Mizuno H, Bisceglia C, Marzi A, Sora N, Guarracini F, Radinovic A, Cireddu M, Sala S, Gulletta S, Paglino G, Mazzone P, Trevisi N, Della Bella P. Noninducibility and late potential abolition: a novel combined prognostic procedural end point for catheter ablation of postinfarction ventricular tachycardia. Circ Arrhythm Electrophysiol. 2014;7:424-435.

7. Jaïs P, Maury P, Khairy P, Sacher F, Nault I, Komatsu Y, Hocini M, Forclaz A, Jadidi AS, Weerasooryia R, Shah A, Derval N, Cochet H, Knecht S, Miyazaki S, Linton N, Rivard L, Wright M, Wilton SB, Scherr D, Pascale P, Roten L, Pederson M, Bordachar P, Laurent F, Kim SJ, Ritter $\mathrm{P}$, Clementy J, Haïssaguerre M. Elimination of local abnormal ventricular activities: a new end point for substrate modification in patients with scar-related ventricular tachycardia. Circulation. 2012;125:2184-2196.

8. Sacher F, Duchateau J, Capellino S, Takigawa M, Derval N, Denis A, Pambrun T, Chaumeil A, Hocini M, Massoulie G, Haissaguerre M, Cochet H, Dubois R, Jais P. Definition of voltage threshold for ventricular substrate mapping using Intellamap Orion mapping catheter. Heart Rhythm. 2017;14 (supplement): S327-S417.

9. Stevenson WG, Khan H, Sager P, Saxon LA, Middlekauff HR, Natterson PD, Wiener I. Identification of reentry circuit sites during catheter mapping and radiofrequency ablation of ventricular tachycardia late after myocardial infarction. Circulation. 1993;88:1647-1670.

10. Berruezo A, Fernández-Armenta J, Andreu D, Penela D, Herczku C, Evertz R, Cipolletta L, Acosta J, Borràs R, Arbelo E, Tolosana JM, Brugada J, Mont L. Scar dechanneling: new method for scar-related left ventricular tachycardia substrate ablation. Circ Arrhythm Electrophysiol. 2015;8:326-336.

11. Tzou WS, Frankel DS, Hegeman T, Supple GE, Garcia FC, Santangeli P, Katz DF, Sauer WH, Marchlinski FE. Core isolation of critical arrhythmia elements for treatment of multiple scarbased ventricular tachycardias. Circ Arrhythm Electrophysiol. 2015;8:353-361.

12. Di Biase L, Burkhardt JD, Lakkireddy D, Carbucicchio C, Mohanty S, Mohanty P, Trivedi C, Santangeli P, Bai R, Forleo G, Horton R, Bailey S, Sanchez J, Al-Ahmad A, Hranitzky P, 
Gallinghouse GJ, Pelargonio G, Hongo RH, Beheiry S, Hao SC, Reddy M, Rossillo A, Themistoclakis S, Dello Russo A, Casella M, Tondo C, Natale A. Ablation of Stable VTs Versus Substrate Ablation in Ischemic Cardiomyopathy: The VISTA Randomized Multicenter Trial. $J$ Am Coll Cardiol. 2015;66:2872-2882.

13. Di Biase L, Santangeli P, Burkhardt DJ, Bai R, Mohanty P, Carbucicchio C, Dello Russo A, Casella M, Mohanty S, Pump A, Hongo R, Beheiry S, Pelargonio G, Santarelli P, Zucchetti M, Horton R, Sanchez JE, Elayi CS, Lakkireddy D, Tondo C, Natale A. Endo-epicardial homogenization of the scar versus limited substrate ablation for the treatment of electrical storms in patients with ischemic cardiomyopathy. J Am Coll Cardiol. 2012;60:132-141.

14. Arenal A, Glez-Torrecilla E, Ortiz M, Villacastín J, Fdez-Portales J, Sousa E, del Castillo S, Perez de Isla L, Jimenez J, Almendral J. Ablation of electrograms with an isolated, delayed component as treatment of unmappable monomorphic ventricular tachycardias in patients with structural heart disease. $J$ Am Coll Cardiol. 2003;41:81-92.

15. Vergara P, Trevisi N, Ricco A, Petracca F, Baratto F, Cireddu M, Bisceglia C, Maccabelli G, Della Bella P. Late potentials abolition as an additional technique for reduction of arrhythmia recurrence in scar related ventricular tachycardia ablation. $J$ Cardiovasc Electrophysiol. 2012;23:621-627.

16. Bogun F, Good E, Reich S, Elmouchi D, Igic P, Lemola K, Tschopp D, Jongnarangsin K, Oral H, Chugh A, Pelosi F, Morady F. Isolated potentials during sinus rhythm and pace-mapping within scars as guides for ablation of post-infarction ventricular tachycardia. J Am Coll Cardiol. 2006;47:2013-2019.

17. Tung R, Josephson ME, Bradfield JS, Shivkumar K. Directional Influences of Ventricular Activation on Myocardial Scar Characterization: Voltage Mapping With Multiple Wavefronts During Ventricular Tachycardia Ablation. Circ Arrhythm Electrophysiol. 2016;9. pii: e004155.

18. Dinov B, Fiedler L, Schönbauer R, Bollmann A, Rolf S, Piorkowski C, Hindricks G, Arya A. Outcomes in catheter ablation of ventricular tachycardia in dilated nonischemic cardiomyopathy compared with ischemic cardiomyopathy: results from the Prospective Heart Centre of Leipzig VT (HELP-VT) Study. Circulation. 2014;129:728-736.

19. Berte B, Relan J, Sacher F, Pillois X, Appetiti A, Yamashita S, Mahida S, Casassus F, Hooks D, Sellal JM, Amraoui S, Denis A, Derval N, Cochet H, Hocini M, Haïssaguerre M, Weerasooriya R, Jaïs P. Impact of electrode type on mapping of scar-related VT. J Cardiovasc Electrophysiol. 2015 July 22 [Epub ahead of print]; doi:10.1111/jce.12761.

20. Yamashita S, Sacher F, Mahida S, Berte B, Lim HS, Komatsu Y, Amraoui S, Denis A, Derval N, Laurent F, Sermesant M, Montaudon M, Hocini M, Haïssaguerre M, Jaïs P, Cochet H. Image Integration to Guide Catheter Ablation in Scar-Related Ventricular Tachycardia. J Cardiovasc Electrophysiol. 2016;27:699-708.

21. Cochet H, Komatsu Y, Sacher F, Jadidi AS, Scherr D, Riffaud M, Derval N, Shah A, Roten L, Pascale P, Relan J, Sermesant M, Ayache N, Montaudon M, Laurent F, Hocini M, Haïssaguerre M, Jaïs P. Integration of merged delayed-enhanced magnetic resonance imaging and multidetector computed tomography for the guidance of ventricular tachycardia ablation: a pilot study. J Cardiovasc Electrophysiol. 2013;24:419-426.

22. Komatsu Y, Cochet H, Jadidi A, Sacher F, Shah A, Derval N, Scherr D, Pascale P, Roten L, Denis A, Ramoul K, Miyazaki S, Daly M, Riffaud M, Sermesant M, Relan J, Ayache N, Kim S, Montaudon M, Laurent F, Hocini M, Haïssaguerre M, Jaïs P. Regional myocardial wall thinning at multidetector computed tomography correlates to arrhythmogenic substrate in postinfarction ventricular tachycardia: assessment of structural and electrical substrate. Circ Arrhythm Electrophysiol. 2013;6:342-350.

23. Vaseghi M, Shivkumar K. Neuroaxial modulation for ventricular arrhythmias: a new hope. Heart Rhythm. 2012;9:1888-1889. 
24. Sapp JL, Beeckler C, Pike R, Parkash R, Gray CJ, Zeppenfeld K, Kuriachan V, Stevenson WG. Initial human feasibility of infusion needle catheter ablation for refractory ventricular tachycardia. Circulation. 2013;128:2289-2295.

25. Koruth JS, Dukkipati S, Miller MA, Neuzil P, d'Avila A, Reddy VY. Bipolar irrigated radiofrequency ablation: a therapeutic option for refractory intramural atrial and ventricular tachycardia circuits. Heart Rhythm. 2012;9:1932-1941.

26. Yokokawa M, Desjardins $\quad$ B, Crawford $\quad$ T, Good $\quad$ E, Morady $\quad$ F, Bogun F. Reasons for recurrent ventricular tachycardia after catheter ablation of post-infarction ventricular tachycardia. J Am Coll Cardiol. 2013;61:66-73. 
Supplemental Table: Predictors for VA-free and overall survival after first ablation procedure.

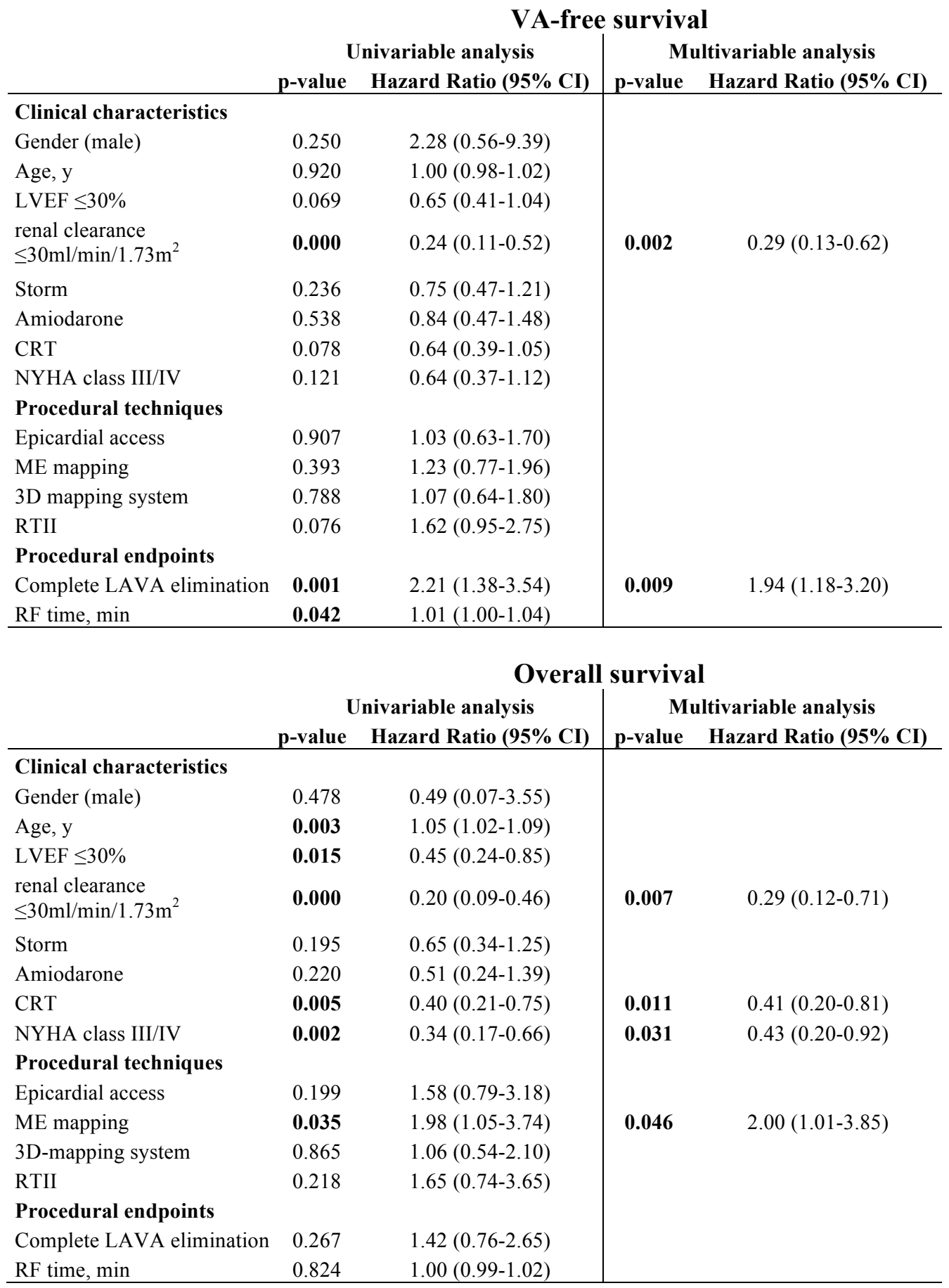


CI: confidence interval; CRT: cardiac resynchronization therapy; LAVA: local abnormal ventricular activities; LVEF: left ventricular ejection fraction; ME: multi-electrode; NYHA: New York Heart Association; RF: radiofrequency; RTII: real-time image integration 

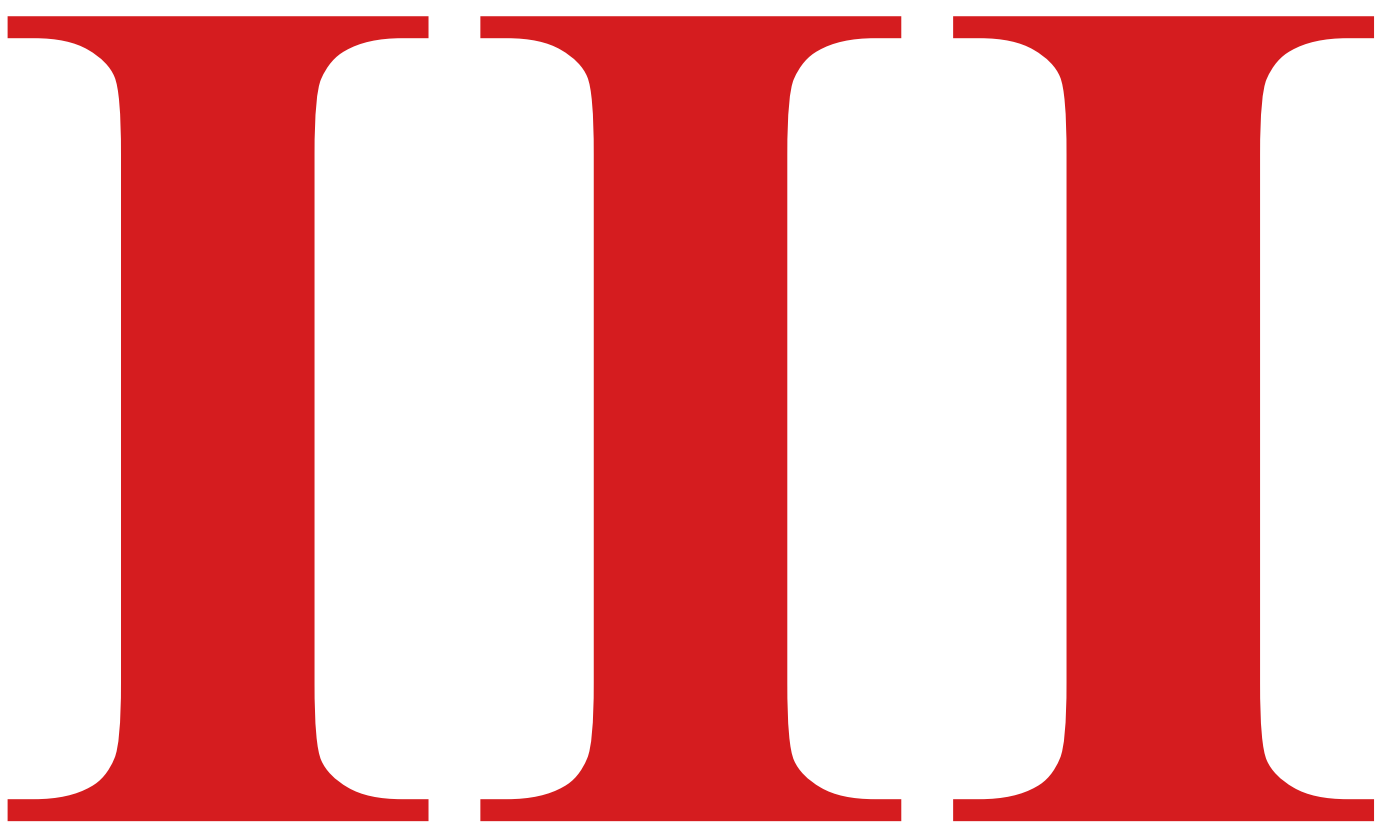
Part III 

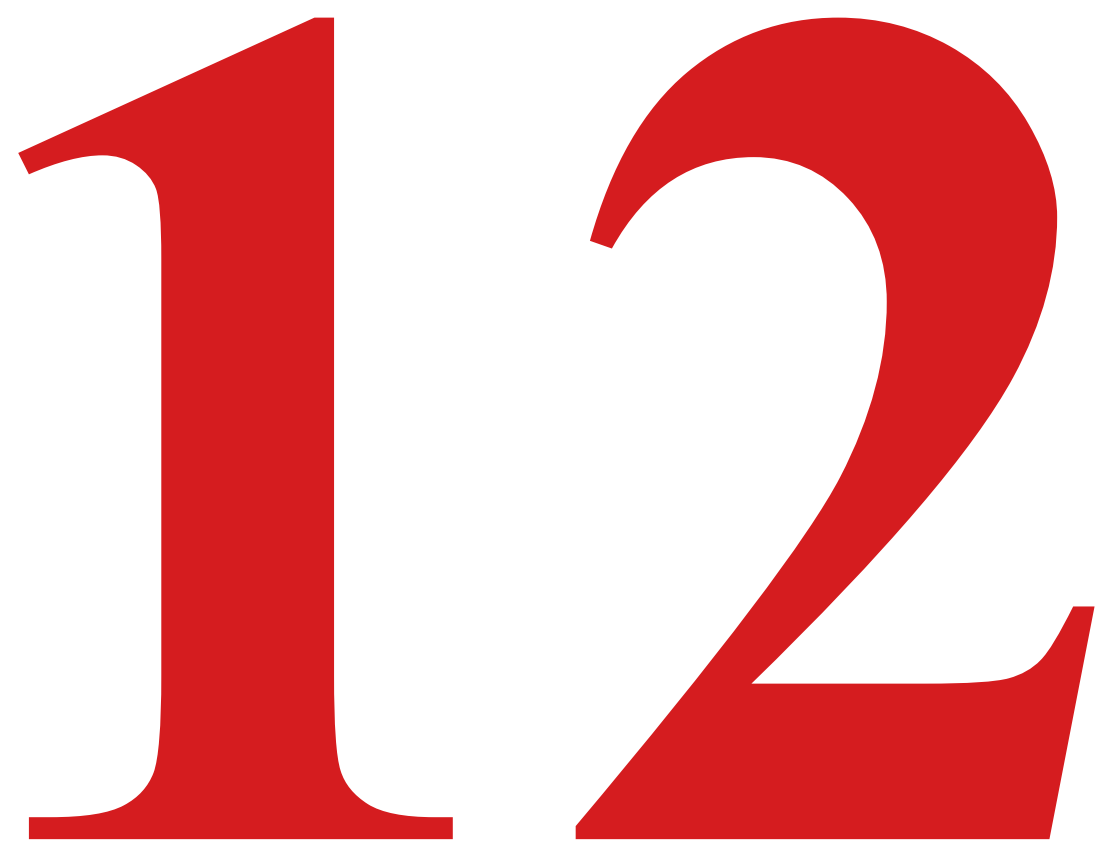


\section{Chapter 12}

General discussion and summary 


\section{ABLATION OF ATRIAL FIBRILLATION}

\section{Scope of the problem and rationale for ablation}

Atrial fibrillation (AF) is a serious public health problem because of its increasing incidence and prevalence in the aging population ${ }^{1}$ and its association with elevated risks of cardiovascular events and death. ${ }^{2-7}$ In addition AF causes a wide variety of symptoms, including fatigue and reduced exercise tolerance, and significantly impairs quality of life. ${ }^{8}$ The morbidity and mortality associated with AF provide a rationale to maintain sinus rhythm. Yet to date, rhythm control strategy has failed to demonstrate a beneficial effect on hard endpoints such as a reduction in stroke or mortality rate. ${ }^{9-11}$ Ablation of atrial fibrillation has proven to reduce symptoms and improve quality of life, and is superior to antiarrhythmic drugs in obtaining rhythm control. ${ }^{11-13}$ Therefore according to current guidelines AF ablation is indicated in symptomatic patients as a second-line treatment after failure of a class I or III antiarrhythmic drug (Class I indication (level of evidence A) in paroxysmal AF, a class IIa indication (level of evidence B) in persistent AF and a class IIb indication (level of evidence C) in long-standing persistent AF. ${ }^{14}$ Given that catheter ablation of $\mathrm{AF}$ as a first-line treatment is associated with a higher freedom from atrial fibrillation compared with drug therapy, ${ }^{15-18}$ current guidelines also state that catheter ablation of AF as a first-line treatment (without a trial of class I of III antiarrhythmic drugs) is indicated in symptomatic paroxysmal (Class IIa - level of evidence B) and persistent AF patients (Class IIa - level of evidence C; and Class IIb - level of evidence C in case of long-standing persistent AF). ${ }^{14}$

\section{Principle of pulmonary vein isolation}

Since Haïssaguerre et al identified the triggers initiating AF to arise predominantly from the muscular sleeves within the pulmonary veins, ${ }^{19}$ electrical isolation of the pulmonary veins has become the cornerstone of catheter ablation of $\mathrm{AF} .{ }^{14}$ Ablation strategies to achieve PV isolation evolved from a segmental ablation at the PV ostium near the earliest activation of the PV muscular sleeves, ${ }^{20}$ to a wider continuous circumferential lesion set around left and right vein pairs, usually guided by a 3D anatomical mapping system. ${ }^{21-23}$ In a recent randomized trial a complete contiguous circumferential ablation lesion around the PVs was found superior with respect to AF recurrence compared to an incomplete ablation circle. ${ }^{24}$

Despite the ability to achieve acute procedural PVI in $>99 \%$ of cases with standard irrigated catheters, permanent, durable isolation of the PVs is only achieved in the minority of cases. Indeed, in studies that performed a repeat procedure irrespective of the clinical outcome after the index PVI, reconduction of at least one PV was observed in 62 to $90 \%$ of cases. ${ }^{24-26}$ Studies that performed a repeat ablation procedure because of AF recurrence after index PVI, observed PV reconduction of at least one PV in $>80 \%$ of patients. ${ }^{26-30}$ This coincides with a suboptimal clinical outcome with AF recurrence in up to $50 \%$ of patients after a single intervention. ${ }^{13,16,17,31}$ 
This is because of insufficient quality of the initial ablation lesion set resulting in parts with latently non-conduction tissue that is still viable and at high risk of restored conduction. Of interest, reconduction of a PV pair after circumferential point-by-point RF PVI could even be attributed to a single ineffective ablation point (principle of the weakest link). It was observed that electrical PV reconduction often occurs fast, with one third of reconnections occurring within 30 minutes after initial isolation. ${ }^{32-34}$ For this reason, one proposed technique in order to reduce the chance of PV reconnection is to install a waiting time of $20-30$ minutes following initial PV isolation (Class IIa level of evidence $\mathrm{B}^{14}$ ). In one prospective randomized trial the benefit of a 30 minute waiting period with 'bonus' lesions was shown. ${ }^{35}$ Another technique aiding to differentiate a durable PVI lesion set with permanent conduction block from a suboptimal lesion set at risk of early reconduction was to administer intravenous adenosine (class IIb indication - level of evidence $\mathrm{B}^{14}$ ). ${ }^{36,37}$ Intravenous adenosine or adenosine triphosphate is able to transiently restore cellular excitability and unmask socalled 'dormant' conduction (viable but latently non-conducting tissue). Not only was it demonstrated that the presence of dormant PV conduction was associated with a higher risk of AF recurrence, but additional adenosine-guided targeted ablation eliminating dormant PV conduction significantly reduced arrhythmia recurrence. ${ }^{36-37}$ The downside of incorporating a waiting phase and adenosine testing into the procedure however, is the prolongation of the left atrial dwell time (increased thromboembolic risk) and procedure time, as well as an increase in cost.

\section{Technological advances to improve the outcome of PVI: contact force sensing}

The likelihood of obtaining permanent PVI is related to the quality of ablation energy delivery and lesion formation. With RF energy the important factors that influence lesion size and transmurality are catheter stability, contact force (CF), RF power, RF duration and temperature.

For point-by-point RF, irrigated-tip catheters are used to avoid excessive tissue heating with subsequent char formation. This allows higher RF power delivery, resulting in increased lesion size, but also increases safety by reducing the risk for steam pops.

The electrode tip - tissue CF is one of the key determinants of lesion size. Without adequate CF, no effective lesions can by generated. Prior to the development of direct CF-sensing catheters, surrogate markers for tip-tissue contact, such as impedance, intracardiac electrogram amplitude, the catheter movement on fluoroscopy and tactile feedback, were used but they proved unreliable to predict actual tip-tissue contact. ${ }^{38-41}$ Ablation catheters using two different technologies have been developed to measure precisely real-time catheter-tissue CF: 1) use of three optical fibres to measure the microdeformation of a deformable body in the catheter tip (TactiCath, St. Jude Medical, Inc.), 2) use of a small spring between the tip electrode and the catheter shaft, with a magnetic transmitter in the tip and magnetic sensors proximal to the tip to measure microdeflection of the spring (ThermoCool SmartTouch, Biosense Webster, Inc.), both corresponding to CF. Even though several clinical studies have shown benefit of CF catheters over non-CF sensing catheters in terms of procedural parameters 
and clinical outcome (lower adenosine-induced dormant conduction and AF recurrence), the two large randomized trial performed were negative, both in terms of (long-term) arrhythmia free survival and rate of complications. ${ }^{42,43}$

On could speculate on theories on why these randomized trials did not prove superiority of CF-guided PVI, while operators perceived they have clearly improved outcomes in daily practice. In the UK Multicenter trial, CF-guided PVI was compared to non-CF guided PVI using the identical ablation catheter (SmartTouch ${ }^{\mathrm{TM}}$, Biosense Webster) with blinding of the online CF-value. ${ }^{42}$ In this study protocol a 60-minute waiting time (+ adenosine) was used to assess acute PV reconnection. Even though CF-guided ablation did result in less acute reconnection ( $22 \%$ vs. $32 \%, p=0.03)$, no difference in 1-year arrhythmia-free survival ( 49 vs. $52 \%, \mathrm{p}=0.90$ ) was observed. One could speculate that if shorter waiting times (e.g. 20 minutes) were applied, which is more in line with reality of the daily clinical practice, less acute reconnections and subsequent re-isolations would have occurred. Therefore the benefit of CF-guided PVI might have been offset by the very long waiting time and higher rate of re-isolation in the non-CF guided PVI group. In the Toccastar Study, CF-guided PVI was performed with the Tacticath ${ }^{\mathrm{TM}}$ catheter and EnSite ${ }^{\mathrm{TM}} \mathrm{NavX}^{\mathrm{TM}}$ mapping system (St. Jude Medical), while in the control group non-CF-guided PVI was performed with the Thermocool Navistar and Carto mapping system (Biosense Webster). Use of different mapping systems might have accounted for lack of superiority of CF-guided PVI (operators were less experienced with the EnSite system). Also during this trial, the target values for CF were not yet established at the onset of this trial.

Despite the absence of prospective, randomized trials proving the superiority of online CF feedback in efficacy and safety, operators worldwide have quickly adopted CF catheters in their routine clinical practice, because they believe that $\mathrm{CF}$ monitoring provides important biomechanical feedback to improve effective lesion formation, durability of PVI and clinical outcomes. ${ }^{14}$ Indeed, both acute adenosine-proof $\mathrm{PV}$ isolation and one-year freedom of AF increased to $72-88 \%{ }^{44-46}$

In Chapter 2 we studied if an extensive 'learning period' with CF catheters with online CF feedback improved the operator's ability to perform PVI when returning to ablation catheters without CF measurement. In September 2013 the Thermocool $^{\circledR}$ SmartTouch $^{\mathrm{TM}}$ catheter was temporarily withdrawn worldwide from clinical use due to technical defaults leading to safety concerns in a small number of catheters. In this transient recall period, the experienced operator involved in the current study returned to the use of the irrigated-tip ablation catheter without CF measurement technology (NaviStar $^{\circledR}$ Thermocool $^{\circledR}$ ablation catheter). Procedural outcome parameters of consecutive PVI procedures performed without $\mathrm{CF}$ measurements during the recall period to a control group undergoing PVI with CF measurement technology were prospectively compared and shown in Table 1 below. 
Table 1:

Procedural outcomes of PVI performed with and without online CF feedback beyond the learning period

CF- group

$(\mathbf{n}=12)$

Number of PV pairs without first-pass isolation

$(\mathrm{n}, \%)$

Number of patients without first-pass isolation

$(\mathrm{n}, \%)$

Number of adenosine-induced reconnected PV

pairs (n, \%)

Total procedure time (min)

Total left atrial time (min)

RF time (min)

Fluoroscopy time (min)
$7 / 24(29.2)$

$7 / 12(58.3)$

$1 / 24(4.2)$

$167.8 \pm 26.0$

$134.1 \pm 25.3$

$31.8 \pm 7.0$

$11.0 \pm 5.8$
$\mathrm{CF}+$ group

$(n=24)$

$2 / 48(4.2)$

$2 / 24(8.3)$

0/48 (0)
0.005

0.002

333

Data are presented as mean \pm standard deviation or absolute number $(n)+$ percentage $(\%)$

CF-: group in which CPVI is performed with the NaviStar ${ }^{\mathbb{B}}$ Thermocool $^{\mathbb{B}}$ ablation catheter; $\mathrm{CF}+$ : control group in which CPVI is performed with the Thermocool ${ }^{\mathbb{B}}$ SmartTouch $^{\mathrm{TM}}$ catheter; PV: pulmonary vein;

RF: radiofrequency

Acknowledging the limitations of this relatively small-sized, non-randomized and single-center, single-operator study design, our results suggest that after gaining experience with CF-measuring catheters, procedural PVI parameters (most importantly first-pass PV isolation) remained superior with this technology when compared to non-CF guided PVI procedures. An extensive training period with real-time direct $\mathrm{CF}$-feedback was not a substitute for real-time direct $\mathrm{CF}$ measurement to maintain this advantage.

\section{A new protocol aiming to optimize radiofrequency pulmonary vein isolation.}

In addition to $\mathrm{CF}$, the lesions depth and quality are also determined by the ablation power and application time. Whereas minimal CF ( $>10$ to $20 \mathrm{~g}$ ) and force-time integral (FTI) (>400gs) previously have shown to predict durable PVI with high sensitivity and durability, ${ }^{47,48}$ both parameters have severe limitations: it is impossible to achieve minimal CF values of $>10-20 \mathrm{~g}$ at each RF site; targeting $\mathrm{CF}>20 \mathrm{~g}$ can lead to catheter dislocation during ablation as well as tissue compression and increase complications (e.g. protrusion of the posterior left atrial wall towards the esophagus). FTI has 2 severe limitations: first, FTI (calculated as the product of force by application time) suggests a linear lesion formation during ablation, whereas it is known that lesion volume growth describes a monoexponential curve. ${ }^{49}$ Second, FTI ignores the important and evident role of power delivery in lesion creation. Therefore the lesion volume of 400 gs FTI applications highly differs depending on 
the power used. This could be one of the reasons why the use of CF feedback and aiming for minimal $\mathrm{CF} / \mathrm{FTI}$ targets did not prove to be superior in randomized trials. ${ }^{42,43}$

The Force-Power-Time Index or Ablation Index (AI)) incorporates CF, ablation power and application time in a logarithmic function, taking into account the greater contribution of power (over CF) and of the initial time of ablation and, unlike mere CF, was found to predict lesion depth in the canine ventricle with high accuracy. ${ }^{50,51}$ The $\mathrm{AI}$ is both a reliable and useful parameters to target durable PVI for the following reasons: 1) lesion quality is expected to be similar at a certain AI value (despite differences in power, force, and time of application required to reach that value), and 2) AI targets can be reached readily at each site within the circle (eg, by increasing power at sites with low but stable contact). However the AI does not take into account lesion contiguity. Logically in order to obtain block across a lesion set, not only the lesion depth of single applications is of importance but also the lesion width ensuring that neighbouring applications are in fact histologically 'contiguous'. Therefore the distance between neighbouring applications is an important parameter to consider, when analysing ablation application effectiveness characteristics. ${ }^{48,52}$

In part IB of this thesis we developed and tested a specific new protocol aiming to improve the pointby-point RF PVI technique by combining strict criteria for both RF application contiguity and quality.

In Chapter 3 we retrospectively analysed 42 CF-guided PVI procedures to define optimal target criteria for RF application quality and contiguity. For each RF tag within the circle, we collected RF characteristics reflecting lesion depth/quality (Ablation Index [AI]) and contiguity (automated interlesion distance [ILD]). To combine both depth (AI) and contiguity (ILD) in one single criterion we developed in Matlab (Mathworks, Natick, MA) a new algorithm to assess ablation line contiguity index (ALCI). ALCI is defined as the ratio of the estimated width of 2 neighboring lesions ( $i$ and $j$ ) over their interlesion distance (ILDij) and calculated as follows:

$$
\mathrm{ALCI}_{\mathrm{ij}}=\frac{\mathrm{OC} \times \operatorname{Conv} \mathrm{x}\left(\frac{\mathrm{AI} \mathrm{I}_{\mathrm{i}}+\mathrm{AI}_{\mathrm{j}}}{2}\right)}{\mathrm{ILD}_{\mathrm{ij}}}
$$

The width of a lesion is defined as the multiplication of estimated lesion depth (AI) and the conversion factor (Conv). The conversion factor is used because experimental studies have shown that maximal lesion width exceeds depth by a factor ranging from 1.5 to 2.0 (ellipsoid nature of RF lesions). In the present study, Conv was 1.5 for the anterior wall (thick tissue) and 2.0 for the posterior wall (thin tissue) lesions. To assure contiguity, we hypothesized that lesions should overlap by $25 \%$. Therefore, 
an overlapping coefficient (OC) of 0.75 was used. This overlap also corrects for the fact that lesion width at the tip-tissue interface is smaller than its maximal width.

In all procedures RF applications were depicted on the anatomical map with automated tagging technology (Carto VisiTag ${ }^{\mathrm{TM}}$, Biosense Webster Inc.) with predefined settings of catheter stability and $\mathrm{CF}$. This automated tagging is a crucial part of this analysis, because 1) it allows to precisely collect the RF characteristics of each tag, and 2) it accurately (i.e. reproducibly) localizes the center of each RF tag, allowing to objectively determine the inter-tag distance (center-to-center). PV circle segments with acute (adenosine or waiting time) and late (at repeat procedures) pulmonary vein reconnections (n $=44)$ were compared to segments without reconnection $(\mathrm{n}=758)$. For each segment, we determined its weakest link by annotating minimal RF time, power, impedance drop, CF, force-time integral (FTI), AI and maximal ILD and minimal ALCI. Segments with reconnections were characterized by comparable power $_{\min }(20$ versus $22 \mathrm{~W} ; \mathrm{P}=0.877)$ and $\Delta$-Imp min $_{\text {( }}(4.8$ versus $7.4 \Omega ; \mathrm{P}=0.08$ ). In contrast, those segments showed significantly lower time $\mathrm{min}_{\text {min }}(33$ versus $35 \mathrm{~s} ; \mathrm{P}=0.025), \mathrm{CF}_{\min }(8.5$ versus $11.8 \mathrm{~g}$; $\mathrm{P}=0.006$ ), $\mathrm{FTI}_{\min }$ (351 versus 473 gs; $\mathrm{P}<0.001$ ), and $\mathrm{AI}_{\min }$ (367 versus 408 au; $\mathrm{P}<0.001$ ). Furthermore, segments with reconnection were characterized by higher $\operatorname{ILD}_{\max }(6.8$ versus $5.5 \mathrm{~mm} ; \mathrm{P}=0.001)$. Also, minimal ALCI per segment was significantly lower in segments with reconnection (74 versus $104 \%$; $\mathrm{P}<0.001)$. In multivariable analysis, FTImin, AImin, and ILDmax remained significantly different between segments with and without reconnection. Overall, ROC curve analysis for all segments together showed the highest diagnostic accuracy for ALCI (area under the curve, AUC=0.73), compared to ILD (0.70), FTI (0.69), CF (0.68), AI (0.67), and $\Delta$-Imp (0.64).

AI and ILD were independently associated with durable lesions, as is illustrated in Figure 1. In those selected segments characterized by the presence of RF tags, all with an ILDmax $<5 \mathrm{~mm}$ (an ILDmax of $<5 \mathrm{~mm}$ was associated with a specificity of $90 \%$ to discriminate durable segments), AImin discriminated between segments with gaps and no gaps ( $\mathrm{P}=0.009$, left). Conversely, in those selected segments characterized by the presence of RF tags, all with an optimal AI (defined as AImin $>550 \mathrm{au}$ on the anterior wall and $>417$ on the posterior wall, both associated with a specificity of $90 \%$ ), ILDmax discriminated between segments with gaps and no gaps $(\mathrm{P}<0.001$, right). The difference in AI target between the anterior and posterior wall reflects the difference in wall thickness.

Combining both ILD and AI in a single index, the ALCI (target $\approx 100 \%$ ) had the highest accuracy to predict durable PVI. We therefore hypothesize that combined ILD- and AI-guided ablation would increase freedom from AF after PVI procedures. 
Segments with tags all with $\mathrm{ILD}_{\max } \leq 5 \mathrm{~mm}$

Ablation index

$\left(\mathbf{A} \mathbf{I}_{\text {min }}\right)$

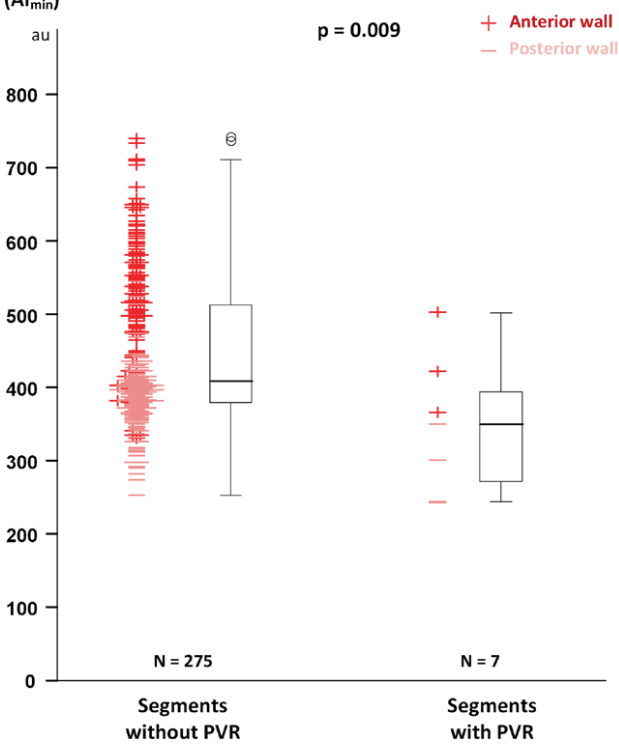

Segments with tags all with $A I_{\min }>417$ (post) or $\mathrm{Al}_{\min }>550$ (ant)

Inter-lesion distance $\left(\right.$ ILD $\left._{\max }\right)$

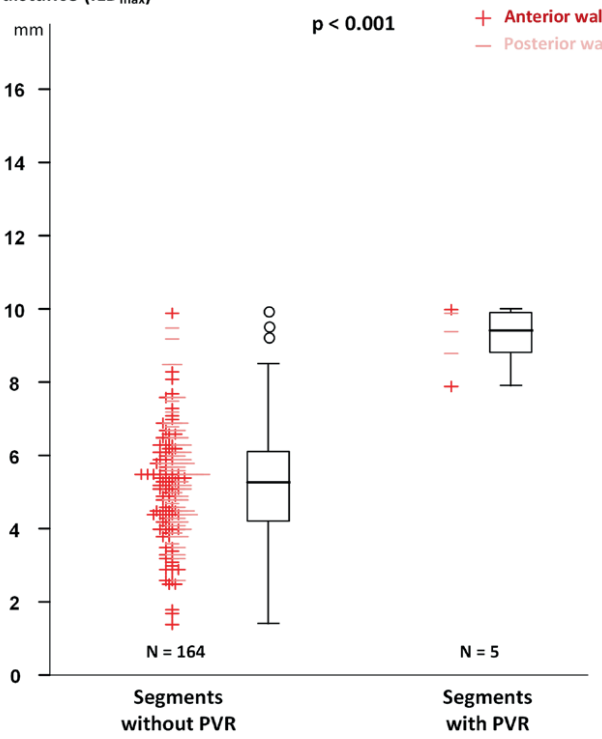

Figure 1: Left, Difference in minimal ablation index (AImin) for segments with maximum automated interlesion distance (ILDmax) $\leq 5 \mathrm{~mm}$. Right, Difference in ILDmax for segments with AImin $>550 \mathrm{~mm}$ (anterior) and $>417$ $\mathrm{mm}$ (posterior). Red scatter markers represent anterior segments. Pink scatter markers represent posterior segments. PVR indicates pulmonary vein reconnection.

\section{Prospective evaluation of the 'CLOSE' protocol: acute and 1-year efficacy outcome}

The analysed targets for RF application quality and contiguity (Chapter 3) were incorporated in a new PVI protocol, aiming at delivering stable, relatively high-power, short-duration RF applications relatively close to the left atrial (LA)-PV junction.

This 'CLOSE' (Contiguous Lesion Optimized pre-Specified Encircling) protocol consisted of the following principles:

1. Detailed mapping of the PV junction (performed with the Thermocool SmartTouch catheter). Applying a prespecified nephroid shaped circle around left and right PV pairs relatively close to the LA-PV junction prior to onset of ablation, based on $\mathrm{CF} /$ stability (identification of carina and ridge).

2. Automated RF tagging using Visitag module, after respiratory gating, with specific settings for lesion stability ( $3 \mathrm{~mm}$ for $8 \mathrm{~s}$ ) and minimal CF (30\% of time $>4 \mathrm{~g})$. Stability was ensured before onset of RF in order to avoid dislocation before reaching the AI target (to avoid edema and ineffective lesions).

3. Contiguity of optimized RF applications: 
- $\mathrm{AI} \geq 550 \mathrm{au}$ on the anterior wall and $\geq 400 \mathrm{au}$ on the posterior wall/roof (300au in case of esophageal temperature rise $>38.5^{\circ} \mathrm{C}$ or excessive pain in awake patients)

- Maximum ILD $\leq 6 \mathrm{~mm}$ (center-to-center) with strict ruling

- In case of dislocation a new application reaching the AI target was applied

4. RF power was delivered in a power-controlled mode (without ramping) at 35 Watts. RF power is only reduced in case of catheter tip temperature rise $>43^{\circ} \mathrm{C}$ or in case of high $\mathrm{CF}(\geq 20 \mathrm{~g})$ that might lead to overshoot of the AI target.

5. Combined endpoint of ALCI $>100 \%$ (except if lower AI due to esophageal temperature rise) on top of electrical PVI.

\section{CLOSE guided PVI}

\section{Ablation index posterior wall} $\geq 400$ au

\section{Ablation index anterior wall $\geq 550$ au \\ Inter-lesion distance $\leq 6 \mathrm{~mm}$}

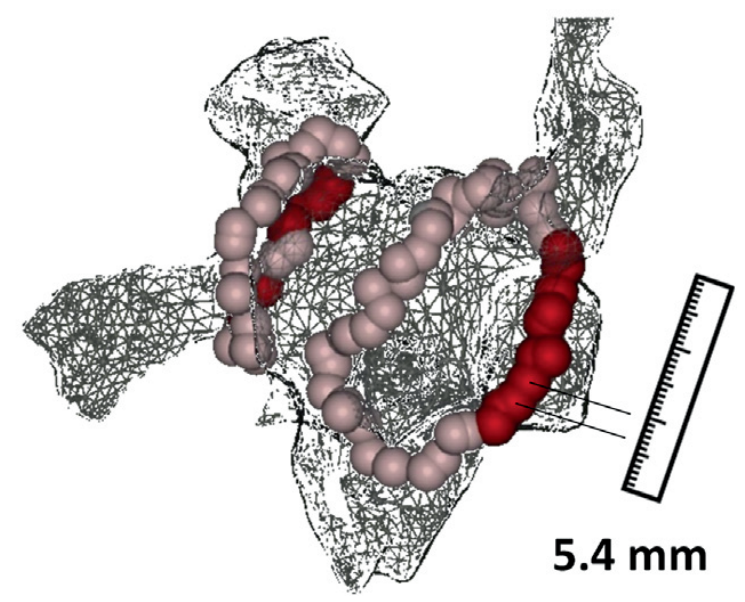

Figure 2: illustration of the CLOSE protocol

In Chapter 4 we provided comparative data on the efficiency, safety, and efficacy of 'CLOSE-PVI' to a historical control group of conventional CF-guided PVI for paroxysmal AF. We analysed the first 50 cases performed according to the 'CLOSE'-protocol and compared results to the last 50 consecutive cases performed with conventional CF-guided PVI. In the CF-guided group the operator was blinded to the AI, and no formal ILD criterion was put forward. CF target was $>10 \mathrm{~g}$ and RF power was delivered at $25 \mathrm{~W}(30 \mathrm{~s})$ at the posterior wall / roof, and at $35 \mathrm{~W}(60 \mathrm{~s})$ at the anterior wall. 


\section{Conventional CF guided PVI}

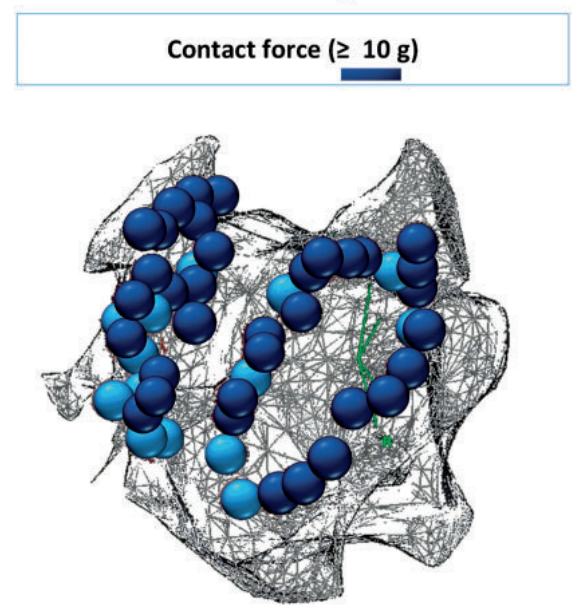

\section{CLOSE guided PVI}

Ablation Index $(\geq 550)$ and ILD guided $(\leq 6 \mathrm{~mm})$

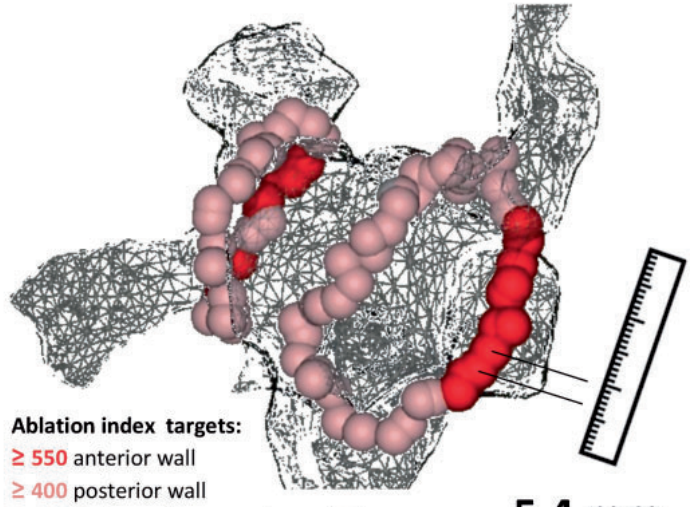

$\geq 300$ if chest pain or esophageal T rise
$5.4 \mathrm{~mm}$

Figure 3: comparison of the deployed circle in CF guided PVI and CLOSE-PVI.

In the CLOSE-PVI group, procedure time was found shorter $(149 \pm 33 \mathrm{~min}$ vs. $192 \pm 42 \mathrm{~min}, \mathrm{P}<$ $0.0001)$, as well as RF time $(17 \pm 3 \mathrm{~min}$ vs. $29 \pm 8 \mathrm{~min}, \mathrm{P}<0.0001$ for the right circles, $19 \pm 4 \mathrm{~min}$ vs. $26 \pm 6 \mathrm{~min}, \mathrm{P}<0.001$ for the left circles). Also in CLOSE-PVI, first pass-isolation ( $98 \%$ vs. $54 \%, \mathrm{P}<$ $0.0001)$ and waiting time/adenosine-proof isolation $(97 \%$ vs. $82 \%, \mathrm{P}<0.001)$ were higher compared to conventional CF-guided PVI. The shorter procedure time in the CLOSE-PVI group was explained by the absence of PV reconnections (no time spent chasing gaps) and a reduction in RF time. The shorter RF time was explained by the markedly shorter applications (use of AI allowing delivery of higher power) and a trend towards fewer applications.

Holter-ECG was performed at 3 months ( 24 h), 6 months ( 24 h), and 12 months ( 7 day) or in case of symptoms. At the end of the blanking period (3 months), 23 patients continued antiarrhythmic drugs (ADT) in the conventional CF-guided group (46\%) vs. 18 patients in the CLOSE-PVI group (36\%). Overall, single-procedure 12 month-freedom from AF, atrial flutter (AFL) or atrial tachycardia (AT) was $94 \%$ in CLOSE-PVI compared to $80 \%$ in conventional CF-guided PVI $(\mathrm{P}<0.05)$. Both in patients with and without ADT, 'CLOSE' was associated with a higher freedom from AF/AT/AFL. No clinical complications occurred in the CLOSE-PVI group. 

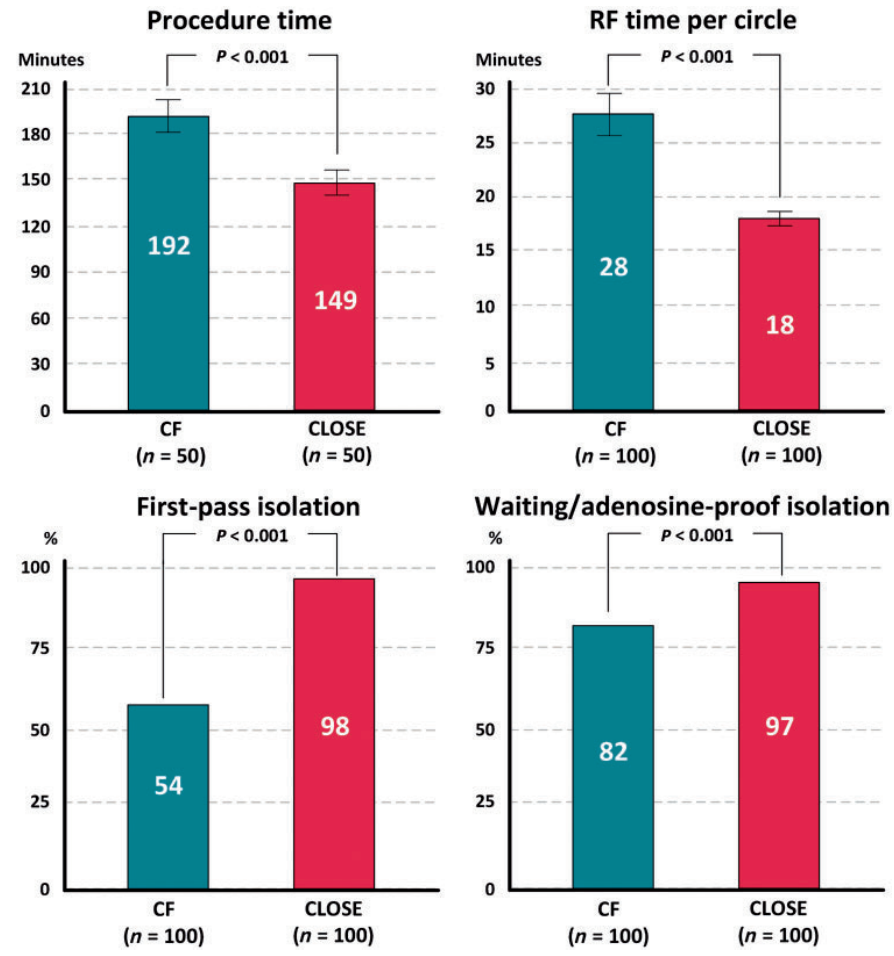

Figure 4: Comparison of procedural outcomes between CLOSE-PVI and conventional CF-guided PVI.
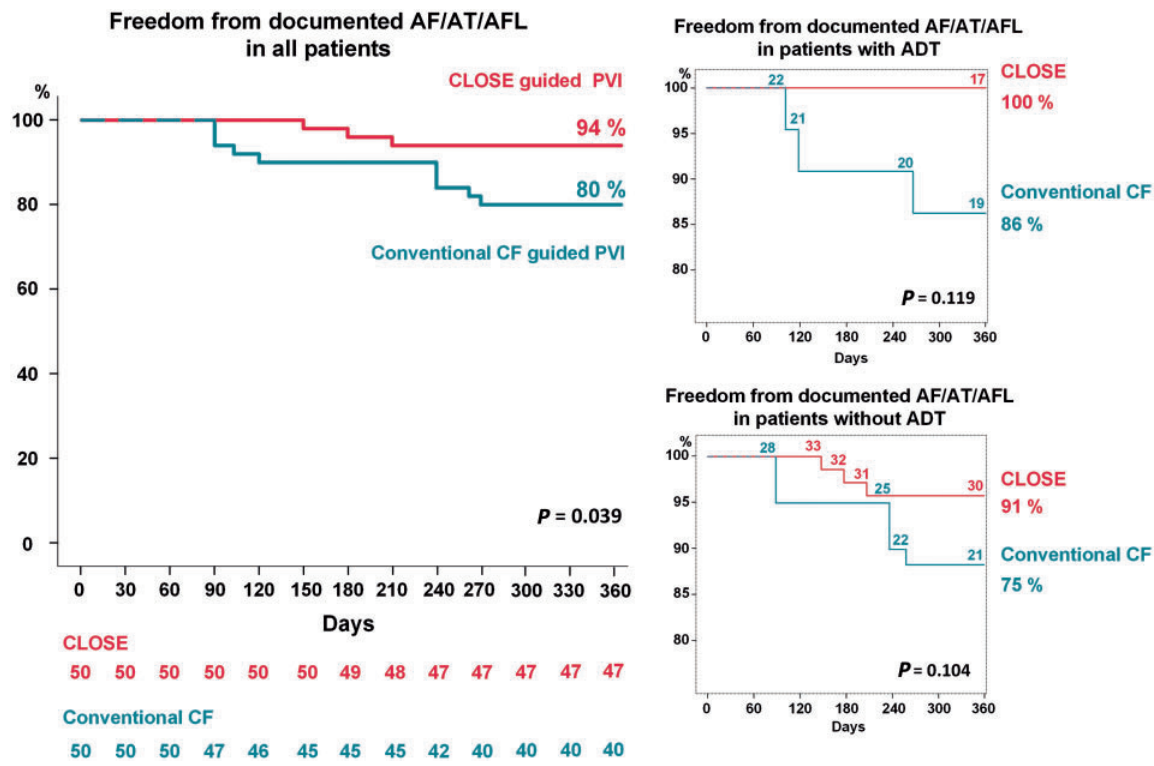

Figure 5: Freedom from atrial fibrillation/tachycardia/flutter after 90 days blanking period in CLOSE PVI (50 patients) versus conventional CF-guided PVI (50 patients). 
Following these promising results, in Chapter 5 we investigated the safety, the acute and 1-year outcome of CLOSE-PVI in a larger patient cohort of 130 consecutive paroxysmal AF patients. Also in this study, the follow-up consisted of Holter-ECGs performed at 3 months ( $24 \mathrm{~h}), 6$ months (24 h), and 12 months ( 7 day) or in case of symptoms. The procedure time was $155 \pm 28 \mathrm{~min}$, with RF time $17 \pm 5$ min per PV circle. Also in this larger cohort, CLOSE-PVI was associated with high incidence of firstpass isolation ( $98 \%$ per circle) and waiting time/adenosine-proof isolation (98\% per circle). Overall, the single-procedure 12-month freedom from AF/AT/AFL was $92.3 \%$, confirming the efficacy of CLOSE-PVI. Throughout the course of the study, 26 patients were on antiarrhythmic drug therapy (ADT). ADT was not systematically stopped and those patients had continued taking ADT after the 3month blanking period. However importantly, no patient was taking ADT to treat a recurrence documented while being off ADT. In those 26 patients on ADT, freedom from AF/AT/AFL was 96.2\%. In those 104 patients off ADT, freedom from AF/AT/AFL was 91.3\%. Aside from one transient ischemic attack that occurred 8 days after the ablation, no adverse events occurred.
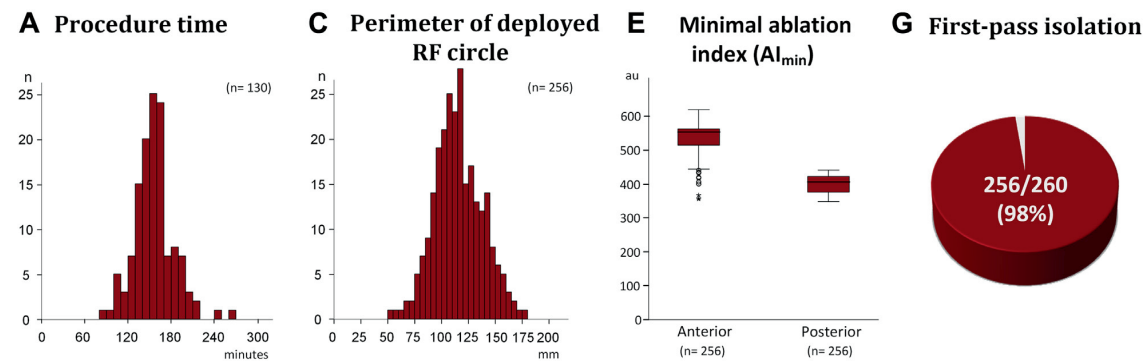

\section{B RF time per circle}
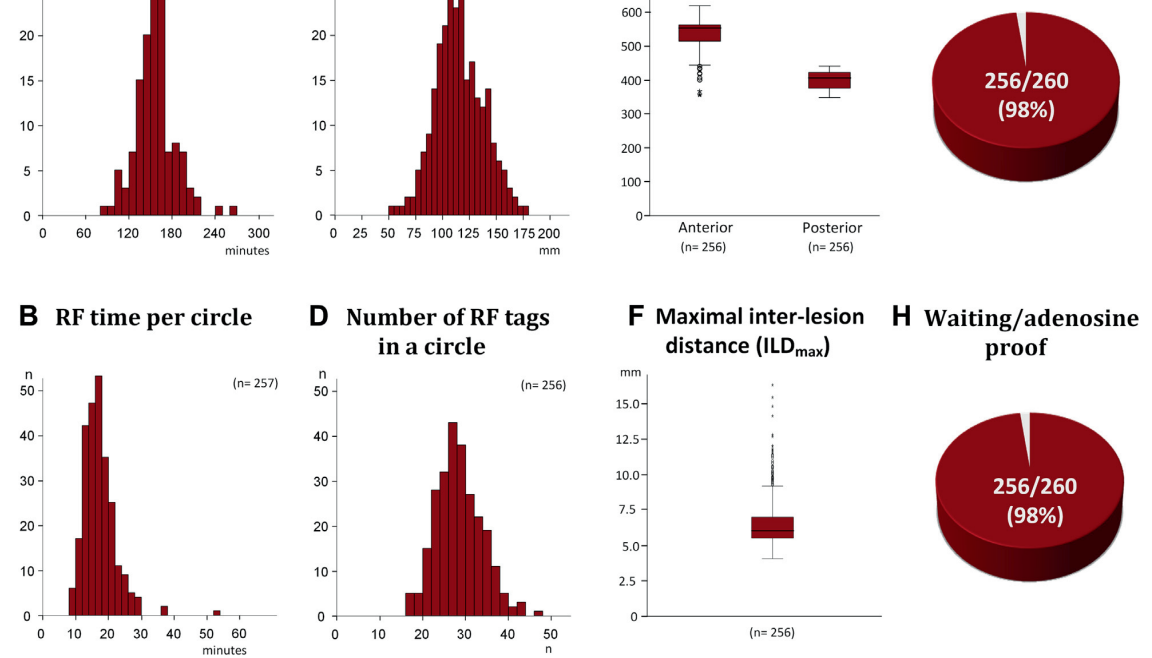

Figure 6: Procedural results of 130 consecutive CLOSE-PVI procedures: (A) Procedure time. (B) Radiofrequency (RF) time per circle. (C) Perimeter of the deployed circle. (D) Number of RF tags within the circle. (E) Obtained minimal ablation index $\left(\mathrm{AI}_{\mathrm{min}}\right)$ per circle. $(\mathrm{F})$ Obtained maximal ILD. $(\mathrm{G})$ Incidence of first-pass isolation (per circle). $(\mathrm{H})$ Incidence of waiting time/adenosine-proof isolation (per circle) 


\section{Freedom from documented AF/AT/AFL in all patients}

( $n=130)$

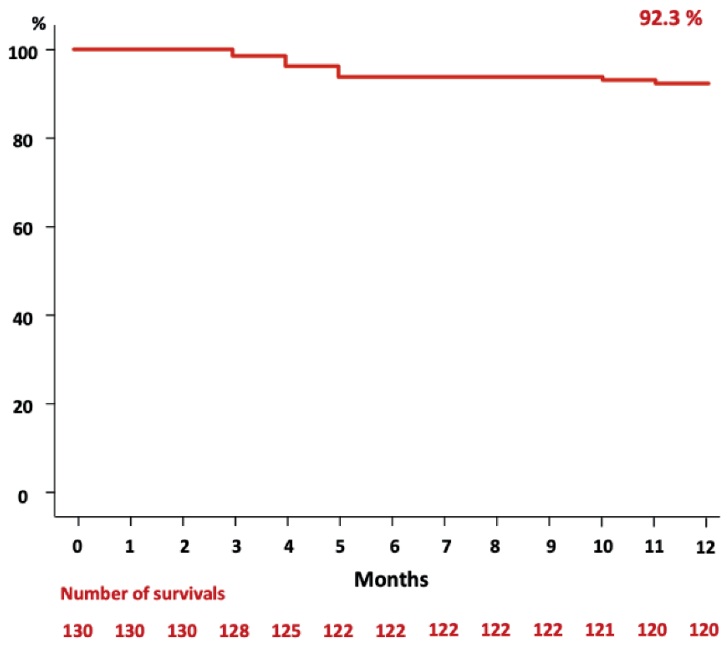

Figure 7: 1-year freedom from recurrence of AF/AFL/AT after a 3-month blanking period in 130 CLOSE-PVI procedures for paroxysmal AF.
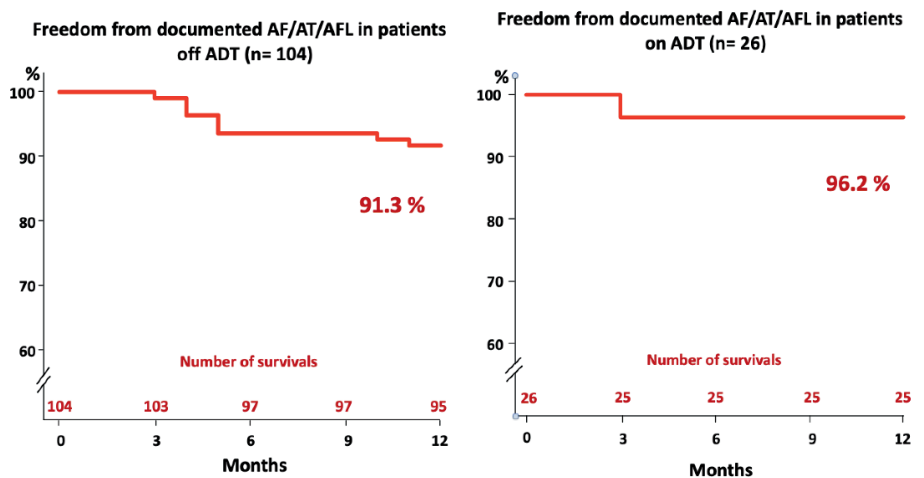

Figure 8: Kaplan-Meier curves depicting time to first recurrence of AF, AFL, or AT in patients undergoing CLOSE-PVI ablation for subgroups of patients not taking antiarrhythmic drug therapy (ADT) (left) and taking ADT (right).

In 72 of 130 patients CLOSE-PVI was performed under general anaesthesia and in 58 patients under conscious sedation. The 1-year arrhythmia-free survival was comparable between groups $(90.3 \%$ with general anesthesia, and $94.8 \%$ with conscious sedation $(\mathrm{p}=0.511)$, demonstrating the effectiveness of the CLOSE protocol irrespective of the sedation used. However use of conscious sedation comes at the expense of a longer (skin-to-skin) procedure time (164 $\pm 31 \mathrm{~min}$, compared to $147 \pm 22 \mathrm{~min}, \mathrm{p}=$ 0.01 ), fluoroscopy time ( $17 \pm 8 \mathrm{~min}$, compared to $16 \pm 7 \mathrm{~min}, \mathrm{p}=0.725)$ and $\mathrm{RF}$ time (for the right circle $18 \pm 4 \min$ vs. $15 \pm 3 \min , \mathrm{p}<0.001$; for the left circle $(21 \pm 7 \min$ vs. $16 \pm 4 \min , \mathrm{p}<0.001)$. 


\section{Safety evaluation of the CLOSE protocol.}

Atrio-esophageal fistula (AEF) is a very rare but often fatal and therefore one of the most feared complications of AF ablation. In order to minimize the risk of AEF, operators often reduce the RF power $(20-25 \mathrm{~W})$ on the posterior wall of the left atrium (or at least over the trajectory of the esophagus). ${ }^{14}$ This comes at the expense of the efficacy and efficiency of PVI procedures, because a lower RF power might result in non-transmural first-pass RF lesions with the need for touch-up applications, and consequently an increased RF and procedure time and suboptimal long-term PVI. Even despite this precaution, the reported incidence of AEF remains 0.02 to $0.11 \%{ }^{14}$ The CLOSE protocol however uses relatively high-power $(35 \mathrm{~W})$ applications to target the prespecified AI, also on the posterior wall. Using higher power is attractive because it allows reaching the target AI faster and therefore reduces RF and procedure time directly but also indirectly by reducing the risk for dislocation prior to reaching the AI target and the need for a second application. Using a higher power along the posterior wall in CLOSE-PVI was assumed not to increase the risk of esophageal injury because at a specific AI value (e.g. 400au on the posterior wall) the lesion depth is expected to be similar despite differences in power (higher power is offset by a shorter RF time). For safety reasons the target $\mathrm{AI}$ is decreased to $\geq 300 \mathrm{au}$ in case of intra-esophageal temperature rise (ITR) $>38.5^{\circ} \mathrm{C}$ (in anesthetized patients with an esophageal temperature probe) or excessive pain in awake patients.

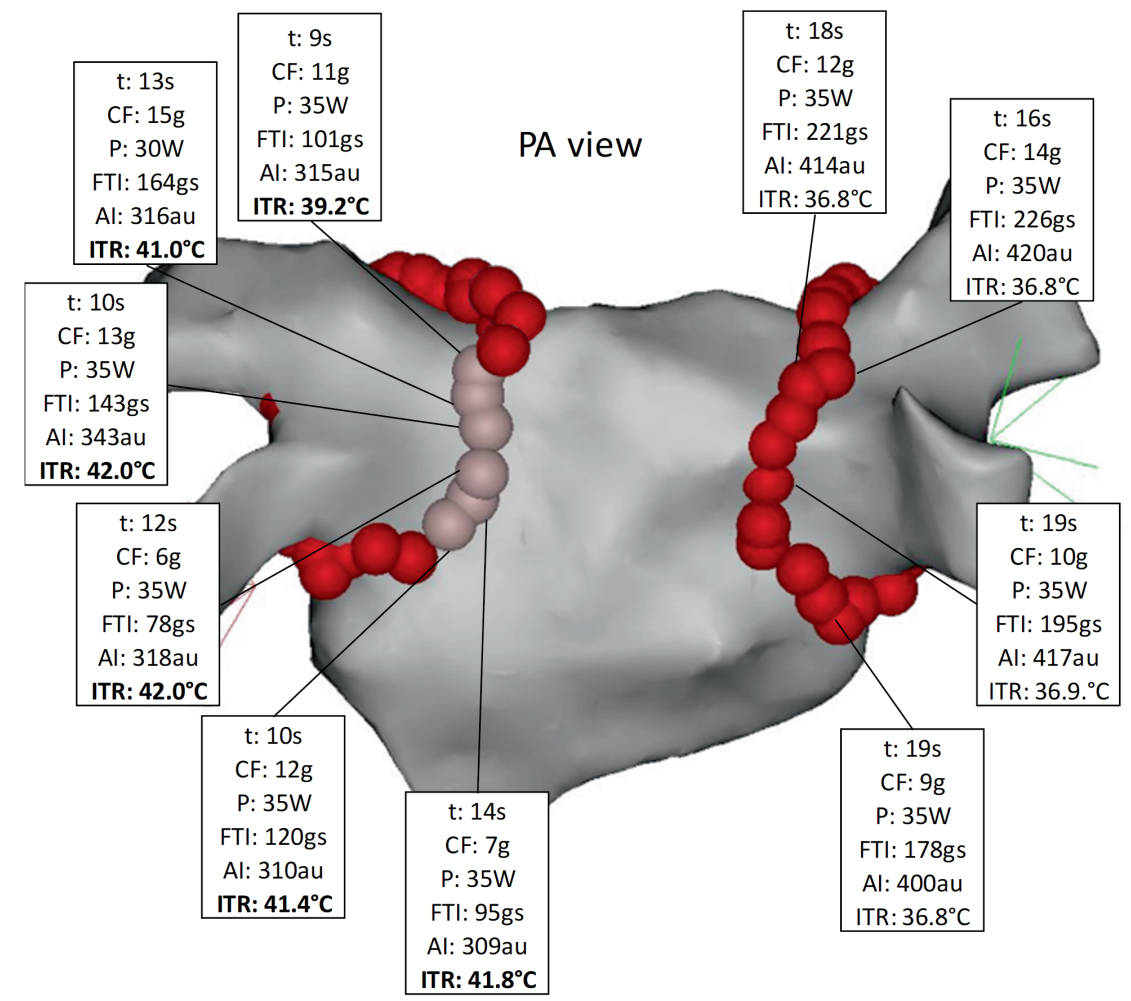

Figure 9: example of CLOSE-PVI: in six applications on the posterior wall of the left circle that resulted in intra-esophageal temperature rise $>38.5^{\circ} \mathrm{C}$, the ablation index target was reduced to $\geq 300$ au (pink Visitags). 
In Chapter 6 we evaluated the safety of the CLOSE protocol with regard to esophageal injury. Whereas the incidence of AEF itself is low (0.02 to $0.11 \%),{ }^{14}$ there is a high (2 to $30 \%$ ) incidence of subclinical esophageal or peri-esophageal injury on endoscopy performed within 7 days after AF ablation. ${ }^{14,53,54}$ Halbfass et al showed that the only risk factor associated with the occurrence of esophageal injury or ulcer is ITR. ${ }^{53}$ Furthermore they found that an esophageal ulcer is a necessary precursor for $\mathrm{AEF} .^{53}$ Therefore, to assess the incidence of esophageal injury (and potential risk of $\mathrm{AEF}$ ) of the CLOSE protocol, we performed echo-endoscopy in 85 patients revealing ITR $>38.5^{\circ} \mathrm{C}$ (max ITR per patient was $39.9^{\circ} \mathrm{C}\left(\left[\mathrm{IQR} 39.2-41.2^{\circ} \mathrm{C}\right]\right.$, with range $\left.38.6-50.0^{\circ} \mathrm{C}\right)$. Additional endoscopic ultrasound evaluation was performed in the first 38 patients. Of interest, with optimal positioning of the esophageal temperature probe, ITR $>38.5^{\circ} \mathrm{C}$ can be observed in $72 \%$ of CLOSE-PVI cases. Endoscopy was performed after mean $9 \pm 4$ days after PVI. In none of the 85 patients an esophageal ulcer was detected. In one patient we observed two erythematous lesions at day 4 after ablation. In none of the 38 patients undergoing additional sonographic evaluation, we observed direct signs of injury to the esophageal wall. In addition, during a median follow-up of 8.9 months [IQR 7.1-11.1] no complications of esophageal fistula, stroke or death were noted.
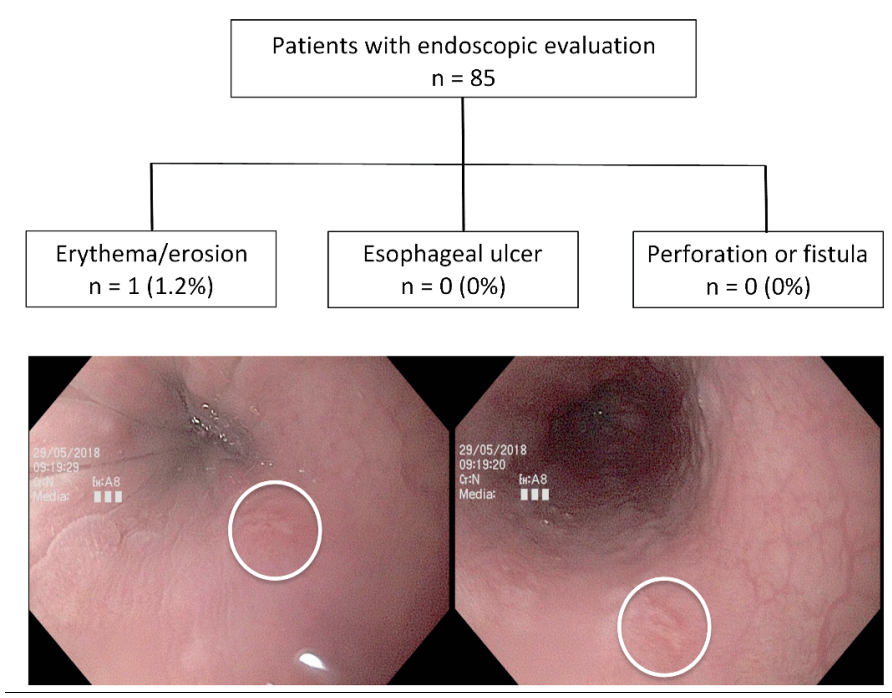

Figure 10: Results of endoscopic evaluation in 85 patients undergoing CLOSE-PVI with intra-esophageal temperature rise > $38.5^{\circ} \mathrm{C}$. Below two examples of esophageal lesions during endoscopy.

We found that the incidence of esophageal injury on endoscopy after CLOSE-PVI is low (1.2\%), despite significant ITR during the procedure. We hypothesized this was due to the specific characteristics of the CLOSE protocol: 
1. Due to the automated ablation tag, stability and contiguity criteria (resulting in limited dislocations and high incidence of first-pass isolation), there is a minimal amount of applications required to isolate the PVs. On average 10 applications per circle cross the posterior wall and only 5 of those are associated with ITR.

2. To reach the pre-specified AI targets at $35 \mathrm{~W}, \mathrm{RF}$ applications at the posterior wall can be as short as 10s (mean 18s) for AI 400au and as short as 8s (mean 14s) for AI 300au. It has been hypothesized that short duration-high power (SD-HP) applications favor the balance in resistive to convective heating thus preventing the risk of collateral damage. ${ }^{55}$ At least in experimental models, SD-HP applications result in more shallow lesions. ${ }^{56,57}$

3. Also inherent to CLOSE-PVI is the concept of striving for high stability rather than high CF. Excessive CF by the ablation catheter (applied in non-CF guided procedures or in earlier CF studies) might result in inadvertent displacement of the posterior wall towards the esophagus. ${ }^{58}$

4. Finally CLOSE-PVI is characterized by ablation at the LA-PV junction. Avoiding a wider encirclement reduces potential overlap with the esophagus and limits the number of procedures in which ITR is observed during both the left and right circle.

It seems unlikely that esophageal injury (and more specifically ulcer) was missed due to the relatively late timing of endoscopy ( $9 \pm 4$ days). In those patients with endoscopy within 7 days we also did not observe any ulceration. Moreover, also in the study by Halbfass et al, reporting $18 \%$ of injury, endoscopy was performed up to 7 days after ablation. ${ }^{53}$ Finally in that latter study, 47 patients with documented esophageal injury underwent a control endoscopy at $12.3 \pm 8.9$ days. Whereas in those patients with initial erythema/erosion, the site of injury was still detectable in $21 \%$ of patients, in those patients with initial ulcer the site of injury was still apparent in $83 \%$ of patients. As such it is unlikely that ulceration was missed in the present study. This hypothesis is further supported by the absence of esophageal or peri-esophageal wall injury on ultrasound.

The absence of esophageal ulceration and lack of changes on ultrasound in our study suggest that the CLOSE protocol is safe with regard to esophageal injury. These findings do require confirmation in a larger patient cohort.

\section{Efficacy evaluation: PV isolation status at repeat procedure}

In Chapter 4 and 5 we demonstrated that CLOSE-PVI was associated with a very high rate of firstpass and adenosine-proof isolation and a high single-procedure arrhythmia-free survival at 1 year, suggesting durable PV isolation. In Chapter 7 we further evaluated the effectiveness of CLOSE-PVI by determining the prevalence of patients presenting with 4 isolated veins at repeat ablation for AF recurrence after CLOSE-PVI. Out of 326 patients who had received a first CLOSE-PVI for paroxysmal AF, 45 patients underwent repeat ablation because of $\mathrm{AF}$ recurrence (ablation occurring at 
$7 \pm 7$ months after the index procedure). Four isolated veins were found in 28 of those 45 patients $(62 \%)$. In patients with PV reconnection $(n=17)$ the mean number of reconnected veins was $2 \pm 1$ with no predilection sites of PV reconnection.

\section{Localisation of gaps in patients with PV reconnection ( $n=27$ gaps, 17 patients)}

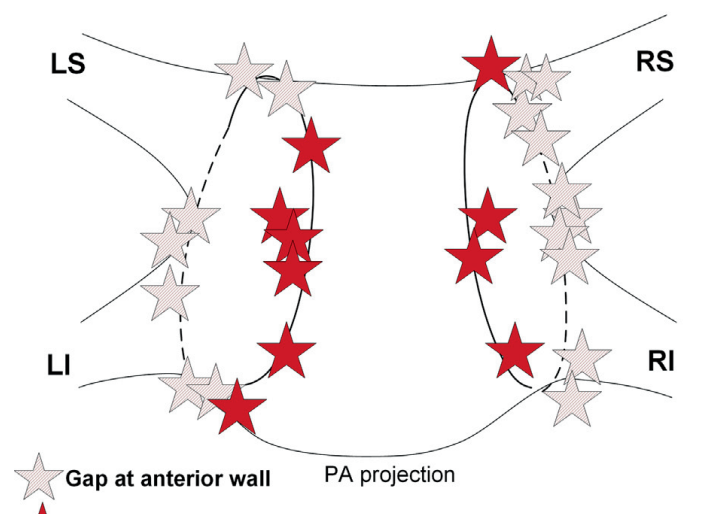

Quality analysis of the radiofrequency circle (circles with versus without reconnection)

\begin{tabular}{|c|c|c|c|}
\hline $\begin{array}{l}\text { Radiofrequency } \\
\text { characteristics }\end{array}$ & $\begin{array}{l}\text { Circles with PV } \\
\text { reconnection }\end{array}$ & $\begin{array}{c}\text { Circles with } \\
\text { isolated veins }\end{array}$ & $\mathrm{p}$ value \\
\hline Time $_{\text {Med }}(\min )$ & $\begin{array}{c}29 \\
{[27: 31]}\end{array}$ & $\begin{array}{c}29 \\
{[26: 32]}\end{array}$ & 0.262 \\
\hline Ablation index $\mathrm{Med}_{\mathrm{M}}(\mathrm{au})$ & $\begin{array}{c}483 \\
{[477: 487]}\end{array}$ & $\begin{array}{c}488 \\
{[478: 496]}\end{array}$ & 0.540 \\
\hline Force Time integral $\left.\right|_{\text {Med }}$ (gs) & $\begin{array}{c}412 \\
{[370: 448]}\end{array}$ & $\begin{array}{c}428 \\
\text { [380:499] }\end{array}$ & 0.244 \\
\hline Inter lesion distance ${ }_{\text {Med }}(\mathrm{mm})$ & $\begin{array}{c}4.1 \\
{[3.7: 4.6]}\end{array}$ & $\begin{array}{c}3.9 \\
{[3.7: 4.3]}\end{array}$ & 0.112 \\
\hline Contact force $_{\text {Med }}(\mathrm{g})$ & $\begin{array}{c}15 \\
{[13: 18]}\end{array}$ & $\begin{array}{c}15 \\
{[13: 18]}\end{array}$ & 0.172 \\
\hline$\Delta$ Impedance $_{\text {Med }}(\mathrm{ohm})$ & $\begin{array}{c}11 \\
{[10: 16]}\end{array}$ & $\begin{array}{c}12 \\
{[11: 13]}\end{array}$ & 0.740 \\
\hline
\end{tabular}

Gap at posterior wall

Figure 11: (Left) Localization of gaps in the ablation circles among patients (17 patients, 27 gaps) with atrial fibrillation recurrence and pulmonary vein (PV) reconnection. No predilection sites of PV reconnection could be identified. (Right) Circles with gaps showed similar lesion quality compared with circles with durable PV isolation.

Patients revealing 4 isolated veins at repeat ablation had similar clinical characteristics as patients with $\mathrm{PV}$ reconnection. Also, the arrhythmic profile of the initial paroxysmal AF was not different, with similar AF burden, both in terms of frequency and duration of AF episodes. Compared with patients with PV reconnection, patients revealing 4 isolated veins had also similar first procedural and RF characteristics. 
Table 2: Characteristics of the first PVI procedure in patients revealing 4 isolated veins at repeat

\begin{tabular}{|c|c|c|c|c|}
\hline & $\begin{array}{c}\text { All repeat } \\
\text { patients }(n=44)\end{array}$ & $\begin{array}{c}\text { Patients with } 4 \\
\text { isolated veins }(n=27)\end{array}$ & $\begin{array}{c}\text { Patients with } \\
\text { reconnected veins }(n=17)\end{array}$ & $\begin{array}{c}\mathbf{p} \\
\text { value }\end{array}$ \\
\hline \multicolumn{5}{|l|}{ Procedure characteristics } \\
\hline General anesthesia, n (\%) & $29(66)$ & $20(74)$ & $9(53)$ & 0.198 \\
\hline Common pulmonary vein, $\mathrm{n}(\%)$ & $4(9)$ & $2(7)$ & $2(12)$ & 0.634 \\
\hline Procedure duration (min) & $162 \pm 37$ & $158 \pm 42$ & $168 \pm 29$ & 0.443 \\
\hline $\mathrm{N}$ of RF applications (points) & $71 \pm 13$ & $69 \pm 13$ & $75 \pm 13$ & 0.148 \\
\hline Total time of RF energy (min) & $33 \pm 7$ & $32 \pm 6$ & $35 \pm 7$ & 0.076 \\
\hline Fluoroscopy time (min) & $15 \pm 8$ & $13 \pm 6$ & $17 \pm 9$ & 0.211 \\
\hline \multicolumn{5}{|l|}{ RF circle characteristics } \\
\hline Time $_{\text {Med }}(\mathrm{s})$ & $29[26: 32]$ & $29[26: 33]$ & $28[27: 31]$ & 0.365 \\
\hline Ablation index $\mathrm{x}_{\mathrm{Med}}(\mathrm{au})$ & $486[478: 494]$ & $484[478: 496]$ & $487[479: 494]$ & 0.689 \\
\hline Force time integral $\mathrm{Med}_{\mathrm{Med}}(\mathrm{gs})$ & $426[379: 484]$ & $426[370: 501]$ & $426[386: 451]$ & 0.567 \\
\hline Inter lesion distance ${ }_{\text {Med }}(\mathrm{mm})$ & $3.9[3.7: 4.4]$ & $4.1[3.7: 4.4]$ & $3.9[3.7: 4.3]$ & 0.907 \\
\hline Contact force $_{\mathrm{Med}}(\mathrm{g})$ & $15[13: 18]$ & $15[13: 18]$ & $16[14: 18]$ & 0.716 \\
\hline$\Delta$ Impedance $_{\text {Med }}(\mathrm{ohm})$ & $12[11: 13]$ & $12[11: 14]$ & $12[11: 13]$ & 0.772 \\
\hline
\end{tabular}

Previously, paroxysmal AF recurrence after PVI was invariably assumed to be caused by reconnection of 1 or more PVs. This was based on numerous prior studies reporting a low incidence of a status of complete vein isolation at repeat ablation for AF recurrence. ${ }^{27-29,59-66}$ Moreover the good outcome after re-isolation of reconnected veins with repeat ablation supports the theory of PV reconnection as the main pathophysiological mechanism of AF recurrence.

In the earlier years (using non-irrigated or irrigated non-CF RF to isolate the veins) the likelihood of complete isolation was as low as $0 \%$ and certainly not exceeding $20 \%$. Although CF-guided ablation is associated with higher percentage of complete isolation in study patients undergoing invasive reevaluation at 3 months regardless of symptoms, ${ }^{47,67}$ more recent data on CF-guided RF PVI still show high reconnection rates at repeat procedure for AF recurrence. ${ }^{64,65}$ Shah et al. showed that the incidence of isolated veins in patients with late AF recurrence ( $>36$ months after first PVI) was $19 \%{ }^{65}$ Buist et al. reported a low incidence of finding 4 veins isolated at repeat for CF-guided RF (only $3 \%)$. ${ }^{64}$ Finally, a recent meta-analysis by Nery et al. reported an overall $14 \%$ likelihood of finding 4 isolated veins in case of a clinically indicated repeat ablation after PVI. ${ }^{68}$ 


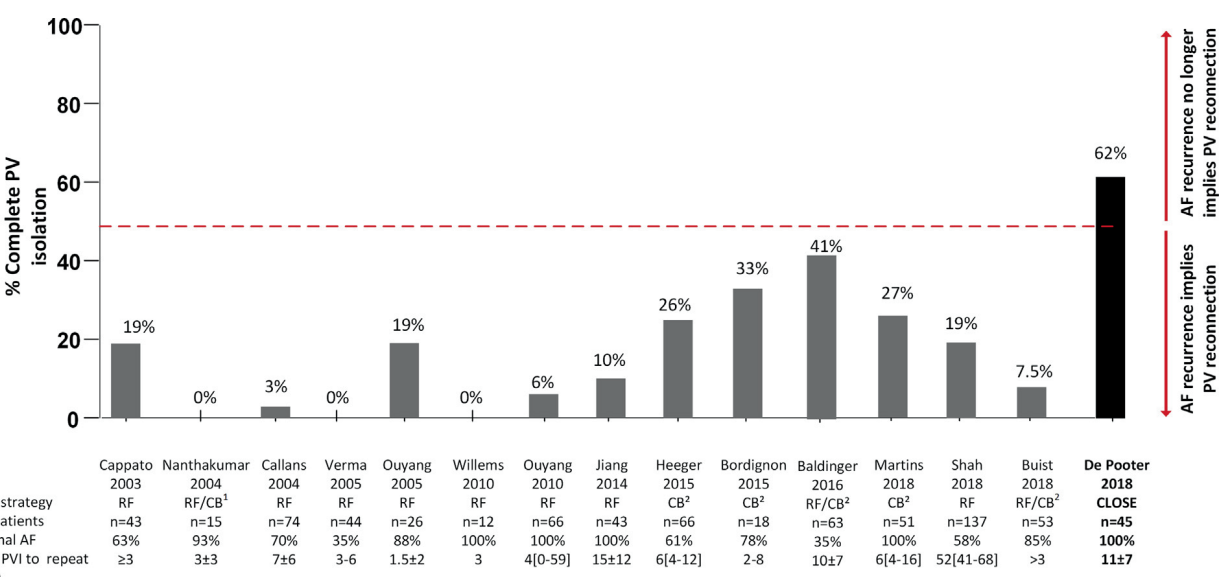

Figure 12: Overview of studies assessing the likelihood of finding 4 isolated veins after single PVI. After CLOSE-guided PVI, AF recurrence no longer implies PV reconnection. (CB: cryoballoon)

The present observation that $62 \%$ of clinically indicated repeat patients reveal a status of complete isolation confirms that CLOSE-PVI, by avoiding weak links in the ablation chain, is associated with durable isolation. This finding is in line with our prior data of favorable acute and 1-year outcome after CLOSE-PVI. Of course, the likelihood of finding 4 isolated veins is expected to be higher when evaluating all CLOSE-PVI patients (i.e. also those without AF recurrence).

This implies that AF recurrence after CLOSE-PVI is more likely to point to non-PV mediated AF. Whereas in the past repeat ablation for paroxysmal AF was straightforward, there are now several issues to consider. Repeat procedures after non-CF or conventional CF-guided PVI were generally shorter than the index procedure, because closing one or more gaps was usually faster than a complete first circumferential lesion set. In addition the clinical outcome of re-isolation of reconnection veins was favorable. In case of AF recurrence with status of four isolated veins, the procedure time can be highly variable (with practical planning issues), the preferred ablation strategy is unknown (unless there is an overt non-PV trigger during the procedure), and the outcome is worse irrespective of which ablation strategy is chosen as we also demonstrated in our patient cohort. Indeed, patients with 4 isolated veins, compared with patients treated for PV reconnection, showed a lower 12-month freedom from $\mathrm{AF}$ after repeat ablation $(61 \%$ vs. $88 \%, \mathrm{p}=0.045)$. 


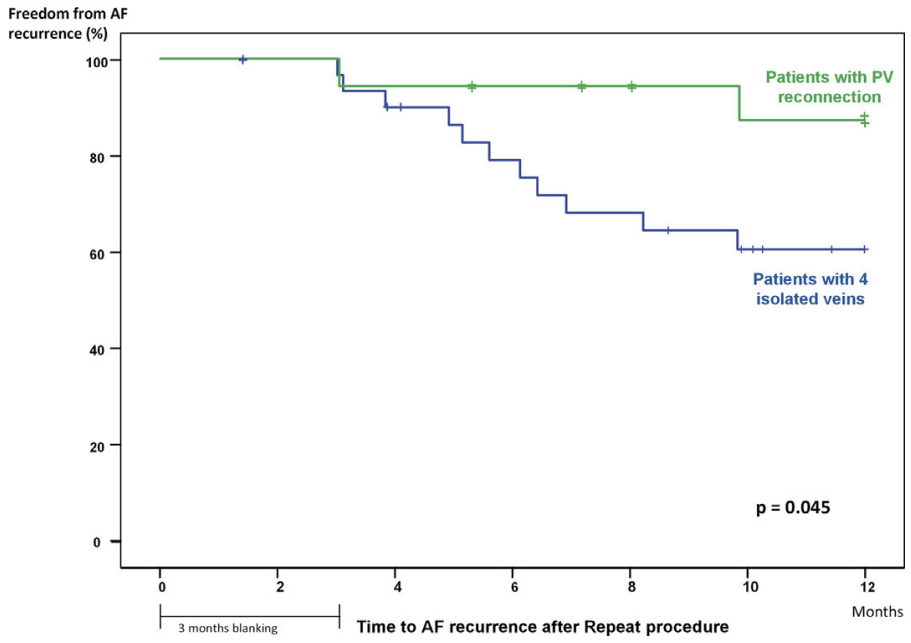

Figure 13: Kaplan-Meier curves depicting time to AF recurrence after repeat ablation for patients with ( $\mathrm{n}=17)$ and without $(n=28)$ PV reconnection at repeat ablation. Patients with all 4 veins being isolated (treated with either empirical trigger ablation or linear substrate ablation) showed worse outcome.

Unfortunately there are no distinct characteristics (neither clinical (e.g. age, sex, LA size) nor in phenotype of paroxysmal AF (e.g. duration and frequency of AF episodes)) that predict the likelihood of finding four isolated veins after AF recurrence. We did observe that in patients with AF recurrence and 4 isolated veins, the incidence of low voltage was significantly higher $(57 \%$ vs. $17 \%$ in reconnected patients, $\mathrm{p}=0.033$ ) and the area of low voltage was significantly larger (median: 4.6 [IQR: $2.3,7.9] \mathrm{cm} 2$ vs. 0.0 [IQR: $0.0,0.0] \mathrm{cm} 2$ in reconnected patients, $\mathrm{p}=0.049$ ). Those low voltage areas are suggestive for atrial fibrosis either as a manifestation of structural heart disease or as a result of an intrinsic fibrotic atrial cardiomyopathy ${ }^{69,70}$ Our findings are in line with prior studies reporting worse single procedure outcome after first PVI in patient presenting with fibrosis or low voltage areas in the LA. ${ }^{71,72}$ The association of low voltage with less favorable outcome after empirical ablation suggests that a patient-specific set-up of repeat ablation might be required to optimize outcome in patients presenting with complete vein isolation.

Efficacy evaluation: arrhythmia-free survival after CLOSE-PVI evaluated with insertable cardiac monitors.

In Chapter 5 we demonstrated that CLOSE-PVI in a cohort of 130 patients with paroxysmal AF was associated with favourable single-procedure, 1-year arrhythmia-free survival, evaluated be intermittent holter monitoring. With intermittent Holter recordings arrhythmia-free survival depends on the extent of monitoring and will always be overestimated as (asymptomatic) recurrences between Holters will be missed. Yet the time-to-first recurrence is an objective outcome parameter, well suited to compare 2 techniques, but does not reflect the most important/relevant clinical efficacy endpoint. The "atrial tachyarrhythmia (ATA) burden" (defined as the \% of time spent in AF/AT/AFL) after an index 
ablation is not limited to the first event, but - more relevantly - it reflects the amount and duration of all recurring events. To illustrate, both a patient with an isolated recurrence and a patient with frequent recurrences are equally deemed clinical trial failures by time-to-first event analysis, yet the impact of failure is completely different from the clinical point of view. Indeed, prior studies suggested a doseeffect relation between ATA burden and symptoms, heart failure and stroke. ${ }^{73-77}$

In Chapter 8, we evaluated the 2-year efficacy of CLOSE-PVI in 105 patients with paroxysmal AF using continuous monitoring with insertable cardiac monitors (ICM) and also evaluated its effect on the ATA burden. To reliably quantify the effect on the ATA burden, all patients were implanted with an ICM at least 2 months before PVI. Within the two-year follow-up period, 0 patients received ADT or cardioversion, 14 patients received a repeat procedure at 150 [100-513] days after PVI.

Even using continuous rhythm monitoring, the single-procedure, off-ADT freedom from any 2-min ATA was very favourable and amounted $87 \%$ after 1 year and $78 \%$ after 2 years.

\section{Freedom from any ICM documented ATA at 2 years follow-up}

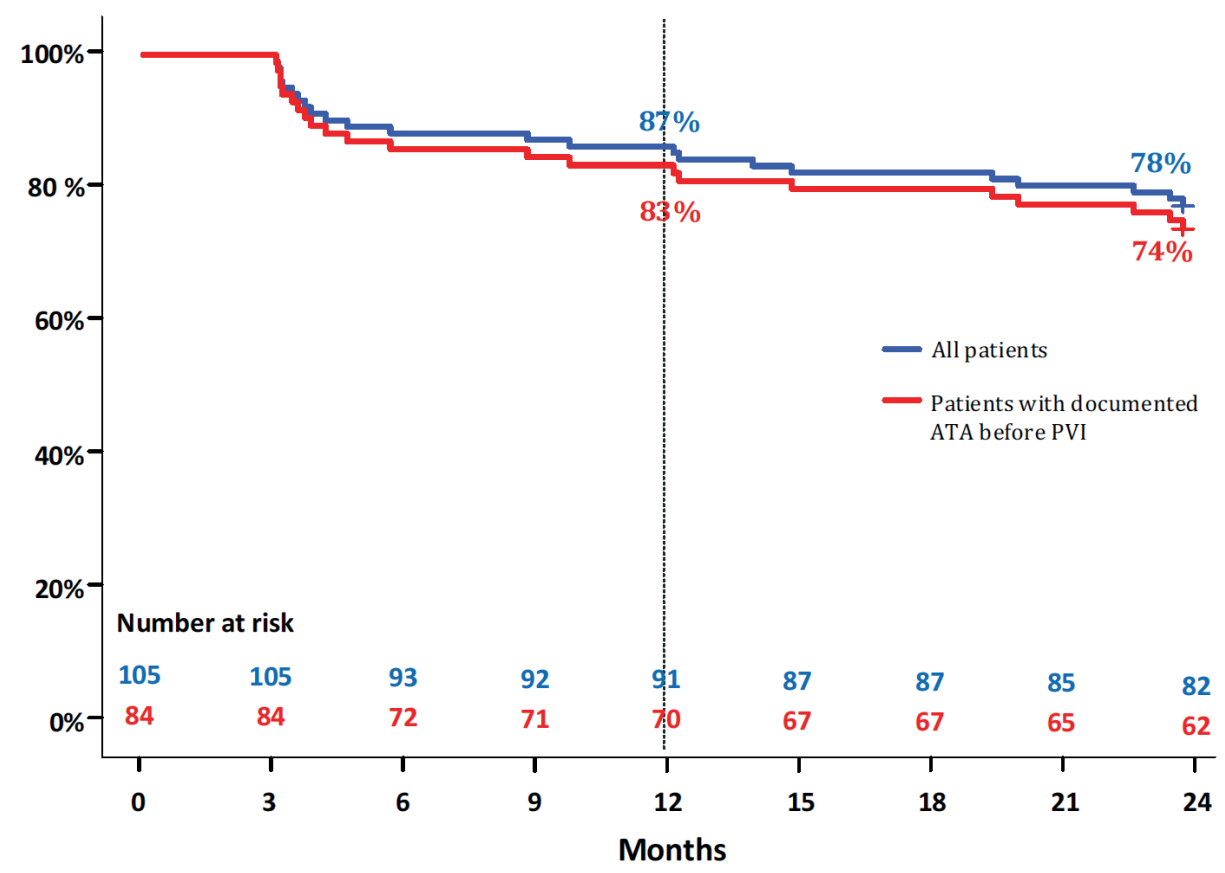

Figure 14: Kaplan-Meier curves depicting time to first recurrence of any ATA during the first 2 years after PVI (blue curve: entire study population; red curve: 84 patients with ILR-documented ATA within 2 months before ablation).

After CLOSE-PVI (1.13 \pm 0.39 procedure per patient throughout the 2-year follow-up), the ATA burden decreased from 2.68 [0.09-15.02] \% at baseline to 0 [0-0] \% during the first year (reduction in 
ATA burden $100[100-100] \%, \mathrm{p}<0.001$ ) and 0 [0-0] \% during the second 2-year (reduction in ATA burden 100 [100-100] \%, p<0.001). Results for the subset of 84 patients with documented ATA during the 2-month monitoring period prior to ablation were similar. The proportion of patients with $>95 \%$ reduction in ATA burden was $94 \%$ and $96 \%$ at 1 and $2-y$ FU. Finally, throughout the first and second year after ablation, only $5(6 \%)$ and $1(1 \%)$ had a residual ATA burden $>0.5 \%$. These results show that CLOSE-PVI (with a low number or repeat procedures and without ADT) results in a major overall reduction in ATA burden and this effect is maintained at longer-term follow-up. There results confirm the efficacy of CLOSE-PVI.

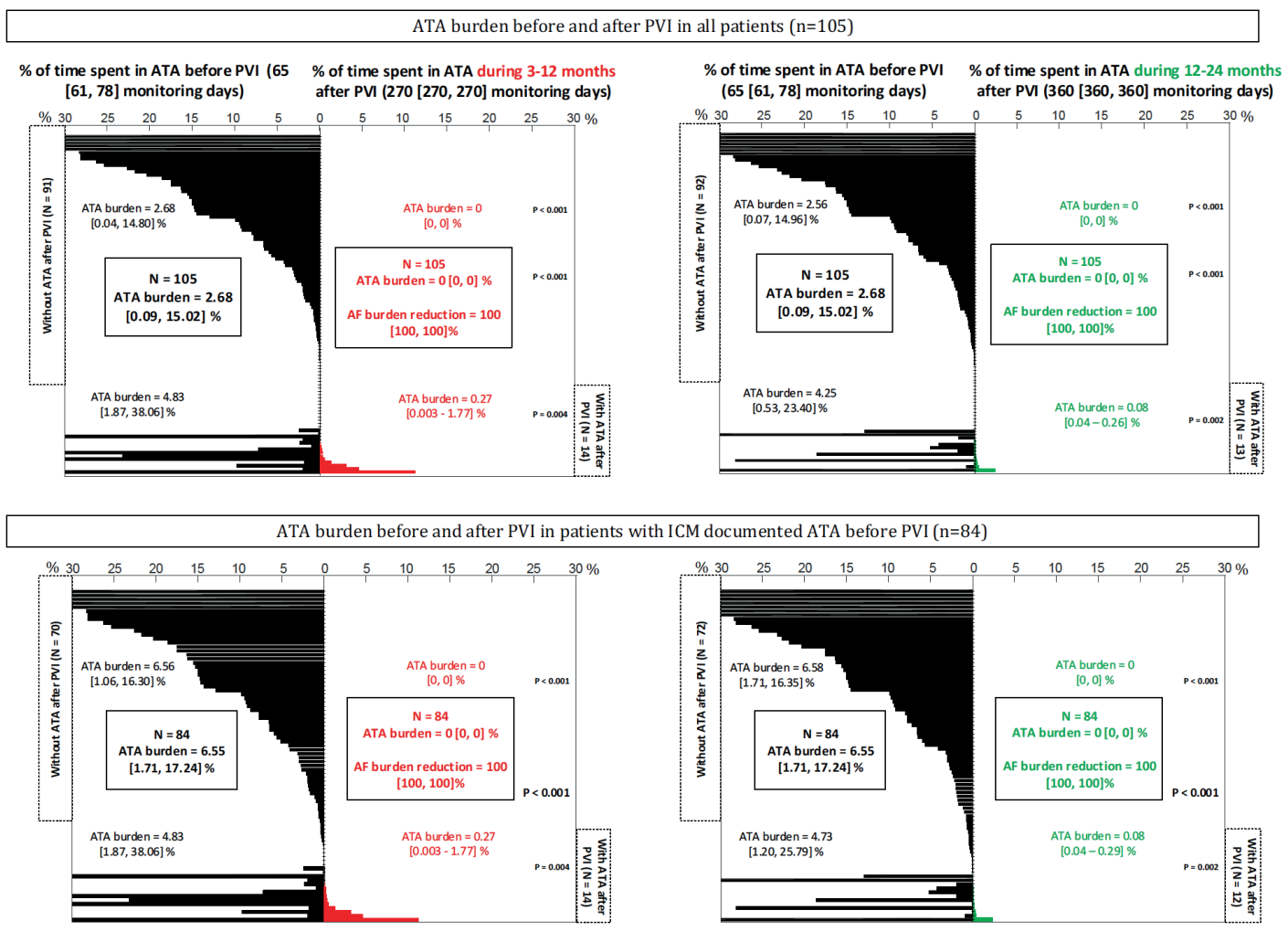

Figure 15: Plots showing the \% of time spent in ATA during the first and second year compared to baseline in the entire population (upper panels) and subpopulation (lower panels). Each line represents an individual patient. Patients are arranged by recurrences during follow-up, ranging from greatest (bottom) to fewest (top).

\section{The CLOSE protocol: conclusion and future perspectives}

Over the past 20 years, there has been a constant effort to improve the PVI technique and achieve durable PV isolation in order to improve the outcome of AF ablation. In this thesis we developed a new PVI protocol after analysing optimal target criteria for lesion quality and contiguity. In singlecenter experience, this CLOSE protocol has demonstrated superior efficacy, even when using 
continuous monitoring to detect AF recurrence, compared to previously published outcomes of PVI performed with either RF or cryo-energy.

Evaluation of a new ablation protocol/technique in a single arm design and comparing its outcome to historical results of older techniques has however inherent pitfalls and limitations. A blinded, randomized controlled trial comparing CLOSE-PVI to conventional CF-guided PVI would be needed to prove the true superiority of the CLOSE protocol. However, after gaining extensive experience with CLOSE-PVI, the principles of RF application quality and contiguity have become so accustomed to the operator, conventional CF-guided PVI results would always be influenced by the gained knowledge and experience and such randomized trial would therefore probably be futile. However, the favourable results on outcome and safety need to be confirmed in larger studies. As such, the multicenter VISTAX study was designed to assess the reproducibility of CLOSE-PVI across 17 European centers and evaluate the outcome in a larger group of 340 paroxysmal AF patients.

Over the last years, PVI with cryo-energy has gained much popularity. This is largely due to two of the major advantages of the Cryoballoon over RF, i.e. the shorter procedure time and the faster learning curve. In the landmark FIRE and ICE trial, non-inferiority of these two treatment modalities was demonstrated, but the results and conclusion were hampered by the use of both non-CF and CF sensing catheters in the RF-arm, and both second and first generation Cryoballoon in the cryo-arm. ${ }^{78}$ However recently the CIRCA-DOSE study evaluating Cryoballoon vs. CF-guided RF PVI for paroxysmal AF confirmed no difference in one-year efficacy assessed by continuous cardiac rhythm monitoring, neither in time-to-first-recurrence nor in reduction of the atrial tachyarrhythmia burden. ${ }^{79}$ Undeniably, the Cryoballoon does have two disadvantages over point-by-point RF. First, the incidence of phrenic nerve palsy is up to $2.7 \%$ with cryo compared to $\sim 0 \%$ with $\mathrm{RF}^{78,79}$ Even if most phrenic nerve palsies recover over time, any (transient) intra-procedural phrenic nerve palsy will limit the applied cryo-application time and rarely even result in omission of an attempt to achieve isolation of the right (superior) PV. Second, the fixed balloon shape is not adaptable to individual anatomical configurations of the left atrial-PV junction. In some cases this results in suboptimal freeze temperatures, too distal PV isolation and rarely incomplete procedural PV isolation. In contrast, with point-by-point RF, the CLOSE protocol targets can be achieved in nearly any anatomical variant. In order to investigate if the CLOSE protocol will finally be able to tip the balance in favour of point-bypoint RF when compared to Cryoballoon PVI, a randomized, multicenter trial would be needed.

The CLOSE protocol has markedly reduced procedure times compared to non-CF en CF guided PVI. Because of the $98 \%$ adenosine and waiting time proof isolation, CLOSE-PVI has allowed omission of the adenosine testing and the 20-minute waiting-time in our institutional ablation protocol. After a learning period, and with general anaesthesia, dislocations during application have become rare, and skin-to-skin procedure times are reproducible around 100 minutes. However in our striving to 
optimize PVI results there is growing interest in increasing the ablation power (high-power, shortduration applications) in order to further decrease the procedure time while maintaining/increasing safety. Given that the lesion depth is similar at a specific AI value (irrespective of the power) using a higher ablation power $(45-50 \mathrm{~W})$ is expected to be feasible. The POWER-AF trial was designed to investigate the efficacy and safety of the CLOSE protocol while using $45 \mathrm{~W}$ applications.

Even when point-by-point ablation is meticulously performed according to the strict established criteria, the CLOSE protocol is still not able to ensure durable PV isolation in $100 \%$ of cases. Indeed, at repeat ablation due to arrhythmia recurrence, we found that $38 \%$ of patients still had at least one reconnected vein. This could be due to variables that are not taken into account, such as the tissue thickness or tissue composition. A patient-tailored strategy with adapted ablation characteristics based on pre- or intra-procedural imaging (e.g. with computed tomography or intra-cardiac echo) as well as intra-procedural lesion verification (e.g. with magnetic resonance imaging) might be the next step to improve CLOSE-PVI results. 


\section{Optimizing left atrial linear ablation}

Left atrial (LA) linear lesions are one of the main techniques described for additional ablation in persistent AF and are also frequently performed for left atrial macroreentrant tachycardia. Roof and mitral isthmus (MI) lines are the most commonly performed LA linear lesions. Even though achieving bidirectional conduction block is challenging ${ }^{80-84}$ (reported block across roof lines of 81 to $95 \%{ }^{81,84,85}$ and for the MI line between 57 to $92 \%{ }^{80-82,86-87}$ ), it is well known that incomplete bidirectional conduction block has been associated with an increase in subsequent arrhythmia during follow-up. ${ }^{81-82}$ On the other hand, increased success in completing linear block is often associated with an increased complication rate from higher ablation power and a higher risk of steam pop, perforation, and tamponade. $^{88}$

In Chapter 9 we applied the same ablation principles of the CLOSE protocol, to assess the efficacy of optimized point-by-point linear RF lesions at the roof and posterior mitral isthmus (MI) in 41 patients.

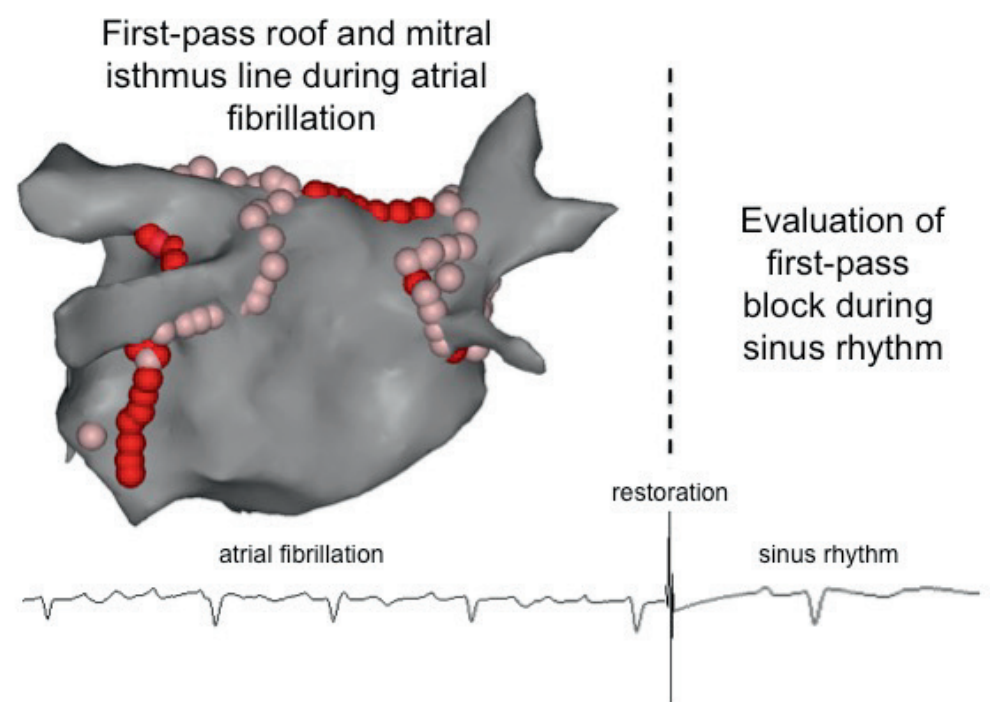

Figure 16: Single anatomical linear lesions at roof and posterior mitral isthmus of contiguous applications with a maximum interlesion distance $\leq 6 \mathrm{~mm}$ (center-to-center) and Ablation Index $(\mathrm{AI}) \geq 550$ (red Visitags).

By only using criteria for contiguity (interlesion distance $\leq 6 \mathrm{~mm})$ and Ablation Index $(\geq 550)$, we achieved complete block in the roof line in $93 \%$ of patients after a single linear lesion set, but only in $23 \%$ at the MI line (without ablation in the coronary sinus) confirming the difference for both lines when applying RF catheter ablation. 


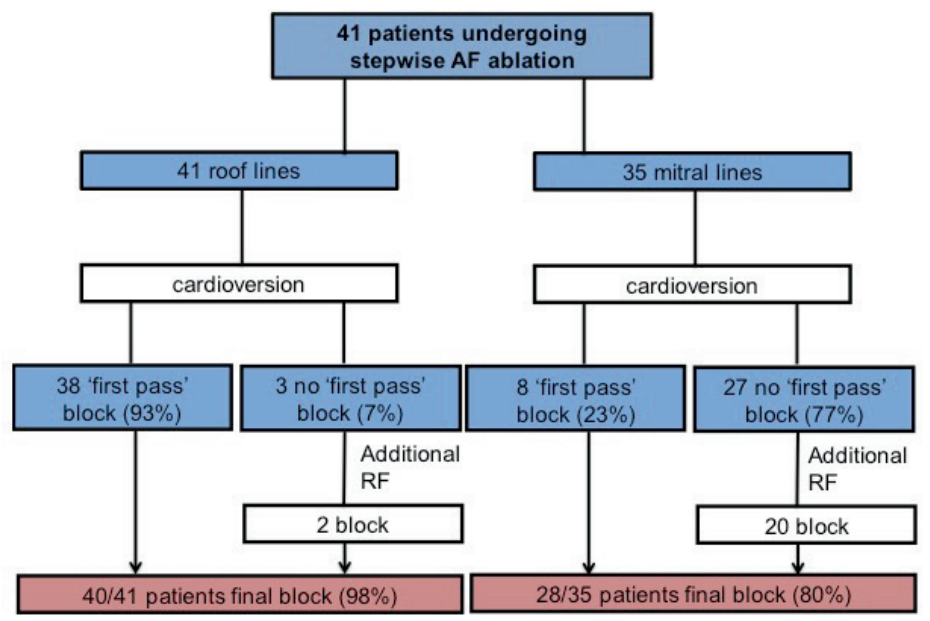

Figure 17: Results of first-pass and final roof and mitral isthmus block.

The very high success rate of first-pass block on the roof in our study (93\%) can probably be explained by the favorable and uniform anatomy of the roof, which renders the roof quite suitable for a strategy aiming at transmurality and contiguity of RF lesions. This was emphasized by the low number of dislocations before reaching Ablation Index target value ( 0 [IQR $0-1]$ per patient). In the case of absence of block after the first-pass roof line and during the redo procedures, all conduction gaps were located in the middle of the roof or near the right PVs (never at the left side of the roof). These locations are characterized by lower stability during RF delivery and also by thicker tissue with interatrial muscular bridges. ${ }^{89}$

Conversely, the success rate for achieving bidirectional conduction block after a first pass linear lesion at the MI was low (23\%). This can probably be explained by the complex 3D anatomical characteristics of the MI region: 1) Post mortem anatomical and in vivo studies have shown that the atrial myocardium at the left mitral line has a wall thickness of up to $10 \mathrm{~mm}$ at the ostium of the left inferior PV. ${ }^{90}$ In comparison, the LA roof is thinner with a range between 3.5 and $6 \mathrm{~mm} .{ }^{89}$ The distance between the endocardium and the overlying inner surface of the coronary sinus (CS) is even larger than the MI thickness, due to the adipose tissue overlaying the CS along the mitral valve, sometimes with interlaying circumflex artery branches. ${ }^{86,91}$ 2) Numerous recesses and cavities along the left mitral isthmus have also been reported ${ }^{18}$ as well as epicardial muscular connections. ${ }^{80,91-92} 3$ ) Blood flow in the CS and circumflex artery may also act as a 'heat sink' and reduce the efficacy of radiofrequency ablation. ${ }^{91}$ 
These anatomical characteristics of the MI region necessitate additional ablation within the CS in the majority of patients (reported in 60 to $84 \%$ of cases ${ }^{80,86-87}$, and to $54 \%$ in our patient cohort) in order to achieve conduction block.

Recovery of conduction has been reported to occur in up to $90 \%$ of MI line and $79 \%$ of the roof lines during repeat evaluation for atrial arrhythmia recurrence ${ }^{93,94}$. In 12 patients conduction recovery was evaluated at repeat ablation for recurrence of atrial tachycardia. For the roof, there was persistent block in 8 out of $12(67 \%)$ evaluated lines. Of the 12 re-evaluated MI lines, 2 were not blocked during the index procedure and were still conducting. Of the remaining 10 evaluated lines, $5 \mathrm{MI}$ lines showed persistent block (50\%) while 5 had re-conduction. Limited RF delivery was necessary to achieve conduction block and was successful in all re-ablated patients for both lines. The different results in durability of achieved block between roof and MI lines was probably again attributable to the differences in anatomy.

As such, anatomical ablation using contiguous and optimized RF lesions appeared very effective for the roof line but not for the MI line. Unlike in CLOSE-PVI, the criteria for Ablation Index and interlesion distance were not based on retrospective analysis, but were arbitrarily chosen for both lines. The chosen criteria were based on previously acquired local experience, and in order to minimize the risk of complications and dislocation (during longer RF applications) which could have impacted the interpretation of the data. In the future, higher Ablation Index or shorter interlesion distance should be evaluated for the MI line given the low amount of first pass block. Also preevaluation with imaging technologies might also improve the results by allowing adaptation of the criteria depending on individual anatomical variations and helping avoid RF under- and overshooting. Although no safety-related issues were observed, larger studies are necessary to evaluate the safety and efficacy of this protocol for linear LA lesions. 


\section{Mapping of drivers in persistent atrial fibrillation}

Despite the advances in technology and our insight in the molecular, ionic, and physiological fundamentals of cardiac electrophysiology, the exact mechanism of AF is still not completely elucidated. Mechanisms how AF is initiated and sustained may differ from patient to patient, and in various AF types (paroxysmal, persistent or permanent). The following concepts have competed trying to explain the pathophysiology of AF: 1) the multiple wavelets hypothesis (Moe ${ }^{95}$ and Allessie $\left.{ }^{96}\right), 2$ ) rapidly discharging automatic foci $\left(\right.$ Waldo $\left.^{97}\right), 3$ ) anatomical localized reentry (Fedorov ${ }^{98}$ ) and 4) functional reentry or rotors $\left(\mathrm{Jalife}^{99}\right)$. Aside from the technical limitations in delivering qualitative ablation lesions, our limited understanding of the mechanisms that initiate and sustain AF withholds us from being able to cure this arrhythmia. While the outcome of $\mathrm{AF}$ ablation in paroxysmal $\mathrm{AF}$ is favorable and ensues from elimination of the triggering firing sources that arise predominantly from the pulmonary veins, the results of PVI are inferior in persistent AF. It is therefore perceived that in these patients (additional) ablation targeting the sustaining atrial substrate is necessary in order to improve ablation outcome. The benefit of non-specific ablation strategies (such as linear ablation or ablation of non-specific complex fragmented atrial electrograms) ${ }^{100-101}$ has proven modest and therefore research is focusing on patient-tailored substrate ablation that is selectively targeting either the patient's specific anatomical substrate (e.g. fibrosis ${ }^{102}$ ) or patient specific mechanistic AF drivers. Indeed intra-procedural identification of focal and reentrant $\mathrm{AF}$ drivers could pose attractive and patient-specific targets for AF ablation. In the last few years, there has been progress in mapping AF sources and mechanisms, mostly using phase-based analysis of epicardial or endocardial potentials. ${ }^{103-}$ ${ }^{109}$ However, phase mapping has limitations as it is known to generate false rotational activities, particularly in cases of low voltage, variable contact and when far-field signals are present. ${ }^{110-111}$ Recently, some studies created AF propagation maps based on direct contact mapping (without phase transformation) using dedicated software (CARTOFINDER ${ }^{\mathrm{TM}}$, Biosense Webster) and a multielectrode basked catheter and were able to reliably and reproducibly identify repetitive activation patterns in the majority of patients. ${ }^{112-115}$ However, as the basket catheter has issues of long interelectrode distance and obtaining stable contact with the atrial wall in some regions leading to underdetection of AF drivers, in Chapter 10 we sought to evaluate the Cartofinder software for automated detection of AF drivers with a new mapping technique using a regional high-density contact mapping catheter (PentaRay ${ }^{\mathrm{TM}}$, Biosense Webster) in sequential positions that overlap the previous recording position with the radius of the PentaRay until the whole biatrial surface is covered. In 14 patients with persistent $\mathrm{AF}$, we characterized repetitive atrial activation patterns (RAAPs), with either focal or rotational activity, during ongoing AF based upon the automated annotation of directly obtained unipolar electrograms. This technique of sequential biatrial mapping proved feasible: a mean of 35.6 \pm 7.6 PentaRay recoding per patient were performed during $44 \pm 6$ min. of mapping with $6 \pm 3$ min radiation exposure. The number of PentaRay recordings displaying RAAP was 9.8 \pm 3.1 per patient (range 3-15), of which 2.4 $\pm 2.4 \mathrm{RAAP}_{\text {rotational }}$ (range 0-7) and 7.4 $\pm 4.4 \mathrm{RAAP}_{\text {focal }}$ (range 1-13). 77\% of 
RAAPs portrayed focal firing. RAAPs were observed both in the RA (35\%) and LA (65\%), with the majority being near the left PVs/appendage (35\% of all RAAPs) and the SVC/right appendage (23\% of all RAAPs). Our study showed that direct endocardial high-density contact mapping EGM analysis during ongoing AF allows identification of RAAPs in all persistent AF patients (predominantly focal firing sources in our study). Our technique of sequential high-density mapping may detect a greater number of RAAPs due to better coverage and mapping density in comparison with basket catheter. The automated annotation software has the potential to provide on-line detection of potential bi-atrial AF drivers annotated on the 3D-geometric bi-atrial shell, making patient-tailored substrate ablation possible. However the effect of ablation of RAAP was not investigated in our study and further studies are needed to investigate if ablation of RAAPs as detected by the system would improve clinical outcome. 
Evolutions in ablation of ventricular tachycardia and impact on long-term outcome results.

Catheter ablation for sustained monomorphic ventricular tachycardia (VT) has emerged as an effective tool for the control of VT recurrence. Ablation strategies have shifted from using activation and entrainment mapping of inducible and hemodynamically tolerated VTs ${ }^{116-117}$ to alternative strategies predominantly based on straightforward substrate modification, such as elimination of LAVA (local abnormal ventricular activities), ${ }^{118}$ late potential abolition, ${ }^{119}$ scar de-channeling, ${ }^{120}$ scar core isolation ${ }^{121}$ and scar homogenization. ${ }^{122,123}$ These strategies not only have the advantage of identifying targets for ablation in patients in whom VT is not inducible or not tolerated, but they may also provide a more comprehensive treatment of the substrate for present but also future VT, as they are not limited to inducible or "clinical" types of VT.

Several groups have tried to modify potentially arrhythmogenic surviving myocardial tissue by targeting late potentials in scar regions. ${ }^{124-126}$ However both the voltage amplitude ${ }^{127}$ and the timing of near-field signals from poorly coupled surviving bundles ${ }^{124}$ are dependent on the activation wavefront, and consequently substrate-based ablation targeting solely late potentials or confined to voltagedefined scar might miss part of the arrhythmogenic substrate. The LAVA approach ${ }^{118}$ aims to overcome these limitations, by not being limited to late potentials (occurring after the QRS) or confined to low-voltage regions.

In Chapter 11 we report long-term outcomes after single and multiple ablation procedures with elimination of LAVA in 159 patients $(65 \pm 11$ y, $92 \%$ ICDs, $54 \%$ storms and $73 \%$ appropriate shocks) with post-myocardial infarction VT. We also the investigated the effect of implementation of evolutionary techniques such as multi-electrode mapping and/or use of real-time image integration on ablation outcomes.

We demonstrated that LAVA can be successfully identified in the vast majority of patients $(92 \%)$ referred for post-MI VT ablation, when using a strategy of meticulous mapping. The endpoint of LAVA elimination in addition to non-inducibility is feasible and effective for arrhythmia control. With a single procedure, $73 \%$ of the patients are free from arrhythmia at 1 year. This success rate decreases over time, but $49 \%$ of patients are still free from any ventricular arrhythmia at 5 years. At the modest cost of repeat procedures in $18 \%$ of patients, this result is improved to $85 \%$ arrhythmiafreedom at 1 year, remaining $62 \%$ after 5 years. 
A

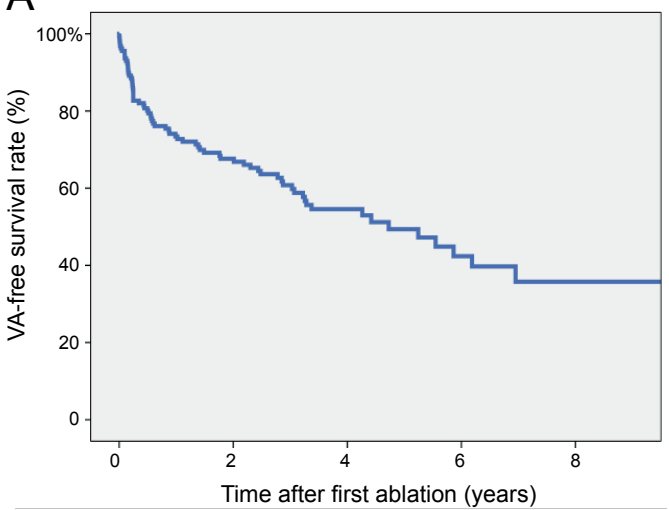

Number at risk

$159 \quad 100$

$44 \quad 21$

B

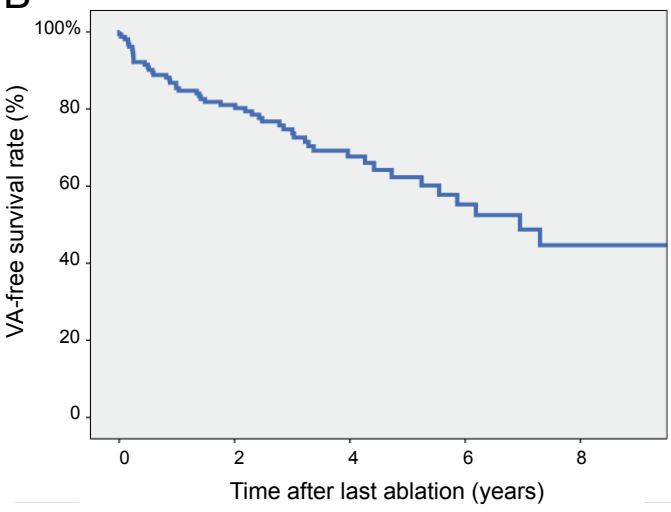

159

87

7

Figure 18: Ventricular arrhythmia (VA)-free survival after single (A) and including repeat (B) ablation procedures in $18 \%$ of patients $(1.3 \pm 0.6$ ablations/patient $)$.

In addition only $9 \%$ of patients had appropriate shocks over a mean of 4 years follow-up. The reduction in VT burden was more than $87 \%$ in $91 \%$ of patients after a single procedure (Figure 19). This strategy allowed for a substantial discontinuation of antiarrhythmic drugs and amiodarone use was reduced by $54 \%$, this benefit being maintained at 5 years. In accordance with previous reports, complete LAVA elimination, which was achieved in $64 \%$ of patients in our cohort, was associated with better outcome.

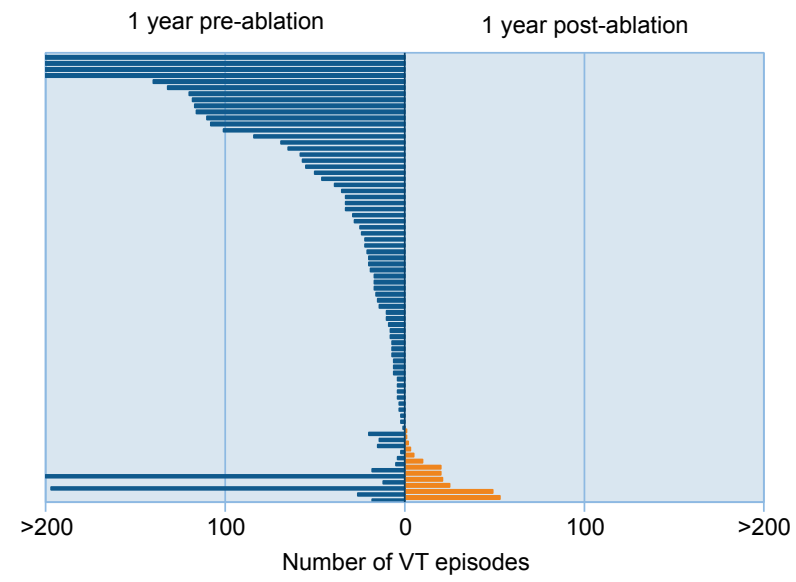

Figure 19: There were 74 patients with implantable cardioverter-defibrillator (ICD) at least 1 year prior to ablation who survived for 1 year. Each line represents an individual patient; blue lines indicate pre-ablation and orange lines post-ablation. Upper boundaries are truncated at 200 episodes. Patients are arranged by recurrences during follow-up, ranging from greatest (bottom) to least (top). In $91 \%$ of these 74 patients, the reduction in VT burden was more than $87 \%$. 


\section{Comparison with other outcome studies}

These results are favorable when compared to reported long-term outcomes of VT ablation in ischemic cardiomyopathy patients, predominantly guided by activation and entrainment mapping of inducible and hemodynamically tolerated VTs. ${ }^{16,117}$ In a single center retrospective series of 358 patients (including repeat ablation in $25 \%$ of patients) the VA-free survival was around $54 \%$ at 5 years $\mathrm{FU}$, compared to $62 \%$ in our study. ${ }^{116}$ Also in the prospective multicenter Post-Approval THERMOCOOL VT trial (249 patients) the single-procedure freedom from arrhythmia was considerably lower when compared to our study amounting only $41 \%$ after 3 years. ${ }^{117}$

Outcomes from substrate-based VT ablation studies have only been reported with limited followup. ${ }^{119,120,122,123,128,129}$ Silberbauer et al reported a VT-free survival of $68 \%$ at 19 months after substrate modification (160 pts, 20\% epicardial mapping) targeting late potential abolition combined with noninducibility. ${ }^{119}$ Proietti et al reported a VA-free survival of $74 \%$ after a mean follow-up of $21 \pm 10$ months in 87 patients undergoing substrate-based VT ablation targeting late potentials in scar regions and regions in the scar-border zone with good pace-map correlation to clinical VTs. ${ }^{129}$ Better shortterm results were reported after more extensive substrate modification targeting not only delayed but all abnormal potentials within and around scar ("scar homogenization"). Di Biase et al reported outcomes of endo- and epicardial scar homogenization in 43 patients with idiopathic dilated cardiomyopathy and electrical storm. ${ }^{122}$ Epicardial access was obtained in all of them, but epicardial ablation was only performed in $33 \%$ if abnormal potentials remained after endocardial ablation. At a mean follow-up of 21 months the VT-free survival was $81 \%$. The results of this ablation approach were confirmed in the VISTA Randomized Multicenter Trial, in which 58 patients underwent extensive ablation of all abnormal electrograms in endocardial scar, but epicardial access was limited to $10 \%$ of patients in whom VT remained inducible after endocardial ablation. ${ }^{123}$ At 12 months followup, the VT-free survival was $84.5 \%$. Both the scar homogenization and LAVA elimination strategy aim to modify the substrate more comprehensively, although the LAVA strategy may allow for less extensive ablation ( $36 \pm 20$ vs. $74 \pm 21$ and $86 \pm 21 \mathrm{~min})$. Short-term freedom from arrhythmia was however $>80 \%$ with the scar homogenization strategy, compared to 73 and $68 \%$ at 1 and 2 years follow-up after a single procedure in our patient series.

\section{Impact of technological evolution}

In our approach, identification of the substrate is contingent on the detectability of LAVA by the mapping catheters used. It was previously demonstrated that the use of multi-electrode (ME) mapping catheters is associated with increased LAVA detection due to reduction of the far-field signal and magnification of the near-field component. ${ }^{130}$ In our study, ME mapping catheters were used in $56 \%$ of patients. Also the added value of real-time integration of preprocedural imaging with DE-MRI or MDCT in post-MI VT ablation was previously reported. ${ }^{131}$ Both DE on MRI as well as wall thinning on MDCT correlate well with low voltage areas on electroanatomic mapping and the presence of 
LAVA. ${ }^{132,133}$ After substrate mapping, real-time integration of image-derived substrate has been shown to motivate the operator to perform additional mapping in the majority of cases with extra LAVA detection in all of them. In addition, in some patients, pre-procedural imaging influenced the decision to perform epicardial access and RTII modified epicardial ablation strategy due to proximity of coronary arteries or phrenic nerve. ${ }^{131}$ In our most recent approach of LAVA ablation, RTII and ME mapping catheters are therefore consistently implemented and resulted in significantly improved outcomes with a VA-free survival of $86 \%$ at 1 year, remaining $65 \%$ at 4 years of follow-up and overall survival of 94 and $91 \%$ at 1 and 4 years respectively.
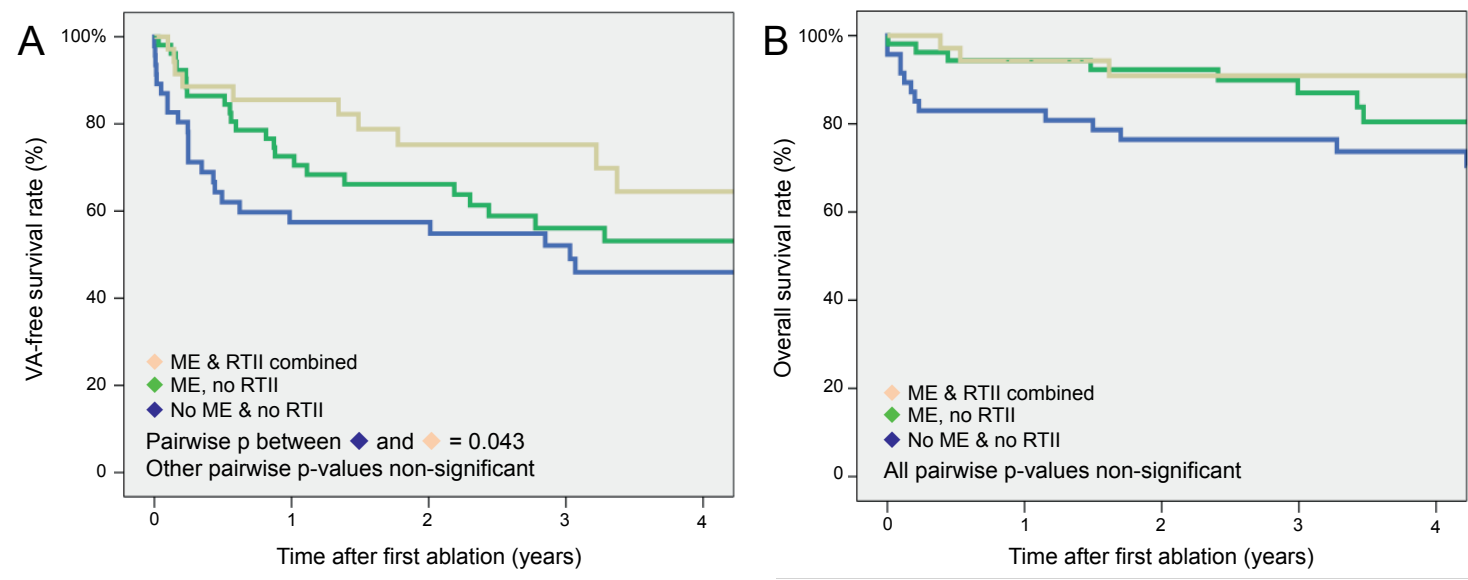

Figure 20: Impact of different mapping technologies. Ventricular arrhythmia (VA)-free (A) and overall (B) survival rate in procedures performed without multi-electrode (ME) mapping and real-time image integration (RTII) (blue, $n=35$ ), with ME mapping but without RTII (green, $n=53$ ), and with combined ME mapping and RTII (yellow, $n=47$ ).

With the contemporary LAVA-strategy, (short-term) results comparable to the scar homogenization strategy are achieved, but with more limited RF delivery. ${ }^{122,123}$ Given these results, a randomized, head-to-head comparison of the contemporary LAVA elimination approach and the scar homogenization approach could be of interest.

\section{Reasons for VA recurrence}

Potential reasons for VA recurrence are 1. inadequate mapping of the substrate, 2. inadequate ablation with lesion regression and reconnection of critical isthmuses, 3. inadequate ablation due to anatomic limitations (mid-myocardial substrate, substrate in vicinity of the coronary arteries or phrenic nerve), 4. progression of the substrate, and 5. progression of neurohormonal and autonomic remodeling. ${ }^{134}$ While inadequate mapping can be improved by triple (both retrograde and transseptal endocardial, and epicardial) LV access and use of ME mapping catheters and RTII, intramyocardial infusion-needle and 
bipolar irrigated RF catheter ablation may offer benefit when conventional RF ablation lesions are of insufficient depth to reach a mid-myocardial substrate. ${ }^{135,136}$ Comparable to previously published series in post-MI VT ablation ${ }^{137}$, recurrent VTs had new morphologies in our study. During repeat ablation, $\geq 1$ VT with different morphology from the index procedure was induced in all evaluable patients and only in a minority, a VT with a same exit as during the index procedure was induced. Despite different VT morphologies, the arrhythmogenic substrate generally remained unchanged between index and repeat procedures. In redo procedures, LAVA were generally (94\%) located in the same area as during the index ablation, either due to recurrence of previously ablated LAVA or residual, unablated LAVA from the index procedure (in several cases possibly missed by mapping with the ablation catheter in the index, but detected by ME mapping in the redo procedure). Without recurrent ischemia, substrate progression in post-MI VT appears to be the exception, as scar areas were unchanged between index and repeat procedures and 'new' LAVA in repeat procedures were very rare.

A

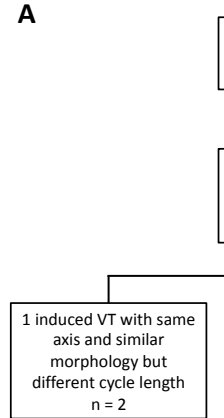

B

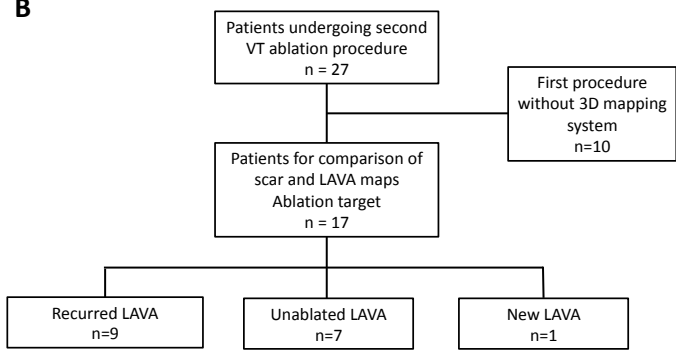

Figure 21: Comparison between index ablation and first repeat ablation procedure of (A) induced ventricular tachycardia morphologies and (B) characteristics of local abnormal ventricular activities.

\section{Conclusion and future clinical perspectives}

Substrate modification by LAVA elimination for post-MI VT resulted in a substantial reduction of VT storm, VT burden and need for ICD shocks. Both short- and long-term outcomes were improved by repeat ablation in a limited number of patients. Implementation of modern mapping technologies such as ME mapping and RTII significantly improved outcomes. However, the recurrence of previously ablated LAVA advocates the importance of effective and durable RF lesions. Therefore, in order to improve outcomes of VT ablation, further research should aim to develop criteria and/or tools for effective RF lesion creation in the ventricle. Secondly, while elimination of all LAVA was associated with a better outcome, many LAVA might be innocent bystanders and are not involved in channels responsible for current or future clinical VTs. Identification of potential VT channels on imaging 
studies (MRI of CT) might significantly reduce the amount of RF delivery, procedures times, avoid unnecessary myocardial destruction and improve arrhythmia-free survival. 


\section{Final conclusion}

Outcomes of ablation of complex atrial and ventricular arrhythmias have significantly improved over the last decade. This can be attributed to technological advances that have resulted in both improved radiofrequency lesion creation and identification of the arrhythmogenic substrate, in combination with robust and innovative clinical ablation strategies based on the pathophysiology of these arrhythmias. 


\section{References}

1. Go AS, et al. Prevalence of diagnosed atrial fibrillation in adults: national implications for rhythm management and stroke prevention: the AnTicoagulation and Risk Factors in Atrial Fibrillation (ATRIA) Study. JAMA. 2001; 285:2370-2375.

2. Wolf PA, et al. Atrial fibrillation as an independent risk factor for stroke: the Framingham Study. Stroke. 1991; 22:983-988.

3. Wang TJ, et al. Temporal relations of atrial fibrillation and congestive heart failure and their joint influence on mortality: the Framingham Heart Study. Circulation. 2003; 107:2920-2925.

4. Benjamin EJ, et al. Impact of atrial fibrillation on the risk of death: the Framingham Heart Study. Circulation. 1998; 98:946-952.

5. Chen LY, et al. Atrial fibrillation and the risk of sudden cardiac death: the Atherosclerosis Risk in Communities Study and Cardiovascular Health Study. JAMA Intern Med. 2013; 173:29-35.

6. Soliman EZ, et al. Atrial fibrillation and the risk of myocardial infarction. JAMA Intern Med. 2014; 174:107-114.

7. Soliman EZ, et al. Atrial fibrillation and risk of ST-segment-elevation versus non-ST-segmentelevation myocardial infarction: the Atherosclerosis Risk in Communities (ARIC) study. Circulation. 2015; 131:1843-1850.

8. Dorian $\mathrm{P}$, et al. The impairment of health-related quality of life in patients with intermittent atrial fibrillation: implications for the assessment of investigational therapy. J Am Coll Cardiol. 2000; 36:1303-1309.

9. Van Gelder IC, et al. Rate Control versus Electrical Cardioversion for Persistent Atrial Fibrillation Study Group. A comparison of rate control and rhythm control in patients with recurrent persistent atrial fibrillation. N Engl J Med. 2002; 347:1834-1840.

10. Wyse DG, et al. A comparison of rate control and rhythm control in patients with atrial fibrillation. N Engl J Med. 2002; 347:1825-1833.

11. Packer DL, et al. Effect of Catheter Ablation vs Antiarrhythmic Drug Therapy on Mortality, Stroke, Bleeding, and Cardiac Arrest Among Patients With Atrial Fibrillation: The CABANA Randomized Clinical Trial. JAMA. 2019; 321:1261-1274.

12. Packer DL, et al. Cryoballoon ablation of pulmonary veins for paroxysmal atrial fibrillation: first results of the North American Arctic Front (STOP AF) pivotal trial. J Am Coll Cardiol. 2013; 61:1713-1723.

13. Wilber DJ, et al. Comparison of antiarrhythmic drug therapy and radiofrequencycatheter ablation in patients with paroxysmal atrial fibrillation: a randomizedcontrolled trial. JAMA. 2010; 303:333-340.

14. Calkins H, et al. 2017 HRS/EHRA/ECAS/APHRS/SOLAECE expert consensus statement on catheter and surgical ablation of atrial fibrillation: Executive summary. Europace. 2018; 20:157208.

15. Wazni OM, et al. Radiofrequency ablation vs antiarrhythmic drugs as first-line treatment of symptomatic atrial fibrillation: a randomized trial. JAMA. 2005; 293:2634-2640.

16. Cosedis Nielsen J, et al. Radiofrequency ablation as initial therapy in paroxysmal atrial fibrillation. N Engl J Med. 2012; 367:1587-1595.

17. Morillo CA, et al. Radiofrequency ablation vs antiarrhythmic drugs as first-line treatment of paroxysmal atrial fibrillation (RAAFT-2): a randomized trial. JAMA. 2014; 311:692-700.

18. Hakalahti A, et al. Radiofrequency ablation vs antiarrhythmic drug therapy as first line treatment of symptomatic atrial fibrillation: systematic review and meta-analysis. Europace. 2015; 17:370378.

19. Haissaguerre $M$, et al. Spontaneous initiation of atrial fibrillation by ectopic beats originating in the pulmonary veins. N Engl J Med. 1998; 339:659-666. 
20. Haissaguerre M, et al. Electrophysiological end point for catheter ablation of atrial fibrillation initiated from multiple pulmonary venous foci. Circulation. 2000; 101:1409-1417.

21. Pappone C, et al. Circumferential radiofrequency ablation of pulmonary vein ostia: A new anatomic approach for curing atrial fibrillation. Circulation. 2000; 102:2619-2628.

22. Pappone $\mathrm{C}$, et al. Atrial electroanatomic remodeling after circumferential radiofrequency pulmonary vein ablation: efficacy of an anatomic approach in a large cohort of patients with atrial fibrillation. Circulation. 2001; 104:2539-2544.

23. Ouyang F, et al. Complete isolation of left atrium surrounding the pulmonary veins: new insights from the double-Lasso technique in paroxysmal atrial fibrillation. Circulation. 2004; 110:2090 2096.

24. Kuck KH, et al. Impact of Complete Versus Incomplete Circumferential Lines Around the Pulmonary Veins During Catheter Ablation of Paroxysmal Atrial Fibrillation: Results From the Gap-Atrial Fibrillation-German Atrial Fibrillation Competence Network 1 Trial. Circ Arrhythm Electrophysiol. 2016; 9:e003337.

25. Pratola C, et al. Radiofrequency ablation of atrial fibrillation: is the persistence of all intraprocedural targets necessary for long-term maintenance of sinus rhythm? Circulation. 2008; 117:136-143.

26. Jiang $\mathrm{RH}$, et al. Incidence of pulmonary vein conduction recovery in patients without clinical recurrence after ablation of paroxysmal atrial fibrillation: mechanistic implications. Heart Rhythm. 2014; 11:969-976.

27. Verma A, et al. Response of atrial fibrillation to pulmonary vein antrum isolation is directly related to resumption and delay of pulmonary vein conduction. Circulation. 2005;112:627-635.

28. Nanthakumar K, et al. Resumption of electrical conduction in previously isolated pulmonary veins: rationale for a different strategy? Circulation. 2004; 109:1226-1229.

29. Ouyang F, et al. Recovered pulmonary vein conduction as a dominant factor for recurrent atrial tachyarrhythmias after complete circular isolation of the pulmonary veins: lessons from double Lasso technique. Circulation. 2005; 111:127-135.

30. Nakamura K, et al. Optimal observation time after completion of circumferential pulmonary vein isolation for atrial fibrillation to prevent chronic pulmonary vein reconnections. Int $\mathrm{J}$ Cardiol $2013 ; 168: 5300-5310$.

31. Oral $\mathrm{H}$, et al. Circumferential pulmonary-vein ablation for chronic atrial fibrillation. $\mathrm{N}$ Engl J Med. 2006; 354:934-41

32. Cheema A, et al. Incidence and time course of early recovery of pulmonary vein conduction after catheter ablation of atrial fibrillation. J Cardiovasc Electrophysiol. 2007;18:387-391.

33. Rajappan K, et al. Acute and chronic pulmonary vein reconnection after atrial fibrillation ablation: a prospective characterization of anatomical sites. Pacing Clin Electrophysiol. 2008; 31:15981605.

34. Bansch D, et al. Circumferential pulmonary vein isolation: wait or stop early after initial successful pulmonary vein isolation? Europace. 2013; 15:183-188.

35. Wang XH, et al. Early identification and treatment of PV re-connections: role of observation time and impact on clinical results of atrial fibrillation ablation. Europace. 2007; 9:481-486.

36. Macle L, et al. Adenosine-guided pulmonary vein isolation for the treatment of paroxysmal atrial fibrillation: an international, multicentre, randomised superiority trial. Lancet. 2015; 386:672-679.

37. Dallaglio PD, et al. The role of adenosine in pulmonary vein isolation: a critical review. Cardiol Res Pract. 2016; 2016:8632509.

38. Wakili R, et al. Impact of real-time contact force and impedance measurement in pulmonary vein isolation procedures for the treatment of atrial fibrillation. Clin Res Cardiol. 2014; 103:97-106. 
39. Di Biase L, et al. Visual, tactile, and contact force feedback: which one is more important for catheter ablation? Results from an in vitro experimental study. Heart Rhythm. 2014; 11:506-513.

40. Ikeda A, et al. Relationship between catheter contact force and radiofrequency lesion size and incidence of steam pop in the beating canine heart: electrogram amplitude, impedance, and electrode temperature are poor predictors of electrode-tissue contact force and lesion size. Circ Arrhythm Electrophysiol. 2014; 7:1174-1180.

41. Nakagawa $\mathrm{H}$, et al. Locations of high contact force during left atrial mapping in atrial fibrillation patients: electrogram amplitude and impedance are poor predictors of electrode-tissue contact force for ablation of atrial fibrillation. Circ Arrhythm Electrophysiol. 2013; 6:746-753.

42. Ullah W, et al. Randomized trial comparing pulmonary vein isolation using the SmartTouch catheter with or without real-time contact force data. Heart Rhythm. 2016; 13:1761-1767.

43. Reddy VY, et al. Randomized, controlled trial of the safety and effectiveness of a contact forcesensing irrigated catheter for ablation of paroxysmal atrial fibrillation: results of the TactiCath Contact Force Ablation Catheter Study for Atrial Fibrillation (TOCCASTAR) study. Circulation. 2015; 132:907-915.

44. Andrade JG, et al. Pulmonary vein isolation using "contact force" ablation: the effect on dormant conduction and long- term freedom from recurrent atrial fibrillation - a prospective study. Heart Rhythm. 2014; 11:1919-1924.

45. Marijon E, et al. Real-time contact force sensing for pulmonary vein isolation in the setting of paroxysmal atrial fibrillation: procedural and 1-year results. J Cardiovasc Electrophysiol. 2014; 25: $130-137$.

46. Natale A, et al. Paroxysmal AF catheter ablation with a contact force sensing catheter: results of the prospective, multicenter SMART-AF trial. J Am Coll Cardiol. 2014; 64:647-656.

47. Neuzil $\mathrm{P}$, et al. Electrical reconnection after pulmonary vein isolation is contingent on contact force during initial treatment: results from the EFFICAS I study. Circ Arrhythm Electrophysiol. 2013; 6:327-333.

48. Park CI, et al. Mechanisms of pulmonary vein reconnection after radiofrequency ablation of atrial fibrillation: the deterministic role of contact force and interlesion distance. J Cardiovasc Electrophysiol. 2014; 1:1-8.

49. Nath S, et al. Biophysics and pathology of catheter energy delivery systems. Prog Cardiovasc Dis. 1995; 37:185-204.

50. Nakagawa H, et al. The Role Of Contact Force In Atrial Fibrillation Ablation. J Atr Fibrillation 2014; 7:1027.

51. Nakagawa $\mathrm{H}$, et al. Prospective study to test the ability to create RF lesions at predicted depths of 3, 5, 7 and $9 \mathrm{~mm}$ using formula incorporating contact force, radiofrequency power and application time (force-power-time index) in the beating canine heart. Heart Rhythm. 2013; 10:S481.

52. Miller MA, et al. Acute electrical isolation is a necessary but insufficient endpoint for achieving durable PV isolation: the importance of closing the visual gap. Europace. 2012; 14:653-660.

53. Halbfass P, et al. Progression From Esophageal Thermal Asymptomatic Lesion to Perforation Complicating Atrial Fibrillation Ablation: A Single-Center Registry. Circ Arrhythm Electrophysiol. 2017; 10(8).pii:e005233.

54. Yarlagadda B, et al. Temporal Relationships between Esophageal Injury Type and Progression in Patients Undergoing Atrial Fibrillation Catheter Ablation. Heart Rhythm. 2018; pii:S15475271(18)31010-5.

55. Barkagan M, Contreras-Valdes FM, Leshem E, Buxton AE, Nakagawa H, Anter E. High-power and short-duration ablation for pulmonary vein isolation: Safety, efficacy, and long-term durability. J Cardiovasc Electrophysiol. 2018; 29:1287-1296. 
56. Leshem E, et al. High-Power and Short Duration Ablation for Pulmonary Vein Isolation: Biophysical Characterization. JACC Clin Electrophysiol. 2018; 4:467-479.

57. Bourier F, et al. High-power short-duration versus standard radiofrequency ablation: Insights on lesion metrics. J Cardiovasc Electrophysiol. 2018; 29:1570-1575.

58. Chelu MG, et al. Durable lesion formation while avoiding esophageal injury during ablation of atrial fibrillation: Lessons learned from late gadolinium MR imaging. J Cardiovasc Electrophysiol. 2018; 29:385-392.

59. Callans DJ, et al. Efficacy of repeat pulmonary vein isolation procedures in patients with recurrent atrial fibrillation. J Cardiovasc Electrophysiol. 2004; 15:1050-5.

60. Cappato R, et al. Prospective assessment of late conduction recurrence across radiofrequency lesions producing electrical disconnection at the pulmonary vein ostium in patients with atrial fibrillation. Circulation. 2003; 108:1599-604.

61. Jiang RH, et al. Incidence of pulmonary vein conduction recovery in patients without clinical recurrence after ablation of paroxysmal atrial fibrillation: mechanistic implications. Heart Rhythm. 2014; 11:969-976.

62. Ouyang F, et al. Long-term results of catheter ablation in paroxysmal atrial fibrillation: lessons from a 5-year follow-up. Circulation. 2010; 122:2368-2377.

63. Willems S, et al. Persistence of pulmonary vein isolation after robotic remote-navigated ablation for atrial fibrillation and its relation to clinical outcome. J Cardiovasc Electrophysiol. 2010; 21:1079-1084.

64. Buist TJ, et al. Arrhythmia-free survival and pulmonary vein reconnection patterns after secondgeneration cryoballoon and contact-force radiofrequency pulmonary vein isolation. Clin Res Cardiol. 2018; 107:498-506.

65. Shah S, et al. Recurrent atrial fibrillation after initial long-term ablation success: electrophysiological findings and outcomes of repeat ablation procedures. Circ Arrhythm Electrophysiol. 2018; 11:e005785.

66. Baldinger $\mathrm{SH}$, et al. Recurrence of atrial arrhythmias despite persistent pulmonary vein isolation after catheter ablation or atrial fibrillation: a case series. J Am Coll Cardiol EP. 2016; 2:723-731.

67. Das M, et al. Pulmonary vein re-isolation as a routine strategy regardless of symptoms: the PRESSURE Randomized Controlled Trial. J Am Coll Cardiol EP. 2017; 3:602-611.

68. Nery PB, et al. Relationship between pulmonary vein reconnection and atrial fibrillation recurrence: a systematic review and meta-analysis. J Am Coll Cardiol EP. 2016; 2:474-483.

69. Kottkamp H, et al. Box Isolation of Fibrotic Areas (BIFA): a patient-tailored substrate modification approach for ablation of atrial fibrillation. J Cardiovasc Electrophysiol. 2016; 27:22-30.

70. Sanders $\mathrm{P}$, et al. Electrical remodeling of the atria in congestive heart failure: electrophysiological and electroanatomic mapping in humans. Circulation. 2003; 108:1461-1468.

71. Marrouche NF, et al. Association of atrial tissue fibrosis identified by delayed enhancement MRI and atrial fibrillation catheter ablation: the DECAAF study. JAMA. 2014; 311:498-506.

72. Verma A, et al. Pre-existent left atrial scarring in patients undergoing pulmonary vein antrum isolation: an independent predictor of procedural failure. J Am Coll Cardiol. 2005; 45: 285-292.

73. Mantovan R, et al. Relationship of quality of life with procedural success of atrial fibrillation (AF) ablation and postablation AF burden: substudy of the STAR AF randomized trial. Can J Cardiol. 2013; 29:1211-1217.

74. Bjorkenheim A, et al. Patient-Reported Outcomes in Relation to Continuously Monitored Rhythm Before and During 2 Years After Atrial Fibrillation Ablation Using a Disease-Specific and a Generic Instrument. J Am Heart Assoc. 2018;7(5). pii: e008362. 
75. Glotzer TV, et al. The relationship between daily atrial tachyarrhythmia burden from implantable device diagnostics and stroke risk: the TRENDS study. Circ Arrhythm Electrophysiol. 2009; 2:474-480.

76. Go A, et al. Association of Burden of Atrial Fibrillation With Risk of Ischemic Stroke in Adults With Paroxysmal Atrial Fibrillation: The KP-RHYTHM Study. JAMA Cardiol.2018; 3:601-608.

77. Marrouche N, et al. Catheter Ablation for Atrial Fibrillation with Heart Failure. N Engl J Med. $2018 ; 378: 417-427$.

78. Kuck KH, et al. Cryoballoon or Radiofrequency Ablation for Paroxysmal Atrial Fibrillation. N Engl J Med. 2016;374:2235-2245.

79. Andrade JG, et al. Cryoballoon or Radiofrequency Ablation for Atrial Fibrillation Assessed by Continuous Monitoring: A Randomized Clinical Trial. Circulation. 2019; 140:1779-1788

80. Jais P, et al. Technique and results of linear ablation at the mitral isthmus. Circulation. 2004; 110 : 2996-3002.

81. Knecht S, et al. Left atrial linear lesions are required for successful treatment of persistent atrial fibrillation. Eur Heart J. 2008; 29:2359-2366.

82. Matsuo S, et al. Completion of mitral isthmus ablation using a steerable sheath: Prospective randomized comparison with a nonsteerable sheath. J Cardiovasc Electrophysiol. 2011; 22:13311338.

83. Miyazaki S, et al. Preprocedural clinical parameters determining perimitral conduction time during mitral isthmus line ablation. Circ Arrhythm Electrophysiol. 2011; 4:287-294.

84. Arbelo E, et al. Benefit of left atrial roof linear ablation in paroxysmal atrial fibrillation: a prospective, randomized study. J Am Heart Assoc. 2014;3:e000877.

85. Wong $\mathrm{T}$, et al. Local activation times at the high posterior wall of the left atrium during left atrial appendage pacing predict roof line block with high specificity and sensitivity. Europace 2011; 13:1243-1249.

86. Latcu DG, et al. Electroanatomic characteristics of the mitral isthmus associated with successful mitral isthmus ablation. Europace. 2016; 18:274-280.

87. Scherr D, et al. Length of the Mitral Isthmus But Not Anatomical Location of Ablation Line Predicts Bidirectional Mitral Isthmus Block in Patients Undergoing Catheter Ablation of Persistent Atrial Fibrillation: A Randomized Controlled Trial. J Cardiovasc Electrophysiol. 2015; 26:629-634.

88. Wong KC, et al. A review of mitral isthmus ablation. Indian Pacing Electrophysiol J. 2012; 12:152-70.

89. Ho SY, et al. Anatomy of the left atrium: implications for radiofrequency ablation or atrial fibrillation. J Cardiovasc Electrophysiol. 1999; 10:1525-1533.

90. Becker AE. Left atrial isthmus: anatomic aspects relevant for linear catheter ablation procedures in humans. J Cardiovasc Electrophysiol. 2004; 15:809-12.

91. Wittkampf $\mathrm{FH}$, et al. Where to draw the mitral isthmus line in catheter ablation of atrial fibrillation: histological analysis. Eur Heart J. 2005; 26:689-695.

92. Fassini G, et al. Long-term results of a prospective randomized study. J Cardiovasc Electrophysiol. 2005; 16:1150-1156.

93. Rostock $\mathrm{T}$, et al. Characterization of conduction recovery across left atrial linear lesions in patients with paroxysmal and persistent atrial fibrillation. J Cardiovasc Electrophysiol. 2006; 17:1106-1111.

94. Kim TH, et al. Challenging Achievement of Bidirectional Block After Linear Ablation Affects the Rhythm Outcome in Patients With Persistent Atrial Fibrillation. J Am Heart Assoc. 2016; 5. pii: e003894. 
95. Moe GK, et al. Atrial fibrillation as a self-sustaining arrhythmia independent of focal discharge. Am Heart J. $1959 ; 58: 59-70$.

96. Allessie MA, et al. Experimental evaluation of Moe's multiple wavelet hypothesis of AF. In: Zipes DP, Jalife J, eds. Cardiac Electrophysiology and Arrhythmias. Orlando, Fla: Grune \& Stratton; 1985: 265-275.

97. Lee S, et al. Simultaneous Biatrial High-Density (510-512 Electrodes) Epicardial Mapping of Persistent and Long-Standing Persistent Atrial Fibrillation in Patients: New Insights Into the Mechanism of Its Maintenance. Circulation. 2015; 132:2108-17.

98. Hansen BJ, et al. Maintenance of Atrial Fibrillation: Are Reentrant Drivers With Spatial Stability the Key? Circ Arrhythm Electrophysiol. 2016;9(10). pii: e004398.

99. Atienza F, et al. Reentry and atrial fibrillation. Heart Rhythm. 2007;4:S13-6.

100. Verma A, et al. Approaches to catheter ablation for persistent atrial fibrillation. N Engl J Med. $2015 ; 372: 1812-1822$.

101. Scherr D, et al. Five-year outcome of catheter ablation of persistent atrial fibrillation using termination of atrial fibrillation as a procedural endpoint. Circ Arrhythm Electrophysiol. 2015; 8:18-24.

102. Rolf S, et al. Tailored atrial substrate modification based on low-voltage areas in catheter ablation of atrial fibrillation. Circ Arrhythm Electrophysiol. 2014; 7:825-833.

103. Narayan SM, et al. Clinical mapping approach to diagnose electrical rotors and focal impulse sources for human atrial fibrillation. J Cardiovasc Electrophysiol. 2012; 23:447-454.

104. Narayan SM, et al. Ablation of rotor and focal sources reduces late recurrence of atrial fibrillation compared with trigger ablation alone: extended follow-up of the CONFIRM trial (Conventional Ablation for Atrial Fibrillation With or Without Focal Impulse and Rotor Modulation). J Am Coll Cardiol. 2014; 63:1761-1768.

105. Haissaguerre $\mathrm{M}$, et al. Driver domains in persistent atrial fibrillation. Circulation. 2014; 130:530-538.

106. Knecht S, et al. Multicentre evaluation of non-invasive biatrial mapping for persistent atrial fibrillation ablation: the AFACART study. Europace. 2017; 19:1302-1309.

107. Kuklik P, et al. Identification of Rotors during Human Atrial Fibrillation Using Contact Mapping and Phase Singularity Detection: Technical Considerations. IEEE Trans Biomed Eng. 2017; 64:310-318.

108. Kik C, et al. Intra-operative mapping of the atria: the first step towards individualization of atrial fibrillation therapy? Expert Rev Cardiovasc Ther. 2017; 15:537-545.

109. Pathik B, et al. Transient Rotor Activity During Prolonged 3-Dimensional Phase Mapping in Human Persistent Atrial Fibrillation. JACC Clin Electrophysiol. 2018; 4:72-83.

110. Benharash P, et al. Quantitative analysis of localized sources identified by focal impulse and rotor modulation mapping in atrial fibrillation. Circ Arrhythm Electrophysiol 2015; 8:554-561.

111. Nattel S, Xiong F, Aguilar M. Demystifying rotors and their place in clinical translation of atrial fibrillation mechanisms. Nat Rev Cardiol. 2017; 14:509-520.

112. Daoud EG, et al. Identification of Repetitive Activation Patterns Using Novel Computational Analysis of Multielectrode Recordings During Atrial Fibrillation and Flutter in Humans. JACC Clin Electrophysiol. 2017; 3:207-216.

113. Honarbakhsh S, et al. A Novel Mapping System for Panoramic Mapping of the Left Atrium: Application to Detect and Characterize Localized Sources Maintaining Atrial Fibrillation. JACC Clin Electrophysiol. 2018; 4:124-134.

114. Honarbakhsh S, et al. Characterization of drivers maintaining atrial fibrillation: Correlation with markers of rapidity and organization on spectral analysis. Heart Rhythm. 2018; 15:12961303. 
115. Verma A, et al. Characterization and significance of localized sources identified by a novel automated algorithm during mapping of human persistent atrial fibrillation. J Cardiovasc Electrophysiol. 2018; 29:1480-1488.

116. Kumar S, et al. Long-term outcomes after catheter ablation of ventricular tachycardia in patients with and without structural heart disease. Heart Rhythm. 2016; 13:1957-1963.

117. Marchlinski FE, et al. Long-Term Success of Irrigated Radiofrequency Catheter Ablation of Sustained Ventricular Tachycardia: Post-Approval THERMOCOOL VT Trial. J Am Coll Cardiol. 2016; 67:674-683.

118. Jaïs $\mathrm{P}$, et al. Elimination of local abnormal ventricular activities: a new end point for substrate modification in patients with scar-related ventricular tachycardia. Circulation. 2012; 125:21842196.

119. Silberbauer J, et al. Noninducibility and late potential abolition: a novel combined prognostic procedural end point for catheter ablation of postinfarction ventricular tachycardia. Circ Arrhythm Electrophysiol. 2014; 7:424-435.

120. Berruezo A, et al. Scar dechanneling: new method for scar-related left ventricular tachycardia substrate ablation. Circ Arrhythm Electrophysiol. 2015; 8:326-336.

121. Tzou WS, et al. Core isolation of critical arrhythmia elements for treatment of multiple scarbased ventricular tachycardias. Circ Arrhythm Electrophysiol. 2015; 8:353-361.

122. Di Biase L, et al. Endo-epicardial homogenization of the scar versus limited substrate ablation for the treatment of electrical storms in patients with ischemic cardiomyopathy. J Am Coll Cardiol. 2012; 60:132-141.

123. Di Biase L, et al. Ablation of Stable VTs Versus Substrate Ablation in Ischemic Cardiomyopathy: The VISTA Randomized Multicenter Trial. J Am Coll Cardiol. 2015; 66:2872_ 2882.

124. Arenal A, et al. Ablation of electrograms with an isolated, delayed component as treatment of unmappable monomorphic ventricular tachycardias in patients with structural heart disease. J Am Coll Cardiol. 2003; 41:81-92.

125. Vergara $\mathrm{P}$, et al Late potentials abolition as an additional technique for reduction of arrhythmia recurrence in scar related ventricular tachycardia ablation. J Cardiovasc Electrophysiol. 2012; 23:621-627.

126. Bogun $\mathrm{F}$, et al. Isolated potentials during sinus rhythm and pace-mapping within scars as guides for ablation of post-infarction ventricular tachycardia. J Am Coll Cardiol. 2006; 47:20132019.

127. Tung R, et al. Directional Influences of Ventricular Activation on Myocardial Scar Characterization: Voltage Mapping With Multiple Wavefronts During Ventricular Tachycardia Ablation. Circ Arrhythm Electrophysiol. 2016;9. pii: e004155.

128. Kumar S, et al. Substrate-Based Ablation Versus Ablation Guided by Activation and Entrainment Mapping for Ventricular Tachycardia: A Systematic Review and Meta-Analysis. J Cardiovasc Electrophysiol. 2016; 27:1437-1447.

129. Proietti R, et al. Substrate-guided ablation of haemodynamically tolerated and untolerated ventricular tachycardia in patients with structural heart disease: effect of cardiomyopathy type and acute success on long-term outcome. Europace. 2015; 17:461-467.

130. Berte B, et al. Impact of electrode type on mapping of scar-related VT. J Cardiovasc Electrophysiol. 2015; 26:1213-1223.

131. Yamashita S, et al. Image Integration to Guide Catheter Ablation in Scar-Related Ventricular Tachycardia. J Cardiovasc Electrophysiol. 2016; 27:699-708. 
132. Cochet $\mathrm{H}$, et al. Integration of merged delayed-enhanced magnetic resonance imaging and multidetector computed tomography for the guidance of ventricular tachycardia ablation: a pilot study. J Cardiovasc Electrophysiol. 2013; 24:419-426.

133. Komatsu Y, et al. Regional myocardial wall thinning at multidetector computed tomography correlates to arrhythmogenic substrate in postinfarction ventricular tachycardia: assessment of structural and electrical substrate. Circ Arrhythm Electrophysiol. 2013; 6:342-350.

134. Vaseghi M, et al. Neuroaxial modulation for ventricular arrhythmias: a new hope. Heart Rhythm. 2012; 9:1888-1889.

135. Sapp JL, et al. Initial human feasibility of infusion needle catheter ablation for refractory ventricular tachycardia. Circulation. 2013; 128:2289-2295.

136. Koruth JS, et al. Bipolar irrigated radiofrequency ablation: a therapeutic option for refractory intramural atrial and ventricular tachycardia circuits. Heart Rhythm. 2012; 9:1932-1941.

137. Yokokawa M, et al. Reasons for recurrent ventricular tachycardia after catheter ablation of post-infarction ventricular tachycardia. J Am Coll Cardiol. 2013; 61:66-73. 


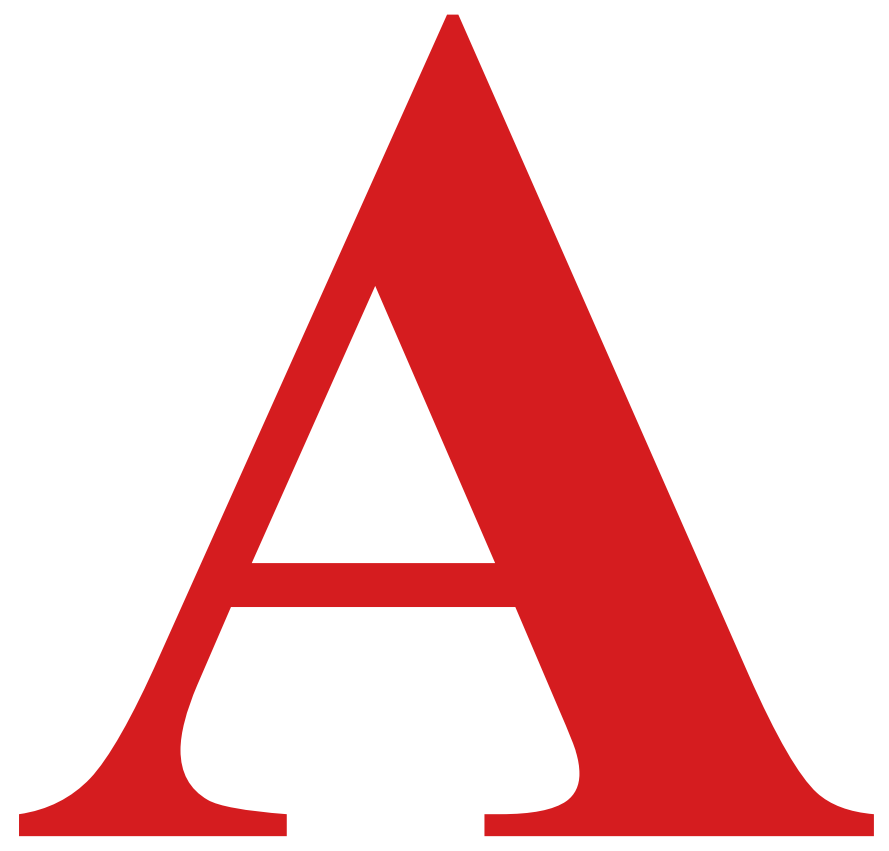




\section{Addenda}

Summary - Samenvatting

Valorization

Aknowledgement - Dankwoord

Curriculum vitae - List of publications 


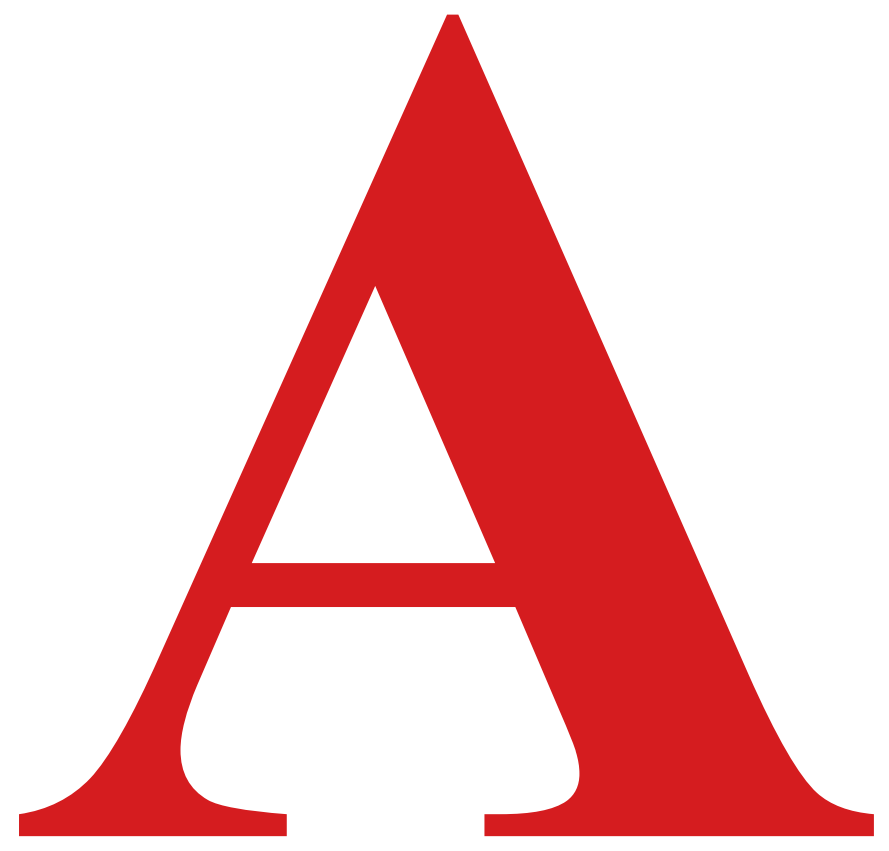


SUMMARY 
This thesis addresses recent evolutions in ablation of complex atrial en ventricular arrhythmias, and how technological advances applied in pathophysiology-based ablation strategies have aided to improve these procedures and their outcome results.

In PART I we aimed to improve outcome results of ablation of atrial fibrillation (AF) and atrial tachycardia. In PART IA (Chapter 2) we investigated and demonstrated that also after having gained extensive experience with online contact force (CF)-measuring catheters, procedural outcomes of pulmonary vein isolation (PVI) remained superior with this technology when compared to non-CF guided PVI. PART IB of this thesis addresses the development of a protocol aiming to improve the point-by-point radiofrequency PVI technique by combining strict criteria for both radiofrequency application contiguity and quality. In Chapter 3 we analysed optimal cut-off criteria for maximum distance $(\leq 6 \mathrm{~mm})$ between neighbouring radiofrequency applications and minimum ablation index targets ( $\geq 400$ au posterior/roof and $\geq 550$ au anterior) associated with durable PVI. These criteria were incorporated into the 'CLOSE' protocol. In Chapter 4 and 5 we investigated the feasibility of CLOSE-PVI in paroxysmal AF patients and observed shorter procedure times, significant improvement in acute PVI durability and high single-procedure arrhythmia-free survival at 1 year, when compared to conventional CF-guided PVI. In Chapter 6 we investigated and demonstrated the safety profile of CLOSE-PVI concerning oesophageal injury. We performed echoendoscopy in those patients revealing intra-oesophageal temperature rise during CLOSE-PVI and found complete absence of esophageal ulceration in all evaluated patients. These findings suggest safety of CLOSE-PVI concerning esophageal injury. The efficacy of CLOSE-PVI was further investigated in Chapters 7 and 8. In Chapter 7 we evaluated the durability of PVI in patients undergoing repeat ablation for AF recurrence after undergoing an index CLOSE-PVI procedure. Durable PV isolation was found in $62 \%$ - higher than previously reported - supporting the efficacy and favorable clinical results of CLOSE-PVI in paroxysmal AF. In Chapter 8 we studied the impact of CLOSE-PVI on AF burden during the first two years after CLOSE-PVI using insertable cardiac monitors. Even when using continuous cardiac monitoring to detect arrhythmia recurrence, the outcome results of CLOSE-PVI for paroxysmal AF remained excellent: the single-procedure freedom of atrial arrhythmia without use of anti-arrhythmic drugs amounted $87 \%$ after 1 year and $78 \%$ after 2 years, with a median atrial arrhythmia burden of 0 [IQR 0-0] \% in the first two years after ablation.

In PART IC (Chapter 9) we focused on the optimization of left atrial linear ablation by applying the same principles of the 'CLOSE' protocol. This strategy of delivering contiguous and optimized radiofrequency lesions applied to linear ablation appeared very effective for the left atrial roof, but not for the (posterior) mitral isthmus, because of its specific anatomical properties.

While the subject of the previous chapters was optimization of ablation, in PART ID (Chapter 10) we aimed to improve our understanding of the pathophysiology of persistent AF. We sought to evaluate a new mapping method to identify and characterize AF drivers (in casu repetitive atrial activation 
patterns) obtained by sequential overlapping recordings with a regional high-density contact mapping catheter (Pentaray) covering the entire bi-atrial endocardial surface, and automatically analyzed with new software (Cartofinder). This mapping method demonstrated the presence of repetitive activation patterns during persistent $\mathrm{AF}$ in all patients. In our series, focal firing was the most frequently observed pattern.

The second part of this thesis (PART II) focused on the evolution and technological advances in ablation of ventricular tachycardia (VT), more specifically in the setting post-myocardial infarction. In Chapter 11 we reported long-term outcomes after single and multiple ablation procedures targeting elimination of local abnormal ventricular activities (LAVA). We also investigated the added value of multi-electrode mapping catheters and of real-time integration of pre-procedural imaging with delayed enhancement-magnetic resonance imaging or multi-detector computed tomography. In our study, this ablation strategy resulted in a substantial reduction of VT storm, VT burden and need for ICD shocks. Both short- and long-term outcomes were improved by repeat ablation in a limited number of patients. Complete LAVA elimination, multi-electrode mapping and real-time image integration were associated with improved ventricular arrhythmia-free survival.

The conclusion of this thesis is that outcomes of ablation of complex atrial and ventricular arrhythmias have significantly improved over the last decade. This can be attributed to technological advances that have resulted in both improved radiofrequency lesion creation and identification of the arrhythmogenic substrate, in combination with robust and innovative clinical ablation strategies based on the pathophysiology of these arrhythmias. 


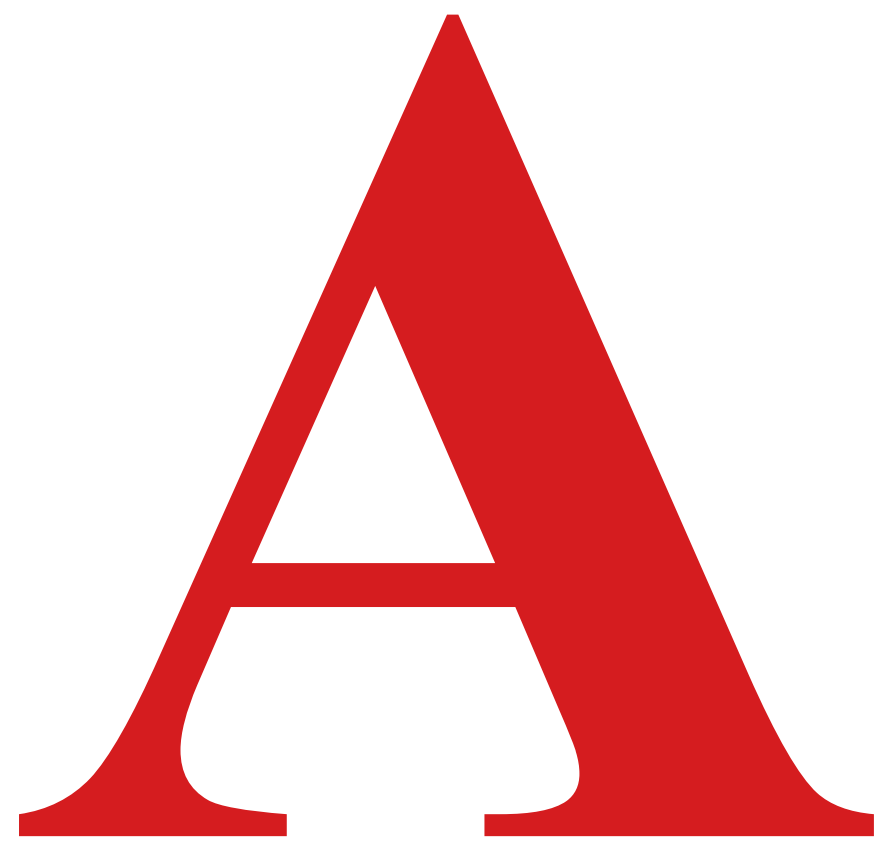


SAMENVATTING 
Deze thesis richt zich op recente evoluties in de ablatie van complexe atriale en ventriculaire ritmestoornissen, en hoe technologische vooruitgang gekoppeld aan pathofysiologische principes deze invasieve therapeutische procedures veiliger en effectiever gemaakt hebben.

DEEL I van deze thesis is gericht op de verbetering van de resultaten van ablatie van atriale fibrillatie (AF) en atriale tachycardie. In DEEL IA (Hoofdstuk 2) onderzochten en toonden we aan dat ook na uitgebreide ervaring te hebben opgebouwd met katheters met online contact force (CF)-feedback, de procedurele resultaten van pulmonale venen isolatie (PVI) met deze technologie superieur blijven in vergelijking met PVI zonder CF-meting. In DEEL IB ontwikkelden we een protocol met als doel de punt-per-punt radiofrequentie PVI techniek te optimaliseren, dit door combinatie van strikte criteria voor zowel contiguïteit als kwaliteit van de radiofrequentie applicaties. In Hoofdstuk 3 analyseerden we optimale cutoff criteria voor maximale afstand $(\leq 6 \mathrm{~mm})$ tussen naburige radiofrequentie applicaties en voor minimale ablatie index waarden ( $\geq 400 \mathrm{au}$ posterior/dak en $\geq 550 \mathrm{au}$ anterior), die geassocieerd waren met duurzame PVI. Deze criteria werden geïncorporeerd in het 'CLOSE' protocol. In Hoofdstuk 4 en 5 onderzochten we de haalbaarheid van CLOSE-PVI bij paroxismale AF patiënten en observeerden we een kortere proceduretijd, een significante verbetering in acute PVI duurzaamheid, en een hoge aritmievrije overleving na 1 jaar na een enkele ablatieprocedure, wanneer vergeleken met conventionele CF-geleide PVI. In Hoofdstuk 6 onderzochten en toonden we het veiligheidsprofiel van CLOSE-PVI aan met betrekking tot slokdarmschade. We voerden endo-echoscopie uit in die patiënten die tijdens CLOSE-PVI een stijging van de intra-oesofagiale temperatuur vertoonden en stelden een volledige afwezigheid van slokdarmulceratie in alle onderzochte patiënten vast. Deze bevindingen suggereren dat CLOSE-PVI veilig is voor wat slokdarmschade betreft. De effectiviteit van CLOSEPVI werd verder onderzocht in Hoofdstukken 7 en 8. In Hoofdstuk 7 evalueerden we de duurzaamheid van PVI in patiënten die een redo-ablatie ondergingen omwille van herval van AF na index CLOSE-PVI te hebben ondergaan. Duurzaamheid van alle vier longvenen werd vastgesteld bij $62 \%$ van deze patiënten - i.e. hoger dan ooit eerder gepubliceerd - wat de effectiviteit en gunstige klinische resultaten van CLOSE-PVI voor paroxismale AF ondersteunt.

In Hoofdstuk 8 bestudeerden we de impact van CLOSE-PVI op de AF burden gedurende de eerste 2 jaar a CLOSE-PVI door gebruik te maken van implanteerbare cardiale monitoren. Zelfs bij gebruik van continue cardiale monitoring om herval van aritmie te detecteren, bleef de uitkomst van CLOSEPVI bij paroxismale AF excellent: de aritmie-vrije overleving na 1 ablatieprocedure, zonder gebruik van antiaritmica bedroeg $87 \%$ na 1 jaar en $78 \%$ na 2 jaar, met een mediane atriale aritmie burden van 0 [IQR 0-0] \% gedurende de eerste 2 jaar na ablatie.

In DEEL IC (Hoofdstuk 9) legden we ons toe op de optimalisatie van lineaire ablatie in het linker atrium door dezelfde principes van het 'CLOSE' protocol toe te passen. Deze strategie van aaneensluitende en geoptimaliseerde radiofrequentie applicaties bleek tevens zeer effectief wanneer 
toegepast voor lineaire ablatie ter hoogte van het dak van het linker atrium, maar niet voor de (posterior) mitraalisthmus, omwille van de specifieke anatomische eigenschappen van deze laatste.

Daar waar optimalisatie van ablatie het onderwerp van de voorgaande hoofdstukken was, legden we ons in DEEL ID (Hoofdstuk 10) toe op het verwerven van meer inzicht in de pathofysiologie van persistente AF. We evalueerden een nieuwe mapping techniek voor de identificatie en typering van $\mathrm{AF}$ drivers (in casu repetitieve atriale activatiepatronen), die gedetecteerd werden door sequentiële overlappende registraties met een regionale high-density mapping katheter (Pentaray) die het hele biatriale oppervlak overspannen en automatisch geanalyseerd werden met nieuwe software (Cartofinder). Deze mappingmethode demonstreerde in alle patiënten de aanwezigheid van repetitieve atriale activaties tijdens persistente AF. In onze reeks werden focale afvurende bronnen het meest frequent geobserveerd.

Het tweede deel van deze thesis (DEEL II) spitst zich toe op de evolutie en technologische vooruitgang in de ablatie van ventrikeltachycardie (VT), meer specifiek bij het substraat na doorgemaakt myocardinfarct. In Hoofdstuk 11 rapporteerden we de lange-termijnsresultaten na enkelvoudige en meervoudige ablatieprocedures gericht op eliminatie van lokale abnormale ventriculaire activiteit (LAVA). We onderzochten tevens de toegevoegde waarde van multi-elektrode mapping katheters en van real-time integratie van pre-procedurele beelden bekomen door middel van magnetische resonantie of multi-detector computertomografie. In onze studie resulteerde deze ablatiestrategie in een substantiële reductie aan VT storm, VT burden en aantal ICD shocks. Het resultaat op zowel korte als lange termijn werd verder verbeterd door additionele ablatieprocedures in een beperkt aantal patiënten. Complete LAVA-eliminatie, multi-elektrode mapping en integratie van real-time beeldvorming was geassocieerd met betere ventriculaire aritmie-vrije overleving.

De conclusie van deze thesis is dat het resultaat van katheterablatie van complexe atriale en ventriculaire aritmie tijdens de laatste decade significant verbeterd is. Dit is deels te danken aan technologische vooruitgang die heeft geleid tot verbetering van radiofrequentie laesie-vorming en identificatie van het aritmogene substraat, en ook aan toepassing van deze nieuwe technologie in innovatieve klinische ablatie strategieën die gebaseerd werden op de pathofysiologie van deze aritmieën. 


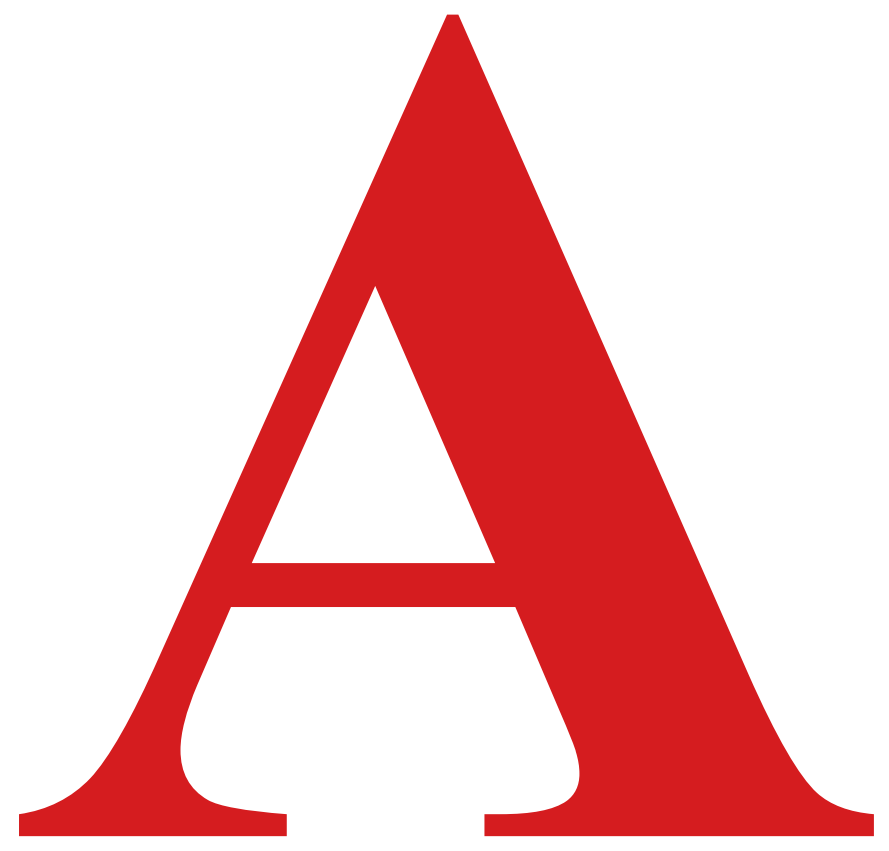


VALORIZATION 


\section{Scope, rationale and socio-economic relevance}

The findings presented in this thesis will boost a significant improvement in the clinical efficacy of catheter ablation for complex arrhythmias such as atrial fibrillation (AF) and ventricular tachycardia (VT). As a consequence more patients may be treated effectively with fewer procedures per patient, thus improving the cost-effectiveness of this costly intervention. The revenue of our strategic physiological application of new ablation technology is even broader: it may not only lead to improved cost-effectiveness, but also it has already formed a firm stepping stone for follow-up observational and randomized controlled studies, enhancing on one hand the spread of our findings across centres in Europe (and the rest of the world), and on the other it generated new data to further enhance efficacy and safety of arrhythmia ablation, and formed a trigger for new technological developments as well.

\section{The scope of atrial fibrillation}

$\mathrm{AF}$ is the most common sustained arrhythmia. In 2010, the estimated worldwide prevalence was 33.5 million. ${ }^{1,2}$ Estimates suggest an overall prevalence of approximately $3 \%$ in adults aged $>20$ years $^{3}$ and its incidence keeps rising due to better detection of silent $\mathrm{AF}^{4-6}$, alongside increasing age and conditions predisposing to AF. ${ }^{7}$ By 2030, 14-17 million AF patients are anticipated in the European Union, with $120000-215000$ newly diagnosed patients per year. ${ }^{3,8,9} \mathrm{AF}$ is a serious public health problem because of its increasing incidence and prevalence in the aging population ${ }^{10}$ and its association with elevated risks of cardiovascular events and death. ${ }^{4,11-15}$ The direct costs of AF already amount to approximately $1 \%$ of total healthcare spending in the UK, and between $6.0-26.0$ billion US dollars in the US for $2008,{ }^{16,17}$ driven by AF-related complications (e.g. stroke) and treatment costs (e.g. hospitalizations). These costs will increase dramatically unless AF is prevented and treated in a timely and effective manner. In addition, AF causes a wide variety of symptoms, including fatigue and reduced exercise tolerance, and significantly impairs quality of life. ${ }^{18}$ The morbidity and mortality associated with AF provide a rationale to maintain sinus rhythm. Yet to date, rhythm control strategy has failed to demonstrate a beneficial effect on hard endpoints such as a reduction in stroke or mortality rate. ${ }^{19-20}$ Ablation of atrial fibrillation has proven to reduce symptoms and improve quality of life, and is superior to antiarrhythmic drugs in obtaining rhythm control. ${ }^{21-23}$ Therefore according to current guidelines AF ablation is indicated in symptomatic patients as a second-line treatment after failure of a class I or III antiarrhythmic drug (Class I indication (level of evidence A) in paroxysmal AF, A class IIa indication (level of evidence B) in persistent AF and a class IIb indication (level of evidence C) in long-standing persistent $\mathrm{AF}^{24}$ Given that catheter ablation of $\mathrm{AF}$ as a first-line treatment is associated with a higher freedom from $\mathrm{AF}$ compared with drug therapy, ${ }^{25-27}$ current guidelines also state that catheter ablation of $\mathrm{AF}$ as a first-line treatment (without a trial of class I of III antiarrhythmic drugs) is indicated in symptomatic paroxysmal (Class IIa - level of evidence B) and 
persistent AF patients (Class IIa - level of evidence C; and Class IIb - level of evidence C in case of long-standing persistent $\mathrm{AF}){ }^{24}$

\section{Pulmonary vein isolation}

Since the initial description of triggers in the pulmonary veins that initiate paroxysmal AF, pulmonary vein isolation (PVI) has become the cornerstone of catheter ablation of $\mathrm{AF}^{24}$ Over the past 20 years, the development of AF ablation technologies has been a constant effort to improve the PVI technique. The goal has been to improve patient outcomes, reducing the need for repeat procedures, to reduce procedure times and to increase the safety of the procedure. However, despite these advances and two decades of experience, long-term procedural success rates at curing AF are still suboptimal and may require repeat procedures or hybrid therapy with antiarrhythmic drugs to improve outcome. The reason for these suboptimal results is twofold; on one side the pathophysiological mechanism of AF is still not completely elucidated, and on the other side the ablation tools and techniques still fail to deliver durable and transmural ablation lesions. The cornerstone of ablation of AF is to achieve durable electrical isolation of the pulmonary veins. ${ }^{24}$ Radiofrequency ablation, the most established ablation technique, aims to achieve PVI by applying circumferential contiguous and transmural point-by-point ablation lesions. ${ }^{29}$ Despite achieving nearly always acute procedural PVI, early and late pulmonary vein reconnections are frequent and are associated with increased AF recurrences and the need for repeat ablation procedures.

\section{Mapping drivers in persistent atrial fibrillation}

Despite the advances in technology and our insight in the molecular, ionic, and physiological fundamentals of cardiac electrophysiology, the exact mechanism of AF is still not completely elucidated. Mechanisms in how AF is initiated and sustained may differ from patient to patient, and in various AF types (paroxysmal, persistent or permanent). Aside from the technical limitations in delivering qualitative ablation lesions, our limited understanding of the mechanisms that initiate and sustain AF withholds us from being able to cure this arrhythmia. While the outcome of AF ablation in paroxysmal $\mathrm{AF}$ is favorable and ensues from elimination of the triggering firing sources that arise predominantly from the pulmonary veins, the results of PVI are inferior in persistent AF. It is therefore perceived that in these patients (additional) ablation targeting the sustaining atrial substrate is necessary in order to improve ablation outcome. The benefit of non-specific ablation strategies (such as linear ablation or ablation of non-specific complex fragmented atrial electrograms) $)^{30-31}$ has proven modest and therefore research is focusing on patient-tailored substrate ablation that is selectively targeting either the patient's specific anatomical substrate (e.g. fibrosis ${ }^{32}$ ) or patient specific mechanistic AF drivers. Indeed intra-procedural identification of focal and reentrant AF drivers could pose attractive and patient-specific targets for AF ablation. 


\section{Ablation of post-myocardial infarction ventricular tachycardia}

For ischemic heart disease patients presenting with VT despite antiarrhythmic drug (AAD) therapy, which often results in ICD therapies (shocks or antitachycardia pacing), therapeutic options include escalating AAD therapy by increasing the dose, changing the drug, adding a new drug, and catheter ablation. In the recent VANISH trial, catheter ablation proved superior to increasing the dose of amiodarone or adding mexiletine. ${ }^{33}$ In addition, in 3 large, prospective, multicenter cohort studies, VT ablation in patients with ischemic heart disease and recurrent VT despite AAD treatment ablation resulted in a consistent reduction of VT episodes. ${ }^{34-36}$ Based on these results VT ablation in ischemic heart disease has become a Class I indication for patients with recurrence of (symptomatic) monomorphic VT despite AAD treatment. ${ }^{37}$ Recently few studies have reported long-term follow-up data of VT ablation, guided predominantly by activation and entrainment mapping, ${ }^{36,38}$ but long-term results of alternative strategies such as substrate modification have not been reported.

\section{The novelty of our research and clinical perspectives}

In PART I we aimed to contribute to the improvement of catheter ablation of AF. In PART IA we were the first to investigate if the benefits on contact force-sensing catheters maintained after acquiring extensive tip-tissue feedback with these advanced catheters. We found that an extensive learning-period still was no substitute for online contact force sensing.

In PART IB we developed a new protocol to optimize the results of pulmonary vein isolation, termed CLOSE-PVI. CLOSE-PVI proved to be associated with a very high single-procedure freedom from atrial arrhythmia recurrence, even when assessed with continuous cardiac monitoring; with more durable isolation at repeat studies; and without compromising safety. Using this novel ablation concept, efficacy is superior compared to previously published outcomes of PVI performed with either radiofrequency or cryo-energy. However, our favourable results on outcome and safety need to be confirmed in larger studies. As such, our multicenter VISTAX study was designed to assess the reproducibility of CLOSE-PVI across 17 European centers and evaluate the outcome in a larger group of 340 paroxysmal AF patients. ${ }^{39}$

In our striving to optimize PVI results there is growing interest in increasing the ablation power (highpower, short-duration applications) in order to further decrease the procedure time while maintaining/increasing safety. Given that the lesion depth is similar at a specific ablation index value (irrespective of the power) using a higher ablation power $(45-50 \mathrm{~W})$ is expected to be feasible. The POWER-AF trial was designed to investigate the efficacy and safety of the 'CLOSE' protocol while using $45 \mathrm{~W}$ applications.

Even when point-by-point ablation is meticulously performed according to the strict established criteria, the 'CLOSE' protocol is still not able to ensure durable PV isolation in $100 \%$ of cases. Indeed, 
at repeat ablation due to arrhythmia recurrence, we found that $38 \%$ of patients still had at least one reconnected vein. This could be due to variables that are not taken into account, such as the tissue thickness or tissue composition. A patient-tailored strategy with adapted ablation characteristics based on pre- or intra-procedural imaging (e.g. with computed tomography or intra-cardiac echo) as well as intra-procedural lesion verification (e.g. with magnetic resonance imaging) might be the next step to improve CLOSE-PVI results.

After optimized index-PVI, even the majority of patients with clinical AF recurrence now present with four isolated pulmonary veins (PVs) during a repeat invasive procedure. On one hand this is the signature of our improvement in achieving durable, effective PVI. On the other hand, this poses several issues. Whereas in the past, repeat ablation for paroxysmal AF generally was straightforward, consisted of closing one or more gaps, had a favorable clinical result and had a shorter, predictable procedure duration. Now, in case of four isolated veins, the preferred ablation strategy is unknown (unless there is an overt non-PV trigger during the procedure), the outcome is worse irrespective of which ablation strategy is chosen, and the procedure time is highly variable (with practical planning issues). Therefore knowing prior to a repeat invasive procedure which patients have a status of four isolated veins is much desired and might - at this time - even lead to deferment of a repeat invasive procedure until all conservative treatment options have been exhausted. Unfortunately there are no distinct non-invasive characteristics that predict the likelihood of finding four isolated veins after AF recurrence. A randomized trial comparing a strategy of detailed invasive evaluation of the atrial substrate (such as high-density voltage mapping, localization/documentation of non-PV atrial extrasystole, ... ) during index-PVI vs. index-PVI without these invasive diagnostics would therefore be of interest.

In PART IC we applied the same principles of the ' $C L O S E$ ' protocol to left atrial linear ablation, and demonstrated that this ablation strategy is also effective at the roof. The applicability of this ablation strategy could therefore be expanded to linear ablation in order to treat macro-reentrant atrial tachycardia or to isolate arrhythmogenic atrial areas. Only at the posterior mitral isthmus this ablation strategy proved unsuccessful, due to its specific anatomical configuration often necessitating epicardial ablation in order to achieve block. Other strategies, such as a combination with alcoholization of the Vein of Marshall, might increase our success in achieving mitral isthmus block.

In PART ID we investigated a new mapping technique with a high yield of online detection of potential bi-atrial drivers in persistent AF. These potential AF drivers can be automatically analyzed and annotated on a 3D-geometric bi-atrial shell, allowing patient-tailored substrate ablation. However the effect of ablation of was not investigated in our study and further studies are needed to investigate if ablation of these repetitive atrial activation sequences would indeed improve clinical outcome. 
In PART II we aimed to contribute to the improvement of catheter ablation of ventricular tachycardia (VT) in ischemic heart disease. Our research was the first to publish long-term outcomes of single and multiple ablation procedures targeting modification of the arrhythmogenic substrate (in casu elimination of all local abnormal ventricular activities (LAVA)). This technique resulted in a substantial reduction of VT storm, VT burden and need for ICD shocks. Outcomes were improved by implementation of modern mapping technologies such as multi-electrode mapping and integration of pre-procedural imaging. However, the recurrence of previously ablated LAVA advocates the importance of effective and durable radiofrequency lesions. Therefore, in order to improve outcomes of VT ablation, further research should aim to develop criteria and/or tools for effective radiofrequency lesion creation in the ventricle. Secondly, while elimination of all LAVA was associated with a better outcome, many LAVA might be innocent bystanders and are not involved in channels responsible for current or future clinical VTs. Identification of potential VT channels on imaging studies (computed tomography or magnetic resonance imaging) might significantly reduce the amount of radiofrequency delivery, procedures times, avoid unnecessary myocardial destruction and improve arrhythmia-free survival. 


\section{References}

1. Chugh SS, et al. Worldwide epidemiology of atrial fibrillation: a Global Burden of Disease 2010 Study. Circulation 2014;129:837-847.

2. Colilla $\mathrm{S}$, et al. Estimates of current and future incidence and prevalence of atrial fibrillation in the U.S. adult population. Am J Cardiol 2013;112:1142-1147.

3. Haim M, et al. Prospective national study of the prevalence, incidence, management and outcome of a large contemporary cohort of patients with incident non-valvular atrial fibrillation. J Am Heart Assoc 2015;4:e001486.

4. Wang TJ, et al. Temporal relations of atrial fibrillation and congestive heart failure and their joint influence on mortality: the Framingham Heart Study. Circulation 2003;107:2920-2925.

5. Kishore A, et al. Detection of atrial fibrillation after ischemic stroke or transient ischemic attack: a systematic re-view and meta-analysis. Stroke 2014;45:520-526.

6. Sanna T, et al., CRYSTAL AF Investigators. Cryptogenic stroke and underlying atrial fibrillation. N Engl J Med 2014;370:2478-2486.

7. Schnabel RB, et al. 50 year trends in atrial fibrillation prevalence, incidence, risk factors, and mortality in the Framingham Heart Study: a cohort study. Lancet 2015;386:154-162.

8. Krijthe BP, et al. Projections on the number of individuals with atrial fib- rillation in the European Union, from 2000 to 2060. Eur Heart J 2013;34: 2746-2751.

9. Zoni-Berisso M, et al. Epidemiology of atrial fibrillation: European perspective. Clin Epidemiol 2014;6:213-220.

10. Go AS, et al. Prevalence of diagnosed atrial fibrillation in adults: national implications for rhythm management and stroke prevention: the AnTicoagulation and Risk Factors in Atrial Fibrillation (ATRIA) Study. JAMA 2001; 285:2370-2375.

11. Wolf PA, et al. Atrial fibrillation as an independent risk factor for stroke: the Framingham Study. Stroke 1991;22:983-988.

12. Benjamin EJ, et al. Impact of atrial fibrillation on the risk of death: the Framingham Heart Study. Circulation 1998;98:946-952.

13. Chen LY, et al. Atrial fibrillation and the risk of sudden cardiac death: the Atherosclerosis Risk in Communities Study and Cardiovascular Health Study. JAMA Intern Med 2013;173:29-35.

14. Soliman EZ, et al. Atrial fibrillation and the risk of myocardial infarction. JAMA Intern Med. 2014; 174:107-114.

15. Soliman EZ, et al. Atrial fibrillation and risk of ST-segment-elevation versus non-ST-segmentelevation myocardial infarction: the Atherosclerosis Risk in Communities (ARIC) study. Circulation 2015;131:1843-1850.

16. Stewart S, et al. Cost of an emerging epidemic: an economic analysis of atrial fibrillation in the UK. Heart 2004;90:286-292.

17. Kim MH, et al. Estimation of total incremental health care costs in patients with atrial fibrillation in the United States. Circ Cardiovasc Qual Outcomes 2011;4:313-320.

18. Dorian $\mathrm{P}$, et al. The impairment of health-related quality of life in patients with intermittent atrial fibrillation: implications for the assessment of investigational therapy. J Am Coll Cardiol 2000; 36:1303-1309.

19. Van Gelder IC, et al. Rate Control versus Electrical Cardioversion for Persistent Atrial Fibrillation Study Group. A comparison of rate control and rhythm control in patients with recurrent persistent atrial fibrillation. N Engl J Med 2002;347:1834-1840.

20. Wyse DG, et al. A comparison of rate control and rhythm control in patients with atrial fibrillation. N Engl J Med 2002;347:1825-1833. 
21. Packer DL, et al. Effect of Catheter Ablation vs Antiarrhythmic Drug Therapy on Mortality, Stroke, Bleeding, and Cardiac Arrest Among Patients With Atrial Fibrillation: The CABANA Randomized Clinical Trial. JAMA 2019;321:1261-1274.

22. Packer DL, et al. Cryoballoon ablation of pulmonary veins for paroxysmal atrial fibrillation: first results of the North American Arctic Front (STOP AF) pivotal trial. J Am Coll Cardiol 2013;61:1713-1723.

23. Wilber DJ, et al. Comparison of antiarrhythmic drug therapy and radiofrequency catheter ablation in patients with paroxysmal atrial fibrillation: a randomized controlled trial. JAMA 2010;303:333-340.

24. Calkins H, et al. $2017 \mathrm{HRS} / \mathrm{EHRA} / \mathrm{ECAS} / \mathrm{APHRS} /$ SOLAECE expert consensus statement on catheter and surgical ablation of atrial fibrillation: Executive summary. Europace 2018;20:157208.

25. Wazni OM, et al. Radiofrequency ablation vs antiarrhythmic drugs as first-line treatment of symptomatic atrial fibrillation: a randomized trial. JAMA 2005;293:2634-2640.

26. Cosedis Nielsen J, et al. Radiofrequency ablation as initial therapy in paroxysmal atrial fibrillation. N Engl J Med 2012;367:1587-1595.

27. Morillo CA, et al. Radiofrequency ablation vs antiarrhythmic drugs as first-line treatment of paroxysmal atrial fibrillation (RAAFT-2): a randomized trial. JAMA 2014;311:692-700.

28. Hakalahti A, et al. Radiofrequency ablation vs antiarrhythmic drug therapy as first line treatment of symptomatic atrial fibrillation: systematic review and meta-analysis. Europace 2015;17:370 378.

29. Ouyang F, et al. Complete isolation of left atrium surrounding the pulmonary veins: new insights from the double-Lasso technique in paroxysmal atrial fibrillation. Circulation 2004;110:20902096.

30. Verma A, et al. Approaches to catheter ablation for persistent atrial fibrillation. N Engl J Med. 2015;372:1812-1822.

31. Scherr D, et al. Five-year outcome of catheter ablation of persistent atrial fibrillation using termination of atrial fibrillation as a procedural endpoint. Circ Arrhythm Electrophysiol 2015; 8:18-24.

32. Rolf $\mathrm{S}$, et al. Tailored atrial substrate modification based on low-voltage areas in catheter ablation of atrial fibrillation. Circ Arrhythm Electrophysiol 2014;7:825-833.

33. Sapp JL, et al. Ventricular tachycardia ablation versus escalation of antiarrhythmic drugs. N Engl J Med 2016;375:111-121.

34. Stevenson WG, et al; Multicenter Thermocool VT Ablation Trial Investigators. Irrigated radiofrequency catheter ablation guided by electroanatomic mapping for recurrent ventricular tachycardia after myocardial infarction: the multicenter thermocool ventricular tachycardia ablation trial. Circulation 2008;118:2773-2782.

35. Tanner $\mathrm{H}$, et al. Catheter ablation of recurrent scar-related ventricular tachycardia using electroanatomical mapping and irrigated ablation technology: results of the prospective multicenter Euro-VT-study. J Cardiovasc Electrophysiol 2010;21:47-53.

36. Marchlinski FE, et al. Long-term success of irrigated radiofrequency catheter ablation of sustained ventricular tachycardia: post-approval THERMOCOOL VT trial. J Am Coll Cardiol 2016;67:674-683.

37. Cronin EM, et al; ESC Scientific Document Group. 2019 HRS/EHRA/APHRS/LAHRS expert consensus statement on catheter ablation of ventricular arrhythmias. Europace 2019;21:11431144.

38. Kumar S, et al. Long-term outcomes after catheter ablation of ventricular tachycardia in patients with and without structural heart disease. Heart Rhythm 2016;13:1957-1963. 
39. Duytschaever M, et al. Reproducibility and acute efficacy of a standardized approach to isolate the pulmonary veins: results from multicenter VISTAX study. European Heart Journal 2018;39(suppl_1):ehy566.P6227 (abstract). 


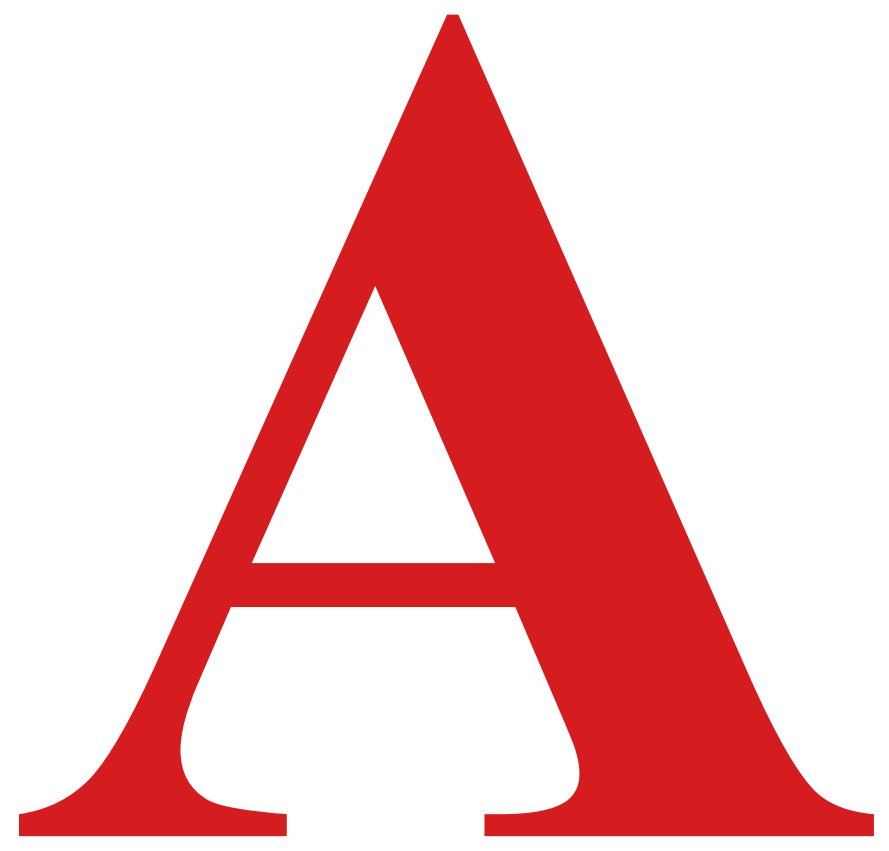


AKNOWLEDGEMENTS / DANKWOORD 
Dit doctoraat was een onmogelijke opdracht geweest zonder de steun en hulp van mijn promotoren, collega's, familie en vrienden.

In de eerste plaats gaat mijn dank uit naar mijn beide promotoren, professor Harry Crijns en professor Mattias Duytschaever. Jullie beiden hebben mij de kans gegeven dit doctoraat te verwezenlijken tijdens mijn bijkomende specialisatie en beginnende professionele activiteit als cardioloogelektrofysioloog. Mijn oprechte dank voor jullie tomeloze inzet, opleiding, begeleiding, motivatie en aanzet tot volharding die geleid hebben tot het bereiken van mijn vooropgestelde professionele, wetenschappelijke en persoonlijke doelstellingen. Mattias, bedankt om mijn mentor te zijn, en voor al je steun en inspiratie. Onze samenwerking is een van mijn meest waardevolle ervaringen.

Secondly, I especially would like to thank Milad El Haddad, who had an incredibly instrumental role throughout my thesis, but also during my additional training in Bruges and in my current scientific work. Your work spirit, attention to detail, kindness and modesty are truly to be admired. More importantly, thank you for also becoming a friend.

Mijn verdere dank gaat uit naar alle overige stafleden met wie onze nauwe samenwerking tot de realisatie van deze thesis geleid heeft: prof. Sébastien Knecht, prof. René Tavernier, dr. Yves Vandekerckhove, prof. Pierre Jaïs, dr. Nicolas Derval, prof. Mélèze Hocini, prof. Michel Haïssaguerre en prof. Andrea Sarkozy. Aside from our scientific collaboration, each of you had a fundamental role in my development as an interventional electrophysiologist, for which I will be eternally grateful.

Further, I would like to thank all national and international co-fellows I had the privilege of working with during my three-year clinical and scientific fellowship period and who have become unique friends for life: Ruan, Manav, Philippe, Rajin, Joël, Tom, Jan, Teresa, Alexandre, Antonio, Masateru, Konstantinos, Ghassen, Ruairidh, Nate, Takeshi, Grégoire and Claire. All different characters and personalities, but all with endless humour and tons of intelligence and inspiration. You were my allies during both good and bad times, what made the whole experience and enrichment for life. I will treasure these memories forever. Jan and Masateru, thank you for always raising the bar, both clinically and scientifically. Antonio, thank you for turning our society of Bordeaux fellows into a family.

A special mention goes out to my co-participants in the DASCAM programme (batch 1). Meeting, working and discussing with young colleagues from all over Europe (and beyond) was again an incredibly enriching experience. Particular thanks go out to Sotiris, for your ubiquitous kindness and your assistance in the final stages of my thesis. 
Dank aan de leden van de beoordelingscommissie voor het reviewen van mijn thesis en het bijwonen van de openbare verdediging.

Graag wil ik alle collega's van het AZ Sint-Jan Brugge, Hôpital Haut-Lévêque-CHU Bordeaux, IHU Liryc Bordeaux, Clinique Saint-Jean Bruxelles en Hartcentrum ZNA Antwerpen bedanken voor de fijne samenwerking gedurende de voorbije 5 jaren. Bijzondere dank aan prof. Yves De Greef, prof. Bruno Schwagten en mijn overige collega's van Hartcentrum ZNA om mij op te nemen in hun elektrofysiologisch team, en mij zo de kans te geven - mede door de bevindingen in deze thesis in de klinische praktijkvoering om te zetten - om samen de beste zorg aan onze hartpatiënten te kunnen bieden.

Verder was uiteraard de steun van mijn familie, partner en vrienden onontbeerlijk. Bedankt voor jullie bijstand, liefde en vriendschap, en voor de nodige ontspanning vóór, tijdens en na dit doctoraat.

Mijn diepste dank gaan uit naar mijn prachtige ouders en zus. Jullie hebben mij steeds alle kansen gegeven en gesteund in het behalen van mijn doelstellingen. Bedankt om altijd in mij te geloven. Deze thesis is aan jullie opgedragen. 


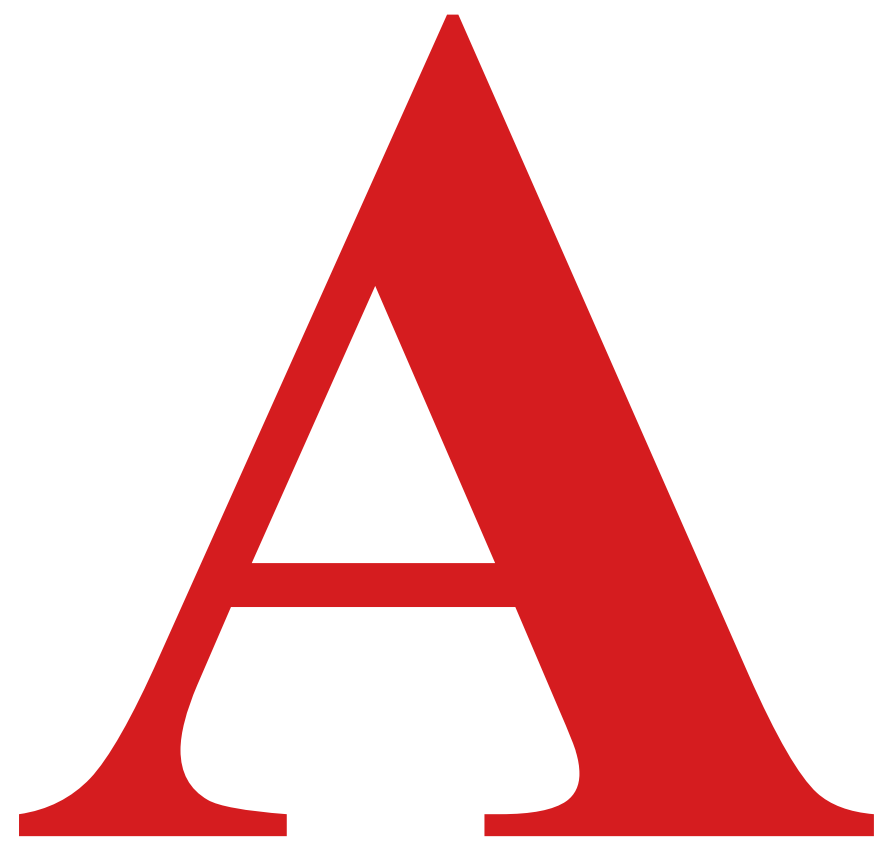




\title{
CURRICULUM VITAE
}

\author{
LIST OF PUBLICATIONS
}




\section{CURRICULUM VITAE}

Michael Wolf was born on January $13^{\text {th }} 1983$ in Lier (Antwerp, Belgium). He obtained his high school diploma at Onze-Lieve-Vrouw-van-Lourdescollege Edegem in 2001. He became Doctor in Medicine summa cum laude at the University of Antwerp in 2008. After cardiology fellowships in ZNA Middelheim and the Antwerp University Hospital, he became a certified cardiologist in 2014. Thereafter, he undertook additional fellowship training in interventional cardiac electrophysiology with professor Mattias Duytschaever in AZ Sint-Jan Bruges (2014-2016) and a fellowship in advanced electrophysiology and ablation of complex arrhythmias with professor Michel Haïssaguerre at Hôpital Cardiologique du Haut-Lévêque - CHU de Bordeaux (2016-2017). During these fellowships, he obtained full accreditation by the European Heart Rhythm Association in interventional electrophysiology and cardiac device therapy (pacing, ICDs and cardiac resynchronization). In addition, he has obtained certification in sports medicine, diving medicine and intensive care medicine. In 2018, he graduated with the first batch of the DASCAM programme (the Diploma of Advanced Studies in Cardiac Arrhythmia Management), at Maastricht University and the European Heart Academy (European Society of Cardiology). As from June 2018, he joined the staff of the Hartcentrum ZNA in Antwerp as a full-time cardiologist-electrophysiologist.

Michael's particular clinical and research interests in the treatment of complex cardiac arrhythmias, such as atrial fibrillation, atrial tachycardia and ventricular tachycardia have resulted in over 50 publications in both national and international peer-reviewed scientific journals, contribution to a book chapter, and several presentations at national and international congresses. In 2016 and 2017, he received the award for best poster presentation at the annual Belgian Heart Rhythm Meeting, and in 2018 the Young EP Best Clinical Case award at the European Heart Rhythm Association congress. 


\section{LIST OF PUBLICATIONS}

1. Wolf Michael, Wibail A, De Jonghe P, de Barsy C, Van Houwe E, Cras P, Parizel P. Delayed hemorrhagic cerebral metastases after atrial myxoma resection: Report of two cases and review of the literature. European Journal of Radiology 2008; 66: 75-79.

2. Wolf Michael, Drubbel V, Hendriks J, Van Schil P. Red blood cell accumulation in a rat model of pulmonary ischemia/reperfusion injury. Journal of Cardiovascular Surgery 2009; 50: 351-356.

3. Wolf Michael, Cnossen W, Rutsaert R, Kersschot I. Chronic total occlusion of the left main coronary artery in a 31-year-old man. Chronische totale hoofdstamocclusie bij een 31-jarige man. (Article in Dutch). Tijdschrift voor Geneeskunde 2011; 67: 141-145.

4. Wolf Michael, Van Offel J, Van de Velde A, Stevens W, De Clerck L. Multicentric plasma cell variant Castleman's disease presenting with cutaneous vasculitis and pulmonary parenchymal involvement in a patient with ankylosing spondylitis: case report and review of the literature. Acta Clinica Belgica 2011; 66: 305-310.

5. Wolf Michael, Heuten H, De Swaef A, de Falleur M, Verpooten G. The evolution of hypertension treatment in Belgium, a pharmacoepidemiological study. Acta Cardiologica 2012; 67: 147-152.

6. Wolf Michael, Vermeersch P, Van Reet B, Van den Branden F. Early Surgical Repair of an Acute Post-Infarction Left Ventricular Pseudoaneurysm Complicated by Second Aneurysm Formation. Acta Cardiologica 2012; 67: 723-726.

7. Wolf Michael. ESC congress 2013: clinical trial update Hot Line II: Updates on intervention and devices. Tijdschrift voor Cardiologie 2013; 25: 372-374.

8. Wolf Michael, Scott B. Left-sided High-Flow Arteriovenous Hemodialysis Fistula Combined with a Persistent Left Superior Vena Cava Causing Coronary Sinus Dilatation. Seminars in Dialysis 2013; 26: E13-E16.

9. Wolf Michael, Van den Brande J, Rodrigus I, Paelinck B. Giant Primary Right Ventricular Synovial Sarcoma. European Heart Journal 2014; 35: 2509.

10. Wolf Michael. $9^{\text {th }}$ Belgian Hearth Rhythm Meeting, 2015: ICD versus ablation as first treatment modality for VT/VF. Tijdschrift voor Cardiologie 2015; 27: 457-462.

11. Wolf Michael. PSCK9i: translating LDL-C lowering into plaque regression and CV event reduction? Tijdschrift voor Cardiologie 2016; 28: 377-381.

12. Wolf Michael, Saenen J, Bories W, Miljoen H, Nullens S, Vrints C, Sarkozy A. Superior Efficacy of Pulmonary Vein Isolation with Online Contact Force Measurement Persists after the Learning Period: a Prospective Case Control Study. Journal of Interventional Cardiac Electrophysiology 2015; 4: 287-296.

13. Sohal M, Choudhury R, Taghji P, Louw R, Wolf Michael, Fedida J, Vandekerckhove Y, Tavernier R, Duytschaever M, Knecht S. Is Mapping of Complex Fractionated Electrograms Obsolete? Arrhythmia \& Electrophysiology Review 2015; 4: 109-115.

14. Wolf Michael. Hotline: Heart Failure and Innovative Approaches. Tijdschrift voor Cardiologie 2016; 28: 345-349.

15. Vandekerckhove J, Wolf Michael. Atrial Fibrillation and Stroke Risk Management. Tijdschrift voor Cardiologie 2016; 28: 103-107.

16. Wolf Michael, El Haddad M, Taghji T, Vandekerckhove Y, Tavernier R, Duytschaever M, Knecht S. Incidense of "First Pass" Block after 'CLOSE' guided Linear Ablation at the Roof and Mitral Isthmus. Heart Rhythm 2016; 13: S427-S514.

17. Wolf Michael. Ablation of Persistent AF: State of the Art. Tijdschrift voor Cardiologie 2016; 28: 445-453.

18. Wolf Michael. Late Breaking Clinical Trials, ESC Congress 2017. Tijdschrift voor Cardiologie 2017; 29: 399-403.

19. Tavernier R, Wolf Michael, Vikas K, Phlips T, Huys R, Taghji P, Louw R, Van Hoeyweghen R, Vandekerckhove Y, Knecht S, Duytschaever M. Screening for Atrial Fibrillation in Hospitalised Geriatric Patients. Heart 2018; 104: 588-593. 
20. El Haddad M, Taghji P, Phlips T, Wolf Michael, Demolder A, Choudhury R, Knecht S, Vandekerckhove Y, Tavernier R, Nakagawa H, Duytschaever M. Determinants of Acute and Late Pulmonary Vein Reconnection in Contact Force-guided Pulmonary Vein Isolation: Identifying the Weakest Link in the Ablation Chain. Circulation: Arrhythmia \& Electrophysiology 2017; 10:pii: e004867.

21. Taghji P, El Haddad M, Phlips T, Wolf Michael, Knecht S, Vandekerckhove Y, Tavernier R, Nakagawa H, Duytschaever M. Evaluation of a strategy to enclose the pulmonary veins with contiguous and optimized RF lesions in paroxysmal atrial fibrillation: a pilot study. JACC: Clinical Electrophysiology 2018; 4: 99-108.

22. Phlips T, Taghji P, El Haddad M, Wolf Michael, Knecht S, Vandekerckhove Y, Tavernier R, Duytschaever M. Improving procedural and 1-year outcome after contact force guided PVI: the role of interlesion distance, ablation index and contact force variability in the 'CLOSE'- protocol. Europace 2018; 20: f419-f427.

23. Wolf Michael, El Haddad M, Fedida J, Taghji P, Van Beeumen K, Strisciuglio T, De Pooter J, Lepièce C, Vandekerckhove Y, Tavernier R, Duytschaever M, Knecht S. Evaluation of left atrial linear ablation using contiguous and optimized radiofrequency lesions: the ALINE study. Europace 2018; 20: f401-f409.

24. De Pooter J, El Haddad M, Wolf Michael, Phlips T, Van Heuverswyn F, Timmers L, Tavernier R, Knecht S, Vandekerckove Y, Duytschaever M. Clinical assessment and comparison of annotation algorithms in high-density mapping of regular atrial tachycardias. Journal of Cardiovascular Electrophysiology 2018; 29: 177-185.

25. Fedida J, Strisciuglio T, Sohal M, Wolf Michael, Van Beeumen K, Neyrinck A, Taghji P, Lepièce C, Almorad A, Vandekerkchove Y, Tavernier R, Duytschaever M, Knecht S. Efficacy of advanced pace mapping technology for idiopathic premature ventricular complexes ablation. Journal of Interventional Cardiovascular Electrophysiology 2018; 51: 271-277.

26. Coeman M, El Haddad M, Wolf Michael, Choudhury R, Vandekerchove Y, Knecht S, Tavernier $\mathrm{R}$, Duytschaever M. 'CLOSE'-guided pulmonary vein isolation and changes in local bipolar and unipolar atrial electograms: observations from the EP lab. Journal of Atrial Fibrillation 2018;10:1794.

27. Wolf Michael, Sacher F, Cochet H, Kitamura T, Takigawa M, Yamashita S, Vlachos K, Cheniti G, Frontera A, Martin R, Thompson N, Massoullié G, Lam A, Martin C, Collot F, Duchateau J, Denis A, Pambrun T, Derval N, Hocini M, Haïssaguerre M, Jaïs P. Long-term Outcome of Substrate Modification in Ablation of Post-Myocardial Infarction Ventricular Tachycardia. Circulation: Arrhythmia \& Electrophysiology 2018; 11(2): e005635.

28. Frontera A, Cheniti G, Martin C, Takigawa M, Duchateau J, Puyo S, Martin R, Thompson N, Kitamura T, Vlachos K, Wolf Michael, Al-Jefairi N, Massouillé G, Pambrun R, Denis A, Sacher F, Derval N, Dubois R, Jaïs P, Hocini M, Haïssaguerre M. Frontiers in non-invasive cardiac mapping: future implications for arrhythmia treatment. Minerva Cardioangiol 2018; 66: 75-82.

29. Takigawa M, Derval N, Frontera A, Martin R, Yamashita S, Cheniti G, Vlachos K, Thompson N, Kitamura T, Wolf Michael, Massoullie G, Martin C, Al-Jefairi N, Amraoui S, Duchateau J, Klotz N, Pambrun T, Denis A, Sacher F, Cochet H, Meleze H, Haissaguierre M, Jais P. Revisiting anatomical macroreentrant tachycardia after atrial fibrillation ablation using ultrahighresolution mapping: Implications for ablation. Heart Rhythm 2018; 15: 326-333.

30. Frontera A, Takigawa M, Martin R, Thompson N, Cheniti G, Massouillé G, Duchateau J, Wielandts JY, Teijera E, Kitamura T, Wolf Michael, Al-Jefairi N, Vlachos K, Yamashita S, Amraoui S, Denis A, Hocini M, Cochet H, Sacher F, Jaïs P, Haïssaguerre M, Derval N. Electrogram signature of specific activation patterns: analysis of atrial tachycardias at high-density endocardial mapping. Heart Rhythm 2018; 15: 28-37.

31. Martin R, Maury P, Bisceglia C, Wong T, Estner H, Meyer C, Dallet C, Martin C, Shi R, Takigawa M, Rollin A, Frontera A, Thompson N, Kitamura T, Vlachos K, Wolf Michael, Cheniti G, Duchateau J, Massoullié G, Pambrun T, Denis A, Derval N, Hocini M, Della Bella P, Haissaguerre M, Jais P, Dubois R, Sacher F. Characteristics of scar related ventricular tachycardia circuits using ultra high-density mapping. Circulation: Arrhythmia \& Electrophysiology 2018;11(10):e006569. 
32. Takigawa M, Relan J, Martin R, Kim S, Kitamura T, Frontera A, Cheniti G, Vlachos K, , Massoullié G, Martin C, Thompson N, Wolf Michael, Bourier F, Lam A, Duchateau J, Klotz N, Pambrun T, Denis A, Derval N, Magat J, Naulin J, Merle M, Collot F, Quesson B, Cochet H, Hocini M, Haïssaguerre M, Sacher F, Jaïs P. Effect of Bipolar Electrode Orientation on Local Electogram Properties. Heart Rhythm 2018; 15: 1853-1861.

33. Kitamura T, Martin R, Denis A, Takigawa M, Duparc A, Rollin A, Frontera A, Thompson N, Massoullié G, Cheniti G, Wolf Michael, Vlachos K, Martin CA, Al Jefairi N, Duchateau J, Klotz N, Pambrun T, Sacher F, Cochet H, Hocini M, Haïssaguerre M, Maury P, Jaïs P, Derval N. Characteristics of single-loop macroreentrant biatrial tachycardia diagnosed by ultrahighresolution mapping system. Circulation: Arrhythmia \& Electrophysiology 2018; 11(2): e005558.

34. Takigawa M, Derval N, Maury P, Martin R, Miyazaki S, Yamashita S, Frontera A, Vlachos K, Kitamura T, Cheniti G, Massoullié G, Thompson N, Martin C, Wolf Michael, Pillois X, Duchateau J, Klotz N, Duparc A, Rollin A, Pambrun T, Sacher F, Cochet H, Hocini M, Haïssaguerre M, Jais P. Comprehensive Multicenter Study of the Common Isthmus in Post-Atrial Fibrillation Ablation Multiple-Loop Atrial Tachycardia. Circulation: Arrhythmia \& Electrophysiology 2018; 11(6): e006019.

35. Gillis K, Wolf Michael. ESC Congress 2018 München: Hot Line session. Tijdschrift voor Cardiologie 2018; 30: 15-20.

36. Tijskens M, Wolf Michael. ESC Congress 2018 München: Hot Line session 1. Tijdschrift voor Cardiologie 2018; 30: 7-13.

37. Wolf Michael. Signaalinterpretatie en ablatie bij persistente atriale fibrillatie. Tijdschrift voor Cardiologie 2018; 30: 45-49.

38. Takigawa M, Derval N, Martin C, Vlachos K, Denis A, Kitamura T, Cheniti G, Bourier F, Lam A, Martin R, Frontera A, Thompson N, Massoullié G, Wolf Michael, Duchateau J, Klotz N, Pambrun T, Sacher F, Cochet H, Hocini M, Haissaguerre M, Jais P. A simple mechanism underlying the behavior of reentrant atrial tachycardia during ablation. Heart Rhythm 2019; 16 : 553-561.

39. Martin C, Takigawa M, Martin R, Maury P, Meyer C, Wong T, Shi R, Gajendragadkar P, Frontera A, Cheniti G, Thompson N, Kitamura T, Vlachos K, Wolf Michael, Bourier F, Lam A, Duchateau J, Massoulié G, Pambrun T, Denis A, Derval N, Hocini Mélèze, Haïssaguerre M, Jais P, Sacher F. Use of novel electrogram 'Lumipoint' algorithm to detect critical isthmus and abnormal potentials for ablation in ventricular tachycardia. JACC: Clinical Electrophysiology 2019; 5: 470-479.

40. Takigawa M, Duchateau J, Sacher F, Martin R, Vlachos K, Kitamura T, Sermesant M, Cedilnik N, Cheniti G, Frontera A, Thompson N, Martin C, Massoullie G, Bourier F, Lam A, Wolf Michael, Escande W, André C, Pambrun T, Denis A, Derval N, Hocini M, Haissaguerre M, Cochet H, Jais P. Are wall thickness channels defined by computed tomography predictive of isthmuses of postinfarction ventricular tachycardia? Heart Rhythm 2019; 16: 1661-1668.

41. Takigawa M, Martin C, Derval N, Denis A, Vlachos K, Kitamura T, Frontera A, Martin R, Cheniti G, Lam A, Bourier F, Thompson N, Wolf Michael, Massoullie G, Escande W, Andre C, Zeng LJ, Nakatani Y, Roux JR, Duchateau J, Pambrun T, Sacher F, Cochet H, Hocini M, Haissaguerre M, Jais P. Insights from atrial surface activation throughout atrial tachycardia cycle length: A new mapping tool. Heart Rhythm 2019; 16: 1652-1660.

42. Takigawa M, Relan J, Kitamura T, Martin C, Kim S, Martin R, Cheniti G, Vlachos K, Massoullié G, Frontera A, Thompson N, Wolf Michael, Bourier F, Lam A, Duchateau J, Pambrun T, Denis A, Derval N, Pillois X, Magat J, Naulin J, Merle M, Collot F, Quesson B, Cochet H, Hocini M, Haïssaguerre M, Sacher F, Jaïs P. Impact of spacing and orientation on the scar threshold in a high-density grid catheter. Circulation: Arrhythmia \& Electrophysiology 2019; 12(9): e007158.

43. Martin CA, Yue A, Martin R, Claridge S, Sawhney V, Maury P, Lowe M, Combes N, Heck P, Begley D, Fynn S, Snowdon R, Seller N, Murray S, Shepherd E, Ezzat V, Gajendragadkar PR, Honarbakhsh S, Takigawa M, Cheniti G, Frontera A, Thompson N, Massouillie G, Kitamura T, Wolf Michael, Duchateau J, Klotz N, Vlachos K, Bourier F, Lam A, Pambrun T, Denis A, Sacher F, Cochet H, Jais P, Hocini M, Haissaguerre M, Iriart X, Thambo JB, Derval N. JACC Clinical Electrophysiology 2019; 5: 1459-1472. 
44. Martin C, Martin R, Maury P, Meyer C, Wong T, Dallet C, Shi R, Gajendragadkar P, Takigawa M, Frontera A, Cheniti G, Thompson N, Kitamura T, Vlachos K, Wolf Michael, Bourier F, Lam A, Duchateau J, Massoullié G, Pambrun T, Denis A, Derval N, Dubois R, Hocini M, Haïssaguerre M, Jaïs P, Sacher F. Effect of activation wavefront on electrogram characteristics during ventricular tachycardia ablation. Circulation: Arrhythmia \& Electrophysiology 2019; 12(6): e007293.

45. Vlachos K, Denis A, Takigawa M, Kitamura T, Martin CA, Frontera A, Martin R, Bazoukis G, Bourier F, Cheniti G, Duchateau J, Thompson N, Massoullie G, Lam A, Wolf Michael, Escande W, Klotz N, Pambrun T, Sacher F, Hocini M, Haissaguerre M, Jais P, Derval N. The role of Marshall bundle epicardial connections in atrial tachycardias after atrial fibrillation ablation. Heart Rhythm 2019; 16: 1341-1347.

46. Takigawa M, Relan J, Martin R, Kim S, Kitamura T, Cheniti G, Vlachos K, Pillois X, Frontera A, Massoullié G, Thompson N, Martin CA, Bourier F, Lam A, Wolf Michael, Duchateau J, Klotz N, Pambrun T, Denis A, Derval N, Magat J, Naulin J, Merle M, Collot F, Quesson B, Cochet H, Hocini M, Haïssaguerre M, Sacher F, Jaïs P. Detailed Analysis of the Relation Between Bipolar Electrode Spacing and Far- and Near-Field Electrograms. JACC Clin Electrophysiol 2019; 5: 6677.

47. Cheniti G, Hocini M, Thompson N, Denis A, Duchateau J, Martin R, Takigawa M, Frontera A, Massoullié G, Vlachos K, Kitamura T, Wolf Michael, Derval N, Sacher F, Jaïs P, Dubois R, Haïssaguerre M. (2019) Mapping and Ablation of Idiopathic Ventricular Fibrillation. In M.E. Josephson (Ed.), Cardiac Mapping, Fifth Edition (pp. 922-933). Oxford, UK: John Wiley \& Sons Ltd.

48. Wolf Michael, El Haddad M, De Wilde V, Phlips T, De Pooter J, Almorad A, Strisciuglio T, Vandekerckhove Y, Tavernier R, Crijns HJ, Knecht S, Duytschaever M. Endoscopic evaluation of the esophagus after catheter ablation of atrial fibrillation using contiguous and optimized radiofrequency applications. Heart Rhythm 2019; 16: 1013-1020.

49. De Pooter J, Strisciuglio T, El Haddad M, Wolf Michael, Phlips T, Vandekerckhove Y, Tavernier R, Knecht S, Duytschaever M. Pulmonary Vein Reconnection No Longer Occurs in the Majority of Patients After a Single Pulmonary Vein Isolation Procedure. JACC: Clinical Electrophysiology 2019; 5: 295-305.

50. Galloo X, Abugattas JP, Tijskens M, Dendale P, Varnavas V, Wolf Michael, De Cocker J, Schwagten B, Sieira J, Ströker E, Chierchia JB, de Asmundis C, De Greef Y. Impact of cryoballoon-guided pulmonary vein isolation on non-invasive autonomic tests in paroxysmal atrial fibrillation. Indian Pacing Electrophysiol Journal 2019; 19: 171-177.

51. Abugattas JP, Schwagten B, De Cocker J, Coutiño HE, Salghetti F, Varnavas V, Ströker E, Sieira J, Wolf Michael, Brugada P, de Asmundis C, Chierchia GB, De Greef Y. Predictors of long-term outcome in patients undergoing a first repeat ablation consisting solely of re-isolation of reconnected pulmonary veins. Journal of Atrial Fibrillation 2019; 11(5): 2114.

52. Wolf Michael, Tavernier R, Zeidan Z, El Haddad M, Vandekerckhove Y, De Pooter J, Phlips T, Strisciuglio T, Almorad A, Kyriakopoulou M, Lycke M, Duytschaever M, Knecht S. Identification of repetitive atrial activation patterns in persistent atrial fibrillation by direct contact high-density electrogram mapping. Journal of Cardiovascular Electrophysiology 2019; 30: 2704-2712.

53. Duytschaever M, De Pooter J, Demolder A, El Haddad M, Phlips T, Strisciuglio T, Debonnaire P, Wolf Michael, Vandekerckhove Y, Knecht S, Tavernier R. Long-term impact of catheter ablation on arrhythmia burden in low-risk patients with paroxysmal atrial fibrillation: the CLOSE to CURE study. Heart Rhythm 2020; 17: 535-543.

54. Takigawa M, Derval N, Martin CA, Vlachos K, Denis A, Nakatani Y, Kitamura T, Cheniti G, Bourier F, Lam A, Martin R Frontera A, Thompson N, Massoullié G, Wolf Michael, Escande W, André C, Zeng LJ, Roux JR, Duchateau J, Pambrun T, Sacher F, Cochet H, Hocini M, Haïssaguerre M, Jaïs P. Mechanism of Recurrence of Atrial Tachycardia: Comparison Between First Versus Redo Procedures in a High-Resolution Mapping System. Circulation: Arrhythmia \& Electrophysiology; 2020; 13(1): e007273. 
55. Kostopoulou A, Zeljko Hrvojka M, Bogossian H, Ciudin R, Costa F, Heijman J, Kochhauser S, Manola S, Scherr D, Sohal M, Wakili R, Wolf Michael, Irfan G; on the behalf of the DAS-CAM participants-2017-2018. Atrial fibrillation-related stroke in women: evidence and inequalities in epidemiology, mechanisms, clinical presentation and management. Clinical Cardiology 2020; 43:14-23.

56. Strisciuglio T, El Haddad M, Debonnaire P, De Pooter J, Demolder A, Wolf Michael, Phlips T, Kyriakopoulou M, Almorad A, Knecht S, Tavernier R, Vandekerckhove Y, Duytschaever M. Paroxysmal AF with high vs low arrhythmia burden: atrial remodeling and ablation outcome. Europace 2020 (accepted for publication, in press)

57. Takigawa M, Vlachos K, Martin C, Bourier F, Denis A, Kitamura T, Cheniti G, Lam A, Martin R, Frontera A, Thompson N, Massoullié G, Wolf Michael, Escande W, André C, Zeng L, Nakatani Y, Nakashima T, Ramirez D, Duchateau J, Pambrun T, Sacher F, Cochet H, Hocini M, Haïssaguerre M, Jaïs P, Derval, N. Does epicardial Marshall Vein Ethanol infusion add benefit over conventional endocardial radiofrequency ablation in perimitral flutter? (submitted) 


\section{ORAL PRESENTATIONS AT NATIONAL AND INTERNATIONAL CONFERENCES}

1. The evolution of hypertension treatment in Belgium. 2011 scientific meeting of the Belgian Hypertension Committee, Liège (8/10/2011)

2. PCI and atrial fibrillation: combination antithrombotic treatment in practice. Cardiology seminar update atrial fibrillation, Antwerp University Hospital (9/10/2012)

3. Paroxysmal AF ablation: Substrate for PV reconnection. State of the art in electrical management of cardiac diseases - Alliance Program, Bordeaux (7-8/3/2017)

4. Comparison of procedural endpoints for ablation of post-myocardial infarction ventricular tachycardia. EHRA Europace - Cardiostim 2017, Vienna (18-22/6/2017)

5. Challenges with post-ablation flutter. $17^{\text {th }}$ Atrial Fibrillation Symposium $-10^{\text {th }}$ Practical Session, Prague (7-9/2/2018)

6. Vein-of-Marshall mediated complex atrial tachycardia. EHRA congress, Barcelona (1820/3/2018) (Young EP Best Clinical Case award)

\section{ABSTRACTS AT NATIONAL AND INTERNATIONAL CONGRESSES}

1. Wolf Michael, et al. Unexpected autoimmune response caused by Castleman's disease in a patient with spondylarthropathy. $14^{\text {th }}$ Belgian Congress on Reumatology, Luxemburg (22-24/9/2010)

2. Wolf Michael, et al. The evolution of hypertension treatment in Belgium. EuroDURG (European Drug Utilization Research Group): joint educational course and scientific meeting, Antwerp $(30 / 11-3 / 12 / 2011)$

3. Wolf Michael, et al. Percutaneous left atrial appendage closure with Amplatzer cardiac plug for stroke prevention in atrial fibrillation patients with contraindication for oral anticoagulation: feasibility, safety and initial results. Belgian Society of Cardiology: $31^{s t}$ Annual Scientific Meeting, Brussels (2-3/2/2012)

4. Wolf Michael, et al. Superior efficacy of pulmonary vein isolation with online contact force measurement persists after the learning period: a prospective case control study. EHRA Europace - Cardiostim congress 2015, Milan (21-24/6/2015); ESC congress 2015, London (29/8-2/9/2015)

5. Wolf Michael, et al. Incidence of "First Pass" Block after 'CLOSE' guided Linear Ablation at the Roof and Mitral Isthmus. Heart Rhythm, 37 th annual scientific sessions, San Francisco (47/5/2016); $10^{\text {th }}$ Belgian Heart Rhythm Meeting, Brussels (6-7/10/2016) (moderated poster - Best Poster award)

6. Wolf Michael, et al. Management of Electrical Storm in Idiopathic Ventricular Fibrillation. 8th Euro VT/VF Meeting 2016, Berlin (2-3/12/2016)

7. Coeman M, et al. Time Course of Changes in Unipolar and Bipolar Electrogram Amplitude and Morphology during Contact Force-guided Pulmonary Vein Isolation. Heart Rhythm, $38^{\text {th }}$ annual scientific sessions, Chicago (10-13/5/2017)

8. Kitamura $\mathrm{T}$, et al. Validation of body surface mapping of atrial tachycardia by novel high-density mapping. Heart Rhythm, $38^{\text {th }}$ annual scientific sessions, Chicago (10-13/5/2017)

9. Haïssaguerre $\mathrm{M}$, et al. Characterization of drivers maintaining ventricular fibrillation in early repolarization syndrome. Heart Rhythm, $38^{\text {th }}$ annual scientific sessions, Chicago (10-13/5/2017)

10. Haïssaguerre $M$, et al. Subclinical arrhythmogenic substrate as a cause of unexplained cardiac death. Heart Rhythm, $38^{\text {th }}$ annual scientific sessions, Chicago (10-13/5/2017)

11. Derval N, et al. Anatomical and electrophysiological characteristics of complex atrial tachycardia: insight from high-resolution mapping. Heart Rhythm, $38^{\text {th }}$ annual scientific sessions, Chicago $(10-13 / 5 / 2017)$

12. Martin R, et al. High-density mapping of conduction barriers in ventricular tachycardia. Heart Rhythm, $38^{\text {th }}$ annual scientific sessions, Chicago (10-13/5/2017) 
13. Martin R, et al. Surface ECG confirmation of isthmus block in Tetralogy of Fallot VT ablation. Heart Rhythm, $38^{\text {th }}$ annual scientific sessions, Chicago (10-13/5/2017)

14. Denis A, et al. High density mapping of atrial reentrant tachycardia in patients without previous AF catheter ablation or cardiac surgery. Heart Rhythm, $38^{\text {th }}$ annual scientific sessions, Chicago $(10-13 / 5 / 2017)$

15. Cheniti G, et al. The right ventricle underlies failed electrical cardioversion in human VF. Heart Rhythm, $38^{\text {th }}$ annual scientific sessions, Chicago (10-13/5/2017)

16. Cheniti G, et al. Spontaneous termination of induced ventricular fibrillation: dominant role of left ventricular drivers. Heart Rhythm, $38^{\text {th }}$ annual scientific sessions, Chicago (10-13/5/2017)

17. Frontera A, et al. Relationship between scar and atrial tachycardia mechanisms: insight from registered magnetic resonance and ultra-high density mapping system. Heart Rhythm, $38^{\text {th }}$ annual scientific sessions, Chicago (10-13/5/2017)

18. Frontera A, et al. EGM fractionation in apparently healthy tissue: time to redefine the voltage threshold for diseased atrium? Heart Rhythm, $38^{\text {th }}$ annual scientific sessions, Chicago (10$13 / 5 / 2017)$

19. Massoullié G, et al. High concentration of sodium-bicarbonate as treatment for ventricular fibrillation storm related to Brugada syndrome. Heart Rhythm, $38^{\text {th }}$ annual scientific sessions, Chicago (10-13/5/2017)

20. Fedida J, et al. Efficacy of advanced pace mapping technology for idiopathic PVC ablation. EHRA Europace - Cardiostim 2017, Vienna (18-22/6/2017)

21. Wolf Michael, et al. Pattern and timing of coronary sinus activation in complex atrial tachycardia. EHRA Europace - Cardiostim 2017, Vienna (18-22/6/2017)

22. Frontera A, et al. Characterization of slow conductions areas in scar-related atrial tachycardia. Insights from high-density mapping system. EHRA Europace - Cardiostim 2017, Vienna (1822/6/2017)

23. Frontera A, et al. Relationship of voltage and EGM duration at sites of fractionation during atrial tachycardias and paced rhythms. EHRA Europace - Cardiostim 2017, Vienna (18-22/6/2017)

24. Takigawa M, et al. Precise circuit of dual-loop reentry and the characteristics of common isthmus in post AF ablation left atrial tachycardia. EHRA Europace - Cardiostim 2017, Vienna (18$22 / 6 / 2017)$

25. Wolf Michael, et al. Long-term Outcome of LAVA Elimination in Ablation of Post-Myocardial Infarction Ventricular Tachycardia. Heart Rhythm, $38^{\text {th }}$ annual scientific sessions, Chicago (1013/05/2016); EHRA Europace - Cardiostim 2017, Vienna (18-22/06/2017) (moderated poster); $11^{\text {th }}$ Belgian Heart Rhythm Meeting, Brussels (5-6/10/2017) (moderated poster - Best Poster award)

26. Duytschaever M, et al. Paroxysmal AF burden measured by continuous monitoring before and after 'CLOSE-guided' PV isolation: preliminary results from the prospective patient-controlled CLOSE-TO-CURE study. Heart Rhythm, 39 ${ }^{\text {th }}$ annual scientific sessions, Boston (9-12/5/2018)

27. Martin C, et al. Multi-center study of ultra-high density activation mapping to aid isthmus identification of atrial tachycardias in congenital heart disease. Heart Rhythm, $39^{\text {th }}$ annual scientific sessions, Boston (9-12/5/2018)

28. Lam A, et al. Are recurrences of post-myocardial infarction ventricular tachycardia due to substrate progression or insufficient index ablation? A 4 years follow-up study. Heart Rhythm, $39^{\text {th }}$ annual scientific sessions, Boston (9-12/5/2018); EHRA - Europace, Barcelona (18-20/3/2018)

29. Kitamura $T$, et al. Does ventricular tachycardia ablation reduce ventricular fibrillation incidence? Heart Rhythm, $39^{\text {th }}$ annual scientific sessions, Boston (9-12/5/2018)

30. Cheniti G, et al. Fat deposition in patients with post-myocardial infarction ventricular tachycardia has distinctive electrophysiological characteristics. Heart Rhythm, $39^{\text {th }}$ annual scientific sessions, Boston $(9-12 / 5 / 2018)$

31. De Pooter J, et al. Predictors of long-term outcome in a large group of patients undergoing a first repeat ablation consisting solely of re-isolation of reconnected pulmonary veins. $12^{\text {th }}$ Belgian Heart Rhythm Meeting, Brussels (4-5/10/2018)

32. De Greef $Y$, et al. Influence of pulmonary vein isolation on autonomic function in patients with paroxysmal atrial fibrillation. $12^{\text {th }}$ Belgian Heart Rhythm Meeting, Brussels (4-5/10/2018) 
33. Strisciuglio $T$, et al. Clinical, echocardiographic and electro-anatomical determinants of the duration of paroxysmal AF episodes. $12^{\text {th }}$ Belgian Heart Rhythm Meeting, Brussels (4-5/10/2018)

34. Wolf Michael, et al. Echo-endoscopic evaluation of the esophagus after enclosing the pulmonary veins with contiguous and optimized RF lesions. $12^{\text {th }}$ Belgian Heart Rhythm Meeting, Brussels (45/10/2018) (moderated poster); EHRA Europace 2019, Lisbon (17-19/3/2019) (moderated poster); Heart Rhythm, $39^{\text {th }}$ annual scientific sessions, San Francisco (8-11/5/2019)

35. Abugattas JP, et al. Predictors of long-term outcome in patients undergoing a first repeat ablation for atrial fibrillation consisting solely of re-isolation of reconnected pulmonary veins. EHRA Europace 2019, Lisbon (17-19/3/2019) 


